\title{
WHOI $-75-8$
}

\author{
DESCRIPTIONS OF WHOI SEDIMENT CORES
}

\author{
Prepared by \\ the Staff of the \\ Sea Floor Samples Laboratory \\ Edited by \\ D. A. Johnson and A. H. Driscoll \\ WOODS HOLE OCEANOGRAPHIC INSTITUTION \\ Woods Hole, Massachusetts 02543
}

May 1975

\begin{abstract}
TECHNICAL REPORT
Prepared for the Office of Naval Research under Contract N00014-74-CO262; NR 083-004 and for the National Science Foundation Grant DES73-06463 (formerly GA-36698).

Reproduction in whole or in part is permitted for any purpose of the United States Govermment. In citing this manuscript in a bibliography, the reference should be followed by the phrase: UNPUBLISHED MANUSCRIPT.

Approved for public release; distribution unzimited.
\end{abstract}


VOLUME I

$\underline{\text { Page }}$

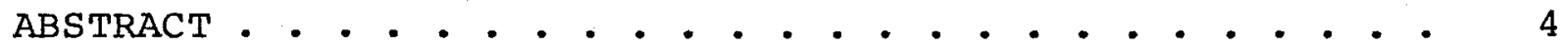

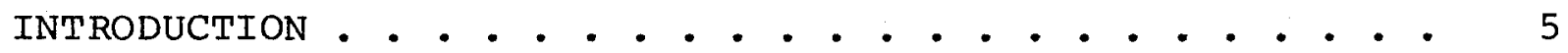

A. Scope and Format of this Report . . . . . . . . 5

B. Core Description Procedures . . . . . . . . . . 7

1. Visual description . . . . . . . . . . 7

2. Smear slide analysis... . . . . . . . 9

3. Designation of sediment type . . . . . . . 16

C. Core Biostratigraphy . . . . . . . . . . . . . . 17

D. Digitization of Geological Sample Data . . . . . 23

E. Procedures for Obtaining Samples and Core Data . . 27

ACKNOWLEDGMENTS • • • • • • • • • • • • • • • • . 30

COMPUTER IISTING OF CORES, ARRANGED BY MARSDEN SQUARES . . 33 Marsden Square Index Map . . . . . . . 34

DESCRIPTIONS OF WHOI SEDIMENT CORES • • • • • • • • • • • 71

R/V ATLANTIS

A 240 . . . . . . . . . . . . . . . . . . . 74

A 246 . . . . . . . . . . . . . . . . 114

A 254 . . . . . . . . . . . . . . . . 163

A 282 •..................... 196

A 296 . . . . . . . . . . . . . . . . . . . . . . 244

A 297 . . . . . . . . . . . . . . . . . . 263

VOLUME II

DESCRIPTIONS OF WHOI SEDIMENT CORES . • . . . . . . • . . 279

$\mathrm{R} / \mathrm{V}$ ATLANTIS II

A II 1 . . . . . . . . . . . . . . . 280

A II 15 . ................. 294

A II 31 . . . . . . . . . . . . . . . 459

A II 32 •................... 544

A II 33 . . . . . . . . . . . . . . . 578

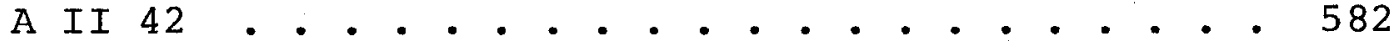

A II 49 . . . . . . . . . . . . . . . . . . 676

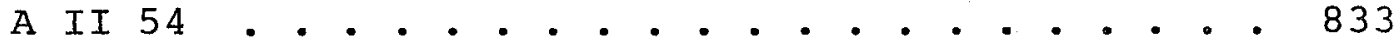

A II 60 .

A II 72 . . . . . . . ........... . 1094

VOLUME III

DESCRIPTIONS OF WHOI SEDIMENT CORES • • • • • • • • . 1196

$\mathrm{R} / \mathrm{V}$ CHAIN

CHAIN 7 . . . . . . . . . . . . . . . . 1197

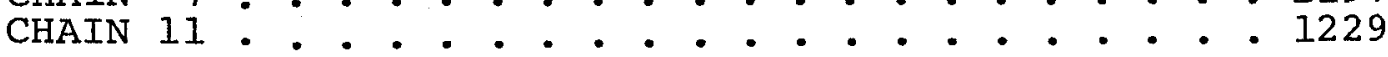

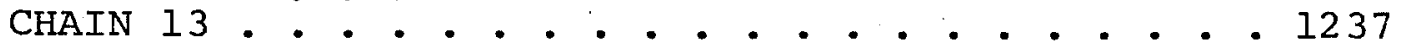


$\mathrm{R} / \mathrm{V}$ CHAIN

$\underline{\text { Page }}$

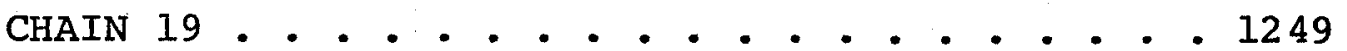

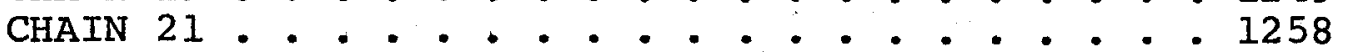

CHAIN 36 . . . . . . . . . . . . . . . 1285

CHAIN 39 . . . . . . . . . . . . . . . . 1310

CHATN 41 . . . . . . . . . . . . . . . 1326

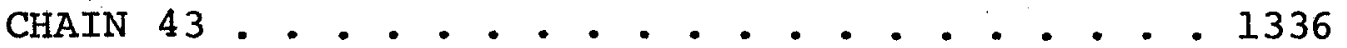

CHAIN 44 . . . . . . . . . . . . . . . 1381

CHAIN 47 . . . . . . . . . . . . . . . . 1391

CHAIN 53 . . . . . . . . . . . . . . . 1399

CHAIN 57 . . . . . . . . . . . . . 1409

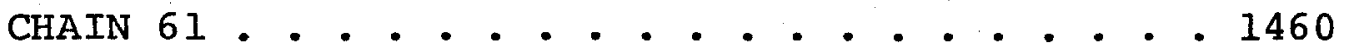

CHAIN 75 . . . . . . . . . . . . . . 1704

CHAIN 82 . . . . . . . . . . . . . . 1875

CHAIN 96 . . . . . . . . . . . . . . 2016

VOLUME IV

DESCRIPTIONS OF WHOI SEDIMENT CORES • • . . . . . • . 2058

$\mathrm{R} / \mathrm{V}$ CHAIN

CHAIN 99 . . . . . • . . . . . . . . 2059

CHAIN 100.. . . . . . . . . . . . . . . 2286

$\mathrm{R} / \mathrm{V}$ KNORR

KNORR 10 .................... 2710

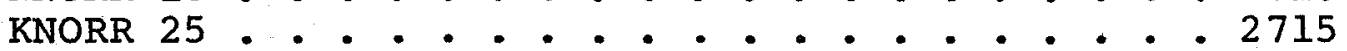

KNORR 27 .................... 2755

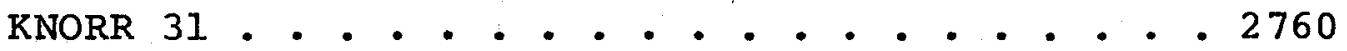

AFRICAN ILAKES . . . . . . . . . . . . . . 2853

IAKE TANGANYIKA 1970 . . . . . . . . . . 2854

LAKE KIVU 1971 . . • . • . . . . . . . . 2875

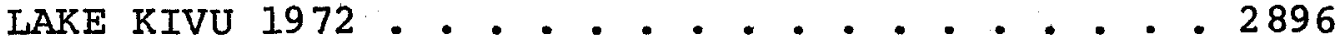

LAKE EDWARD 1972 . . . . . . . . . . . . . . 2918

IAKE ALBERT 1972 . . . . . . . . . . . . 2930-293\%

\section{LIST OF TABLES AND FIGURES}

Table 1: Sediment classification system . . . . . . . 18

Table 2: List of Tertiary cores . . . . . . . . . . 22

Table 3: Explanation of MUDDIE Four-Digit Code . . . . 24

Figure la: Sediment classification system . . . . . . . 19

Figure lb: Sediment classification system . . . . . . . 20

Figure 2: Lithologic symbols . . . . . . . . . . . 21

\section{APPENDIX}

Appendix I: Sample request form . . . . . . . . . . 32 
$\mathfrak{f}$
$\vdots$
1
1
$\vdots$
1
1

l.

\{

I

!

\{

\{

I

1

l

1 


\begin{abstract}
This report presentsvisual core descriptions and smear slide analyses for all cores in the Woods Hole Oceanographic Institution geological samples collection which were obtained prior to November 1973. Approximately 1000 coring stations from the Atlantic, Indian and Pacific oceans and adjacent seas are represented. Charts of ships' track and computer listings of all cores are also included.
\end{abstract}




\section{INTRODUCTION}

\section{A. Scope and Format of this Report}

This report presents visual core descriptions and smear slide analyses for all cores in the Woods Hole geological samples collection which were obtained prior to November 1973 . This compilation represents approximately 1000 coring stations from the Atlantic, Indian and Pacific oceans and adjacent seas. Core descriptions have been grouped according to ship and cruise number and are arranged chronologically for each cruise. A computer listing of the cores taken on each cruise, together with a chart indicating the ship's track, precedes the core descriptions.

When this project was initiated in mid-1972, we quickly discovered that the cores obtained prior to this time had been labeled, cataloged, and described with varying degrees of accuracy and thoroughness. Consequently, we devoted a major effort toward cross-checking all logistical data for each core and verifying that the cores have been properly labeled. Any inconsistencies or contradictions that could not be resolved are indicated on the visual description sheets.

The numbers assigned to the geological samples are followed by a letter (or letters) to indicate the method used in obtaining the samples. The letters which have been used are:

$$
\begin{aligned}
& \text { GC - Gravity core } \\
& \text { PC - Piston core } \\
& \text { GPC - Giant piston core } \\
& \text { GGC - Giant gravity core } \\
& \text { PG - Pilot gravity core } \\
& \text { FF - Free-fall core } \\
& \text { KC - Camera (pogo) core } \\
& \text { HC - Hydro core } \\
& \text { KK - Kasten core } \\
& \text { D - Dredge }
\end{aligned}
$$

All cores in the wHOI collection except the giant cores and Kasten cores were obtained with conventional PVC core liner. These cores have been split and stored at room temperature in sealed polystyrene D-tubes, with moisture-saturated spongy 
\{
\{
\{
\{

(

I

\{

(

(

1

I

1

i

1

1

1

\{

| 
material sealed inside to retard the loss of moisture from the cores. The giant piston cores and giant gravity cores were obtained without the use of core liner inside the core barrels. Upon recovery these cores were extruded into half-round cylindrical shells (150-cm length) and split longitudinally into working and archive halves. Each half was then sealed in plastic sleeving.

We have retained the original stations numbers and core numbers insofar as possible. A gap in the core or station numbering sequence for any given expedition indicates either that the core is not now in the WHOI geological collection, or that such a core was never obtained. In some instances, the letter designations for the type of sampling device have been amended or deleted for purposes of clarity and consistency within the collection. For example, a core now labeled as a "10a-PC" was originally labeled "10a"; the "PC" has been added to identify it as a piston core, and the "a" has been retained to insure proper correlation with the original coring records.

In spite of the varied condition of the core collection when this project was begun, we have applied uniform procedures in completing the core descriptions and microscopic analyses of smear slides. A more detailed discussion of the W.H.O.I. procedures for shipboard core handling, core archiving, core describing, and core photography is included in the following technical reports:

(1) Johnson, D. A. and Driscoll, A. H. (1972), "The curating of WHOI's geological collections", Woods Hole Oceanogr. Inst. Tech. Memorandum WHOI-2-72, $20 \mathrm{pp}$.

(2) Mountain, G. S. (1973), "Procedures for description of WHOI sediment cores", Woods Hole Oceanogr. Inst. Tech. Memorandum WHOI-7-73, $25 \mathrm{pp}$.

(3) Gilman, J. A. (1973), "Procedures for photographing WHOI sediment cores", Woods Hole Oceanogr. Inst. Tech. Memorandum WHOI-1-73, $11 \mathrm{pp}$.

We anticipate that these volumes of core descriptions will be updated periodically with subsequent volumes for cores obtained following November 1973. These updates will be distributed to all persons receiving this initial set of volumes. 
In the following sections we explain the procedures, symbols, and abbreviations used in preparing core description and smear slide information.

\section{B. Core Description Procedures}

1. Visual description

Core analysis begins with a visual description. The entire core is laid out in the correct order of sections, and proper labeling is verified. The core describer then subdivides the core into units, which may be distinguished from each other by lithology, color, texture, or special features.

A unit, once decided upon, is described macroscopically. The bottom contact is classified as gradational (G) or sharp. (S), the latter being defined as a transition to the next lower unit within one $\mathrm{cm}$. Sharp bottom contacts are further described as horizontal (H), inclined (I), mottled, irregular, or convex upward.

The color of the unit is systematically identified by comparison with the Munsell Soil Color Chart. Both the color name and its tonal composition number are recorded (e.g. pale brown, loYR 7/3). In some cases, more than one color may be dominant, excluding mottles or burrows; each noteworthy color is recorded in such an instance. When a multitude of fine laminations is present, only the dominant color is recorded.

Mottles are often present and may occur in sizes ranging from small specks several $\mathrm{mm}$ across to irregular patches with dimensions of several $\mathrm{cm}$. Mottles are commonly round or oblong, with a color and/or texture differing from that of the surrounding sediment. Many of these are worm burrows but are identified as such only when the core describer is very certain of this. All mottles are described as to depth in core, color, size and abundance (few, common, extensive).

Textural notation includes several descriptive parameters, such as grain size and the amount of dehydration, compaction, and lithification. Sediment characteristics such as "dry", "hard", "soupy", or "firm" are given on the basis of feel and visual appearance. In many cases these descriptive terms may reflect the handling and storage history of the core, rather than conditions at the site of deposition. Grain size 
parameters used are: lutite $(<4 \mu)$, silt $(4 \mu-62 \mu)$; sand $(62 \mu-2 \mathrm{~mm})$, and gravel (>2 mm). Although a unit occasionally contains only one size range, grain size often varies enough to require a combination of the individual textural terms. In this instance the predominant size is indicated along with a modifying adjective. For example, "silty lutite" indicates that lutite predominates with a lesser amount of terrigenous detritus, authigenic minerals, volcanic shards, or microfossil tests that are silt-sized. In the majority of WHOI sediment cores lutite-sized sediment does predominate, with minor components of silt most readily detected by noting a gritty texture when a small sample is placed between the teeth. Subsequent microscopic examination of smear slides is used to determine the lithology and relative abundance of siltand sand-sized components. Sand and gravel are distinguishable through a magnifying glass and to the unaided eye. Estimation of sizes within the sand range is accomplished by comparison with vials of sieved, standardized sands. Relative abundance of forams is estimated based on macroscopic core examination if the core is moist and fresh, and it is designated by one of the following relative abundance terms: "scattered" "few", "common", "abundant", or "foram sand".

A number of additional observations that may be of significance fall under the heading of "special features". Graded bedding may be observed in silt-sized or coarsergrained sediment. The range in grain size and the depth interval over which grading occurs are noted. Graded beds are often burrowed in the fine upper section and have sharp, eroded bottom contacts. Many graded beds may be turbidites, but this generic term is not used in the descriptions of graded beds.

Cross-bedding is rarely observed and is generally restricted to silt- or sand-sized sediment. Beds of alternating colors or textures which truncate each other on a scale as small as one $\mathrm{mm}$ are described as cross bedding. Laminations and microlaminations (up to $1 \mathrm{~mm}$ thickness) are observed and noted. occasionally a unit is totally composed of very thin laminations. Since description of individual laminations would be far too tedious, the overall color(s), texture, and any rhythmic sequence of the laminations are noted.

Poor core recovery, washed sediment, or flow-in are also included as special features. Washed sediment usually occurs at the top of a core and commonly results from excess water and subsequent disturbance of the sediment. Poor core recovery 
or extreme post-coring desiccation may also be indicated by voids, distorted sediments, or pieces of lithified sediment that have obviously become disoriented. Flow-in may occur in piston cores, and is usually found at the bottom of the core, but may occasionally occur in upper sections as well. It results from insufficient core penetration and subsequent sucking action of the piston upon core pull-out. Tell-tale signs of flow-in are thin, linear features (variations in color or texture) that extend vertically for several $\mathrm{cm}$ or more. Lengths of extreme homogeneity in a core may also suggest flow-in. Verification of flow-in can be obtained by $x$-radiography if flow-in is suspected but cannot be demonstrated visually. Occasional multiple penetrations of either the pilot core or the piston core have been noted.

"Special features" also includes manganese nodules, organic-rich sapropel-likelayers, shell fragments, pyritized worm burrows, and other unusual lithologic features that may be encountered.

\section{Smear slide analysis}

Smear slides have been prepared and analyzed for all cores with the exception of those from the hot brine areas of the Red sea which contain amorphous metallic oxides. Slides are routinely taken from the top and bottom of each core, at intervals of approximately one meter within the core, and from each major lithologic unit when closer sampling is required. Smear slides are prepared by smearing a small amount of sediment on a 1" $x$ 3" glass slide, drying on a hot plate, and covering with Caedax and a cover slip. Slides are then placed in a $65^{\circ} \mathrm{C}$ oven overnight for permanent curing.

The smear slides are examined through a polarizing binocular microscope, commonly with a magnification of $80 \mathrm{x}-320 \mathrm{x}$. The slide is first scanned at low power for a general indication of its composition. The describer then estimates the percentages of the various components, referring to the guideIines for component identification which are outlined on the following pages. Standardized smear slides and frequent confirmations between describers give some assurance that percentages are being estimated with some degree of accuracy and consistency. However, the data tabulated on the smear slide forms should be used only qualitatively as an indication of the relative proportion of various components, and how the 
abundance of each component appears to change within a given core. Quantitative usage of these numbers, particularly for core-to-core comparisons, is not justified and may be misleading. The coarser components in particular, such as whole foraminiferal tests, are generally under-represented in smear slide preparation, and consequently a considerable bias may be introduced during sample preparation itself.

The following sediment components represent those most commonly encountered in smear slide analysis, and they are used in identifying the sediment type:

Inorganic Components

(a) Detrital grains

(b) Micronodules

(c) Zeolites

(d) Volcanic shards

(e) Pyrite

(f) Clay
Biogenic Components

Calcareous

(g) Foraminifera

(h) Nannofossils

(i) Discoasters

(j) Pteropods

(k) Others
(I) Diatoms

(m) Radiolaria

(n) Sponges

(o) Silico-

flagellates

(a) DETRITAI GRAINS - Mineral grains eroded from a source rock, transported and deposited; may be derived from either terrestrial or marine sources. Fine-grained material may be transported by wind over long distances.

Occurrence - Downcurrent or downslope from sources; common near continental margins and volcanically active regions.

Size - Silt through sand.

Color - Generally speaking, exact identification of mineral grains is more difficult when viewing whole grains than when viewing thin sections. Characteristic colors will be found under plane polarized light, but color and birefringence under crossed nicols are unpredictable due to variable grain thickness. Quartz is perhaps the most common of the detrital minerals, with feldspars and pyroxenes also encountered. Opaque minerals may be common, particularly near continental margins.

Distinctive features - Shape, color, birefringence, relief, refractive index.

(b) MICRONODULES - Microscopic aggregates of chemically precipitated oxides of $\mathrm{Mn}, \mathrm{Fe}, \mathrm{Ni}, \mathrm{Co}, \mathrm{Cu}$, and other metals.

Occurrence - Widespread; most abundant in areas of slow deposition, beneath strong bottom currents, and near submarine volcanoes. 


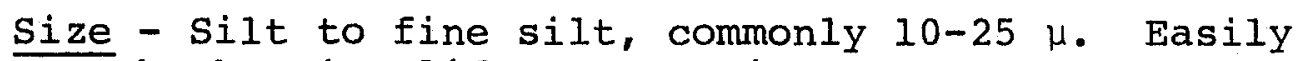
broken in slide preparation.

Color - Thickest grains are opaque; faintly yellowish to reddish brown in thinner grains.

Form - Grains are subrounded, often with ragged edges. Distinctive Features - Color and form.

(c) ZEOLITES - Euhedral crystals of hydrous sodium-calciumaluminum silicates.

Occurrence - Formed in pillow basalts, weathered and deposited in sediments (rare). Also may be formed authigenically in clay-rich sediments, in which case abundance usually increases with depth in in the core.

Size - Normally fine to coarse silt.

Color - Colorless under plane polarized light, but may be pinkish due to presence of iron oxides. Optical properties under crossed nicols are varied. Some appear faintly yellowish gray; smaller grains are gray. Some exhibit parallel extinction. Quite small (clay-sized) particles do not transmit cross-polarized light.

Form - Predominantly elongated, needle-like crystals occasionally showing interpenetrating twins; radiating crystal aggregates are common.

Distinctive Features - Form and size. Low relief and birefringence.

(d) VOLCANIC SHARDS - Amorphous mineral grains (mineraloids) formed at chilled contacts of lava; may be derived from pumice, or directly from volcanic ash falls.

Occurrence - Near volcanic sources, e.g. continental volcanoes, island arcs, and oceanic ridges, transported by wind or water. Fine fraction may be distributed world-wide.

Size - Silt to medium sand.

Color - Generally colorless, but can be yellowish, yellowish brown, brown, or greenish when altered (hydrated) to form the mineraloid palagonite.

Form - Angular, irregular to conchoidal fracture; no cleavage; sometimes thin and sheet-like.

Distinctive Features - Low relief; isotropic; birefringence very rare - only in strained glass. 
(e) PYRITE - Iron sulphide mineral formed in situ during incomplete decomposition of organic material in a reducing environment.

Occurrence - Anoxic basins contain the greatest concentrations of pyrite, but any marine sediment low in free oxygen and containing large amounts of organic material can produce pyrite. Sometimes seen as a partial to total replacement of microfossil tests.

Size - Fine silt.

Color - Completely opaque; spherical forms show a thin gold dispersion halo when viewed with a substage converging lens.

Form - Spherical, cubic, or irregular; often grouped together in raspberry-like framboids. Interpenetration twins are also common.

Distinctive Features - All sizes and shapes are opaque, distinguishing these from manganese micronodules. Brassy under reflected light.

(f) CLAY - For purposes of smear slide work, "clay" is a size term that refers to both clay minerals and other components of size $<4 \mu$.

Occurrence - All marine environments, but most significant near terrigenous source-areas and within central gyres away from productive regions.

Size $-<4 \mu$

Color - Those grains that can be resolved are grayish, brownish, or at least form a faint groundmass. Quartz, feldspar or calcite can sometimes be resolved in clay sizes.

Form - Smooth and irregular in largest sizes; majority of clays produce a groundmass at 100-400x. Distinctive Features - Size, first-order gray birefringence under crossed nicols.

\section{BIOGENIC COMPONENTS}

(g) FORAMINIFERA - One-celled microscopic to macroscopic zooplankton that construct chambered tests of calcium carbonate or aggregated mineral grains.

Occurrence - All marine environments down to 4 or $5 \mathrm{~km}$ water depth. Calcareous forms generally absent below $5 \mathrm{~km}$. 
Size - Range of 25 to $1000 \mu$; usually $25-100 \mu$ in smear slides.

Color - Carbonate tests are colorless to white, sometimes faintly yellowish brown in largest sizes. High relief. High birefringence. Agglutinated forams have the characteristics of the detrital grains used in their construction.

Form - Several chambers, often rounded to subrounded, which may be arranged in a great variety of ways. Distinctive Features - Almost all calcareous species exhibit high birefringence with a N-S, E-W oriented extinction cross under cross-polarized light. This extinction pattern perhaps diminishes in forams from water depths near the carbonate compensation depth. Agglutinated tests will have the extinction characteristics of the component grains.

(h) NANNOFOSSILS - A collective term for coccoliths and other small algal fossils, usually calcareous.

Occurrence - All marine environments above carbonate compensation depth. Absent below $\sim 5 \mathrm{~km}$ in many regions.

Size $-2-20 \mu$.

Color - Colorless with low relief.

Form - Discoidal or toroidal plates, generally perforated. Distinctive Features - With a bright light source and cross-polarized light, coccoliths are gray with a distorted extinction cross; the horseshore-shaped ceratoliths which indicate a late Neogene age, are white to very pale reddish yellow under crossed nicols.

(i) DISCOASTERS - Calcareous nannofossils that are remains of pre-Pleistocene organisms.

Occurrence - Paleocene to late Pliocene sediments; rarely preserved below $5 \mathrm{~km}$ water depth. Often reworked into Pleistocene sediments; consequently their presence is not necessarily indicative of a pre-Pleistocene age of deposition.

Size - 10-35 $\mu$; usually about $20 \mu$ in diameter, some older species are slightly larger.

Color - Colorless, high relief.

Form - Star-shaped or rosette-shaped with four, five, six or more points, the tips sometimes bifurcated. Distinctive Features - High relief, but show up only very faintly gray under bright cross-polarized light. Very distinctive forms. Useful for biostratigraphy in Tertiary sediments. Often not visible under crossed nicols. 
(j) PTEROPODS - Pelagic moliluscs with conically shaped aragonite tests up to several $\mathrm{mm}$ in length.

Occurrence - Generally in shallow $(<2600 \mathrm{~m})$ depths, with abundance decreasing with' water depth, due to dissolution.

Size $-5 \mu-5 \mathrm{~mm}$; silt to fine sand in smear slides (easily broken in slide preparation).

Color - One type is colorless with high relief, the other is greenish brown.

Form - Two forms, at least, are found: the colorless type breaks up into regular, rectangular "slivers"; the colored species are larger, platey and irregularly shaped, and have a fine, grainy surface texture with many cracks.

Distinctive Features - The regular fragments resemble calcite, with high birefringence; the others are very faint gray and show four extinction bars as the sample is rotated under cross-polarized light. Usually fairly large, irregularly-shaped fragments.

(k) OTHERS - This category is reserved for calcareous fragments that appear biogenic, but which have been altered by fragmentation, dissolution, or recrystallization so as to preclude precise identification.

(1) DIATOMS - Microscopic plants that grow tests of amorphous silica.

Occurrence - To some extent in almost all marine environments, but in greatest abundance in polar waters and in areas of upwelling.

Size - 5-150 $\mu$.

Color - Colorless.

Form - Varied; large circular discs or plates with regularly-spaced hexagonal holes; others are elongated, elliptical, over $100 \mu$, with internal structuring; some species are triangular, others semicircular. Symmetry is either radial or bilateral.

Distinctive Features - Form; do not transmit crosspolarized light.

(m) RADIOLARIA - Microscopic siliceous zooplankton.

Occurrence - Worldwide; especially common in polar regions and areas of upwelling.

Size - silt; $50 \mu$ to $200 \mu$, with rare forms up to $1 \mathrm{~mm}$. Color - Colorless, with high relief. 
Form - Extremely varied. Often spherical, discoid, or conical. Skeletons have holes.

Distinctive Features - Often higher relief than diatoms. Rads almost always show a third dimension, and have less obvious symmetry than diatoms. Do not transmit cross-polarized light. Thickness of skeletal bars in relation to pore size is much greater than for silicoflagellates.

(n) SPONGES - Multi-celled marine invertebrates. Nearly all sponges possess internal skeletal elements, termed spicules, which may be either calcareous, siliceous, or organic. Individual siliceous spicules are the forms most commonly found preserved in marine sediments.

Occurrence - Siliceous spicules may be present in sediments from all depths of water; most common in deep regions where solution has removed the calcareous components.

Size - Up to $500 \mu$ long.

Form - Slender and smooth spines, with straight edges, often with a canal extending lengthwise along the center; some spicules have several points. Several forms of spicules may be attached to spherules of silica. These spherules frequently break away and are frequently mistaken for tektites. They are, however, not tear-shaped but perfect spheres. Distinctive Features - Form; may be distinguished from radiolarian spines by the presence of the central canal, siliceous species do not transmit crosspolarized light.

(o) SILICOFLAGELLATES - Siliceous phytoplankton. Occurrence - May be found in small numbers along with diatoms.

Size - 5-60 $\mu$; silt.

Color - Colorless with high relief.

Form - Simple arrangement of arcs, spines, and thin rings. Common taxa are diamond-shaped within a spike at each of the four corners.

Distinctive Features - Unique form. Can generally be distinguished from radiolaria by the relatively thin tubular skeletal bars and wide intervening pores. 
There are a number of uncertainties associated with the identification of sediment components in smear slides and the estimation of the relative abundances of these components. Precise identification of individual opaque minerals is difficult without additional techniques; consequently the opaque minerals are grouped together under the category "detrital grains". Estimates of the abundance of manganese micronodules, pyrite, and other near-opaque grains of low abundance (generally <5\%) are of uncertain accuracy. Grains of inorganic clay-sized material are generally impossible to resolve and to identify mineralogically by routine examination, and therefore the estimation of the percentage of clay is only approximate.

Pteropod and foraminiferal debris of small size $(5 \mu-30 \mu)$ may be included as "calcareous, others", since diagnostic morphologic features may not be present in such small fragments. Rhombohedral calcite crystals, which may be either primary or detrital in origin, are occasionally present. Their presence is either included in the category "detrital grains" or a separate column labelled "calcite" may be added.

Occasionally smear slides contain a dense matrix of claysized dark material that appears as a cloud behind the other sediment components. This mass is often associated with pyrite framboids in sapropel-like sediments which are common in reducing environments such as the Black Sea and African Lakes. However, $\mathrm{H}_{2} \mathrm{O}_{2}$ has little or no effect on the material, and its exact nature is uncertain. A separate column has been added to note the presence of this material.

\section{Designation of sediment type}

After the smear slide analyses for a core have been completed, a sediment name is assigned to each sample according to the sediment classification scheme summarized in Table 1 and Figure 1 (see following pages). These sediment names are recorded on the smear slide description sheet, and serve as a basis for designating one or more sediment types for each lithologic unit.

Each principal lithologic unit is described on the visual description sheets, using a format similar to the following: 
$0-112$

CALC OOZE

10 YR $6 / 4$ light yellowish brown

common dark brown mottling throughout

firm, slightly silty lutite

2 Mn nodules, $2 \mathrm{~cm}$ diam., $95-100 \mathrm{~cm}$

$S$, inclined $10^{\circ}$

Depth interval $(\mathrm{cm})$

Sediment type

Color

Mottling (if present)

Texture

Special features

Basal contact

In the lithologic log on the left side of the visual description sheets, appropriate symbols are used to summarize the lithology and any special features which are readily observed macroscopically. A key to the symbols used in the lithologic logs is presented in Figure 2 (see following pages).

\section{Core Biostratigraphy}

Calcareous nannofossil assemblages were examined to assign a biostratigraphic age to the top and bottom of each core. Martini's (1971) "Standard Tertiary and Quaternary calcareous nannoplankton zonation" served as the reference manual for age determinations. An epoch name (e.g. Pleistocene, Pliocene, etc) was designated for each sample examined; no attempt was made to determine the specific nannofossil zones. Of the 1252 cores in the collection (including pilot cores), 86\% contain sufficient nannofossils to allow an age determination. Only 38 of the cores (see Table 2) contain sediment identified to be of Tertiary age. The large majority of the cores, approximately 95\%, contain only Pleistocene or Recent forms. No nannofossils, and consequently no age determinations, are present for the African Lakes and Red Sea hot brine cores.

Each visual description sheet contains the age of the top and bottom of the core in the margin next to the lithologic log. Any age which is in question (e.g., Pliocene?) indicates possible reworking, to account for an assemblage of nannofossils which have non-overlapping stratigraphic ranges. In cores where nannofossils were absent in the bottom smear slide but were identified in overlying samples, the basal age is assumed to be the same as that of the deepest nannofossil-bearing sample which was examined. In this case, the notation used is an asterisk (e.g., Pliocene*). 
TABIE I: Sediment Classification System

I. MAJOR SEDIMENT NAME: Based on relative proportion of biogenic material $\left(\mathrm{CaCO}_{3}+\mathrm{SiO}_{2}\right)$ and inorganic material.

A. Oozes: Total biogenic material $\geqslant 30 \%$

Calcareous ooze: $\quad \mathrm{CaCO}_{3}>>\mathrm{SiO}_{2}$

Calcareous-siliceous ooze: $\mathrm{CaCO}_{3}>\mathrm{SiO}_{2}, \mathrm{SiO}_{2}>5 \%$

Siliceous-calcareous ooze: $\mathrm{SiO}_{2}>\mathrm{CaCO}_{3}, \mathrm{CaCO}_{3}>5 \%$

Siliceous ooze: $\quad \mathrm{SiO}_{2}>\mathrm{CaCO}_{3}$

B. Clays: Total biogenic material $<30 \%$

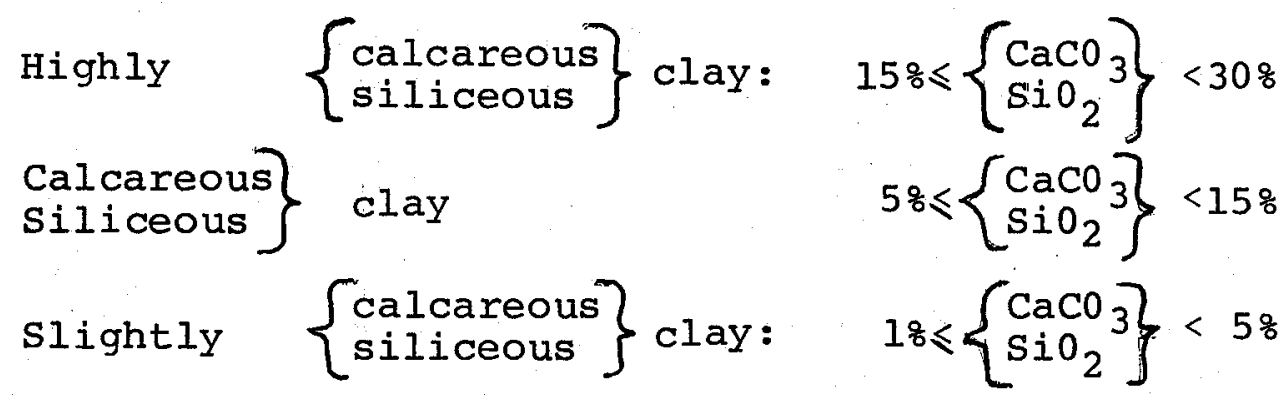

II. SECONDARY SEDIMENT NAMES: Included when silt- or sandsized inorganic components are present in excess of $15 \%$.

(MAJOR SEDIMENT NAME) with $\left\{\begin{array}{l}\text { detrital grains } \\ \text { Mn micronodules } \\ \text { zeolites } \\ \text { volcanic ash } \\ \text { etc. }\end{array}\right\}: 15 \%\left\{\begin{array}{l}\text { detrital grains } \\ \text { Mn micronodules } \\ \text { eolites } \\ \text { volcanic ash } \\ \text { etc. }\end{array}\right\}:\{30 \%$
(MAJOR SEDIMENT NAME) $I\} \geqslant 30 \%$ 
FIGURE 1a: Sediment Classification System

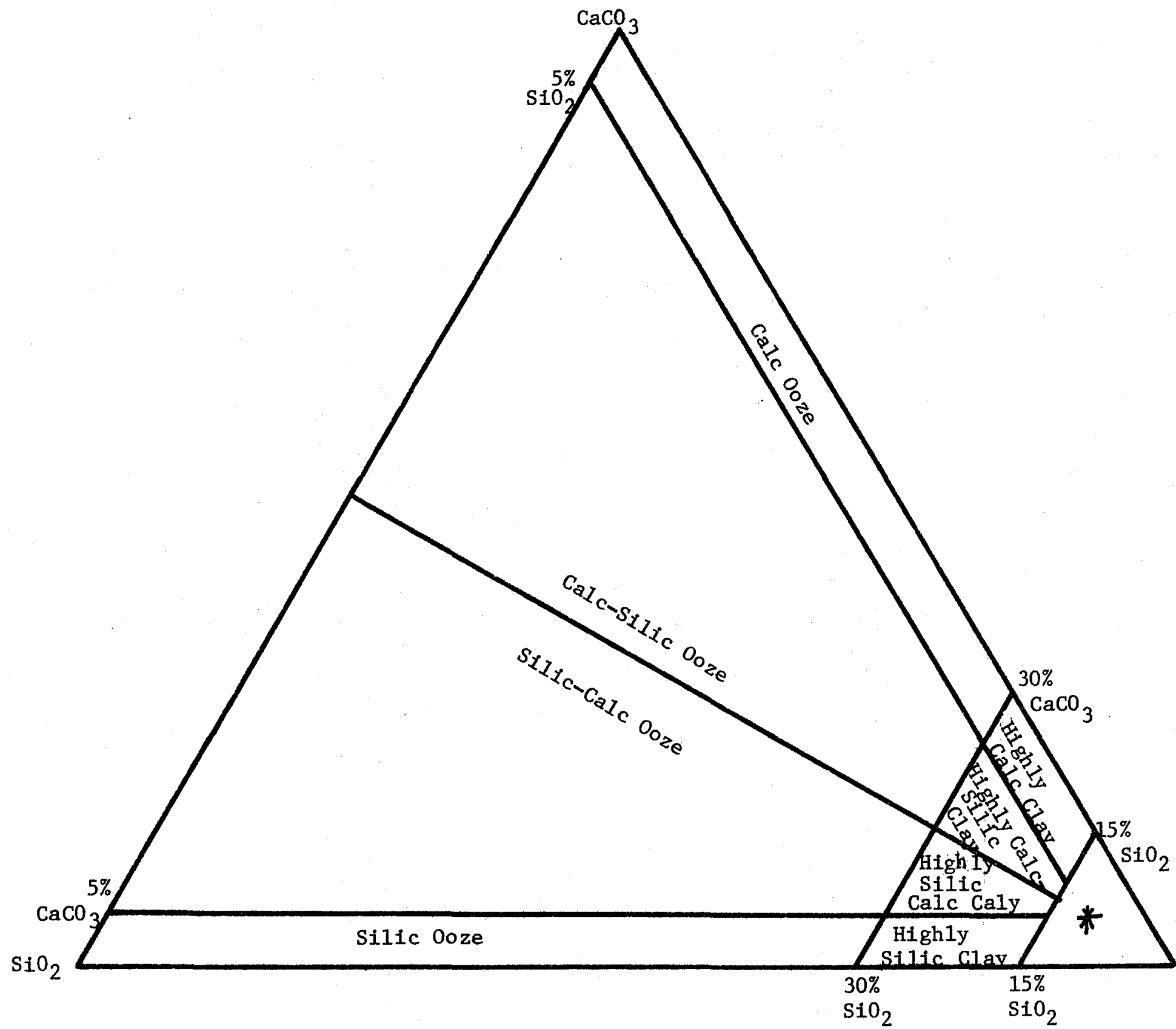

Tota1

Inorganic

* (see Figure 1b) 
FIGURE 1b: Sediment Classification System

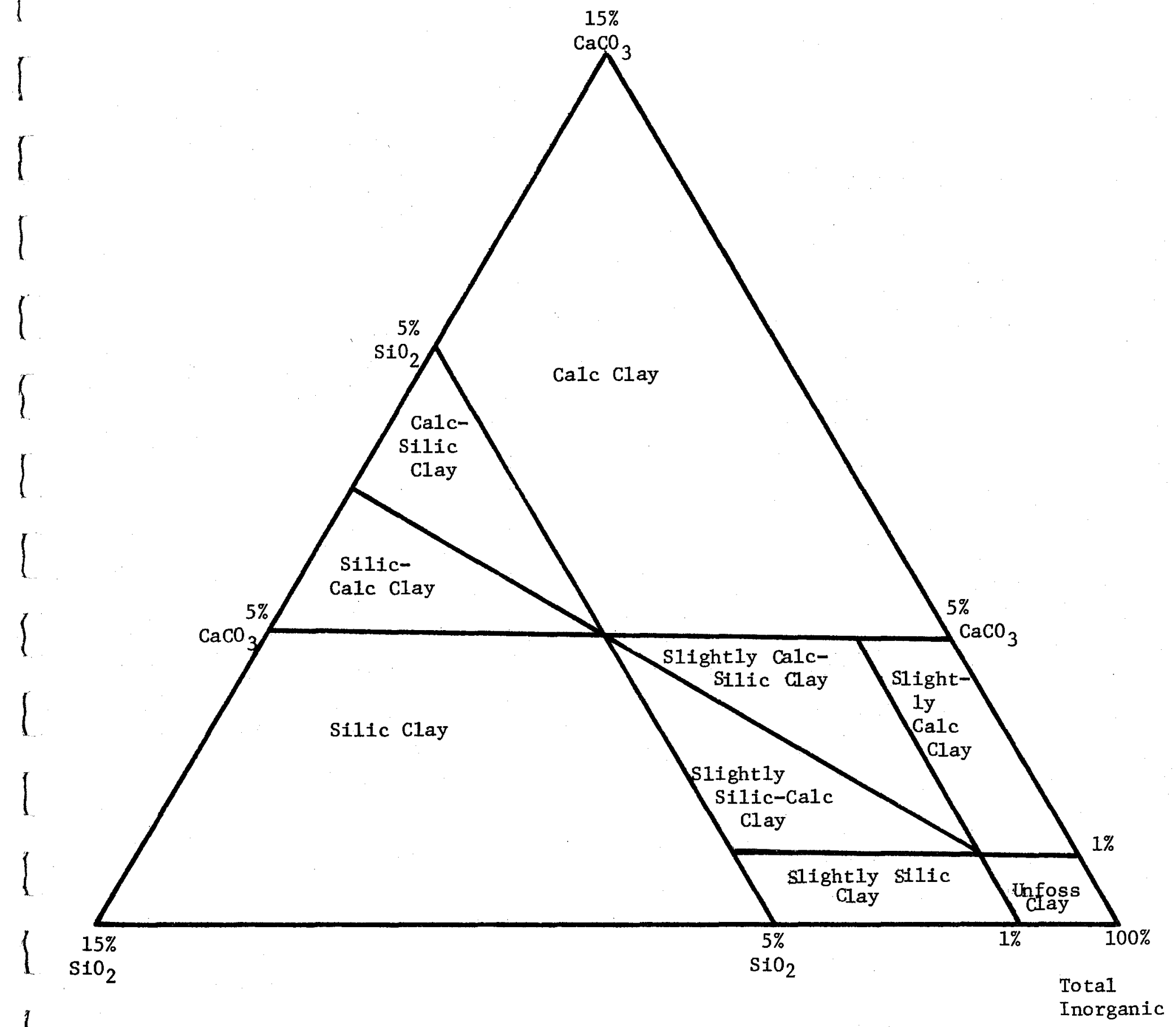


FIGURE 2: Lithologic Symbols

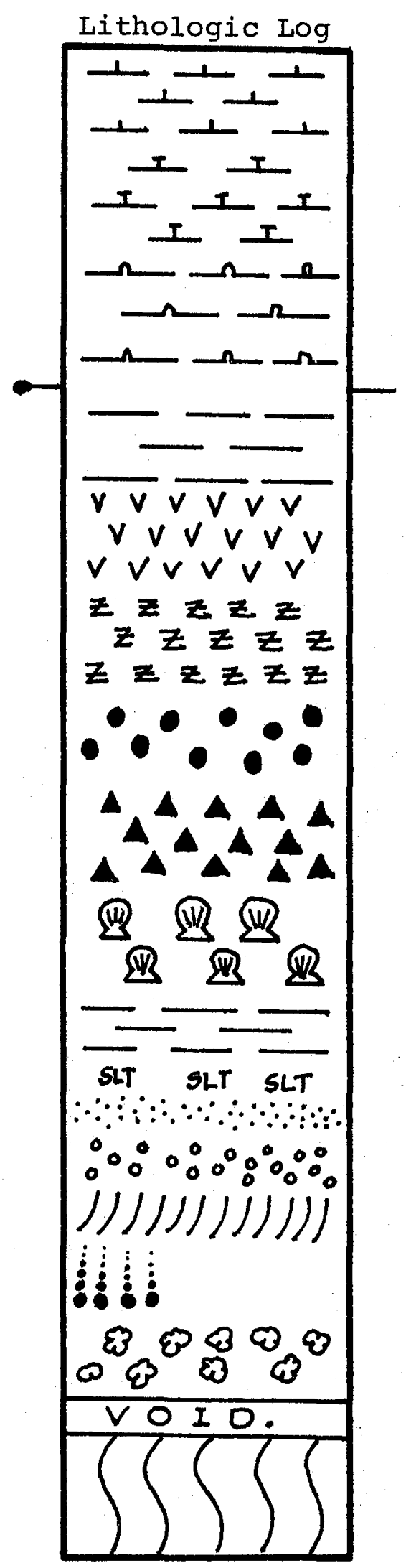

\section{Lithologic symbols}

Calcareous ooze

Foram sand

Siliceous ooze

Unit Contact Mark

Clay

Sediments

Volcanic ash

Zeolites

Mn nodules

Pyrite

Shell Hash

Clay

silt

Sand

Granules, pebbles

Cross-bedding

Graded bedding

Mottling (burrowing)

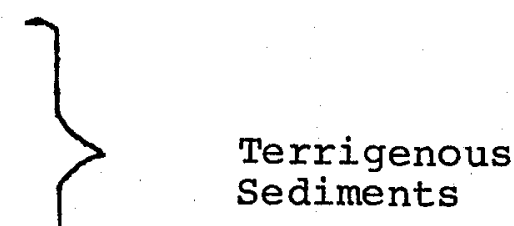

Sediment missing

Flow-in

Special Features 
TABLE 2: Tertiary Cores in WHOI Core Collection **

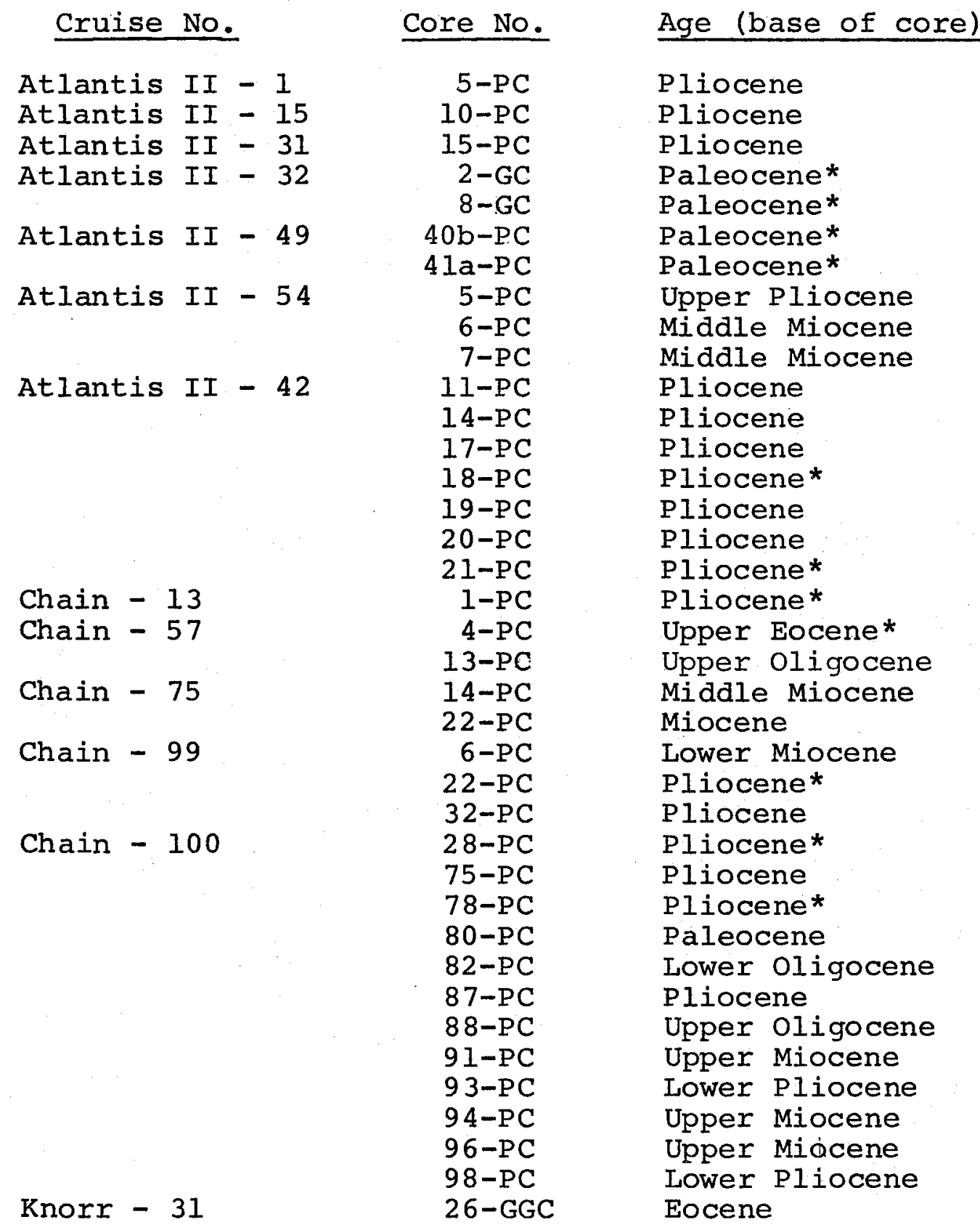

* Bottom sample in core is devoid of nannofossils. Basal age is assumed equal to that of lowermost microfossil-bearing horizon.

** Includes all cores obtained prior to November 1973. 
Since many cores in the WHOI collection are Pleistocene in age, a project was recently initiated (by $C$. Denham and G.P. Lohmann) to date the Pleistocene cores more precisely using paleomagnetic stratigraphy and carbonate analyses. The paleomagnetic work will hopefully locate the Brunhes/ Matuyama boundary (approx. 700,000 y.b.p.) in those cores which penetrated into pre-Brunhes sediment. Carbonate analyses will be performed on closely-spaced samples from cores in which no Brunhes/Matuyama transition is found. Major fluctuations in carbonate content of late Pleistocene cores from widespread geographic areas appear to be climatically controlled, and occur with a periodicity of approximately 100,000 years. These paleomagnetic and carbonate analyses may therefore provide a more precise chronology for many of the WHOI Pleistocene cores.

\section{Digitization of Geological Sample Data}

All logistical information about geological samples in the WHOI core collection is stored on magnetic tape and accessible through computer program MUDDIE. In addition to these data, a summary of the descriptive information for each core has been put into digital form to allow rapid retrieval. This four-digit code summarizes the descriptive information according to primary sediment type, secondary sediment type, relationship of the two, and special features (see Table 3). Stored information about the samples may be retrieved according to combinations of any of the following parameters: ship, cruise, and leg number; latitude and longitude limits; Marsden square number(s); water depth interval; core length; specific or general sampling device; physiographic province; and rock or sediment type.

A complete listing and documentation of the computer program MUDDIE is included in a recent WHOI technical report ("WHOI Geological Samples Data File", A. H. Driscoll and S. M. Rush, in preparation).

The MUDDIE program enables one to obtain either a complete print-out of the data stored for each sample, or an abbreviated version ("mini-listing") which lists the data in a semi-coded format (one line per sample). An example of both the full listing and the "mini-listing" is given on the next two pages. A "mini-listing" precedes the core description sheets for each cruise in the remainder of this report. In addition, a complete mini-listing sorted by Marsden squares and a world index map are found at the end of the introductory section (page 33). 
Table 3

EXPLANATION OF MUDDIE FOUR-DIGIT CODE

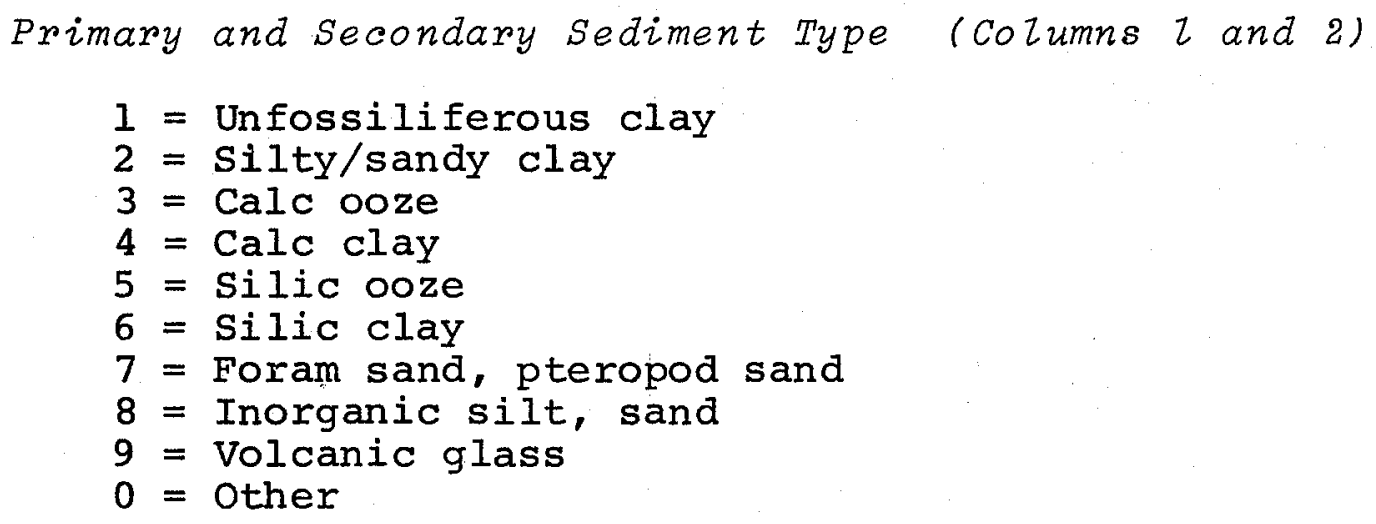


CHN 100 HES 4: 3TATIA:IDIVE \# 0043 SAMF \# OODO DATE: 71/ 4/15 MARSDEN \#: 31.32 3.137N 52.233E FIX: DEAD RECKENING * COHE \# OO31 DEFTH $=5115 \mathrm{M}$ CQRE LENOTH $254 \mathrm{CM}$

- Device: fisten ceje

* vita code: with camera, heat flow, cempass

* PRIMAFY SEDIMENT TYPE: SILIC gOZE

* PRQVINCE: aBYSSAL PLAIN

* REMARKS:

* Secondary sediment tYPe: calc geze

* SPECIAL FEATURES: NONE

CHN 100 LES 4: STATION/CIVE \# 0043 SAMP \# O000 PATE: 71/4/15 MARSOEN \#: 31.32 3.137N 52.233E FIX: DEAD RECKONING

* DERE H OO31 DEPTH $5115 \mathrm{M}$ CGRE LENGTH O CM PILOT LENGTH 0104

- vita cade: with camera, heat flow, compass

* PRQVINCE: aBYSSAL PLAIN

* vita coDe; Nith camera, heat flg

* * SECGNDARY SEDIMENT TYPE: SILIC g日ZE

* sec relationghip: centained in same litholggic unit

* SPECIAL FEATURES: NQNE

CHN 100 LEG 4: STATIQN/DIVE O OU44 SAMP \# OOOO DATE: 71/ 4/16 MARSDEN \#: 31.32 3.142N 52.410E FIX: DEAD RECKONING * CFFE \# OD3? DEPTH = 51 ?3 M CORE LENGTH $959 \mathrm{CM}$

* ofvice: pisten cora

* vita code: with camera, heat flow, compass

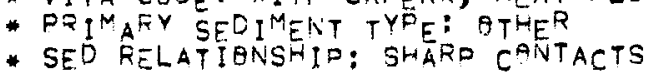

* PRQVINCE: TRENCH - CONTINENTAL MARGIN

* REMARKS:

* SECANDARY SEDIMENT TYPei calC goz

* SPECIAL featuRes: gRaded bedDing or cRess bedDing

CHN 100 LEG 4: STATIEN,JIVE \# OO44 SAMP \# OOOO DATE: 71

* CoRE h o032 DEPTH = 5123

* DeVICE: pilgt gravity CORe

* Vita code: laspecifje?

- pitmary sediment type: other

* sed relationship: gráatignal contacts

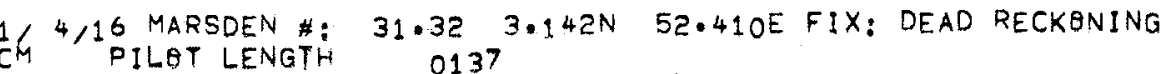
* PREVINCE: TRENCH - OCONTINENTAL MARGIN

* REMARKS:

* secendary sediment type: calc geze

* SPECIAL FEATURES: NeNE

CHN $100 L_{E G}$ 4: STATIGN/SIVE \# 0245 SAMP \# 0000 DATE: 71/4/16 MARSDEN \#: 31.32 3.08ON 52.383E FIX: DEAD RECKENING * CGRE * OC33 OEPTH = $5126 \mathrm{M}$ CQRE LENGTH $1014 \mathrm{CM}$

* device: piston cepe

* Vita code: nith camera, heat fleqw, compass

* primary sediment tyfe: calc egze

* prevince: abyssal plain

* seo relationship! sharp cantacts

* SEcendary sediment type: sil ic agze

* SPECIAL FEATURES! GRADED BEDDiNG oR CRESS BEDDING

CHN 100 LEG 4: STATIQA/LIVE \# 0045 SAMP \# DOOO DATE: 71/4/16 MARSDEN \#: 31.32 3.08ON 52.383E FIX: DEAD RECKONING * CRE \# OO33 DEPTH = $5126 \mathrm{M}$ CQRE LENGTH

$0 \mathrm{CM}$ PILOT LENGTH 0152

* Device: pilet gravity care

- vita code: hith camera, heat flow, compass

* PRQVINCE:

* pejmarit sediment type: siljc egze

* secondar sediment type: calc goze

- Sec relationship: ConTaINED in SAME Lithologic UNIT

* SPECiaL FEATURES: NANE

CHN 100 LEG 4: STATIGH/2IVE \# 0046 SAMP \# OOOO DATE: 71/4/17 MARSDEN \#: 31.20 2.201N 50.130E FIX: DEAD RECKONING * CORE H OO34 DEFTH $=5004 \mathrm{M}$ CQRE LENGTH $895 \mathrm{~cm}$

* DFvice: piston coFe

* vita cede: inith camera, heat flow, compass

* PRQVINCE: UNSPECIFIED

* primatr secipent type: calc goze

* REMARKS:

* SECGNDARY SEDIMENT TYPE: SILIC goZe

- sec felationship. cantained in same lithglggic unit

* SPECIAL FEATURES: PARTIALLY LiTHIFIED SEDIMENT 


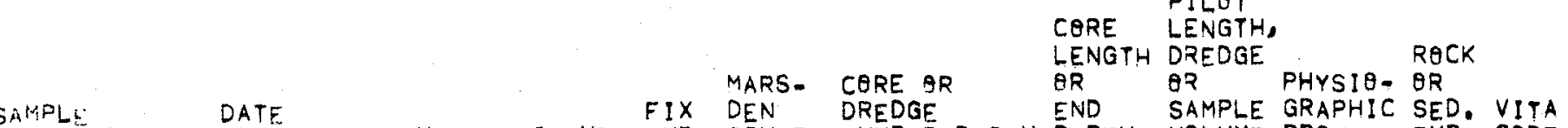

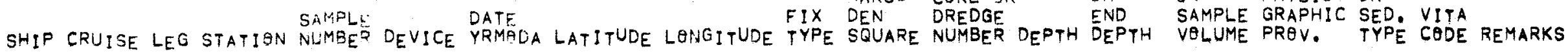

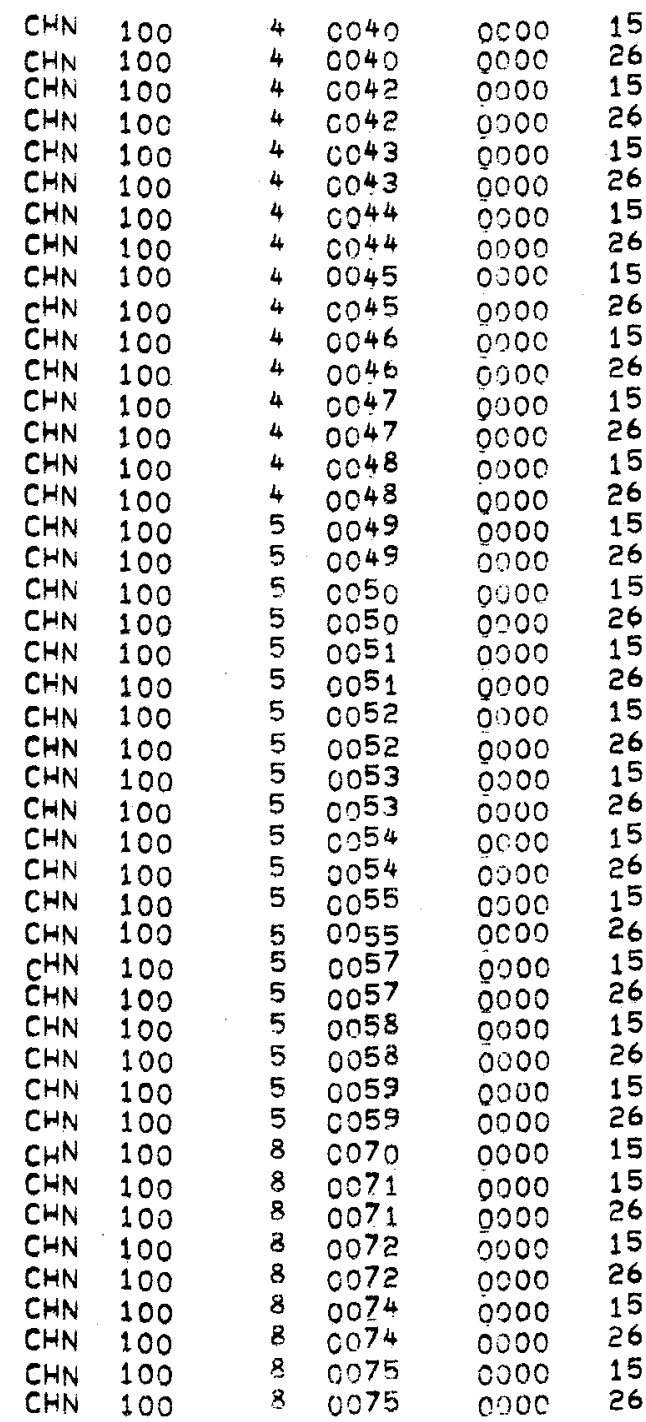

\begin{tabular}{|c|c|c|}
\hline $\begin{array}{lll}1 & 4 & 8 \\
1 & 4 & 8 \\
1 & 413 \\
1 & 413 \\
1 & 415 \\
1 & 415 \\
1 & 416 \\
1 & 416 \\
1 & 416 \\
1 & 416 \\
1 & 417 \\
1 & 417 \\
1 & 418 \\
1 & 418 \\
1 & 420 \\
1 & 420 \\
1 & 427 \\
1 & 427 \\
1 & 428 \\
1 & 428 \\
1 & 429 \\
1 & 429 \\
1 & 430 \\
1 & 430 \\
1 & 5 & 2 \\
1 & 5 & 2 \\
1 & 5 & 3 \\
1 & 5 & 3 \\
1 & 5 & 4 \\
1 & 5 & 4 \\
1 & 5 & 6 \\
1 & 5 & 6 \\
1 & 5 & 7 \\
1 & 5 & 7 \\
1 & 5 & 9 \\
1 & 5 & 9 \\
1 & 727 \\
1 & 728 \\
1 & 728 \\
1 & 728 \\
1 & 728 \\
1 & 730 \\
1 & 730 \\
1 & 731 \\
1 & 731\end{array}$ & 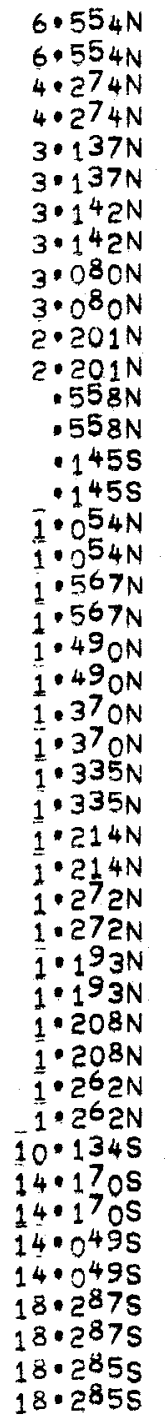 & 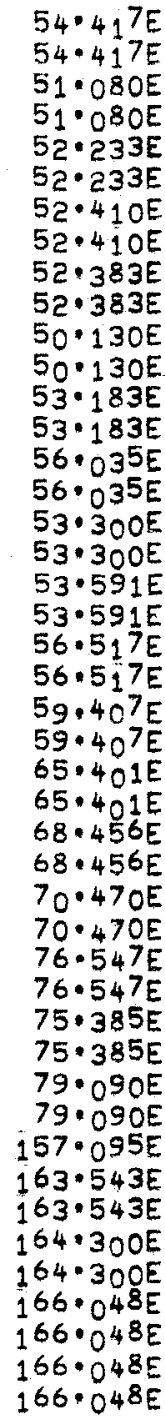 \\
\hline
\end{tabular}

$31 \cdot 64002^{9}$ 31.640029 31.410030 31.410030 $31 \cdot 32 \quad 0031$ 31.320031 $31 \cdot 320032$ $31 \cdot 320033$ $31 \cdot 32 \quad 0033$ $31 \cdot 20 \quad 0034$ $31 \cdot 20 \quad 0034$ $31 \cdot 030035$ $31 \cdot 030035$ 330.060036 $330 \cdot 060036$ $31 \cdot 130037$ 31.130038 31.130038 $31 \cdot 160039$ 31.160039 31.190040 31.190040 $30 \cdot 150041$ $30 \cdot 15.0041$ $30 \cdot 180042$ $30 \cdot 180042$ $30 \cdot 100043$ 30.100043 29.160044 $29 \cdot 160044$ 29.150045 $29 \cdot 150045$ 29.190046 $29 \cdot 190046$ 356.070048 355.430049 355.440050 355.44
355.440050
355.80 355.860051 355.860051 $355.86 \quad 0052$ 355.860052

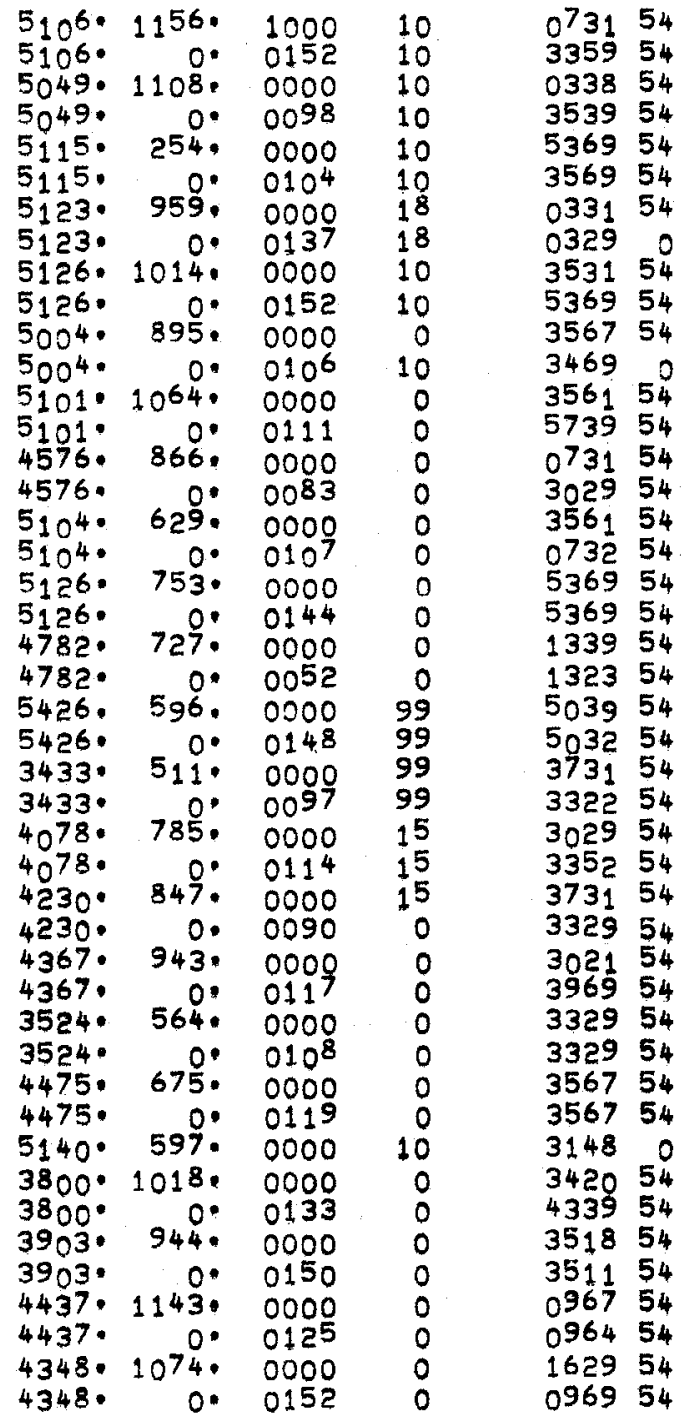


E. Procedures for Obtaining Samples and Additional Core Data

The WHOI Sea Floor Samples Laboratory is prepared to furnish sediment samples and data to interested scientists, researchers, and students inside or outside WHOI who express a legitimate interest and need. Sediment sampling is normally permitted in reasonable quantitites, though sampling of recently acquired cores (taken during the preceding two years) is subject to the approval of the appropriate cruise chief scientist or collector of the samples.

The following procedures will serve as a guide to individuals requesting samples:

PROCEDURES FOR REQUESTING SAMPLES

(1) Requests for samples may be sent directly to the staff scientist engaged in research on the samples, if this person is known. If not known, sample requests should be sent to the Curator's Office, Data and Earth Sample Center, Woods Hole Oceanographic Institution, Woods Hole, Massachusetts 02543 .

(2) A request for samples should include a brief summary of the type of research to be undertaken, the nature of the laboratory facilities available, and the source of financial support available for the work. The names of associated investigators should be given, and the nature of their research, facilities, and funding should be indicated if different from that of the applicant.

(3) If the material requested is within the 2-year period of proprietary access, sample requests will be referred to the appropriate scientists for approval. Otherwise, sample requests will be reviewed by the curator's office.

(4) The curator's office, in consultation with the appropriate WHOI staff scientist, will advise on the availability of material and on any other conditions that may be appropriate to ensure effective utilization of the material. 


\section{RESPONSIBILITIES OF PERSONS RECEIVING SAMPLES}

(1) The original alpha-numeric samples label should be used in published papers, or any departure from this scheme should be clearly equated with the original labelling system in published papers or data summaries. This labelling system will be explained in the information supplied with the samples.

(2) Published papers should acknowledge the source of samples and the appropriate grant or funding agency which supported the cruise recovering the samples. This information will be supplied at the time the samples are sent. These papers should also acknowledge the financial support responsible for maintaining the Woods Hole geological samples (NSF Grant DES73-06463 A02, and ONR Contract N00014-74-C-0262).

(3) Copies of all published papers, reports, or data summaries utilizing Woods Hole samples should be sent to the appropriate WHOI staff scientist and the WHOI curator.

(4) The researcher should return all unused samples or portions of samples to the curator at the completion of $\mathrm{his}$ work.

(5) Recipients of samples should not co-opt the services of other investigators or undertake research projects which differ substantially from work originally proposed, withoutobtaining the approval of the curator and the appropriate staff scientist.

Standardized sampling request sheets are used to maintain consistency and to aid in identifying previous investigators and the type of study done. A sample request form is included in the Appendix of this report. This form may be copied, completed, and returned to the WHOI Core Laboratory when requesting samples. Approval of the appropriate chief scientist(s), when required, will be obtained by the core lab staff. All other information on the sample request form should be completed.

Persons sampling cores should exercise caution in avoiding contamination from sediment directly adjacent to the Iiner, which may have been displaced by frictional effects during the coring process or during subsequent core handling. 
Computer capabilities, as outlined in the previous section, are available for outside use. Requests for listings will be processed as quickly as the appropriate retrieval program is written and computer time is obtained.

Cores are routinely photographed, and a complete set of negatives and 8" $x 10 "$ glossy prints is on file. Persons who wish to receive prints as a prelude to sampling or as a separate part of their research should send a written request for the photographs to the Core Curator, DESC Building, Woods Hole Oceanographic Institution. There is no charge for providing core photographs, as long as the number of prints required is not excessive.

A facility for $x$-radiography of the cores has recently been installed at the core Laboratory and should be fully operational by late 1975. Special studies using $\mathrm{x}$-radiography may be undertaken after that date. This facility will be available to investigators from WHOI and from outside institutions.

Requests for extensive sediment sampling, computer data retrieval, photographs, or X-rays may be subject to a charge for labor, materials, or computer time.

All correspondence regarding core lab operations should be addressed to the Core Curator, DESC Building, Woods Hole Oceanographic Institution. 


\section{ACKNOWIEDGMENTS}

This project could not have been initiated without the support and encouragement of J.R. Heirtzler and R.W. Morse, and an initial grant from the Woods Hole Ocean Industry Program. Continuing support of the W.H.O.I. core lab operations has been provided (since January 1973) by the Office of Naval Research under Contract No0014-74-C0262, NR 083-004; and by the National Science Foundation under Grant No. DES73-06463 (formerly GA-36698).

We have benefited from useful discussions with numerous colleagues concerning procedures for completing the descriptive work and presenting the results. Particular appreciation is expressed to C.D. Hollister, D.A. Ross, W.B. Bryan, P.A. Jezek, C.O. Bowin, K.O. Emery, A.H. Bouma, and W.R. Riedel for their suggestions. We thank J.V. Gardner, W.D. Gardner, R.I. Houghton, and A.N. Shor for critically reviewing the introductory section. Thanks are also due to Bob Groman for writing program MUDDIE and to Bill Dunkle for assistance in locating cruise records and station data.

Full credit for the successful completion of this project is due to the careful and comprehensive work of the W.H.O.I. core lab staff over the past three years. Particular recognition is due to Greg Mountain, Jim Broda, Frank Shephard, Bob McGirr and sue Rush for their efforts in seeing this project through to completion. 


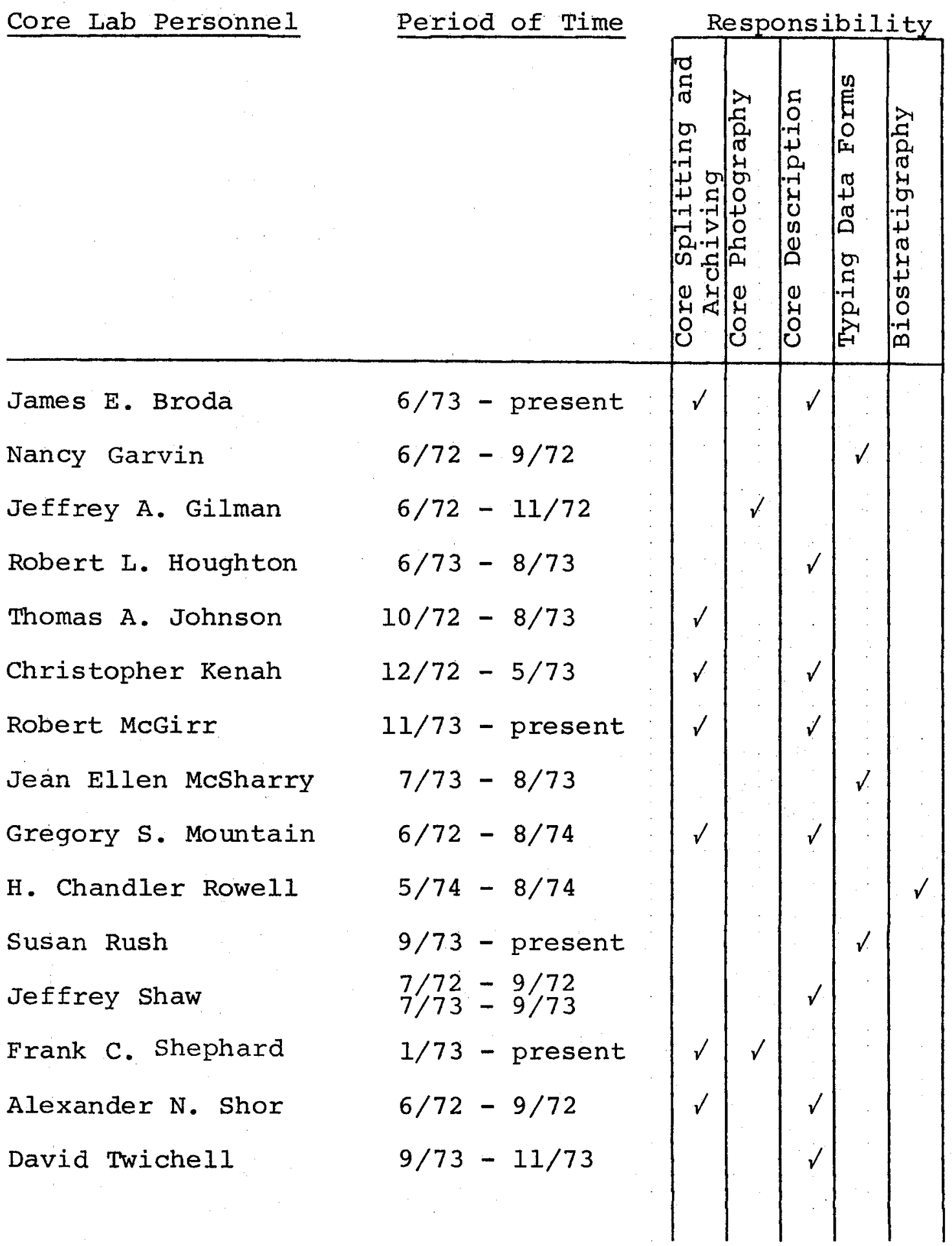


APPENDIX I

W. H.O.I. SAMPLING SHEET

Name

Institution

Position

Purpose of sampling Address

Cruise Leg_L_ Station___ Core or dredge

Chief Scientist

Type of sample taken

Note: chief scientist of cruise must approve sampling.

\begin{tabular}{|l|l|l|}
\hline $\begin{array}{c}\text { Sample depth } \\
\text { in core }\end{array}$ & $\begin{array}{c}\text { Sample } \\
\text { size }\end{array}$ & Comments (include proposed analysis) \\
\hline & & \\
\hline & & \\
\hline & & \\
\hline & & \\
\hline & & \\
\hline & & \\
\hline & & \\
\hline & & \\
\hline & & \\
\hline
\end{tabular}

If first time sampling, please include position, institution, address, and purpose, 
COMPUTER LISTING OF WHOI SEDIMENT CORES (ARRANGED BY MARSDEN SQUARES) 


\section{4}

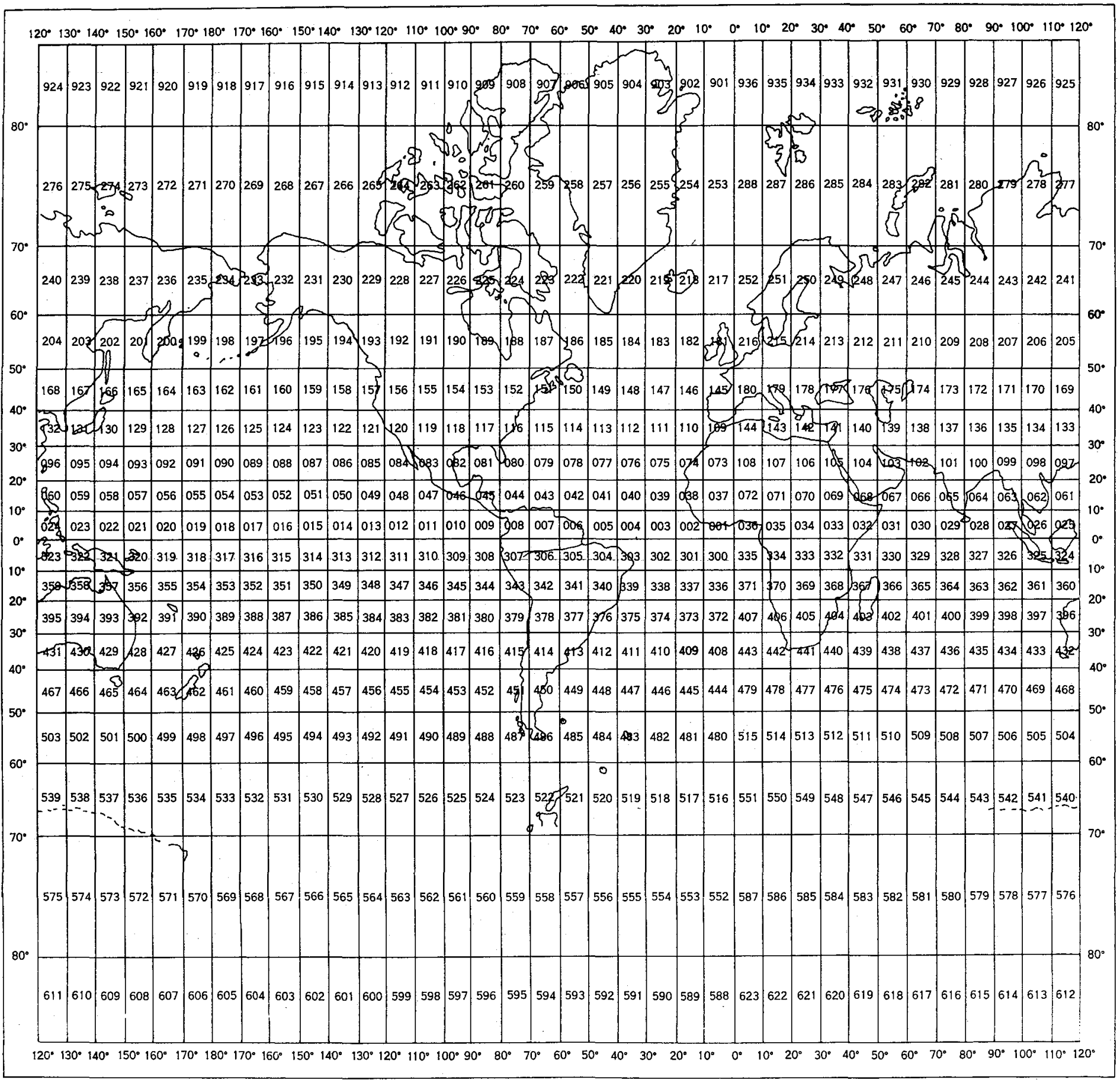




\section{5}

$* * * * * * * * * * * * * * *)$

STATION CATA RETRIEVAL DATE: $12: 36$ MAY 16,175

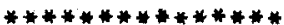

$* * * * * * * * * * * * *$
PAGE 1

**WHOI**

MARS- CQRE $Q$ LR LENGTH DREDGE PHYSIO.

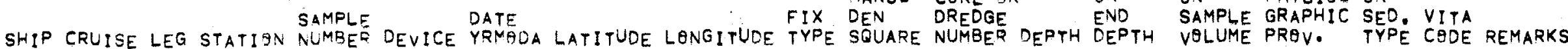

MARSDEN SQUARE \# 2

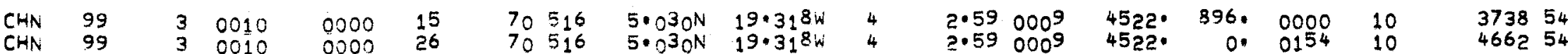

MARSDEN SQUARE \# 3

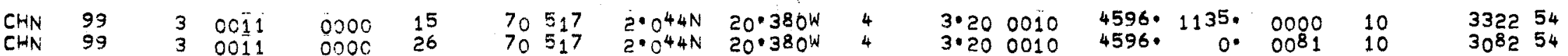

MARSDEN: SQUARE \# 4

$\begin{array}{llllllllllllllllll}\text { Al1 } & 60 & 2 & 0006 & 0000 & 26 & 71218 & .520 \mathrm{~N} & 38 \cdot 210 \mathrm{~W} & 1 & 4 \cdot 58 & 0006 & 4488 \cdot & 0 . & 0049 & 11 & 3932 & 0 \\ A 11 & 60 & 2 & 000^{6} & 0000 & 15 & 71218 & .520 \mathrm{~N} & 38 \cdot 210 \mathrm{~W} & 1 & 4 \cdot 58 & 000^{6} & 4488 . & 290 . & 0000 & 11 & 3932 & 0\end{array}$

MARSDEN SQUARE * 5

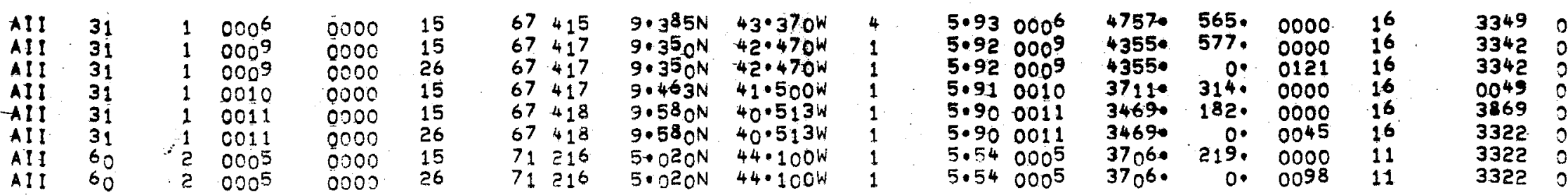

MARSDEN SQUARE \#9

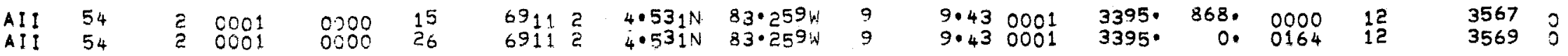

MARSDEN SQUARE \#10

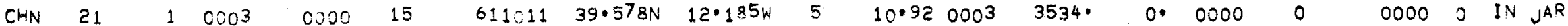




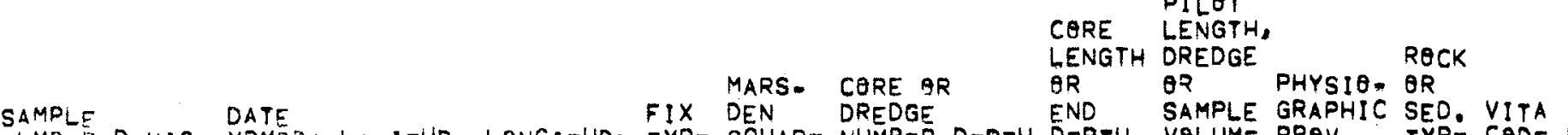

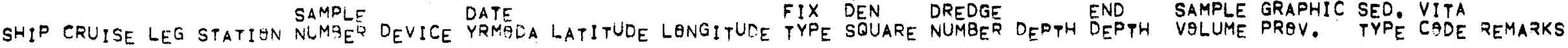

MARSDEN SQUARE \# 29

$\begin{array}{ll}\text { AI! } & 15 \\ \text { AII } & 15 \\ \text { AI! } & 15 \\ A ! I & 15 \\ \text { A!I } & 15 \\ A ! I & 15 \\ \text { AII } & 15 \\ \text { CHN } & 100 \\ \text { CHN } & 100 \\ \text { CHN } & 100 \\ \text { CHN } & 100 \\ \text { CHN } & 100 \\ \text { CHN } & 100\end{array}$

$\begin{array}{lll}5 & c^{614} & 0000 \\ 5 & c^{614} & 0000 \\ 5 & 0^{614} & 0000 \\ 5 & 0^{6} 15 & 0000 \\ 5 & 0617 & 0000 \\ 5 & 0^{6} 18 & 0000 \\ 6 & 0629 & 0000 \\ 5 & 0057 & 0000 \\ 5 & 0057 & 0000 \\ 5 & 0058 & 0000 \\ 5 & 0058 & 0000 \\ 5 & 0059 & 0000 \\ 5 & 0059 & 0000\end{array}$

13
15
26
13
13
13
13
15
26
15
26
15
26

$\begin{array}{lll}65 & 4 & 6 \\ 65 & 4 & 6 \\ 65 & 4 & 6 \\ 65 & 4 & 6 \\ 65 & 4 & 7 \\ 65 & 4 & 7 \\ 65 & 4 & 5 \\ 71 & 5 & 6 \\ 71 & 5 & 6 \\ 71 & 5 & 7 \\ 71 & 5 & 7 \\ 71 & 5 & 9 \\ 71 & 5 & 9\end{array}$

$\begin{array}{ll}9.57 \mathrm{ON} & 74.110 \mathrm{E} \\ 9.54 \mathrm{~N} & 74.110 \mathrm{E} \\ 9.54 \mathrm{ON} & 74.110 \mathrm{E} \\ 9.52 \mathrm{~N} & 75.190 \mathrm{E} \\ 7.57 \mathrm{ON} & 74.080 \mathrm{E} \\ 7.015 \mathrm{~N} & 73.240 \mathrm{E} \\ 4.59 \mathrm{~N} & 71.140 \mathrm{E} \\ 1.193 \mathrm{~N} & 76.547 \mathrm{E} \\ 1.193 \mathrm{~N} & 76.547 \mathrm{E} \\ 1.208 \mathrm{~N} & 75.385 \mathrm{E} \\ 1.208 \mathrm{~N} & 75.385 \mathrm{E} \\ 1.262 \mathrm{~N} & 79.090 \mathrm{E} \\ 1.262 \mathrm{~N} & 79.090 \mathrm{E}\end{array}$

$\begin{array}{ll}29.94 & 0614 \\ 29.94 & 0021 \\ 29.94 & 0021 \\ 29.95 & 0615 \\ 29.74 & 0617 \\ 29 \cdot 73 & 0618 \\ 29.47 & 0629 \\ 29 \cdot 16 & 0044 \\ 29 \cdot 16 & 0044 \\ 29 \cdot 15 & 0045 \\ 29.15 & 0045 \\ 29 \cdot 19 & 0046 \\ 29.19 & 0046\end{array}$

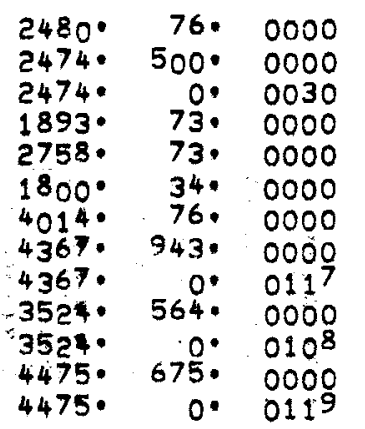

$\begin{array}{lll}6 & 3469 & 0 \\ 6 & 3439 & 0 \\ 6 & 0355 & 0 \\ 6 & 4452 & 0 \\ 6 & 4349 & 0 \\ 6 & 3059 & 0 \\ 0 & 4032 & 0 \\ 0 & 3021 & 54 \\ 0 & 3969 & 54 \\ 0 & 3329 & 54 \\ 0 & 3329 & 54 \\ 0 & 3567 & 54 \\ 0 & 3567 & 54\end{array}$

AlI 15

CHN 100

CHN 100

CHN 100

CHN 100

CHN 100

$\begin{array}{llll}4 & c^{57 E} & 0000 & 18 \\ 5 & 0053 & 0000 & 15 \\ 5 & 0053 & 0000 & 26 \\ 5 & 0054 & 0000 & 15 \\ 5 & 0054 & 0000 & 26 \\ 5 & 0055 & 0000 & 15 \\ 5 & 0055 & 0000 & 26\end{array}$

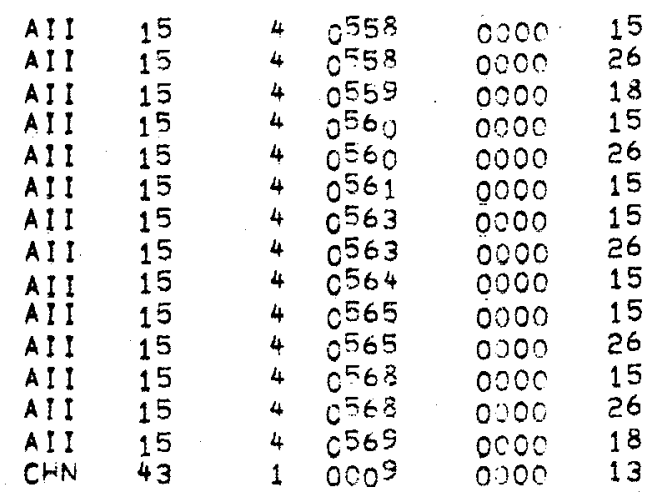

$\begin{array}{lll}9 \cdot 130 N & 60.130 E & 9 \\ 1 \cdot 335 N & 65.401 E & 1 \\ 1 \cdot 335 N & 65.401 E & 1 \\ 1.214 N & 68.456 E & 1 \\ 1.214 N & 68.456 E & 1 \\ 1.272 N & 70.470 E & 1 \\ 1.272 N & 70.470 E & 1\end{array}$

MARSDEN SOUARE \# 31

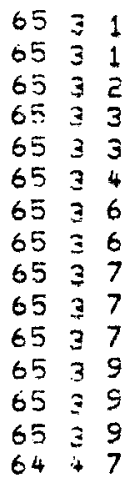

$8 \cdot 59$ ON $51.440 \mathrm{E}$ $8.59 \mathrm{CN} \quad 51.440 \mathrm{OE}$ $\begin{array}{ll}8.540 N & 51 \cdot 370 E \\ 8.585 N & 52.020 E\end{array}$ $8.585 \mathrm{~N} \quad 52.020 \mathrm{E}$ $8 \cdot 585 \mathrm{~N} \quad 52.020 E$ $8 \cdot 58 \mathrm{ON} \quad 52 \cdot 2 \mathrm{COE}$ $9 \cdot 11$ CN $52 \cdot 235 \mathrm{E}$ $9 \cdot 110 \mathrm{~N} \quad 52 \cdot 235 \mathrm{E}$ $9.050 \mathrm{~N} 53.060 \mathrm{~N}$ $9 \cdot 020 N \quad 53 \cdot 405 E$ $9.02 \mathrm{CN} \quad 53 \cdot 40^{5 E}$ $8 \cdot 59$ ON $54.470 E$ $8.590 \mathrm{ON} \quad 54.470 \mathrm{E}$ $8.585 \mathrm{~N} \quad 56.020 E$ $.55 \mathrm{ON} 51.380 \mathrm{E}$
$30^{.9} 0 \quad 0^{572}$ $30 \cdot 150041$ $30 \cdot 150041$ 30.180042 30.180042 30.100043 30.100043

3705. $100^{\circ} 0000$ $34330^{\circ} 511$. 0000 4078 . 785. 0000 $4078 . \quad 0.0114$ $\begin{array}{rrr}4078 \cdot & 0 . & 0114 \\ 4230 \cdot 847 . & 0000\end{array}$ $\begin{array}{rrr}4230^{\circ} & 847 . & 0000 \\ 4230^{\circ} & 0 . & 0090\end{array}$

$\begin{array}{rll}15 & 3322 & 0 \\ 99 & 3731 & 54 \\ 99 & 3322 & 54 \\ 15 & 3029 & 54 \\ 15 & 3352 & 54 \\ 15 & 3731 & 54 \\ 0 & 3329 & 54\end{array}$

$31 \cdot 81000^{9}$ $31 \cdot 810009$ $31 \cdot 810559$ 31.820010 31.820010 31.820011 31.920012 31.920012 31.930013 $31 \cdot 930014$ 31.930014 $31 \cdot 840015$ 31.840015 $31.01000^{4}$
3985. $870^{\circ} 0000$ $\begin{array}{rrr}3985 . & 870^{\circ} & 0000 \\ 3797 . & 0039\end{array}$ 3797. 104, 0000 4350. 665. 0000 $4350^{\circ} \quad 0.0023$ 442. $999^{\circ}, 0000$ 4499.0 .0039 4824.00000 $4852 \cdot 1050 \cdot 0000$ $\begin{array}{rrr}4852^{\circ} & 1050 . & 0000 \\ 4852 . & 0 . & 0069\end{array}$ $4950^{\circ} 1179.0000$ $4950^{\circ} 0.0055$ $\begin{array}{lll}4001^{\circ} & 105 . & 0000 \\ 514^{\circ} & 145 . & 0000\end{array}$

$\begin{array}{lll}10 & 0019 & 0 \\ 10 & 0029 & 0 \\ 15 & 3959 & 0 \\ 15 & 3342 & 0 \\ 15 & 3959 & 0 \\ 15 & 0649 & 0 \\ 15 & 0342 & 0 \\ 15 & 0059 & 0 \\ 15 & 0000 & 0 \\ 15 & 0049 & 0 \\ 15 & 3029 & 0 \\ 10 & 4661 & 0 \\ 10 & 0059 & 0 \\ 10 & 0369 & 0 \\ 10 & 3569 & 0\end{array}$




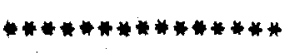

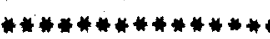

STATIEN, DATA RETRIEVAL DATE: $12 / 36$ MAY 16,'75

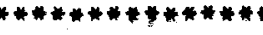

PACE 3

**WHQ !**

\section{LENGTH DREDGE ROCK \\ MARS- CORE GR OR OR PHYSIO. OR}

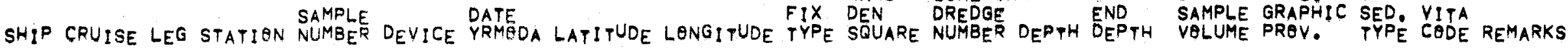

MARSDEN SQUUARE \# 31

$\begin{array}{llllll}\text { CHN } & 43 & 1 & 001 C & 0000 & 13 \\ C H N & 43 & 1 & 0069 & 0000 & 15 \\ \text { CHN } & 100 & 4 & 0036 & 0000 & 15 \\ C H N & 100 & 4 & 0036 & 0000 & 26 \\ \text { CHN } & 100 & 4 & 0037 & 0000 & 15 \\ \text { CHN } & 100 & 4 & 0037 & 0000 & 26 \\ \text { CHN } & 100 & 4 & 0038 & 0000 & 15 \\ \text { CHN } & 100 & 4 & 0038 & 0000 & 26 \\ \text { CHN } & 100 & 4 & 0040 & 0000 & 15 \\ C H N & 100 & 4 & 0040 & 0000 & 26 \\ \text { CHN } & 100 & 4 & 0042 & 0000 & 15 \\ \text { CHN } & 100 & 4 & 0042 & 0000 & 26 \\ \text { CHN } & 100 & 4 & 0043 & 0000 & 15 \\ \text { CHN } & 100 & 4 & 0043 & 0000 & 26 \\ \text { CHN } & 100 & 4 & 0044 & 0000 & 15 \\ \text { EHN } & 100 & 4 & 0044 & 0000 & 26 \\ \text { CHN } & 100 & 4 & 0045 & 0000 & 15 \\ \text { CHN } & 100 & 4 & 0045 & 0000 & 26 \\ \text { CHN } & 100 & 4 & 0046 & 0000 & 15 \\ \text { CHN } & 100 & 4 & 0046 & 0000 & 26 \\ \text { CHN } & 100 & 4 & 0047 & 0000 & 15 \\ \text { CHN } & 100 & 4 & 0047 & 0000 & 26 \\ C H N & 100 & 5 & 0049 & 0000 & 15 \\ \text { CHN } & 100 & 5 & 0049 & 0000 & 26 \\ \text { CHN } & 100 & 5 & 0050 & 0000 & 15 \\ \text { CHN } & 100 & 5 & 0050 & 0000 & 26 \\ \text { CHN } & 100 & 5 & 0051 & 0000 & 15 \\ \text { CHN } & 100 & 5 & 0051 & 0000 & 26 \\ \text { CHN } & 100 & 5 & 0052 & 0000 & 15 \\ \text { CHN } & 100 & 5 & 0052 & 0000 & 26 \\ & & & & & \end{array}$

$\begin{array}{lll}64 & 4 & 8 \\ 64 & 530 \\ 71 & 4 & 6 \\ 71 & 4 & 6 \\ 71 & 4 & 7 \\ 71 & 4 & 7 \\ 71 & 4 & 7 \\ 71 & 4 & 7 \\ 71 & 4 & 8 \\ 71 & 4 & 8 \\ 71 & 413 \\ 71 & 413 \\ 71 & 415 \\ 71 & 415 \\ 71 & 416 \\ 71 & 416 \\ 71 & 416 \\ 71 & 416 \\ 71 & 417 \\ 71 & 417 \\ 71 & 418 \\ 71 & 418 \\ 71 & 427 \\ 71 & 427 \\ 71 & 428 \\ 71 & 428 \\ 71 & 429 \\ 71 & 429 \\ 71 & 430 \\ 71 & 430\end{array}$

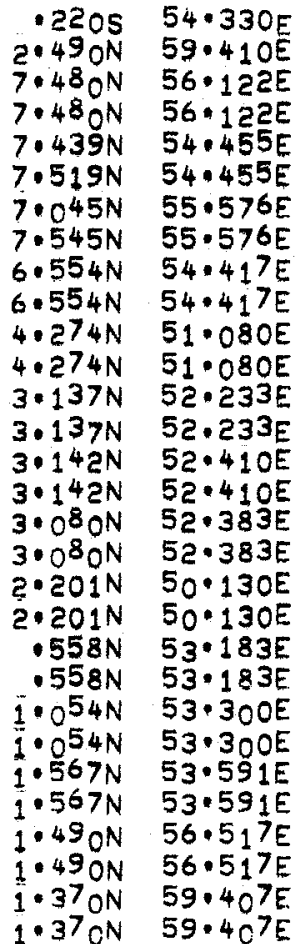

5
5
1
1
1
1
1
1
1
1
1
1
1
1
1
1
1
1
1
1
1
1
1
1
1
1
1
1
9
9

4864, 264, 0000 4215. 325. 0000 $4680^{\circ} 1142 \cdot 0000$ $4680^{\circ}, 0.0112$ 5102. 1149. 0000 5102.00 .0145 4250: 431, 0000 $5250^{\circ}, 156^{\circ} 0100$ 5106. 0. 0152 5049.1108 .0000 5049. 1108. 0009 $\begin{array}{rrr}5049 . & 0 . & 0098 \\ 5115 . & 254 . & 0000\end{array}$ 5115. 0.0104 5123. 959. 0000 5123. 0.0137 5126. 1014. 0000 5126. 0. 0152 5004 . 895. 0000 5004 . 0.0106 $\begin{array}{rrr}5004 . & 0 . & 0106 \\ 5101 \cdot 1064 . & 0000\end{array}$ 5101.0 .0111 5104. 629. 0000 5104.010107 5126. 753. 0000 $5126 . \quad 0.0144$ 4782. 727. 0000 4782. 0. 0052 5426. 596. 0000 $5426 . \quad 0.0148$

$\begin{array}{llll}10 & 3029 & 0 \\ 15 & & 4969 & 46 \\ 99 & 3562 & 54 \text { CHN RIDGE } \\ 99 & & 3569 & 54 \text { CHN RIDGE } \\ 10 & & 3021 & 54 \\ 10 & & 3569 & 54 \\ 10 & & 3328 & 54 \\ 10 & & 3322 & 54 \\ 10 & & 5731 & 54 \\ 10 & 3359 & 54 \\ 10 & & 0338 & 54 \\ 10 & 3539 & 54 \\ 10 & 5369 & 54 \\ 10 & 3569 & 54 \\ 18 & & 0331 & 54 \\ 18 & 0329 & 0 \\ 10 & 3531 & 54 \\ 10 & 5369 & 54 \\ 0 & 3567 & 54 \\ 10 & 3469 & 0 \\ 0 & 3561 & 54 \\ 0 & 5739 & 54 \\ 0 & 3561 & 54 \\ 0 & 0732 & 54 \\ 0 & 5369 & 54 \\ 0 & 5369 & 54 \\ 0 & 1339 & 54 \\ 0 & 1323 & 54 \\ 99 & 5039 & 54 \\ 99 & 5032 & 54\end{array}$

40. 532. 0000 22 55. 484. 0000 22 $\begin{array}{llll}50 . & 499 . & 0000 & 22 \\ 55 . & 499 . & 0000 & 22\end{array}$
5545 LAKE ALBER 5645 O LAKE ALBERT 5038 O LAKE ALBERT 5048 O LAKE ALBERT

MARSDEN SQUARE \# 38

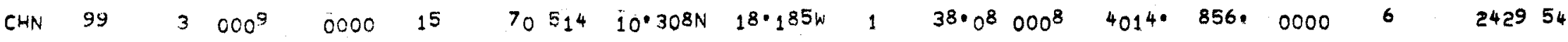




\section{8}

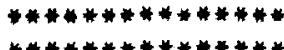

$* * * * * * * * * * * * * * *$
STATION DATA RETRIEVAL OATE: 12:36 MAY 16,175

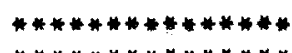

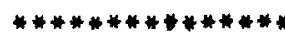

PAGE

* WHOI**

\section{$\begin{array}{lll}\text { LENGTH DREDGE } & \text { RQCK } \\ \text { OR } & \text { QR } & \text { PHYSIO } \\ \text { OR }\end{array}$

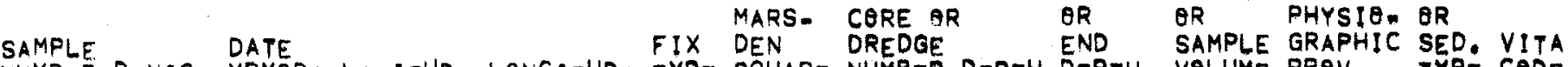

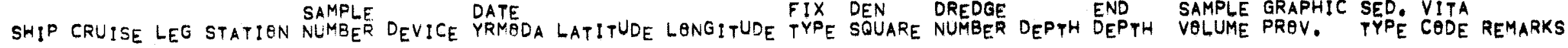

MARSOEN SQUARE \#38

MARSDEN SQUARE \# 39

\begin{tabular}{|c|c|c|c|c|c|c|c|c|c|c|c|c|c|c|c|c|c|}
\hline $\begin{array}{l}A ! I \\
A ! ! \\
A ! ! \\
A ! I\end{array}$ & $\begin{array}{l}42 \\
42 \\
42 \\
42\end{array}$ & $\begin{array}{l}1 \\
1 \\
1 \\
1\end{array}$ & $\begin{array}{l}0001 \\
0002 \\
0003 \\
000^{4}\end{array}$ & $\begin{array}{l}0000 \\
0000 \\
0000 \\
0000\end{array}$ & $\begin{array}{l}15 \\
15 \\
15 \\
15\end{array}$ & $\begin{array}{l}68 \\
68 \\
68 \\
68\end{array}$ & $\begin{array}{l}629 \\
630 \\
71 \\
72\end{array}$ & $\begin{array}{l}16.2^{6} \mathrm{ON} \\
18.02 \mathrm{ON} \\
19 \cdot 41 \mathrm{ON}^{2} \\
19.435 \mathrm{~N}\end{array}$ & $\begin{array}{l}21.430 \mathrm{~W} \\
24 \cdot 270 \mathrm{~W} \\
26 \cdot 090 \mathrm{~W} \\
29 \cdot 020 \mathrm{~W}\end{array}$ & $\begin{array}{l}1 \\
1 \\
1 \\
1\end{array}$ & $\begin{array}{l}39.61 \\
39.84 \\
39.96 \\
39.99\end{array}$ & $\begin{array}{l}0001 \\
0002 \\
000^{3} \\
000^{4}\end{array}$ & $\begin{array}{l}3696^{\circ} \\
3696^{\circ} \\
4550^{\circ} \\
4659^{\circ}\end{array}$ & $\begin{array}{r}258, \\
600 \\
1200^{\circ} \\
1135\end{array}$ & $\begin{array}{l}0000 \\
0000 \\
0000 \\
0000\end{array}$ & $\begin{array}{l}8 \\
8 \\
8 \\
8\end{array}$ & $\begin{array}{l}3739 \\
3241 \\
3329 \\
3429\end{array}$ \\
\hline
\end{tabular}

MARSDEN SQUARE \#40

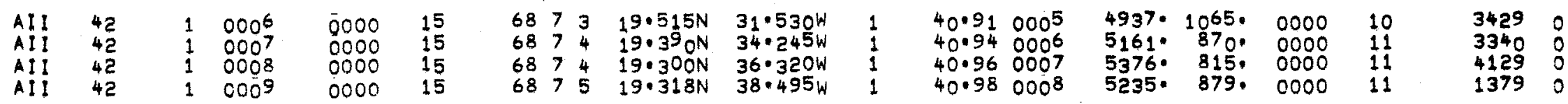

MARSDEN SQUARE \# 41

\begin{tabular}{|c|c|c|c|c|c|c|c|c|c|c|c|c|c|c|c|c|c|}
\hline 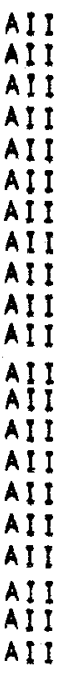 & $\begin{array}{l}31 \\
31 \\
31 \\
31 \\
31 \\
31 \\
31 \\
31 \\
31 \\
31 \\
31 \\
31 \\
31 \\
31 \\
31 \\
31 \\
31 \\
31 \\
31 \\
31 \\
31\end{array}$ & $\begin{array}{l}1 \\
1 \\
1 \\
1 \\
1 \\
1 \\
1 \\
1 \\
1 \\
1 \\
1 \\
1 \\
1 \\
1 \\
1 \\
1 \\
1 \\
1 \\
1 \\
1 \\
1\end{array}$ & $\begin{array}{l}0001 \\
0001 \\
0002 \\
0002 \\
0003 \\
0003 \\
0004 \\
0004 \\
0005 \\
0007 \\
0007 \\
0008 \\
0012 \\
0012 \\
0013 \\
0013 \\
0014 \\
0014 \\
0015 \\
0015 \\
0016\end{array}$ & $\begin{array}{l}0000 \\
0000 \\
0000 \\
0000 \\
0000 \\
0000 \\
0000 \\
0000 \\
0000 \\
0000 \\
0000 \\
0000 \\
0000 \\
0000 \\
0000 \\
0000 \\
0000 \\
0000 \\
0000 \\
0000 \\
0000\end{array}$ & $\begin{array}{l}15 \\
26 \\
15 \\
26 \\
15 \\
26 \\
15 \\
26 \\
15 \\
15 \\
26 \\
15 \\
15 \\
26 \\
15 \\
26 \\
15 \\
26 \\
15 \\
26 \\
15\end{array}$ & $\begin{array}{l}67 \\
67 \\
67 \\
67 \\
67 \\
67 \\
67 \\
67 \\
67 \\
67 \\
67 \\
67 \\
67 \\
67 \\
67 \\
67 \\
67 \\
67 \\
67 \\
67 \\
67\end{array}$ & $\begin{array}{l}49 \\
49 \\
412 \\
412 \\
413 \\
413 \\
415 \\
415 \\
414 \\
416 \\
416 \\
416 \\
418 \\
418 \\
419 \\
419 \\
420 \\
420 \\
420 \\
420 \\
421\end{array}$ & $\begin{array}{l}10.450 \mathrm{~N} \\
10.45 \mathrm{ON} \\
10.35 \mathrm{ON} \\
10.350 \mathrm{~N} \\
10.545 \mathrm{~N} \\
10.545 \mathrm{~N} \\
10.49 \mathrm{ON} \\
10.49 \mathrm{ON} \\
10.220 \mathrm{~N} \\
10.29 \mathrm{ON} \\
10.29 \mathrm{~N} \\
10.485 \mathrm{~N} \\
10.200 \mathrm{~N} \\
10.200 \mathrm{~N} \\
11.203 \mathrm{~N} \\
11.203 \mathrm{~N} \\
11.32 \mathrm{ON} \\
11.320 \mathrm{~N} \\
11.531 \mathrm{~N} \\
11.531 \mathrm{~N} \\
11.575 \mathrm{~N}\end{array}$ & $\begin{array}{l}44 \cdot 510 \mathrm{~W} \\
44 \cdot 510 \mathrm{~W} \\
44 \cdot 510 \mathrm{~W} \\
44 \cdot 510 \mathrm{~W} \\
44 \cdot 070 \mathrm{~W} \\
44 \cdot 070 \mathrm{~W} \\
44 \cdot 100 \mathrm{~W} \\
44 \cdot 100 \mathrm{~W} \\
44 \cdot 180 \mathrm{~W} \\
43 \cdot 415 \mathrm{~W} \\
43 \cdot 415 \mathrm{~W} \\
42 \cdot 560 \mathrm{~W} \\
41 \cdot 175 \mathrm{~W} \\
41 \cdot 175 \mathrm{~W} \\
41 \cdot 518 \mathrm{~W} \\
41 \cdot 518 \mathrm{~W} \\
42 \cdot 425 \mathrm{~W} \\
42 \cdot 425 \mathrm{~W} \\
43 \cdot 474 \mathrm{~W} \\
43 \cdot 474 \mathrm{~W} \\
46 \cdot 100 \mathrm{~W}\end{array}$ & $\begin{array}{l}4 \\
4 \\
4 \\
4 \\
4 \\
4 \\
4 \\
4 \\
1 \\
1 \\
1 \\
1 \\
1 \\
1 \\
1 \\
1 \\
1\end{array}$ & $\begin{array}{l}41 \cdot 04 \\
41 \cdot 04 \\
41 \cdot 04 \\
41 \cdot 04 \\
41 \cdot 04 \\
41 \cdot 04 \\
41 \cdot 04 \\
41 \cdot 04 \\
41 \cdot 04 \\
41 \cdot 03 \\
41 \cdot 03 \\
41 \cdot 02 \\
41 \cdot 01 \\
41 \cdot 01 \\
41 \cdot 11 \\
41 \cdot 11 \\
41 \cdot 12 \\
41 \cdot 12 \\
41 \cdot 13 \\
41 \cdot 13 \\
41 \cdot 16\end{array}$ & $\begin{array}{l}0001 \\
0001 \\
0002 \\
0002 \\
0003 \\
0003 \\
0004 \\
0004 \\
0005 \\
0007 \\
0007 \\
0008 \\
0012 \\
0012 \\
0013 \\
0013 \\
0014 \\
0014 \\
0015 \\
0015 \\
0016\end{array}$ & 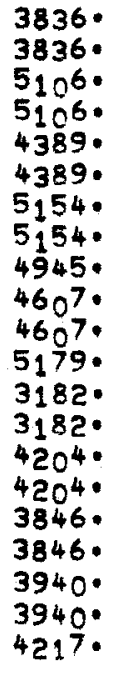 & 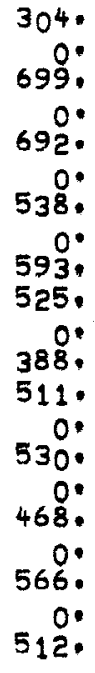 & $\begin{array}{l}0000 \\
0063 \\
0000 \\
0018 \\
0000 \\
0053 \\
0000 \\
0098 \\
0000 \\
0000 \\
0022 \\
0000 \\
0000 \\
0078 \\
0000 \\
0119 \\
0000 \\
0069 \\
0000 \\
0083 \\
0000\end{array}$ & $\begin{array}{l}16 \\
16 \\
16 \\
16 \\
16 \\
16\end{array}$ & $\begin{array}{l}3849 \\
3849 \\
8240 \\
1249 \\
2839 \\
2349 \\
2349 \\
2349 \\
2329 \\
3662 \\
3662 \\
0079 \\
3349 \\
3322 \\
3029 \\
3329 \\
3329 \\
3329 \\
3340 \\
3739 \\
3322\end{array}$ \\
\hline
\end{tabular}




\section{9}

$* * * * * * * * * * * * * *$

$* * * * * * * * * * * * * * *$
STATIEN DATA RETRIEVAL DATE: 12:36 MAY 16,!75
$* * * * * * * * * * * * * *$
$* * * * * * * * * * * * * * * * *)$
PAGE 5

**WH9I**

MARS - CQRE OR LENGTH DREDGE PHYSIO- $Q$ OR

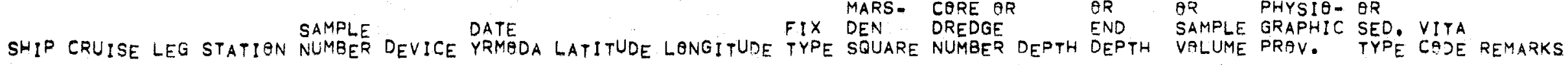

MARSDEN SQUARE \# 41

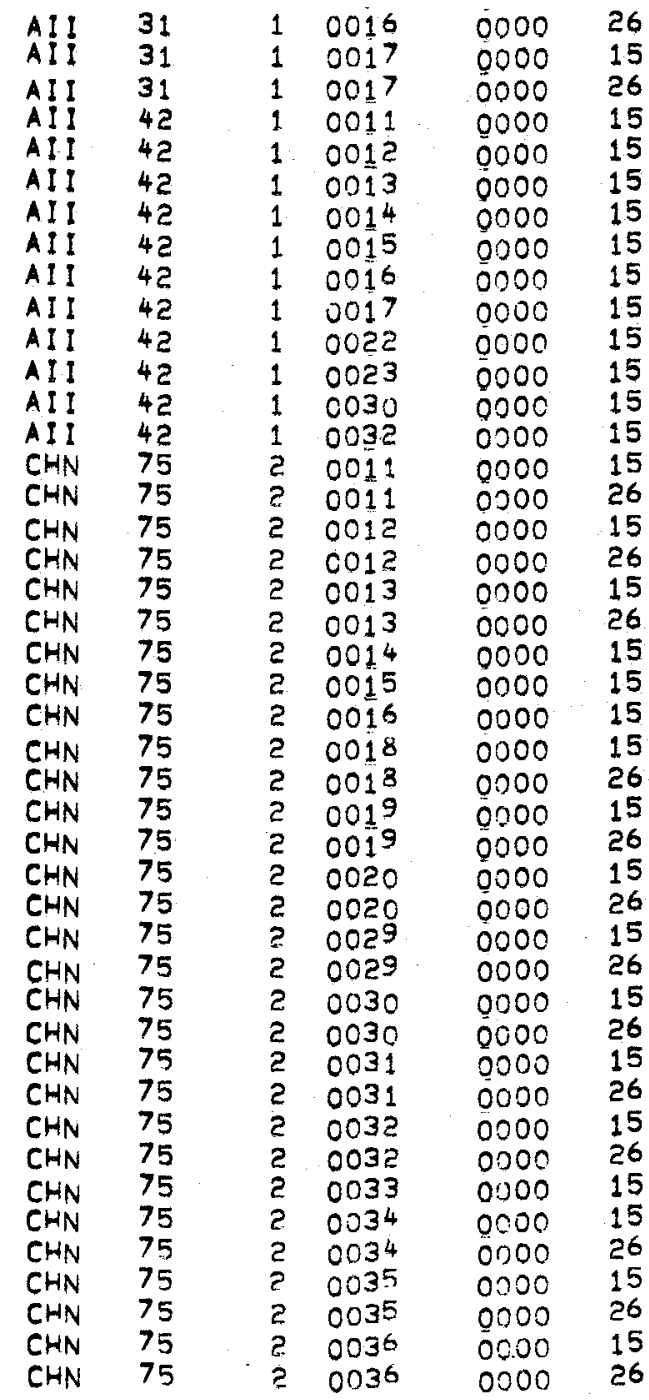

\begin{tabular}{|c|c|c|}
\hline $\begin{array}{l}421 \\
422 \\
422\end{array}$ & 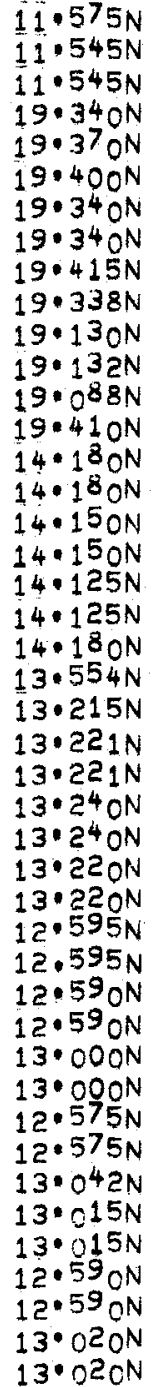 & 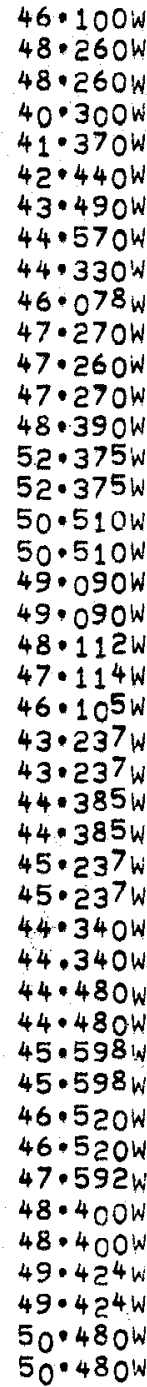 \\
\hline
\end{tabular}

$41 \cdot 160016$ $41 \cdot 18 \quad 0017$ $41 \cdot 180017$

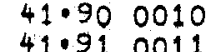
41.910011 $41 \cdot 920012$ 41.340014 41.940015 41.960016 41.970017 41.970017 41.970018
41.970019 41.980020 41.420009 41.420009 41.400010 41.400010 41.490011 41.490011
41.48 $41 \cdot 48$
41.37 .0012
$41 \cdot 36$ $41 \cdot 36 \quad 0014$ $41 \cdot 330016$ $41 \cdot 330016$ $41 \cdot 340017$ $41 \cdot 340017$ $41 \cdot 350018$ $41 \cdot 350018$
41.350018 41.350018
41.240019 41.240019 $41 \cdot 240020$ $41 \cdot 240020$ $41 \cdot 35002$ $41 \cdot 350021$ $41 \cdot 26$
41.26 0022 $\begin{array}{lll}41.26 & 0022 \\ 41 \cdot 37 & 0023\end{array}$ $41 \cdot 38 \quad 0024$ $41 \cdot 38 \quad 002^{4}$ $41 \cdot 290025$ $41 \cdot 290025$ $41 \cdot 300026$ $41 \cdot 30002^{6}$

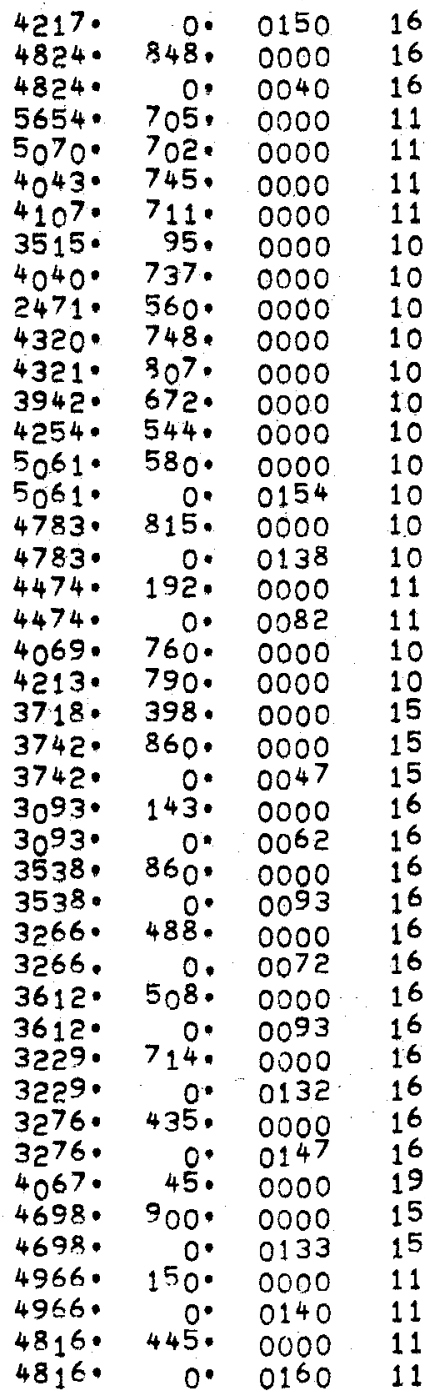

3329 3426 ? 3136 2279 3432 3349

3322

3379

3349

3349

3731

3341

3322

3320
343254

343254 142954 132954 332954 375254 373954 302954 332954 3729.54 332954 332954 332954 332954 332954 000054 0002.54 332454 332954 372254 332254 332954 332954 085354 343254 332954 3430.54 332954 432954 
MARS - CORE OR LENGTH DREDGE RQCK

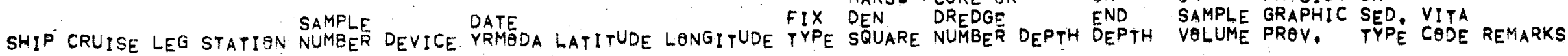

MARSDEN SOUARE \#42

$\begin{array}{llllll}A I l & 31 & 1 & 0019 & 0000 & 15 \\ A I I & 31 & 1 & 0019 & 0000 & 26 \\ A I I & 31 & 1 & 0019 & 0000 & 26 \\ \text { CHN } & 36 & 1 & 0005 & 0000 & 15 \\ \text { CHN } & 36 & 1 & 0006 & 0000 & 15 \\ \text { CHN } & 36 & 1 & 0007 & 0000 & 15 \\ \text { CHN } & 36 & 1 & 0009 & 0000 & 15 \\ \text { CHN } & 36 & 1 & 0010 & 0000 & 15 \\ \text { CHN } & 36 & 1 & 0011 & 0000 & 15 \\ \text { CHN } & 36 & 1 & 0013 & 0000 & 15 \\ \text { CHN } & 36 & 1 & 0014 & 0000 & 15 \\ \text { CHN } & 44 & 1 & 0033 & 0000 & 13 \\ \text { CHN } & 44 & 1 & 0035 & 0000 & 15 \\ \text { CHN } & 44 & 1 & 0036 & 0000 & 13 \\ \text { CHN } & 44 & 1 & 0038 & 0000 & 13 \\ \text { CHN } & 75 & 2 & 0006 & 0000 & 15 \\ \text { CHN } & 75 & 2 & 0007 & 0000 & 15 \\ \text { CHN } & 75 & 2 & 0007 & 0000 & 26 \\ \text { CHN } & 75 & 2 & 0003 & 0000 & 15 \\ \text { CHN } & 75 & 2 & 0008 & 0000 & 26 \\ \text { CHN } & 75 & 2 & 0009 & 0000 & 15 \\ \text { CHN } & 75 & 2 & 0009 & 0000 & 26 \\ \text { CHN } & 75 & 2 & 0010 & 0000 & 15 \\ \text { CHN } & 75 & 2 & 0010 & 0000 & 26 \\ \text { CHN } & 75 & 2 & 0037 & 0000 & 15 \\ \text { CHN } & 75 & 2 & 0037 & 0000 & 26 \\ \text { CHN } & 75 & 2 & 0038 & 0000 & 15 \\ \text { CHN } & 75 & 2 & 0039 & 0000 & 15 \\ \text { CHN } & 75 & 2 & 0039 & 0000 & 26 \\ \text { CHN } & 75 & 2 & 0040 & 0000 & 15 \\ \text { CHN } & 75 & 2 & 0041 & 0000 & 15 \\ \text { CHN } & 75 & 2 & 0041 & 0000 & 26 \\ \text { CHN } & 75 & 2 & 0042 & 0000 & 15 \\ \text { CHN } & 75 & 2 & 0043 & 0000 & 15 \\ \text { CHN } & 75 & 3 & 0044 & 0000 & 15 \\ \text { CHN } & 75 & 3 & 0045 & 0000 & 15 \\ \text { CHN } & 75 & 3 & 0045 & 0000 & 15 \\ \text { CHN } & 75 & 3 & 0045 & 0000 & 26 \\ \text { CHN } & 75 & 3 & 0046 & 0000 & 15 \\ \text { CHN } & 75 & 3 & 0045 & 0000 & 26 \\ & & & & & \end{array}$

42.890018 42.890018 42.890018 42.790004 42.670005 42.670006 42.670007 42.670008 $42 \cdot 680009$ 42.680010 42.680011 42.680001 42.780003
42.780004 42.770006 42.490004 $42 \cdot 480005$ $42.48000^{5}$ 42.470006 42.470006 42.550007 42.550007
42.550007 $42 \cdot 550007$
42.440008 $42 \cdot 440008$ $42 \cdot 310027$ $42 \cdot 310027$ $42 \cdot 220028$ $42 \cdot 240029$ $42 \cdot 240029$ 42.250030 $42 \cdot 26 \quad 0031$

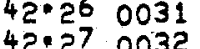
$42 \cdot 290033$ 42.570034 42.670035 42.670036 $\begin{array}{ll}42 \cdot 67 & 0036 \\ 42.77 & 0037\end{array}$ 42.770037

\begin{tabular}{|c|c|c|c|c|}
\hline 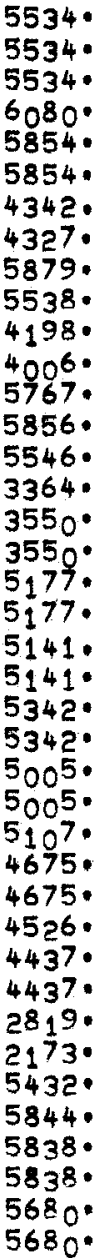 & 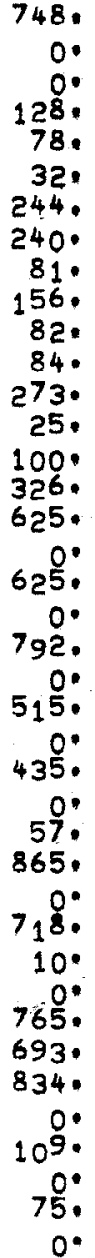 & $\begin{array}{l}0000 \\
0098 \\
0098 \\
0000 \\
0000 \\
0000 \\
0000 \\
0000 \\
0000 \\
0000 \\
0000 \\
0000 \\
0000 \\
0000 \\
0000 \\
0000 \\
0000 \\
0056 \\
0000 \\
0092 \\
0000 \\
0173 \\
0000 \\
0089 \\
0000 \\
0180 \\
0000 \\
0000 \\
0178 \\
0000 \\
0000 \\
0149 \\
0000 \\
0000 \\
0000 \\
0111 \\
0000 \\
0145 \\
0000 \\
0145\end{array}$ & $\begin{array}{l}16 \\
16 \\
16 \\
17 \\
19 \\
19 \\
19 \\
19 \\
19 \\
19 \\
19 \\
19 \\
199 \\
19 \\
19 \\
99 \\
99 \\
99 \\
10 \\
10 \\
10 \\
10 \\
10 \\
10 \\
10 \\
10 \\
10 \\
6 \\
6 \\
6 \\
6 \\
6 \\
4 \\
19 \\
19 \\
19 \\
19 \\
19\end{array}$ & $\begin{array}{l}1223 \\
6639 \\
6639 \\
2839 \\
1839 \\
2839 \\
3332 \\
3329 \\
1239 \\
1939 \\
3329 \\
3969 \\
1923 \\
2969 \\
1129 \\
3739 \\
3969 \\
3969 \\
1629 \\
3969 \\
1429 \\
3322 \\
9469 \\
9469 \\
3929 \\
3969 \\
4969 \\
3420 \\
3329 \\
3939 \\
3359 \\
3339 \\
3335 \\
3932 \\
1969 \\
1969 \\
9169 \\
9169 \\
9469 \\
9469\end{array}$ \\
\hline
\end{tabular}


0041

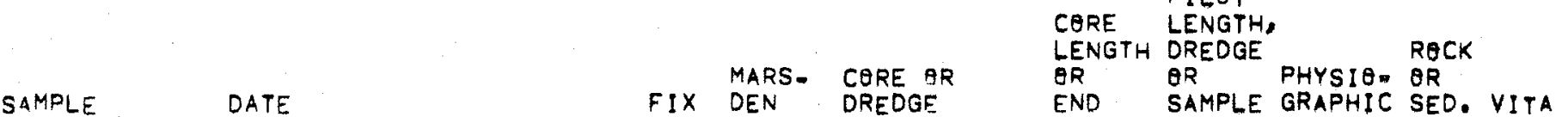

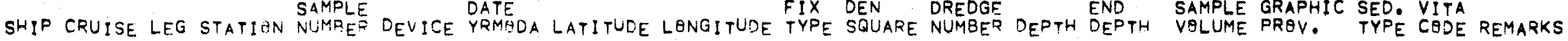

MARSDEN SQUARE \# 43

$\begin{array}{llllll}\text { ATL } & 240 & 1 & 0007 & 0000 & 15 \\ \text { ATL } & 240 & 1 & 0008 & 0000 & 15 \\ \text { ATL } & 240 & 1 & 0009 & 0000 & 15 \\ \text { ATL } & 240 & 1 & 0010 & 0000 & 15 \\ \text { ATL } & 240 & 1 & 0011 & 0000 & 15 \\ \text { ATL } & 240 & 1 & 0012 & 0000 & 15 \\ \text { ATL } & 240 & 1 & 0013 & 0000 & 15 \\ \text { ATL } & 240 & 1 & 0014 & 0000 & 15 \\ \text { ATL } & 240 & 1 & 0015 & 0000 & 15 \\ \text { ATL } & 240 & 1 & 0019 & 0000 & 15 \\ \text { ATL } & 240 & 1 & 0017 & 0000 & 15 \\ \text { ATL } & 240 & 1 & 0018 & 0000 & 15 \\ \text { ATL } & 240 & 1 & 0019 & 0000 & 15 \\ \text { ATL } & 240 & 1 & 0020 & 0000 & 15 \\ \text { ATL } & 240 & 1 & 0021 & 0000 & 15 \\ \text { ATL } & 240 & 1 & 0022 & 0000 & 15 \\ \text { ATL } & 240 & 1 & 0023 & 0000 & 15 \\ \text { ATL } & 246 & 0 & 0062 & 0000 & 15 \\ \text { ATL } & 246 & 0 & 0062 & 0000 & 26 \\ \text { ATL } & 246 & 0 & 0063 & 0000 & 15 \\ \text { ATL } & 246 & 0 & 0063 & 0000 & 26 \\ \text { ATL } & 246 & 0 & 0064 & 0000 & 15 \\ \text { ATL } & 246 & 0 & 0064 & 0000 & 26 \\ \text { ATL } & 246 & 0 & 0065 & 0000 & 15 \\ \text { ATL } & 246 & 0 & 0065 & 0000 & 26 \\ \text { ATL } & 246 & 0 & 0066 & 0000 & 15 \\ \text { ATL } & 246 & 0 & 0066 & 0000 & 26 \\ \text { ATL } & 246 & 0 & 0068 & 0000 & 15 \\ \text { ATL } & 246 & 0 & 0068 & 0000 & 26 \\ \text { ATL } & 246 & 0 & 0069 & 0000 & 15 \\ \text { ATL } & 246 & 0 & 0069 & 0000 & 26 \\ \text { ATL } & 246 & 0 & 0070 & 0000 & 15 \\ \text { ATL } & 246 & 0 & 0070 & 0000 & 26 \\ \text { ATL } & 246 & 0 & 0071 & 0000 & 26 \\ \text { CHN } & 11 & 1 & 0011 & 0000 & 15 \\ \text { CHN } & 19 & 1 & 0002 & 0000 & 15 \\ \text { CHN } & 19 & 1 & 0003 & 0000 & 15 \\ \text { CHN } & 36 & 1 & 0004 & 0000 & 15 \\ \text { CHN } & 41 & 1 & 0002 & 0000 & 13 \\ \text { CHN } & 57 & 1 & 0014 & 0000 & 15 \\ \text { CHN } & 57 & 1 & 0114 & 0000 & 26 \\ \text { CHN } & 57 & 1 & 0016 & 0000 & 26 \\ \text { CHN } & 57 & 1 & 0016 & 0000 & 26 \\ \text { CHN } & 57 & 1 & 0018 & 0000 & 15 \\ \text { CHN } & 57 & 1 & 0021 & 0000 & 15 \\ & & & & & \end{array}$

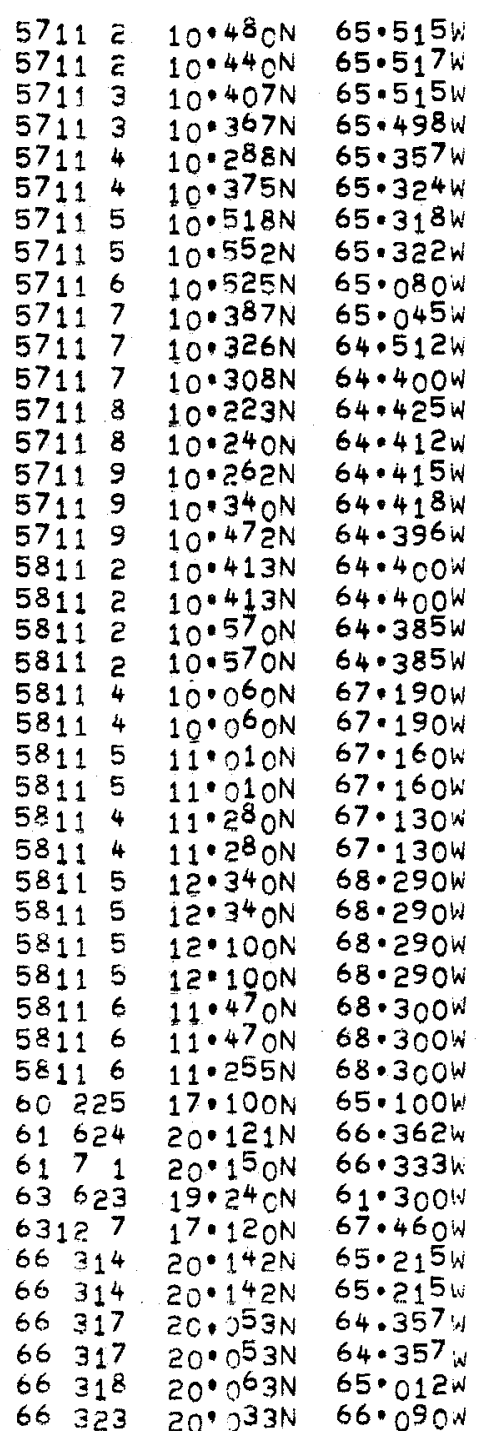

$43 \cdot 050007$ $43 \cdot 050008$ $43 \cdot 050009$ $43 \cdot 050010$ $43 \cdot 050011$ $43 \cdot 050012$ $43 \cdot 050013$ $43 \cdot 050014$ $43 \cdot 050015$ 43.050016 43.060018 43.040019 43.040019 43.040020 43.040021 $43 \cdot 040022$ $43 \cdot 040023$ $43 \cdot 040024$ $43.0^{4} 002^{4}$ 43.040025 43.040025 43.070026 $43 \cdot 070026$ $43 \cdot 170027$ $43 \cdot 170027$ $43 \cdot 170028$ $43 \cdot 170028$ $43 \cdot 280029$ $43 \cdot 280029$ $43 \cdot 280030$ $43 \cdot 280030$ $43 \cdot 18.0031$ $43 \cdot 18 \quad 0031$ $43 \cdot 180032$ 43.750011 $43.06 \quad 0002$ 43.060003 43.910003 43.770002 43.050001 $43 \cdot 050001$ 43.040002 43.040002 $43 \cdot 060004$

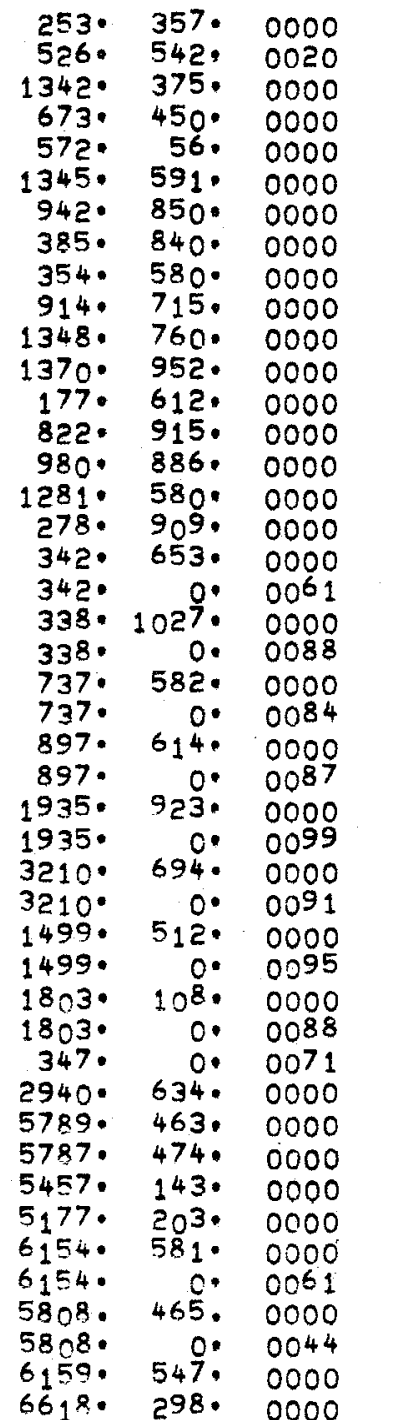

$\begin{array}{lll}18 & 3769 & 0 \\ 8 & 3769 & 0 \\ 18 & 2348 & 0 \\ 18 & 3349 & 0 \\ 18 & 4446 & 0 \\ 18 & 3865 & 0 \\ 18 & 4446 & 0 \\ 18 & 4329 & 0 \\ 18 & 8455 & 0 \\ 18 & 3075 & 0 \\ 18 & 4731 & 0 \\ 18 & 4839 & 0 \\ 18 & 3845 & 0 \\ 18 & 4176 & 0 \\ 18 & 3029 & 0 \\ 18 & 4378 & 0 \\ 18 & 3075 & 0 \\ 19 & 3479 & 0 \\ 19 & 3555 & 0 \\ 19 & 4029 & 0 \\ 19 & 4029 & 0 \\ 19 & 4859 & 0 \\ 19 & 4859 & 0 \\ 19 & 3859 & 0 \\ 19 & 3859 & 0 \\ 19 & 4355 & 0 \\ 19 & 4355 & 0 \\ 19 & 3355 & 0 \\ 19 & 3355 & 0 \\ 19 & 3355 & 0 \\ 19 & 3569 & 0 \\ 19 & 3359 & 0 \\ 19 & 3569 & 0 \\ 19 & 4425 & 0 \\ 2 & 3739 & 0 \\ 13 & 1150 & 0 \\ 13 & 1150 & 0 \\ 17 & 1129 & 0 \\ 13 & 3732 & 0 \\ 17 & 6559 & 54 \\ 17 & 6659 & 54 \\ 17 & 1629 & 54 \\ 17 & 1629 & 54 \\ 17 & 1129 & 54 \\ 17 & 0000 & 54 \\ & & \end{array}$




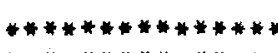

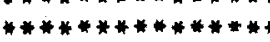

STATION CATA RETRIEVAL DATE: 12:36 MAY 16,175
***************

$* * * * * * * * * * * * * *$
PAGE 8

**WHQI**

CORE LENGTH,

LENGTH DREDGE

OR OR PHYSIO- OR

SAMPLE DATE FIX MARS - CORE TR

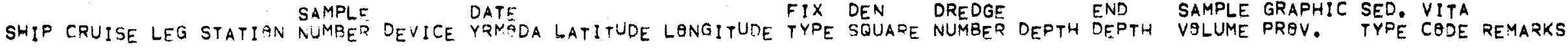

$\begin{array}{llllll}\text { CHN } & 57 & 1 & 0028 & 0000 & 15 \\ \text { CHN } & 57 & 1 & 0029 & 0000 & 15 \\ \text { CHN } & 57 & 1 & 0032 & 0000 & 15 \\ \text { CHN } & 57 & 1 & 0036 & 0000 & 15 \\ \text { CHN } & 57 & 1 & 0037 & 0000 & 15 \\ \text { CHN } & 75 & 1 & 0001 & 0000 & 15 \\ \text { CHN } & 75 & 1 & 0002 & 0000 & 15 \\ \text { CHN } & 75 & 1 & 0002 & 0000 & 26 \\ \text { CHN } & 75 & 1 & 000^{4} & 0000 & 15\end{array}$

$\begin{array}{lrllll}\text { ATL } & 246 & 0 & 0214 & 0000 & 26 \\ \text { ATL } & 246 & 0 & 0215 & 0000 & 26 \\ \text { ATL } & 246 & 0 & 0216 & 0000 & 26 \\ \text { ATL } & 246 & 0 & 0217 & 0000 & 26 \\ \text { ATL } & 246 & 0 & 0213 & 0000 & 26 \\ \text { ATL } & 246 & 0 & 0213 & 0000 & 26 \\ \text { CHN } & 41 & 1 & 0002 & 0000 & 15 \\ \text { CHN } & 41 & 1 & 0004 & 0000 & 13\end{array}$

$\begin{array}{ll}\text { ATL } & 254 \\ \text { ATL } & 254 \\ \text { ATL } & 254 \\ \text { ATL } & 254 \\ \text { ATL } & 254 \\ \text { ATL } & 254 \\ \text { ATL } & 254 \\ \text { ATL } & 254 \\ \text { ATL } & 254\end{array}$

0324
0330
0330
0331
0331
0333
0333
0334
0335

$$
\begin{aligned}
& 0000 \\
& 0000 \\
& 0000 \\
& 0000 \\
& 0000 \\
& 0000 \\
& 0000 \\
& 0000
\end{aligned}
$$

000000015

15
15
26
15
26
15
26
15
15

$\begin{array}{lll}66 & 46 & 20 \cdot 000 N \\ 66 & 46 & 20 \cdot 962 N \\ 66 & 47 & 19 \cdot 560 N \\ 66 & 410 & 20 \cdot 114 N \\ 66 & 410 & 19 \cdot 590 N \\ 671023 & 19 \cdot 240 N \\ 671026 & 19 \cdot 295 N \\ 671026 & 19 \cdot 295 N \\ 671030 & 14 \cdot 447 N\end{array}$

$64 \cdot 178 \mathrm{~W}$

$65 \cdot 505 \mathrm{~W}$

$67.010 W$

$68 \cdot 021 \mathrm{w}$

$65 \cdot 070 \mathrm{~W}$

$60.465 \mathrm{~W}$

$60.465 \mathrm{~W}$

$63.590 \mathrm{~W}$

\begin{abstract}
$43 \cdot 040009$ $43 \cdot 050010$ $43 \cdot 970011$ $43 \cdot 070012$ $43 \cdot 980013$

\begin{tabular}{|c|c|c|}
\hline $\begin{array}{l}581120 \\
581121 \\
581121 \\
581121 \\
581121 \\
581121 \\
63128 \\
631212\end{array}$ & $\begin{array}{l}11 \cdot 15 \mathrm{ON} \\
11 \cdot 265 \mathrm{~N} \\
11 \cdot 34 \mathrm{ON} \\
11 \cdot 46 \mathrm{ON} \\
11 \cdot 46 \mathrm{ON} \\
11 \cdot 45 \mathrm{ON} \\
17 \cdot 18 \mathrm{ON} \\
19 \cdot 37 \mathrm{ON}\end{array}$ & $\begin{array}{l}71 \cdot 350 \mathrm{~W} \\
71 \cdot 365 \mathrm{~W} \\
71 \cdot 355 \mathrm{~W} \\
71 \cdot 130 \mathrm{~W} \\
71 \cdot 050 \mathrm{~W} \\
70 \cdot 490 \mathrm{~W} \\
72 \cdot 110 \mathrm{~W} \\
76 \cdot 270 \mathrm{~W}\end{array}$ \\
\hline
\end{tabular}
$43 \cdot 950001$ $43 \cdot 900002$ $43 \cdot 900002$

$43 \cdot 43000^{3}$
\end{abstract}

MARSDEN SQUARE \# 44

$44 \cdot 110033$ $44 \cdot 110034$ $44 \cdot 110035$ $44 \cdot 110036$ 44.110037 $44 \cdot 100038$ $44 \cdot 720002$ $44.96000^{4}$

\begin{tabular}{|c|c|c|}
\hline $\begin{array}{ll}60 & 215 \\
60 & 220 \\
60 & 220 \\
60 & 221 \\
60 & 221 \\
60 & 222 \\
60 & 222 \\
60 & 222\end{array}$ & $\begin{array}{l}21.32 \mathrm{ON} \\
19 \cdot 350 \mathrm{ON} \\
19 \cdot 350 \mathrm{ON} \\
19 \cdot 12 \mathrm{ON} \\
19 \cdot 12 \mathrm{ON} \\
18 \cdot 29 \mathrm{ON} \\
18 \cdot 29 \mathrm{ON} \\
17.52 \mathrm{ON}\end{array}$ & $\begin{array}{l}83 \cdot 100 \mathrm{~W} \\
84 \cdot 510 \mathrm{~W} \\
84 \cdot 510 \mathrm{~W} \\
86 \cdot 440 \mathrm{~W} \\
86 \cdot 440 \mathrm{~W} \\
86 \cdot 200 \mathrm{~W} \\
86 \cdot 200 \mathrm{~W} \\
86 \cdot 15 \mathrm{WW} \\
86 \cdot 345 \mathrm{~W}\end{array}$ \\
\hline
\end{tabular}

$\begin{array}{lll}6414 \cdot & 700: & 0000 \\ 7074 \cdot & 604 \cdot & 0000 \\ 6907 \cdot & 593: & 0000 \\ 5547 \cdot & 532 \cdot & 0000 \\ 5384 \cdot & 502 \cdot & 0000 \\ 5690^{\circ} & 156: & 0000 \\ 5262 \cdot & 361: & 0000 \\ 5262 \cdot & 0 \cdot & 0115 \\ 2376 \cdot & 452 \cdot & 0000\end{array}$

$\begin{array}{rll}2 & 1629 & 54 \\ 2 & 1139 & 54 \\ 2 & 1129 & 54 \\ 2 & 1968 & 54 \\ 2 & 0000 & 54 \\ 18 & 3344 & 54 \\ 17 & 1479 & 54 \\ 17 & 4459 & 54 \\ 14 & 3029 & 54\end{array}$

302954

$\begin{array}{rrrrrr}18 \cdot & 0 \cdot & 0110 & 19 & 2255 & 0 \\ 24 \cdot & 0: & 0063 & 19 & 2255 & 0 \\ 22 \cdot & 0 \cdot & 0028 & 19 & 8855 & 0 \\ 18 \cdot & 0 \cdot & 0087 & 19 & 1155 & 0 \\ 16 \cdot & 0 \cdot & 0087 & 19 & 1155 & 0 \\ 37 \cdot & 0 \cdot & 0055 & 19 & 2255 & 0 \\ 4296 \cdot & 513 \cdot & 0000 & 13 & 3739 & 0 \\ 6752 \cdot & 121 \cdot & 0000 & 13 & 3739 & 0\end{array}$

\section{MARSDEN SQUARE \# 45}

$45 \cdot 130001$ $45 \cdot 51000^{6}$ 45.510006 $\begin{array}{ll}45.96 & 0007 \\ 45.96 & 000^{7}\end{array}$ $45.96000^{7}$
$45.86000^{8}$ $45 \cdot 86000^{8}$ 45.860008 45.66000

3655. 601. 0000 4579. 605. 0000 4579. 0.0034 4526. 300. 0000 4526. 0.0067 $4402 \cdot 292 \cdot 0000$ $44022^{\circ} \quad 0.0076$ $2440^{\circ} \quad 0 \cdot 0061$

$\begin{array}{lll}0 & 3735 & 0 \\ 2 & 3339 & 0 \\ 2 & 3339 & 0 \\ 2 & 7339 & 0 \\ 2 & 3739 & 0 \\ 2 & 3849 & 0 \\ 2 & 3329 & 0 \\ 2 & 3759 & 0 \\ 2 & 3355 & 0\end{array}$

MARSDEN SQUARE \#65

Al1 $15 \quad 5 \quad 0^{6} 12 \quad 0000 \quad 13 \quad 6544 \quad 13 \cdot 35 \mathrm{CN} \quad 71 \cdot 335 \mathrm{E} \quad 9 \quad 65 \cdot 310612 \quad 1697 \cdot 70.0000 \quad 6 \quad 3355 \quad 0$ 


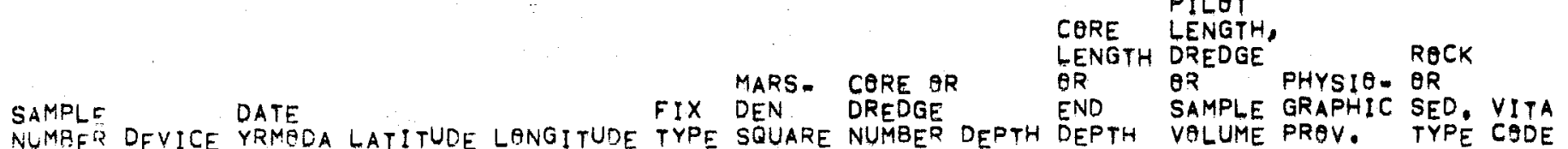

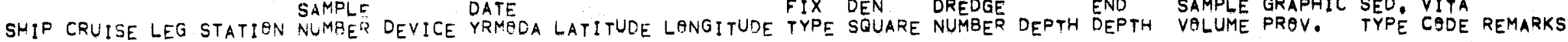

MARSDEN SQUARE \# 66

$\begin{array}{llllll}A ! 1 & 15 & 5 & 0596 & 0000 & 18 \\ A ! & 15 & 5 & 0605 & 0000 & 26 \\ A ! 1 ! & 15 & 5 & 0507 & 0000 & 18 \\ A ! 15 & 15 & 5 & 0608 & 0000 & 13 \\ A & 15 & 5 & 0610 & 0000 & 13\end{array}$

\begin{tabular}{|c|c|}
\hline $\begin{array}{lll}65 & 327 \\
65 & 4 & 2 \\
65 & 4 & 2 \\
65 & 4 & 3 \\
65 & 4 & 3\end{array}$ & $\begin{array}{l}18 \cdot 56 \mathrm{ON} \\
14 \cdot 25 \mathrm{ON} \\
14 \cdot 16 \mathrm{ON} \\
14 \cdot 07 \mathrm{ON} \\
14.020 \mathrm{~N}\end{array}$ \\
\hline
\end{tabular}

\subsection{6} 66.430020 $66 \cdot 44060 ?$
66.45 .0608 66.480610

$\begin{array}{rrr}3694^{\circ} & 120: & 0000 \\ 4023^{\circ} & 0: & 0063 \\ 39655^{\circ} & 87: & 0000 \\ 3957^{\circ} & 74 \cdot & 0000 \\ 4075 \cdot & 28 . & 0000\end{array}$

$\begin{array}{rrr}6 & 3359 & 0 \\ 21 & 3432 & 0 \\ 21 & 3359 & 0 \\ 21 & 3339 & 0 \\ 21 & 4359 & 0\end{array}$

MARSDEN SQUARE \#67

$\begin{array}{llllll}\text { AII } & 15 & 4 & 0547 & 0000 & 15 \\ \text { III } & 15 & 4 & 0547 & 0000 & 26 \\ \text { AII } & 15 & 4 & 0552 & 0000 & 15 \\ \text { AII } & 15 & 4 & 0552 & 0000 & 26 \\ \text { AII } & 15 & 5 & 0597 & 0000 & 18 \\ \text { III } & 15 & 5 & 0597 & 0000 & 13 \\ \text { AI! } & 15 & 5 & 0597 & 0000 & 18 \\ \text { AII } & 15 & 5 & 0597 & 0000 & 15 \\ \text { A!I } & 15 & 5 & 0599 & 0000 & 18 \\ \text { A!I } & 15 & 5 & 0600 & 0000 & 18 \\ \text { AII } & 15 & 5 & 0602 & 0000 & 18 \\ \text { CHN } & 100 & 4 & 0035 & 0000 & 15 \\ \text { CHN } 100 & 4 & 0035 & 0000 & 26\end{array}$

$\begin{array}{llll}65 & 226 & 12 \cdot 00 O N & 51 \cdot 540 E \\ 65 & 226 & 12 \cdot 00 O N & 51 \cdot 540 E \\ 65 & 227 & 10 \cdot 15 O N & 53 \cdot 100 E \\ 65 & 227 & 10 \cdot 15 O N & 53 \cdot 100 E \\ 65 & 329 & 17 \cdot 26 O N & 57 \cdot 110 E \\ 65 & 329 & 17 \cdot 260 N & 57 \cdot 110 E \\ 65 & 329 & 16 \cdot 140 N & 54 \cdot 460 E \\ 65 & 329 & 16 \cdot 140 N & 54 \cdot 460 E \\ 65 & 330 & 15 \cdot 220 N & 53 \cdot 110 E \\ 65 & 330 & 15 \cdot 16 O N & 54 \cdot 380 E \\ 65 & 331 & 14 \cdot 56 O N & 57 \cdot 210 E \\ 71 & 44 & 14 \cdot 017 N & 51 \cdot 486 E \\ 71 & 44 & 14 \cdot 017 N & 51 \cdot 486 E\end{array}$

$67 \cdot 210007$ $67 \cdot 210007$ 67.030008 67.030008
$67.77597 A$ $67.77 \quad 0597$ 67.645978 67.640019 67.530599 67.540600 67.54
67.470600 67.470602 67.410025

1602. 190. 0000

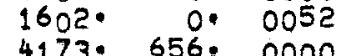
4173. 656. 0000 4173. 0.0039 $1805^{\circ}$ 86. 0000 1805.17 .0000 922. 90. 0000 2939. 670. 0000 2292. $110 \cdot 0000$ 899. 100. 0000 3357. 106. 0000 539. 570. 000 5329. 0.0119

MARSDEN SOUARE \#68

$\begin{array}{lrrlll}\text { AlI } & 15 & 3 & 0545 & 0000 & 18 \\ \text { AII } & 15 & 4 & 0546 & 0000 & 15 \\ \text { CHN } & 43 & 1 & 0004 & 0000 & 13 \\ \text { CHN } & 43 & 1 & 0005 & 0000 & 13 \\ \text { CHN } & 100 & 4 & 0032 & 0000 & 15\end{array}$

$\begin{array}{llll}65 & 219 & 16 \cdot 34 \mathrm{ON} & 41.03 O E \\ 65 & 223 & 11.53 \mathrm{ON} & 48.373 \mathrm{E} \\ 64 & 326 & 17.39 \mathrm{ON} & 40.100 \mathrm{E} \\ 64 & 326 & 17.39 \mathrm{ON} & 40.100 \mathrm{O} \\ 71 & 41 & 12.234 \mathrm{~N} & 43.422 \mathrm{E}\end{array}$

$68 \cdot 610^{545}$ $68 \cdot 18000^{6}$

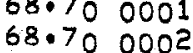
$68 \cdot 23002^{4}$

$\begin{array}{rrr}19810^{\circ} & 42 \cdot & 0000 \\ 2136 \cdot & 160^{\circ} & 0000 \\ 1296 \cdot & 90^{\circ} & 0000 \\ 1470^{\circ} & 233 \cdot & 0000 \\ 313^{\circ} & 90 \cdot & 0000\end{array}$

$\begin{array}{lll}16 & 3439 & 0 \\ 16 & 3459 & 0 \\ 16 & 3326 & 0\end{array}$

33260

3328
8468 54 GULF ADEN

\section{MARSDEN SQUARE \#69}

$\begin{array}{lrllll}\text { CHN } & 61 & 7 & 0152 & 0000 & 15 \\ \text { CHN } & 61 & 7 & 0152 & 0200 & 26 \\ \text { CHN } & 61 & 7 & 0155 & 0000 & 15 \\ \text { CHN } & 61 & 7 & 0155 & 0000 & 26 \\ \text { CHN } & 100 & 3 & 0001 & 000 & 13 \\ \text { CHN } & 100 & 3 & 0002 & 0000 & 13 \\ \text { CHN } & 100 & 3 & 0.03 & 0000 & 15\end{array}$

$69 \cdot 980152$ $69 \cdot 980152$ $69 \cdot 98 \quad 0155$ 69.980155 69.790001 69.700002 69.890003

$\begin{array}{rrrr}2359 \cdot & 711: & 0000 & 10 \\ 2359 \cdot & 0: & 0045 & 10 \\ 2046 \cdot & 424 \cdot & 0000 & 10 \\ 2046 . & 0 \cdot & 0117 & 10 \\ 176 . & 42 \cdot & 0000 & 99 \\ 169 . & 97 \cdot & 0000 & 99 \\ 1374 . & 798 . & 0000 & 16\end{array}$

77590

37310

48170

4867 .

3359 O RED SEA

3725 O RED SEA

386854 


\section{4}

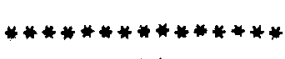

$* * * * * * * * * * * * * * *$

STATION CATA RETRIEVAL

DATE: $12: 36$ MAY 16,175

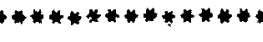

$* * * * * * * * * * * * * *$

CQRE LENGTH

LENGTH DREDGE

MARS - CORE OR

OR $\quad$ GR

PHYSIO- OR

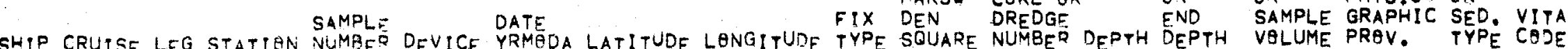

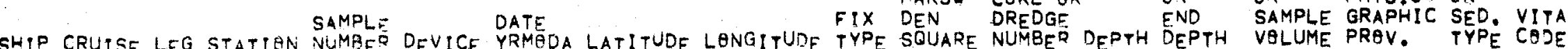
MARSDEN SQUARE \# 69

$\begin{array}{llllll}\text { CHN } & 100 & 3 & 0003 & 0000 & 15 \\ \text { CHN } & 100 & 3 & 0005 & 0000 & 15 \\ \text { CHN } & 100 & 3 & 0005 & 0000 & 26 \\ \text { CHN } & 100 & 3 & 0006 & 0000 & 13 \\ \text { CHN } & 100 & 3 & 0007 & 0000 & 15\end{array}$

$\begin{array}{lllll}71 & 3 & 3 & 18.090 \mathrm{ON} & 39 \cdot 530 \mathrm{E} \\ 71 & 3 & 4 & 19 \cdot 05 \mathrm{ON}^{\mathrm{N}} & 39 \cdot 595 \mathrm{E} \\ 71 & 3 & 4 & 19 \cdot 050 \mathrm{~N} & 39 \cdot 595 \mathrm{E} \\ 71 & 3 & 5 & 19 \cdot 38 \mathrm{ON} & 38 \cdot 362 \mathrm{E} \\ 71 & 3 & 7 & 20.277 \mathrm{~N} & 39 \cdot 139 \mathrm{E}\end{array}$

69.890003 $69.99000^{4}$ $69.99000^{4}$ 69.980005 69.090006

$\begin{array}{rrrr}1374 \cdot & 0 & 0112 & 16 \\ 328: & 577: & 0000 & 16 \\ 328 \cdot & 0 \cdot & 0108 & 16 \\ 2010^{\circ} & 1011^{\circ} & 0000 & 16 \\ 646 \cdot & 636 \cdot & 0000 & 99\end{array}$

323254 323854 RED SEA 332954 RED SEA $\begin{array}{lll}3865 & 0 & \\ 3328 & 54 & R E D\end{array}$

\section{MARSDEN SQUARE \# 75}

\section{CHN $\quad 96 \quad 1^{4} 0001$ \\ $0000 \quad 15$ \\ $69116 \quad 27 \cdot 215 \mathrm{~N} \quad 21 \cdot 580^{N}$ \\ MARSDEN SQUARE \# 76}

$75 \cdot 710001$

4879. $750^{\circ} 0000$

6

$332^{9} 54$

$\begin{array}{llll}\text { CHN } & 96 & 14 & 0003 \\ \text { CHN } & 96 & 14 & 0003 \\ \text { CHN } & 99 & 2 & 00 C^{7} \\ \text { CHN } & 99 & 2 & 000^{8}\end{array}$

$\begin{array}{ll}0000 & 15 \\ 0000 & 26 \\ 0000 & 15\end{array}$

$\begin{array}{lll}69119 & 29 \cdot 105 \mathrm{~N} & 38.286 \mathrm{~W}\end{array}$ $69119 \quad 29.105 \mathrm{~N} \quad 38.286 \mathrm{~W}$ $70429 \quad 29 \cdot 184 \mathrm{~N} \quad 36 \cdot 367 \mathrm{~W}$ $0000 \quad 15$
$76 \cdot 980003$ 76.980003 $76 \cdot 960006$ $76 \cdot 92000^{7}$ $\begin{array}{rrrr}4760^{\circ} & 558: & 0000 & 15 \\ 4760^{\circ} & 0 & 0109 & 15 \\ 39366^{\circ} & 732 . & 0000 & 14 \\ 430^{\circ} & 719 . & 0000 & 19\end{array}$

$\begin{array}{lll}15 & 3731 & 54 \\ 15 & 3329 & 54 \\ 14 & 3739 & 54 \\ 19 & 3329 & 54\end{array}$

332954
PAGE 10

*WHSI**

OCK
MARSDEN SQUARE \# 77

$\begin{array}{llll}A I I & 42 & 1 & 0033 \\ C H N & 21 & 1 & 0002 \\ C H N & 21 & 1 & 0003 \\ C H N & 21 & 1 & 0003 \\ C H N & 21 & 1 & 0004 \\ C H N & 21 & 1 & 000^{5} \\ C H N & 21 & 1 & 000^{6} \\ C H N & 21 & 1 & 0006 \\ C H N & 21 & 1 & 0007 \\ C H N & 21 & 1 & 000^{7} \\ C H N & 21 & 1 & 000^{8} \\ C H N & 21 & 1 & 000^{9} \\ C H N & 21 & 1 & 000^{9} \\ C H N & 21 & 1 & 0010 \\ C H N & 21 & 1 & 0010 \\ C H N & 21 & 1 & 0011 \\ C H N & 21 & 1 & 0011 \\ C H N & 21 & 1 & 0012 \\ C H N & 21 & 1 & C 013 \\ C H N & 61 & 10 & 0174\end{array}$

$\begin{array}{ll}0000 & 15 \\ 0000 & 13 \\ 0000 & 15 \\ 0000 & 13 \\ 0000 & 13 \\ 0000 & 15 \\ 0000 & 15 \\ 0000 & 13 \\ 0000 & 15 \\ 0000 & 13 \\ 0000 & 15 \\ 0000 & 15 \\ 0000 & 13 \\ 0000 & 15 \\ 0000 & 13 \\ 0000 & 15 \\ 0000 & 13 \\ 0000 & 15 \\ 0000 & 15 \\ 0000 & 15\end{array}$

\begin{tabular}{|c|c|c|c|}
\hline \multirow{2}{*}{\multicolumn{4}{|c|}{$8.565 \mathrm{~W}$}} \\
\hline & & & \\
\hline 1 & 829 & $2^{9} \cdot 000 \mathrm{~N}$ & $47 \cdot 220 \mathrm{~W}$ \\
\hline $31+2>$ & $82^{9}$ & $29.000 \mathrm{~N}$ & $47 \cdot 220$ \\
\hline 61 & $\begin{array}{l}930 \\
830\end{array}$ & $28 \cdot 505 N$ & $44.563 \mathrm{~W}$ \\
\hline 1 & 1 & $29 \cdot 0^{59} \mathrm{~N}$ & $44 \cdot 332 W$ \\
\hline 1 & 91 & $29.259 \mathrm{~N}$ & $44 \cdot 332 W$ \\
\hline 51 & 2 & $29 \cdot 0^{47} \mathrm{~N}$ & $44 \cdot 162 \mathrm{~W}$ \\
\hline 3 & 2 & $29 \cdot 0^{47} \mathrm{~N}$ & $44 \cdot 162 W$ \\
\hline 1 & 2 & $29 \cdot 0^{5} 0^{N}$ & $44^{\circ} 111^{N} \mathrm{~N}$ \\
\hline s & 2 & HON & $43^{\circ} 3636$ \\
\hline ?] & 93 & $29 \cdot-32 N$ & $43 \cdot 110 w$ \\
\hline 61 & & $29.032 \mathrm{~N}$ & $43^{\circ} 110 \mathrm{~W}$ \\
\hline & 3 & 28 & Oon \\
\hline & 3 & $28.52 \mathrm{2} N$ & $42 \cdot 540 \mathrm{~N}$ \\
\hline & 94 & $28 \cdot 513 N$ & $42 \cdot 484 \mathrm{~h}$ \\
\hline & 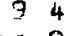 & 29 & 41.095 \\
\hline & & & $45 \cdot 550 \mathrm{~W}$ \\
\hline
\end{tabular}

$\begin{array}{ll}77.09 & 0021 \\ 77.88 & 0002 \\ 77.97 & 0003 \\ 77.97 & 0003 \\ 77.86 & 0004 \\ 77.84 & 0005 \\ 77.94 & 0006 \\ 77.94 & 0006 \\ 77.94 & 0007 \\ 77.94 & 0007 \\ 77.94 & 0008 \\ 77.83 & 0009 \\ 77.83 & 0009 \\ 77.93 & 0010 \\ 77.93 & 0010 \\ 77.82 & 0011 \\ 77.82 & 0011 \\ 77.82 & 0012 \\ 77.91 & 0013 \\ 77.75 & 0174\end{array}$

4598 $4654^{\circ}$ $47,5$. 4376 . 3923. 3923. 3328. 3328. $3777^{\circ}$ 3798 . $3771^{\circ}$ $2944^{\circ}$ 3072 . 3065. $3500^{\circ}$ $3500^{\circ}$ $3529^{\circ}$ $\begin{array}{lll}3474 \cdot & 80 \\ 4027 \cdot & 10^{8}: & 0000 \\ 4 & 48000\end{array}$ $\begin{array}{lll}4148 \cdot 708 \cdot & 10000 \\ & & \end{array}$ 
$* * * * * * * * * * * * * *)$ CORE PILOT
LENGTH DREDTH, MARS- CORE $Q R$ QR OR OR PHYSIO- OR SAMPLF. DATE FIX DEN DREDGE END SAMPLE GRAPHIC SED. VITA

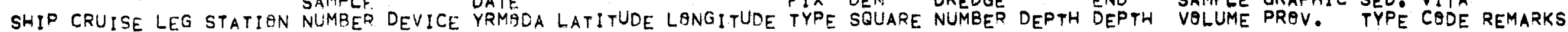

MARSDEN SQUARE \# 77

$\begin{array}{llll}\text { CHN } & 61 & 10 & 0175 \\ \text { CHN } & 61 & 10 & 0177 \\ \text { CHN } & 61 & 10 & 0178 \\ \text { CHN } & 96 & 14 & 0004 \\ \text { CHN } & 96 & 14 & 0004 \\ \text { CHN } & 99 & 2 & 000^{4} \\ \text { CHN } & 99 & 2 & 0006\end{array}$

$\begin{array}{ll}0000 & 15 \\ 0200 & 15 \\ 0000 & 15 \\ 0000 & 15 \\ 0000 & 26 \\ 0000 & 15 \\ 0000 & 15\end{array}$

$\begin{array}{lll}66129 & 27.525 N & 45 \cdot 105 W \\ 661211 & 28.19 O N & 45.320 W \\ 661211 & 28.435 N & 46.480 W \\ 691112 & 29.528 N & 41.194 W \\ 691112 & 29 \cdot 528 N & 41.194 W \\ 70427 & 29 \cdot 278 N & 41.343 W \\ 70427 & 29 \cdot 221 N & 40.513 W\end{array}$

$\begin{array}{ll}77.75 & 0175 \\ 77.85 & 0000 \\ 77.86 & 0000 \\ 77.91 & 000^{4} \\ 77.91 & 000^{4} \\ 77.91 & 0004 \\ 77.90 & 000^{5}\end{array}$

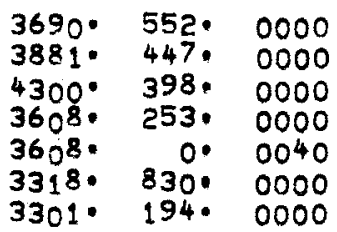

10
99
99
14
14
14
14

$\begin{array}{lll}3329 & 0 & \\ 3359 & 54 & \\ 3969 & 54 & \\ 3329 & 54 & \\ 3329 & 54 & \\ 3731 & 54 & \text { MAR } \\ 3323 & 54 & \text { MAR }\end{array}$

MARSDEN SQUARE \# 78

$\begin{array}{llllll}A ! I & 31 & 1 & 0020 & 0000 & 15 \\ A ! & 31 & 1 & 0020 & 0000 & 26 \\ A ! I & 42 & 1 & 0034 & 0000 & 15 \\ A ! ! & 42 & 1 & 0035 & 0000 & 15 \\ A ! I & 42 & 1 & 0038 & 0000 & 15 \\ A ! I & 42 & 1 & 0039 & 0000 & 15 \\ A ! I & 42 & 1 & 0041 & 0000 & 15 \\ A ! ! & 42 & 1 & 0042 & 0000 & 15 \\ A ! I & 42 & 1 & 0042 & 0000 & 26 \\ A ! ! & 42 & 1 & 0373 & 0000 & 13 \\ \text { CHN } & 21 & 1 & 0001 & 0000 & 13 \\ \text { CHN } & 39 & 1 & 0001 & 0000 & 13 \\ \text { CHN } & 39 & 1 & 0004 & 0000 & 13 \\ \text { CHN } & 39 & 1 & 0007 & 0000 & 13 \\ \text { CHN } & 39 & 1 & 0009 & 0000 & 13 \\ \text { CHN } & 39 & 1 & 0010 & 0000 & 13\end{array}$

$\begin{array}{lllll}67 & 426 & 21 \cdot 285 \mathrm{~N} & 60 \cdot 290 \mathrm{~W} & 1 \\ 67 & 426 & 21 \cdot 285 \mathrm{~N} & 60 \cdot 290 \mathrm{~W} & 1 \\ 68 & 719 & 20 \cdot 205 \mathrm{~N} & 50 \cdot 518 \mathrm{~W} & 1 \\ 68 & 719 & 20 \cdot 375 \mathrm{~N} & 51 \cdot 515 \mathrm{~W} & 1 \\ 68 & 720 & 21 \cdot 185 \mathrm{~N} & 53 \cdot 580 \mathrm{~W} & 1 \\ 68 & 721 & 21 \cdot 440 \mathrm{~N} & 55 \cdot 020 \mathrm{~W} & 1 \\ 68 & 721 & 22 \cdot 140 \mathrm{~N} & 56 \cdot 390 \mathrm{~W} & 1 \\ 68 & 722 & 24 \cdot 160 \mathrm{~N} & 58 \cdot 230 \mathrm{~W} & 1 \\ 68 & 722 & 24 \cdot 16 \mathrm{~N} & 58 \cdot 230 \mathrm{~W} & 1 \\ 68 & 720 & 21 \cdot 000 \mathrm{~N} & 52 \cdot 560 \mathrm{~W} & 1 \\ 61 & 827 & 29 \cdot 510 \mathrm{~N} & 54 \cdot 352 \mathrm{~W} & 5 \\ 63 & 96 & 29 \cdot 000 \mathrm{~N} & 59 \cdot 130 \mathrm{~W} & 5 \\ 63 & 910 & 25 \cdot 180 \mathrm{~N} & 55 \cdot 445 \mathrm{~W} & 5 \\ 63 & 911 & 24 \cdot 033 \mathrm{~N} & 55 \cdot 150 \mathrm{~W} & 5 \\ 63 & 913 & 27 \cdot 555 \mathrm{~N} & 57 \cdot 00 \mathrm{~W} & 5 \\ 63 & 914 & 28 \cdot 300 \mathrm{~N} & 57 \cdot 590 \mathrm{~W} & 5\end{array}$

$78 \cdot 100020$ $78 \cdot 100020$ 78.000022 78.010023 $78.13002^{4}$ $78 \cdot 150025$ $78 \cdot 26 \quad 0027$ $78.48 \quad 0028$ $78 \cdot 48002^{8}$ 78.120001 78.940001 78.990001

78.550002

78.450003 78.45
78.77 0003 $78.87000^{5}$

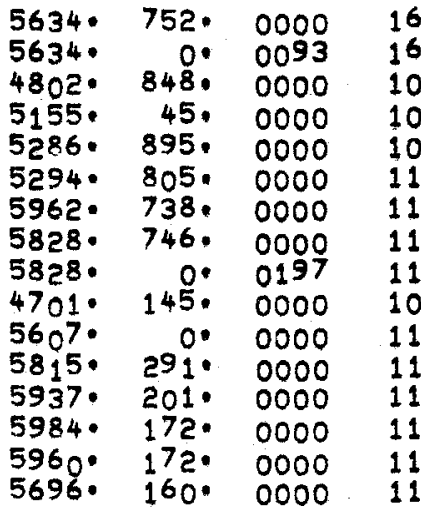

MARSDEN SQUARE \# 79

\begin{tabular}{|c|c|c|c|c|}
\hline $\begin{array}{l}A ! ! \\
A ! I \\
A ! ! \\
A ! I \\
A ! ! \\
A ! ! \\
A ! ! \\
A ! ! \\
A ! I \\
A ! I \\
A ! ! \\
A ! !\end{array}$ & $\begin{array}{l}31 \\
31 \\
42 \\
42 \\
42 \\
60 \\
60 \\
60 \\
60 \\
60 \\
60 \\
60\end{array}$ & $\begin{array}{l}1 \\
1 \\
1 \\
1 \\
1 \\
1 \\
8 \\
8 \\
8 \\
8 \\
8 \\
8 \\
8\end{array}$ & $\begin{array}{l}0021 \\
0022 \\
0043 \\
0043 \\
0044 \\
0003 \\
000^{5} \\
000^{6} \\
000^{7} \\
000^{8} \\
000^{8} \\
000^{9}\end{array}$ & $\begin{array}{l}0000 \\
0000 \\
0000 \\
0000 \\
0000 \\
0000 \\
0000 \\
0200 \\
0200 \\
0000 \\
0200 \\
0000\end{array}$ \\
\hline
\end{tabular}

79.610021 79.730022

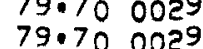
79.700029 79.920030 $79 \cdot 39002 C$ $79.48003 \mathrm{C}$ $79 \cdot 380001$ 79.380002 79.380004 79.380003 $79 \cdot 390004$
5884. 521. $0000 \quad 16$ 5380. 92. 000010 5629. 257. 000010 5265. 911: 0000.10 5566. 61. $0000 \cdot 13$ 5349. $96.0000: 13$ 5204. 203. 0000 13 5202. 92. 000013 5398. 89. $0000 \quad 13$ 5629. 0.016510 5768.270 .0000
PAGE 11

332354 MAR
$7136 \quad 23.35 \mathrm{ON} 68.540 \mathrm{~W}$

$7136 \quad 23 \cdot 35 \mathrm{cN} \quad 68 \cdot 540$

$23 \cdot 256 \mathrm{~N} \quad 69 \cdot 048 \mathrm{~W}$ 
0046

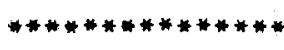

$* * * * * * * * * * * * *$

DATE: $12 \$ 36$ MAY 16,175
STATION DATA RETRIEVAL

PAGE 12

$*$ WHOI**

PILQT

LERE LENGTH,

CENGTH DREDGE ROCK

MARS - CORE OR OR OR PHYSIO. OR

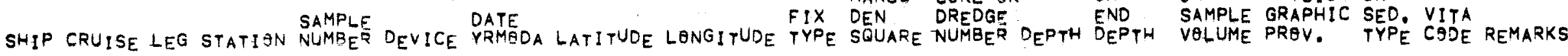

MARSDEN SQUARE \# 79

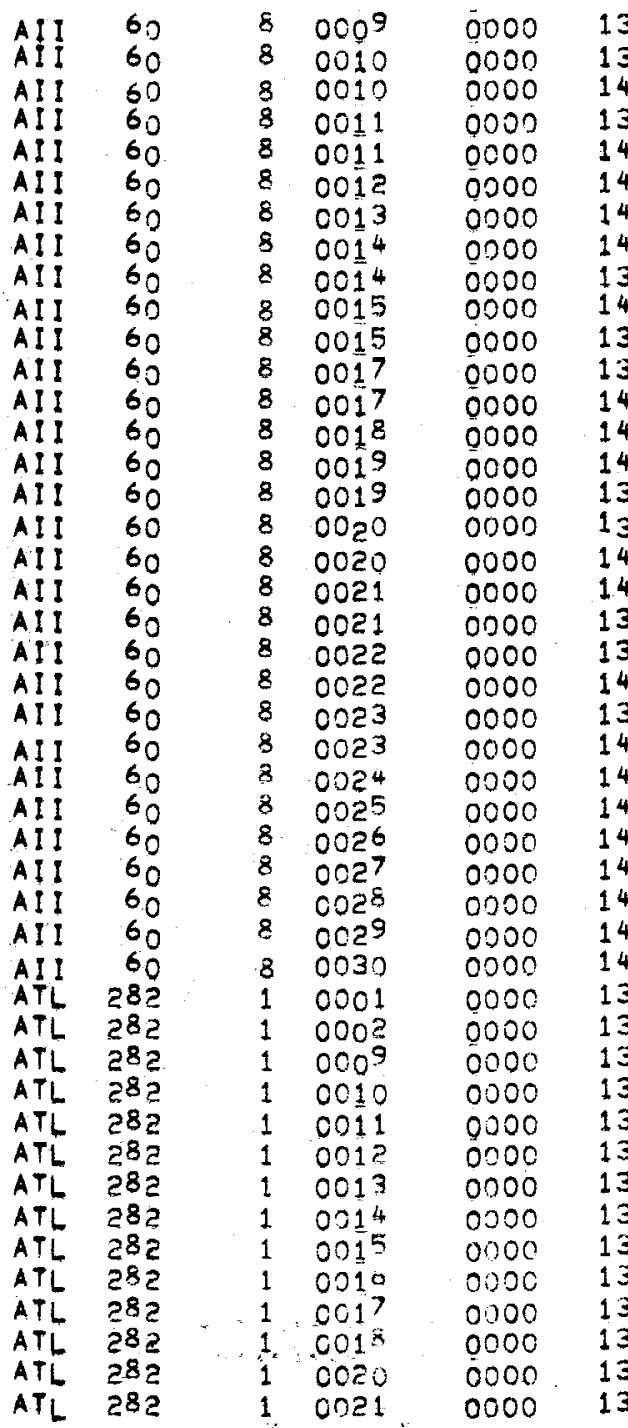

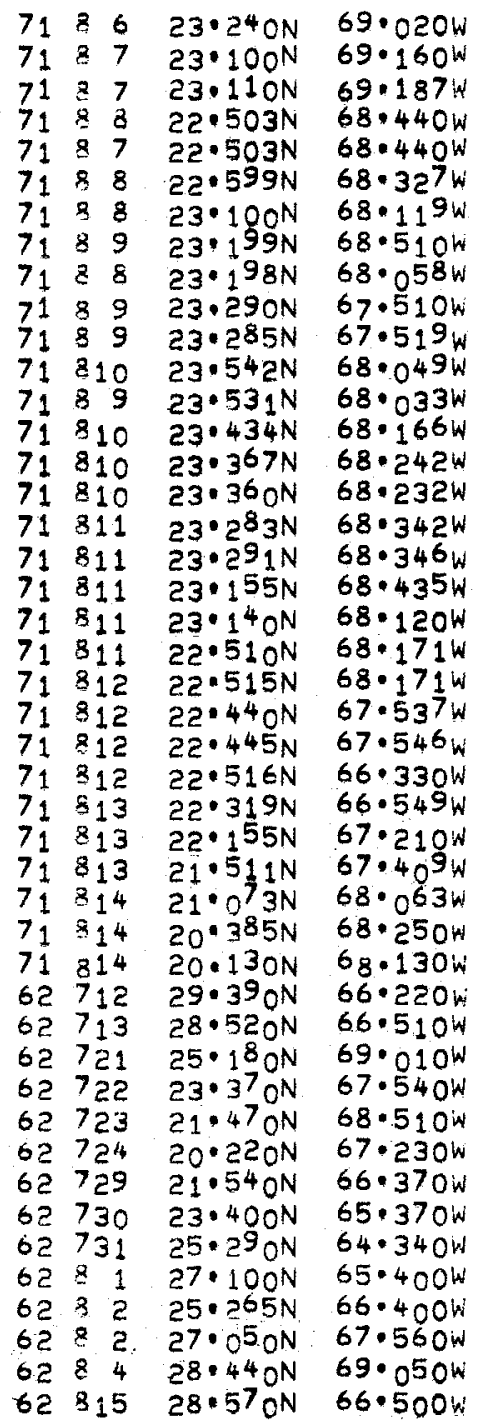

$\begin{array}{ll}79 \cdot 39 & 0005 \\ 79 \cdot 39 & 0006 \\ 79 \cdot 39 & 0005 \\ 79 \cdot 28 & 0007 \\ 79 \cdot 28 & 0006 \\ 79 \cdot 28 & 0007 \\ 79 \cdot 38 & 0008 \\ 79 \cdot 38 & 0009 \\ 79 \cdot 38 & 0010 \\ 79 \cdot 37 & 0010 \\ 79 \cdot 37 & 0011 \\ 79 \cdot 38 & 0013 \\ 79 \cdot 38 & 0012 \\ 79 \cdot 38 & 0013 \\ 79 \cdot 38 & 0014 \\ 79 \cdot 38 & 0015 \\ 79 \cdot 38 & 0016 \\ 79 \cdot 38 & 0015 \\ 79 \cdot 38 & 0016 \\ 79 \cdot 38 & 0017 \\ 79 \cdot 28 & 0018 \\ 79 \cdot 28 & 0017 \\ 79 \cdot 27 & 0019 \\ 79 \cdot 27 & 0018 \\ 79 \cdot 27 & 0019 \\ 79 \cdot 26 & 0020 \\ 79 \cdot 27 & 0021 \\ 79 \cdot 17 & 0022 \\ 79 \cdot 18 & 0023 \\ 79 \cdot 18 & 0024 \\ 79 \cdot 08 & 0025 \\ 79 \cdot 96 & 0001 \\ 79 \cdot 86 & 0002 \\ 79 \cdot 59 & 0009 \\ 79 \cdot 37 & 0010 \\ 79 \cdot 18 & 0011 \\ 79 \cdot 07 & 0012 \\ 79 \cdot 16 & 0013 \\ 79 \cdot 35 & 0014 \\ 79 \cdot 54 & 0015 \\ 79 \cdot 75 & 0016 \\ 79 \cdot 56 & 0017 \\ 79 \cdot 77 & 0018 \\ 79 \cdot 89 & 0020 \\ 79 \cdot 86 & 0021 \\ 7 & \end{array}$

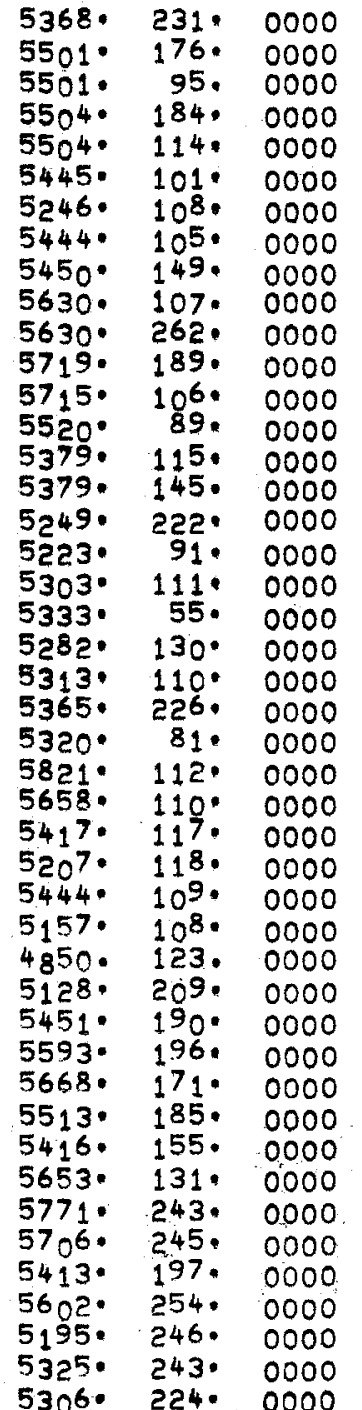

$\begin{array}{lll}13 & 3436 & 0 \\ 13 & 4139 & 0 \\ 13 & 4459 & 0 \\ 13 & 4439 & 0 \\ 13 & 4459 & 0 \\ 13 & 4969 & 0 \\ 13 & 3329 & 0 \\ 13 & 3359 & 0 \\ 13 & 4429 & 0 \\ 13 & 4969 & 0 \\ 13 & 4339 & 0 \\ 13 & 1439 & 0 \\ 13 & 3359 & 0 \\ 13 & 3359 & 0 \\ 13 & 3359 & 0 \\ 13 & 4329 & 0 \\ 13 & 4316 & 0 \\ 13 & 3359 & 0 \\ 13 & 3969 & 0 \\ 13 & 3359 & 0 \\ 13 & 4969 & 0 \\ 13 & 3359 & 0 \\ 13 & 3969 & 0 \\ 13 & 3359 & 0 \\ 13 & 4339 & 0 \\ 13 & 4459 & 0 \\ 13 & 4969 & 0 \\ 13 & 3359 & 0 \\ 13 & 3359 & 0 \\ 13 & 3359 & 0 \\ 13 & 3359 & 0 \\ 13 & 4129 & 0 \\ 13 & 3459 & 0 \\ 10 & 4159 & 0 \\ 10 & 4159 & 0 \\ 10 & 1859 & 0 \\ 10 & 4143 & 0 \\ 10 & 4159 & 0 \\ 10 & 1159 & 0 \\ 10 & 1159 & 0 \\ 13 & 1453 & 0 \\ 13 & 4153 & 0 \\ 13 & 1449 & 0 \\ 13 & 4129 & 0 \\ 13 & 1429 & 0 \\ & & \end{array}$




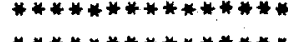

$* * * * * * * * * * * * * *$
PAGE 13

**WHQI**

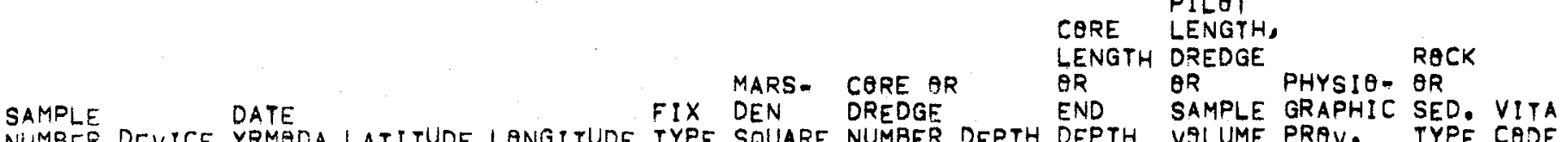

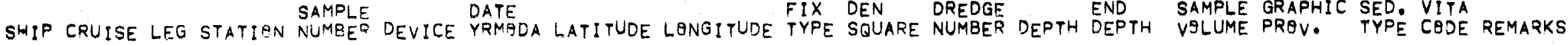

MARSDEN SQUARE \# 79

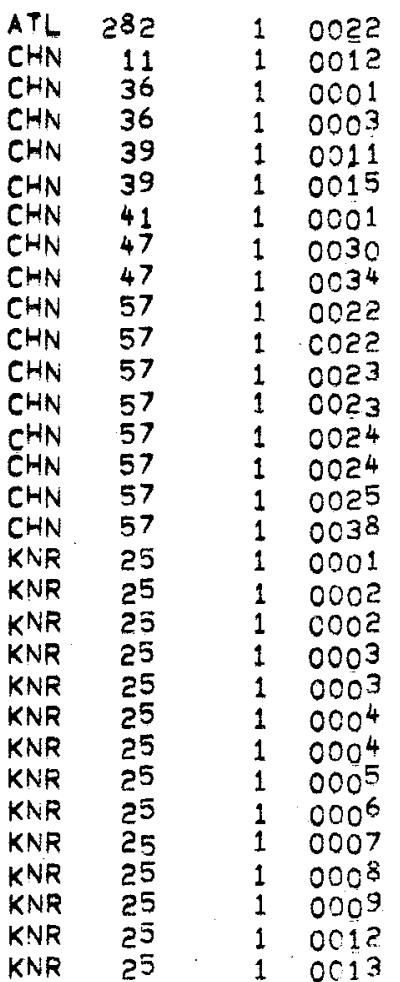

$\begin{array}{ll}0000 & 13 \\ 0000 & 15 \\ 0000 & 15 \\ 0000 & 15 \\ 0000 & 13 \\ 0000 & 13 \\ 0000 & 15 \\ 0000 & 18 \\ 0000 & 18 \\ 0000 & 15 \\ 0000 & 26 \\ 0000 & 15 \\ 0000 & 26 \\ 0000 & 15 \\ 0000 & 26 \\ 0000 & 15 \\ 0000 & 15 \\ 0000 & 14 \\ 0000 & 14 \\ 0000 & 16 \\ 0000 & 14 \\ 0000 & 17 \\ 0000 & 14 \\ 0000 & 16 \\ 0000 & 14 \\ 0000 & 14 \\ 0000 & 14 \\ 0000 & 14 \\ 0000 & 14 \\ 0000 & 13 \\ 0000 & 13\end{array}$

$628628.54 \mathrm{ON} 64.390 \mathrm{~W}$

$63620 \quad 21.33 \mathrm{~N} 65 . \mathrm{c25W}$

$63622 \quad 20.180 \mathrm{~N} \quad 63.395 \mathrm{~W}$

6391629.55 oN $60.315 \mathrm{~W}$

$6391929.465 \mathrm{~N} 62.115 \mathrm{~W}$

631 ? $521.530 \mathrm{~N} \quad 70 \cdot 160 \mathrm{~W}$

$655429.305 \mathrm{~N} \quad 67.094 \mathrm{~W}$

$\begin{array}{lllll}65 & 5 & 5 & 30 \cdot 307 \mathrm{~N} & 66 \cdot 520 \mathrm{~W}\end{array}$

$66 \quad 375 \quad 22 \cdot 407 N$

$\begin{array}{llll}66 & 325 & 22.407 N & 66.296 \mathrm{~W}\end{array}$

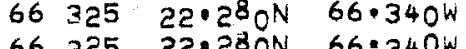

$6632522.280 \mathrm{~N} 66.340 \mathrm{~W}$

$66 \quad 329 \quad 22.423 \mathrm{~N} 67.422 \mathrm{~W}$

$66329 \quad 22.400 \mathrm{~N} \quad 67.410 \mathrm{~W}$

$6641121 \cdot 308 \mathrm{~N} \quad 68.147 \mathrm{~W}$

$72210 \quad 25.015 \mathrm{~N} \quad 68.035 \mathrm{~W}$

$72210 \quad 24.422 \mathrm{~N} 68.080 \mathrm{~W}$

$72215 \quad 23.556 \mathrm{~N} \quad 68 \cdot 364 \mathrm{~W}$

$\begin{array}{llll}72 & 211 & 24 \cdot 238 \mathrm{~N} & 68 \cdot 114 \mathrm{~W}\end{array}$

$72316 \quad 23.452 \mathrm{~N} \quad 69.40^{8} \mathrm{~W}$

$72216 \quad 21 \cdot 300 \mathrm{~N} \quad 67 \cdot 310 \mathrm{~W}$

$72 \geq 12 \quad 23.485 \mathrm{~N} \quad 69.545 \mathrm{~N}$

72 2.12 23.526N 68.351

$72213 \quad 23.435 \mathrm{~N} \quad 68.415 \mathrm{~W}$

$\begin{array}{llll}72 & 213 & 23.572 \mathrm{~N} & 68.590 \mathrm{~W} \\ 72 & 213 & 23.24 \mathrm{ON} & 69.061 \mathrm{~W}\end{array}$

$7221423.467 \mathrm{~N} \quad 69.423 \mathrm{~W}$

$72215 \quad 22 \cdot 154 \mathrm{~N} \quad 67.569 \mathrm{~N}$

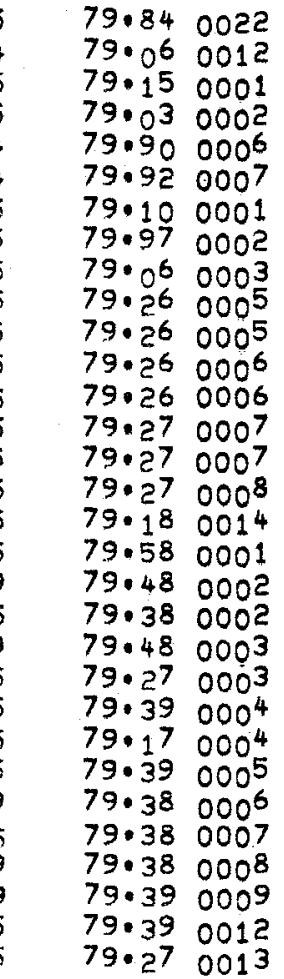

MARSDEN SOUARE \# 80

$\begin{array}{lrllll}\text { AII } & 1 & 1 & 0005 & 0000 & 15 \\ \text { ATL } & 882 & 1 & 0003 & 0000 & 13 \\ \text { ATL } & 282 & 1 & 0005 & 0000 & 13 \\ \text { ATL } & 282 & 1 & 0006 & 0000 & 13 \\ \text { ATL } & 282 & 1 & 0007 & 0000 & 13 \\ \text { ATL } & 282 & 1 & 0009 & 0000 & 13 \\ \text { ATL } & 282 & 1 & 0019 & 0000 & 13 \\ \text { CHN } & 53 & 1 & 0001 & 0000 & 18 \\ \text { CHN } & 53 & 1 & 0002 & 0000 & 18\end{array}$

$63228 \quad 29 \cdot 100 \mathrm{~N} \quad 76 \cdot 220 \mathrm{w}$

$\begin{array}{llll}62 & 716 & 23.325 \mathrm{~N} & 70.020 \mathrm{~W} \\ 62 & 717 & 23.28 \mathrm{~N} & 72.185 \mathrm{~W}\end{array}$

$6271825.135 \mathrm{~N} 73.160 \mathrm{~N}$

6271926.59 oN 72.130N

$62720 \quad 25 \cdot 110 \mathrm{~N} \quad 71.160 \mathrm{~W}$

$62: 3 \quad 27.740 \mathrm{~N} 70.100 \mathrm{~W}$

$651220 \quad 28.490 \mathrm{~N} 70.525 \mathrm{~W}$

$651 \mathrm{C21} 28.530 \mathrm{~N} \quad 70.545 \mathrm{~W}$
$80 \cdot 960005$ $\begin{array}{ll}80 \cdot 30 & 0003 \\ 80.32 & 0005\end{array}$ 80.530006 80.620007 $80.51000^{8}$ $80 \cdot 700019$ 80.800001 80.800002
$60229 \quad 20.500 \mathrm{~N} \quad 66.2704$
4846. 301. 0000

$5306 \cdot 173.0000$

5728.41 .0000

5705.193 .0000

4897. 212. 0000

5492. 0.0000

5096. 100. 0000

$50^{\circ} \cdot 100^{\circ}, 0000$

$5820^{\circ} \quad 0.0085$

5613. 816. 0000

$5613^{\circ} \quad 0.0088$

5531. 895. 0000

5531. 0.0080

5389. 927. 0000

551. 0000

5523. 61. 0000

5689. 147. 0000

5515. 832. 0000

5729. 150. 0000

5374. 1139. 0000

5392. 136. 0000

$5163 \cdot 2159 \cdot 0000$

$540^{9} \cdot 140 \cdot 0000$

5486. 145. 0000

$5306 \cdot 145 \cdot 0000$

5451 . 142. 0000

5411.147 .0000

$\begin{array}{lll}5419^{\circ} & 68 . & 0000 \\ 5374 . & 39 . & 0000\end{array}$
4548. 550. 0000

$\begin{array}{llll}13 & 3469 & 0 & \\ 17 & 1179 & 0 & \\ 17 & 4122 & 0 & \\ 17 & 1223 & 0 & \\ 11 & 1143 & 0 & \\ 11 & 3149 & 0 & \\ 13 & 0000 & 0 & \text { IN JAR } \\ 99 & 3422 & 54 & \text { SW BERMUDA } \\ 99 & 1349 & 54 & \text { SW BERMUDA }\end{array}$

134954 SW BERMUDA

184954

41290

142954 NARES BASIN

115954 NARES BASIN

483954 NARES BASIN

143954 NARES BASIN

242954

087954

000040

000040

00000

000040

$\begin{array}{ll}2260 & 0 \\ 4459 & 0\end{array}$

00120

001040

001040

001040

001040
001040

001040

0010

4994. 285. 0000
5492. 120. 0000 5287. 164. 0000 5316. 188, 0000 $5154^{\circ} \quad 228 \cdot 0000$ $5520^{\circ} 30^{\circ} 0000$ $5482 \cdot 113.0000$ 2949.43 .0000 3791 . 66. 0000

$\begin{array}{rrr}10 & 3739 & 0 \\ 10 & 4841 & 0 \\ 10 & 4149 & 0 \\ 10 & 4859 & 0 \\ 10 & 4129 & 0 \\ 10 & 4869 & 0 \\ 13 & 4249 & 0 \\ 99 & 2839 & 0 \\ 2 & 4839 & 0\end{array}$


0048

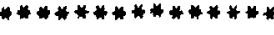

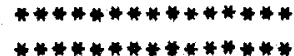

PAGE 14

**WHQ I**

\section{CORE LENGTH,
MENS - CORE OR RATH DREDGE POCK \\ SAMPLF DATE FIX DEN DREDGE END SAMPLE GRAPHIC SED. VITA}

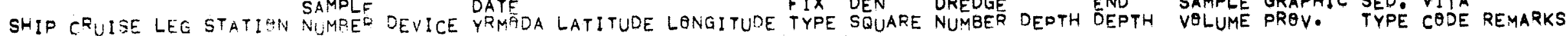

MARSDEN SQUARE \# 80

$\begin{array}{llllll}\text { CHN } & 53 & 1 & 0003 & 0000 & 18 \\ \text { CHN } & 53 & 1 & 0004 & 0000 & 18 \\ \text { KNR } & 25 & 6 & 0225 & 0000 & 13 \\ \text { KNR } & 31 & 4 & 0007 & 0000 & 16 \\ \text { KNR } & 31 & 4 & 0008 & 0000 & 16 \\ \text { KNR } & 31 & 4 & 0009 & 0000 & 16 \\ \text { KNR } & 31 & 5 & 0010 & 0000 & 14 \\ \text { KNR } & 31 & 5 & 0011 & 0000 & 16 \\ \text { KNR } & 31 & 5 & 0012 & 0000 & 16 \\ \text { KNR } & 31 & 5 & 0013 & 0000 & 14 \\ \text { KNR } & 31 & 5 & 0014 & 0000 & 14 \\ \text { KNR } & 31 & 5 & 0016 & 0000 & 18 \\ \text { KNR } & 31 & 5 & 0016 & 0000 & 18 \\ \text { KNR } & 31 & 5 & 0016 & 0000 & 18\end{array}$

\begin{tabular}{|c|c|c|}
\hline 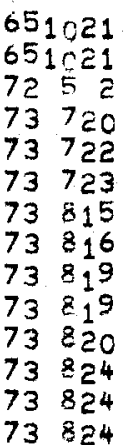 & $\begin{array}{l}28 \cdot 500 \mathrm{~N} \\
28 \cdot 440 \mathrm{~N} \\
22 \cdot 480 \mathrm{~N} \\
28 \cdot 179 \mathrm{~N} \\
28 \cdot 417 \mathrm{~N} \\
28 \cdot 147 \mathrm{~N} \\
28 \cdot 368 \mathrm{~N} \\
28 \cdot 38 \mathrm{ON} \\
28 \cdot 356 \mathrm{~N} \\
28 \cdot 357 \mathrm{~N} \\
28 \cdot 15 \mathrm{ON} \\
28.161 \mathrm{~N} \\
28.161 \mathrm{~N} \\
28 \cdot 161 \mathrm{~N}\end{array}$ & $\begin{array}{l}70 \cdot 540 w \\
70 \cdot 556 w \\
71 \cdot 300 w \\
72 \cdot 178 w \\
75 \cdot 160 W \\
74 \cdot 264 w \\
75 \cdot 195 w \\
75 \cdot 215 w \\
75 \cdot 273 w \\
75 \cdot 255 W \\
75 \cdot 245 w \\
75 \cdot 253 w \\
75 \cdot 253 w \\
75 \cdot 253 w\end{array}$ \\
\hline
\end{tabular}

80.800003 $80 \cdot 800004$ 80.210001 $80.82 \quad 0007$ 80.850008 80.840009 80.850010 80.850011 80.850012 80.850013 80.850014 80.850016 80.850018 80.850019

3957. 34, 0000 4428. 26, 0000 5152. 110. 0000 4935. 4158:0000 4962. 2946. 0000 4758. 3689, 0000 4967. 99, 0000 4967, 2464, 0000 498. 1399. 0000 4982. 106, 0000 4765. 110. 0000 $4780^{\circ}$ 76, 0000 4780. 76. 0000 4773. 96. 0000

$\begin{array}{lll}2 & 2849 & 0 \\ 2 & 2439 & 0 \\ 13 & 3269 & 0 \\ 13 & 4139 & 0 \\ 13 & 4331 & 0 \\ 13 & 4139 & 0 \\ 13 & 4439 & 0 \\ 13 & 4339 & 0 \\ 10 & 4331 & 0 \\ 10 & 3339 & 0 \\ 13 & 3359 & 0 \\ 13 & 3433 & 0 \\ 13 & 3439 & 0 \\ 13 & 4269 & 0\end{array}$

$\begin{array}{llllll}\text { ATL } & 254 & 3 & 0325 & 0000 & 15 \\ \text { ATL } & 254 & 3 & 0325 & 0000 & 26 \\ \text { ATL } & 254 & 3 & 0326 & 0000 & 15 \\ \text { ATL } & 254 & 3 & 0325 & 0000 & 26 \\ \text { ATL } & 254 & 3 & 0327 & 0000 & 15 \\ \text { ATL } & 254 & 3 & 0328 & 0000 & 26\end{array}$

MARSDEN SQUARE \# 81

\begin{tabular}{|c|c|c|c|c|}
\hline $\begin{array}{ll}60 & 216 \\
60 & 216 \\
60 & 216 \\
60 & 216 \\
60 & 217 \\
60 & 217\end{array}$ & $\begin{array}{l}21.110 N \\
21.110 N \\
21.110 N \\
21.110 N\end{array}$ & $\begin{array}{l}82 . \\
82 . \\
82, \\
84 . \\
84 . \\
84 .\end{array}$ & $\begin{array}{l}5 \\
5 \\
5 \\
5\end{array}$ & 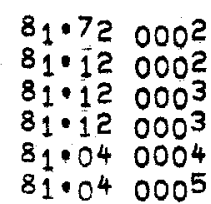 \\
\hline
\end{tabular}

4463. 218. 0000

4463. $530^{\circ} 0055$

3596. $03 \% 0000$

4500. 498. 0000

4568. 0.0072

$\begin{array}{lll}0 & 3725 & 0 \\ 0 & 3359 & 0 \\ 2 & 3090 & 0 \\ 2 & 3759 & 0 \\ 2 & 7439 & 0 \\ 2 & 3329 & 0\end{array}$

MARSDEN SQUARE \#102

$\begin{array}{llllll}A I I & 15 & 4 & 0585 & 0000 & 13 \\ \text { AII } & 15 & 5 & 0586 & 0000 & 15 \\ \text { AII } & 15 & 5 & 0586 & 0000 & 26 \\ \text { AII } & 15 & 5 & 0586 & 0000 & 13 \\ \text { A!I } & 15 & 5 & 0592 & 0000 & 18 \\ & 15 & 5 & 0594 & 0000 & 15\end{array}$

\begin{tabular}{|c|c|c|}
\hline $\begin{array}{ll}65 & 321 \\
65 & 321 \\
65 & 321 \\
65 & 321 \\
65 & 325 \\
65 & 325\end{array}$ & $\begin{array}{l}20 \cdot 090 \mathrm{~N} \\
20 \cdot 070 \mathrm{~N} \\
20.07 \mathrm{~N} \\
20 \cdot 075 \mathrm{~N} \\
20 \cdot 500 \mathrm{~N} \\
20 \cdot 35 \mathrm{ON}\end{array}$ & $\begin{array}{l}69 \cdot 260 E \\
67 \cdot 550 E \\
67 \cdot 550 E \\
67 \cdot 560 E \\
61 \cdot 010 E \\
63 \cdot 530 E\end{array}$ \\
\hline
\end{tabular}

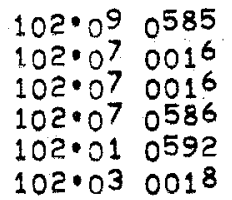

216. 80. 0000 $3047.1210^{\circ} 0000$ $30470^{\circ} \quad 0102$ 3628 87, 0000 3338 . 990. 0000

$\begin{array}{lll}2 & 3355 & 0 \\ 6 & 3422 & 0 \\ 6 & 3332 & 0 \\ 6 & 3332 & 0 \\ 6 & 3359 & 0 \\ 6 & 3860 & 0\end{array}$

MARSDEN SQUARE \# 103

$\begin{array}{llllll}A ! 1 & 15 & 5 & 0589 & 0000 & 15 \\ A ! 1 & 15 & 5 & 0589 & 0000 & 13 \\ A ! I & 15 & 5 & 0590 & 0000 & 13 \\ A ! 1 & 15 & 5 & 0591 & 0000 & 13\end{array}$

$\begin{array}{lllllll}65 & 324 & 24 \cdot 02 O N & 59.531 E & 9 & 103.49 & 0017 \\ 65 & 324 & 24 \cdot 020 N & 59.531 E & 9 & 103.49 & 0589 \\ 65 & 324 & 23.070 N & 59 \cdot 220 E & 9 & 103.39 & 0590 \\ 65 & 325 & 21 \cdot 000 N & 59.330 E & 9 & 103.19 & 0591\end{array}$

3341. 742, 0000 $3341,82,0000$ 1805. 42. 0000 1267.70 .0000

\section{c}

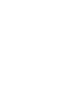


0049

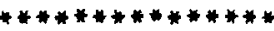
****************10
STATIEN CATA RETRIEVAL OATE: $12: 36$ MAY 16,175
***************
PAGE 15

DHHO I5
CORE LILOT

ENGTH DREDGE
RQCK

SAMPLE DATE FIX DEN DREDGE END SAMPLE GRAPHIC SED. VITA

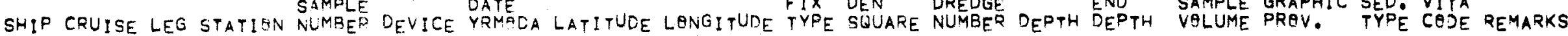

MARSDEN SQUARE \#104

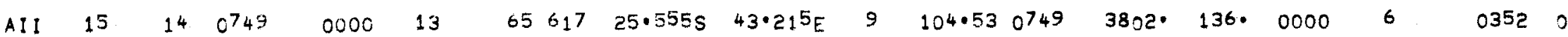

MARSDEN SQUARE \# 105

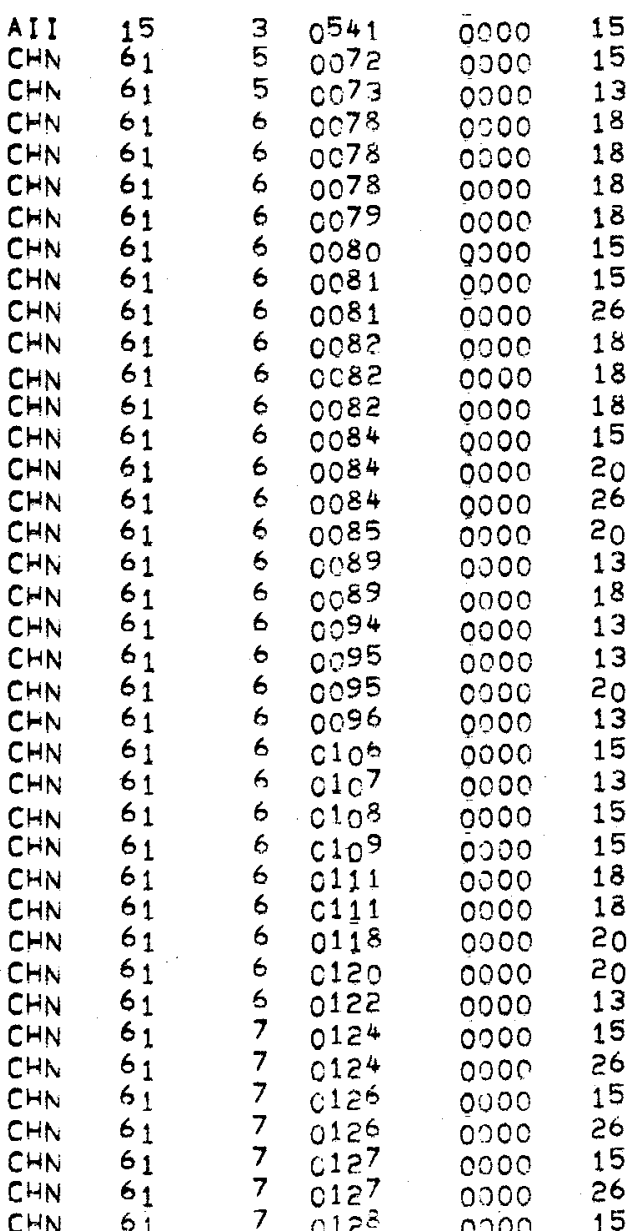

\begin{tabular}{|c|c|c|c|c|c|}
\hline & $\begin{array}{l}21 \cdot 170 \mathrm{~N} \\
21 \cdot 178 \mathrm{~N} \\
21 \cdot 147 \mathrm{~N} \\
21 \cdot 228 \mathrm{~N} \\
21 \cdot 225 \mathrm{~N} \\
21 \cdot 225 \mathrm{~N} \\
21 \cdot 213 \mathrm{~N} \\
21 \cdot 169 \mathrm{~N} \\
21 \cdot 17 \mathrm{~N} \\
21 \cdot 170 \mathrm{~N} \\
21 \cdot 188 \mathrm{~N} \\
21 \cdot 188 \mathrm{~N} \\
21 \cdot 188 \mathrm{~N} \\
21 \cdot 210 \mathrm{~N} \\
21 \cdot 210 \mathrm{~N} \\
21 \cdot 21 \mathrm{~N} \\
21 \cdot 188 \mathrm{~N} \\
21 \cdot 233 \mathrm{~N} \\
21 \cdot 228 \mathrm{~N} \\
21 \cdot 208 \mathrm{~N} \\
21 \cdot 233 \mathrm{~N} \\
21 \cdot 234 \mathrm{~N} \\
21 \cdot 265 \mathrm{~N} \\
21 \cdot 212 \mathrm{~N} \\
21 \cdot 217 \mathrm{~N} \\
21 \cdot 224 \mathrm{~N} \\
21 \cdot 241 \mathrm{~N} \\
21 \cdot 216 \mathrm{~N} \\
21 \cdot 216 \mathrm{~N} \\
21 \cdot 144 \mathrm{~N} \\
21 \cdot 226 \mathrm{~N} \\
21 \cdot 176 \mathrm{~N} \\
21 \cdot 232 \mathrm{~N} \\
21 \cdot 232 \mathrm{~N} \\
21 \cdot 219 \mathrm{~N} \\
21 \cdot 219 \mathrm{~N} \\
21 \cdot 224 \mathrm{~N} \\
21 \cdot 224 \mathrm{~N} \\
21 \cdot 254 \mathrm{~N}\end{array}$ & $\begin{array}{l}38 \cdot 020 E \\
38 \cdot 028 E \\
38.047 E \\
38.005 E \\
38.040 E \\
38.047 E \\
38.036 E \\
38.020 E \\
38.021 E \\
38.021 E \\
38 \cdot 038 E \\
38.035 E \\
38.032 E \\
38.038 E \\
38.038 E \\
38.038 E \\
38.039 E \\
38.034 E \\
38.023 E \\
38.039 E \\
38.031 E \\
38.033 E \\
38.030 E \\
38.035 E \\
38.033 E \\
38.047 E \\
38.040 E \\
38.055 E \\
38.053 E \\
38.043 E \\
38.045 E \\
38.015 E \\
38.043 E \\
38.043 E \\
38.044 E \\
38.044 E \\
38 \cdot 029 E \\
38.029 E \\
38.034 E\end{array}$ & $\begin{array}{l}6 \\
0 \\
0 \\
0 \\
0 \\
0 \\
6 \\
1 \\
6 \\
1 \\
6 \\
0 \\
0 \\
0 \\
1 \\
0 \\
0 \\
0 \\
0 \\
0 \\
0 \\
0 \\
1 \\
1 \\
0 \\
6\end{array}$ & $\begin{array}{l}105 \cdot 18 \\
105 \cdot 18 \\
105 \cdot 18 \\
105 \cdot 18 \\
105 \cdot 18 \\
105 \cdot 18 \\
105 \cdot 18 \\
105 \cdot 18 \\
105 \cdot 18 \\
105 \cdot 18 \\
105 \cdot 18 \\
105 \cdot 18 \\
105 \cdot 18 \\
105 \cdot 18 \\
105 \cdot 18 \\
105 \cdot 18 \\
105 \cdot 18 \\
105 \cdot 18 \\
105 \cdot 18 \\
105 \cdot 18 \\
105 \cdot 18 \\
105 \cdot 18 \\
105 \cdot 18 \\
105 \cdot 18 \\
105 \cdot 18 \\
105 \cdot 18 \\
105 \cdot 18 \\
105 \cdot 18 \\
105 \cdot 18 \\
105 \cdot 18 \\
105 \cdot 18 \\
105 \cdot 18 \\
105 \cdot 18 \\
105 \cdot 18 \\
105 \cdot 18 \\
105 \cdot 18 \\
105 \cdot 18 \\
105 \cdot 18 \\
105 \cdot 18\end{array}$ & $\begin{array}{l}0004 \\
0072 \\
0073 \\
0 F F A \\
0 F F C \\
0 F F D \\
0079 \\
0000 \\
0081 \\
0081 \\
0 F F B \\
0 F F C \\
000 D \\
0000 \\
0084 \\
0000 \\
0085 \\
000 B \\
000 D \\
0094 \\
0095 \\
0095 \\
0096 \\
0106 \\
0107 \\
0108 \\
0109 \\
1110 \\
111 C \\
0118 \\
0120 \\
0122 \\
0000 \\
0000 \\
0000 \\
0000 \\
0000 \\
0000 \\
0000\end{array}$ \\
\hline
\end{tabular}

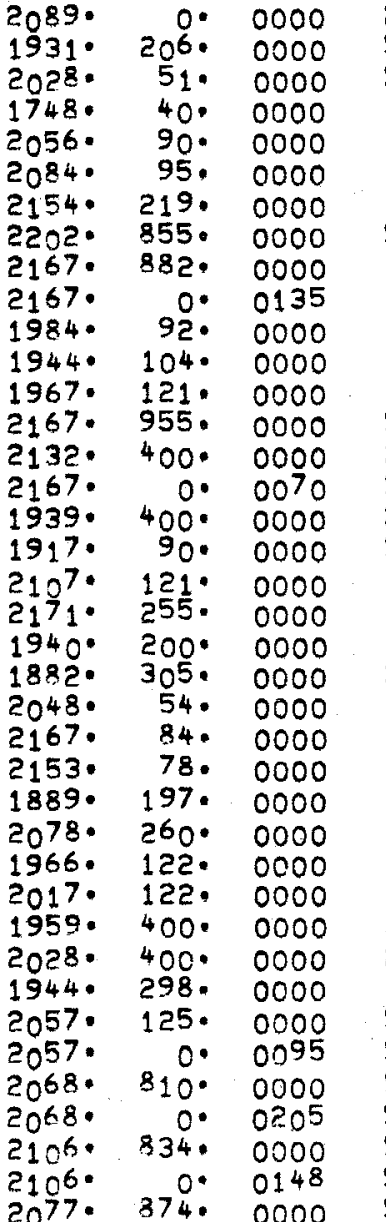

00005

3 BAGS

343254

37290

00595

00592

486954

43410

4079

0019

4459

0439 ?

001954

$0000 \quad 5$

00000

043254

00590

0039 ?

00390

00000

00590

00390

00392

0039

0039
0339

03390

00000

00000

$\begin{array}{ll}0331 & 0 \\ 4459 & 54\end{array}$

003954

003954

00002

044954

005954

033954 
SAMPLE

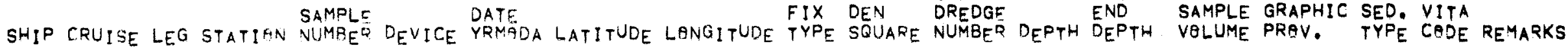

FIX MERS - CORE QR

CQRE LENGTH

LENGTH DREDGE

ROCK

$\begin{array}{lll}\text { QR } & \text { OR } & \text { PHYSI }=\text { OR } \\ \text { END } & \text { SAMPLE GRAPHIC SE }\end{array}$

MARSDEN SQUARE * 105

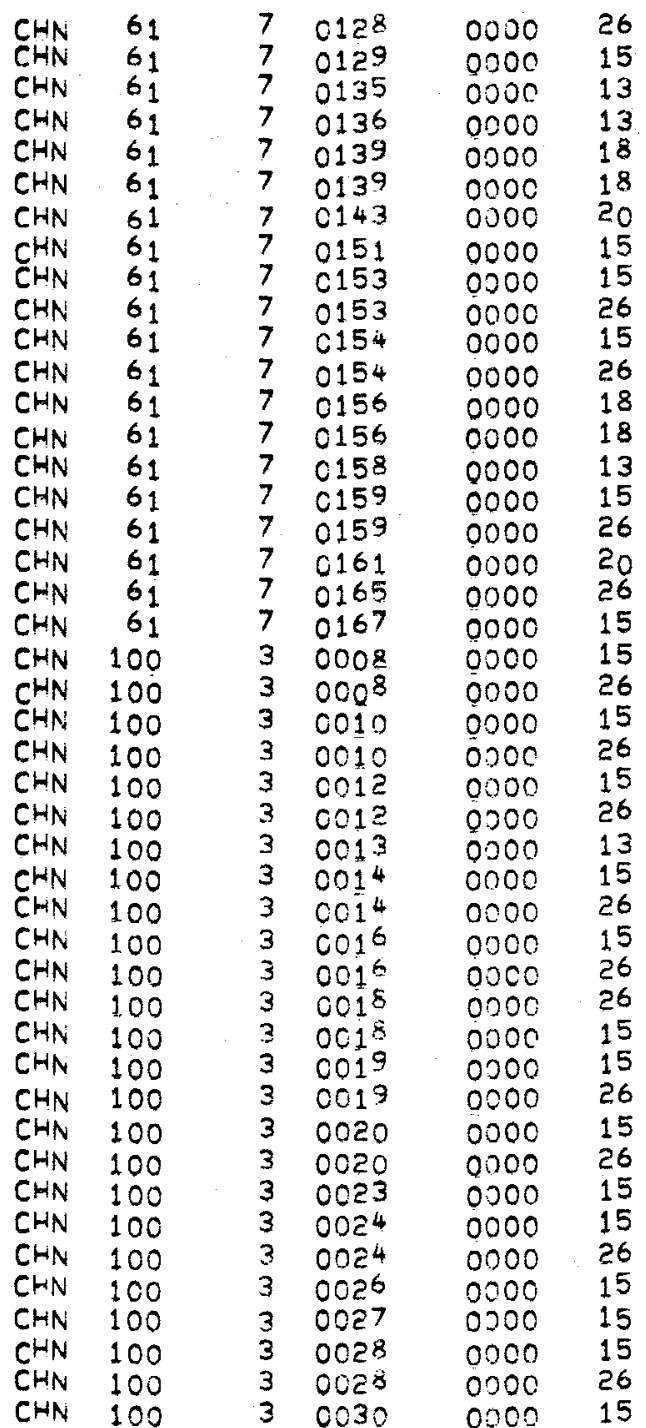

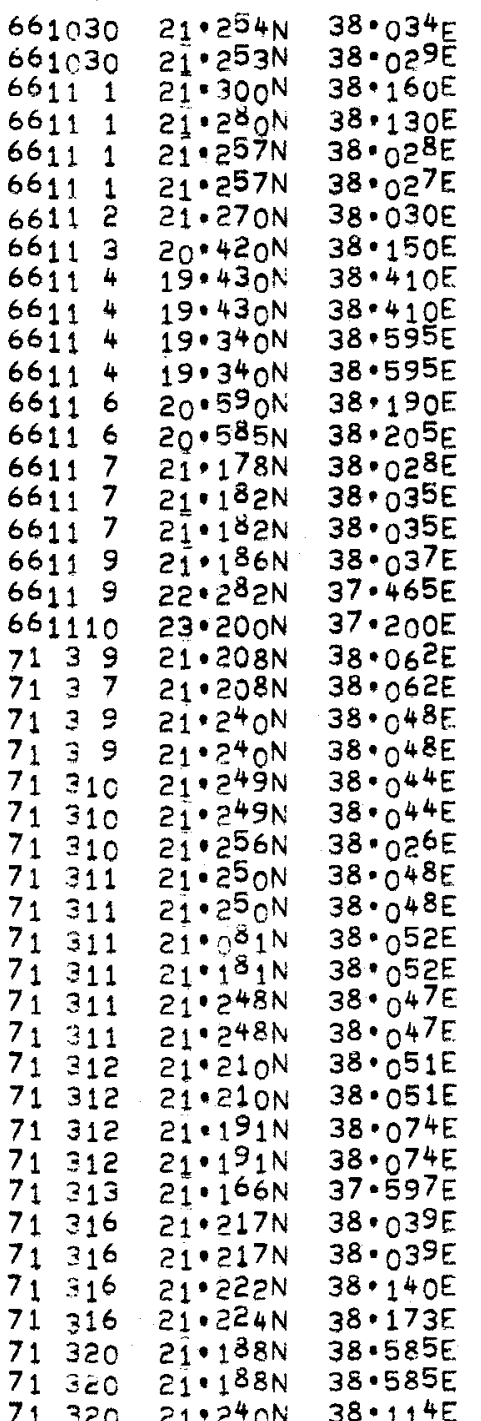

$105 \cdot 180000$ $105 \cdot 180000$ $105 \cdot 180135$ $105 \cdot 180136$ $105.18000 A$ $105 \cdot 18000^{\mathrm{B}}$ $105 \cdot 180143$ $105 \cdot 080151$ 105.980000 $105 \cdot 980000$
105.980000 105.980000 105.980000 105.08 OFFB $105 \cdot 180158$ $105 \cdot 180000$ $105 \cdot 180000$ $105 \cdot 180161$ $\begin{array}{ll}105 \cdot 27 & 0165 \\ 105.37 & 0167\end{array}$ 105.180007 $105 \cdot 180007$ $105 \cdot 180009$ $105 \cdot 180009$ 105.180010 105.180010 105.180011 $105 \cdot 180012$ $105 \cdot 180012$ $105 \cdot 180013$ $105 \cdot 180013$ $105 \cdot 180014$ $105 \cdot 180014$ $105 \cdot 18$
105.180015 $105 \cdot 180016$ $105 \cdot 180016$ $105 \cdot 170017$ $105 \cdot 180018$ $105 \cdot 180018$ $05 \cdot 180019$ $105 \cdot 180020$ $105 \cdot 180021$ $105 \cdot 180021$
105.180022

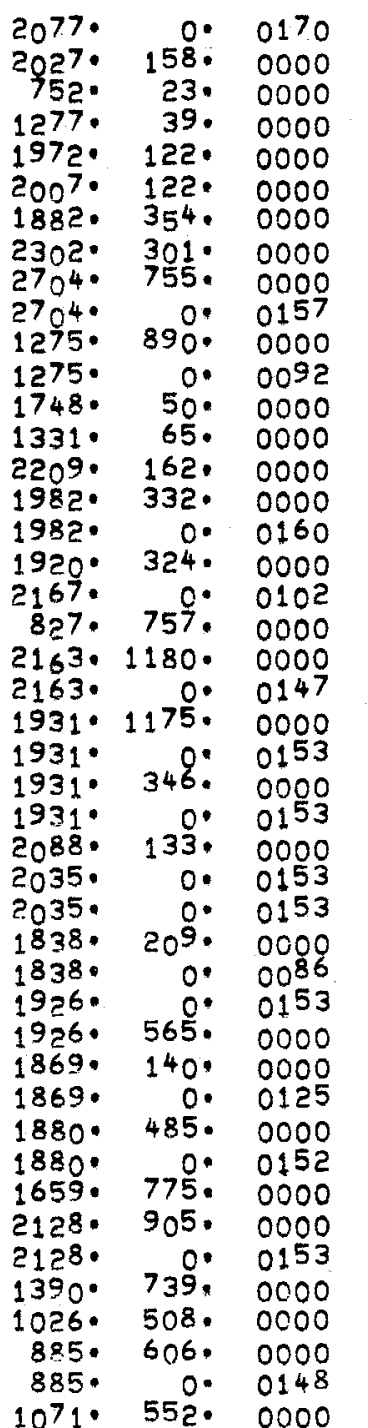

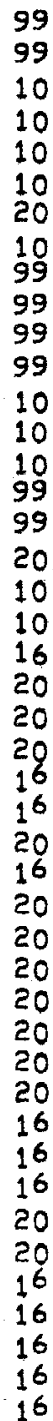

033954 444754 49690 $\begin{array}{ll}3352 & 0 \\ 0447 & 0\end{array}$

00170

00000

43450 $\begin{array}{ll}4345 & 5 \\ 3439 & 54\end{array}$ 373254 343954 332954 34290 3439 4962 03370

00000

00000

3329

000054

00000 UNSPL!T 0000 U UNSPLIT 00000 UNSPLIT 000054 000054 $0000 \quad 0$ 00000 $0000 \quad 0$ UNSPLIT 0000 O UNSPL!T 00000 UNSPLIT $0000 \quad 0$ UNSPLIT 00000 UNSPLIT $0000 \quad 0$ UNSPLIT 342154 332754 332754 00000 UNSPLIT $0000 \%$ UNSPLIT 383854 373854 $\begin{array}{ll}3739 & 54 \\ 3338 & 54\end{array}$ 


\section{1}

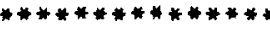

$* * * * * * * * * * * * * * *$

STATION DATA RETRIEVAL

DATE: $12: 36$ MAY 16,175

\section{$4 * * * * * * * * * * * *$

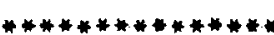

PAGE 17

* WHO I*

LENGTH DREDGE RQCK

CARE LILOT

MARS- CORE QR LENGTH DREDGE PHYSIO- OR

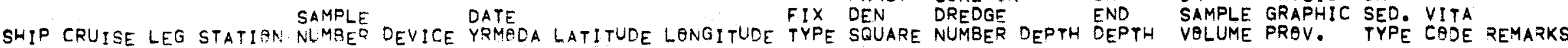

MARSDEN SQUARE \# 105

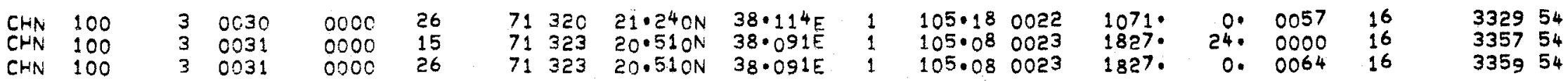

MARSDEN SQUARE \#109

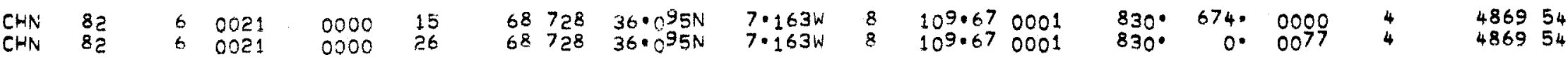

MARSDEN SQUARE \#110

$\begin{array}{llllll}C H N & 21 & 1 & 0014 & 0000 & 15 \\ C H N & 21 & 1 & 0014 & 0000 & 13 \\ C H N & 21 & 1 & 0015 & 0000 & 13 \\ C H N & 82 & 6 & 0022 & 0000 & 15 \\ C H N & 82 & 6 & 0022 & 0000 & 26\end{array}$

$\begin{array}{lllllll}61 & 915 & 33 \cdot 320 \mathrm{~N} & 18 \cdot 110 \mathrm{~W} & 5 & 110.38 & 0014 \\ 61 & 915 & 33.320 \mathrm{~N} & 18.110 \mathrm{~W} & 5 & 110.38 & 0014 \\ 61 & 915 & 34.00 \mathrm{~N} & 15.510 \mathrm{~W} & 5 & 110.45 & 0015 \\ 68 & 731 & 35.39 \mathrm{ON} & 13.420 \mathrm{~W} & 8 & 110.53 & 0002 \\ 68 & 731 & 35.390 \mathrm{~N} & 13.420 \mathrm{~W} & 8 & 110.53 & 0002\end{array}$

$3813^{\circ}$

3835. 0.000

3929. 167. 0000

4853. 708. 0000

4853. $\quad 0.0060$

10
0
1
4
4

00000

0000 O IN JAR

33500

383154

383154

\section{MARSDEN SQUARE \# 111}

$\begin{array}{llll}\text { CHN } & 7 & 8 & 0011 \\ \text { CHN } & 61 & 10 & 0171 \\ \text { CHN } & 61 & 10 & 0172 \\ \text { CHN } & 96 & 14 & 0008 \\ \text { CHN } & 96 & 14 & 0010 \\ \text { CHN } & 96 & 14 & 0010\end{array}$

$0000 \quad 15$

$0000 \quad 15$

$0000 \quad 15$

0000

000015

$0.000 \quad 26$

$\begin{array}{lll}59617 & 30 \cdot 300 \mathrm{~N} & 28 \cdot 230 \mathrm{~W} \\ 661111 & 26 \cdot 42 \mathrm{~N} & 39 \cdot 230 \mathrm{~W} \\ 66126 & 26 \cdot 33 \mathrm{~N} & 39 \cdot 585 \mathrm{~W} \\ 691119 & 30 \cdot 313 \mathrm{~N} & 20 \cdot 197 \mathrm{~W} \\ 69123 & 32 \cdot 361 \mathrm{~N} & 21 \cdot 262 \mathrm{~W} \\ 69123 & 32 \cdot 361 \mathrm{~N} & 21 \cdot 262 \mathrm{~W}\end{array}$

$111 \cdot 080011$ $\begin{array}{ll}111.69 & 0171 \\ 111.69 & 0172\end{array}$ $111 \cdot 000008$ 111.000008 $111 \cdot 210010$

4204. 73. 0000

4279. 760. 0000

4356. 160. 0000

4818. 0.0055

5129. 840.0000

$5129 . \quad 0.0103$

MARSDEN SQUARE \# 11E

KNR

30001

$0000 \quad 16$

7362

$36 \cdot 2^{68 N} \quad 32.001 \mathrm{~N}$

$9 \quad 112.620001$

$2829 \cdot 10^{83} \cdot 0000$

1

33240

MARSDEN SQUARE \#113

$\begin{array}{llllll}\text { CHN } & 96 & 14 & 0006 & 0000 & 15 \\ \text { CHN } & 96 & 14 & 0006 & 0000 & 26 \\ \text { CHN } & 96 & 15 & 0012 & 0000 & 15\end{array}$

$691113 \quad 30 \cdot 184 \mathrm{~N} \quad 42 \cdot 378 \mathrm{~W}$

$691113 \quad 30 \cdot 184 \mathrm{~N} \quad 42 \cdot 378 \mathrm{~N}$

$3 \mathrm{C} \cdot 157 \mathrm{~N} \quad 43 \cdot 189 \mathrm{~W}$ $113^{\circ} 02000^{6}$ $113 \cdot 030012$ $\begin{array}{rrrrr}3205 \cdot & 536 . & 0000 & 14 & 372154 \\ 3205 \cdot & 0 . & 0082 & 14 & 332954\end{array}$

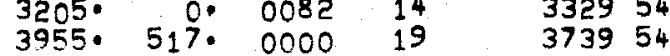




\section{2}

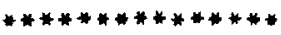

$* * * * * * * * * * * * * * *$

STATION DATA RETRIEVAL DATE: $12: 36$ MAY 16,175
$* * * * * * * * * * * * * *$

$* * * * * * * * * * * * * *$
PAGE 18

**WHOI**

PILOT

CORE LENGTH.

MARS. CORE $Q R$ LENGTH DREDGE PHYSIO. RQRK

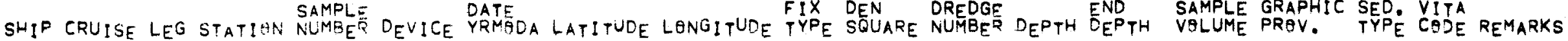

MARSDEN SQUARE \# 113

$\begin{array}{llllll}C H N & 96 & 15 & 0013 & 0000 & 13 \\ C H N & 96 & 15 & 0014 & 0000 & 15 \\ C H N & 96 & 15 & 0015 & 0000 & 15 \\ C H N & 99 & 2 & 0002 & 0000 & 15 \\ C H N & 99 & 2 & 0003 & 0000 & 15 \\ C H N & 99 & 2 & 0004 & 0000 & 15\end{array}$

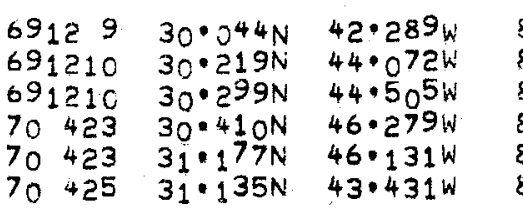

MARSDEN SQUARE \# 114

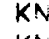

KNR $31 \quad 3 \quad 0003$

KNR $31 \quad 3 \quad 000$

KNR $31 \quad 3 \quad 000^{6}$

0000

0000

0000
$113 \cdot 020013$ $113 \cdot 040014$ $113 \cdot 040015$ $113 \cdot 060001$ 113.160002 $113 \cdot 13000^{3}$
5216.

293: 0000

4083. 286. 0000

4294. 800. 0000

4358. 525. 0000

3857.673 .0000

$\begin{array}{llr}19 & 8339 & 54 \\ 19 & 3329 & 54 \\ 19 & 3731 & 54 \\ 19 & 3329 & 54 \\ 19 & 3329 & 54 \\ 19 & 3322 & 0\end{array}$

33220

$\begin{array}{lrllll}\text { AII } & 1 & 1 & 000 & 0000 & 15 \\ \text { AII } & 42 & 1 & 0045 & 0000 & 15 \\ \text { ATL } & 282 & 1 & 0001 & 0000 & 13 \\ \text { ATL } & 282 & 1 & 0023 & 0000 & 15 \\ \text { ATL } & 296 & 0 & 0001 & 0000 & 13 \\ \text { ATL } & 296 & 0 & 000 ? & 0000 & 13 \\ \text { ATL } & 296 & 0 & 0004 & 0000 & 13 \\ \text { ATL } & 296 & 0 & 0005 & 0000 & 13 \\ \text { ATL } & 296 & 0 & 0006 & 0000 & 13 \\ \text { ATL } & 296 & 0 & 0007 & 0900 & 13 \\ \text { ATL } & 296 & 0 & 0008 & 0000 & 13 \\ \text { ATL } & 296 & 0 & 0009 & 0000 & 13 \\ \text { ATL } & 297 & 1 & 6375 & 0000 & 13 \\ \text { ATL } & 297 & 1 & 6377 & 0000 & 13 \\ \text { ATL } & 297 & 1 & 6380 & 0000 & 13 \\ \text { ATL } & 297 & 1 & 6381 & 0000 & 13 \\ \text { CHN } & 13 & 1 & 0001 & 0000 & 15 \\ \text { CHN } & 13 & 1 & 000 Z & 0000 & 15 \\ \text { CHN } & 19 & 1 & 0001 & 0000 & 15 \\ \text { CHN } & 47 & 1 & 0004 & 0000 & 18 \\ \text { KNR } & 31 & 5 & 0017 & 0000 & 18 \\ \text { KNR } & 31 & 5 & 0017 & 0000 & 18 \\ \text { KNR } & 31 & 5 & 0018 & 0000 & 18 \\ \text { KNR } & 31 & 5 & 0019 & 0000 & 18 \\ \text { KNR } & 31 & 5 & 0020 & 0000 & 18\end{array}$

\section{MARSDEN SQUARE \# 115}

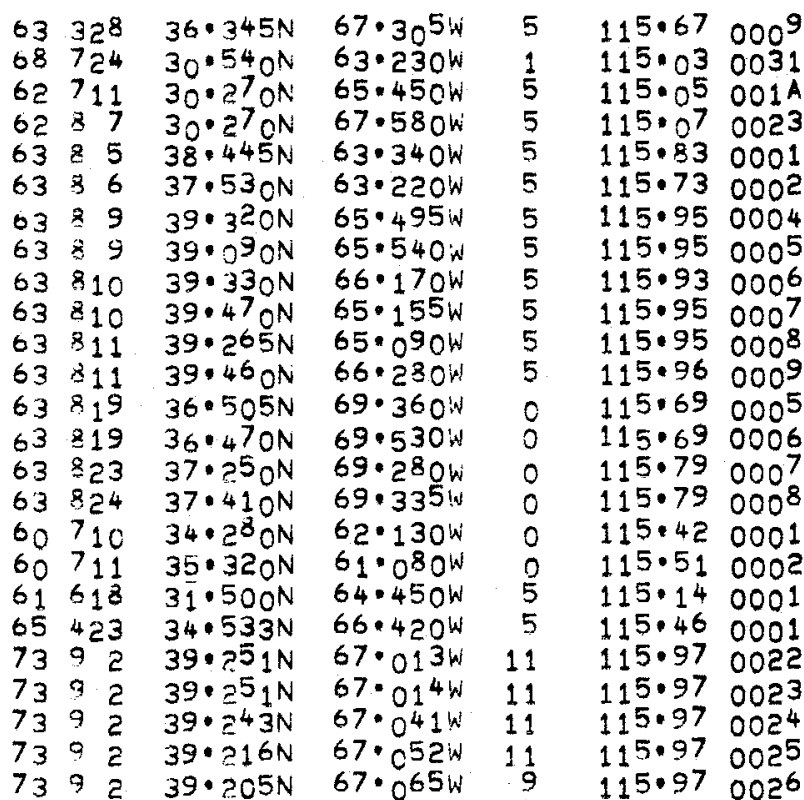

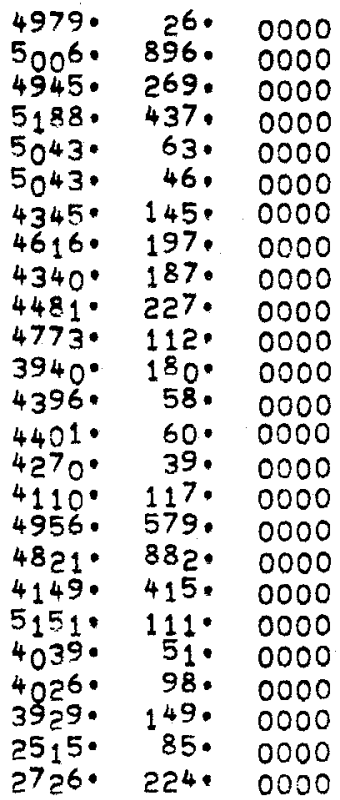

4459

34310

3869

2379

8339

3839

4229

4239

1339

2839

28390

2839

4459

4459

3359

3359

3129

3332

$\begin{array}{ll}3735 & 0 \\ 3279 & 54\end{array}$

0259

2028

$\begin{array}{ll}2028 & 0 \\ 0000 & 0\end{array}$

48140

00000 


\begin{tabular}{|c|c|c|}
\hline $\begin{array}{l}63225 \\
632225 \\
63226 \\
6333 \\
721021 \\
721021 \\
721021 \\
721021 \\
721021 \\
721021 \\
721021 \\
721021 \\
721021 \\
721022 \\
721022 \\
721022 \\
721022 \\
721023 \\
721023 \\
721023 \\
721023 \\
721023 \\
721023 \\
721026 \\
721026 \\
721026 \\
721028 \\
721028 \\
721028 \\
721029 \\
721029 \\
721029 \\
721029 \\
721029 \\
721029 \\
721029 \\
721030 \\
721030 \\
721030 \\
721031 \\
721031 \\
721031 \\
72113 \\
72113 \\
72113\end{array}$ & $\begin{array}{l}32 \cdot 020 N \\
31 \cdot 150 N \\
30 \cdot 560 N \\
37 \cdot 100 N \\
39 \cdot 585 N \\
39 \cdot 499 N \\
39 \cdot 499 N \\
39 \cdot 436 N \\
39 \cdot 436 N \\
39 \cdot 398 N \\
39 \cdot 398 N \\
39 \cdot 406 N \\
39 \cdot 406 N \\
39 \cdot 495 N \\
39 \cdot 495 N \\
39 \cdot 376 N \\
39 \cdot 376 N \\
39 \cdot 555 N \\
39 \cdot 555 N \\
39 \cdot 489 N \\
39 \cdot 489 N \\
39 \cdot 410 N \\
39 \cdot 410 N \\
39 \cdot 281 N \\
39 \cdot 281 N \\
39 \cdot 435 N \\
32 \cdot 320 N \\
32 \cdot 320 N \\
32 \cdot 517 N \\
34 \cdot 105 N \\
34 \cdot 105 N \\
34 \cdot 130 N \\
34 \cdot 130 N \\
34 \cdot 022 N \\
34 \cdot 322 N \\
34 \cdot 032 N \\
34 \cdot 010 N \\
33 \cdot 495 N \\
33 \cdot 495 N \\
33 \cdot 269 N \\
33 \cdot 269 N \\
33 \cdot 17 \\
34 \cdot 138 N \\
34 \cdot 145 N \\
34 \cdot 138 N\end{array}$ & 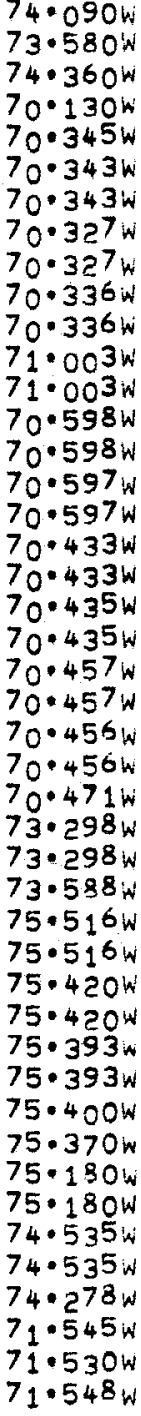 \\
\hline
\end{tabular}

(3)

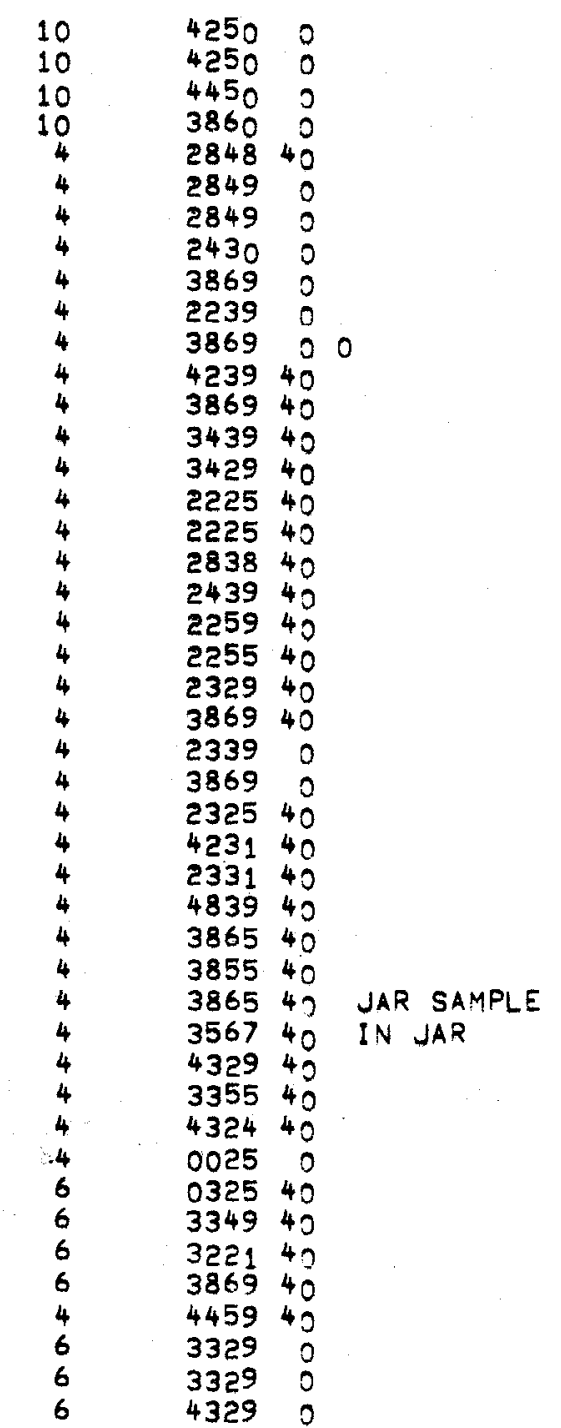




\section{4}

$* * * * * * * * * * * * *$
$* * * * * * * * * * * * * *$
STATIEN DATA RETRIEVAL DATE: $12: 36$ MAY 16,175
***************
PAGE 20

PILOT

CORE LENGTH

LENGTH DREDGE

MARS- CORE $9 R$ OR QR PHYSIO. QR

SAMPLE FIX DEN DREDGE END SAMPLE GRAPHIC SED, YITA

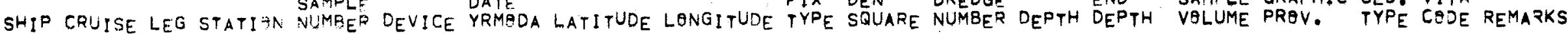

MARSDEN SQUARE \# 116

$\begin{array}{lrllll}\text { AII } & 72 & 1 & 0031 & 0000 & 14 \\ \text { ATL } & 297 & 1 & 6369 & 0000 & 13 \\ \text { ATL } & 297 & 1 & 6371 & 0000 & 13 \\ \text { ATL } & 297 & 1 & 6372 & 0000 & 13 \\ \text { CHN } & 11 & 1 & 0013 & 0000 & 15\end{array}$

$7211434 \cdot 150 \mathrm{~N} \quad 71 \cdot 515 \mathrm{~W} \quad 5 \quad 116 \cdot 410027$

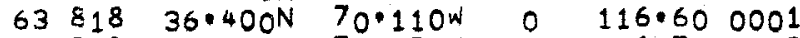
$63818 \quad 37.100 \mathrm{~N} 70.250 \mathrm{~W} \quad 0 \quad 116.700002$

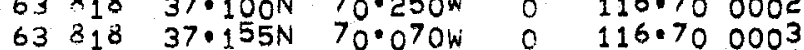
$60320 \quad 33 \cdot 000 \mathrm{~N} 72 \cdot 150 \mathrm{~W} 4 \quad 116.320013$

\section{MARSDEN SQUARE \#141}

$\begin{array}{llllll}\text { AII } & 15 & 3 & 0522 & 0000 & 13 \\ \text { AII } & 15 & 3 & 0522 & 0000 & 13 \\ \text { AII } & 15 & 3 & 0522 & 0000 & 13 \\ \text { CHN } & 61 & 4 & 0055 & 0000 & 15 \\ \text { CHN } & 61 & 4 & 0055 & 0000 & 26 \\ \text { CHN } & 61 & 4 & 0056 & 0000 & 15 \\ \text { CHN } & 61 & 4 & 0056 & 0000 & 26 \\ \text { CHN } & 61 & 4 & 0057 & 0000 & 15 \\ \text { CHN } & 61 & 4 & 0057 & 0000 & 26 \\ \text { CHN } & 61 & 4 & 0058 & 0000 & 15 \\ \text { CHN } & 61 & 4 & 0058 & 0000 & 26 \\ \text { CHN } & 61 & 4 & 0059 & 0000 & 15 \\ \text { CHN } & 61 & 4 & 0059 & 0000 & 26 \\ \text { CHN } & 61 & 4 & 0062 & 0000 & 15 \\ \text { CHN } & 61 & 4 & 0062 & 0000 & 26 \\ \text { CHN } & 61 & 4 & 0063 & 0200 & 15 \\ \text { CHN } & 61 & 4 & 0063 & 0000 & 26 \\ \text { CHN } & 61 & 4 & 0064 & 0000 & 15 \\ \text { CHN } & 61 & 4 & 0064 & 0000 & 26 \\ \text { CHN } & 61 & 4 & 0065 & 0000 & 15 \\ \text { CHN } & 61 & 4 & 0065 & 0000 & 26 \\ \text { CHN } & 61 & 4 & 0666 & 0200 & 15 \\ \text { CHN } & 61 & 4 & 0066 & 0000 & 26 \\ \text { CHN } & 61 & 4 & 0067 & 0000 & 15 \\ \text { CHN } & 61 & 4 & 0067 & 0000 & 26 \\ \text { CHN } & 61 & 4 & 0063 & 0000 & 15\end{array}$

$141 \cdot 02$ OBL1 141.02 OBL2 141.02 OBL3 141.410000 $141 \cdot 410000$ $141 \cdot 210000$ $141 \cdot 210000$ $141 \cdot 320000$ $141 \cdot 320000$ $141 \cdot 320000$ $141 \cdot 320000$ $141 \cdot 330000$ $141 \cdot 330000$ $141 \cdot 610000$ 141.610000 $141 \cdot 520000$ 141.520000 141.540000 141.540000 141.550000 141.550000 $141 \cdot 440000$ 1.41 .440000 141.430000 $141 \cdot 430000$
$141 \cdot 440068$
4418.111 .0000

40.0000 $243^{\circ} 21.20000$ $3010^{\circ} 610^{\circ} 0000$
444940

38390

34590

3831

$\begin{array}{rrr}6 & 4449 & 40 \\ 2 & 3839 & 0 \\ 2 & 4459 & 0 \\ 2 & 3359 & 0 \\ 10 & 3831 & 0\end{array}$

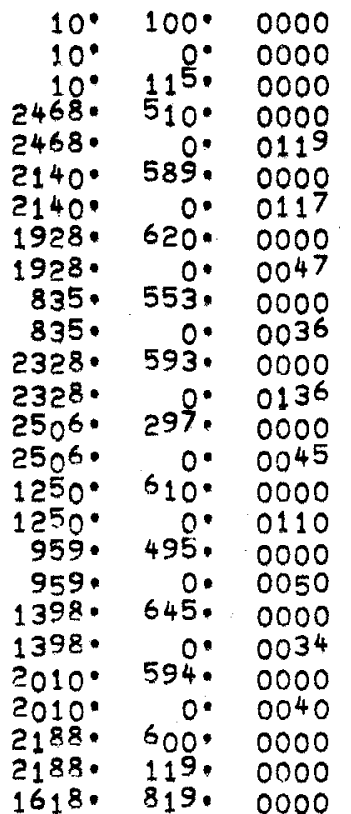

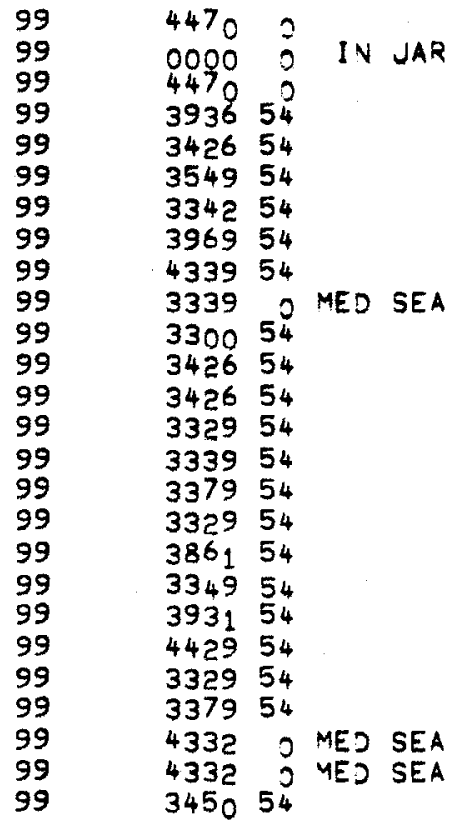

MARSDEN SQUARE \# 142

$\begin{array}{llllll}\text { CHN } & 7 & 3 & 0002 & 0.00 & 15 \\ \text { CHN } & 7 & 3 & 0003 & 0000 & 15 \\ \text { CHN } & 7 & 3 & 0004 & 0009 & 15 \\ \text { CHN } & 7 & 4 & 0005 & 0000 & 15\end{array}$

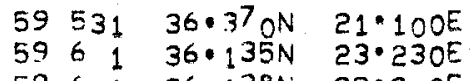
$596136 \cdot 138 \mathrm{~N} \quad 23 \cdot 200 \mathrm{E}$ $5965.36 \cdot 118 \mathrm{~N} \quad 23 \cdot 220 \mathrm{E}$

$\begin{array}{ll}142.61 & 0002 \\ 142 \cdot 63 & 0003 \\ 142.63 & 0004 \\ 142.63 & 000^{5}\end{array}$
4976. $151 \cdot 0000$ 1249. 157. 0000 1249. 122. 0000

$\begin{array}{lll}21 & 3350 & 0 \\ 21 & 3330 & 0 \\ 21 & 3350 & 0 \\ 21 & 3350 & 0\end{array}$




\section{5}

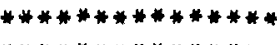

$* * * * * * * * * * * * * *$
STATION DATA RETRIEVAL DATE: $12: 36$ MAY 16,175

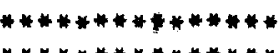

PAGE 21

PILOT

CQRE LENGTH

LENGTH DREDGE

MARS- CORE QR

SAMPLE DATE FIX DEN DREDGE END SAMPLE GRAPHIC SED. VITA

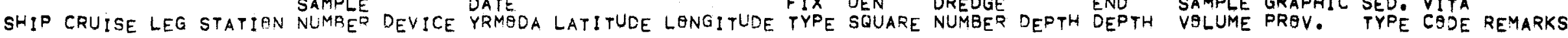

MARSDEN SQUARE \# 142

$\begin{array}{llllll}C H N & 7 & 4 & 0006 & 0000 & 15 \\ C H N & 61 & 3 & 0035 & 0000 & 15 \\ C H N & 61 & 3 & 0037 & 0000 & 15 \\ C H N & 61 & 3 & 0039 & 0000 & 15 \\ C H N & 61 & 3 & 0040 & 0000 & 15 \\ C H N & 61 & 3 & 0041 & 0000 & 15 \\ C H N & 61 & 3 & 0042 & 0000 & 15 \\ C H N & 61 & 3 & 0043 & 0000 & 15 \\ C H N & 61 & 3 & 0046 & 0000 & 15 \\ C H N & 61 & 3 & 0050 & 0000 & 15 \\ C H N & 61 & 4 & 0051 & 0000 & 15 \\ C H N & 61 & 4 & 0051 & 0000 & 26 \\ C H N & 61 & 4 & 0052 & 0000 & 15 \\ C H N & 61 & 4 & 0053 & 0000 & 15 \\ C H N & 61 & 4 & 0053 & 0000 & 26 \\ C H N & 61 & 4 & 0054 & 0000 & 15 \\ C H N & 61 & 4 & 0054 & 0000 & 26\end{array}$

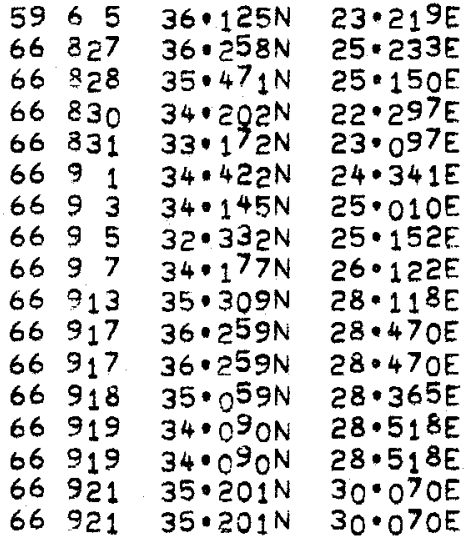
$6692135.201 N \quad 30.070 E$

$\begin{array}{lrrlll}\text { AII } & 49 & 5 & A 003 & 0000 & 15 \\ \text { AII } & 49 & 5 & B 003 & 0000 & 26 \\ \text { CHN } & 7 & 3 & 0001 & 0000 & 15 \\ \text { CHN } & 7 & 4 & 0001 & 0000 & 15 \\ \text { CHN } & 7 & 4 & 000 ? & 0000 & 15 \\ \text { CHN } & 7 & 5 & 0003 & 0000 & 15 \\ \text { CHN } & 7 & 8 & 0009 & 0000 & 15 \\ \text { CHN } & 43 & 1 & 0085 & 0000 & 13 \\ \text { CHN } & 61 & 2 & 0025 & 0000 & 15 \\ \text { CHN } & 61 & 2 & 0029 & 0000 & 15 \\ \text { CHN } & 61 & 2 & 0031 & 0000 & 15 \\ \text { CHN } & 61 & 3 & C 045 & 0000 & 15\end{array}$

$\begin{array}{llll}69 & 521 & 34 \cdot 055 \mathrm{~N} & 26 \cdot 490 \mathrm{E} \\ 69 & 521 & 33 \cdot 20 \mathrm{~N} & 19 \cdot 250 \mathrm{E} \\ 59 & 531 & 36 \cdot 55 \mathrm{~N} & 19 \cdot 502 \mathrm{E} \\ 59 & 611 & 40 \cdot 160 \mathrm{~N} & 12 \cdot 375 \mathrm{E} \\ 59 & 613 & 40 \cdot 020 \mathrm{~N} & 12 \cdot 165 \mathrm{E} \\ 59 & 617 & 40 \cdot 080 \mathrm{~N} & 12 \cdot 190 \mathrm{E} \\ 59 & 617 & 40 \cdot 100 \mathrm{~N} & 12 \cdot 150 \mathrm{E} \\ 64 & 627 & 38 \cdot 473 \mathrm{~N} & 15 \cdot 030 \mathrm{E} \\ 66 & 812 & 35 \cdot 123 \mathrm{~N} & 16 \cdot 311 \mathrm{E} \\ 66 & 316 & 35 \cdot 553 \mathrm{~N} & 19 \cdot 360 \mathrm{E} \\ 66 & 817 & 33 \cdot 305 \mathrm{~N} & 19 \cdot 498 \mathrm{E} \\ 66 & 97 & 33 \cdot 147 \mathrm{~N} & 27.519 \mathrm{E}\end{array}$
3612. 792. 0000
$142 \cdot 630006$ $142 \cdot 650000$ $142 \cdot 550000$ $142 \cdot 420000$ $142 \cdot 330000$ $142 \cdot 44,0000$ $142 \cdot 100000$ $142 \cdot 250000$ $142 \cdot 46 \quad 0000$ $142 \cdot 58.0050$ 142.680000 142.680000 $142 \cdot 580000$ $142 \cdot 480000$ $142 \cdot 48 \quad 0000$ $142 \cdot 50 \quad 0000$

\section{MARSDEN SQUARE \# 143}

$\begin{array}{lll}5 & 143.46 & 03-1 \\ 5 & 143.39 & 03-2 \\ 4 & 143.69 & 0001 \\ 4 & 143.02 & 0001 \\ 0 & 143.02 & 0002 \\ 4 & 143.02 & 0003 \\ 4 & 143.02 & 0009 \\ 5 & 143.85 & 0027 \\ 6 & 143.56 & 0000 \\ 6 & 143.59 & 0000 \\ 6 & 143.39 & 0000 \\ 6 & 143.37 & 0045\end{array}$

2637. 100. 0000 $\begin{array}{lll}27880^{\circ} & 1000^{\circ} & 0239 \\ 3677 . & 144 . & 0000\end{array}$ 3677. 144. 0000 3529. 595: 0000 $3610^{\circ} 874.0000$ $3405 \cdot 3190 \cdot 0000$ 0.81 .0000 1460.732 .0000 3955. 910.0000 $110^{8} \cdot 338.0000$ 3038 . 435. 0000

$\begin{array}{llll}21 & 3330 & 0 & \text { SC } \\ 99 & 9864 & 54 & \\ 99 & 4969 & 54 & \text { AEGEAN SEA } \\ 99 & 3329 & 54 \\ 99 & 3818 & 54 \\ 99 & 3279 & 54 \\ 99 & 3866 & 54 \\ 99 & 3349 & 54 \\ 99 & 3036 & 54 \\ 99 & 3969 & 54 \\ 99 & 3429 & 54 \\ 99 & 3969 & 54 \\ 99 & 3359 & 54 \\ 99 & 3969 & 54 \\ 99 & 3969 & 54 \\ 99 & 3349 & 54 \\ 99 & 334954\end{array}$

MARSOEN SQUARE \# 144 


\section{6}

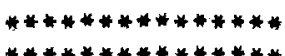

STATION DATA RETRIEVAL

DATE: $12: 36$ MAY 16,175

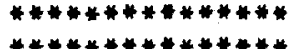

CORE LENGTH,

LENGTH DREDGE

MARS- CQRE $Q R$

PHYSIO - OR

SAMPLE

PAGE 22

*WHOI**

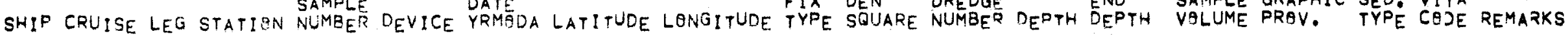

MARSDEN SQUARE \# 145

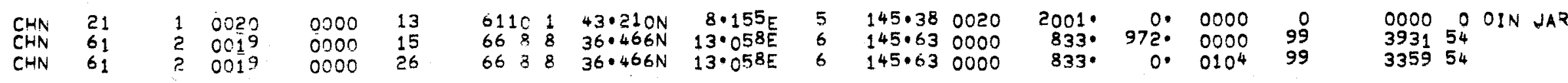

MARSDEN SQUARE \# 147

\begin{tabular}{|c|c|c|c|c|}
\hline $\begin{array}{l}\text { CHN } \\
\text { CHN } \\
\text { CHN } \\
\text { CHN } \\
\text { CHN } \\
\text { CHN } \\
\text { CHN } \\
\text { CHN } \\
\text { CHN } \\
\text { CHN } \\
\text { CHN }\end{array}$ & $\begin{array}{l}43 \\
43 \\
43 \\
43 \\
82 \\
82 \\
82 \\
82 \\
82 \\
82 \\
82\end{array}$ & $\begin{array}{l}1 \\
1 \\
1 \\
1 \\
6 \\
6 \\
6 \\
6 \\
6 \\
6 \\
6\end{array}$ & $\begin{array}{l}0102 \\
010 \\
0109 \\
0115 \\
0023 \\
0023 \\
0025 \\
0025 \\
0027 \\
0027 \\
0028\end{array}$ & $\begin{array}{l}0000 \\
0000 \\
0000 \\
0000 \\
0000 \\
0000 \\
0000 \\
0000 \\
0000 \\
0000 \\
0000\end{array}$ \\
\hline $\begin{array}{l}\text { CHN } \\
\text { CHN } \\
C H N \\
C H N \\
C H N \\
C H N \\
C H N\end{array}$ & $\begin{array}{l}82 \\
82 \\
82 \\
82 \\
82 \\
82 \\
82\end{array}$ & $\begin{array}{l}6 \\
6 \\
6 \\
6 \\
6 \\
6 \\
6\end{array}$ & $\begin{array}{l}0028 \\
0030 \\
0030 \\
0030 \\
0032 \\
0032 \\
0033\end{array}$ & $\begin{array}{l}0000 \\
0000 \\
0000 \\
0000 \\
0000 \\
0000 \\
0000\end{array}$ \\
\hline $\begin{array}{l}\mathrm{CHN} \\
\mathrm{CHN}\end{array}$ & $\begin{array}{l}82 \\
82\end{array}$ & $\begin{array}{l}6 \\
6\end{array}$ & $\begin{array}{l}0033 \\
0036\end{array}$ & $\begin{array}{l}0000 \\
0000\end{array}$ \\
\hline CHN & 82 & 6 & 0041 & 0500 \\
\hline $\mathrm{CHN}$ & 82 & 6 & 0041 & 0000 \\
\hline $\begin{array}{l}\text { CHN } \\
\text { CHN }\end{array}$ & $\begin{array}{l}82 \\
8 ?\end{array}$ & 6 & 0042 & 0000 \\
\hline CHN & 82 & 6 & $\begin{array}{l}00^{44} \\
00^{4 / 4}\end{array}$ & 0000 \\
\hline $\mathrm{CHN}$ & 82 & 8 & 0045 & 0000 \\
\hline $\begin{array}{l}C H N \\
C H N\end{array}$ & $\begin{array}{l}82 \\
82\end{array}$ & $\begin{array}{l}8 \\
8\end{array}$ & $\begin{array}{l}0049 \\
0049\end{array}$ & 0000 \\
\hline $\mathrm{CHN}$ & 82 & 8 & 0050 & 0000 \\
\hline $\mathrm{CHN}$ & 83 & $\stackrel{8}{8}$ & 0050 & 0000 \\
\hline CHN & 82 & 8 & 0051 & 0000 \\
\hline CHN & 8 & 8 & 0051 & 0000 \\
\hline$C H N$ & 82 & 8 & 0054 & 0000 \\
\hline ctins & 82 & 8 & 0057 & 0000 \\
\hline & 82 & 8 & & 0000 \\
\hline
\end{tabular}

\begin{tabular}{|c|c|c|c|c|c|c|}
\hline & & & & & & \\
\hline 64 & 38 & $45 \cdot 300^{N}$ & $27 \cdot 500 \mathrm{~W}$ & 5 & $147 \cdot 57$ & $22^{8}$ \\
\hline $\begin{array}{l}64 \\
64\end{array}$ & $\begin{array}{l}89 \\
819\end{array}$ & $\begin{array}{l}44 \cdot 36 \mathrm{O}^{\mathrm{N}} \\
42 \cdot 37 \mathrm{IN}^{\mathrm{N}}\end{array}$ & $\begin{array}{l}28.100 \mathrm{~W} \\
28.448 \mathrm{~W}\end{array}$ & $\begin{array}{l}5 \\
5\end{array}$ & $\begin{array}{l}147.48 \\
147.38\end{array}$ & 0030 \\
\hline 64 & 812 & $42 \cdot 381 \mathrm{~N}$ & $28.484 W$ & 5 & $147 \cdot 28$ & 0033 \\
\hline 68 & & $41.38 \mathrm{ON}$ & $27 \cdot 200 w$ & & 147 & $000^{3}$ \\
\hline 68 & & $41 \cdot 380 \mathrm{~N}$ & $27.200 \mathrm{~W}$ & 8 & 147.17 & 0003 \\
\hline $\begin{array}{l}68 \\
68\end{array}$ & & $\begin{array}{l}87 \mathrm{~N} \\
87 \mathrm{~N}\end{array}$ & $\begin{array}{l}28 \cdot 345 W \\
38 \cdot 345 w\end{array}$ & 8 & $\begin{array}{l}147 \cdot 2^{8} \\
147 \cdot 38\end{array}$ & 0 \\
\hline 00 & 89 & $42 \cdot 0^{6} 0^{N}$ & $28 \cdot 160 \mathrm{~W}$ & & & \\
\hline 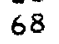 & 89 & $42 \cdot 0^{6} O^{N}$ & $160 \mathrm{~W}$ & & 14 & \\
\hline 68 & 810 & $42 \cdot 000 \mathrm{~N}$ & $540 \mathrm{~W}$ & & 147 & \\
\hline 68 & 810 & $42 \cdot 000 \mathrm{~N}$ & $29.540 \mathrm{w}$ & & & $0^{8}$ \\
\hline 68 & 817 & $40^{\circ} 48 \mathrm{2N}$ & $26.270 \mathrm{w}$ & 8 & & 8 \\
\hline 68 & $\begin{array}{l}816 \\
816\end{array}$ & $\begin{array}{l}41.506 N \\
41.506 N\end{array}$ & $\begin{array}{l}26 \cdot 270 \mathrm{~W} \\
26 \cdot 270 \mathrm{~W}\end{array}$ & 8 & & 000 \\
\hline 8 & 820 & $43.45 \mathrm{ON}$ & $27.465 \mathrm{~W}$ & 8 & & 00 \\
\hline 68 & 820 & 43 & $65 \mathrm{~W}$ & 8 & & \\
\hline 68 & 822 & $42 \cdot 285 \mathrm{~N}$ & $28.400 \mathrm{~W}$ & 8 & & 0 \\
\hline 8 & 822 & $42 \cdot 2^{8} 5 \mathrm{~N}$ & $28.400 \mathrm{~W}$ & 8 & 14 & 001 \\
\hline$\gamma$ & 80 & 42 & $85 w$ & 8 & & 0 \\
\hline 8 & 826 & 43. & $3^{9} \mathrm{w}$ & 8 & & 0 \\
\hline 58 & 826 & $43.223 \mathrm{~N}$ & $28 \cdot 13^{9} \mathrm{k}$ & 8 & 14 & 0 \\
\hline 68 & 826 & $43 \cdot 196 \mathrm{~N}$ & $46 W$ & & & \\
\hline 68 & 831 & $40^{.577 N}$ & $115 \mathrm{~W}$ & 8 & & 00 \\
\hline$=$ & 831 & $40.577 \mathrm{~N}$ & $\because 5 \mathrm{w}$ & & 7 & \\
\hline 68 & 831 & $41 \cdot 3$ & $70 \mathrm{w}$ & 0 & 147 & \\
\hline 68 & 93 & $43 \cdot 2$ & $44 k !$ & 0 & 14 & \\
\hline 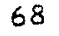 & & $43 \cdot z$ & & 4 & & \\
\hline 68 & 94 & $43 \cdot 2^{99 N}$ & $520 \mathrm{~W}$ & 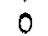 & 14 & \\
\hline 00 & 94 & $43^{\circ}$ & 29 & 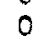 & & \\
\hline 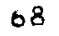 & & $43 \cdot 173 N$ & 25 & 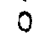 & & \\
\hline 58 & ${ }^{4}$ & $43 \cdot 1$ & $498 \mathrm{~W}$ & & $147 \cdot 39$ & \\
\hline$g=$ & 6 & $43 \cdot 5$ & $27.575 \mathrm{w}$ & 8 & 147 & \\
\hline 68 & & $43 \cdot 2$ & $30.396 \mathrm{w}$ & 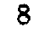 & & \\
\hline & 97 & $43 \cdot 3^{9} 5 \mathrm{~N}$ & $30 \cdot 130 \mathrm{~W}$ & 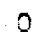 & 147 & \\
\hline
\end{tabular}

\begin{tabular}{|c|c|c|}
\hline 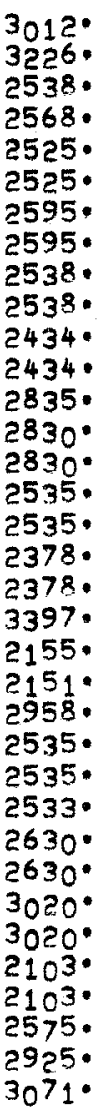 & 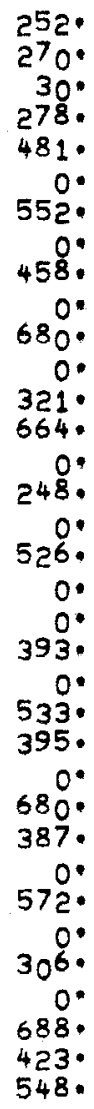 & $\begin{array}{l}0000 \\
0000 \\
0000 \\
0000 \\
0000 \\
0062 \\
0000 \\
0020 \\
0000 \\
0075 \\
0000 \\
0065 \\
0000 \\
0000 \\
0064 \\
0000 \\
0079 \\
0000 \\
0077 \\
0036 \\
0000 \\
0122 \\
0000 \\
0000 \\
0111 \\
0000 \\
0000 \\
0045 \\
0000 \\
0066 \\
0000 \\
0084 \\
0000 \\
0000 \\
0000\end{array}$ \\
\hline
\end{tabular}

3964

396946

33590

$\begin{array}{ll}0000 & 2 \\ 3938 & 54\end{array}$

332954

332454

332454

335954

$\begin{array}{ll}3328 & 54 \\ 3322 & 54\end{array}$

332254

332954

332954

393254

393254

333854

333854

$\begin{array}{ll}3339 & 54 \\ 3334 & 54\end{array}$

$3334 \quad 54$

333854

335956

302856

332456

373856

303456

$\begin{array}{rr}3034 & 56 \\ 3738 & 0\end{array}$

$\begin{array}{ll}3738 & 0 \\ 3322 & 0\end{array}$

3322
3369

3838

$\begin{array}{ll}3029 & 0 \\ 3738 & 0\end{array}$

3738

303854

$\begin{array}{ll}3038 & 54 \\ 3328 & 54\end{array}$

$\begin{array}{rr}3328 & 54 \\ 3738 & 0\end{array}$ 
0057

$* * * * * * * * * * * * * *$
STATIEN DATA RETRIEVAL

DATE: 12:36 MAY 16,175

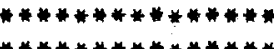 \\ $* * * * * * * * * * * * * * *$}

PAGE 23

**WHOI**

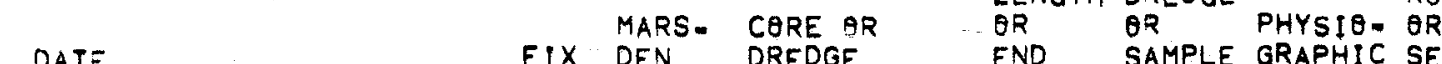

\begin{tabular}{lll} 
CORE & LENGTH, & \multicolumn{2}{l}{ ROCK } \\
LENGTH & DREDGE & PHYSIO- \\
OR & OR & PHYIO- \\
END & SAMPLE GRAPHIC SED. VIYA
\end{tabular}

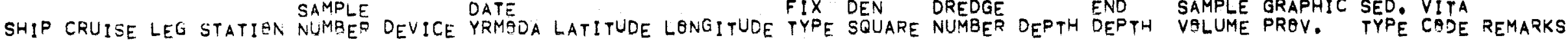

MARSDEN SQUARE \# 148

$\begin{array}{llllll}C H N & 13 & 1 & 0003 & 0000 & 15 \\ C H N & 82 & 6 & 0024 & 0000 & 26 \\ C H N & 82 & 6 & 0024 & 0000 & 15 \\ C H N & 82 & 6 & 0026 & 0000 & 15 \\ C H N & 82 & 6 & 0026 & 0000 & 26 \\ C H N & 82 & 6 & 0031 & 0000 & 15 \\ C H N & 82 & 6 & 0031 & 0000 & 26 \\ C H N & 82 & 8 & 0055 & 0000 & 15 \\ C H N & 82 & 8 & 0059 & 0000 & 15\end{array}$

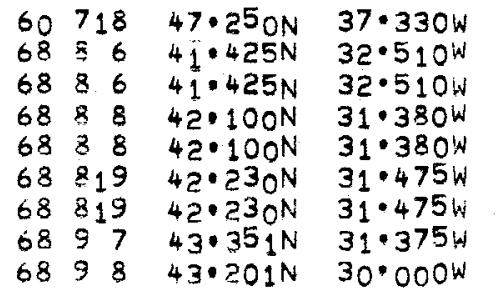

148.770003 $148 \cdot 12000^{4}$ $148 \cdot 12.0004$ $148 \cdot 210006$ $148 \cdot 210006$ $148 \cdot 210011$ $148 \cdot 210011$ $148 \cdot 310023$ $148 \cdot 300026$

$\begin{array}{rrr}2716: & 847: & 0000 \\ 3427: & 0: & 0077 \\ 3427: & 669: & 0000 \\ 3153: & 394: & 0000 \\ 3132: & 0 \cdot & 0086 \\ 3209: & 541: & 0000 \\ 3209 \cdot & 0: & 0086 \\ 3406: & 621: & 0000 \\ 2760 . & 495 \cdot & 0000\end{array}$

$3864 \quad 0$ 332254 332954 332854 332554 333854 $3868 \quad 54$ $3338 \quad 54$

032954

MARSDEN SQUARE \# 151

$\begin{array}{llllll}\text { AII } & 33 & 1 & 0001 & 0000 & 15 \\ \text { KNR } & 27 & 1 & 0001 & 0000 & 16\end{array}$

$67728 \cdot 43.010 \mathrm{~N} 70.260 \mathrm{~W} \quad 5 \quad 151.30 .0001$

105. 468, 0000

$4839 \quad 0$

MARSDEN SQUARE \# 152

$\begin{array}{llllll}\text { A!I } & 72 & 1 & 0001 & 0000 & 15 \\ \text { AII } & 72 & 1 & 0001 & 0000 & 26 \\ \text { AII } & 72 & 1 & 0010 & 0000 & 15 \\ \text { AII } & 72 & 1 & 0012 & 0000 & 15 \\ \text { KNR } & 10 & 1 & 0001 & 0000 & 16\end{array}$

$\begin{array}{llll}721021 & 40.077 \mathrm{~N} & 70.344 \mathrm{~W} & 5 \\ 721021 & 40.077 \mathrm{~N} & 70.344 \mathrm{~W} & 5 \\ 721022 & 40.044 \mathrm{~N} & 70.595 \mathrm{~W} & 5 \\ 721023 & 40.013 \mathrm{~N} & 70.444 \mathrm{~W} & 9 \\ 7099 & 42.246 \mathrm{~N} & 70.343 \mathrm{~W} & 3\end{array}$

$152 \cdot 470001$ $152 \cdot 470001$ $152 \cdot 000009$ 152.000010 $152 \cdot 200001$

$\begin{array}{rrrrrr}117 \cdot & 468 \cdot & 0000 & 2 & 8855 & 0 \\ 117 \cdot & 0 \cdot & 0037 & 2 & 2255 & 0 \\ 214 \cdot & 364 \cdot & 0000 & 4 & 8855 & 0 \\ 214 \cdot & 375 \cdot & 0000 & 4 & 2838 & 0 \\ 77 . & 2174 \cdot & 0000 & 2 & 0010 & 0\end{array}$

MARSDEN SQUARE \# 177

$\begin{array}{llllll}\text { AII } & 49 & 1 & 1433 & 0000 & 15 \\ \text { AII } & 49 & 3 & 1431 & 0000 & 15 \\ \text { AII } & 49 & 3 & 1432 & 0000 & 13 \\ \text { AII } & 49 & 3 & 1434 & 0000 & 15 \\ \text { AI1 } & 49 & 3 & 1435 & 0000 & 13 \\ \text { AII } & 49 & 3 & 1436 & 0000 & 15 \\ \text { AII } & 49 & 3 & 1436 & 0000 & 26 \\ \text { AII } & 49 & 3 & 1437 & 0000 & 13 \\ \text { AII } & 49 & 3 & 1438 & 0000 & 13 \\ \text { AII } & 49 & 3 & 1439 & 0000 & 15 \\ \text { AI1 } & 49 & 3 & 1440 & 0000 & 13 \\ \text { AII } & 49 & 3 & 1440 & 0000 & 15 \\ \text { AII } & 49 & 3 & 1440 & 0000 & 26 \\ \text { AII } & 49 & 3 & 1442 & 0000 & 15\end{array}$

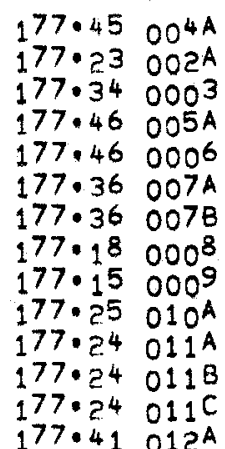

$2225 \cdot 493 \cdot 0000$ $2136.491 \% 0000$ $1466.124 \circ 0000$ 1033. 160. 0000 2158.535 .0000 2158.0 .0119 973.62 .0000 $\begin{array}{lll}973^{\circ} & 62^{\circ} & 0000 \\ 284^{\circ} & 145^{\circ} & 0000\end{array}$ 1943. $700 \cdot 0000$ 264. 131. 0000 $207 \cdot 740 \cdot 0000$

$20^{7} \cdot 128 \cdot 0000$ $420^{\circ} 278.0000$

$\begin{array}{lll}21 & 4311 & 0 \\ 21 & 3226 & 0 \\ 21 & 3419 & 0 \\ 21 & 2319 & 0 \\ 21 & 2329 & 0 \\ 21 & 2431 & 0 \\ 21 & 4349 & 0 \\ 21 & 3219 & 0 \\ 21 & 3316 & 0 \\ 21 & 2815 & 0 \\ 21 & 4319 & 0 \\ 21 & 3125 & 0 \\ 21 & 0059 & 0 \\ 21 & 4839 & 0\end{array}$


0058

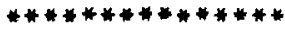

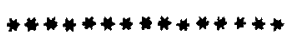

STATION DATA RETRIEVAL

DATE: $12: 36$ MAY 16:175

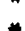

$* * 4 * * * * * * * * * *$

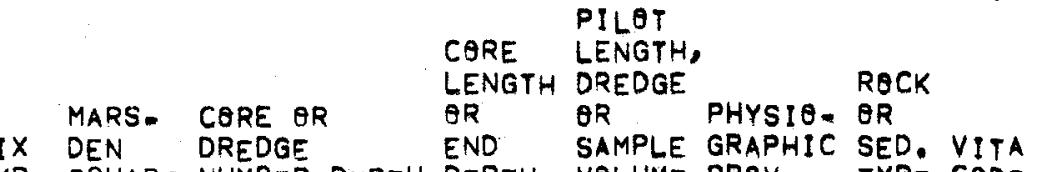

PAGE 24

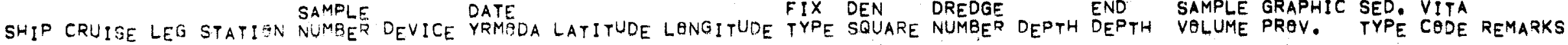

MARSDEN SQUARE \# 177

\begin{tabular}{|c|c|c|c|c|}
\hline$A I I$ & 49 & 3 & 1442 & 0000 \\
\hline AII & 49 & 3 & 1442 & 0000 \\
\hline AII & 49 & 3 & 1443 & 0000 \\
\hline Al I & 49 & 3 & 1443 & 0000 \\
\hline AII & 49 & 3 & 1444 & 0000 \\
\hline A II & 49 & 3 & 1445 & 0000 \\
\hline$A\lfloor I$ & 49 & 3 & 1445 & 0000 \\
\hline AII & 49 & 3 & 1446 & 0000 \\
\hline AII & 49 & 3 & 1446 & 0000 \\
\hline AII & 49 & 3 & 1447 & 0000 \\
\hline AII & 49 & 3 & 1447 & 0000 \\
\hline A II & 49 & 3 & 1450 & 0000 \\
\hline AII & 49 & 3 & 1462 & 0000 \\
\hline Ali & 49 & 4 & 1462 & 0000 \\
\hline AII & 49 & 4 & 1462 & 0000 \\
\hline AII & 49 & 4 & 1462 & 0000 \\
\hline$A \perp I$ & 49 & 4 & 1464 & 0000 \\
\hline$A ! !$ & 49 & 4 & 1464 & 0000 \\
\hline A II & 49 & 4 & 1464 & 0000 \\
\hline A I I & 49 & 4 & 1464 & 0000 \\
\hline$A \llbracket !$ & 49 & 4 & 1464 & 0000 \\
\hline AI! & 49 & 4 & 1466 & 0000 \\
\hline AII & 49 & 4 & 1466 & 0000 \\
\hline AII & 49 & 4 & 1466 & 0000 \\
\hline$A] I$ & 49 & 4 & 1468 & 0000 \\
\hline A II & 49 & 4 & 1470 & 0000 \\
\hline A!I & 49 & 4 & 1472 & 0000 \\
\hline AII & 49 & 4 & 147.3 & 0000 \\
\hline AII & 49 & 4 & $1 * 74$ & 0000 \\
\hline AII & 49 & 4 & 1474 & 0000 \\
\hline II & 49 & 4 & 1474 & 0000 \\
\hline Ali & 49 & 4 & 1476 & 0000 \\
\hline AII & 49 & 4 & 1476 & 0000 \\
\hline AII & 49 & 4 & 1477 & 0000 \\
\hline AII & 49 & 4 & 1478 & 0000 \\
\hline$A !$ & 49 & 4 & 1478 & 0000 \\
\hline A I & 49 & 4 & 1479 & 0000 \\
\hline AII & 49 & 4 & 1479 & 0000 \\
\hline AI & 49 & 4 & 1480 & 0000 \\
\hline AI & 49 & 4 & 1480 & 0000 \\
\hline AI & 49 & 4 & 1481 & 0000 \\
\hline AI & 49 & 4 & 1481 & 0000 \\
\hline AI & 49 & 4 & 1484 & 0000 \\
\hline A! & 49 & 4 & 1484 & 0000 \\
\hline$A I$ & 49 & 4 & 1485 & 0000 \\
\hline
\end{tabular}

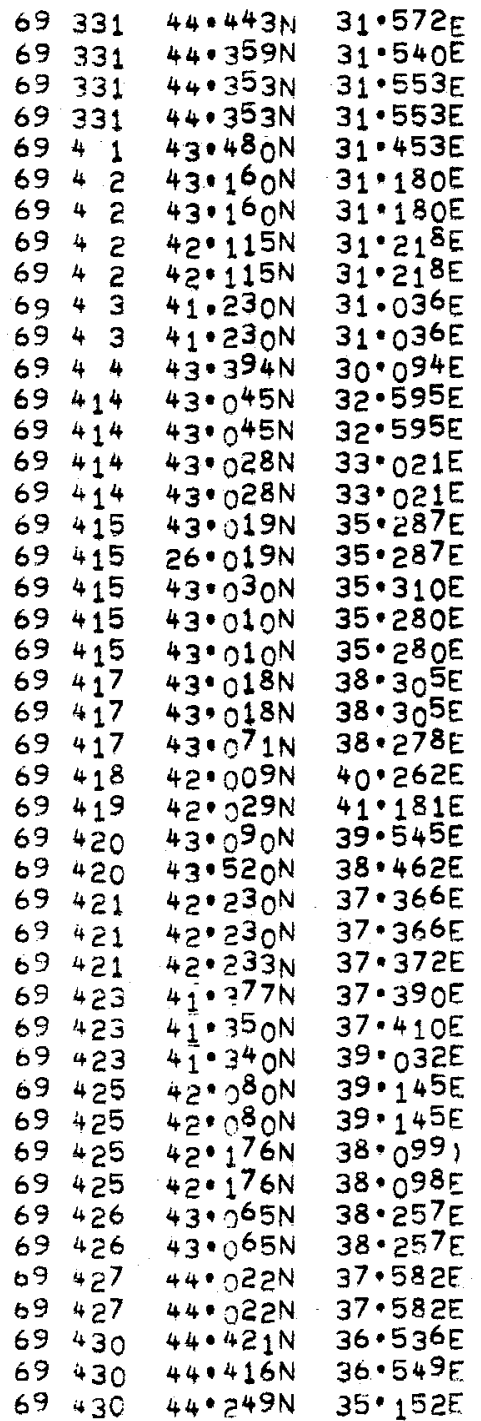

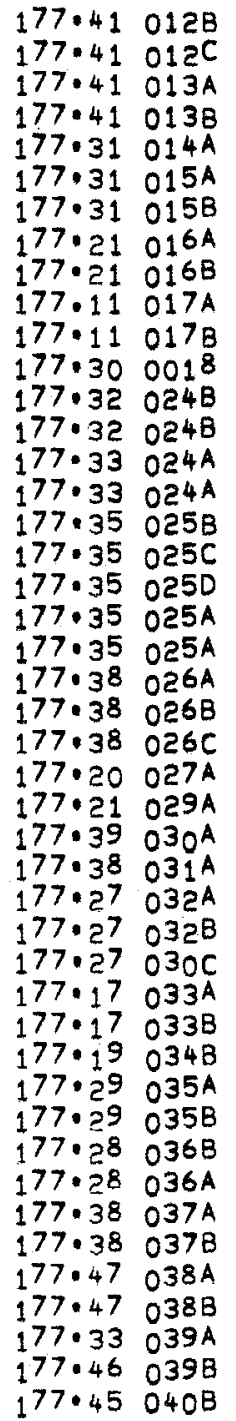

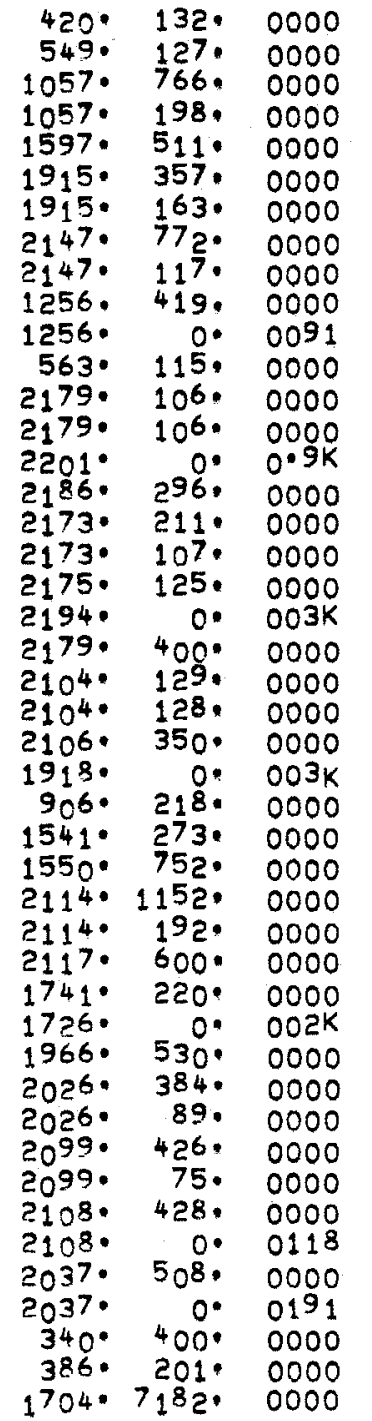

4326

3419

$\begin{array}{ll}2416 & 0 \\ 3429 & 0\end{array}$

42160

32160

34190

$\begin{array}{ll}4831 & 0 \\ 0239 & 0\end{array}$

$\begin{array}{ll}0239 & 0 \\ 3219 & 0\end{array}$

46690

34160

43190

0000 UNSPLIT

3449

23190

23190

$8000 \quad 0$

34190

00000 UNSPLIT

0000

0

00000

2250

2339

23390

2319

00000

82490

2349

28690

23190

$\begin{array}{ll}2311 & 0 \\ 3419 & 0\end{array}$

23190

23190

34590

2219 
0059

$* * * * * * * * * * * * * *$

\section{$\begin{array}{llll} & \text { MARS - CORE OR } & \text { OR } & \text { OR } \\ \text { SAMPLE DATE PHYSIOE OR } & \text { FIX DEN OREDGE } & \text { END SAMPE GRAPHIC SED. VITA }\end{array}$}

$\begin{array}{lll}\text { CORE } & \text { LENGTH, } \\ \text { LENGTH DREDGE } & \text { ROCK } \\ \text { OR } & \text { OR } & \text { PHYSIO- } \\ \text { END } & \text { SAMPLE GRAPHIC SED. VITA }\end{array}$

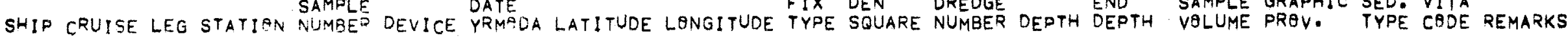

MARSDEN SQUARE $\# 177$

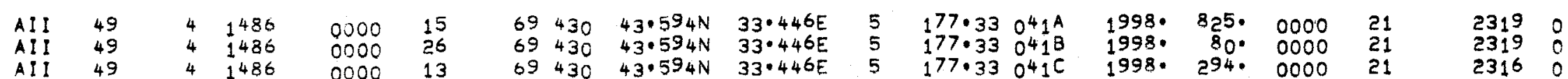

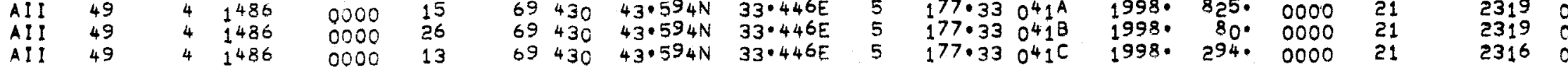

MARSDEN SQUARE \# 178

$\begin{array}{llllll}A ! I & 49 & 3 & 1430 & 0000 & 13 \\ A ! ! & 49 & 3 & 1451 & 0000 & 13 \\ A ! ! & 49 & 3 & 1452 & 0000 & 15 \\ A ! ! & 49 & 3 & 1453 & 0000 & 13 \\ A ! I & 49 & 3 & 1461 & 0000 & 15\end{array}$

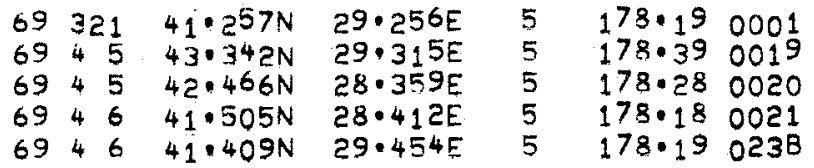

663. $119 \cdot 0000$

$760^{\circ} \quad 82 \cdot 0000$

728. 848,0000

$\begin{array}{rrr}7550^{\circ} & 53 \cdot & 0000 \\ 1788 . & 890 \cdot & 0000\end{array}$

$\begin{array}{lll}21 & 2079 & 0 \\ 21 & 3419 & 0 \\ 21 & 4329 & 0 \\ 21 & 0819 & 0 \\ 21 & 0811 & 0\end{array}$

MARSDEN SQUARE \# 180

$\begin{array}{llllll}C H N & 7 & 7 & 0006 & 0000 & 15 \\ \text { CHN } & 21 & 1 & 0019 & 0000 & 13 \\ \text { CHN } & 21 & 1 & 0020 & 0000 & 15\end{array}$

$5971141 \cdot 350 \mathrm{~N}$

$61930 \quad 42 \cdot 16 \mathrm{CN}$

$4 \cdot 520 \mathrm{E}$

$6110143 \cdot 210 \mathrm{~N}$

$7 \cdot 105 E$
$8 \cdot 155 E$

$180 \cdot 140006$
$180 \cdot 270019$

2499. 764. 0000

2728. 191. 0000

$\begin{array}{rrr}2043 . & 0.0000\end{array}$

$21 \quad 3230 \quad 0$

$180 \cdot 380020$

MARSDEN SQUARE \#183

CHN $\quad 13 \quad 1000^{4} \quad 0000 \quad 15$

$6072353.530 \mathrm{~N} \quad 24 \cdot 120 \mathrm{~W} \quad 0 \quad 183.34000^{4}$

3375. 195. 0000

16

35620

MARSDEN SQUARE \# 215

$\begin{array}{llllll}A I I & 32 & 1 & 000^{4} & 0000 & 13 \\ A ! I & 32 & 1 & 0006 & 0000 & 13 \\ A ! I & 32 & 1 & 000^{7} & 0000 & 13 \\ A I I & 32 & 1 & 0008 & 0000 & 13 \\ A I ! & 32 & 1 & 0011 & 0000 & 13 \\ A ! I & 32 & 1 & 0012 & 0200 & 13 \\ A ! I & 32 & 1 & 0013 & 0000 & 13 \\ A I ! & 32 & 1 & 0017 & 0000 & 13 \\ A ! I & 32 & 1 & 0018 & 0000 & 13 \\ A ! I & 32 & 2 & 0020 & 0000 & 13 \\ A ! I & 32 & 2 & 0021 & 0000 & 13 \\ A ! I & 32 & 2 & 0028 & 0000 & 13 \\ A ! I & 32 & 2 & 0029 & 0000 & 13\end{array}$

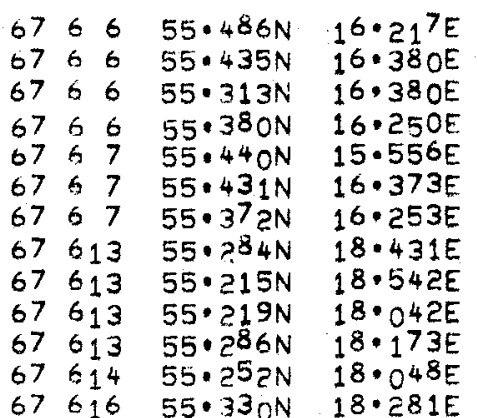

215.560002 $215.56000^{4}$ $215 \cdot 560005$ $215 \cdot 560006$ 215.550007 $215.56000^{8}$ 215.560009 $215.58 \quad 0010$ 215.580010 215.580011 215.580012 215.580013 215.580014

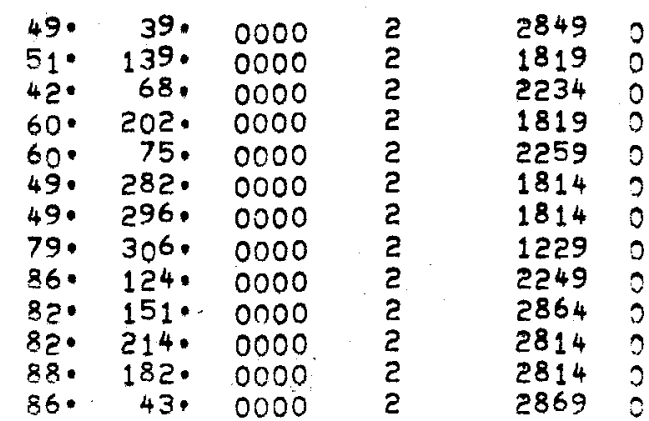




\section{0}

***************

$* * * * * * * * * * * * * * *$
STATION DATA RETRIEVAL

DATE: 12:36 MAY 16,175

\section{***************}

PAGE 26

PILOT

CORE LENGTH

LENGTH DREDGE

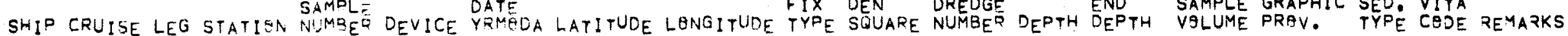

MARSDEN SQUARE \# 215

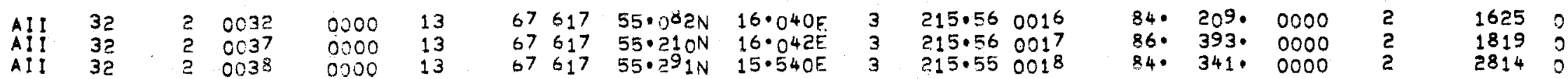

MARSDEN SQUARE \#300

$\begin{array}{llllll}\text { CHN } & 99 & 3 & 0029 & 0000 & 1 \\ C H N & 99 & 3 & 0030 & 0.000 & 1 \\ C H N & 99 & 3 & 0031 & 0000 & 1 \\ C H N & 99 & 3 & 0032 & 0000 & 1 \\ C H N & 99 & 3 & 0033 & 0000 & 1 \\ \text { CHN } & 99 & 3 & 0034 & 0000 & 1 \\ C H N & 99 & 3 & 0034 & 0000 & 26 \\ C H N & 99 & 3 & 0036 & 0000 & 1 \\ \text { CHN } & 99 & 3 & 0036 & 0000 & 26\end{array}$

$\begin{array}{llllll}C H N & 99 & 3 & 0012 & 0000 & 13 \\ C H N & 99 & 3 & 0014 & 0000 & 15 \\ C H N & 99 & 3 & 0014 & 0000 & 26 \\ C H N & 99 & 3 & 0015 & 0000 & 15 \\ C H N & 99 & 3 & 0015 & 0000 & 26 \\ C H N & 99 & 3 & 0016 & 0000 & 15 \\ C H N & 99 & 3 & 0015 & 0000 & 26 \\ C H N & 99 & 3 & 0019 & 0000 & 15 \\ C H N & 99 & 3 & 0019 & 0000 & 26 \\ C H N & 99 & 3 & 0021 & 0000 & 15 \\ C H N & 99 & 3 & 0021 & 0000 & 26 \\ C H N & 99 & 3 & 0023 & 0000 & 15 \\ C H N & 99 & 3 & 002^{4} & 0000 & 15 \\ C H N & 99 & 3 & 002^{4} & 0000 & 15 \\ C H N & 99 & 3 & 0028 & 0000 & 15\end{array}$

$\begin{array}{llllll}\text { AII } & 54 & 2 & 0003 & 0000 & 1 \\ \text { AII } & 54 & 2 & 0003 & 0000 & 2 \\ \text { AII } & 54 & 2 & 0053 & 0000 & 1\end{array}$

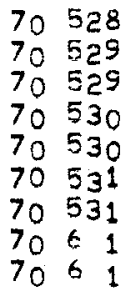

$8 \cdot 4005$
$8 \cdot 3785$
$8 \cdot 4285$
$8 \cdot 4305$
$8 \cdot 4185$
$8 \cdot 4425$
$8 \cdot 4425$
$8 \cdot 4455$
$8 \cdot 4455$

$9 \cdot 250 w$
$8 \cdot 058 w$
$6 \cdot 255 w$
$4 \cdot 587 w$
$3 \cdot 325 w$
$1 \cdot 525 w$
$1 \cdot 525 w$
$: 116 w$
$-116 w$
7061

\section{MARSDEN SQUARE \#301}

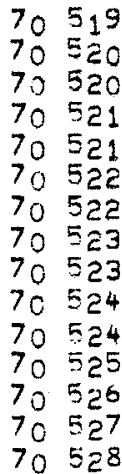

$\begin{array}{ll}\cdot 090 \mathrm{~S} & 19 \cdot 079 \mathrm{~W} \\ 2 \cdot 3495 & 19 \cdot 052 \mathrm{~W} \\ 2 \cdot 3495 & 19 \cdot 052 \mathrm{~W} \\ 4 \cdot 360 \mathrm{~S} & 19 \cdot 030 \mathrm{~W} \\ 4 \cdot 360 \mathrm{~S} & 19 \cdot 030 \mathrm{~W} \\ 6 \cdot 3915 & 18 \cdot 553 \mathrm{~W} \\ 6 \cdot 391 \mathrm{~S} & 18 \cdot 553 \mathrm{~W} \\ 8 \cdot 150 \mathrm{~S} & 17 \cdot 400 \mathrm{~W} \\ 8 \cdot 150 \mathrm{~S} & 17 \cdot 400 \mathrm{~W} \\ 8 \cdot 1035 & 15 \cdot 269 \mathrm{~W} \\ 8 \cdot 1035 & 15 \cdot 269 \mathrm{~W} \\ 6 \cdot 4675 & 12 \cdot 470 \mathrm{~W} \\ 7 \cdot 2015 & 14 \cdot 050 \mathrm{~W} \\ 8 \cdot 2305 & 13 \cdot 036 \mathrm{~W} \\ 8 \cdot 3975 & 10 \cdot 580 \mathrm{~W}\end{array}$

MARSDEN SOUARE \#308

$5.3745 \quad 87.213 \mathrm{~W}$

69115
69115 69115
691129 $\begin{array}{ll}5 \cdot 3745 & 87 \cdot 213 \\ 5 \cdot 3745 & 87 \cdot 213\end{array}$ $\begin{array}{ll}5 \cdot 3745 & 87 \cdot 213 w \\ 2 \cdot 450 S & 86 \cdot 460 w\end{array}$
$300 \cdot 890020$ $300: 880021$ $300 \cdot 86 \quad 0022$ $300 \cdot 840023$ $300 \cdot 830024$ 300.810025 $300 \cdot 810025$ 300.800026

$301 \cdot 0^{9} 0001$ 301.290011 $301 \cdot 290011$ 301.490012 301.490012 $301 \cdot 680013$ $301 \cdot 680013$ $301 \cdot 870014$ $301 \cdot 870014$ $301 \cdot 850015$ $301 \cdot 850015$ 301.620016 301.740017 301.740017

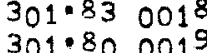

3733. 1129. 0000 4075. 926. 0000 $4660^{\circ} 509.0000$ $4404 \cdot 837.0000$ $4414 \cdot 1066$ : 0000 $4896 \cdot 1076 \cdot 0000$ 4896. $0.0176 \quad 15$ $\begin{array}{rrrr}4914^{\circ} & 1190^{\circ} & 0000 & 15 \\ 494^{\circ} & 0^{\circ} & 0191 & 15\end{array}$

15
15 15

5

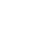

$\begin{array}{lll}3329 & 54 & \text { MAR } \\ 3329 & 54 & \text { MAR } \\ 1339 & 54 & \text { MAR } \\ 3738 & 54 & \text { MAR } \\ 3932 & 54 & \\ 3532 & 54 & \text { MAR } \\ 0549 & 54 & \text { MAR } \\ 3022 & 54 & \\ 3032 & 54 & \end{array}$
$\begin{array}{ll}3022 & 54 \\ 3032 & 54\end{array}$

4195. 31. 0000 $5457 \cdot 1097,0000$ $5457 . \quad 0.0185$ 4353. 1095. 0000 4353. 0.0188 4762.1184. 0000 4762. 0.0186 4029. 1037. 0000 4029.00000 3652. 0.0081 $3286.858: 0000$ 3722. 829. 0000 $3722^{\circ} 829^{\circ} 0000$ $31160^{\circ} 756^{\circ}$. 0000

3339

3339
1432 372954 332254 335954 302254 MAR 334254 MAR 373154 MAR 332954 MAR 332954 MAR 335954 MAR 372954 MAR 372154 ASCENSION 372954 372954 MAR

\section{$5991+5$}

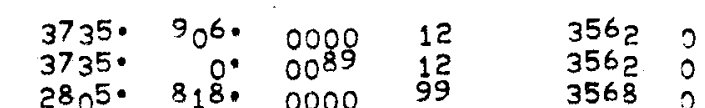


****

\section{$\begin{array}{ll}\text { CORE } & \text { LENGTH, } \\ \text { LENGTH DREDGE } & \text { ROCK } \\ \text { OR } & \text { OR }\end{array}$ \\ END SAMPLE GRAPHIC OR}

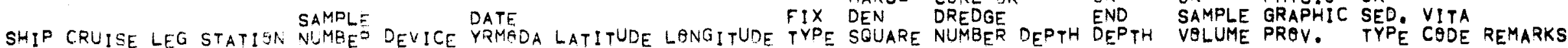

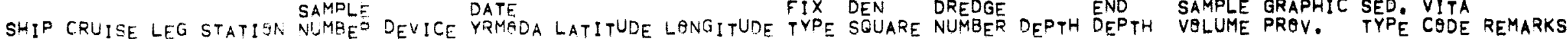

MARSDEN SQUJARE \# 308

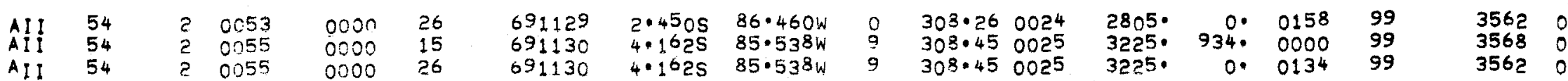

MARSDEN SQUARE \#309

\begin{tabular}{|c|c|c|c|}
\hline$A ! !$ & 2 & 0005 & 0000 \\
\hline 54 & 2 & 0005 & 0000 \\
\hline 54 & 2 & $000^{7}$ & 0000 \\
\hline 54 & 2 & $000^{7}$ & 0000 \\
\hline 54 & 2 & $000^{9}$ & 0000 \\
\hline 54 & 2 & 0009 & 0000 \\
\hline 54 & 2 & 0011 & 0000 \\
\hline 54 & 2 & 0011 & 0000 \\
\hline 54 & 2 & 0012 & 0000 \\
\hline 54 & 2 & 0012 & 0000 \\
\hline 54 & 2 & 0042 & 0900 \\
\hline 54 & 2 & 0042 & 0000 \\
\hline 54 & 2 & 0045 & 0000 \\
\hline 5 & 2 & 0045 & 0000 \\
\hline & 2 & 0047 & 0000 \\
\hline 5 & 2 & 0047 & 0000 \\
\hline & 2 & 0050 & 0000 \\
\hline & 2 & 0050 & 0000 \\
\hline 5 & 2 & 0052 & 0000 \\
\hline 54 & 2 & 0052 & 0000 \\
\hline 100 & 11 & 0140 & 0000 \\
\hline 100 & 11 & 0140 & 0000 \\
\hline 10 & 11 & 0141 & 0000 \\
\hline 100 & 11 & 0141 & 0000 \\
\hline 100 & 11 & 0142 & 0000 \\
\hline 100 & 11 & 0142 & 0000 \\
\hline
\end{tabular}

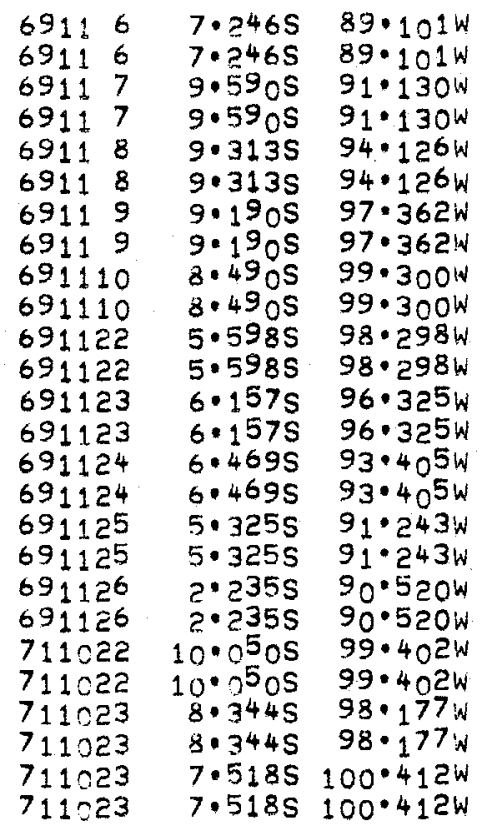

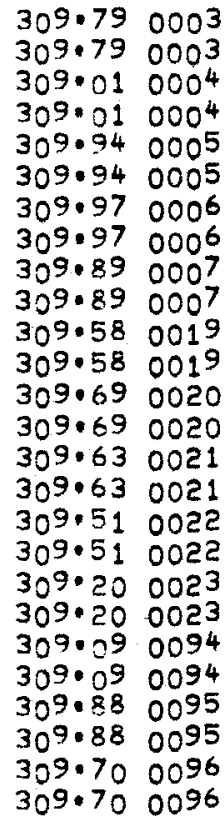

4165. 122, 0000

4165. 0.0140

4115. 428. 0000

$41150^{\circ} \quad 0^{\circ} 0085$

3960. 771. 0000

$3960^{\circ} \quad 0.0091$

4287. 881. 0000

4287.0140

799. 0000

4375. 0.0130

3659. 961. 0000

$\begin{array}{rrr}3659^{\circ} & 00^{\circ} & 0127 \\ 37850^{\circ} & 966 . & 0000\end{array}$

3785.00 .0145

3985. 832. 0000

3985. 0.0155

4075. $1020 \cdot 0000$

$4075 . \quad 0.0160$

$3230^{\circ} 10^{46}$. 0000

$3230^{\circ} 0.006$

4314. 739. 0000

4314. $00^{\circ} 0154$

627.0000

4201: 833. 0000

MARSDEN SQUARE \# 310

$\begin{array}{llllll}\text { AII } & 54 & 2 & 0014 & 0000 & 15 \\ \text { AII } & 54 & 2 & 0014 & 0000 & 26 \\ \text { AII } & 54 & 2 & 0016 & 0000 & 15 \\ \text { AII } & 54 & 2 & 0015 & 0100 & 26 \\ \text { AII } & 54 & 2 & 0013 & 0000 & 15 \\ \text { AII } & 54 & 2 & 0018 & 0700 & 26\end{array}$

691111

691111

691112

691112

691112

$8 \cdot 8^{6} 5 \mathrm{~s} \quad 102 \cdot 120 \mathrm{~W}$ $8 \cdot 2625102 \cdot 120 \mathrm{~W}$ $8 \cdot 0^{7} 4510^{4} \cdot 188 \mathrm{~W}$ $8 \cdot 0745 \quad 104 \cdot 188 \mathrm{~W}$ $7 \cdot 5855 \quad 106 \cdot 325 \mathrm{~W}$ $7 \cdot 585 \mathrm{~s} 106 \cdot 32^{5} \mathrm{w}$

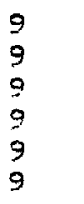

310.820008 310.820008 $310 \cdot 840009$ 310.840009 310.760010 310.760010

$\begin{array}{rrrrrr}3984^{\circ} & 709 \cdot & 0000 & 12 & 4339 & 0 \\ 39840^{\circ} & 0 \cdot & 0129 & 12 & 3339 & 0 \\ 3560^{\circ} & 230^{\circ} & 0000 & 12 & 3324 & 0 \\ 3560^{\circ} & 0 \cdot & 0140 & 12 & 3359 & 0 \\ 34050^{\circ} & 815, & 0000 & 12 & 3562 & 0 \\ 340^{\circ} & 0 . & 0143 & 12 & 3569 & 0\end{array}$

69690

35690

33290

35390

35620

04290

3562

3050

$\begin{array}{ll}3032 & 0 \\ 5369 & 0\end{array}$

35690

35690

03220

03220

3562

35680

35620

35690

$\begin{array}{ll}3569 & 5 \\ 1422 & 54\end{array}$

115254

032254

356254

432954 SAME AS 141

103954 SAME AS 141 


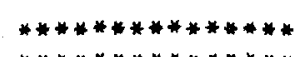
$* * * * * * * * * * * * * *$
STATION DATA RETRIEVAL DATE: $12: 36$ MAY 16,175

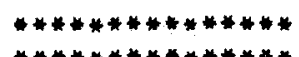

PAGE 28

PILOT

CORE LENGTH

LENGTH DREDGE

ReCX

MARS- CORE OR

SAMPL

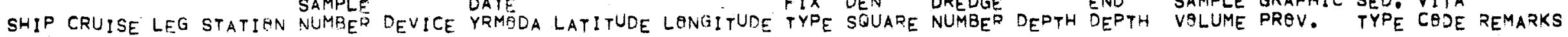

MARSDEN SOUARE \# 310

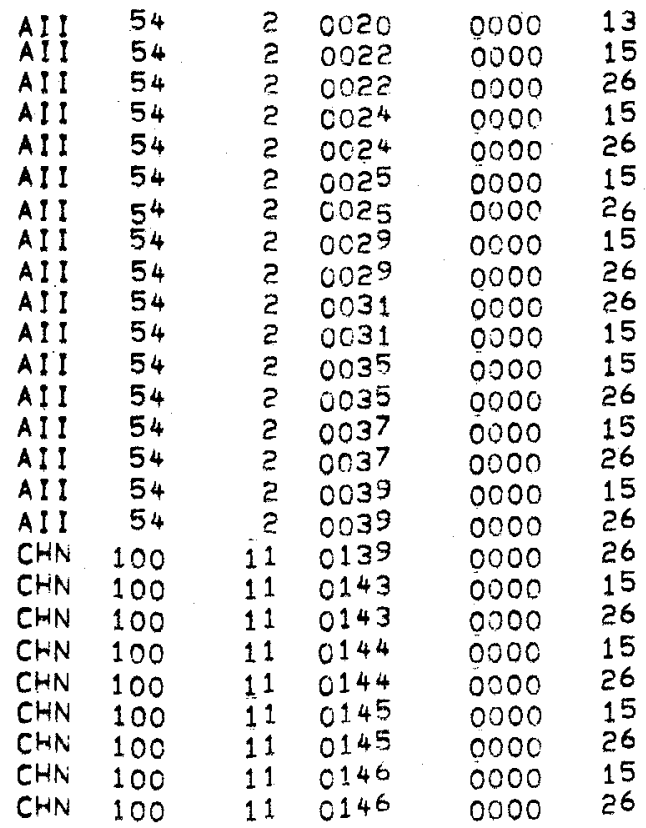

691113
691114
691114
691115
691115
691116
691116
691117
691117
691118
691118
691119
691119
691120
691120
691121
691121
711022
711024
711024
711024
711024
711024
711224
711025
711025

$7 \cdot 260 S 108 \cdot 150 \mathrm{~W}$ $5 \cdot 1975108.483 \mathrm{~W}$ $5 \cdot 1975,108 \cdot 483 \mathrm{~W}$
$5 \cdot 2595108 \cdot 151 \mathrm{~W}$ $5.2595108 \cdot 151 \mathrm{~W}$ $5 \cdot 4415107 \cdot 272 \mathrm{~W}$ $5 \cdot 4415107 \cdot 272 \mathrm{~W}$ $5 \cdot 4395107 \cdot 341 w$ $5 \cdot 4395107 \cdot 341 \mathrm{~W}$ $6 \cdot 2425 \quad 105 \cdot 473 \mathrm{~W}$ $6.2425105 .473 \mathrm{~W}$ $6 \cdot 19$ OS $105 \cdot 397 \mathrm{~W}$ $6 \cdot 1905105.397 \mathrm{~W}$ $5 \cdot 3065102 \cdot 432 \mathrm{~W}$ $5 \cdot 3065102 \cdot 432 \mathrm{~W}$ $5 \cdot 3835101 \cdot 248 \mathrm{~W}$ $5 \cdot 3835101 \cdot 248 \mathrm{~W}$ $0.2285101 .008 \mathrm{~W}$ $7.4485101 \cdot 246 \mathrm{~W}$ $7.4485101 \cdot 246 \mathrm{~W}$ $7 \cdot 3785 \quad 101 \cdot 554 w$ $7 \cdot 3785 \quad 101 \cdot 554 \mathrm{~W}$ $7 \cdot 178 \mathrm{~S} \quad 102 \cdot 582 \mathrm{~W}$ $7 \cdot 1785 \quad 102.582 \mathrm{~W}$ $5 \cdot 5365102 \cdot 388 \mathrm{~W}$ $5 \cdot 536 \mathrm{~S} 102 \cdot 388 \mathrm{~W}$
$310 \cdot 780001$ $310 \cdot 580011$ $310 \cdot 580011$ $310.58 \quad 0012$ 310.570013 310.570013 310.570014 310.570014 310.650015 310.650015 310.650016 $310 \cdot 650016$ 310.520017 $310 \cdot 520017$ 310.510018 310.510018 310.010093 $310 \cdot 710097$ 310.710097 310.710098 310.710098 310.720099 $310 \cdot 720099$ 310.520100 $310.52 \quad 0100$ 310.58001

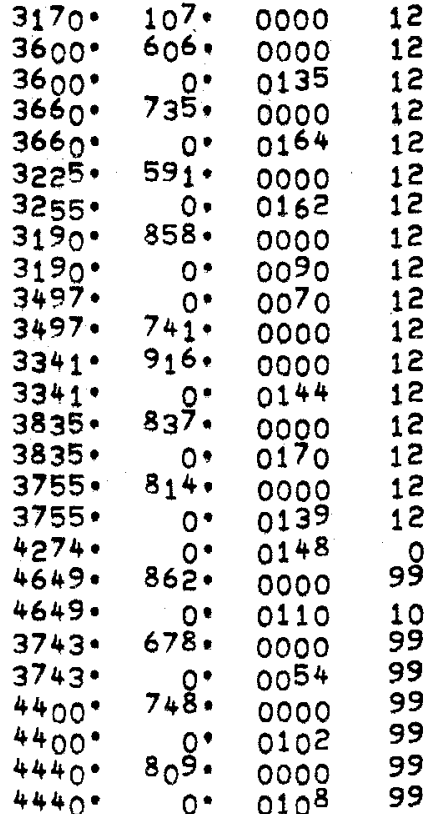

MARSOEN SQUARE \# 316

$\begin{array}{llllll}\text { CHN } & 100 & 10 & 0106 & 0000 & 15 \\ \text { CHN } & 100 & 10 & 0107 & 0000 & 15 \\ C H N & 100 & 10 & 0109 & 0000 & 15 \\ \text { CHN } & 100 & 10 & 0109 & 0000 & 26 \\ C H N & 100 & 10 & 0110 & 0000 & 15 \\ C H N & 100 & 10 & 0110 & 0000 & 26 \\ \text { CHN } & 100 & 10 & 0111 & 0000 & 15 \\ C H N & 100 & 10 & 0111 & 0000 & 26 \\ C H N & 100 & 10 & 0113 & 0000 & 15 \\ C H N & 100 & 10 & 0113 & 0000 & 26 \\ \text { CHN } & 100 & 10 & 0114 & 0000 & 26 \\ \text { CHN } & 100 & 10 & 0116 & 0000 & 15 \\ \text { CHN } & 100 & 10 & 0116 & 0000 & 26 \\ \text { CHN } & 100 & 10 & 0117 & 0000 & 15\end{array}$

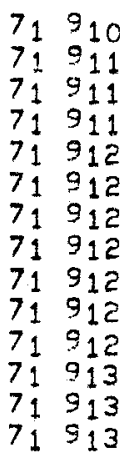

$8 \cdot 0375 \quad 168.341 \mathrm{~W}$ $8 \cdot 0305 \quad 168 \cdot 345 \mathrm{~W}$ $8 \cdot 2825168 \cdot 438 \mathrm{~W}$ $8 \cdot 2825 \quad 168 \cdot 438 \mathrm{~W}$ $8 \cdot 3^{7} 1 \mathrm{~S} 168 \cdot 357 \mathrm{~W}$ $8 \cdot 37$ is $168 \cdot 357 w$ $8.4055,168.551 \mathrm{~W}$ $8.405 \mathrm{~s} 168.551 \mathrm{~W}$ $8 \cdot 2225168 \cdot 517 \mathrm{~h}$ $8.2225168 \cdot 517 \mathrm{~W}$ $8 \cdot 2625168.000 \%$ $8.2035168 .460 \mathrm{~W}$ $8 \cdot 2035168 \cdot 460 \mathrm{~W}$
$316.88 \quad 0072$ $316.88 \quad 0073$ $316 \cdot 88 \quad 0074$ 316.880075 $316.88 \quad 0075$ 316.880076 316.880076 316.880077 $\begin{array}{lll}316.88 & 0077\end{array}$ $316 \cdot 88 \quad 0078$ $316 \cdot 88 \quad 0079$ $316.88 \quad 0079$ $316 \cdot 8800^{80}$ 316.880074

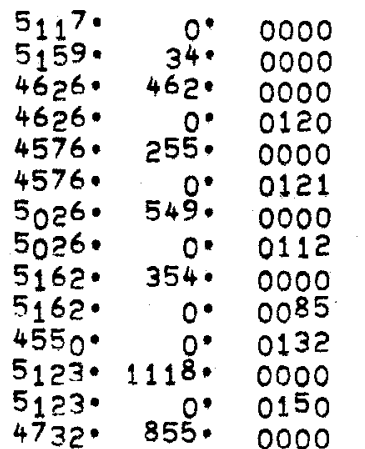

99 99 99 99 99 99 99 99 99 99 99 99 99

33290

03290

03290

03220

03220

03220

03220

3562

0325

0355

03220

03290

03220

33320

35620

3569

342254 SAME AS 138 353854 SAME AS 141 66690

302254

033954

103254 SAME AS 144 536254 SAYE AS 144 536254 SAME AS 144 000054 SAME AS 144

$\begin{array}{lll}8000 & 0 & \text { IN JAR } \\ 8853 & 0 & \\ 1023 & 54 & \\ 1023 & 54 \\ 3123 & 54 \\ 3129 & 54 \\ 1323 & 54 & \\ 1323 & 54 & \text { SAMOAN PAS } \\ 1323 & 54 & \\ 1323 & 54 \\ 3423 & 54 \\ 1153 & 0 \\ 1653 & 0 \\ 1323 & 54 & \end{array}$


$* * * * * * * * * * * * * * *$

$* * * * * * * * * * * * * *$
STATION DATA RETRIEVAL DATE: $12: 36$ MAY 16,175

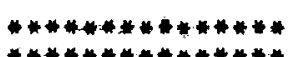

PAGE 29

CORE LENGTH

MARS - CORE $9 R$ LENGTH DREDGE PHYSIO- RQR

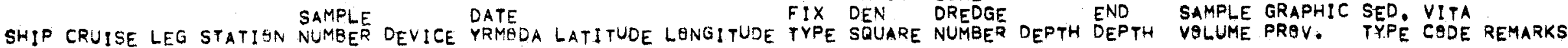

\section{MARSDEN SQUARE \# 316}

$\begin{array}{llllll}\text { CHN } & 100 & 10 & 0117 & 0000 & 26 \\ \text { CHN } & 100 & 10 & C 119 & 0000 & 15 \\ \text { CHN } & 100 & 10 & 0118 & 0000 & 26 \\ \text { CHN } & 100 & 10 & 0123 & 0000 & 14 \\ \text { CHN } & 100 & 10 & 0124 & 0000 & 15 \\ \text { CHN } & 100 & 10 & 1124 & 0000 & 26 \\ \text { CHN } & 100 & 10 & 0126 & 0000 & 15 \\ \text { CHN } & 100 & 10 & 0125 & 0000 & 26 \\ \text { CHN } & 100 & 10 & 0127 & 0000 & 15 \\ \text { CHN } & 100 & 10 & C 128 & 0000 & 15 \\ \text { CHN } & 100 & 10 & 0129 & 0000 & 26 \\ \text { CHN } & 100 & 10 & C 129 & 0000 & 15 \\ \text { CHN } & 100 & 10 & 0131 & 0000 & 15 \\ \text { CHN } & 100 & 10 & 0131 & 0000 & 26 \\ \text { CHN } & 100 & 10 & 0132 & 0000 & 15 \\ \text { CHN } & 100 & 10 & 0132 & 0000 & 26\end{array}$

$\begin{array}{ll}71 & 913 \\ 71 & 914 \\ 71 & 914 \\ 71 & 916 \\ 71 & 916 \\ 71 & 916 \\ 71 & 916 \\ 71 & 916 \\ 71 & 917 \\ 71 & 917 \\ 71 & 918 \\ 71 & 918 \\ 71 & 918 \\ 71 & 918 \\ 71 & 918 \\ 71 & 918\end{array}$

$8 \cdot 1885168 \cdot 323 w \quad 1$ $8 \cdot 1525168 \cdot 398 \mathrm{~W}$ $8.1525168 \cdot 398 \mathrm{~W}$ $8.1605168 .269 \mathrm{~W}$ $7.1905168 .216 \mathrm{~W}$ $7 \cdot 120 \mathrm{~s} 168.216 \mathrm{~W}$ $7 \cdot 1905168 \cdot 216 \mathrm{~W}$ $7 \cdot 2585168 \cdot 320 \mathrm{~W}$ $7 \cdot 2585168 \cdot 320 \mathrm{~W}$ $7 \cdot 2355168 \cdot 413 \mathrm{w}$ $7 \cdot 3635 \quad 167.57$ ow $7 \cdot 3635167 \cdot 570 \mathrm{w}$ $7.3675168 .106 \mathrm{~W}$ $7 \cdot 3675168.106 \mathrm{~W}$ $7 \cdot 3675168 \cdot 106 \mathrm{~W}$ $\begin{array}{ll}7 \cdot 2725 & 168 \cdot 067 W \\ 7 \cdot 2725 & 168 \cdot 067 W\end{array}$ $7 \cdot 2125 \quad 168 \cdot 312 \mathrm{~W}$

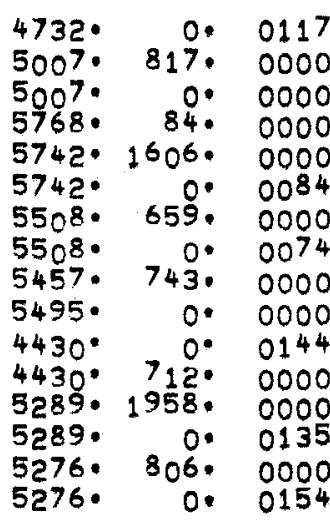

132354 SAMGAN PAS 1123 O SAMOAN PAS 14330 363154 115954 11630 16630 11390 0000 O IN JAR 3469

372354

633954

152954

16630 $\begin{array}{ll}1663 & 0 \\ 1163 & 0\end{array}$

MARSOEN SQUARE \# 319

$\begin{array}{llllllll}71912 & 8.2^{6} 2 S & 168.000 \mathrm{~W} & 1 & 319.88 & 0078\end{array}$

MARSDEN SQUARE \# 328

$\begin{array}{llllll}\text { Al1 } & 15 & 15 & 0^{769} & 0000 & 13 \\ & & & & & \\ & & & & & \\ \text { CHN } & 43 & 1 & 0068 & 0000 & 15 \\ & & & & & \\ & & & & & \\ \text { CHN } & 43 & 1 & 0007 & 0000 & 15 \\ \text { CHN } & 43 & 1 & 0012 & 0000 & 13 \\ \text { CHN } & 43 & 1 & 0015 & 0000 & 13 \\ \text { CHN } & 43 & 1 & 0053 & 0200 & 13 \\ \text { CHN } & 100 & 4 & 0548 & 0000 & 15 \\ \text { CHN } & 100 & 4 & 0.48 & 0000 & 26\end{array}$

$\begin{array}{llllllll}0576 & 31.58 & 5 & 70 \cdot 420 E & 9 & 328 \cdot 30 & 0769\end{array}$

MARSDEN SQUARE \# 329

$64528 \quad 1 \cdot 0255 \quad 6_{1} \cdot 120 E \quad 5 \quad 329 \cdot 110022$

MARSDEN SQUARE \# 330

$\begin{array}{rrrr}64 & 47 & 5 \cdot 52 O N & 53.510 E \\ 6449 & 1.38 O S & 53.2 O C E \\ 64411 & 2.55 O S & 55.430 E \\ 64517 & 7.1645 & 60.320 E \\ 71 & 420 & .145 S & 56.035 E \\ 71 & 420 & .1455 & 56.035 E\end{array}$

330.530001 $330 \cdot 130006$ $330 \cdot 25000^{8}$ 330.700018 $330.06 \quad 0036$ $330 \cdot 06 \quad 0036$
4944. 582,0000 3697. 305. 0000 3791. 114. 0000 4576. 866 . 0000 4576. 0.0083
342354

$0053 \quad 0$

$3449 \cdot 0$

34690

35690

35690

3569
073154

302954 
MARS. CORE SR LENGTH DREDGE PHSIO ROCK

SAMPLF FIX DEN DREDGE END SAMPLE GRAPHIC SED. VITA

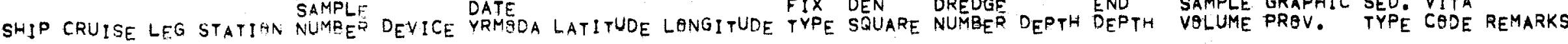

MARSDEN SOUARE \# 331

$\begin{array}{llllll}C H N & 99 & 8 & 0058 & 0000 & 15 \\ C H N & 99 & 8 & 0059 & 0000 & 15 \\ C H N & 99 & 8 & 0059 & 0000 & 26 \\ C H N & 99 & 8 & 0060 & 0000 & 15 \\ C H N & 99 & 8 & 0060 & 0000 & 26 \\ C H N & 99 & 8 & 0061 & 0000 & 15 \\ C H N & 99 & 8 & 0061 & 0000 & 26 \\ C H N & 99 & 8 & 0062 & 0000 & 15 \\ C H N & 99 & 8 & 0062 & 0000 & 26 \\ C H N & 99 & 8 & 0063 & 0000 & 15 \\ C H N & 99 & 8 & 0063 & 0000 & 26\end{array}$

$\begin{array}{llll}701125 & 3 \cdot 324 S & 42 \cdot 283 E & 9 \\ 701126 & 3 \cdot 240 S & 45 \cdot 005 E & 9 \\ 701126 & 3 \cdot 2405 & 45 \cdot 005 E & 9 \\ 701128 & 3 \cdot 306 S & 47 \cdot 004 E & 9 \\ 701128 & 3 \cdot 3065 & 47 \cdot 004 E & 9 \\ 701129 & 3 \cdot 2665 & 49 \cdot 492 E & 9 \\ 701129 & 3 \cdot 2665 & 49 \cdot 492 E & 9 \\ 70121 & 6 \cdot 464 S & 48.418 E & 9 \\ 70121 & 6.464 S & 48.418 E & 9 \\ 70122 & 10 \cdot 530 S & 47.377 E & 9 \\ 70122 & 10.5305 & 47 \cdot 377 E & 9\end{array}$

$331 \cdot 320039$ $331 \cdot 350040$ $331 \cdot 350040$ $331 \cdot 370041$ $331 \cdot 370041$ 331.390042 $331 \cdot 390042$ $331 \cdot 68 \quad 0043$ $331.68 \quad 0043$ $331 \cdot 070044$ $331 \cdot 070044$

\begin{tabular}{|c|c|c|}
\hline $\begin{array}{l}3467^{\circ} \\
44440^{\circ} \\
44440^{\circ} \\
4832^{\circ} \\
4832^{\circ} \\
4890^{\circ} \\
4890^{\circ} \\
4425^{\circ} \\
4425^{\circ} \\
40050^{\circ} \\
40050^{\circ}\end{array}$ & $\begin{array}{r}0^{\circ} \\
840^{\circ} \\
0^{\circ} \\
811^{\circ} \\
90^{\circ} \\
990^{\circ} \\
959^{\circ} \\
80^{\circ} \\
88^{\circ}\end{array}$ & $\begin{array}{l}0133 \\
0000 \\
0178 \\
0000 \\
0176 \\
0000 \\
0188 \\
0000 \\
0157 \\
0000 \\
0047\end{array}$ \\
\hline
\end{tabular}

332954

356954

483154

483154

303954

$\begin{array}{ll}3831 & 54 \\ 3569 & 54\end{array}$

373254

143954

383954

MARSDEN SQUARE \# 332

$\begin{array}{llllll}Z Z Z & 70 & 0 & 0001 & 0000 & 15 \\ Z Z Z & 70 & 0 & 000^{4} & 0000 & 15 \\ & & & & & \\ Z Z Z & 70 & 0 & 0006 & 0000 & 15 \\ Z Z Z & 70 & 0 & 0009 & 0000 & 15 \\ Z Z Z & 70 & 0 & 0010 & 0000 & 15 \\ Z Z Z & 70 & 0 & 0011 & 0000 & 15 \\ Z Z Z & 70 & 0 & 0014 & 0000 & 15 \\ Z Z Z & 70 & 0 & 0015 & 0000 & 15 \\ Z Z Z & 70 & 0 & 0018 & 0000 & 15 \\ Z Z Z & 70 & 0 & 0019 & 0000 & 15 \\ Z Z Z & 71 & 0 & 0001 & 0000 & 15 \\ Z Z Z & 71 & 0 & 0004 & 0000 & 13 \\ Z Z Z & 71 & 0 & 0005 & 0000 & 13 \\ Z Z Z & 71 & 0 & 0007 & 0000 & 13 \\ Z Z Z & 71 & 0 & 0009 & 0000 & 15 \\ Z Z Z & 71 & 0 & 0010 & 0000 & 15 \\ z Z Z & 71 & 0 & 0011 & 0000 & 15 \\ Z Z Z & 71 & 0 & 0013 & 0000 & 15 \\ Z Z Z & 71 & 0 & 0014 & 0000 & 15 \\ Z Z Z & 72 & 1 & 0001 & 0000 & 15 \\ Z Z Z & 72 & 1 & 0003 & 0000 & 15 \\ Z Z Z & 72 & 1 & 0004 & 0000 & 15 \\ Z Z Z & 72 & 1 & 0010 & 0000 & 15 \\ Z Z Z & 72 & 1 & 0011 & 0000 & 15 \\ & & & & & \end{array}$

MARSDEN SQUARE \#333

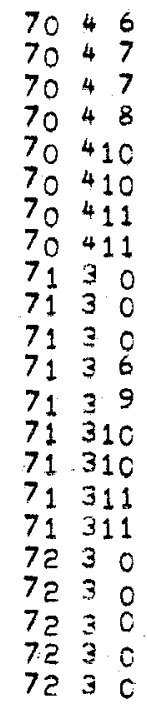

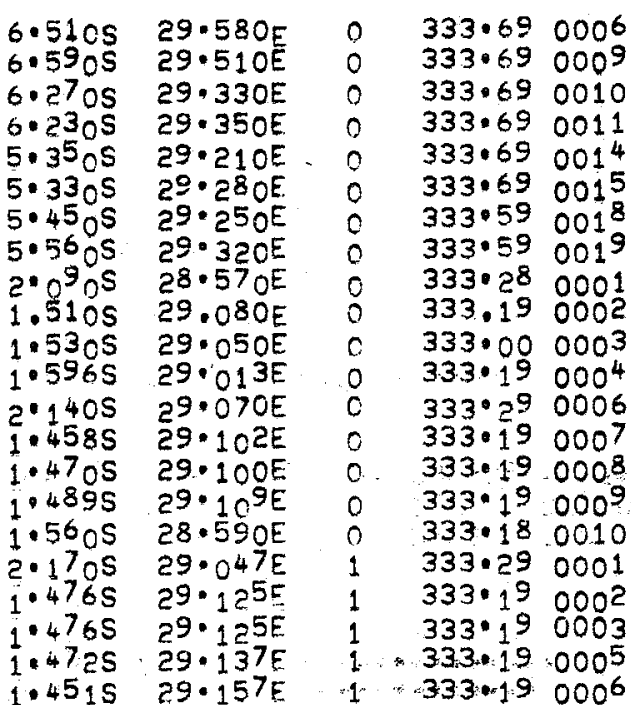

$\begin{array}{rrr}77^{\circ} & 120^{\circ} & 0000 \\ 33 & 0000\end{array}$

22
22

6626 O TANGANYIKA

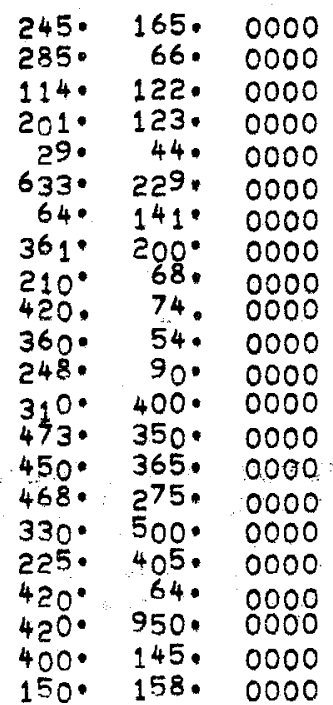

22
22
22
22
22
22
22
22
22
22
22
22
22
22
22
22
22
22
22
22
22
22

$\begin{array}{ll}1530 & 0 \text { IANGANYIKA } \\ 5211 & 0 \text { TANGANYIKA } \\ 5220 & 0 \text { TANGANYIKA } \\ 1519 & 0 \text { TANGANYIKA } \\ 1229 & 0 \text { TANGANYIKA } \\ 6519 & 0 \text { TANGANYIKA } \\ 6519 & 0 \text { TANGANYIKA } \\ 6819 & 0 \text { TANGANYIKA } \\ 5519 & 0 \text { LAKE KIVU } \\ 9469 & 0 \text { LAKE KIVU } \\ 5516 & 0 \text { LAKE KIVU } 1 \\ 5529 & 0 \text { LAKE KIVU } 1 \\ 5846 & 0 \text { LAKE KIVU } \\ 5516 & 0 \text { LAKE KIVU } \\ 5826 & 0 \text { LAKE KIVU } \\ 5246 & 0 \text { LAKE KIVU } \\ 2544 & 0 \text { LAKE KIVU } 1 \\ 5519 & 0 \text { LAKE KIVU } 2 \\ 5836 & \text { DAKE KIVU } \\ 0000 & 0 \text { LAKE KIVU } \\ 5816 & 0 \text { LAKE KIVU } \\ 5819 & 0 \text { LAKE KIVU }\end{array}$




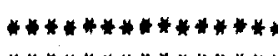

$* * * * * * * * * * * * * *$
STATION DATA RETRIEVAL

DATE: $12: 36$ MAY 16,175
ค***************

$* * * * * * * * * * * * * *$
PAGE 31

CORE LENGTH

LENGTH OREDGE

MARS. CORE QR OR OR PHYSIOH OR

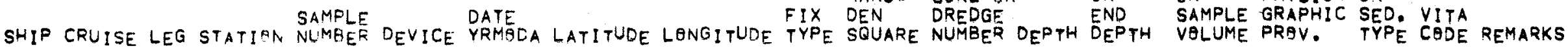

MARSDEN SQUARE \# 333

$\begin{array}{llllll}Z Z Z & 72 & 1 & 0012 & 0000 & 15 \\ Z Z Z & 72 & 1 & 0013 & 0000 & 15 \\ Z Z 2 & 72 & 1 & 0015 & 0000 & 15 \\ Z Z 2 & 72 & 1 & 0019 & 0000 & 15 \\ Z Z 2 & 72 & 2 & 0001 & 0000 & 15 \\ Z Z Z & 72 & 2 & 0002 & 0000 & 15 \\ Z Z 2 & 72 & 2 & 0003 & 0000 & 15 \\ Z Z 2 & 72 & 2 & 0004 & 0000 & 15 \\ Z Z Z & 72 & 2 & 000^{5} & 0000 & 15\end{array}$

$\begin{array}{lll}72 & 3 & 0 \\ 72 & 3 & 0 \\ 72 & 3 & 0 \\ 72 & 3 & 0 \\ 72 & 3 & 0 \\ 72 & 3 & 0 \\ 72 & 3 & 0 \\ 72 & 3 & 0 \\ 72 & 3 & 0\end{array}$

$\begin{array}{ll}1 \cdot 477 S & 29 \cdot 156 E \\ 2 \cdot 2595 & 28 \cdot 516 E \\ 2 \cdot 1775 & 28 \cdot 588 E \\ 1 \cdot 375 S & 29 \cdot 036 E \\ \cdot 335 S & 29 \cdot 264 E \\ \cdot 2925 & 29 \cdot 278 E \\ : 2695 & 29 \cdot 275 E \\ \cdot 211 S & 29 \cdot 270 E \\ \cdot 171 S & 29 \cdot 283 E\end{array}$

$333 \cdot 190007$ $333 \cdot 280008$ 333.280009 $333.1900 \% 0$ 333.090001 333.090002 333.090002 333.090003 333.090004

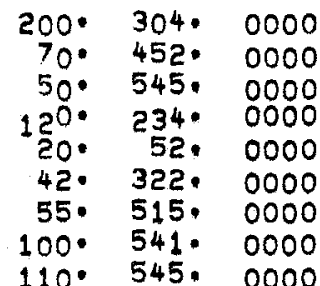

5816 O LAKE KIVU ? 5816 O LAKE KIVU

0000 O LAKE KIVU

2669 D LAKE KIVU

5025 O LAKE EOWARD

5659 D LAKE EDWARD

5279 O LAKE EDWARD

5519 O LAKE EDWARD

5519 O LAKE EDWARD

\section{MARSDEN SQUARE \# 334}

$\begin{array}{llllll}\text { CHN } & 99 & 3 & 0041 & 0000 & 15 \\ \text { CHN } & 99 & 3 & 0041 & 0000 & 26 \\ \text { CHN } & 99 & 3 & 0042 & 0000 & 15 \\ \text { CHN } & 99 & 4 & 0046 & 0000 & 15 \\ \text { CHN } & 99 & 4 & 0046 & 0000 & 26\end{array}$

$\begin{array}{lll}70 & 6 & 5 \\ 70 & 6 & 5 \\ 70 & 6 & 6 \\ 70 & 6 & 6 \\ 70 & 6 & 6\end{array}$

$\begin{array}{ll}8.408 S & 10.268 E \\ 8.408 S & 10.268 E \\ 8.405 S & 11.495 E \\ 8.505 S & 11.492 E\end{array}$

$334 \cdot 800031$ $334.80 \quad 0031$ 334.510032 $334 \cdot 810033$

7066

$8.505 \mathrm{~S} \quad 11.492 E$

$334 \cdot 810033$

MARSDEN SQUARE \# 335

$\begin{array}{llll}\text { CHN } & 99 & 3 & 0037 \\ \text { CHN } & 99 & 3 & 0037 \\ \text { CHN } & 99 & 3 & 0038 \\ C H N & 99 & 3 & 0038 \\ \text { CHN } & 99 & 3 & 0039 \\ \text { CHN } & 99 & 3 & 0039 \\ \text { CHN } & 99 & 3 & 0040 \\ \text { CHN } & 99 & 3 & 0040\end{array}$

$\begin{array}{ll}0000 & 15 \\ 0000 & 26 \\ 0000 & 15 \\ 0000 & 26 \\ 0000 & 15 \\ 0000 & 26 \\ 0000 & 15 \\ 0000 & 26\end{array}$

$\begin{array}{lll}70 & 6 & 2 \\ 70 & 6 & 2 \\ 70 & 6 & 3 \\ 70 & 6 & 3 \\ 70 & 6 & 3 \\ 70 & 6 & 3 \\ 70 & 6 & 4 \\ 70 & 6 & 4\end{array}$

$8 \cdot 3^{8} \mathrm{OS}$

$8 \cdot 3805$

$8 \cdot 3715$
$8 \cdot 3715$

$8 \cdot 4275$

$8 \cdot 4275$

8.4105

8.4105
8.4105

$2 \cdot 045 E$
$2 \cdot 045 E$
$4 \cdot 248 E$
$4 \cdot 248 E$
$6 \cdot 300 E$
$6 \cdot 300 E$
$8 \cdot 310 E$
$8 \cdot 310 E$

$8 \cdot 310 E$

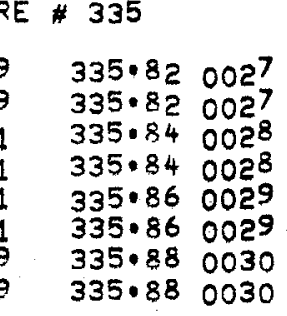

3855.1144. 0000

3855. 0.0124

1945. 633. 0000

$2209 \cdot 1019 \cdot 0000$

2209. 0.0155

MARSDEN SQUARE \# 336

$0000 \quad 13$

$64427 \cdot 17 \cdot 220 S 60 \cdot 240 E \quad 5 \quad 336.700012$

$5658 \cdot 1104 \cdot 0000$

$5658.170^{\circ} 0186$

$5371 . \quad 0 \cdot 0188$

4938.1111 .0000

4938. 0.0187

4515. 1163. 0000

4515. 0.0185

865354

242954

335954

012954
442954

442954

MARSDEN SQUARE \# 346

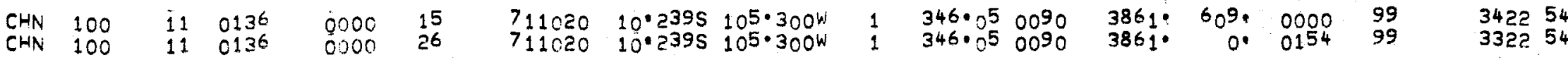




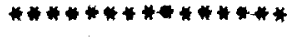

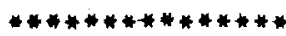

STATION DATA RETRIEVAL

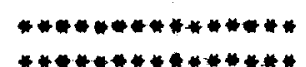

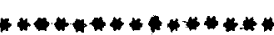

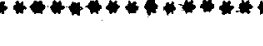

PAGE 32

**WHOI**

MARS - LORE OR

OR

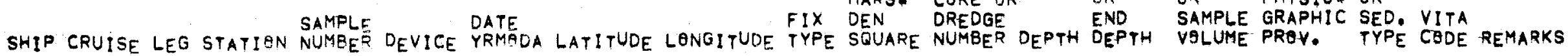

MARSDEN SQUARE \# 346

$\begin{array}{llll}\text { CHN } & 100 & 11 & 0137 \\ C H N & 100 & 11 & 0137 \\ C H N & 100 & 11 & 0138 \\ \text { CHN } & 100 & 11 & 0138 \\ \text { CHN } & 100 & 11 & 0139\end{array}$

$0000 \quad 15$

$0000 \quad 26$

$0000 \quad 15$

000015 $711021 \quad 10 \cdot 2325103 \cdot 482 \mathrm{~W}$

$346 \cdot 030091$ 346.030091 346.020092 346.020092 346.010093

MARSDEN SQUARE \# 354

$\begin{array}{ll}\text { CHN } & 100 \\ C H N & 100 \\ C H N & 100 \\ C H N & 100 \\ C H N & 100 \\ C H N & 100 \\ C H N & 100 \\ C H N & 100 \\ C H N & 100 \\ C H N & 100 \\ C H N & 100\end{array}$

$\begin{array}{ll}9 & 0077 \\ 9 & 0078 \\ 9 & 0078 \\ 9 & 0096 \\ 9 & 0096 \\ 9 & 0097 \\ 9 & 0097 \\ 9 & 0101 \\ 9 & 0101 \\ 9 & 0102 \\ 9 & 0102\end{array}$

0000 0000 0000 0000 0000 0000 0000 0000 0000

0000

15
15
26
15
26
15
26
15
26
15
26

$\begin{array}{lll}71 & 8 & 5 \\ 71 & 8 & 6 \\ 71 & 8 & 6 \\ 71 & 821 \\ 71 & 821 \\ 71 & 822 \\ 71 & 822 \\ 71 & 824 \\ 71 & 824 \\ 71 & 824 \\ 71 & 824\end{array}$

$\begin{array}{ll}17 \cdot 1065 & 170 \cdot 087 E \\ 16 \cdot 5585 & 170.590 E\end{array}$ $16 \cdot 5585$ 170.59OE $13 \cdot 2005 \quad 170 \cdot 354 E$ $13 \cdot 2005170 \cdot 354 \mathrm{E}$ $14 \cdot 488 S \quad 170 \cdot 447 E$ $1494885+170 \cdot 447 \mathrm{E}$ $15 \cdot 286 S \quad 171 \cdot 225 E$ $15 \cdot 2865 \quad 171 \cdot 225 E$ $71824 \quad 15.2865172 .068 E$ $\begin{array}{llll}7 & 824 & 15.2865 \quad 172.068 E\end{array}$

\section{MARSDEN SQUARE \# 355}

$\begin{array}{llllll}\text { CHN } & 100 & 8 & 0071 & 0000 & 15 \\ \text { CHN } & 100 & 8 & 0071 & 0000 & 26 \\ \text { CHN } & 100 & 8 & 0072 & 0000 & 15 \\ \text { CHN } & 100 & 8 & 0072 & 0000 & 26 \\ \text { CHN } & 100 & 8 & 0074 & 0000 & 15 \\ \text { CHN } & 100 & 8 & 0074 & 0000 & 26 \\ \text { CHN } & 100 & 8 & 0075 & 0000 & 15 \\ \text { CHN } & 100 & 8 & 0075 & 0000 & 26 \\ \text { CHN } & 100 & 9 & 0076 & 0000 & 15 \\ \text { CHN } & 100 & 9 & 0076 & 0000 & 26 \\ \text { CHN } & 100 & 9 & 0079 & 0000 & 15 \\ \text { CHN } & 100 & 9 & 0080 & 0000 & 15 \\ \text { CHN } & 100 & 9 & 0080 & 0000 & 26 \\ \text { CHN } & 100 & 9 & 0082 & 0000 & 15 \\ \text { CHN } & 100 & 9 & 0082 & 0000 & 26 \\ \text { CHN } & 100 & 9 & 0 C 84 & 0000 & 15 \\ \text { CHN } & 100 & 9 & 0084 & 0200 & 26 \\ \text { CHN } & 100 & 9 & 0087 & 0000 & 15 \\ \text { CHN } & 100 & 9 & 0087 & 0000 & 26\end{array}$
$7172^{8} \quad 14 \cdot 1705 \cdot 163 \cdot 543 E$ $\begin{array}{llll}71 & 72^{8} & 14 \cdot 1705 & 163 \cdot 543 E \\ 71 & 728 & 14 \cdot 0495 & 164 \cdot 300 E\end{array}$ $\begin{array}{llll}71 & 728 & 14 \cdot 0495 & 164 \cdot 300 E \\ 71 & 728 & 14 \cdot 0495 & 164 \cdot 300 E\end{array}$ $71730 \quad 18.2875 \quad 166.048 E$ $\begin{array}{llll}71 & 730 & 18 \cdot 287 \mathrm{~S} & 166 \cdot 048 \mathrm{E}\end{array}$ $\begin{array}{llll}71731 & 18.2855 & 166 \cdot 048 E\end{array}$ $\begin{array}{lllll}71 & 731 & 18.2855 & 166.048 E\end{array}$

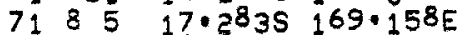
$71 \& 5 \quad 17.2835169 \cdot 158 \mathrm{E}$ 7188 18.338S $167 \cdot 159 \mathrm{E}$ $7188 \quad 18 \cdot 0345 \quad 167 \cdot 114 \mathrm{E}$ $7188 \quad 18 \cdot 0345 \quad 167 \cdot 114 \mathrm{E}$ $71810 \quad 15.564 S \quad 169.412 E$ $71810 \quad 15 \cdot 5645 \quad 169.412 \mathrm{E}$ $71811 \quad 16 \cdot 1635 \quad 166 \cdot 117$ $\begin{array}{lllll}71 & 811 & 16 \cdot 1635 & 166 \cdot 117 E \\ 71 & 14 & 12 \cdot 5075 & 168 \cdot 381 E\end{array}$ $\begin{array}{llll}71 & 814 & 12 \cdot 5075 & 168 \cdot 381 E\end{array}$
$711 \mathrm{C21} 10 \cdot 2325103 \cdot 482 \mathrm{~W}$ $711 \mathrm{C21} 10 \cdot 220 \mathrm{~S} 102 \cdot 380 \mathrm{~W}$
$711022 \quad 10 \cdot 2285101 \cdot 00^{8} \mathrm{~W}$

\begin{abstract}
$\begin{array}{ll}354 \cdot 70 & 0054 \\ 354 \cdot 60 & 0055\end{array}$ $354 \cdot 600055$ $354 \cdot 600055$ $354 \cdot 300066$ $354 \cdot 300066$ $354.40 \quad 0067$ 354.400067 354.570070 354.57 .0070 354.520071 $354 \cdot 520071$
\end{abstract}

3069. 508. 0000 3316. 553, 0000 3316. 50: 0126 3385. 558. 0000 3385. 0.0123 3515. 360. 0000 3515. 0.0035 3312, 536: 0000 3312. 0.0114 $33000^{\circ} 442.0000$ 3300.010145

$355.430049 \quad 3800^{\circ} 101^{8} \cdot 0000$ 355.430049 355.440050 355.440050
355.86 355.860051 355.860051 355.86 .0052 355.860052 355.790053 355.790053 355.870056 355.87 .0057 355.870057 355.590058 355.590058 $355.66 \quad 0059$ $355.66 \quad 0059$ $355,28,0060$ 355.280060 $390^{\circ}, \quad 940^{\circ}, 0133$ $393^{\circ}, 00^{\circ} 0150$ 4437. 1143, 0000 4437. 0.0125 4348. 1074, 0000 4348. 0.0152 1971 . 173, 0000 1971. 0.0142 4526. 335. 0000 4215. 541, 0000 4215. 0.0098 3237. 119. 0000 3237. 0.0045 4490. 862. 0000 $4490^{\circ} \quad 0^{\circ} 0148$ 3473. 855. 0000 $3473^{\circ} 0.0152$
132954

145954

115954

115954

312254 SAME AS 138

$\begin{array}{rll}99 & 132954 \\ 99 & 145954 \\ 99 & 115954 \\ 99 & 115954 \\ 0 & 312254 \text { SAME AS } 138\end{array}$

$\begin{array}{rrrr}0 & & 0932 & 54 \\ 0 & & 0931 & 54 \\ 0 & & 0229 & 54 \\ 0 & & 3560 & 54 \\ 0 & & 3569 & 54 \\ 0 & & 0931 & 54 \\ 0 & & 3569 & 54 \\ 10 & & 3931 & 0 \\ 10 & & 3560 & 0 \\ 0 & & 3560 & 54 \\ 0 & & 3560 & 54\end{array}$

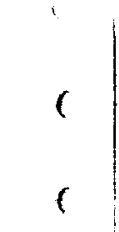

(

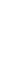

(


LENGTH LENGTH,

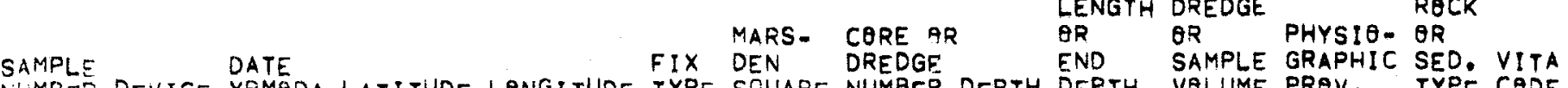

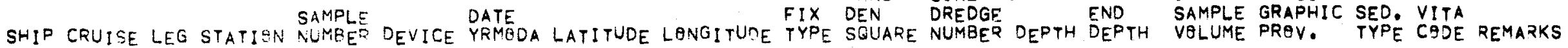

MARSDEN SQUARE \# 355

$\begin{array}{llllll}\text { CHN } & 100 & 9 & 0088 & 0000 & 15 \\ \text { CHN } & 100 & 9 & 0088 & 0000 & 26 \\ \text { CHN } & 100 & 9 & 0089 & 0000 & 15 \\ \text { CHN } & 100 & 9 & 0092 & 0000 & 15 \\ \text { CHN } & 100 & 9 & 0092 & 0000 & 26 \\ \text { CHN } & 100 & 9 & 0094 & 0000 & 15 \\ \text { CHN } & 100 & 9 & 0094 & 0000 & 26 \\ \text { CHN } & 100 & 9 & 0095 & 0000 & 15 \\ \text { CHN } & 100 & 9 & 0095 & 0000 & 26 \\ \text { CHN } & 100 & 9 & 0098 & 0000 & 15 \\ \text { CHN } & 100 & 9 & 0098 & 0000 & 26 \\ \text { CHN } & 100 & 9 & 0099 & 0000 & 15 \\ \text { CHN } & 100 & 9 & 0099 & 0000 & 26\end{array}$

$\begin{array}{llll}71 & 815 & 13 \cdot 045 S & 167 \cdot 520 E \\ 71 & 815 & 13 \cdot 045 S & 167 \cdot 520 E \\ 71 & 815 & 13 \cdot 338 S & 166 \cdot 165 E \\ 71 & 818 & 11 \cdot 325 S & 167 \cdot 347 E \\ 71 & 818 & 11 \cdot 325 S & 167 \cdot 347 E \\ 71 & 819 & 12 \cdot 114 S & 167 \cdot 134 E \\ 71 & 819 & 12 \cdot 114 S & 167 \cdot 134 E \\ 71 & 820 & 11 \cdot 571 S & 169 \cdot 341 E \\ 71 & 820 & 11 \cdot 571 S & 169.341 E \\ 71 & 822 & 14 \cdot 015 S & 169 \cdot 511 E \\ 71 & 822 & 14 \cdot 015 S & 169 \cdot 511 E \\ 71 & 823 & 14 \cdot 554 S & 169 \cdot 108 E \\ 71 & 823 & 14 \cdot 554 S & 169 \cdot 10^{8 E}\end{array}$

$\begin{array}{ll}355 \cdot 37 & 0061 \\ 355 \cdot 37 & 0061 \\ 355 \cdot 36 & 0062 \\ 355 \cdot 17 & 0063 \\ 355 \cdot 17 & 0063 \\ 355 \cdot 27 & 0064 \\ 355 \cdot 27 & 0064 \\ 355 \cdot 19 & 0065 \\ 355 \cdot 19 & 0065 \\ 355 \cdot 49 & 0068 \\ 355 \cdot 49 & 0068 \\ 355 \cdot 49 & 0069 \\ 355 \cdot 49 & 0069\end{array}$

$2811 \cdot 520 \cdot 0000$

2811 . 0.0120

5851 . 285. 0000

$2640^{\circ} 550^{\circ} 0000$

$2640^{\circ} \quad 0^{\circ} \quad 0452$

1805. 298. 0000

$1805^{\circ} \quad 0.0144$

$3280^{\circ} \quad 4870^{\circ} \quad 0000$

$3670^{\circ} 390.0000$

$\begin{array}{rrr}3670^{\circ} & 390^{\circ} & 0 \\ 3670^{\circ} & 0 . & 0147\end{array}$

$\begin{array}{rr}3248 \cdot & 330 \cdot 0000 \\ 3248 . & 0.0137\end{array}$

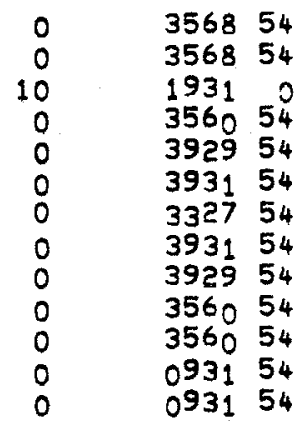

MARSDEN SQUARE * 356

CHN 100

0000

$71727 \quad 10 \cdot 1345 \quad 157 \cdot 095 \mathrm{E}$

$356 \cdot 070048$

5140

597

0000

10

$3148 \quad 0$

MARSDEN SQUARE \# 365

\section{MARSDEN SQUARE \# 366}

$\begin{array}{rrrrrr}\text { AII } & 15 & 13 & 0732 & 0000 & 13 \\ \text { AII } & 15 & 13 & 0733 & 0000 & 13 \\ \text { AII } & 15 & 13 & 0737 & 0000 & 13 \\ \text { CHN } & 43 & 1 & 0723 & 0000 & 13 \\ \text { CHN } & 43 & 1 & 0030 & 0000 & 13 \\ \text { CHN } & 43 & 1 & 0033 & 0000 & 13\end{array}$

$\begin{array}{llll}65 & 611 & 19 \cdot 530 S & 56 \cdot 48 O E \\ 65 & 611 & 19.56 O S & 55 \cdot 170 E \\ 65 & 612 & 19 \cdot 595 S & 50 \cdot 065 E \\ 64 & 423 & 17.270 S & 58.055 E \\ 64 & 430 & 11 \cdot 30 O S & 58.240 E \\ 64 & 52 & 18.040 S & 58 \cdot 240 E\end{array}$

$366 \cdot 96 \quad 0732$ $366.95 \quad 0733$ $366 \cdot 90 \quad 0737$ 366.780011 $366 \cdot 180014$ $366 \cdot 880016$

4382. 118. 0000 4382. 39. 0000 $4356^{\circ} 80^{\circ} 0000$ 4038.127 .0000 $4095 \cdot 262 \cdot 0000$ 3869. 163. 0000

$\begin{array}{rrr}6 & 0362 & 0 \\ 6 & 4869 & 0 \\ 6 & 0459 & 0 \\ 15 & 3329 & 0 \\ 10 & 3469 & 0 \\ 15 & 0329 & 0\end{array}$

\section{MARSDEN SQUARE \#367}

$\begin{array}{llllll}\text { AII } & 15 & 7 & 0673 & 0000 & 13 \\ \text { AII } & 15 & 7 & 0675 & 0000 & 13 \\ \text { CHN } & 99 & 8 & 0064 & 0000 & 15 \\ \text { CHN } & 99 & 8 & 0064 & 0000 & 26\end{array}$




\section{8}

$* * * * * * * * * * * * * *$

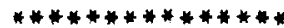

STATION DATA RETRIEVAL

DATE: 12:36 MAY 16,175

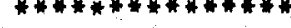

*****************)
PAGE 34

*WHOI**

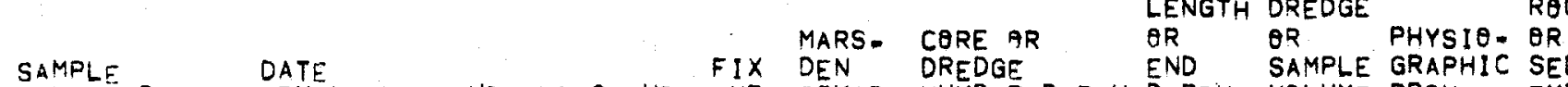
CORE LENGTH,

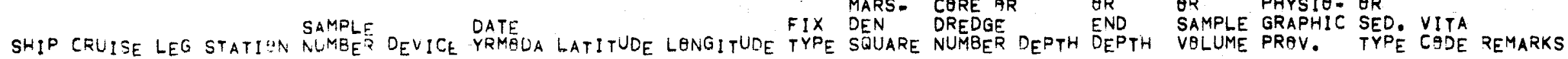

MARSDEN SQUARE 367

$\begin{array}{llllll}C H N & 99 & 8 & 0065 & 0000 & 15 \\ C H N & 99 & 8 & 0065 & 0000 & 26 \\ C H N & 99 & 8 & 0066 & 0000 & 15 \\ C H N & 99 & 8 & 0066 & 0000 & 26 \\ \text { CHN } & 99 & 8 & 0067 & 0000 & 15 \\ \text { CHN } & 99 & 8 & 0067 & 0000 & 26 \\ \text { CHN } & 99 & 8 & 0068 & 0000 & 15 \\ \text { CHN } & 99 & 8 & 0068 & 0000 & 26 \\ \text { CHN } & 99 & 8 & 0069 & 0000 & 15 \\ \text { CHN } & 99 & 8 & 0070 & 0000 & 15 \\ \text { CHN } & 99 & 8 & 007 C & 0000 & 26\end{array}$

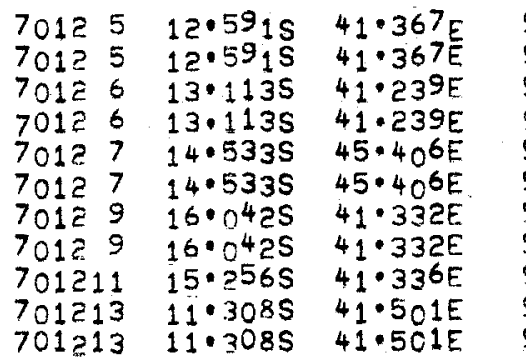

$367 \cdot 210046$ 367.210046 $367 \cdot 310047$ 367.310047 $367 \cdot 450048$ $367 \cdot 450048$ $367 \cdot 610049$ 367.610049 367.510050 367.510050 $367 \cdot 110051$

3540. 896. $0000 \quad 99$ $3540^{\circ} \quad 0^{\circ} 0069 \quad 99$ $2350 \cdot 875.000099$ 2350. $0.0158 \quad 99$ 2941. 804. 000021 2941. 0.0067 2758.840 .0000 2758.010 .0183 $2085 \cdot 161.0000$ $2085 \cdot 161 \cdot 0000$ 2151. 0.0035

372154 333954 335954 335954 232954 342954 373154 483154 783854 30854 $\begin{array}{ll}3039 & 54 \\ 3329 & 54\end{array}$

MARSDEN SQUARE \# 370

$\begin{array}{llllll}\text { CHN } & 99 & 4 & 0048 & 0000 & 1 \\ \text { CHN } & 99 & 4 & 0 C 48 & 0000 & 2 \\ \text { CHN } & 99 & 4 & 0.49 & 0000 & 1 \\ \text { CHN } & 99 & 4 & 0049 & 0000 & 2\end{array}$

15
26
15
26

$\begin{array}{llll}70 & 613 & 11 \cdot 050 S & 10 \cdot 440 E \\ 70 & 513 & 11 \cdot 050 S & 10.440 E \\ 70 & 616 & 19 \cdot 0075 & 10.040 E \\ 70 & 616 & 19 \cdot 0075 & 10.040 E\end{array}$

$370 \cdot 100034$

3961 1122, 0000 $3961^{\circ} 0^{\circ} 0188 \quad 10$ $4130^{\circ} 973.0000 \quad 1$

422954 343954 062954 042954

MARSDEN SQUARE \# 371

\section{$\begin{array}{rrrrrrrrr}\text { CHN } 99 \quad 40051 \quad 0000 \quad 15 & 70617 & 19.5845 & 9.218 E & 1 & 371.99 \\ \text { MARSDEN SQUARE \# } 375\end{array}$}

$\begin{array}{llllll}A ! 1 & 60 & 2 & 000^{7} & 0000 & 15 \\ A ! 1 & 60 & 2 & 000 & 0000 & 15 \\ A ! 1 & 60 & 2 & 009 & 0200 & 13 \\ \text { AII } & 60 & 2 & 0010 & 0200 & 15\end{array}$

$\begin{array}{lllllll}71 & 226 & 26 \cdot 399 S & 34 \cdot 000 W & 9 & 375 \cdot 64 & 000^{7} \\ 71 & 228 & 29 \cdot 083 S & 34 \cdot 346 \mathrm{~W} & 9 & 375.94 & 009 \mathrm{~A} \\ 71 & 22^{8} & 29 \cdot 0^{46 S} & 34 \cdot 40^{6} \mathrm{~W} & 9 & 375 \cdot 94 & 009 \mathrm{~B}\end{array}$

4626. 164. 0000 $3990^{\circ} 273.0000$ $1840^{\circ} 120^{\circ} 0000$

$\begin{array}{lll}\mathbf{5} & 1937 & 0 \\ 5 & 3327 & 0 \\ 5 & 3329 & 0 \\ 5 & 3329 & 0\end{array}$

MARSDEN SQUARE \#402

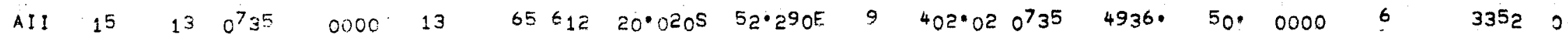


0069

$* * * * * * * * * * * * * * *$

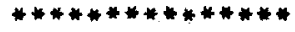

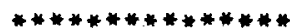

PAGE 35

**WHOI**

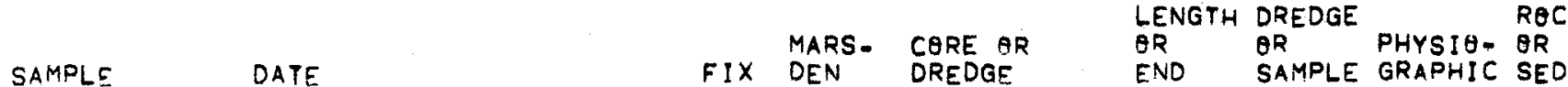

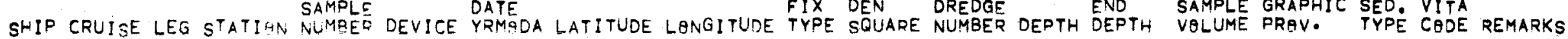

MARSDEN SQUARE \#403

$\begin{array}{llllll}A ! I & 15 & 13 & 0^{744} & 0000 & 13 \\ A ! I & 15 & 13 & 0745 & 0000 & 13 \\ A ! I & 15 & 13 & 0746 & 0000 & 13 \\ A ! I & 15 & 14 & 0747 & 0000 & 13 \\ A ! I & 15 & 14 & 0748 & 0000 & 13 \\ A ! I & 15 & 14 & C^{7} 51 & 0000 & 13\end{array}$

$\begin{array}{llll}65 & 615 & 24 \cdot 540 S & 48 \cdot 110 E \\ 65 & 615 & 24 \cdot 590 S & 47 \cdot 450 E \\ 65 & 616 & 25 \cdot 031 S & 47.264 E \\ 65 & 616 & 26 \cdot 010 S & 44 \cdot 320 E \\ 65 & 617 & 26 \cdot 0105 & 43.570 E \\ 65 & 618 & 26 \cdot 070 S & 41 \cdot 160 E\end{array}$

\section{$403 \cdot 48 \quad 0744$} $403 \cdot 470745$ $403.57 \quad 0746$ $403 \cdot 640747$

$\begin{array}{ll}403 \cdot 63 & 0748 \\ 403 \cdot 61 & 0751\end{array}$ $656.18 \quad 26 \cdot 070 S \quad 41 \cdot 160 E$

4039. $138 \cdot 0000$ 3269. 22: 0000 1307.72 .0000 $12860^{\circ} 70^{\circ} 0000$ 48.3. 78. 0000

MARSDEN SQUARE \# 404

$\begin{array}{llllll}A ! 1 & 15 & 14 & 0753 & 0000 & 13 \\ A ! & 15 & 14 & 0755 & 0000 & 13 \\ \text { A!I } & 15 & 14 & 0756 & 0000 & 13 \\ \text { AII } & 15 & 14 & 0758 & 0000 & 13\end{array}$

$\begin{array}{lllllll}65 & 618 & 25.550 S & 38.530 E & 9 & 404 \cdot 58 & 0753 \\ 65 & 619 & 25.565 S & 36.420 E & 9 & 404 \cdot 56 & 0755 \\ 65 & 619 & 25.540 S & 36.090 E & 9 & 404.56 & 0756 \\ 65 & 620 & 25.5605 & 34.450 E & 9 & 404 \cdot 54 & 0758\end{array}$

3884. 20. 0000 AII $15 \quad 14 \quad 0758$

MARSDEN SQUARE \# 407

$\begin{array}{llllll}\text { CHN } & 99 & 4 & 0050 & 0000 & 15 \\ \text { CHN } & 99 & 4 & 0050 & 0000 & 26 \\ \text { CHN } & 99 & 4 & 0052 & 0000 & 15 \\ \text { CHN } & 99 & 4 & 0052 & 0000 & 26\end{array}$

CHN $99 \quad 40052 \quad 000026$

$\begin{array}{llllll}\text { AlI } & 60 & 2 & 0013 & 0000 & 15 \\ \text { AlI } & 60 & 2 & 0013 & 0000 & 15 \\ \text { AlI } & 60 & 2 & 0014 & 0000 & 15\end{array}$

$\begin{array}{lll}70 & 617 & 20 \cdot 4905 \\ 70 & 617 & 20 \cdot 4905 \\ 70 & 619 & 21 \cdot 4485 \\ 70 & 619 & 21 \cdot 4485\end{array}$

$9 \cdot 563 E$
$9 \cdot 563 E$
$8 \cdot 300 E$
$8 \cdot 300 E$

$$
\begin{aligned}
& 407 \cdot 090036 \\
& 407 \cdot 090036 \\
& 407 \cdot 180038 \\
& 407 \cdot 180038
\end{aligned}
$$

MARSDEN SQUARE \# 411

$\begin{array}{llll}71 & 3 & 1 & 31 \cdot 5915 \\ 71 & 3 & 2 & 32 \cdot 2995 \\ 71 & 3 & 2 & 32 \cdot 3405\end{array}$

$36 \cdot 388 \mathrm{~W}$
$38 \cdot 519 \mathrm{~W}$

\section{$411 \cdot 16 \quad 013 \mathrm{~A}$ $411 \cdot 28013 B$}

2739. 207. 0000 3122. 219. 0000 4463. 162. 0000

MARSDEN SQUARE \# 412

AII $60 \quad 2 \quad 0015 \quad 0000 \quad 15 \quad 7133 \quad 32 \cdot 2705 \quad 40 \cdot 277 \mathrm{~W} \quad 9 \quad 412 \cdot 20015 \mathrm{~A}$

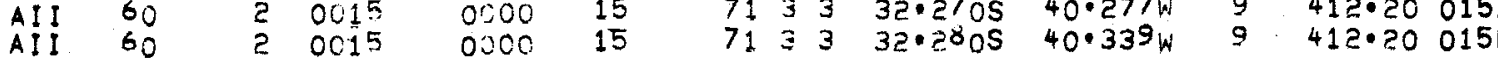

MARSDEN SQUARE \# 434

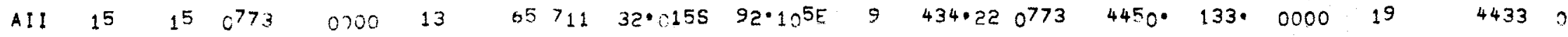




\section{0}

************* STATION DATA RETRIEVAL

************* DATE! 12:36 MAY 16:175

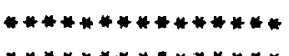

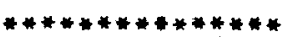

PAGE 36

$*$ WHOI**

\section{LENGTH DREDGE RGCK}

OR OQ PHYSIO- OR

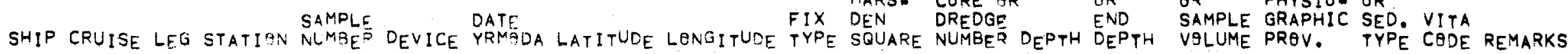

MARSDEN SQUARE \# 438

\begin{tabular}{|c|c|c|c|c|c|c|c|c|c|c|c|c|c|c|c|c|c|}
\hline AlI & 15 & 15 & 0766 & 0000 & 13 & 65 & 73 & $32 \cdot 0005$ & $55 \cdot 070 E$ & 9 & $438 \cdot 25$ & 0766 & 4417 & 32. & 0000 & 13 & 4359 \\
\hline & & & & & & & & & RSDEN SO & RE & $\# 439$ & & & & & & \\
\hline AII & $\begin{array}{l}15 \\
15\end{array}$ & $\begin{array}{l}15 \\
15\end{array}$ & $\begin{array}{l}0763 \\
0765\end{array}$ & $\begin{array}{l}0,000 \\
0000\end{array}$ & $\begin{array}{l}13 \\
13\end{array}$ & $\begin{array}{l}65 \\
65\end{array}$ & $\begin{array}{l}630 \\
72\end{array}$ & $\begin{array}{l}32 \cdot 0105 \\
32 \cdot 0155\end{array}$ & $\begin{array}{l}40 \cdot 491 E \\
49 \cdot 555 E\end{array}$ & $\begin{array}{l}9 \\
9\end{array}$ & $\begin{array}{l}439 \cdot 20 \\
439.29\end{array}$ & $\begin{array}{l}0763 \\
0765\end{array}$ & $\begin{array}{l}45499^{\circ} \\
3698^{\circ}\end{array}$ & $\begin{array}{l}124 . \\
117 .\end{array}$ & $\begin{array}{l}0000 \\
0000\end{array}$ & $\begin{array}{l}19 \\
14\end{array}$ & $\begin{array}{l}4432 \\
3359\end{array}$ \\
\hline & & & & & & & & & PSDEN SO & & $\# 440$ & & & & & & \\
\hline$A ! I$ & 15 & 15 & 0761 & 0200 & 13 & 65 & 628 & $31 \cdot 3265$ & $32 \cdot 346 E$ & 9 & $440 \cdot 14$ & 0761 & 2916. & 55. & 0000 & 6 & 3359 \\
\hline
\end{tabular}

THERE WERE 1165 ITEMS THAT MET YOUR REQUIREMENTS.

THANK YOU FOR USING PRGGRAM MUDDIE.

*STOP* THAT IS ALL FER NEW 
DESCRIPTIONS OF W.H.O.I. SEDIMENT CORES

VOLUME I Of IV 


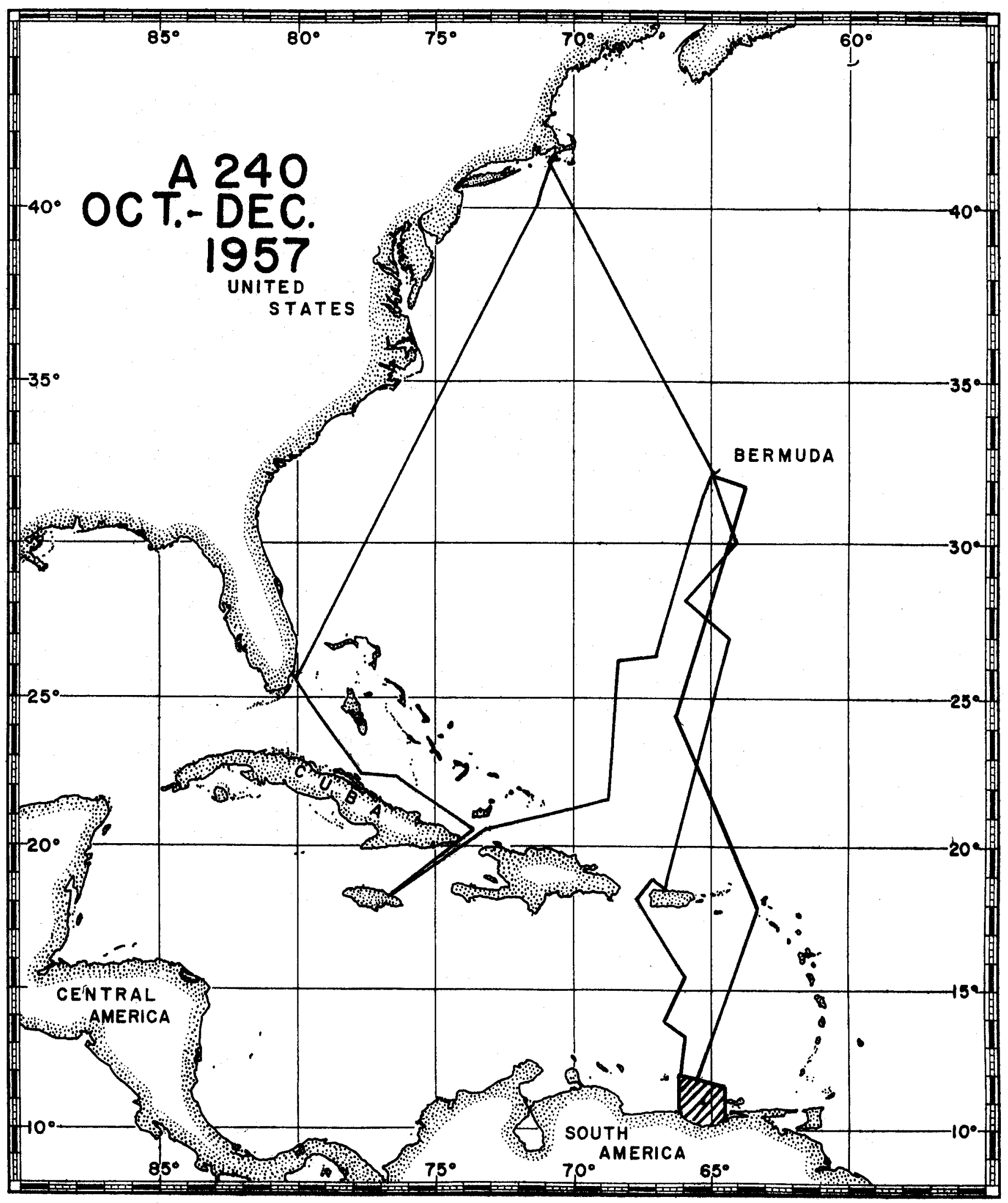




\section{3}

STATION DATA RETRIEYAL OATEI 06132 MAY 13:175

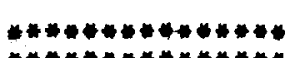

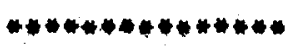

10
PAGE I OF 1

PILOT

CORE LENGTH.

LENGYH DREDGE

OR OR

MARS. CORE OR

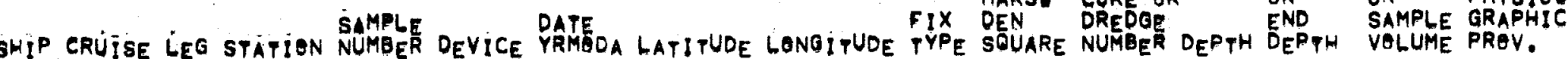
Rock

\begin{tabular}{|c|c|c|c|}
\hline $\begin{array}{l}A T L \\
A T L \\
A T L \\
A T L \\
A T L \\
A T L \\
A T L \\
A T L \\
A T L \\
A T L \\
A T L \\
A T L \\
A T L\end{array}$ & $\begin{array}{l}240 \\
240 \\
240 \\
240 \\
240 \\
240 \\
240 \\
240 \\
240 \\
240 \\
240 \\
240 \\
240 \\
240 \\
240 \\
240 \\
240\end{array}$ & 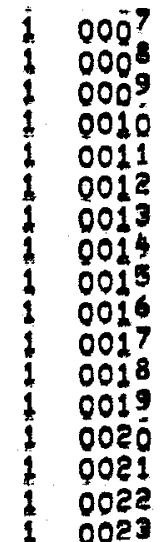 & $\begin{array}{l}0000 \\
0000 \\
0000 \\
0000 \\
0000 \\
0000 \\
0000 \\
0000 \\
0000 \\
0000 \\
0000 \\
0000 \\
0000 \\
0000 \\
0000 \\
0000 \\
0000 \\
0000 \\
0000\end{array}$ \\
\hline
\end{tabular}

\begin{tabular}{|c|c|c|}
\hline $\begin{array}{l}5711 \\
5711 \\
5711 \\
5711 \\
5711 \\
5711 \\
5711 \\
5711 \\
5711 \\
5711 \\
5711 \\
5711 \\
5711 \\
5711 \\
5719 \\
5711 \\
5711\end{array}$ & $\begin{array}{l}10.480 \mathrm{~N} \\
10.440 \mathrm{~N} \\
10.407 \mathrm{~N} \\
10.367 \mathrm{~N} \\
10.288 \mathrm{~N} \\
10.375 \mathrm{~N} \\
10.518 \mathrm{~N} \\
10.552 \mathrm{~N} \\
10.525 \mathrm{~N} \\
10.387 \mathrm{~N} \\
10.326 \mathrm{~N} \\
10.308 \mathrm{~N} \\
10.223 \mathrm{~N} \\
10.24 \mathrm{ON} \\
10.26 \mathrm{2N} \\
10.340 \mathrm{~N} \\
10.47 \mathrm{gN}\end{array}$ & 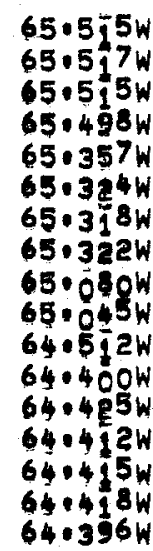 \\
\hline
\end{tabular}

$43 \cdot 050007$
$43 \cdot 05000$
$43 \cdot 050009$
$43 \cdot 050010$
$43 \cdot 050011$
$43 \cdot 050012$
43.050013
43.050014
$43 \cdot 050015$
43.050016
43.040017
43.060018
43.040019
$43 \cdot 040020$
43.040021
43.040022
43.040023

\begin{tabular}{|c|c|c|}
\hline $\begin{array}{r}2530 \\
5260 \\
1342 \\
6730 \\
572 \\
13450 \\
942 \\
3850 \\
3540 \\
9140 \\
1348 \\
1370 \\
1770 \\
822 \\
980 \\
1281 \\
278\end{array}$ & $\begin{array}{r}357 \\
542 \\
375 \\
450 \\
56 \\
591 \\
850 \\
860 \\
580 \\
715 \\
760\end{array}$ & $\begin{array}{l}0000 \\
0020 \\
0000 \\
0000 \\
0000 \\
0000 \\
0000 \\
0000 \\
0000 \\
0000 \\
0000 \\
0000 \\
0000 \\
0000 \\
0000 \\
0000 \\
0000\end{array}$ \\
\hline
\end{tabular}

18
18
18
18
18
18
18
18
18
18
18
18
18
18
18
18
18

3769

3769

23480

33490

4446

$3865:$

43290

8455

8455
3075

3075

4839

38450

41760

30290

43780

30750

THERE WERE 17 ITEMS THAT MET YOUR REQUIREMENTS

THANK YOÜ FOR USING PROGRAM MUDDIE.

-stop. that is all for now RUN 
$\ldots$

3 
0074

VISUAL CORE DESCRIPTION

Poge__ 1 of _ _ 1

Ship $A$ Cruise 240 Leg__ Sto. 7 Core No. $7 P C$ Total Length $3.35 \mathrm{~cm}$. Lot. $10^{\circ} 48.0^{\prime} \mathrm{N}$ Long. $65^{\circ} 51.5^{\prime} \mathrm{W}$ Depth $253 \mathrm{CORR} \mathrm{m}$ Core condition fairly moist; goed Date Described $4 / 27 / 74$ by $B M^{C}$ Gir Physiographic location $N$ Slope of W Basin of Carieco Trench

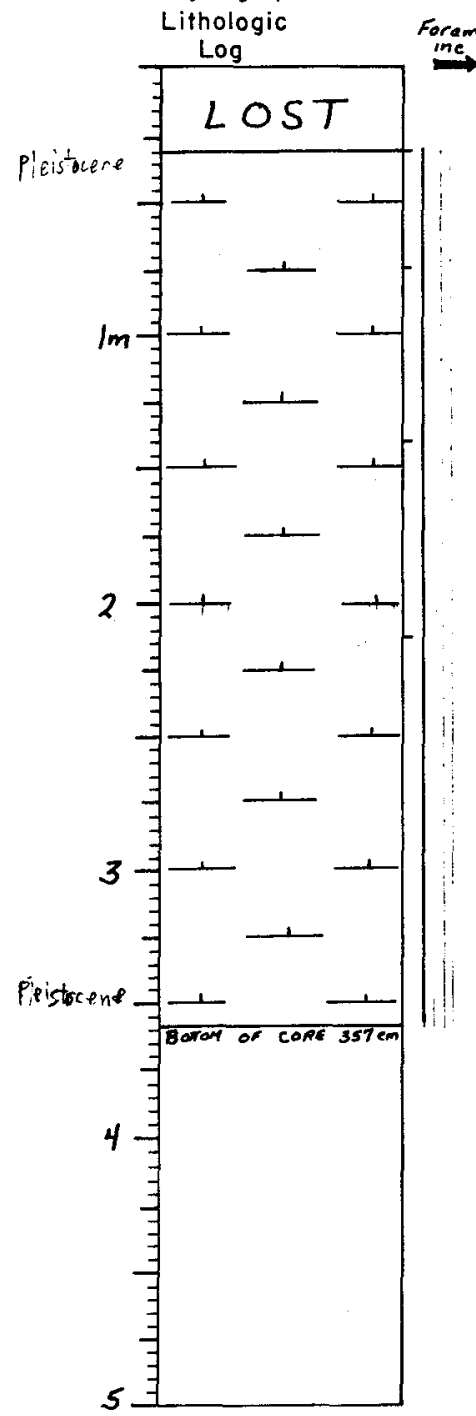

Detoiled Description

$0-30$ LOST

0-74

$5 \mathrm{Y} 4 / 2$ olive gray

firm $v$. silty lutite, few forams

v. thin faint lams throughout

74-140

CALC OOZE

$5 Y 4 / 2$ olive gray

tite, few forams

lams throughout

140-213

CALC OOZE

firm v. silty lutite, few forams

. thin faint lams throughout, somewhat crumbly throughou pteropod testa visible

213-357

CALC OOZE

firm silty lutite, few forams

somewhat crumb ly $230-266 \mathrm{~cm}$
$5 Y 3 / 2 \mathrm{dk}$ ollive gray
0075

SMEAR SLIDE DESCRIPTIONS - W.H.O.I. SEDIMENT CORES

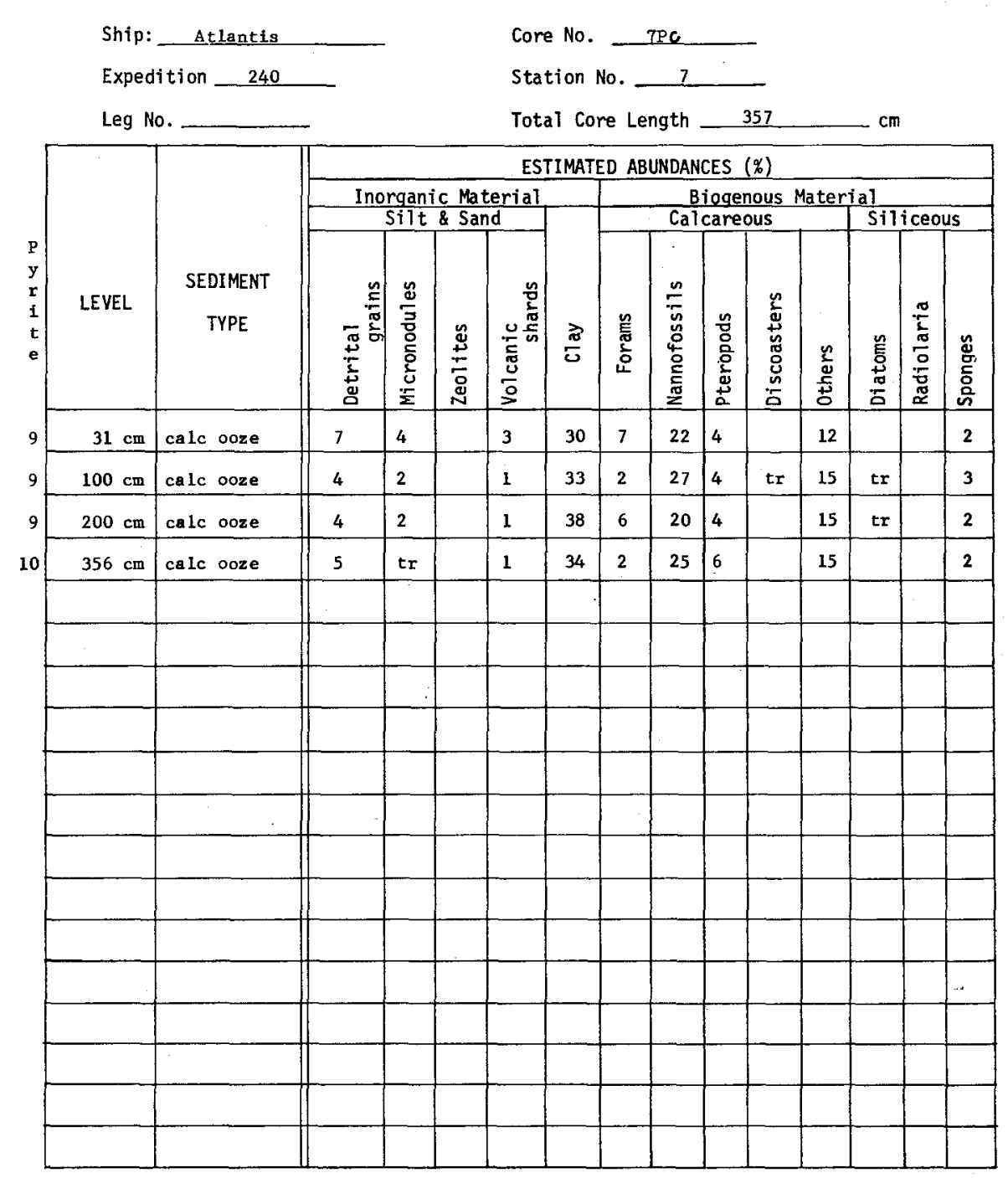


Ship A Cruise 240 Leg__ Sta. $\frac{8}{2}$ Core No. 8 PC Total Length $542 \mathrm{~cm}$. Lat. $10^{\circ} 44.0^{\circ} \mathrm{N}$ Long. $65^{\circ} 51.7^{\prime} \mathrm{W}$ Depth 526 CORR m Core condition moict; Very gead Date Described $4 / 17 / 24$ by BMCGire

Physiographic location N. Slope of $W$. Basin of the Cariace Trench

Lithologic

Log

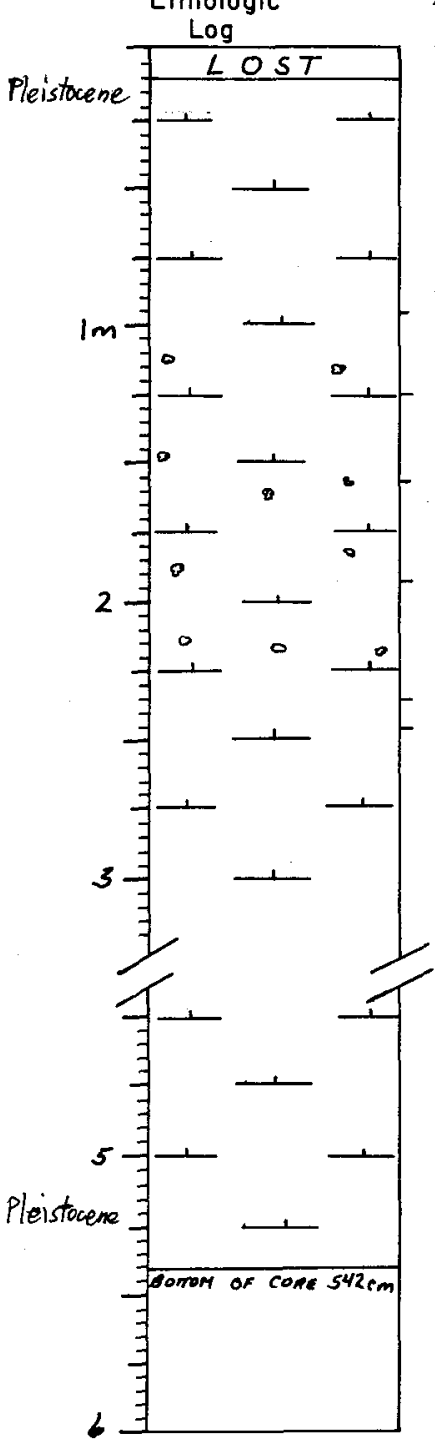

\section{Foroms Detailed Description} LOST

CALC OOZE

$5 Y 4 / 2,3 / 2$ olive gray to dk olive gray

firm, silty lutite, few forams

pteropod fragments scattered, dk at bottom of unit $96-125$

\section{CAIC OOZE}

scattered mottling 109-116 cm, sma11, brown

firm, silty lutite, few forams

v. faint thin lams

$25-157$

CALC OOZE

$5 Y 4 / 2$ olive gray

scattered mottling $145-157 \mathrm{~cm}$, brown

fim, silty lutite, few forams

7 193

\section{CALC OOZE}

5 Y 5/2 olive gray

scattered mottling throughout, brown

firm silty lutite, Eew forams

193-235

CALC OOZE

5Y. $5 / 3$ olive

fixm silty lutite, few forams

211-214 cm 1 irregular patch olive gray

235- 245

CALC OOZE

firm, silty lutite, few forams

245-542

CALC OOZE

$5 Y 5 / 2,4 / 2$ olive gray

firm silty lutite, few forams

sl variations in color throughout unit, gypsum crystals scattered from $42,0-543 \mathrm{~cm}$
$5 Y 4 / 2$ olive gray
SMEAR SLIDE DESCRIPTIONS - W.H.O.I. SEDIMENT CORES

\begin{tabular}{|c|c|c|c|c|c|c|c|c|c|c|c|c|c|c|}
\hline \multicolumn{2}{|c|}{ Ship: Atlantis } & & & & \multicolumn{2}{|c|}{ Core No. } & \multicolumn{4}{|c|}{870} & & & & \\
\hline \multicolumn{2}{|c|}{ Expedition $\quad 240$} & & & & \multicolumn{6}{|c|}{ Station No. 8} & & \multirow{2}{*}{\multicolumn{2}{|c|}{$\mathrm{cm}$}} & \\
\hline \multicolumn{3}{|c|}{ Leg No. _ } & & & \multicolumn{7}{|c|}{ Total Core Length $\quad 542$} & & & \\
\hline \multirow[b]{4}{*}{ LEVEL } & \multirow[b]{4}{*}{$\begin{array}{c}\text { SEDIMENT } \\
\text { TYPE }\end{array}$} & \multicolumn{13}{|c|}{ ESTIMATED ABUNDANCES $(\%)$} \\
\hline & & Ino & gani & Mat & erial & & & & oge & ous & ter & & & \\
\hline & & & $i 1 t$ & Sar & & & & Cal & are & & & Sil & ceo & \\
\hline & & 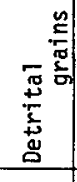 & $\begin{array}{l}\frac{y}{5} \\
\frac{7}{0} \\
\frac{0}{0} \\
\frac{0}{\Sigma}\end{array}$ & $\begin{array}{l}\stackrel{n}{ \pm} \\
\stackrel{ \pm}{8} \\
\mathbb{N}\end{array}$ & $\begin{array}{r}\frac{n}{0} \\
\frac{0}{0} \\
\frac{0}{5} \\
\frac{5}{5} \\
\frac{0}{5} \\
\frac{5}{5}\end{array}$ & $\frac{\overrightarrow{\mathrm{t}}}{\mathrm{\sigma}}$ & 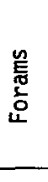 & 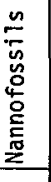 & 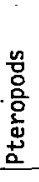 & 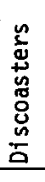 & 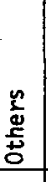 & 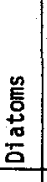 & 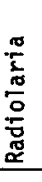 & 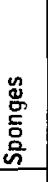 \\
\hline $12 \mathrm{~cm}$ & calc ooze & 4 & 1 & & 2 & 54 & 4 & 15 & 2 & & 10 & $\mathbf{t r}$ & & $\operatorname{tr}$ \\
\hline $100 \mathrm{~cm}$ & calc ooze & 15 & 1 & & 1 & 45 & 3 & 17 & 4 & & 10 & & & tr \\
\hline $175 \mathrm{~cm}$ & calc ooze & 7 & tr & & 2 & 35 & 3 & 25 & 3 & & 10 & & & $\operatorname{tr}$ \\
\hline $200 \mathrm{~cm}$ & calc ooze & 7 & 2 & & 2 & 30 & 2 & 34 & 4 & & 15 & & & tr \\
\hline $400 \mathrm{~cm}$ & calc ooze & 4 & 2 & & 1 & 38 & 2 & 35 & 4 & & 10 & $\therefore$ & & \\
\hline $541 \mathrm{~cm}$ & calc ooze & 4 & 2 & & 1 & 40 & 4. & 18 & 3 & & 20 & & & \\
\hline & & & & & & & & & & & & & & \\
\hline & & & & & & & & & & & & & & \\
\hline & & & & & & & & & & & & & & \\
\hline & & & & & & & & & & & & & & \\
\hline & & & & & & & & & & & & & & \\
\hline & & & & & & & & & & & & & & \\
\hline & & & & & & & & & & & & & & ..* \\
\hline & & & & & & & & & & & & & & \\
\hline & & & & & & & & & & & & & & \\
\hline & & & & & & & & & & & & & & \\
\hline & & & & & & & & & & & & & & \\
\hline
\end{tabular}


0078

VISUAL CORE DESCRIPTION

Page 1 of 2

Ship A Cruise 240 Leg Sta. 9 Core No. 9PC

Total Length $375 \mathrm{~cm}$. Lat. $10^{\circ} 40.7^{\prime} \mathrm{N}$ Long. $65^{\circ} \mathrm{S} / .5^{\prime} \mathrm{W}$ Depth $1342 \mathrm{CoRRm}$

Core condition fairly moist; good Date Described 4/18/74 by $B$ MCGire

Physiographic location Near the battom of the West Basin, Carraco Trench

Lithologic

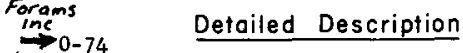

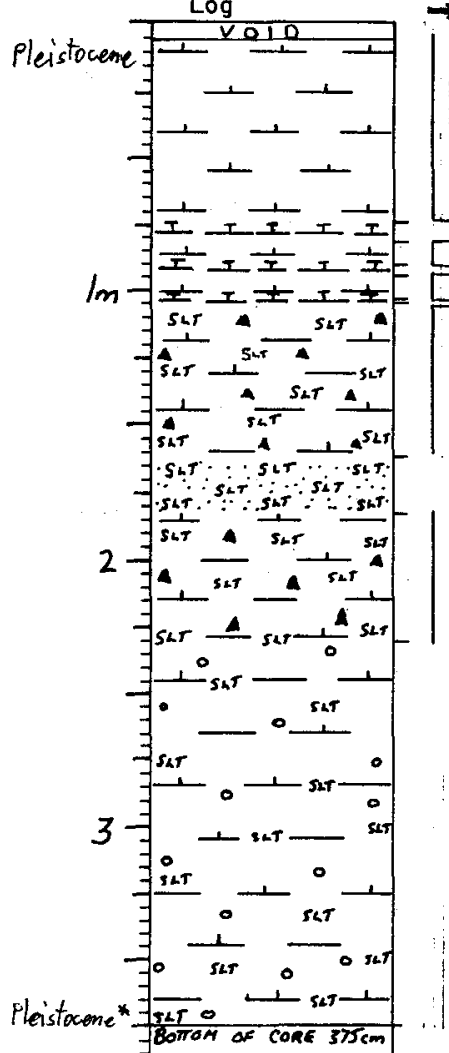

4

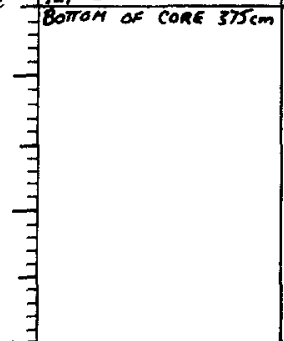

CALC OOZE

$3 \mathrm{Y} 3 / 2 \mathrm{dk}$ olive gray

$4-82$ irregular

FORAM SAND

$5 Y$ 7/3 pale yellow

silt-medium sand, foram san

82-91

CAIC OOZE

$5 \mathrm{Y} 3 / 2 \mathrm{dk}$ olive gray

firm v. silty lutite, forams scattered

$S$ irregular

$1-95$

FORAM SAND

$5 \mathrm{Y} 7 / 3$ pale yellow

silt to medium sand, foram sand

95-103

CALC OOZE

$5 Y 3 / 2 \mathrm{dk}$ olive gray

firm v. silty lutite, forams scattered 5 irregular

103-105

FORAM SAND

Y $7 / 3$ pale yellow

ilt to medium sand, foram sand

105-162

HLY CALC CLAY/DET WITH PYRITE

$5 Y 3 / 2$ dk ollve gray

firm v. silty lutite, forams scattered

faint, thin lams throughout

$S$ i
$162-183$

\section{DETRITUS}

lutite to coarse sand with some pebbles and shell hash appears to be slumped in from above

183-231

HLY CALC CIAY/DET WITH PYRITE

$5 Y 3 / 2$ dk olive gray

$1 \mathrm{~mm}$, v. silty lutite, forams scattere

some v. thin silt lams, sl convex up

231-375

HLY CALC CLAY WITH DET TO HLY CALC CLAY/DET WITH PEBBLES $5 Y 4 / 2,3 / 2$ olive gray to dk olive gray
0079

VISUAL CORE DESCRIPTION

Page 2 of 2

Ship A_C Cruise 240 Leg _ Sta._. Core No. gece

Lithologic

Log

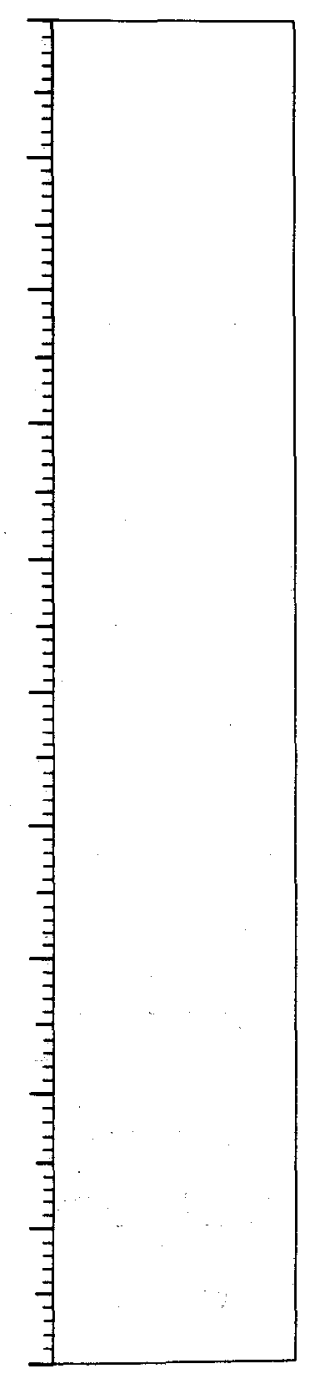

firm silty lutite with sand and pebbles scattered throughout v. poorly sorted unit 
0080

SMEAR SLIDE DESCRIPTIONS - W.H.O.I. SEDIMENT CORES

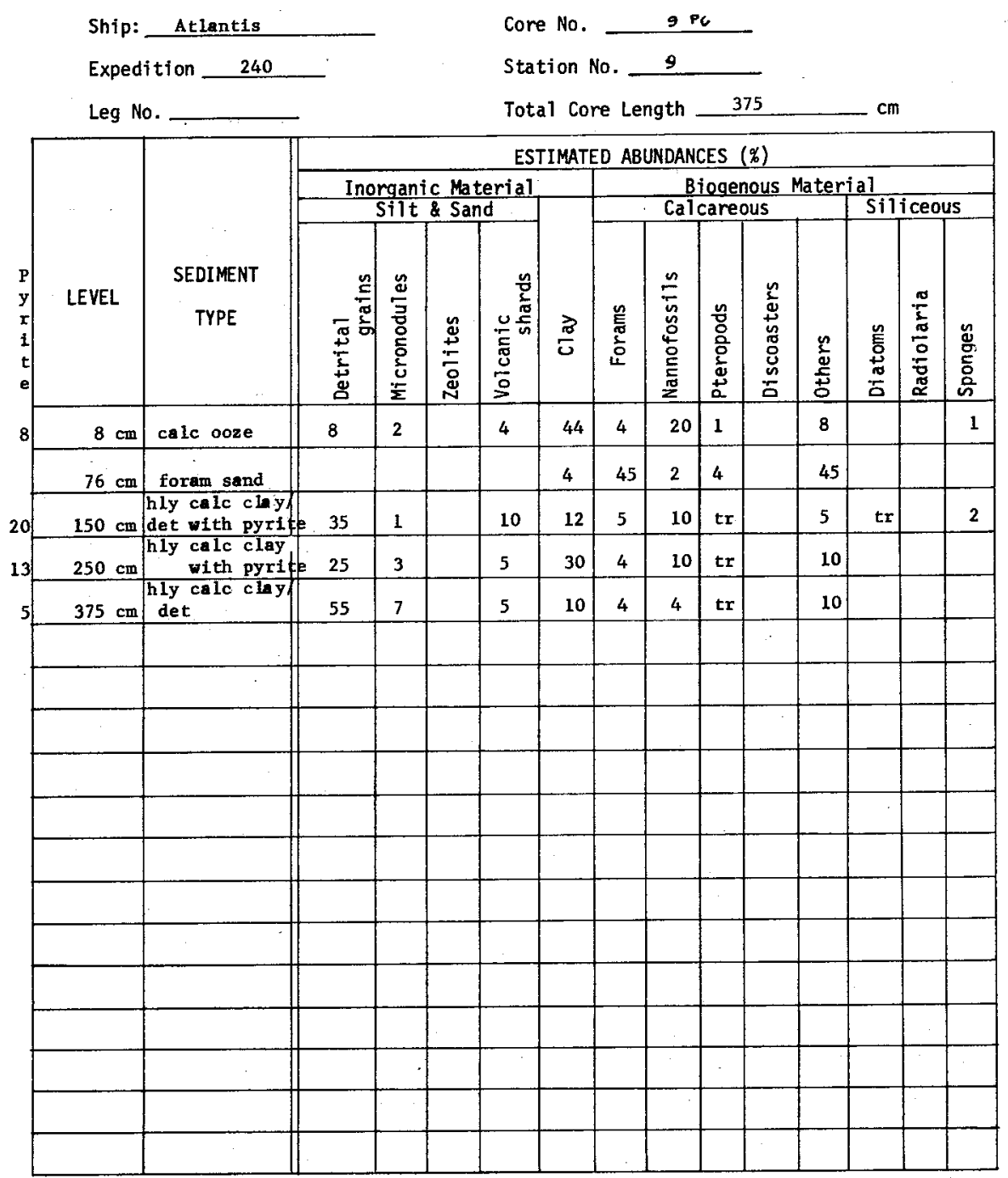

0081

VISUAL CORE DESCRIPTION

Page_L of 1

Ship $A$ Cruise 240 Leg_Sta. 10 Core No. 10 PC Ship 150 Lat. $10^{\circ} 347^{\circ N}$ Long. $45^{\circ} 49.8^{\prime} \mathrm{W}$ Depth $1.73 \mathrm{CORP} m$ Core condition maist; good Date Described $4 / 18 / 74$ by B MeGirr Physiographic locotion W. Basia, Cariace. Trench

Lithologic

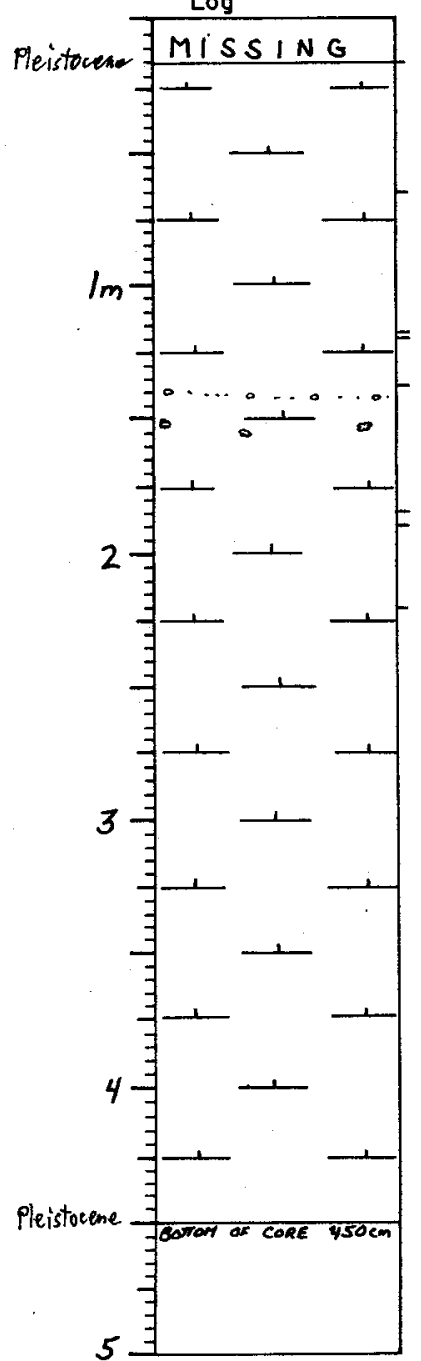

Detailed Description

$0-16$

VOI

CALC OOZE

$5 Y 3 / 2$ dark olive gray

firm silty lutite.

folor variations within unit

$\underset{65-118}{\text { very } G}$

CALC OOZI

5Y $3 / 2$ dark olive gros

firm silty lutit

3-85cm.. olive gray lutite layer no forams, sharp contact S irregular

CALC

CALC OOZE

5 T $4 / 2$ olive gray

s irregular

$120-139$

CALC OOZE

5/1 gray

Irm slightly silty lutite.. no foram

very last $2 \mathrm{~cm}$ of unit have sandy-pebbley material included

30 irsegular

$139-185$

CALC OOZE

sceittered small olive gray mottling.. 150-60

firm slightly silty lutite.. no forams

S irregular'

CALC 007 (85-191

$5 Y 4 / 2$ olive gray

Pirm slight? sy sity lutite. no forams

gypsum? crystals scattered throughout unit

irregular

CAIC OOZ

$5 Y$ 5/2 olive gray

firm slightiy silty lutit

$S$ bottom of section 3

$220-450$
CALC

CALC OOZE

$5 Y$ 4/2 olive gray

fer eorams

color variations within unit

vas originally listed as being $543 \mathrm{~cm}$ long.

It has since been remeasured.. but one section has eviden-

tly been lost.

END OF CORE 
0082

SMEAR SLIDE DESCRIPTIONS - W.H.O.I. SEDIMENT CORES

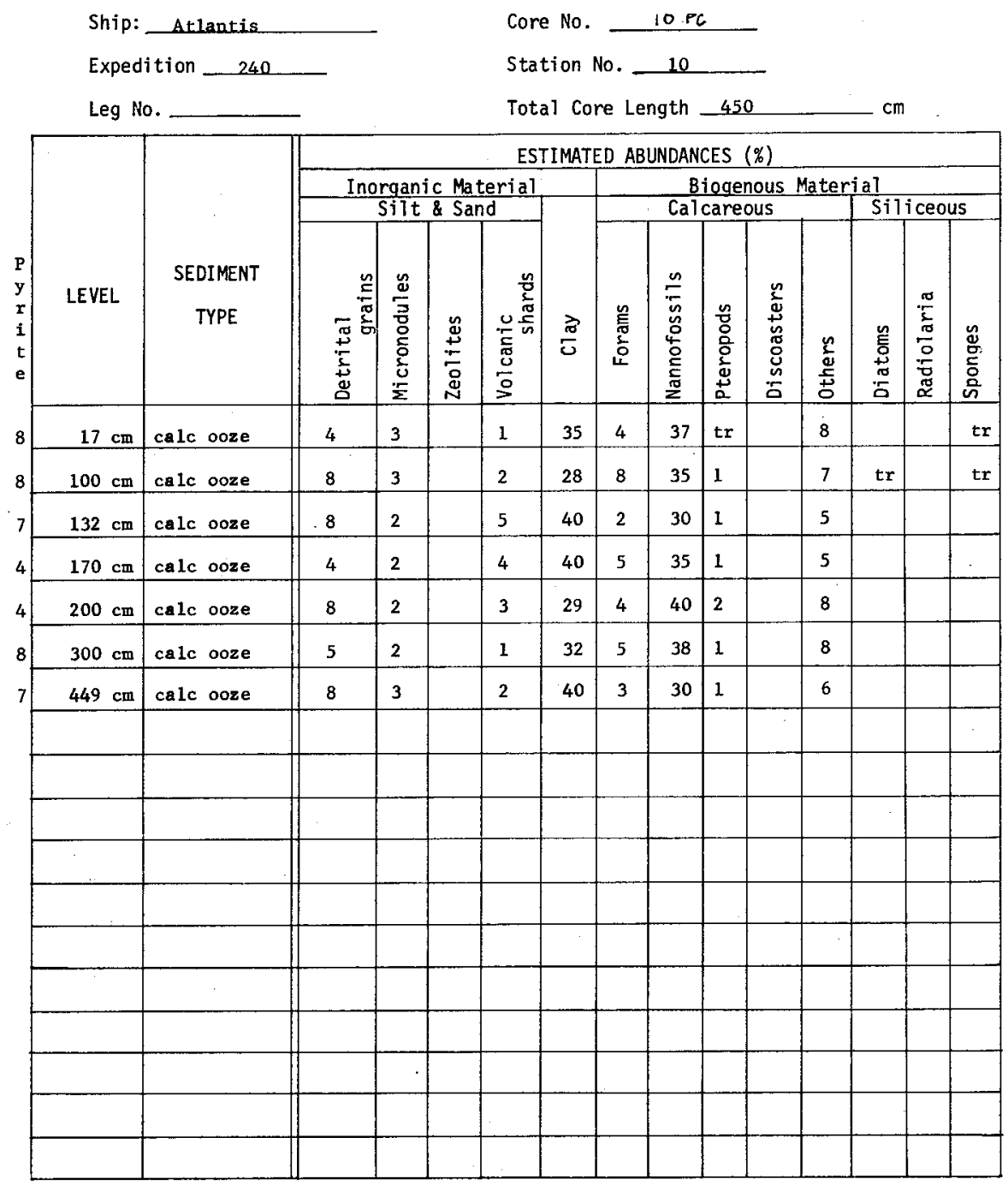

0083

VISUAL CORE DESCRIPTION

Page 1 of 1

Ship A Cruise 240 Leg Sta. 11 Core No. IIPC Totol Length 56 cm. Lat. $10^{\circ} 28.8^{\prime} \mathrm{N}$ Long. $65^{\circ} 35.3^{\prime} \mathrm{W}$ Depth 572 cara M

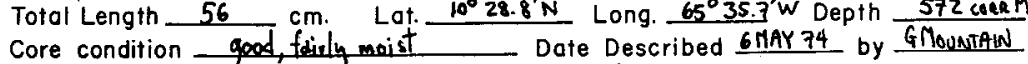
Physiographic location CARIACO TRENCH

Lithologic

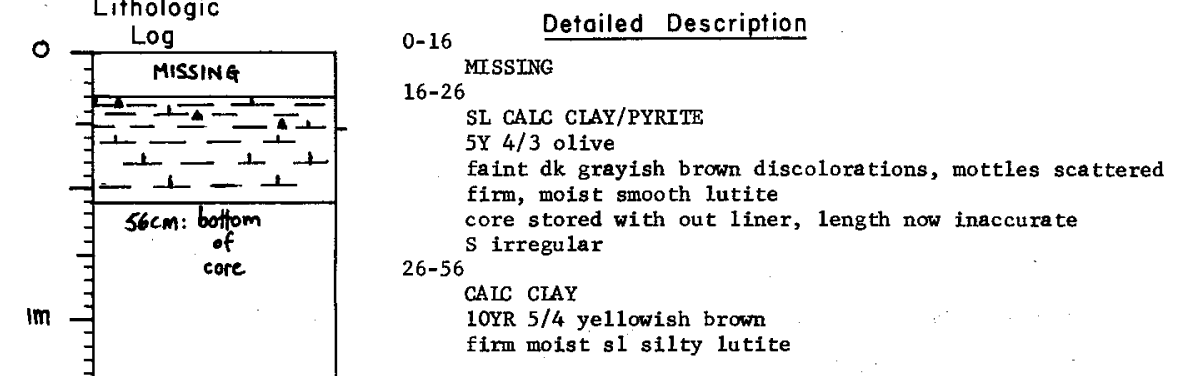

- MISSING firm moist si silty lutite
MISSING SL CALC CLAY/PYRITE

scattered cim, molat smooth lutite CAIC CIAY 
0084

SMEAR SLIDE DESCRIPTIONS - W.H.O.I. SEDIMENT CORES

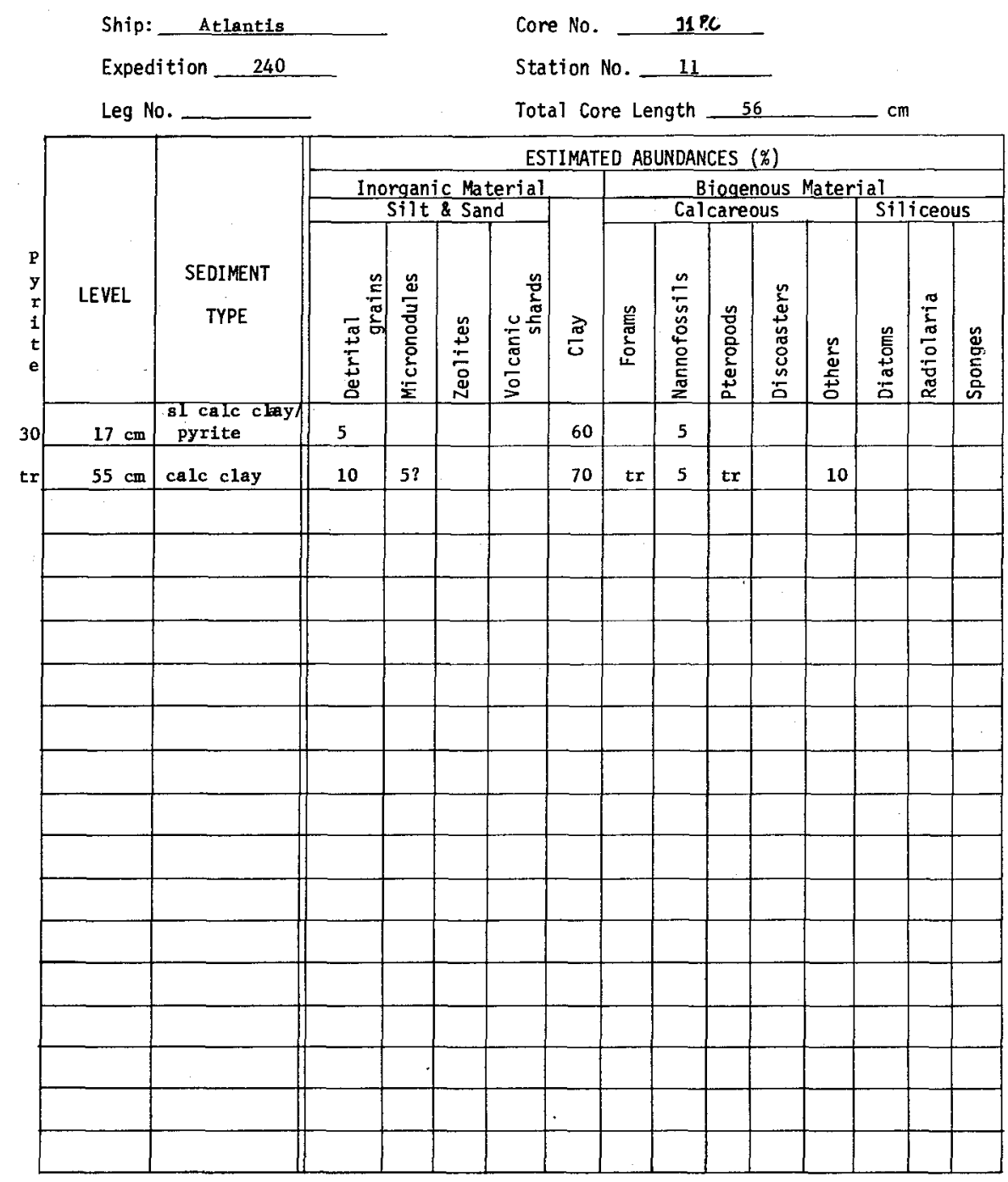

0085

VISUAL CORE DESCRIPTION

Page 1 of 1

Ship $A$ Cruise 240 Leg Sta. 12 Core No. $12 \not C$ Total Length $591 \mathrm{~cm}$. Lat. $10^{\circ} 37.5^{\circ} \mathrm{N}$ Long. $65^{\circ} 324 \mathrm{~W}$ Depth $1345 \mathrm{coRR}$ Core condition most; good

Physiographic location Cariaco Trench

Lithologic

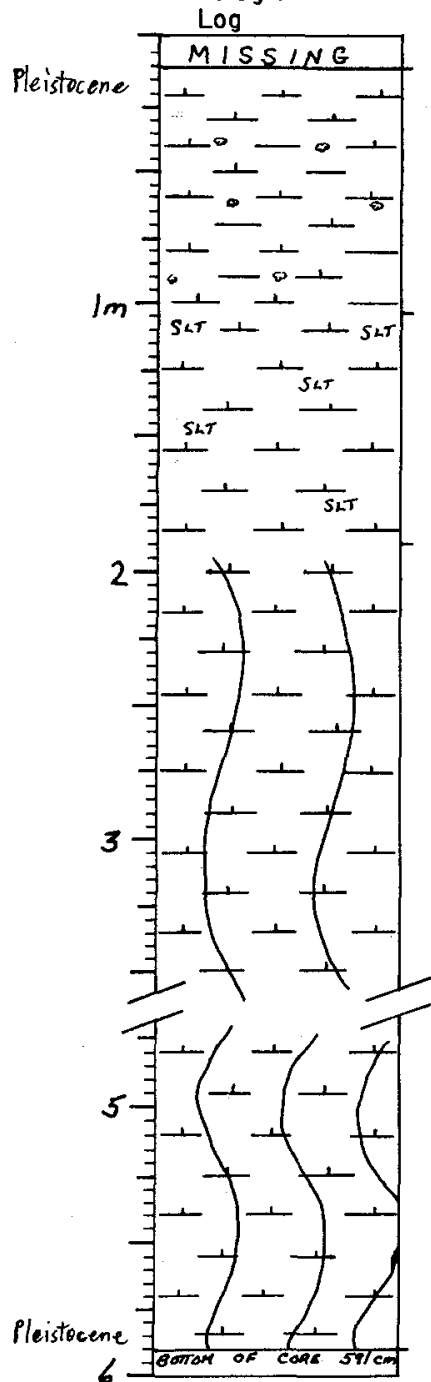

0-11 MISSING

11-103

CALC OOZE AND HLY CALC CLAY

Y $4 / 2,3 / 2$ olive gray and dk olive gray

comon mottiling in lutite

int la sequence of 2 al ternating layers. one is $v$.

sheli fratents in tilty lutite. silty late, white

$11-37,50-53,59-65,68-74,85-86 \mathrm{~cm}$, si silty lutite-

$37-50 \mathrm{~cm}, 53-59,65-68,74-85,86-103 \mathrm{~cm}$ shaxp contacts between layers

03-190

CALC OOZE/DET

$5 \mathrm{Y} 3 / 2$ dk ollve gray

firm silty lutite

ave been sampled, no indication as to

this section, $103-190 \mathrm{~cm}$, patches of

clumps scattered throughout unit, some

190-591 inera1 (chalcedony?)

CALC DOZB

$5 Y 3 / 2$ dk olive gray

$281-293 \mathrm{~cm}$ mlssing, $500-503 \mathrm{~cm}$ missing, this unit is $\mathrm{v}$. hat patches rom 300-411 and possibly even higher up the core. this strongly suggests suck up in the lower part of the core, and possibly from $190 \mathrm{~cm}$ to the v. bottom 
SMEAR SLIDE DESCRIPTIONS - W.H.O.I. SEDIMENT CORES

Ship: Atlantis

Expedition 240

Leg No.

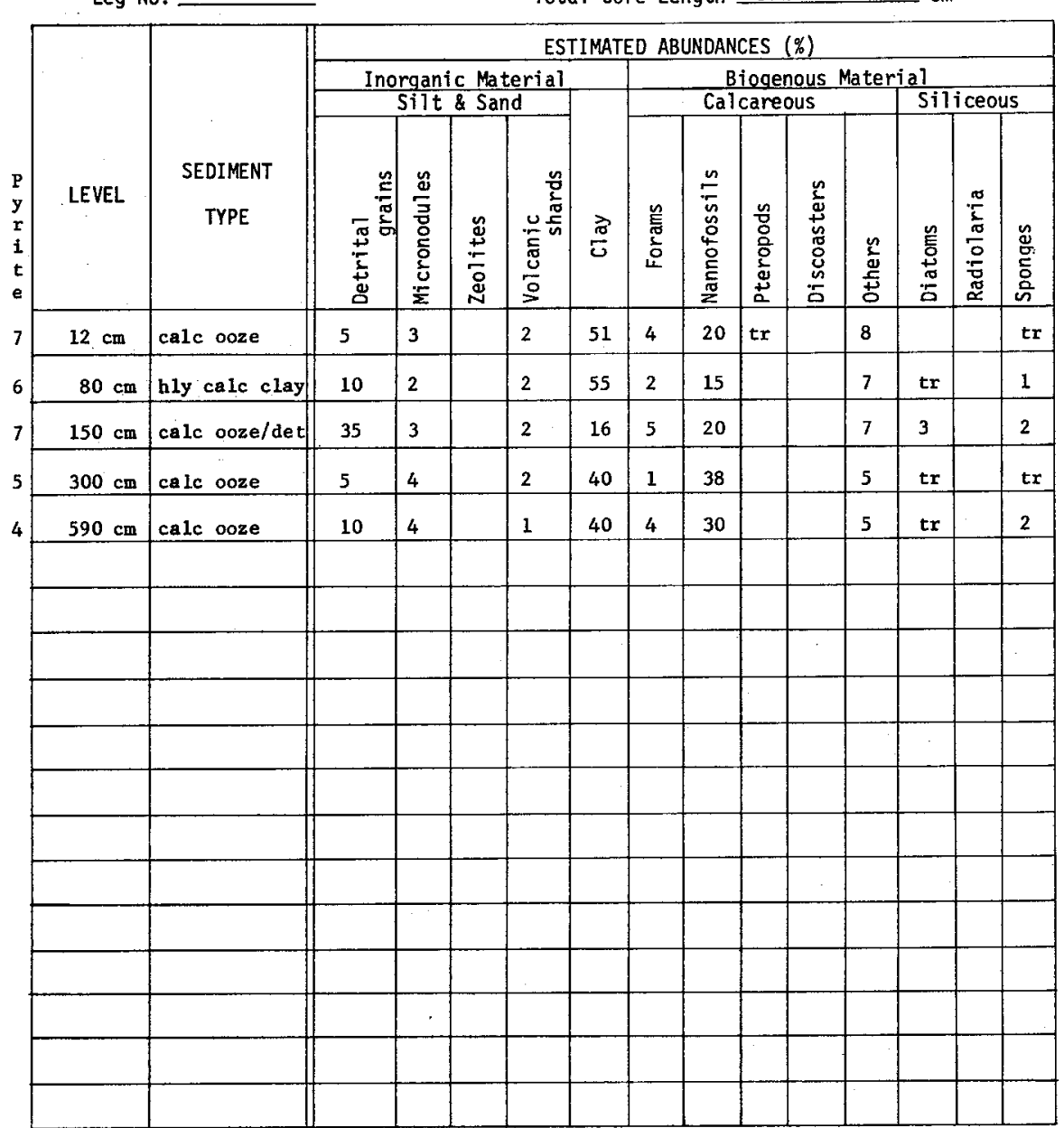

Core No. 12 ec

Station No.

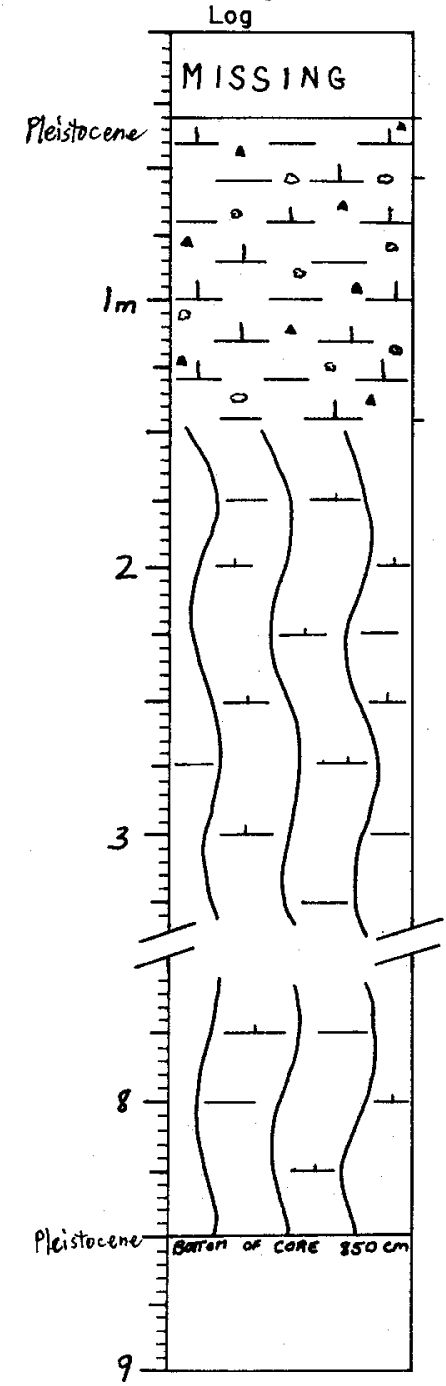

Lithologic
Ship $A$ Cruise 240 Sta 13 Core No $13 \mathrm{PC}$

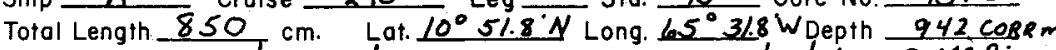
Core condition moist; good Date Described $4 / 23 / 74$ by $B$ MeGirr Physiogrophic location Cariaco Trench 0-31 MISSING

31-54 HLY CALC CIAY WITH PYRIT
$5 Y 3 / 2 \mathrm{dk}$ olive gray

1 patch of mottling below unit at $50 \mathrm{~cm}$

color variations within unit.hint of horizontal lam $31-40 \mathrm{~cm}$ void

$54-147$ 77 PYRITE $5 \mathrm{Y} 4 / 2$ olive gray
scattered through scatcered throughe, mottling $d k$ olive gra gypsum crystals $s$ irregular

$147-850$

HLY CALC CIAY

$5 Y 4 / 3,4 / 4$ olive gra

firm silty lutite

this core to $795 \mathrm{~cm}$ has vertical layers, usually with one color along the edges and a secon across this contact. From this, it is concluded that the rest of the core is most probably suck up.
HLY CALC CIAY WITH
$5 Y 4 / 2$ olive gray 


\section{8}

SMEAR SLIDE DESCRIPTIONS - W.H.O.I. SEDIMENT CORES

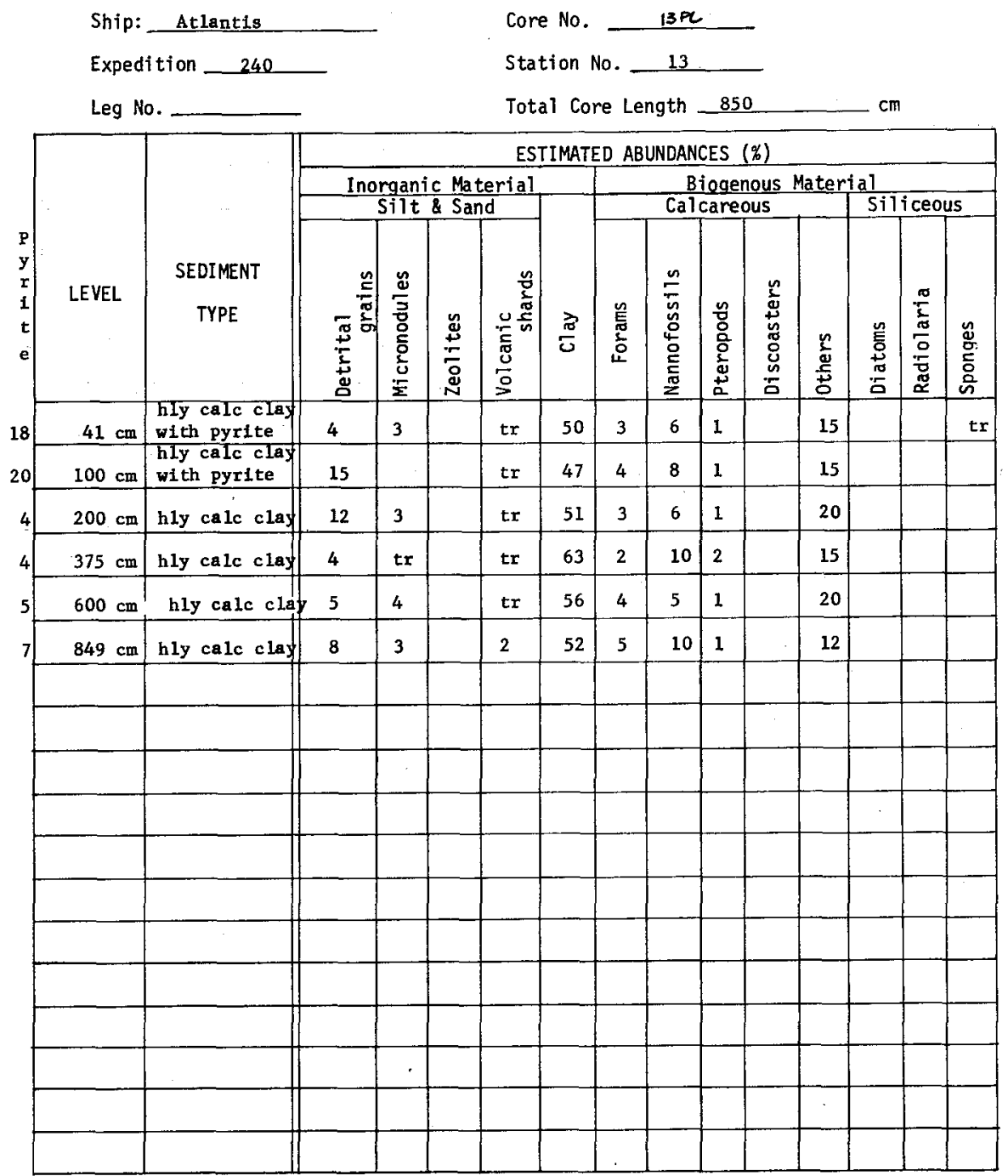

0089

VISUAL CORE DESCRIPTION

Page 1 of 1

Ship A Cruise 240 Sta. 14 Core No. $14 \mathrm{PC}$ Total Length $840 \mathrm{~cm}$. Lat. $10^{\circ} 55.2^{\prime} \mathrm{N}$ Long. $65^{\circ} 32.2 \mathrm{~W}$ Depth $385 \mathrm{cose} \mathrm{m}$ Core condition mest; good Date Described $4 / 23 / 24$ by $B M^{e}$ Gire Physiographic location N. Slope, Cariace Trench
Lithologic

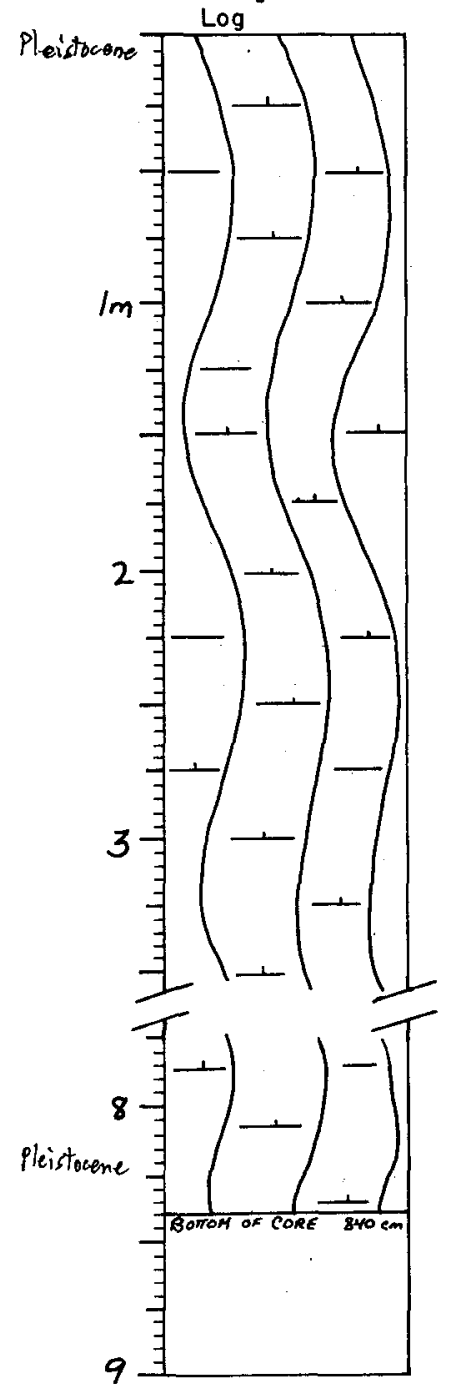

Detailed Descriptio length being recorded as $20 \mathrm{~cm}$ too long. Therefore, because (1) the difference is only $20 \mathrm{~cm}$, and (2) the $0-840$ HLY CALC CLAY TO CALC OOZE $5 \mathrm{Y} 4 / 2$ olive gray

$10-51 \mathrm{~cm}$ missing. $s 1$ color variations within unit,

as vertical streaks mostly $\mathrm{y}$, homogeneous, with several patches of siltier lutite- bottom $5 \mathrm{~cm} d \mathrm{k}$ and silt ler than rest of core 1ote; Section $\%$ length being recorded as $20 \mathrm{~cm}$ too long. Therefore, al core sections have not been remeasured and relabell entire core is homogeneous and most probably all suck up 
0090

SMEAR SLIDE DESCRIPTIONS - W.H.O.I. SEDIMENT CORES

Ship: Atlantis

Expedition 240

Core No. $14 \mathrm{PC}$

Leg No.

Station No. 14

Total Core Length $840 \_\mathrm{cm}$

\begin{tabular}{|c|c|c|c|c|c|c|c|c|c|c|c|c|c|c|}
\hline \multirow[b]{3}{*}{ LEVEL } & \multirow[b]{3}{*}{$\begin{array}{c}\text { SEDIMENT } \\
\text { TYPE }\end{array}$} & \multicolumn{13}{|c|}{ ESTIMATED ABUNDANCES $(\%)$} \\
\hline & & \multicolumn{4}{|c|}{$\frac{\text { Inorganic Material }}{\text { Silt \& Sand }}$} & & \multicolumn{5}{|c|}{$\begin{array}{l}\text { Biogenous Mater } \\
\text { Calcareous }\end{array}$} & \multicolumn{3}{|c|}{$\frac{\mathrm{al}}{\text { Siliceous }}$} \\
\hline & & 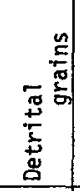 & 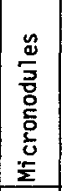 & 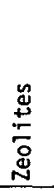 & 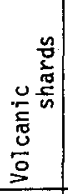 & $\frac{\vec{\pi}}{0}$ & 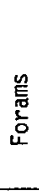 & 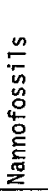 & 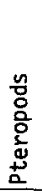 & 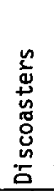 & $\begin{array}{l}\frac{n}{2} \\
\frac{m}{2} \\
0 \\
0\end{array}$ & $\begin{array}{l}0 \\
\overline{0} \\
+0 \\
.0 \\
\vdots \\
0\end{array}$ & 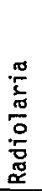 & 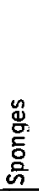 \\
\hline $52 \mathrm{~cm}$ & hly calc clay & 5 & 2 & & tr & 58 & 3 & 5 & & & 20 & & & \\
\hline $300 \mathrm{~cm}$ & hly calc clay & 7 & 3 & & tr & 55 & 3 & 4 & $\mathrm{tr}$ & & 20 & & & \\
\hline $500 \mathrm{~cm}$ & calc ooze & 6 & 2 & & 1 & 50 & 3 & 6 & $\operatorname{tr}$ & & 25 & & & \\
\hline $839 \mathrm{~cm}$ & calc ooze & 8 & 6 & & 1 & 30 & 5 & 15 & $t x$ & & 30 & & & \\
\hline & & & & & & & & & & & & & & \\
\hline & & & & & & & & & & & & & & \\
\hline & & & & & & & & & & & & & & \\
\hline & & & & & & & & & & & & & & \\
\hline & & & & & & & & & & & & & & \\
\hline & & & & & & & & & & & & & & \\
\hline & & & & & & & & & & & & & & \\
\hline & & & & & & & & & & & & & & \\
\hline & & & & & & & & & & & & & & \\
\hline & & & & & & & & & & & & & & \\
\hline & & & & & & & & & & & & & & \\
\hline & & & & & & & & & & & & & & \\
\hline & & & & & & & & & & & & & & \\
\hline & & & & & & & & & & & & & & \\
\hline
\end{tabular}

0091

VISUAL CORE DESCRIPTION

Page _ L of 1

Ship $A$ Cruise 240 Leg_ Sta. 15 Core No. 15 PC Total Length $580 \mathrm{~cm}$. Lat. $10^{\circ} 52.5^{\prime} \mathrm{N}$ Long. $15^{\circ} 08.0 \mathrm{WDepth} 354 \mathrm{CoRR}$ Core condition dey to fairlymoist; feir Date Described $4 / 24 / 74$ by $B M^{C} G$ irr

Physiographic locotion Carien Trench

Lithologic

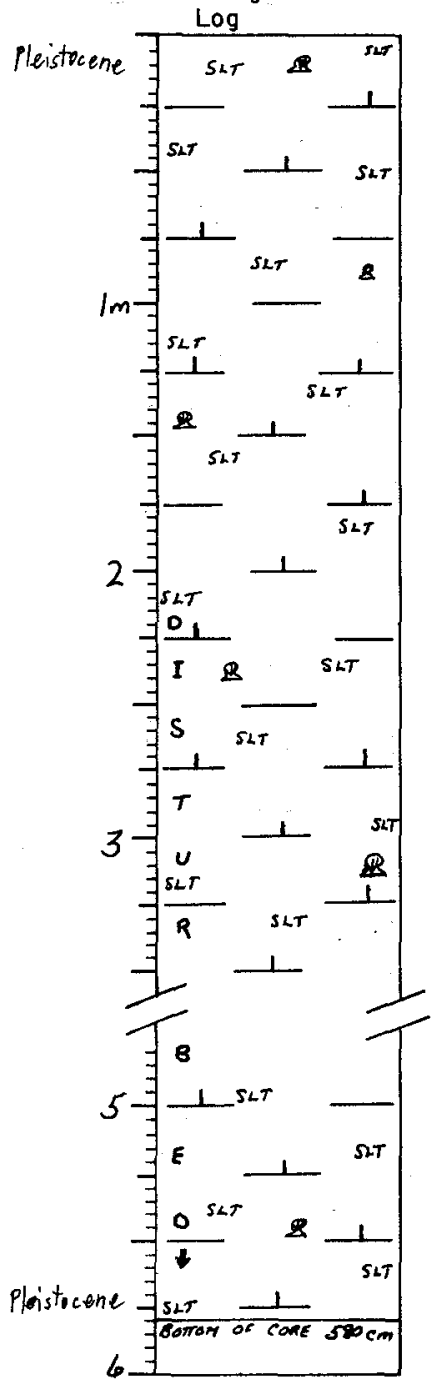

Detailed Description 
SMEAR SLIDE DESCRIPTIONS - W.H.O.I. SEDIMENT CORES

Ship: Atlantis

Expedition 240

Leg No.

\begin{tabular}{|c|c|c|c|c|c|c|c|c|c|c|c|c|c|c|c|}
\hline & \multicolumn{14}{|c|}{10} \\
\hline \multirow[b]{4}{*}{ LEVEL. } & \multirow[b]{4}{*}{$\begin{array}{c}\text { SEDIMENT } \\
\text { TYPE }\end{array}$} & \multicolumn{14}{|c|}{ ESTIMATED ABUNDANCES (\%) } \\
\hline & & \multirow{2}{*}{\multicolumn{4}{|c|}{$\frac{\text { Inorganic Material }}{\text { Silt \& Sand }}$}} & \multirow[b]{3}{*}{$\frac{\pi}{0}$} & \multirow{2}{*}{\multicolumn{6}{|c|}{$\begin{array}{l}\text { Biogenous Mater } \\
\text { Calcareous }\end{array}$}} & \multirow{2}{*}{\multicolumn{3}{|c|}{ Siliceous }} \\
\hline & & & & & & & & & & & & & & & \\
\hline & & 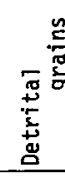 & 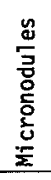 & 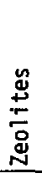 & 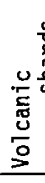 & & & 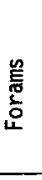 & 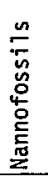 & 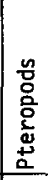 & 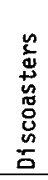 & 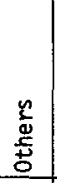 & 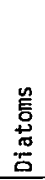 & $\begin{array}{l}\frac{\pi}{2} \\
\frac{\pi}{\sigma} \\
\frac{\pi}{0} \\
\frac{\pi}{\pi} \\
\end{array}$ & $\begin{array}{l}0 \\
0 \\
0 \\
\vdots \\
0 \\
0 \\
0\end{array}$ \\
\hline $1 \mathrm{~cm}$ & $\begin{array}{l}\text { hly calc clay } \\
\text { det }\end{array}$ & 45 & 2 & & 1 & 2 & & 1 & 5 & tr & & 15 & & & \\
\hline $200 \mathrm{~cm}$ & $\begin{array}{l}\text { hIy calc clay } \\
\text { with det }\end{array}$ & 20 & 2 & & tr & 4 & & $\mathrm{I}$ & 8 & 1 & & 20 & & & \\
\hline $400 \mathrm{~cm}$ & $\begin{array}{l}\text { hly calc clas } \\
/ \text { det }\end{array}$ & 60 & 2 & & tr & 1 & & $\operatorname{tr}$ & 2 & 1 & & 20 & & & \\
\hline $579 \mathrm{~cm}$ & \begin{tabular}{|l} 
hly calc \\
clay/det
\end{tabular} & 55 & 1 & & tr & 1 & & 1 & 3 & & & 20 & & & \\
\hline & & & & & & & & & & & & & & & \\
\hline & & & & & & & & & & & & & & & \\
\hline & & & & & & & & & & & & & & & \\
\hline & & & & & & & & & & & & & & & . \\
\hline & & & & & & & & & & & & & & & \\
\hline & & & & & & & & & & & & & & & \\
\hline & & & & & & & & & & & & & & & \\
\hline & & & & & & & & & & & & & & & \\
\hline & & & & & & & & & & & & & & & \\
\hline & & & & & & & & & & & & & & & \\
\hline & & & & & & & & & & & & & & & \\
\hline & & & . & & & & & & & & & & & & \\
\hline & & & & & & & & & & & & & & & \\
\hline & & & & & & & & & & & & & & & \\
\hline
\end{tabular}

Core No. ISPC

Station No. 15

Total Core Length 580
Pritacas

Pleistocene

firm silty lutite, forams common at top to few at bottom fragments and pteropods scattered throughout unit1 color changes from section to section, $1 \mathrm{~mm}$ to $1 / 2 \mathrm{~cm}$ hick horizontal foram sand layers at $58 \mathrm{~cm}, 116,135$, $194,218,245,259,312,353,359,368,430,447, \mathrm{~cm}-$
$0-126 \mathrm{~cm}$, unit is homogeneous, $260-533 \mathrm{~cm}$, unft composed $0-126 \mathrm{~cm}$, unit is homogeneous, $260-533 \mathrm{~cm}$, unit composed
of v. fine, faint, horizontal lams $s$

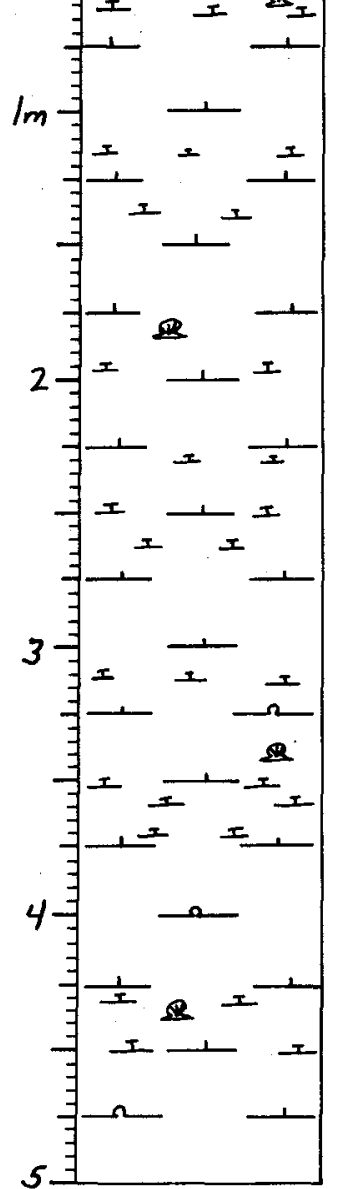

ILIC CAIC OOZE

$5 \mathrm{Y} 4 / 3$ and $4 / 4$ olive

firm s1 silty lu tite, no forams

vine, horizontal lam throughout unit, in a varve-like pettern. occassions 1 lengths of homogeneous sediment 
0094

VISUAL CORE DESCRIPTION

Ship A Cruise 240 Leg_Sto. 16 Core No. 16 PC

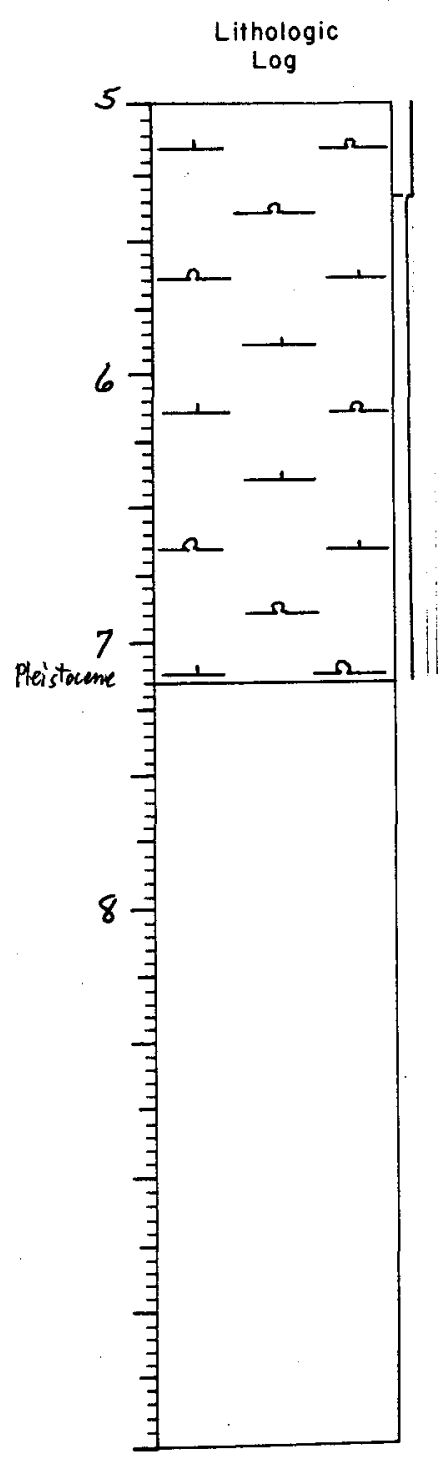

0095

SMEAR SLIDE DESCRIPTIONS - W.H.O.I. SEDIMENT CORES

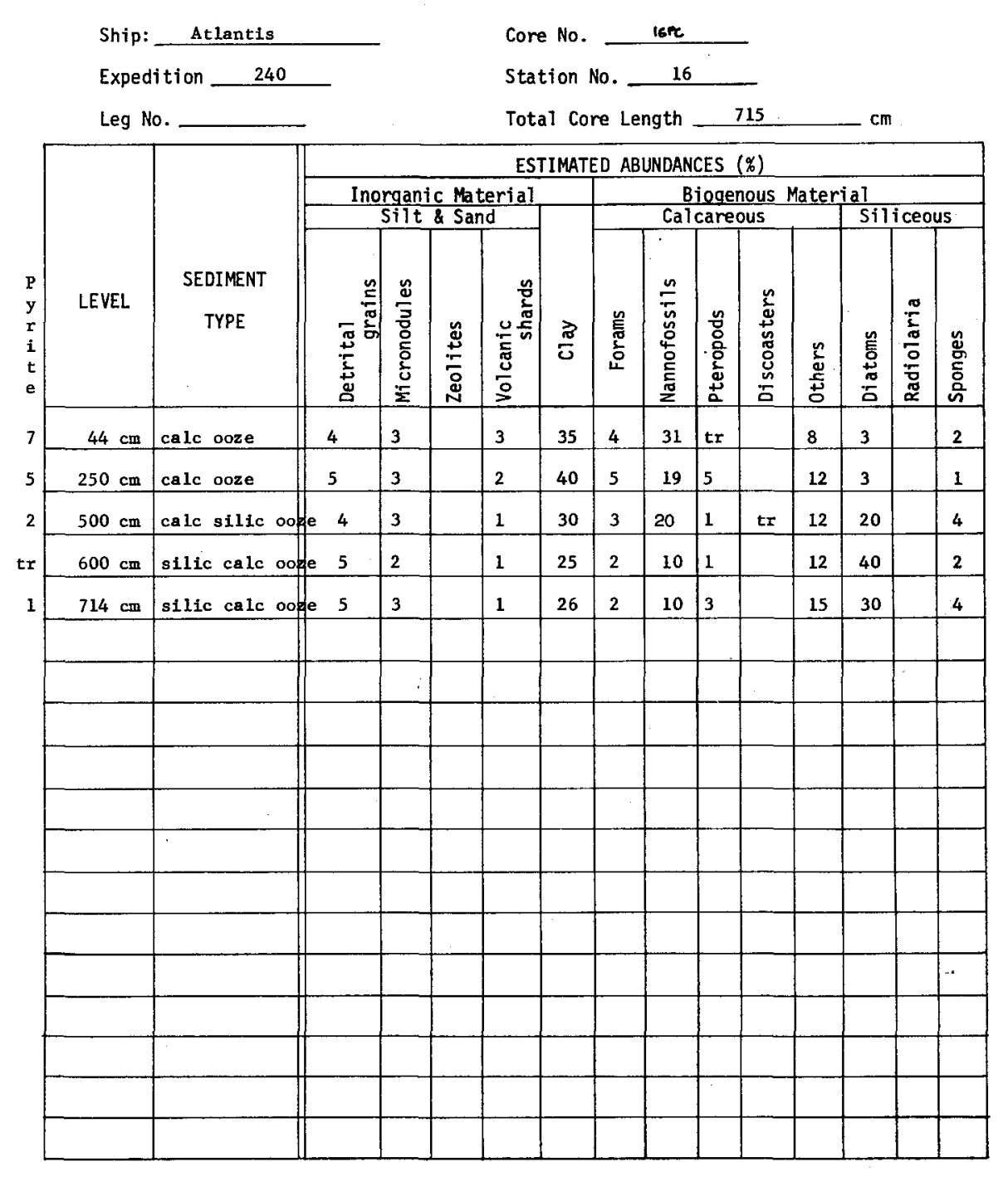


0096

VISUAL CORE DESCRIPTION

Page_ 1 of 1

Ship $A$ Cruise 240 Leg_Sta. 17 Core No. 17 PC

Total Length $759 \mathrm{~cm}$. Lat. $10^{\circ} 32.6^{\top} \mathrm{N}$ Long. 64. $51.2^{\prime} \mathrm{W}$ Pepth $1348 \mathrm{mCORR}$.

Core condition fair to goed Date Described 25APR 74 by GMounTain

Physiogrophic locotion CARIACO TRENCH

Lithologic

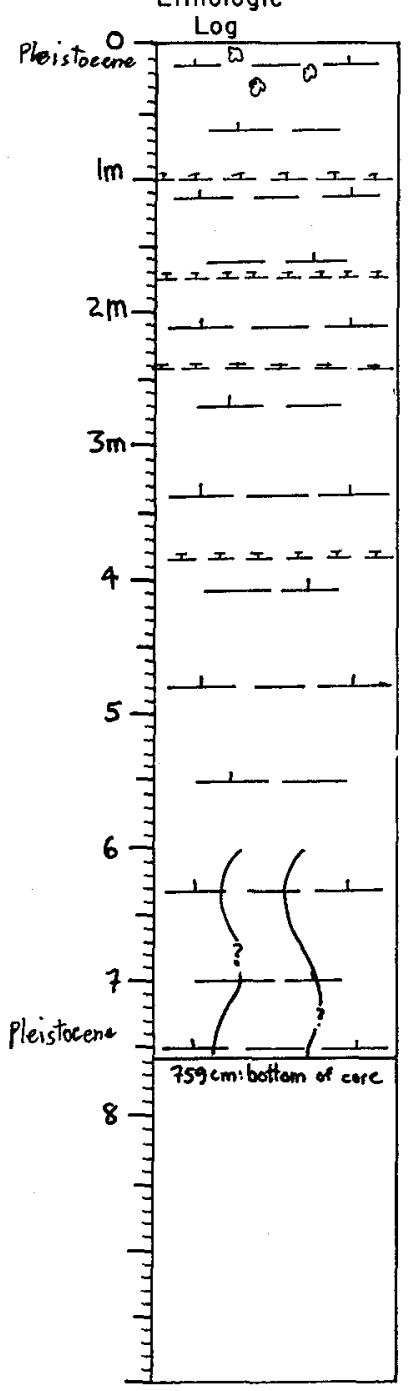
$0-600$

HLY CALC CLAY

$5 \mathrm{X} 3 / 2$ dk olive gray

$\begin{array}{lll} & & \end{array}$

(

crystals; mottling, scattered at bottom of core.

600-759 stripes of silty white mineral (authigenic?)
0097

SMEAR SLIDE DESCRIPTIONS - W.H.O.I. SEDIMENT CORES

Note; core $\frac{\text { Defailed Description }}{\text { stored }}$ sedjment changed shape and

grayish brown foram sand layers throughout core, esp $0-400$

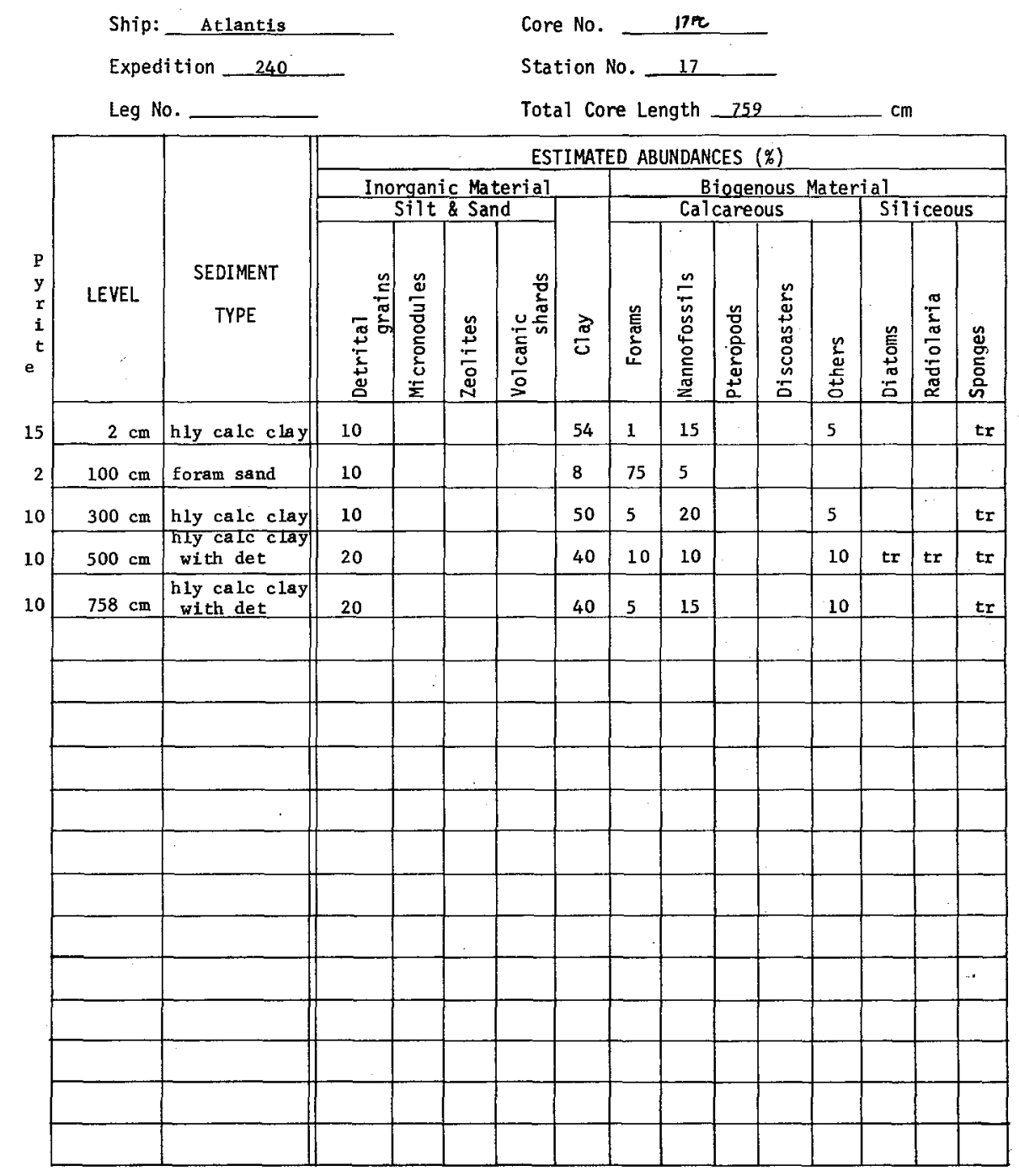


0098

VISUAL CORE DESCRIPTION

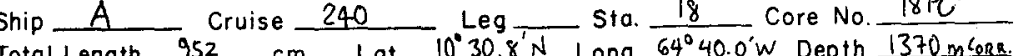

Described 24 APR 74 by GMOUNTAN

Physiographic locótion. EAST Basin Cariaco TrenCH

Lithologic

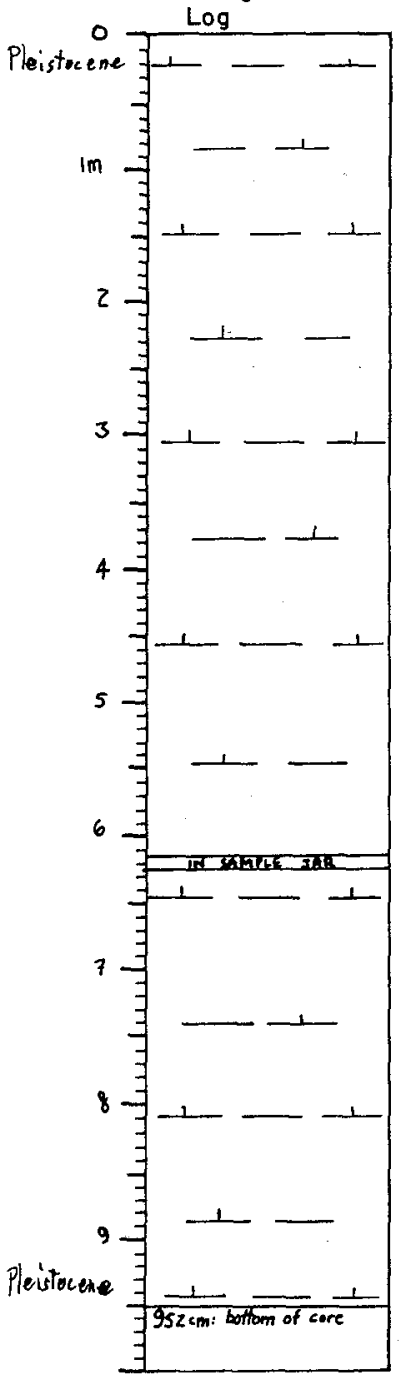

\section{Detailed Description} HLY CALC CIAY

$5 \mathrm{Y} 4 / 2$ olive gray

moist, fim silty luti te, forams scattered to common,

is occasional silty layers; faint homogeneous, structureless
0099

SMEAR SLIDE DESCRIPTIONS - W.H.O.I. SEDIMENT CORES

Ship: Atlantis

Expedition 240

Core No. 18Pe

Leg No.

Total Core Length 952

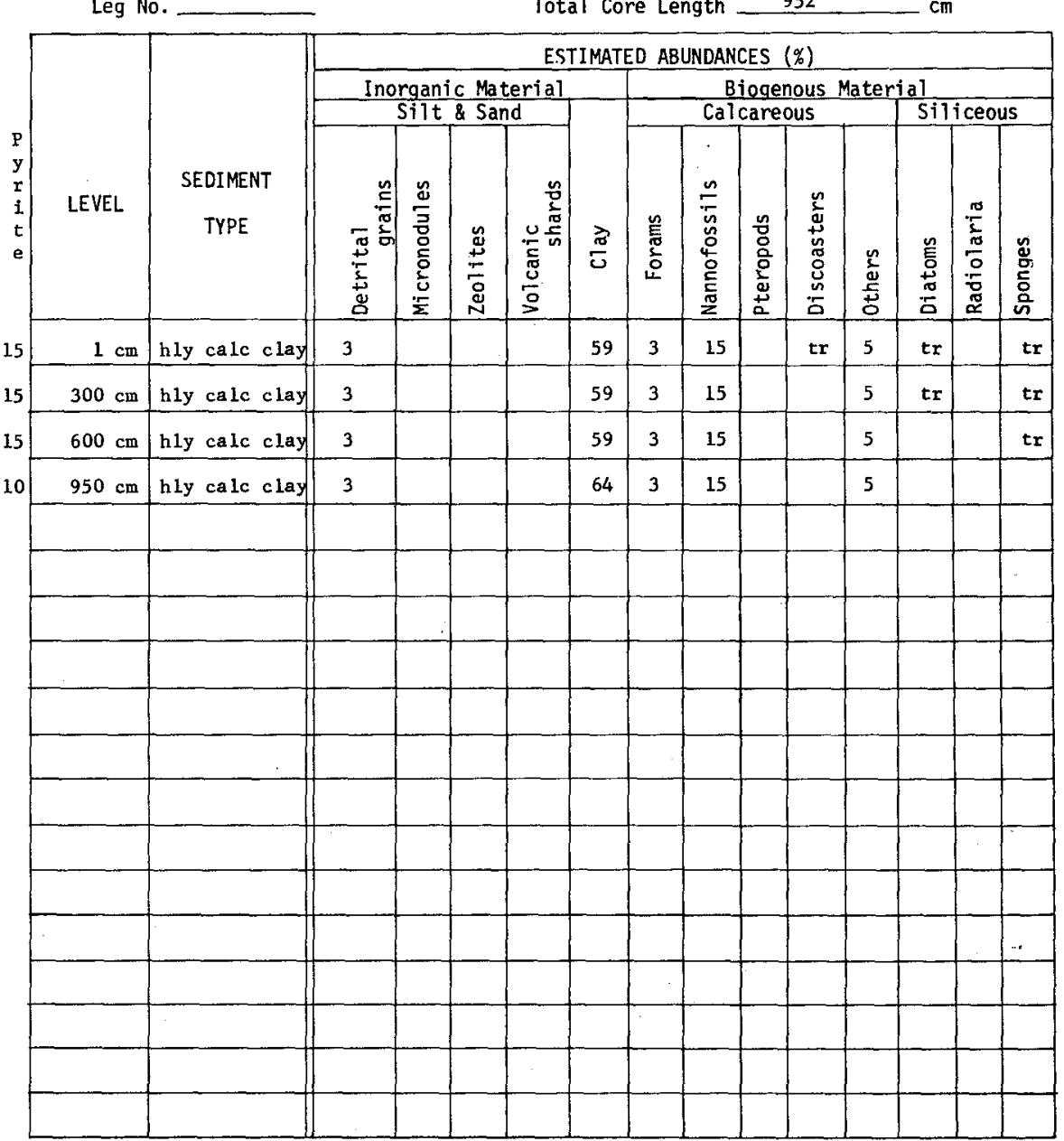


0100

VISUAL CORE DESCRIPTION

Page 1 of 2

Ship $A$ Cruise 240 Leg__ Sta. 19 Core No. $19 \mathrm{PC}$ Totol Length $612 \mathrm{~cm}$. Lat. $10^{\circ} 22.3^{\circ} \mathrm{N}$ Long. $64^{\circ} 42.5^{\prime} \mathrm{w}$ Depth $177 \mathrm{~m} \mathrm{cos}$. Core condition good Date Described 18ARRL, ZY by GMouNTAIN

Physiographic location INSHORE END, EAST BASIN, CARIACO TRENCI-

Lithologic

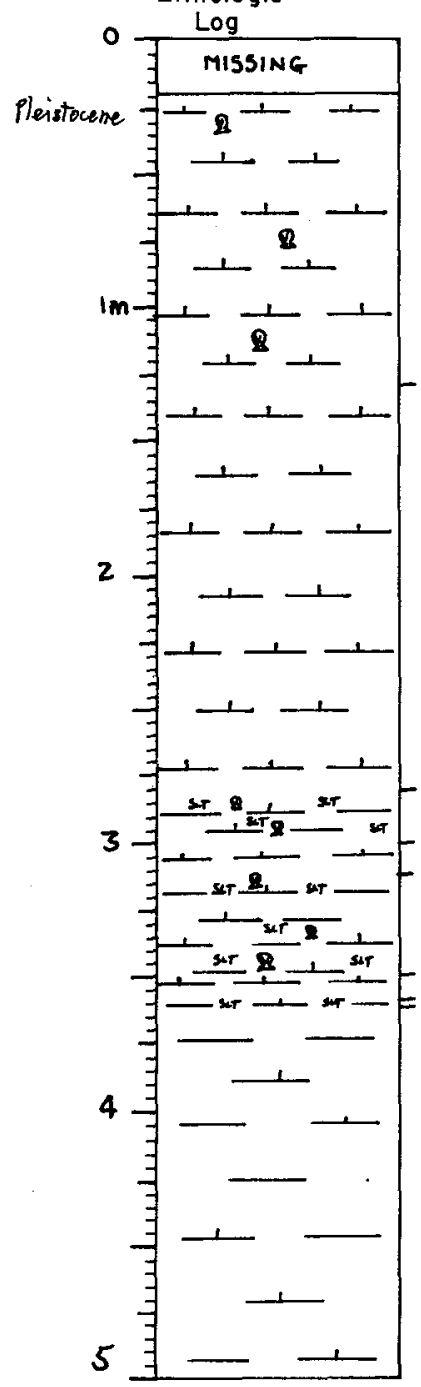

\section{Detailed Description}

MISSING

The; sediment was stored some time without liner; changed shape; measurements somewhat inaccurate.

CALC OOZE

$5 \mathrm{Y} 3 / 3 \mathrm{dk}$ olive

firm, moist silty lutite, common forams, pteropods, gastropods occastonal gypsum crystals

$130-281$

$$
\text { CALC OOZE }
$$

$5 Y 5 / 2$ olive gray

more pronounced than the color change from the above unit is the near absence of shell fragments

281-301

HLY CALC CLAY/DET

$5 Y 4 / 2$ olive gray

dry, firm silt; scattered shell fragments

S convex upward

CALC OOZE

$5 Y 5 / 2$ olive gray

Lutite

$312-351$

HLY CALC CLAY/DET

$5 \mathrm{Y} 4 / 2$ olive gray

dry firm silt, pteropod, gastropod fragments, common incress. Ing downwa

351-359 S irregula

CAIC OOZE

moist sl silty lutite

smooth, homogeneous

359-362

\section{HLY CALC CLAY/DET}

dry firm silt, shell fragments

$362-612$

5 inclined

CALC CIAY

57 $5 / 3$ olive

mo1st sl silty lutite

列

section $1,522-612 \mathrm{~cm}$ and occassional black pyrite
0101

VISUAL CORE DESCRIPTION

Ship A Cruise 240 Leg Sto. 19_ Core No. $19 \mathrm{PC}$

Lithologic

Log

Detailed Description
Page 2 of 2

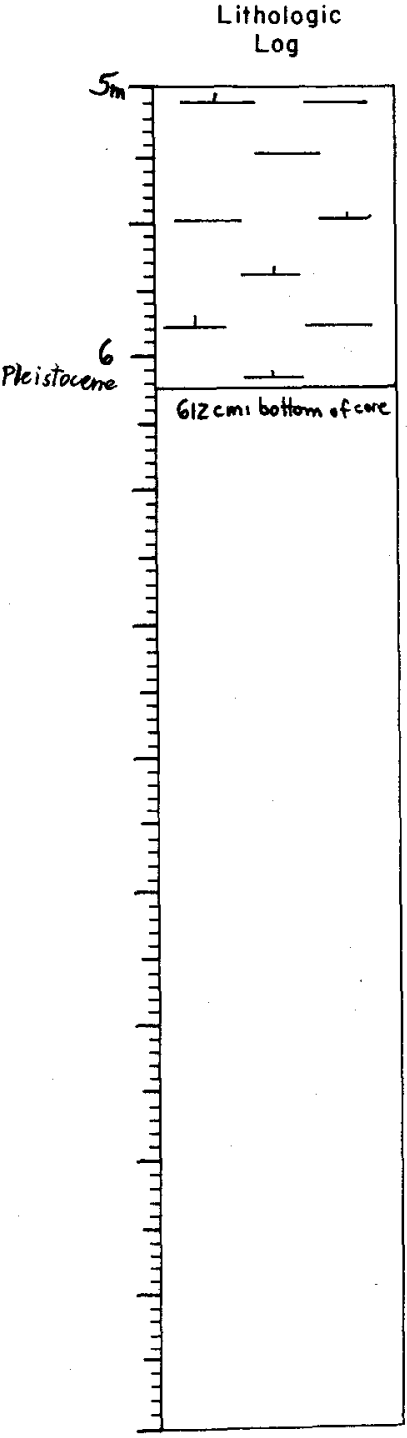


SMEAR SLIDE DESCRIPTIONS - W.H.O.I. SEDIMENT CORES

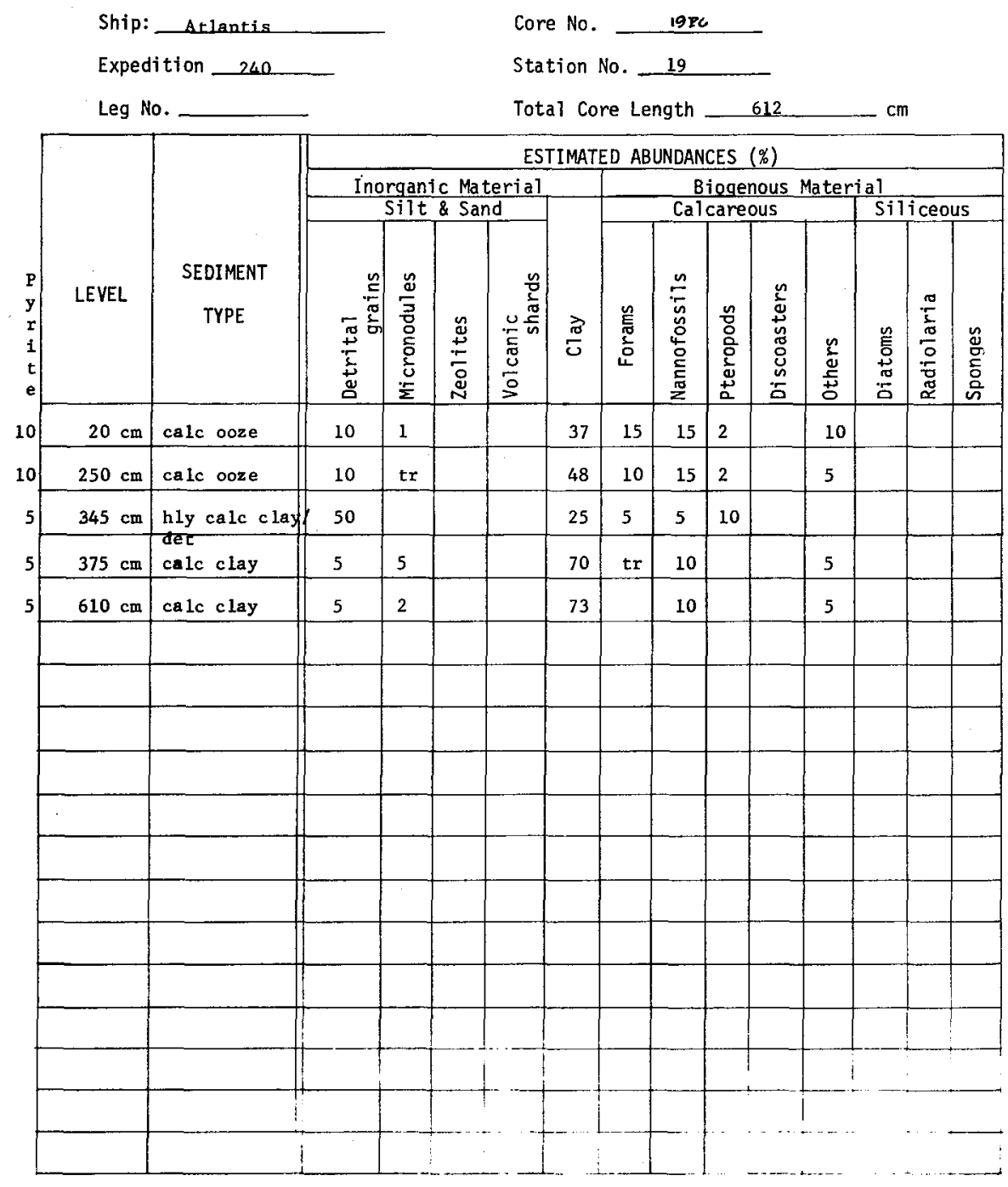

Ship A Cruise 240 Leg_ Sta. 20 Core No. $20 \mathrm{PC}$ Total Length $915 \mathrm{~cm}$. Lat. $10^{\circ} 24.0^{\circ} \mathrm{N}$ Long. $64^{\circ} 41.2^{\prime} \mathrm{W}$ Depth $822 \mathrm{mCow}$

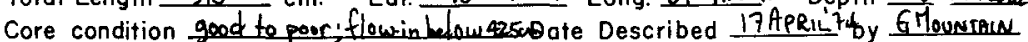
Physiographic location Capiace Trench

Lithologic

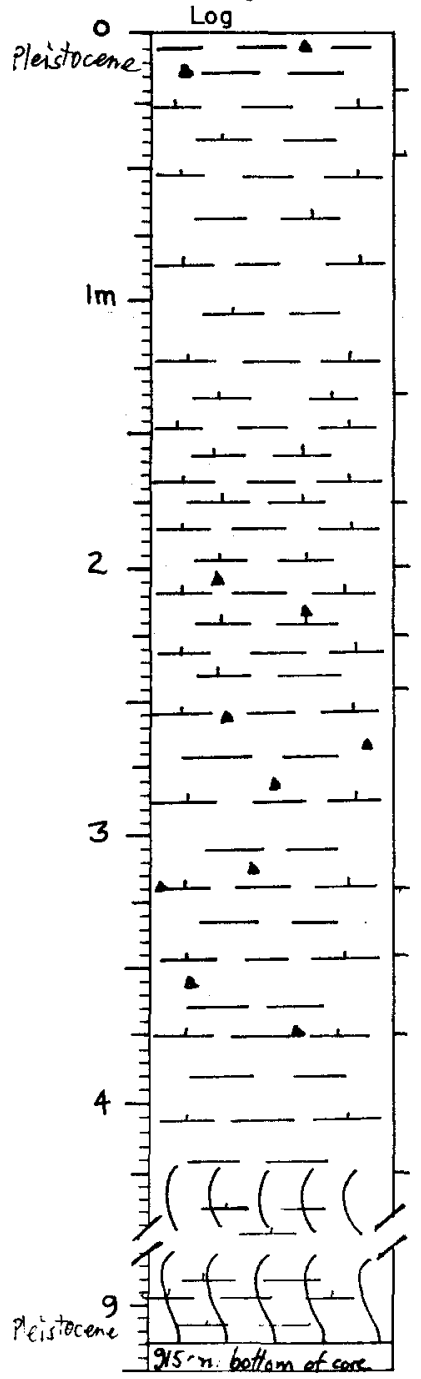

Detailed Description

core was stored without liners; measurements now inaccurat 1.e, section $10,1-45 \mathrm{~cm}$, now in $28 \mathrm{~cm}$ liner

UNFOSS CIAY WITH PYRITE

$5 Y 4 / 1 \mathrm{dk}$ gray

white crystals abunt. on surface of this dry unit (salt?)

(gypsum?)

$20-45$

\section{CALC CIAY}

$5 Y 6 / 3$ pale olive

lithified lutite, fragmented

pteropods visible .. bottom of section 10

CALC CIAY

$5 Y 5 / 3$ olive

oist silty lutite

for black specks

sample

SA-135

CALC CIAY

$2.5 Y 4 / 4,5 / 3$ olive brown and olive

dry firm silty lutite $100 \mathrm{~cm}$. fragmented below that

HLY CALC CLAY

$5 Y 5 / 3$ olive

homogeneous

$S$ irregular

175-200

HLY CALC CIA Y

$2.5 \mathrm{Y} 5 / 2$ grayish brow

moist silty lutite

200-225

HLY CAIC CIAY WITH PYRITE

$5 Y 4 / 1$ dk gray

gypsum crystals throughout

$225-245$

C CIAY

2. 5 Y $5 / 2$ grayish beown

3. jray ajue tretlin

$\sum$ ir $1 \%$ 
0104

VISUAL CORE DESCRIPTION

Page 2 of 2

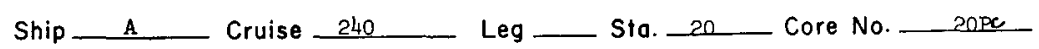

Lithologic

Log

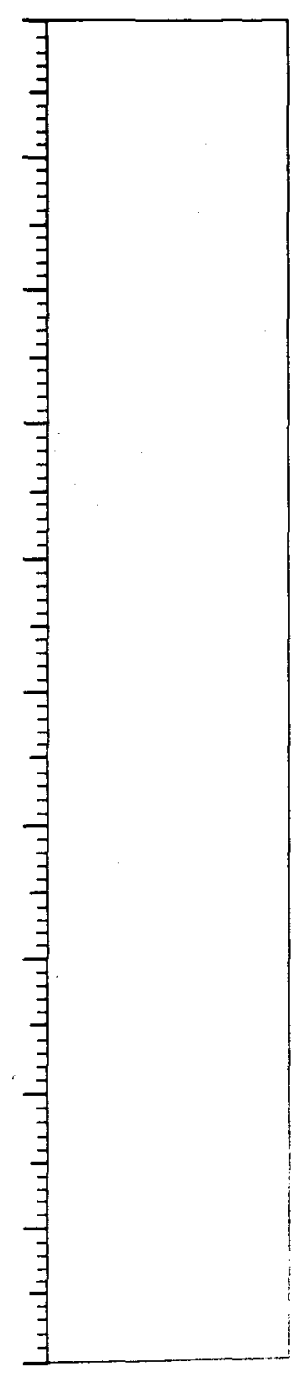

Detailed Description

245-320

CALC CLAY WITH PYRIT

$5 Y 4 / 1$ dk gray
firm silty lutite

Som inht dry and crumbled, white, calc worm tubes, cemented

in a cluster, $290 \mathrm{~cm}$ $20-345$

CALC CLAY

2.5Y $5 / 2$ grayish brown

firm silty lutite

vague bottom contact

CALC CLAY WITH PYRITE

$5 Y 4 / 1 d k$ gray

abunt. white crystals (gypsum?) beginning in this unit top of section 6 and continuing downwards $S$ inclined $45^{\circ}$

$375-425$

CALC CLAY

$5 Y 5 / 1$ gray

Degaced mottles, discolorations show that disturbance high as $375 \mathrm{~cm}$

425-915

HLY CALC CIAY

$5 Y 5 / 3$ olive

flow in

\section{5}

SMEAR SLIDE DESCRIPTIONS - W.H.O.I. SEDIMENT CORES

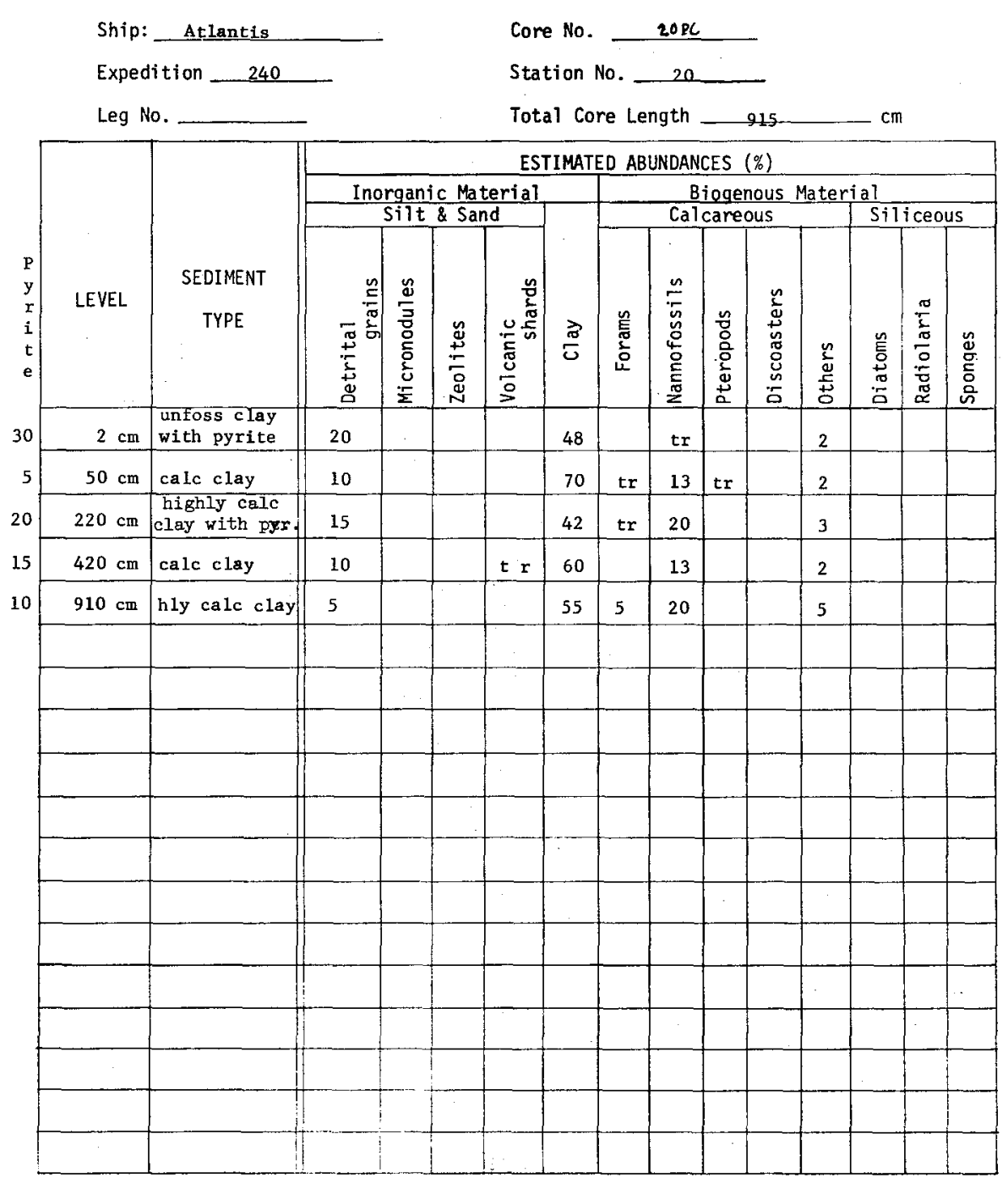


0100

VISUAL CORE DESCRIPTION

Poge 1 of 1

Ship_A Cruise 240 Leg__ Sta. 21 Core No. 2IPC

Total Length $886^{2} \mathrm{~cm}$. Lat. $10^{\circ} 26.2^{\prime} \mathrm{N}$ Long. $64^{\circ} 41.5^{\prime} \mathrm{W}$ Depth $980 \mathrm{mCos}$

Core condition good downto $4 \mathrm{~m}$ Date Described 23 APR 74 by GROUNTAN

Physiographic location Lowest Put Landward Slope of E Basin - CARIACO TRENCH

Lithologic

0 Log

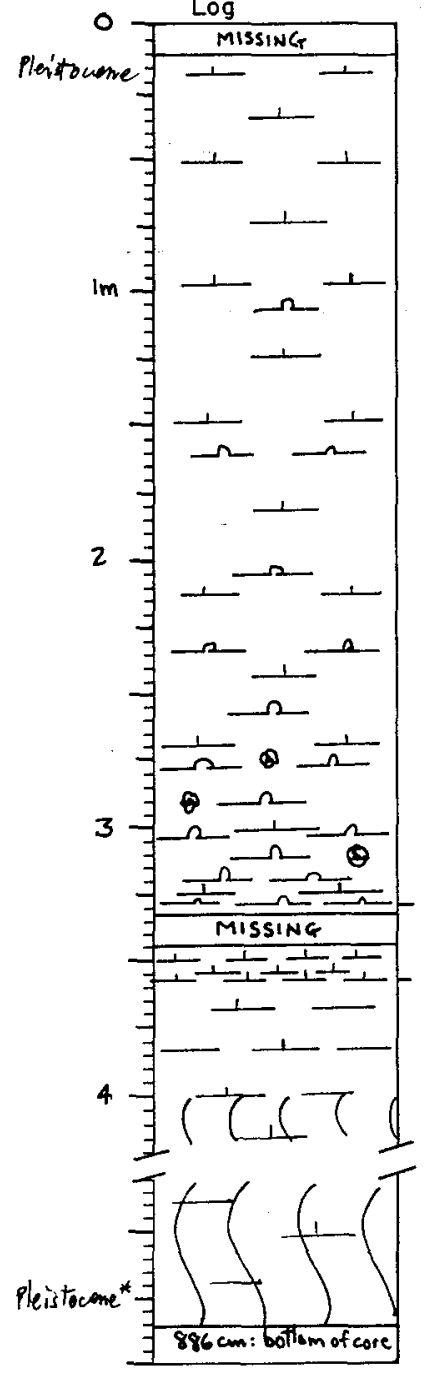

0107

SMEAR SLIDE DESCRIPTIONS - W.H.O.I. SEDIMENT CORES

Ship: Atlantis

Expedition 240

Leg No.

MISSING

CALC OOZE GRADES TO SILIC CAIC OOZE

$5 \mathrm{Y} 4 / 2$ olive gray

ttles (pyrite?)

Lams below $100 \mathrm{~cm}$, dk olive gray layering

at bottom of unit.

330-333

CAIC OOZE

333-345

MISSINC

CAIC OOZE

$5 \mathrm{Y} 5 / 1$ gray

firm moist lutite

appears to be continuation of above unit, considerable white surficialdiscoloration (secondary?). gypsum crystals $358-400$

HLY CALC CLAY

$2.5 \mathrm{Y} 5 / 4$ 1t olive brow $\mathrm{n}$

moist firm lutite, homogeneous

hard to say where flow in begins; hly irregular olive,

chalky inclusion with dk gray pyriteurich sed of thent also, 400-886

FLOW IN

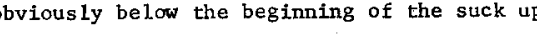

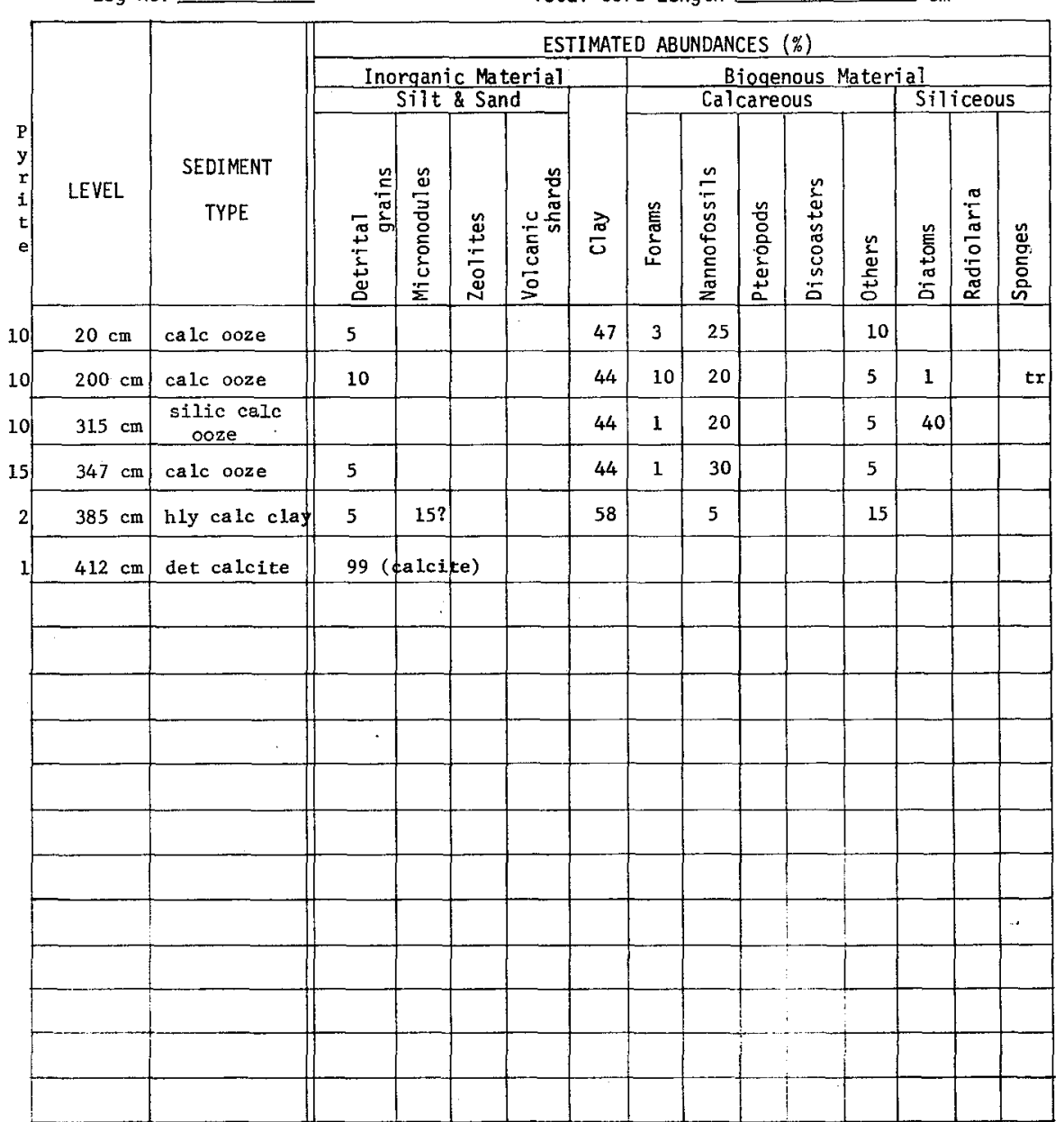

Core No. 21PC

Station No. 21

Total Core Length 886 
0108

VISUAL CORE DESCRIPTION Ship A Cruise 240 Leg___ Sta. 22 Core No. 22 PC Total Length $580 \mathrm{~cm}$. Lat. $10^{\circ} 34.0 \mathrm{~N}$ Long. $64^{\circ} 41.8^{\prime} \mathrm{w}$ Depth $1281 \mathrm{mGat}$

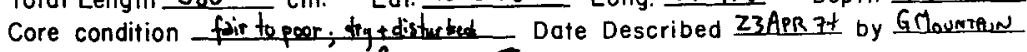
Physiographic location Carafo Trewat

Lithologic

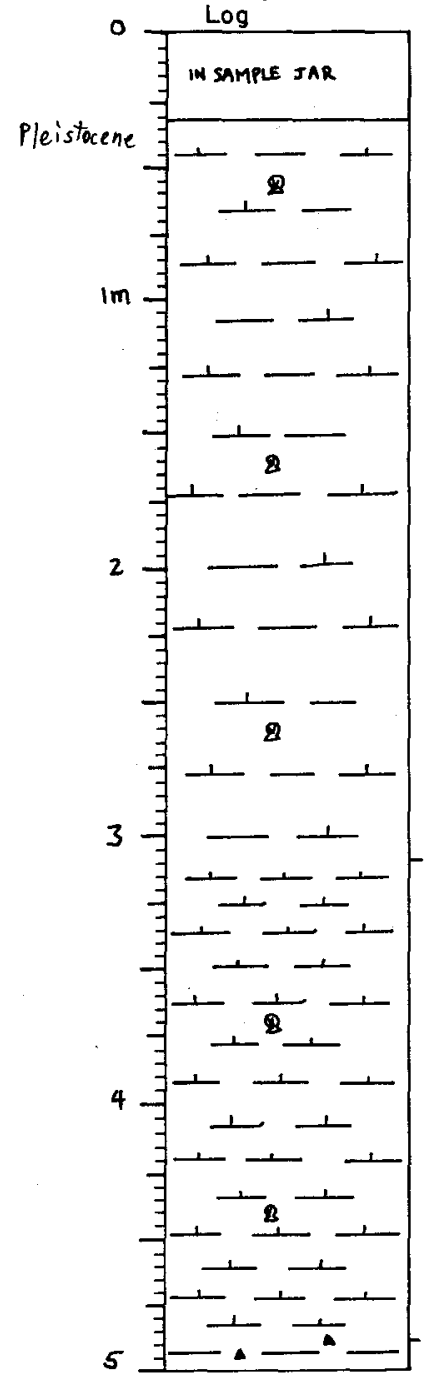

core v. dry and fragmented; was stored without liner, mea$32-310$

HY $3 / 1,6 / 2 \mathrm{v}$. dk gray. gray grades to $1 \mathrm{t}$ olive gray white irregular inclusions, probably secondar

ard, fragmented lutite, forams common, scattered shell

dk color (only at top of each section) probably due to molsture from oasis, v. fine pale/olive lams visible at but "schistose" texture of core indicates finely lamed sediment throughout; scattered gypsum crystals.

$310-490$ CALC OOZE

$5 \mathrm{Y} 3 / 2 \mathrm{dk}$ olive gray

extensive mottling pale yellow, surficial mottles, (secondary $430-470 \mathrm{~cm}$

firm moist, silty lutite, forams common, with pterepods and gastropod fragments

Which indicate that this $350-400$ all concave upwards 490-525

ust colored fine sand in an irregular inclusion 475-48

UNFOSS CIAY/PYRITE

1OYR $4 / 2 \mathrm{dk}$ grayish brow

white secondary surficial mottlin

firm moist lutite

gray and brown

525-527

UNFOSS CIAY/PYRIT

$2.5 Y 5 / 2$ grayish brown

firm moist silty lutit

5 irregular

$527-530$

UNDO SS CLAY/PYRTTE

$5 \times 5 / 1$ gray

silty lutite

530-532

DETRITAL SILT

$2.5 Y 5 / 4$ it olive brown

dry coarse silt

$532-580$

CAC OOZE

1OYR $5 / 4$ yellowish brown

tann molst latice

layers
0109

VISUAL CORE DESCRIPTION

Ship A Cruise 240 Leg Sta. 22 Core No. $22 \mathrm{PC}$
Detoiled Description

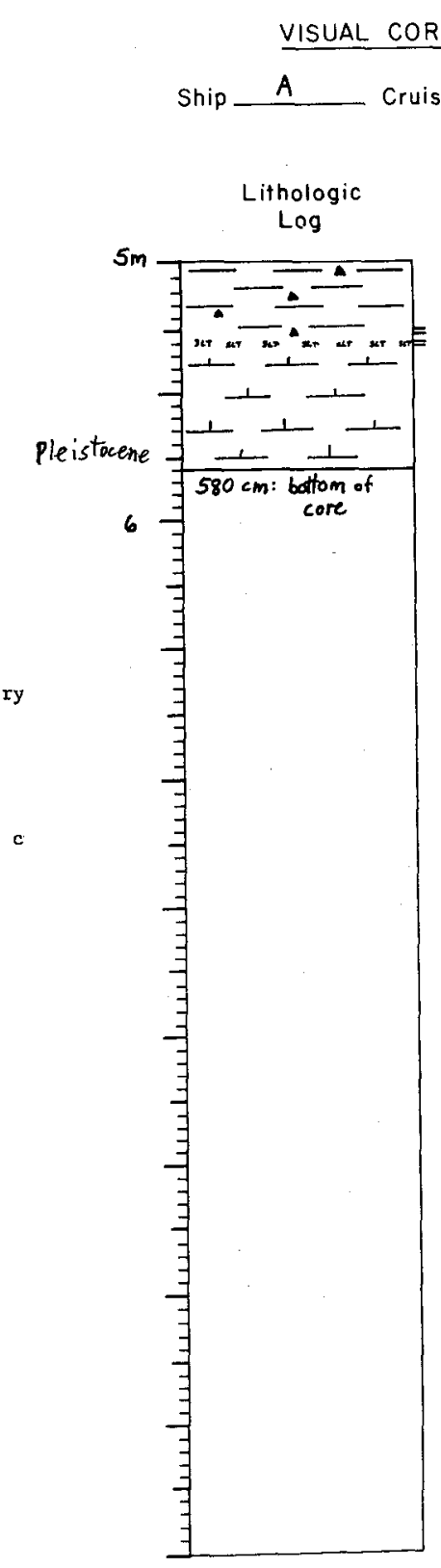

Page 2 of 2

toiled Descrip 
SMEAR SLIDE DESCRIPTIONS - W.H.O.I. SEDIMENT CORES

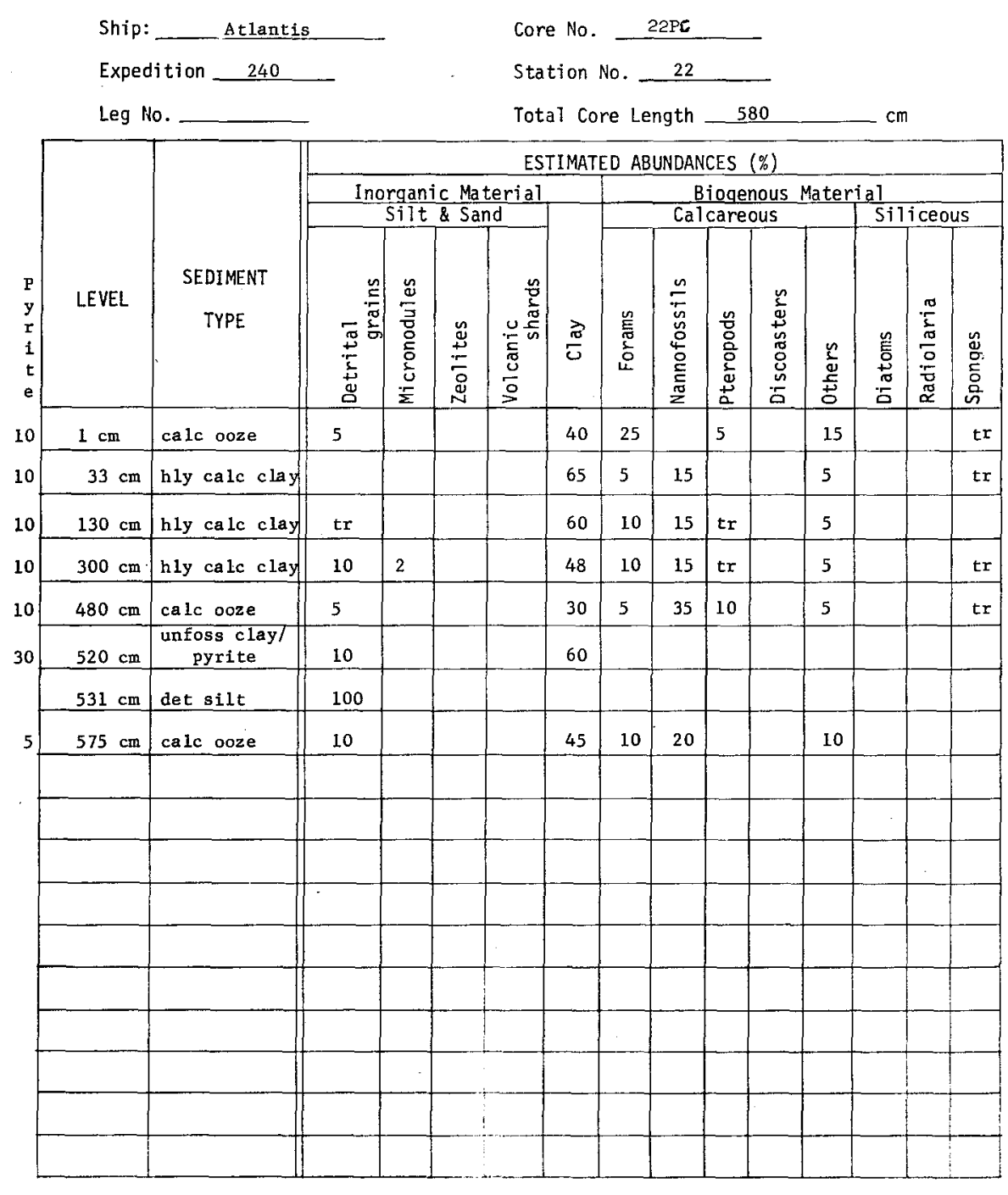

Ship_A _ Cruise 240_Leg_Sta. 23 Core No. 23 PC Total Length $909^{\circ}$ Lat. $10^{\circ} 47.2^{\prime} \mathrm{N}$ Long. $64^{\circ} 396^{\prime} \mathrm{W}^{\mathrm{W}}$ Depth $278 \mathrm{~m} \operatorname{Cos} \mathrm{B}$ Core condition fairtegood Date Described 19APRLL'74 by GMOUWAAIN Physiographic location Maw Basin, Carace Trency

Lithologic

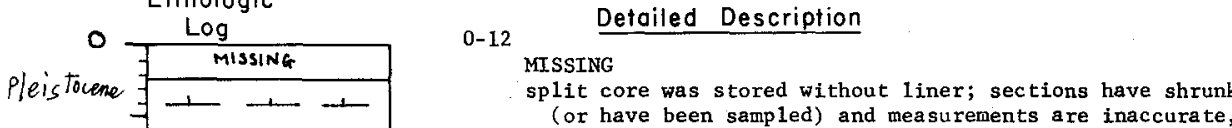

$$
\begin{aligned}
& \text { 12-52 CALC 0OZE } \\
& \begin{array}{l}
\text { CAIC 0OZE } \\
5 Y \text { Y } 5 / 2 \text { olive gray }
\end{array}
\end{aligned}
$$

52-95 fragmen

$$
\text { CALC OOZE }
$$

$5 Y 3 / 2 \mathrm{dk}$ olive gray

hard, fragmented lutite, abnt. forams, scattered shell fragm probably same stratigraphic unit as above and below; color change due to differential drying

95-110

VOID
$110-145$

\section{CALC-SILIC OOZE}

$5 Y 5 / 2$ olive gray

olive brown discoloration

hard, fragmented lutite, ahunt. forams, scattered shell frag-

CALC-SILIC OOZE GRADES TO SILIC-CALC OOZE

fr $3 / 2$ dk olive gray

tilty lutite, pteropods visible crystals concentrated in pockets

230-365

SILIC-CALC OOZ

SY $4 / 2$ olive gray

th $7 / 2$ to 1 t olive gray $6 / 2$, especially intense $250-275 \mathrm{~cm}$

$365-410$

CALC OOZE

$5 Y 5 / 2$ olive gray

dry, fragmented s1 silty lutite, gastropod fragments and whole shells

$\underset{410-420}{\text { samp } 1 \text { ed? }}$ 
0112

VISUAL CORE DESCRIPTION

Ship A Cruise 240 Leg_ Sta. 23 Core No. 23 PC

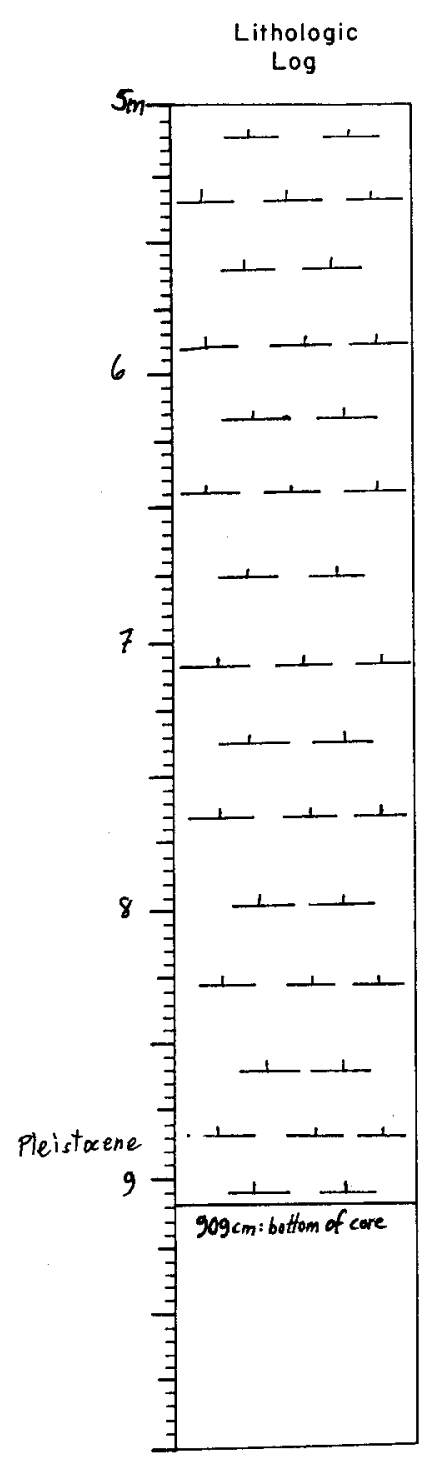

Detailed Description

420-909

CAIC OOZE

ragmented but moist silty lutite, gastropod fragments

several claw fragments (crabs?) $440 \mathrm{~cm}$, white gypsum (?)

crystals concentrated $650-670 \mathrm{~cm}, 730 \mathrm{~cm}$, single v, shar white lam inclined $45^{\circ} 560 \mathrm{~cm}$.

0113

SMEAR SLIDE DESCRIPTIONS - W.H.O.I. SEDIMENT CORES

Page 2 of 2 


\section{U114}

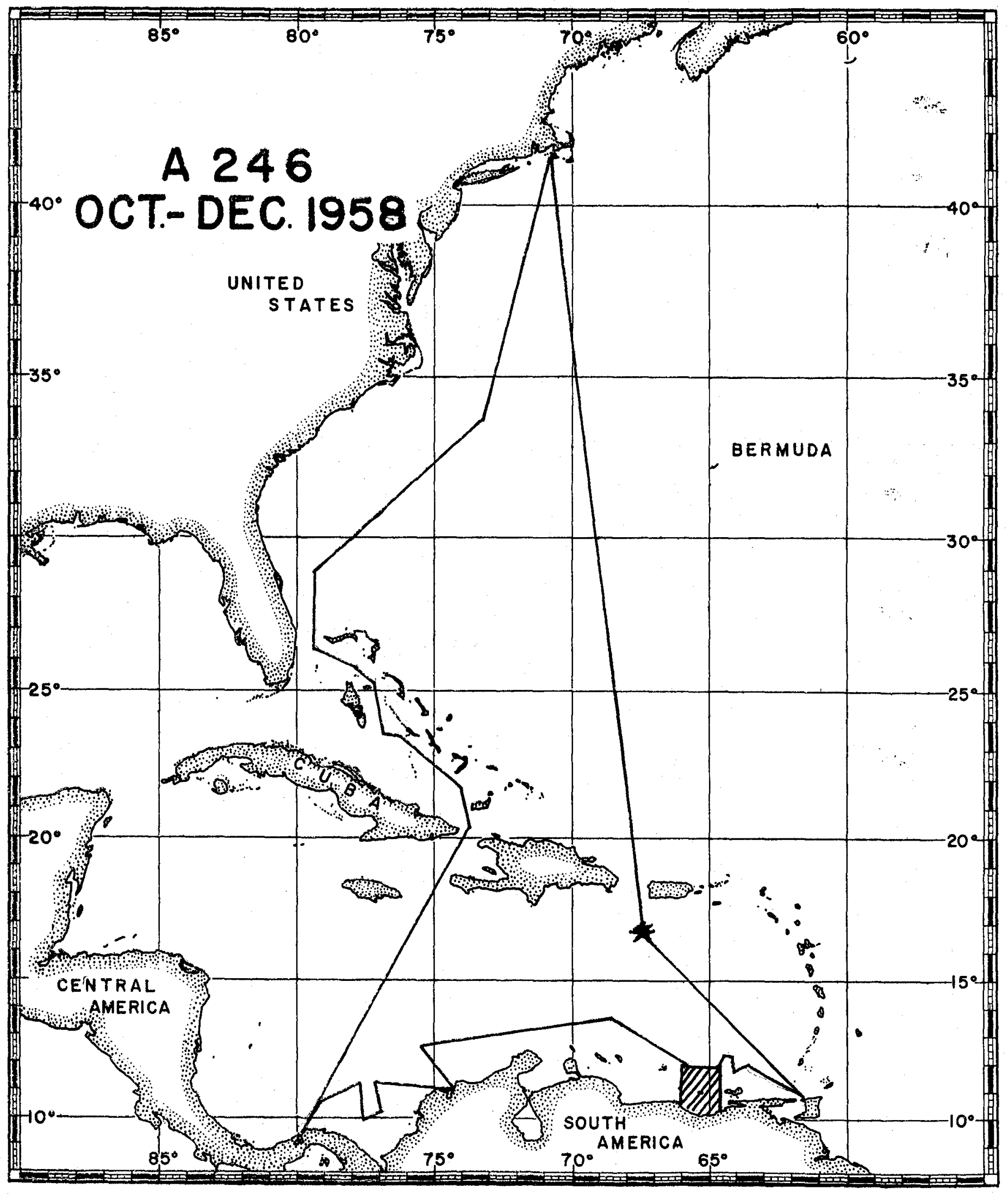




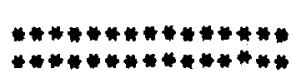

STATION DATA RETRIEYAL" DATE: 06132 MAY 13,175

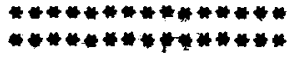

PAGE 1 QF $i$

$*$ WHO I**

MARS: CORE GR LENGTH OREDGE OR ROCK

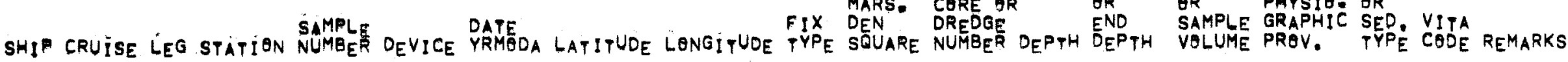

$\begin{array}{lllll}\text { ATL } & 246 & 0 & 0062 & 0000 \\ \text { ATL } & 246 & 0 & 0062 & 0000 \\ \text { ATL } 246 & 0 & 0063 & 0000 \\ \text { ATL } 246 & 0 & 0063 & 0000 \\ \text { ATL } 246 & 0 & 0064 & 0000 \\ \text { ATL } 246 & 0 & 0064 & 0000 \\ \text { ATL } 246 & 0 & 0065 & 0000 \\ \text { ATL } 246 & 0 & 0065 & 0000 \\ \text { ATL } 246 & 0 & 0066 & 0000 \\ \text { ATL } 246 & 0 & 0066 & 0000 \\ \text { ATL } 246 & 0 & 0068 & 0000 \\ \text { ATL } 246 & 0 & 0068 & 0000 \\ \text { ATL } 246 & 0 & 0069 & 0000 \\ \text { ATL } 246 & 0 & 0069 & 0000 \\ \text { ATL } 246 & 0 & 0020 & 0000 \\ \text { ATL } 246 & 0 & 0070 & 0000 \\ \text { ATL } 246 & 0 & 0071 & 0000 \\ \text { ATL } 246 & 0 & 0214 & 0000 \\ \text { ATL } 246 & 0 & 0215 & 0000 \\ \text { ATL } 246 & 0 & 0216 & 0000 \\ \text { ATL } 246 & 0 & 0217 & 0000 \\ \text { ATL } 246 & 246 & 0 & 0218 & 0000 \\ \text { ATL } 246 & 0 & 0219 & 0000\end{array}$

15
26
15
26
15
26
15
26
15
26
15
26
15
26
15
26
26
26
26
26
26
26
26

\begin{tabular}{|c|c|c|}
\hline $\begin{array}{lll}5811 & 2 \\
5811 & 2 \\
5811 & 2 \\
5811 & 2 \\
5811 & \\
5811 & 4 \\
5811 & 5 \\
5811 & 5 \\
5811 & 4 \\
5811 & 4 \\
5811 & 5 \\
5811 & 5 \\
5811 & 5 \\
5811 & 5 \\
581 & 6 \\
5811 & 6 \\
5811 & 6 \\
581120 & \\
581121 \\
581121 \\
581121\end{array}$ & 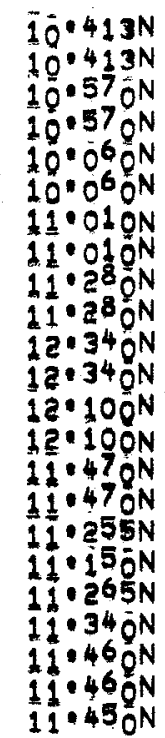 & $\begin{array}{l}64 \cdot 400 \mathrm{~W} \\
64 \cdot 400 \mathrm{~W} \\
64 \cdot 385 \mathrm{~W} \\
64 \cdot 385 \mathrm{~W} \\
67 \cdot 190 \mathrm{~W} \\
67 \cdot 190 \mathrm{~W} \\
67 \cdot 160 \mathrm{~W} \\
67 \cdot 160 \mathrm{~W} \\
67 \cdot 130 \mathrm{~W} \\
67 \cdot 130 \mathrm{~W} \\
68 \cdot 29 \mathrm{~W} \\
68 \cdot 290 \mathrm{~W} \\
68 \cdot 290 \mathrm{~W} \\
68 \cdot 290 \mathrm{~W} \\
68 \cdot 300 \mathrm{~W} \\
68 \cdot 300 \mathrm{~W} \\
68 \cdot 300 \mathrm{~W} \\
71 \cdot 350 \mathrm{~W} \\
71 \cdot 365 \mathrm{~W} \\
71 \cdot 355 \mathrm{~W} \\
71 \cdot 3 \\
71 \cdot 130 \mathrm{~W} \\
70.050 \mathrm{~W} \\
70 \cdot 490 \mathrm{~W}\end{array}$ \\
\hline
\end{tabular}

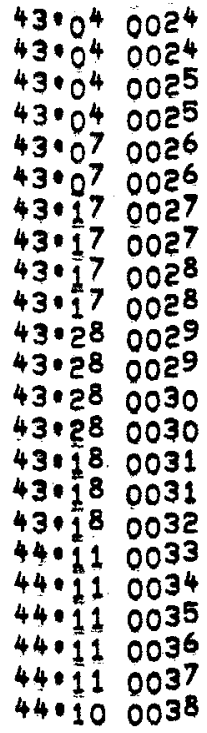

$\begin{array}{rrr}342: & 653: & 0000 \\ 342: & 0: & 0061 \\ 338: & 1027: & 0000 \\ 338: & 0: & 0088 \\ 737: & 582: & 0000 \\ 737: & 0: & 0084 \\ 897: & 614: & 0000 \\ 897: & 0: & 0087 \\ 1935: & 923: & 0008 \\ 1935: & 0: & 0093 \\ 3210: & 694: & 0000 \\ 3210: & 0: & 0091 \\ 1499: & 512: & 0000 \\ 1499: & 0: & 0095 \\ 18030 & 108: & 0000 \\ 1803: & 0: & 0088 \\ 347: & 0: & 0071 \\ 18: & 0: & 0110 \\ 24: & 0: & 0063 \\ 22: & 0: & 0028 \\ 18: & 0: & 0087 \\ 16: & 0: & 0087 \\ 37: & 0 \cdot & 0055\end{array}$

$\begin{array}{lll}19 & 3479 & 0 \\ 19 & 3555 & 0 \\ 19 & 4029 & 0 \\ 19 & 4029 & 0 \\ 19 & 4859 & 0 \\ 19 & 4859 & 0 \\ 19 & 3859 & 0 \\ 19 & 3859 & 0 \\ 19 & 4355 & 0 \\ 19 & 4355 & 0 \\ 19 & 3355 & 0 \\ 19 & 3355 & 0 \\ 19 & 3355 & 0 \\ 19 & 3569 & 0 \\ 19 & 3359 & 0 \\ 19 & 359 & 0 \\ 19 & 425 & 0 \\ 19 & 4425 \\ 19 & 2255 & 0 \\ 19 & 2255 & 0 \\ 19 & 8855 & 0 \\ 19 & 1155 & 0 \\ 19 & 1155 & 0 \\ 19 & 2255 & 0\end{array}$

THERE WERE 23 ITEMS THAT MET YOUR REQUIREMENTS.

THANK YOU FER USING PREGRAM MUDDIE.

"STOP* THAT is aLL FOR NGW RUN 
0116

VISUAL CORE DESCRIPTION

Poge 1 of 2

Ship A Cruise 246 Leg_Sta. 62 Core No. 24 PC Total Length $653 \mathrm{~cm}$. Lat. $10^{\circ} 41.3^{\prime} \mathrm{N}$ Long. $64^{\circ} 40.0^{\circ} \mathrm{W}$ Depth 342 CORR Core condition vecy doy; paor Date Described $4 / 13 / 74$ by $B$ MeGiec Physiographic location Caciaca. Treoch

Lithologic

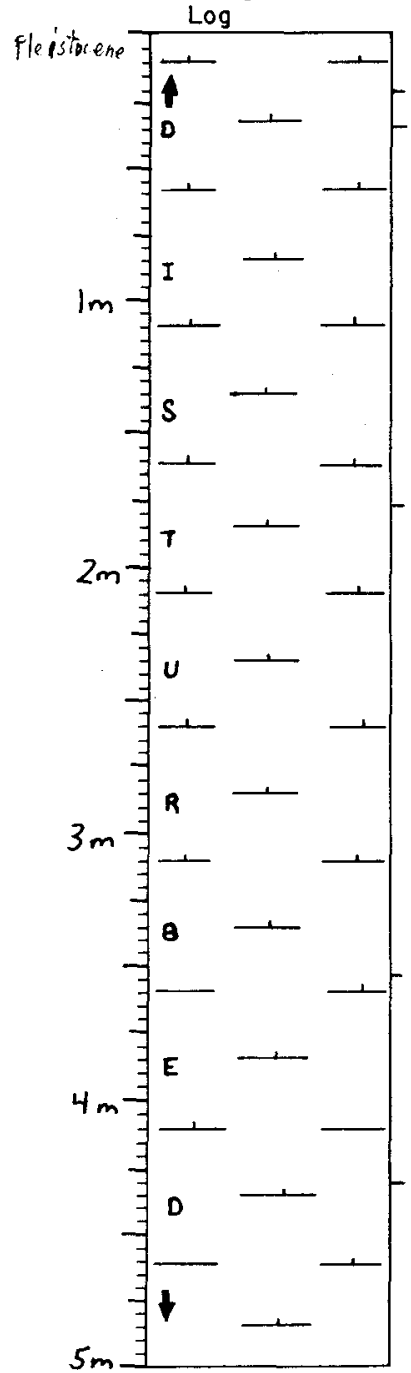

\section{Detailed Description}

Note; This entire core is disturbed, v. dry and crumbly throughout. Description is nearly meaningless due to the poorcondition. Obvious stratigraphic units colncide preduct by differing amounts of desiccation from section to section. conversely, true stratigraphic changes may be masked by the core's present crumbly condition. The core description done shortly after the core was taken is inoluded here also to better Indicate the

"The sediment of the upper $73 \mathrm{~cm}$ of core 24 is composed primarily of a hly fossiliferous, finely lamed, grayis olive silty clay. Between 73 and 77 can there 15 a fossiliferous sandy silt. Below $77 \mathrm{~cm}$ the sediment is essentially untformly homogeneous, moderately fossiliferous, dk grayish olive silty clay. This extends to the bottom of the core at approximately $691 \mathrm{~cm}$. This is probably a much better description of the sediment in core $24 \mathrm{PC}$. One posstble explanation of the difference in total length is that the original descut $40 \mathrm{~cm}$ consice suggested the upper $40 \mathrm{~cm}$ of the piston core were $10 \mathrm{st}$ The core has since been remeasured, considering the

$0-20$ op of the core to be at $0 \mathrm{~cm}$

CALC OOZE

$5 \mathrm{Y} 3 / 1 \mathrm{v}$. dk gray

silty lutilejcore too dry and disturbed to permit visua approximation of foram content $0-5 \mathrm{~cm}$ missing; entire core disturbed, crumbly

20-34

CALC OOZE

$5 Y 4 / 3,7 / 2$ olive and 1 t gray

silty lutite

thin, sl concave up lams of the 2 color

34-177

177

CALC DOZE

$5 \mathrm{Y} 4 / 2$ ollive gray

especially crumbly 90-160

$\mathrm{s}$ bottom of section?

177-353

CALC OOZE

5Y $3 / 2$ dk olive gray

silty lutite

somewhat moister than most of core
$S$ bottom of section 5
0117.

VISUAL CORE DESCRIPTION

Poge 2 of 2

Ship_A Cruise 246 Leg__ Sta. 62 Core No. $24 P C$

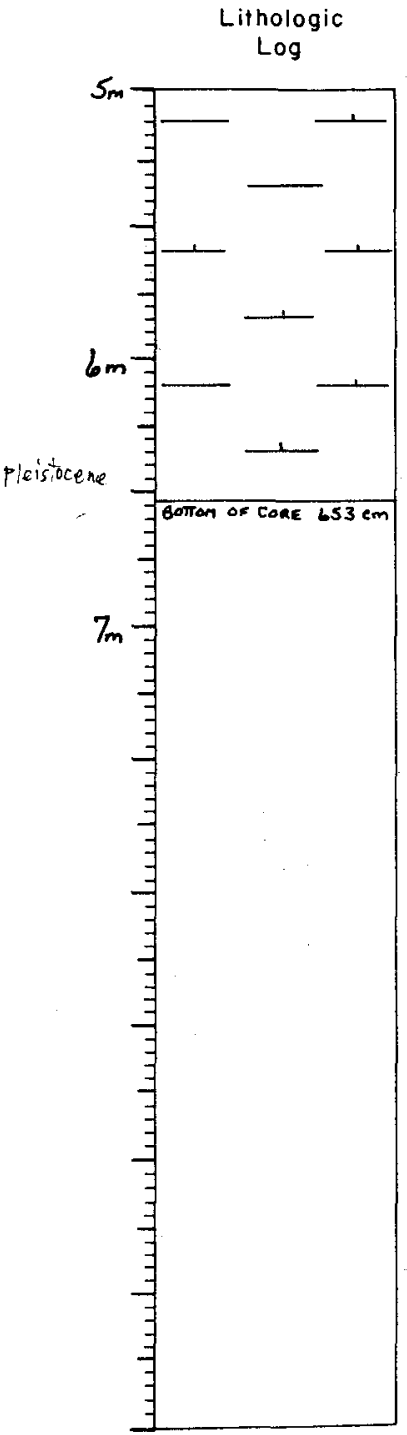

Detailed Description

353-430

HLY CALC CLAY

Y $4 / 2$ olive gray

v. dry, crumbly

bottom of section

430-653

HLY CALC CLAY

$5 \mathrm{Y} 3 / 2 \mathrm{dk}$ olive gray

silty lutite

section 2 relatively moist, section 3 crumbly, section $1 \mathrm{v}$. do

is real, it indicates suck up in bottom $90 \mathrm{~cm}$.

end of core 
0118

SMEAR SLIDE DESCRIPTIONS - W.H.O.I. SEDIMENT CORES

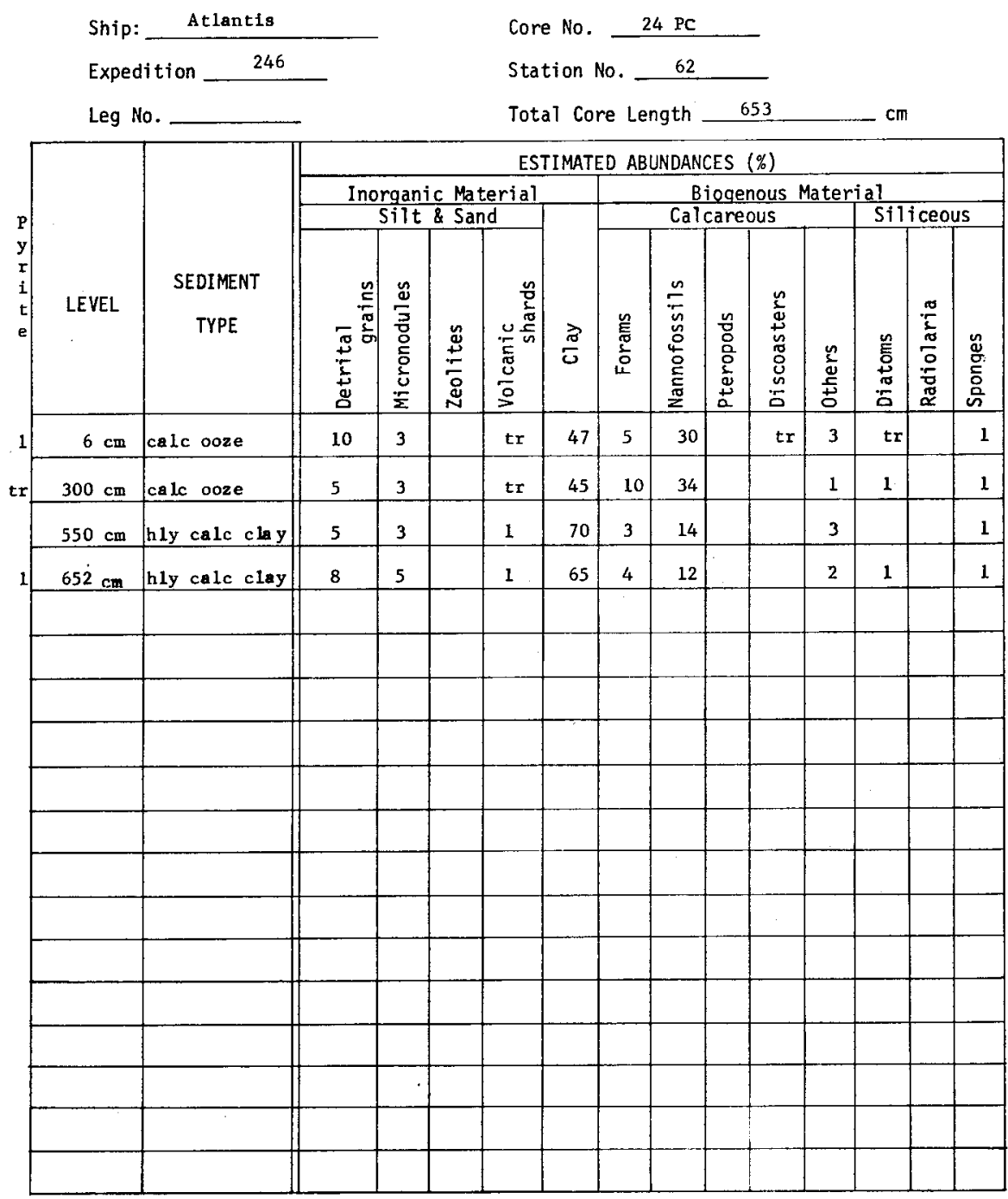

0119

VISUAL CORE DESCRIPTION

Poge 1 of 1

Ship_A Cruise 246 Leg_Sta. 62 Core No. $24 \mathrm{PG}$ Totol Length $61 \mathrm{~cm}$. Lat. $10^{\circ} 41.3^{\prime} \mathrm{N}$ Long. $64^{\circ} 40.0^{\circ} \mathrm{W}$ Depth $342 \mathrm{mcorR}$ Core condition dry fair Date Described 6 MAY 74 by Gllountain Core condition dry, far Date CARIACO TRENCH

Lithologic

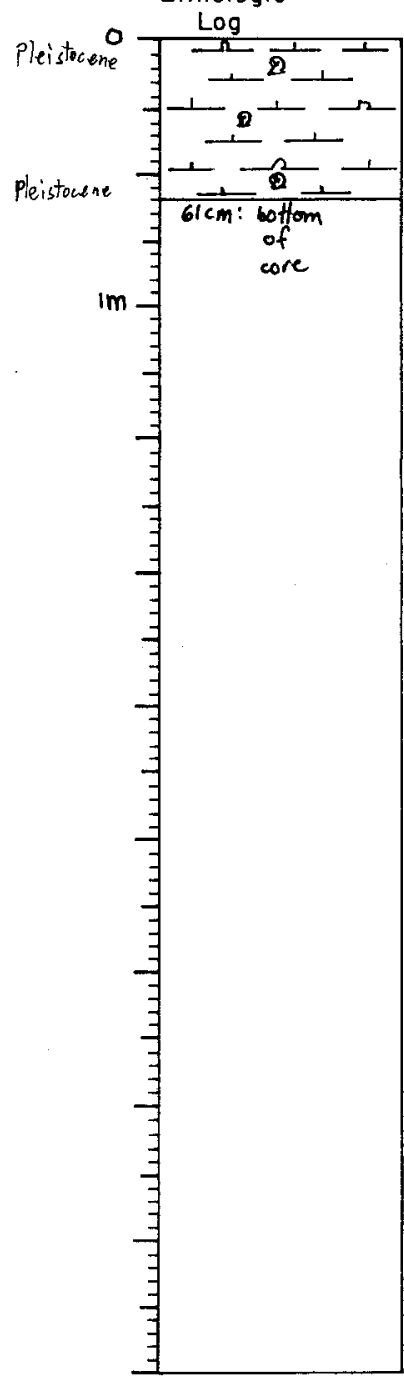

Description

CALC SILIC OOZE

$5 Y 6 / 211$ olfve gray

dry, hard, and crumbling with mollusc shells and fragments

and forams scattered throughout

apparently all one unit

end of core 
$01: 20$

SMEAR SLISE DESCRIPTIONi -

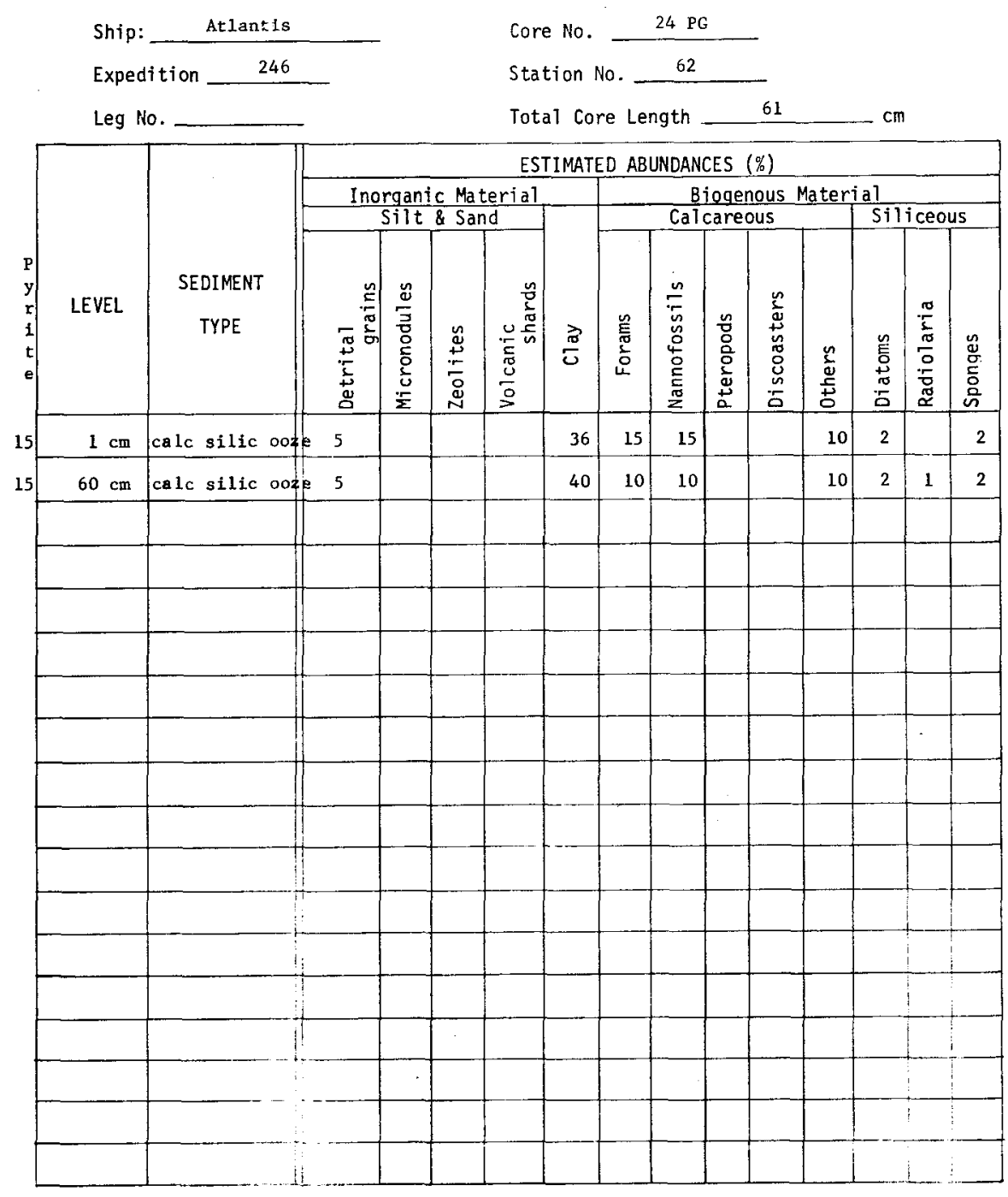

0121

VISUAL CORE DESCRIPTION

Poge 1 of 1

Ship Cruise 246 Leg_Sta. 63 Core No. 25 PC Total Length $1027 \mathrm{~cm}$. Lat. $10^{\circ} 57.0^{\circ} \mathrm{N}$ Long. $64^{\circ} 38.5^{\prime} \mathrm{W}$ Depth $338 \mathrm{cose} . \mathrm{m}$ Core condition very dryi peor Date Described $4 / 44 / 14$ by $R M^{2} G$,er Physiographic locotion Cariaco Trench

Lithologic

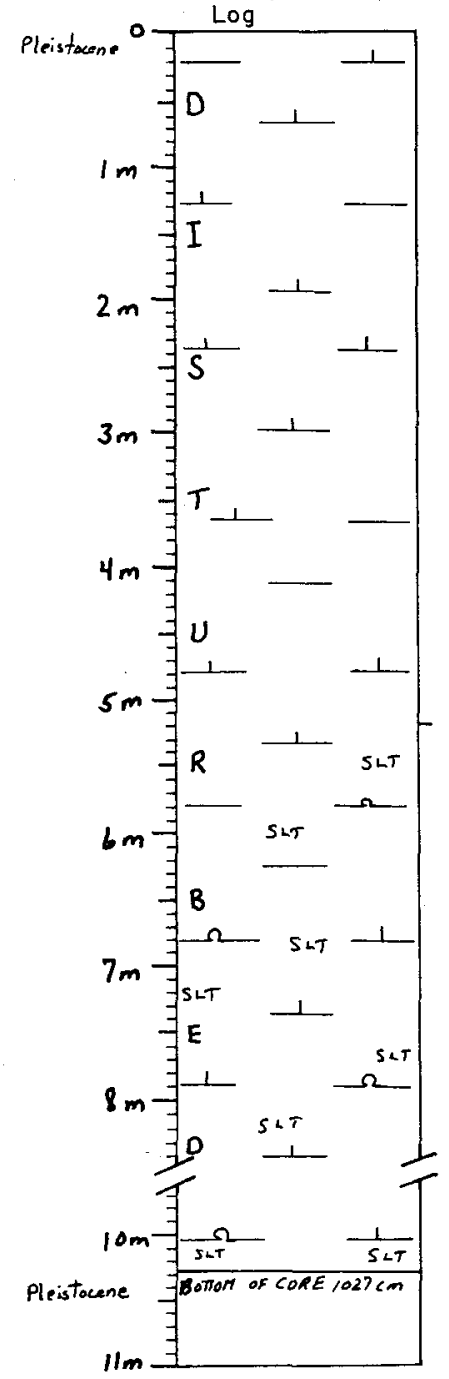

Detailed Description

Note; This entire core is $v$. dry, hard, and cracked. It is disturbed throughout. Original stratigraphic units are probably concealed by the poor conditionand in othe places it is broken by by the poor corly horizional

th a luminum foil $0-520$

HLY CAIC CIAY

$5 Y 5 / 2,4 / 2$ olive gray

v. silty lutite, core too dry and disturbed to permit

v. crumbly throughout, appears to have a lot of organic matter; $0-25$

HLY CALC SILIC CLAY WITH DET TO CALC SILIC OOZE WITH DET $5 \mathrm{Y} 6 / 2$ 1t olive gray

silty lutite

many desiccation cracks, appears to have some organic

matter; faint, $1 \mathrm{t}$ and $\mathrm{dk} 2 \mathrm{~mm}$ thick, lams appear in part of this unit

end of core 
$012 !$

SMEAR SLIDE DESCRIPTIONS - W.H.O.I. SEDIMENT S.ES

Ship: Atlantis

Expedition 246

Leg No.

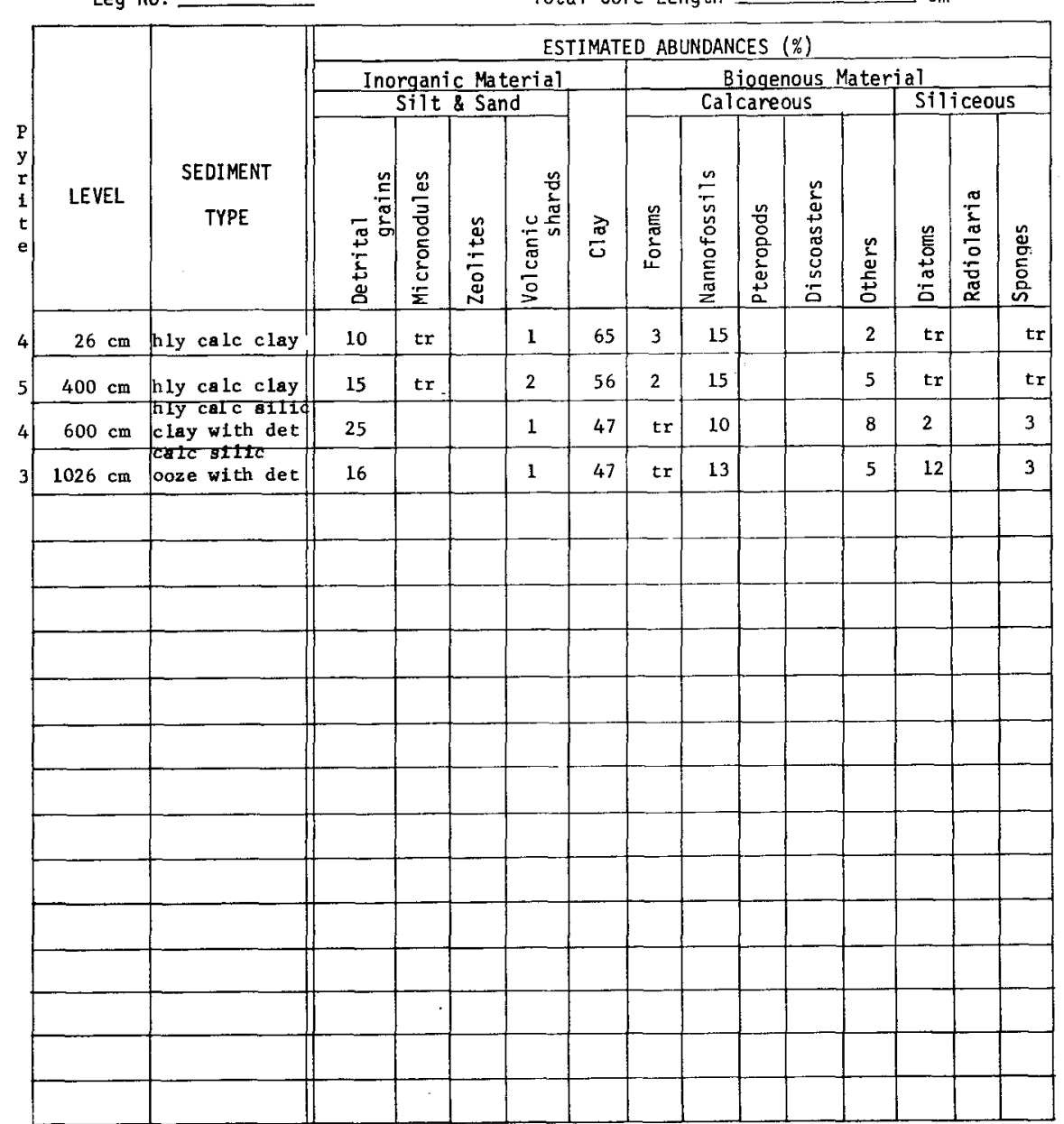

0123

VISUAL CORE DESCRIPTION

Page 1 of 1

Ship _A Cruise 246 Leg___ Sta. 63 Core No. $25 P G$ Total Length $88 \mathrm{~cm}$. Lat. $10^{\circ} 57.0^{\circ} \mathrm{N}$ Long. $64^{\circ} 38.5^{\prime} \mathrm{W}$. Depth $338 \mathrm{mcarR}$ Core dry crumbled. poos Physiogrophic locotion CARIACO TRENCH

Lithologic

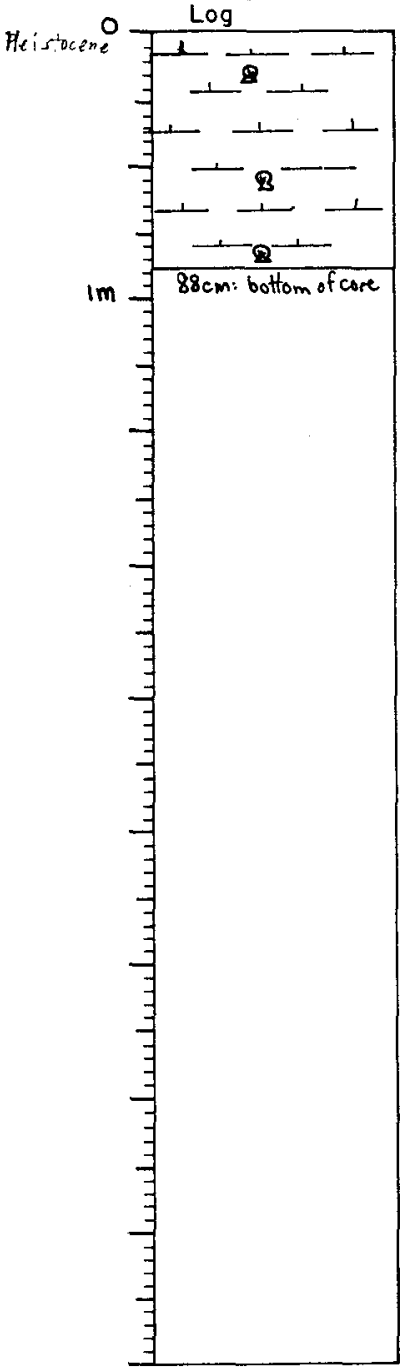

\section{Detailed Description}

$0-88$ CALC OOZE

Y $5 / 2$ olive gray

(a) mollusc fragments and at tered throughout

considerably less than $88 \mathrm{~cm}$ of sediment now remaining end of core 


\section{4}

SMEAR SLIDE DESCRIPTIONS - W.H.O.I. SEDIMENT CORES

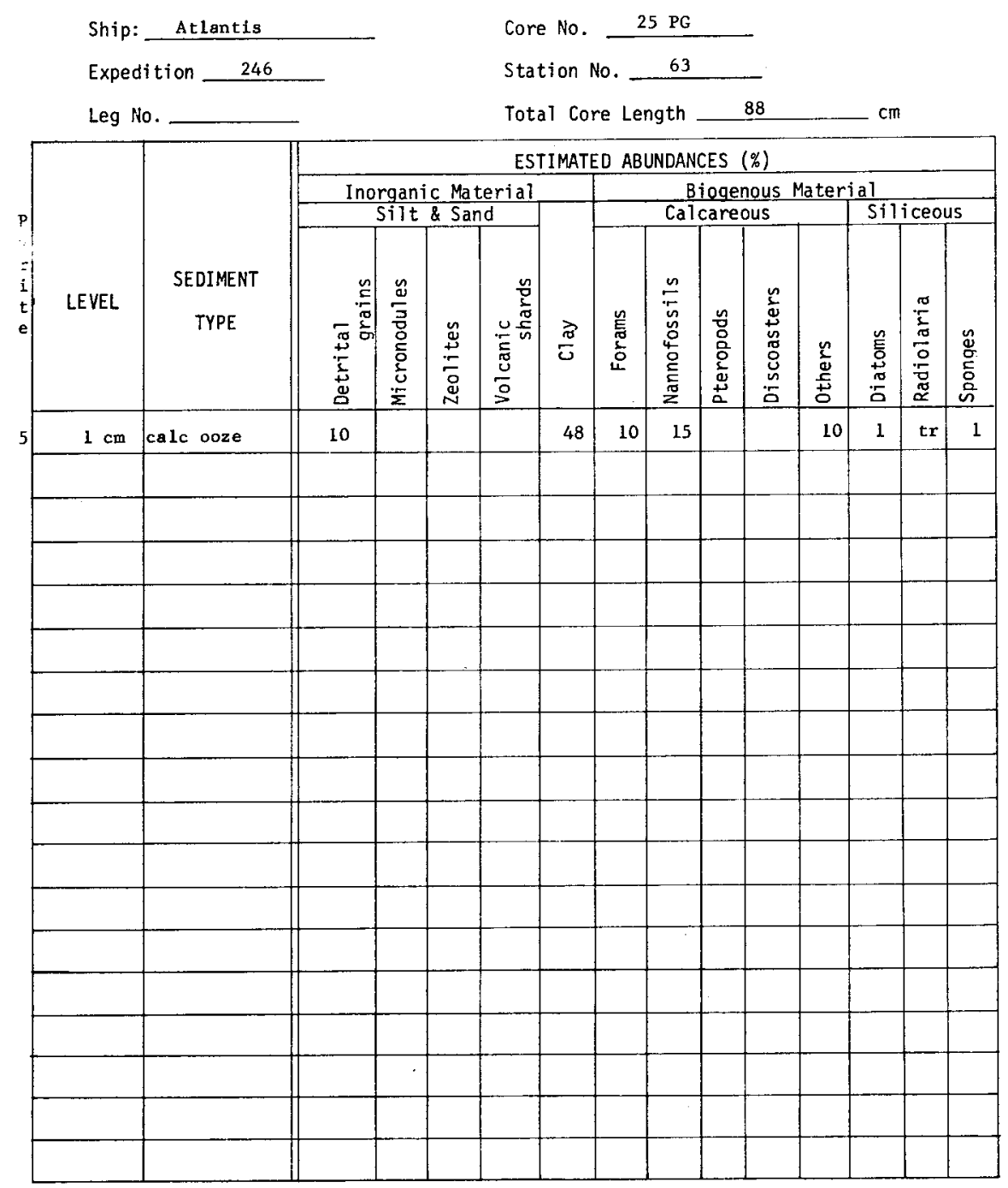

0125

VISUAL CORE DESCRIPTION

Page _ L of _ 1

Ship A Cruise 246 Leg_ Sto. 64 Core No. 26 PC Total Length $582 \mathrm{~cm}$. Lat. $10^{\circ} 06.0^{\circ} \mathrm{N}$ Long. $67^{\circ} 19^{\prime} \mathrm{W}$ Depth 737 Core $\mathrm{m}$ Core condition very dry; poer Date Described $4 / 13 / 24$ by $B$ MeGicr

Lithologic

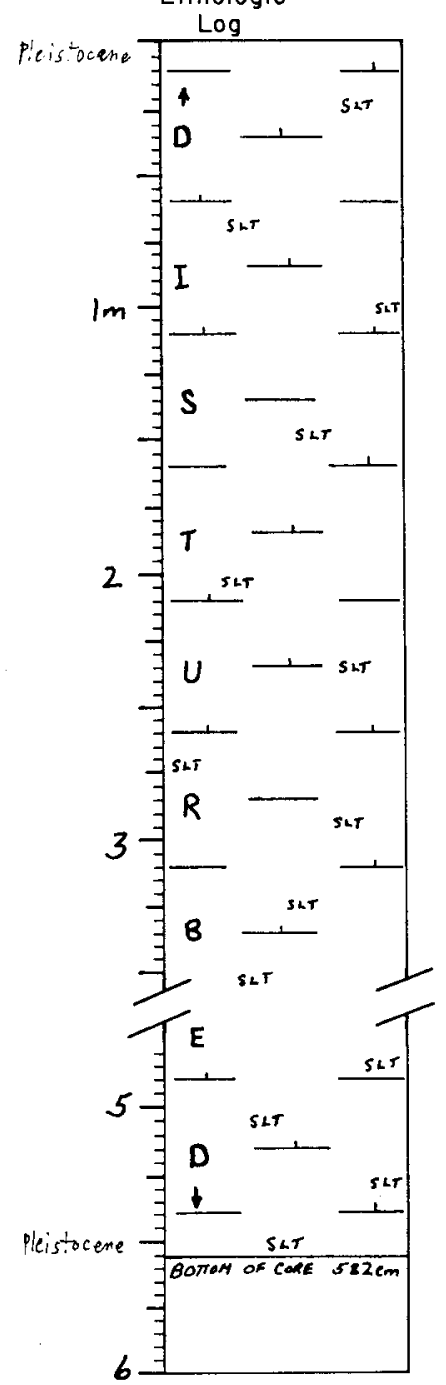

\section{Detciled Description}

Note; This entire core is $\mathrm{v}$. dry and hard. There are man desiccation cracks, all approximately on horizontal

bedding planes. The core is somewhat disturbed throughout, and in places pleces of aluminum foll are stuek in the sediment. The core condition prohibits recognition $0-582$

of true stratigraphic

(1t olive gray to olive gray

silty lutite, core too dry to permit visual approximation f foram content.

core v. dry, disturbed throughout

nd of core
HLY CATC CIAY WTTY DET 
0126

SMEAR SLIDE DESCRIPTIONS - W.H.0.I. SEDIMENT CORES

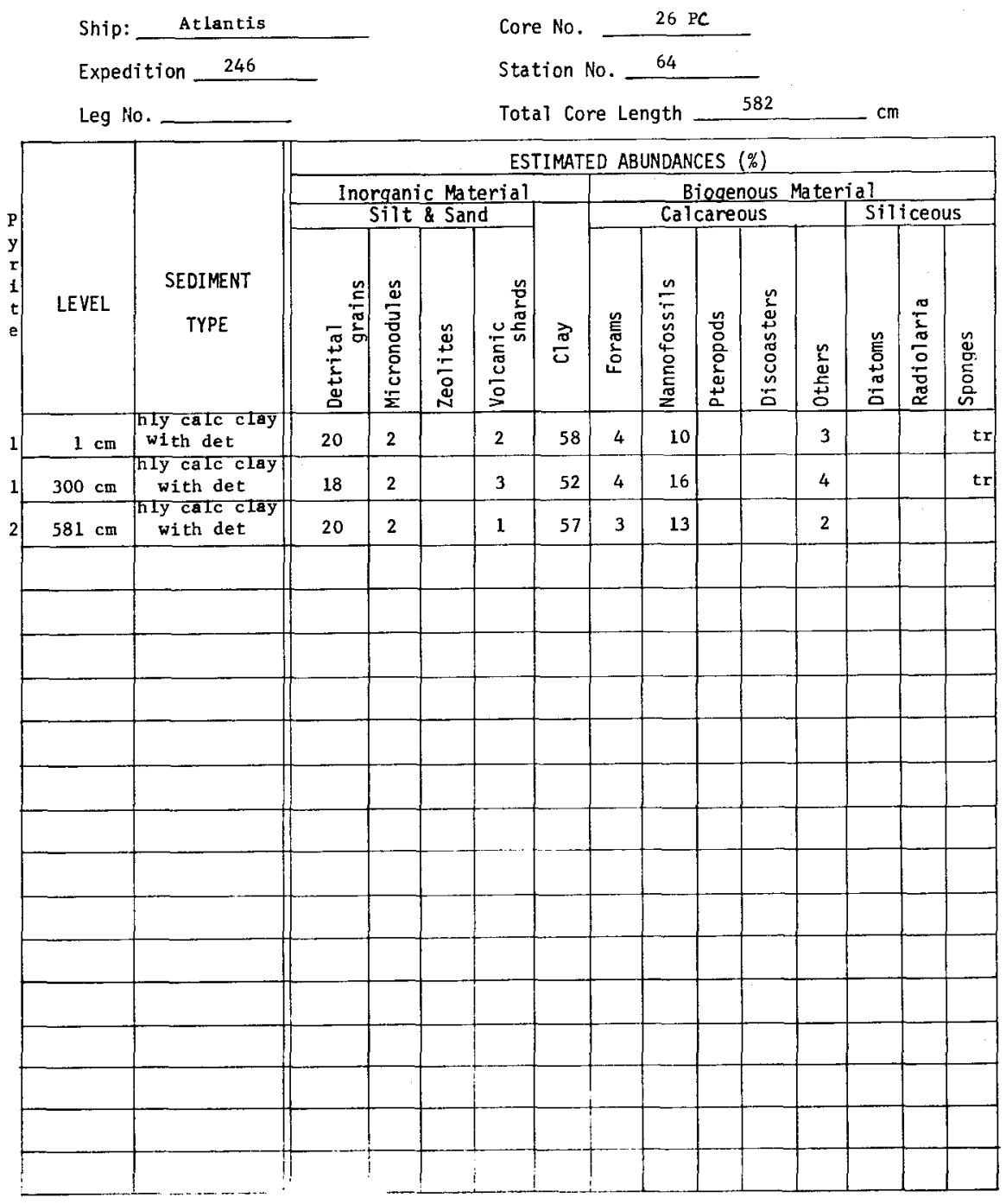

0127

VISUAL CORE DESCRIPTION

Page 1 of 1 Ship A Cruise 246 Leg__ Sto. 64_Core No. 26 PG Total Length $84 \mathrm{~cm}$. Lat. $10^{\circ} \mathrm{O} 60^{\prime} \mathrm{N}$ Long. $67^{\circ} \mathrm{lg} \mathrm{W}$ Depth $737 \mathrm{mcoRR}$. Core condition dry; good Date Described 6MAY, 74 by GHOUNTAN Physiographic location CARIBBEAN, JUST OFF VENEZUELAN COAST Lithologic

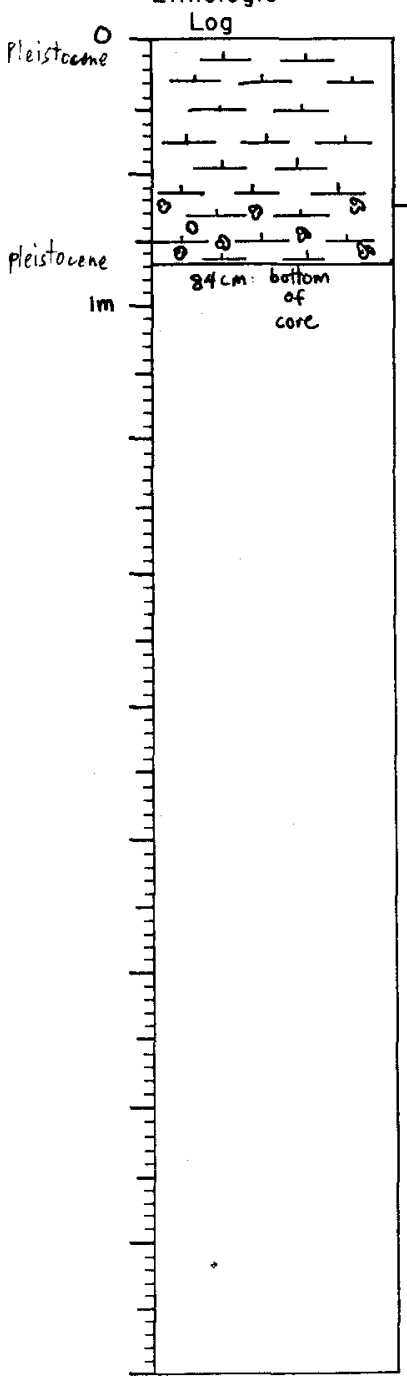

\section{Detailed Description}

$0-63$ CAIC OOZE CALC OOZE

dry,

core dry and cracked, has shrunk about 10-15 cm

63-84

CALC OOZE

5 Y $6 / 2$ 1t olive gray

rrowing throughout color of above uni

compact

end of core 
SMEAF SLIDE DESCRIFTIONS - W.H.O.I. STDIMENT CORES

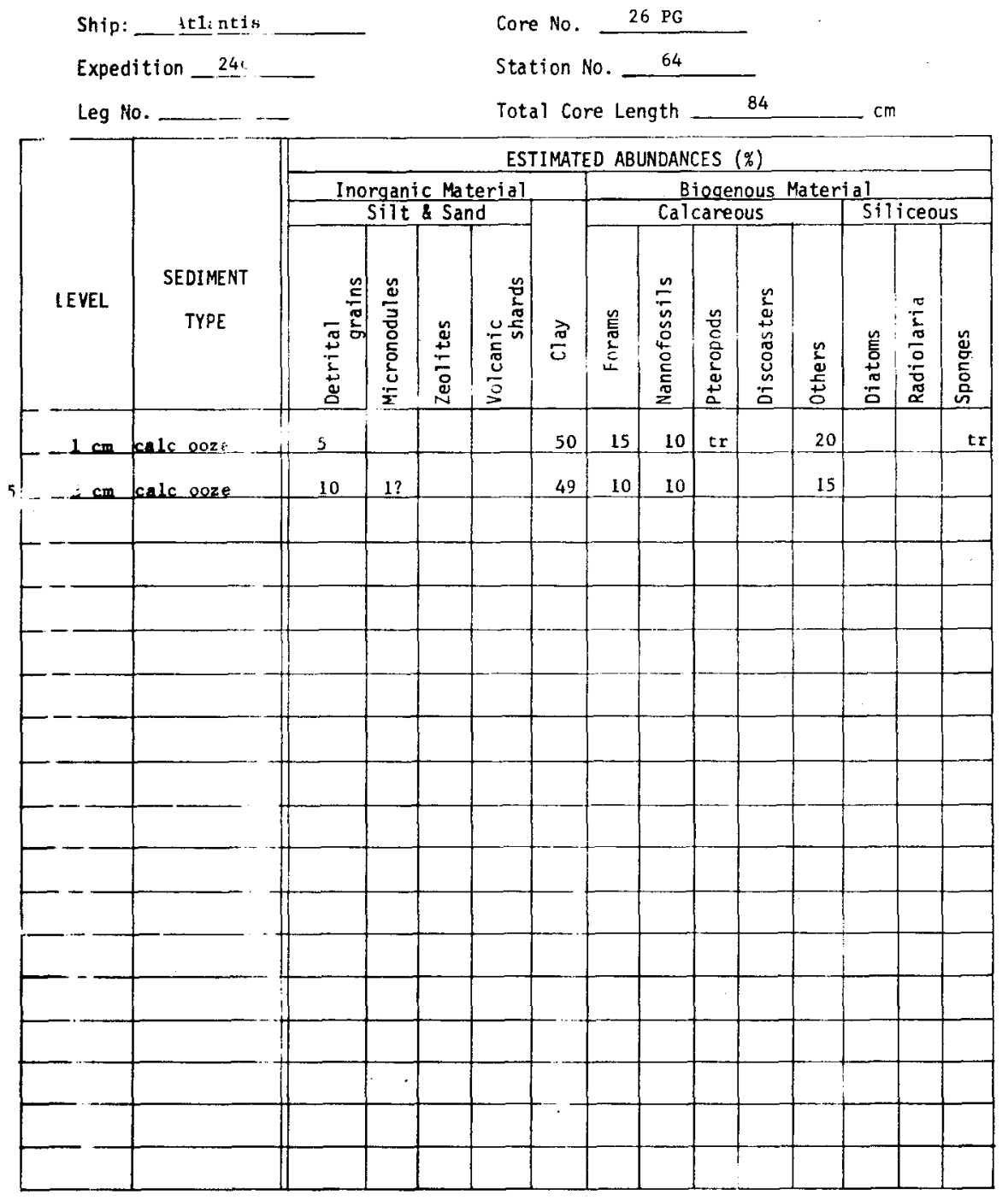

VISUAL CORE DESCRIPTION

Poge 1 of 1

Ship A Cruise $346 \quad$ Leg 45 Core No $27 P C$ Total Length $6.614 \mathrm{~cm}$. Lat. $11^{\circ}$ ol. $N$ Lang. $47^{\circ} 16^{\circ} \mathrm{W}$ Depth $897 \mathrm{CoPR}$ Core condition Very dry; poor__ Dote Described $4 / 15 / 74$ by B MeGire

Physiographic location Cacibbean Sea, jist off Venezuelan coast Lithologic

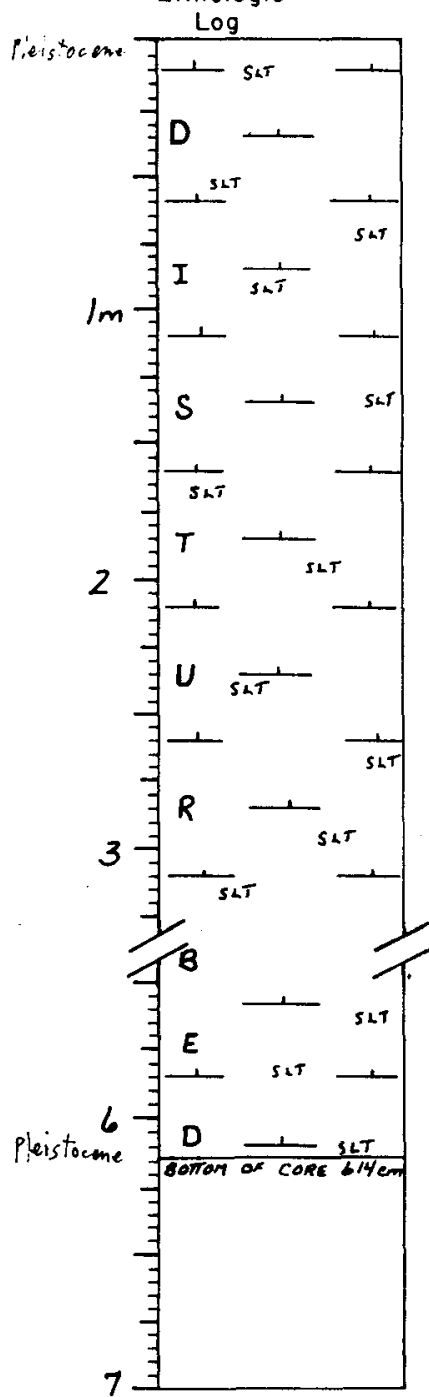

Detailed Description

Note. This enttre core is v dry, hard, cracked, and disturbed. Many desiccation cracks are nearly horizontal. but many others are randomly oriented. Blts of a luminum foll contaminate the core surface in places. The poor core condition seriously hinders any visual description. True stratigraphic units and contacts are probably obscured by the ary, crumbled hature of the was fresh, so that no comparison can now be made. $0-614$

CALC OOZE WITH DET of foram content

entire core somewhat disturbed

$S$

end of core 
SMEAR SLIDE DESCRIPTIONS - «.H.O.I. SEDIMENT CORES

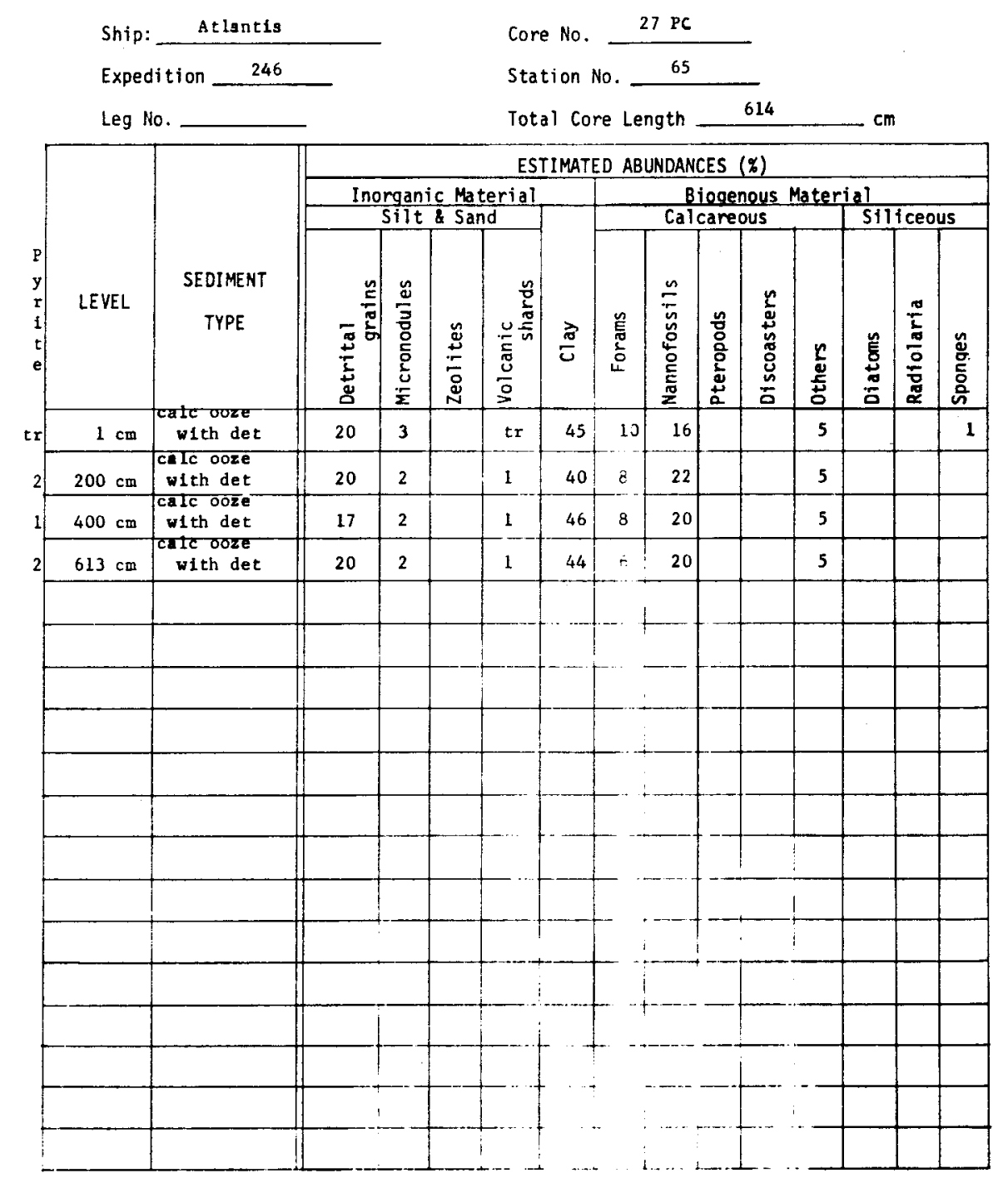

Ship A Cruise 246 L L S Sta. 65 _ Core No. $27 \mathrm{PS}$ Total Length $87 \mathrm{~cm}$. Lat. $110^{101 . N}$ Long. $67^{\circ} 16^{\prime} \mathrm{W}$ Depth $897 \mathrm{mba}$ Core condition dry. Dat Described Ghay 74 by Gllowitaw Physiogrophic locotion GeaseAs, JUST Off VENEZUELAN COAST

Lithologic

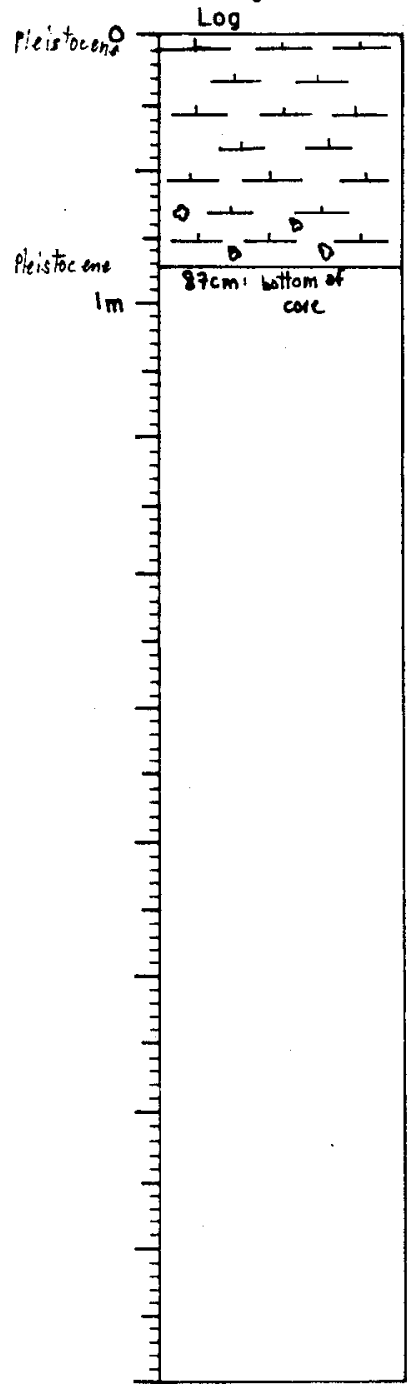

CALC DOZE $5 Y 7 / 2$ 1t grey

faint dk gray mottling, fine scale mottling towards

hard, dry crumbled lutite, forame common, scattered molluse fregents

end of core
Detailed Description 
0132

SMEAR SLIDE DESCRIPTIONS - W.H.O.I. SEDIMENT CORES

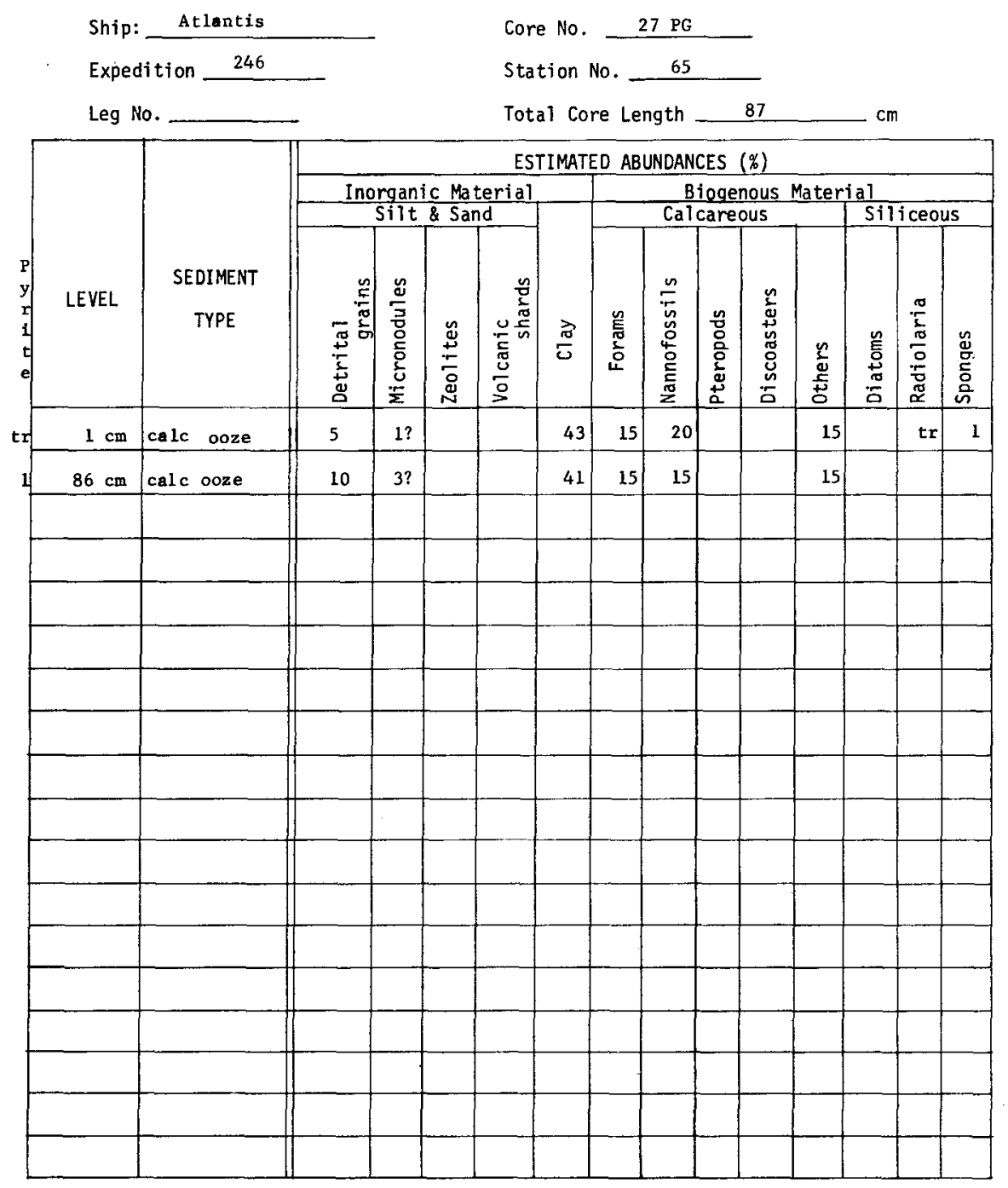

0133

VISUAL CORE DESCRIPTION

Page 1 of 1

Ship A Cruise 24h Leg Sto b6 Core No. $28 \mathrm{PC}$

Total Length $923 \mathrm{~cm}$. Lat. $11^{\circ} 28^{\prime} \mathrm{N}$ Long. $67^{\circ} 13^{\prime} \mathrm{W}$ Depth $1935 \mathrm{coBP} \mathrm{m}$

Core condition vecy diy; poor Date Described $4 / 16 / 74$ by B Me Girr

Physiographic location Cacibbean Sea, off Venezvela

Lithologic

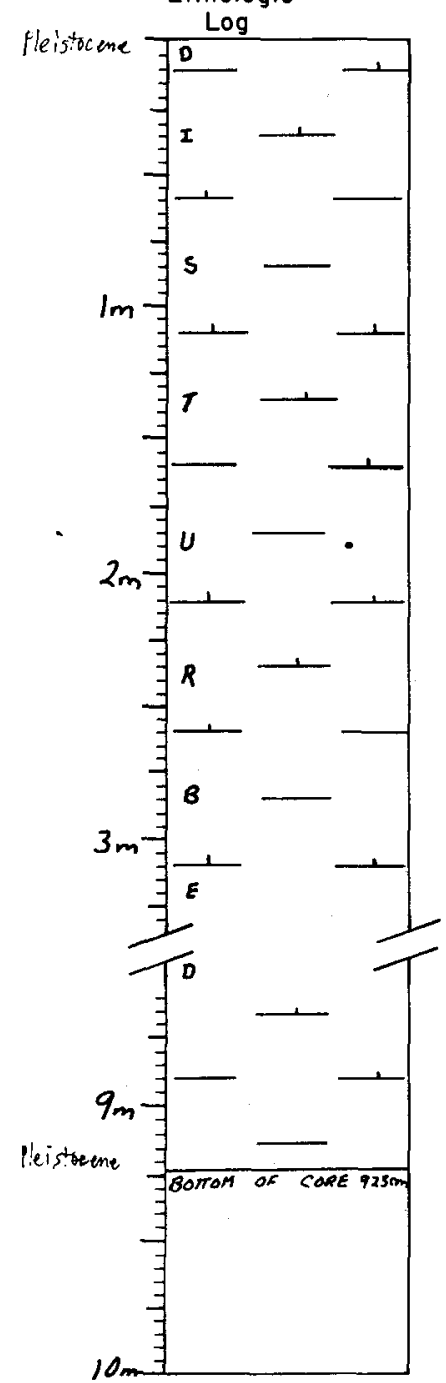

Note; This entire core is $v$. dry, hard, and most of the core is disturbed. In many places the core is crumbly. The poor condition probably masks true stratigraphic units and contacts. There is no record of description $0-923$ when the core was fresh.

HLY CALC CLAY OR CALC OOZE

$5 Y 6 / 2$ lt olive gray

is too dry to permit visual

of foram conten

$42 \mathrm{~cm} v$. crumbly, disturbed; $420-550 \mathrm{~cm}$, fairly solid: $550-820 \mathrm{~cm}$,desiccation cracks mostly horizontal, core mostly undisturbed; 820-923 core mostly solld-random drying cracks; $725 \mathrm{~cm}, 1 \mathrm{~cm}$ diameter iron oxide nodule; a few scattered shell fragnents

end of core 
0134

SMEAR SLIDE DESCRIPTIONS - W.H.O.I. SEDIMENT CORES

Ship: Atlantis

Expedition 246

Leg No.

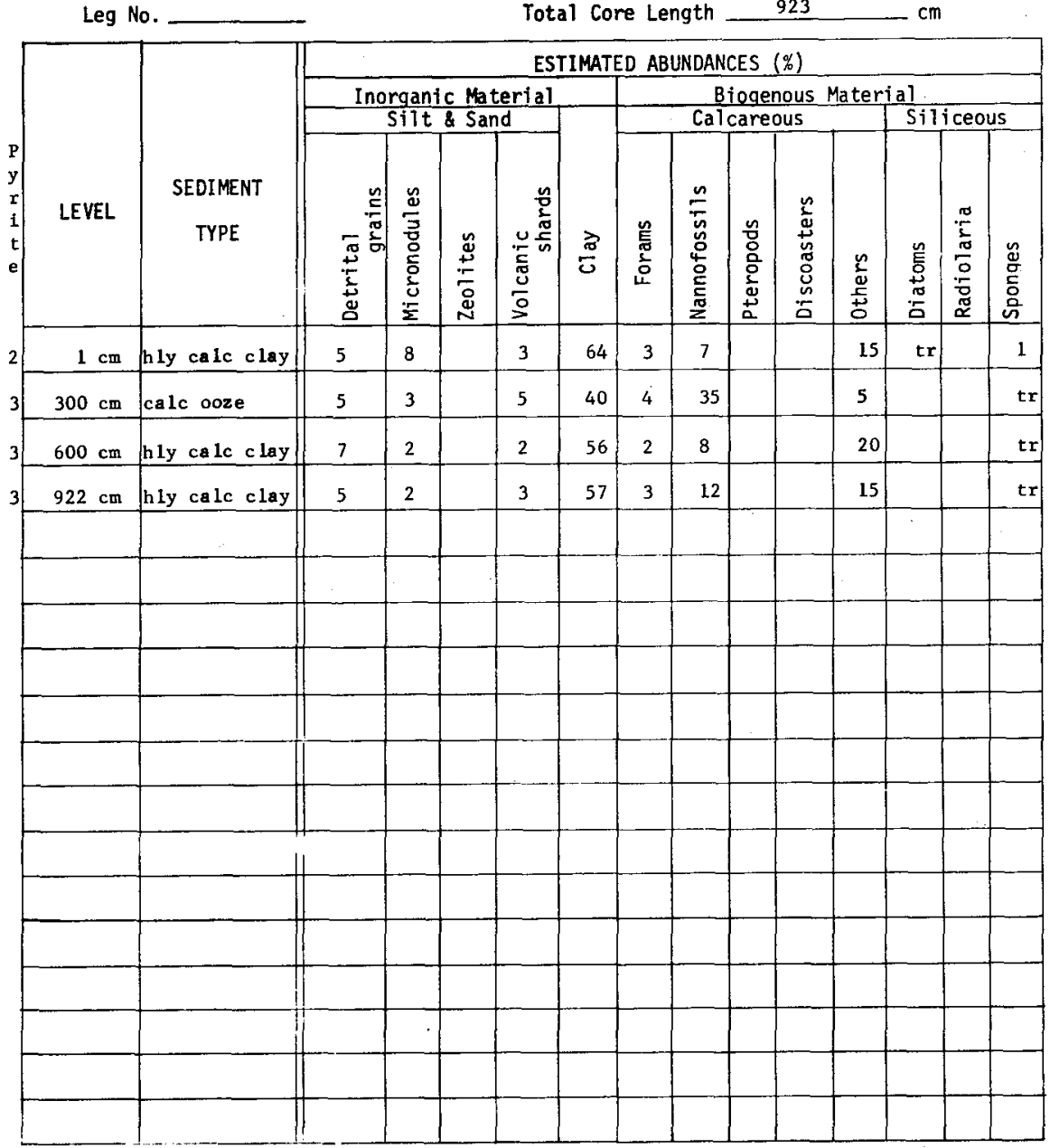

0135

VISUAL CORE DESCRIPTION
Page 1 of 1

Ship A 246 Leg 66 Core No. 28 PG

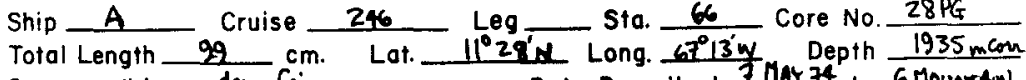
Core condition dry; fair Dote Described IMaY 74 by G Gountain Physiographic location CARIBBEAN, OFF VENEZUELA

Lithologic

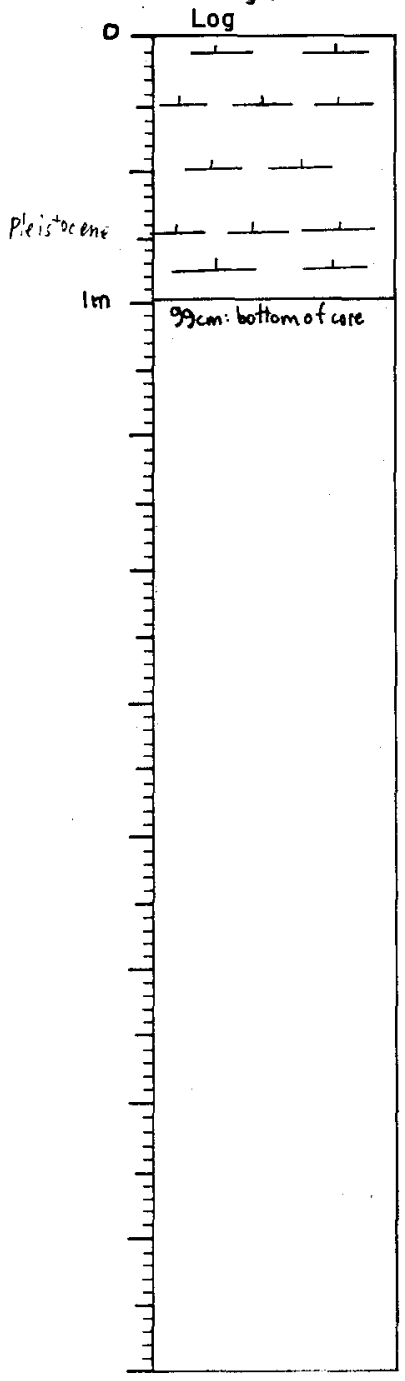

\section{Detailed Description}

0-99

CALC OOZE

dry, crumbled lutite, forams common

core dry, crumbled, shrunk; what remains appears to be quite homogeneous 
0138

SMEAR SLIDE DESCRIPTIONS - W.H.O.I. SEDIMENT CORES

Ship: Atlantis

Expedition 246

Leg No. .

\begin{tabular}{|c|c|c|c|c|c|c|c|c|c|c|c|c|c|c|}
\hline \multirow[b]{4}{*}{ LEVEL } & \multirow[b]{4}{*}{$\begin{array}{c}\text { SEDIMENT } \\
\text { TYPE }\end{array}$} & \multicolumn{13}{|c|}{ ESTIMATED ABUNDANCES (\%) } \\
\hline & & \multirow{2}{*}{\multicolumn{4}{|c|}{$\begin{array}{c}\text { Inorganic Material } \\
\text { Silt \& Sand }\end{array}$}} & & \multirow{2}{*}{\multicolumn{5}{|c|}{$\begin{array}{l}\text { Biogenous Mater } \\
\text { Calcareous }\end{array}$}} & \multirow{2}{*}{\multicolumn{3}{|c|}{ al }} \\
\hline & & & & & & & & & & & & & & \\
\hline & & 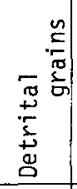 & 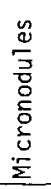 & 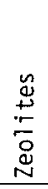 & 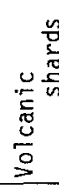 & $\frac{\sqrt{0}}{6}$ & 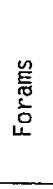 & 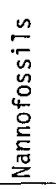 & 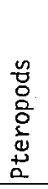 & 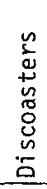 & $\begin{array}{l}n \\
\frac{n}{2} \\
\frac{1}{5} \\
0\end{array}$ & 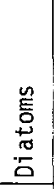 & 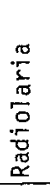 & 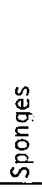 \\
\hline $1 \mathrm{~cm}$ & calc ooze & & 3 & & & 29 & 15 & 45 & & & 5 & $\operatorname{tr}$ & tr & 1 \\
\hline $300 \mathrm{~cm}$ & calc ooz e & & & & & 22 & 25 & 45 & 3 & & 5 & & & \\
\hline $600 \mathrm{~cm}$ & calc ooze & & & & & 38 & 15 & 35 & 5 & & 5 & & & \\
\hline $690 \mathrm{~cm}$ & calc ooze & & & & & 48 & 15 & 30 & & & 5 & & & \\
\hline & & & & & & & & & & & & & & \\
\hline & & & & & & & & & & & & & & \\
\hline & & & & & & & & & & & & & & \\
\hline & & & & & & & & & & & & & & \\
\hline & & & & & & & & & & & & & & \\
\hline & & & & & & & & & & & & & & \\
\hline & & & & & & & & & & & & & & \\
\hline & & & & & & & & & & & & & & \\
\hline & & & & & & & & & & & & & & \\
\hline & & & & & & & & & & & & & & \\
\hline & & & & & & & & & & & & & & \\
\hline & & & & & & & & & & & & & & \\
\hline & & & & & & & & & & & & & & \\
\hline & & & & & & & & & & & & & & \\
\hline
\end{tabular}

Station No. 68
0139

VISUAL CORE DESCRIPTION
Page 1 of 1

Ship A Cruise 246 Leg__ Sto. 68 Core No. 2996 Total Length $91 \quad \mathrm{~cm}$. Lat. $12^{\circ} 34^{\prime} \mathrm{N}$ Long. $68^{\circ} 29^{\prime} \mathrm{w}$ Depth $3210 \mathrm{mCon}$ Core condition dry; good Date Described 7 MAY 74 by G Jounten Core condition Physiographic location Lithologic

Core No. 29 PE

Log CARIBBEAN NW OF BONAIRE ISLAND

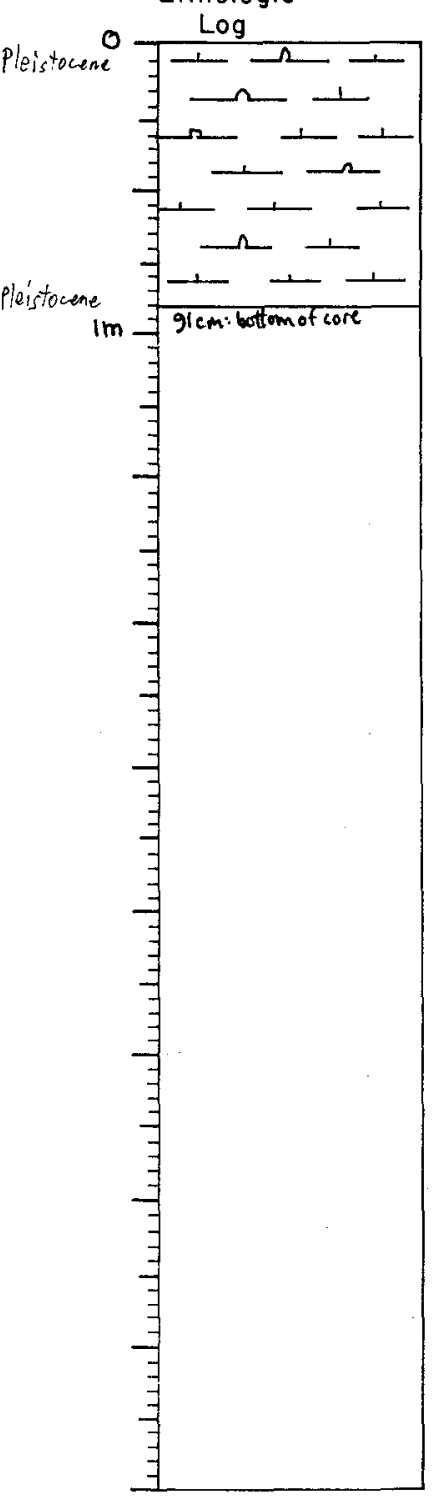

$0-91$ Detailed Description

CALC SILIC OOZE GRADES TO CALC OOZE $2.5 \mathrm{Y} 7 / 2$ 1t gray

dry, crumbled lutite, forams and pteropods common $\mathrm{s}$

end of core 
0136

SMEAR SLIDE DESCRIPTIONS - W.H.O.I. SEDIMENT CORES



0137

VISUAL CORE DESCRIPTION

Page 1 of 1

Ship A Cruise 246 Leg_Sta. 68 Core No. 29 PC

Total Length $694 \mathrm{~cm}$. Lat. $12^{\circ} 34^{\circ} \mathrm{N}$ Long. $68^{\circ} 29^{\prime} \mathrm{W}$ Depth $3210 \mathrm{mCaRR}$.

Core condition dry crumbled poor Dote Described 16 APRLL 74 by GMounTAind

Physiographic location CARIBBEAN, NW OF BONAIRF ISLAND

Lithologic

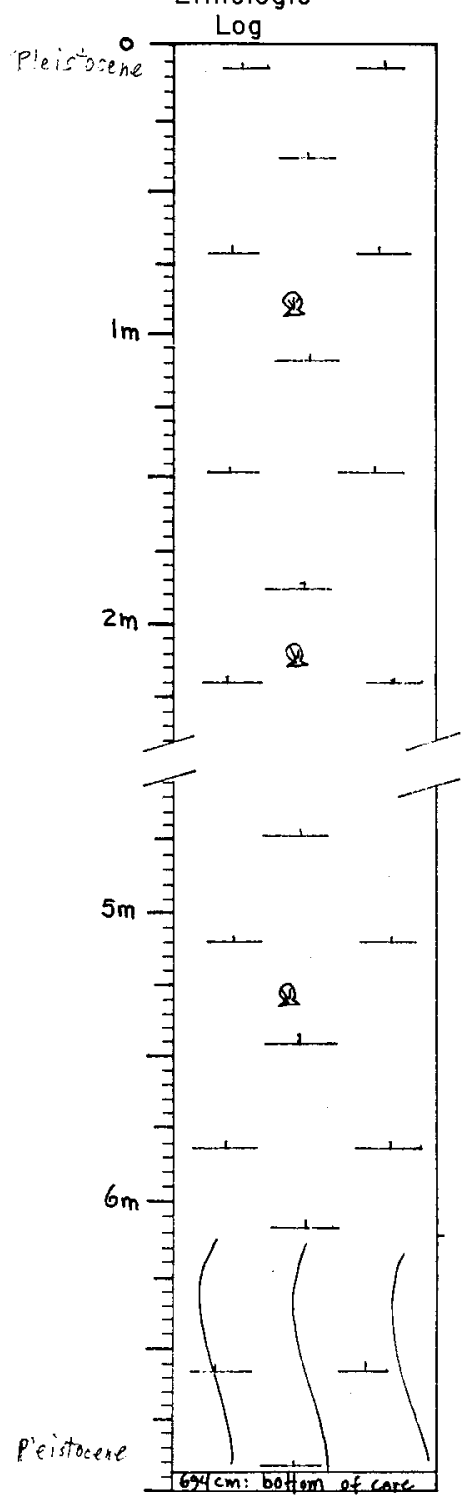


0141

VISUAL CORE DESCRIPTION

Ship A Cruise 246 Leg_ Sta. 69 Core No. 30 PC

Total Length $512 \mathrm{~cm}$. Lat. $12^{\circ} 10.0^{\circ} \mathrm{N}$ Long $6829.0^{\prime} \mathrm{W}$ Depth $1499 \mathrm{mager}$

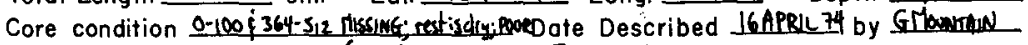

Physiographic location 6 Mires W. Of Bonalies Is A AND

Ship: ___ Atlantis

Expedition 246

Leg No.

\begin{tabular}{|c|c|c|c|c|c|c|c|c|c|c|c|c|c|c|}
\hline \multirow[b]{4}{*}{ LEVEL } & \multirow[b]{4}{*}{$\begin{array}{c}\text { SEDIMENT } \\
\text { TYPE }\end{array}$} & \multicolumn{13}{|c|}{ ESTIMATED ABUNDANCES $(\%)$} \\
\hline & & \multirow{2}{*}{\multicolumn{4}{|c|}{$\frac{\text { Inorganic Material }}{\text { Silt \& Sand }}$}} & \multirow[b]{3}{*}{$\frac{\pi}{\omega}$} & \multirow{2}{*}{\multicolumn{5}{|c|}{$\begin{array}{l}\text { Biogenous Mater } \\
\text { Calcareous }\end{array}$}} & \multirow{2}{*}{\multicolumn{3}{|c|}{$\frac{\text { al }}{\text { Siliceous }}$}} \\
\hline & & & & & & & & & & & & & & \\
\hline & & 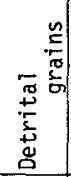 & 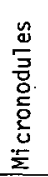 & $\begin{array}{l}\stackrel{\Xi}{ \pm} \\
\frac{\mathbb{N}}{N} \\
N\end{array}$ & 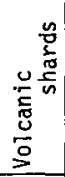 & & 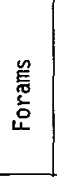 & 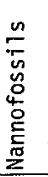 & 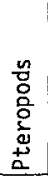 & 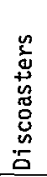 & 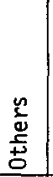 & 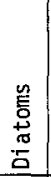 & 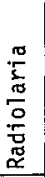 & 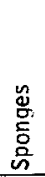 \\
\hline $1 \mathrm{~cm}$ & calc silic 002 & $\operatorname{tr}$ & $3 ?$ & & & 39 & 15 & 15 & & & 15 & 10 & 1 & 2 \\
\hline $90 \mathrm{~cm}$ & calc ooze & $\operatorname{tr}$ & $3 ?$ & & & 37 & 15 & 30 & 5 & & 10 & $\mathrm{tr}$ & & \\
\hline & & & & & & & & & & & & & & \\
\hline & & & & & & & & & & & & & & \\
\hline & & & & & & & & & & & & & & \\
\hline & & & & & & & & & & & & & & \\
\hline & & & & & & & & & & & & & & \\
\hline & & & & & & & & & & & & & & \\
\hline & & & & & & & & & & & & & & \\
\hline & & & & & & & & & & & & & & \\
\hline & & & & & & & & & & & & & & \\
\hline & & & & & & & & & & & & & & \\
\hline & & & & & & & & & & & & & & \\
\hline & & & & & & & & & & & & & & \\
\hline & & & & & & & & & & & & & & \\
\hline & & & & & & & & & & & & & & \\
\hline & & & & & & & & & & & & & & \\
\hline & & & & & & & & & & & & & & \\
\hline
\end{tabular}

Core No. 29 PG

Station No. 68

Lithologic

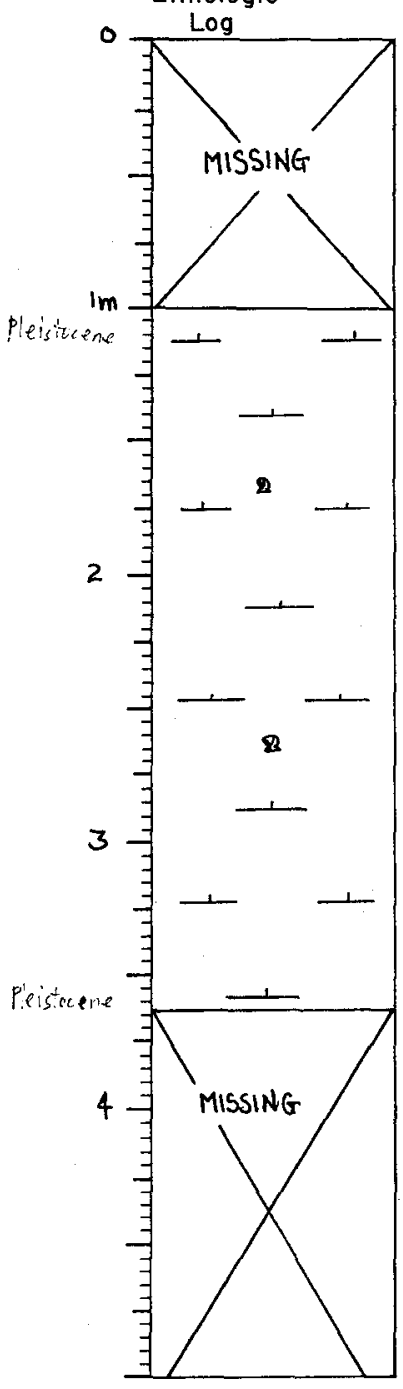

Detailed Description

0-100

100-364

CALC OOZE

$2.5 Y 7.5 / 2$ it gray

hard dry, crumbling lutite; forams, gastropods, pteropods

dry, and speckled with alumintm foil, visual description of minimal value $364-512 \mathrm{~cm}$.. MISSING. 
0142

SMEAR SLIDE DESCRIPTIONS - W.H.O.I. SEDIMENT CORES

Ship: Atlantis

Expedition 246

Leg No.

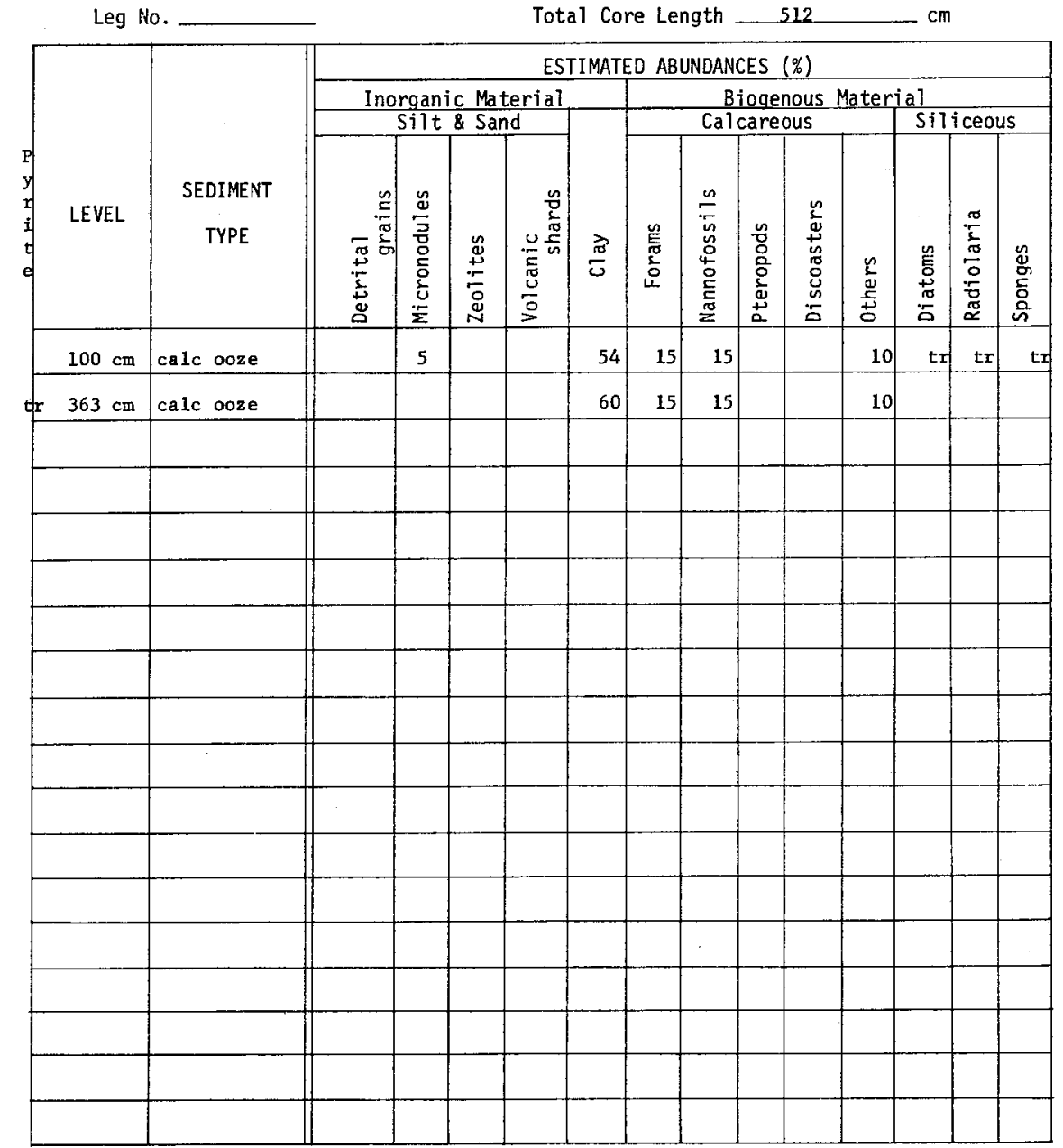

0143

VISUAL CORE. DESCRIPTION

Page 1 of 1

Ship A Cruise 246 Leg_ Sta. 69 Core No. $30 \mathrm{PG}$

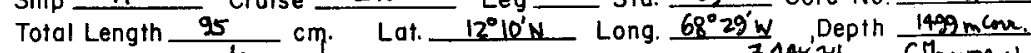
Core condition dry good Date Described 7 1Ar 74 by GMountan

Physiographic locotion SIX TllLES WEST OF BONAIRE ISLAND

Lithologic

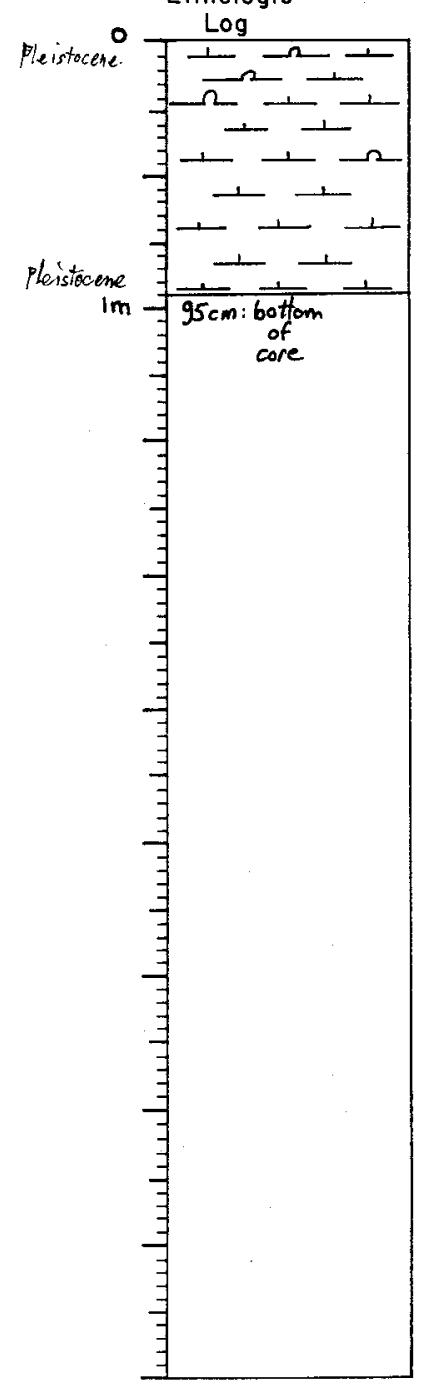

Detailed Description

$0-95$ CALC SILIC OOZE GRADES TO CALC OOZE $2.5 Y^{7} 7 / 21$ t gray

dry, crumbling lutite, forams common, pteropods scattered

end of core 


\section{4}

SMEAR SLIDE DESCRIPTIONS - W.H.O.I. SEDIMENT CORES

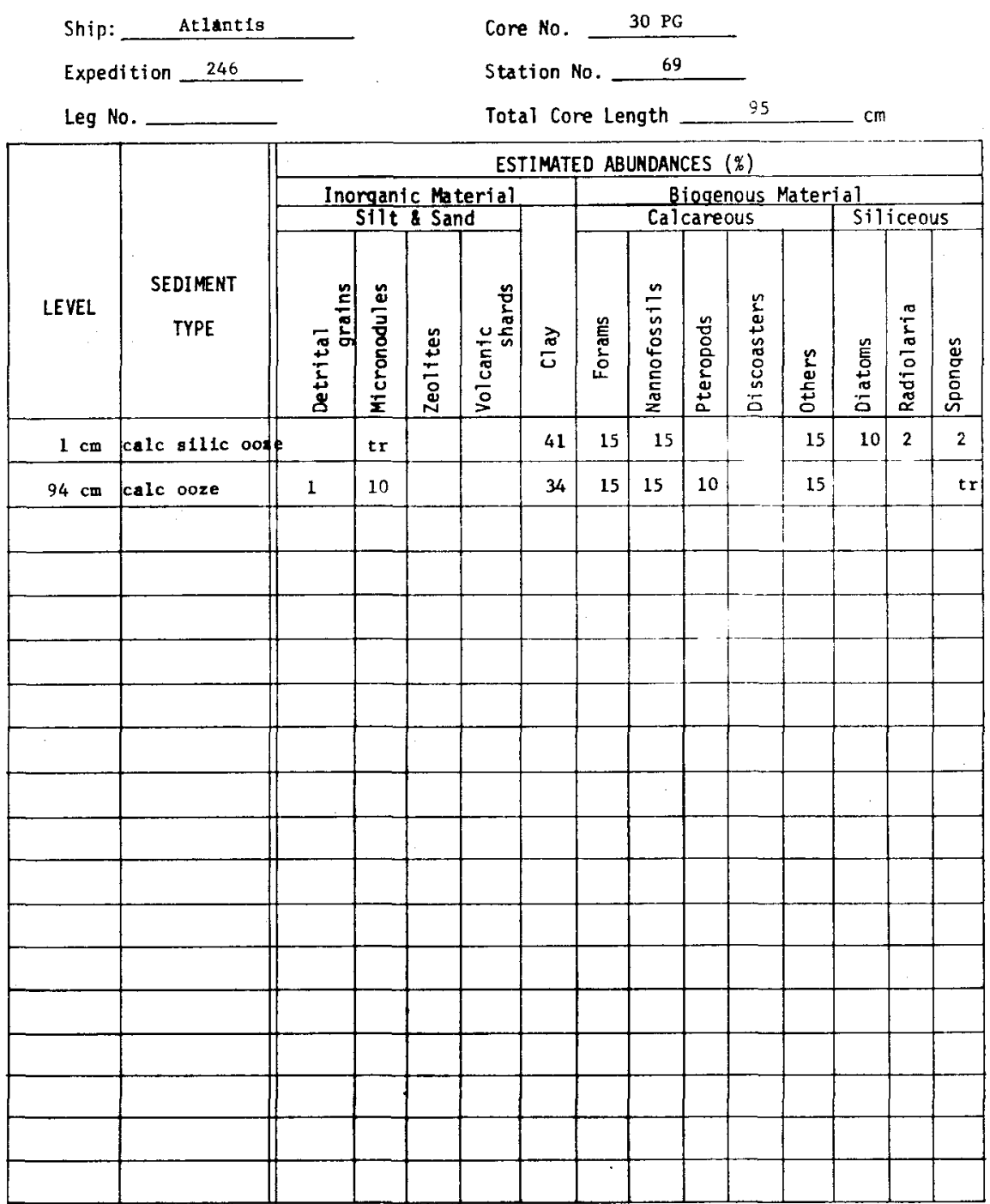

0145

VISUAL CORE DESCRIPTION

Poge 1 of 1

Ship A Cruise 246 Leg_ Sta. 70 Core No 3IPC Total Length $108 . \mathrm{cm}$. Lot. $11^{\circ} 47^{\prime} \mathrm{N}$ Long. $68^{\circ} 30^{\circ} \mathrm{m}$ Depth $1803 \mathrm{mmom}$. Core condition dy, fair Date Described IMAY 74 by Ghowntain Physiographic locotion SE OF KLEN CURACAO

Lithologic

$$
\begin{gathered}
\text { Pleistacene } \\
\text { Log }
\end{gathered}
$$

fte+

$\exists-1$

$\ln$

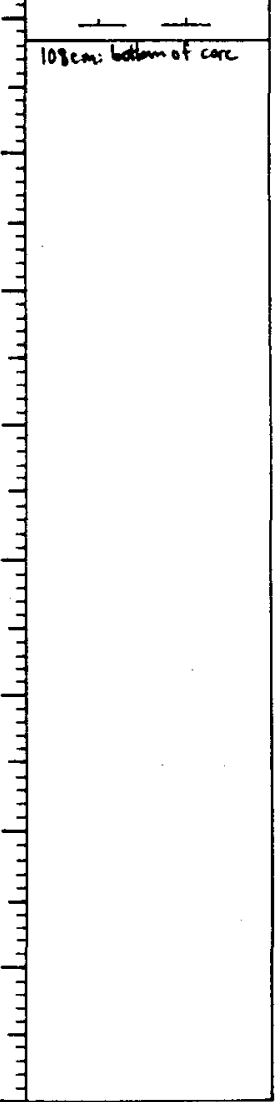


0146

SMEAR SLIDE DESCRIPTIONS - W.H.O.I. SEDIMENT CORES

Ship: Atlantis

Expedition 246

Leg No.



0147

VISUAL CORE . DESCRIPTION

Page 1 of 1

Ship A Cruise 246 Leg_Sto. 70 Core No. 31PG

Total Length $\frac{88}{\mathrm{~cm}}$. Lat. $11^{\circ} 47^{\mathrm{in}}$ Long. $\frac{68^{\circ} 30^{\prime} \mathrm{w}}{7}$ Depth $1803 \mathrm{cmm}$

Core condition dryjgond Date Described 7 MAY 74 by G Gowntin

Physiographic location

Lithologic

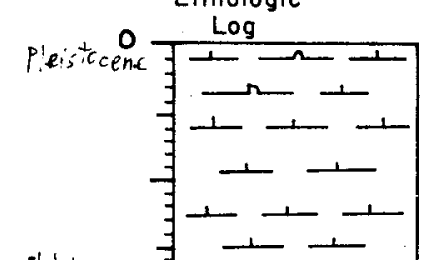

SE of KLEIN CuRncAO

Detailed Description

$0-88$ CALC SILIC OOZE TO CALC OOZE $2.5 Y$ Y $7 / 2$ it gray

dry, crumbled lutite, forms common, few pteropods $\mathrm{s}$ end of core

reistocene

Ini

$88 \mathrm{~cm}$ : bottom of core

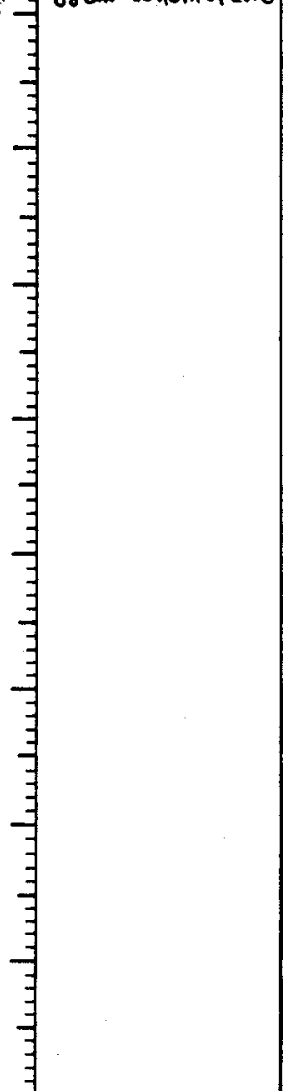


0148

SMEAR SLIDE DESCRIPTIONS - W.H.O.I. SEDIMENT CORES

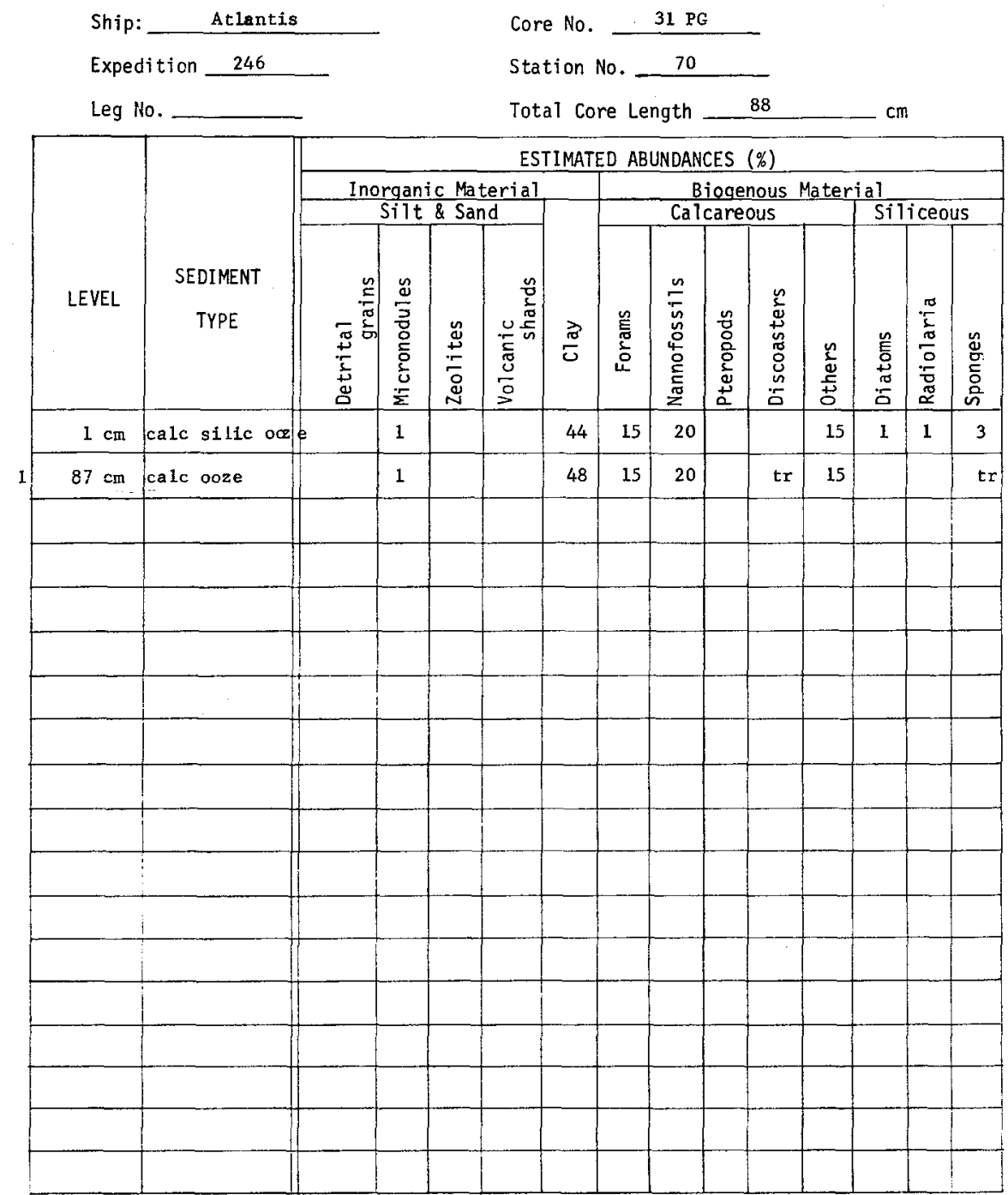

0149

VISUAL CORE .DESCRIPTION

Poge 1 of 1

Ship A Cruise 246 Leg Sta. 71 Core No. 32PG Total Length 71 cm. Lat. $11^{\circ} 25.5^{\prime} \mathrm{N}$ Long. $68^{\circ} 30^{\prime} \mathrm{W}$ Depth $347 \mathrm{mcous}$ Core condition dry; fair Date Described 8 MAY 74 by GHowewtain Physiographic location off VeNEzUELA Lithologic

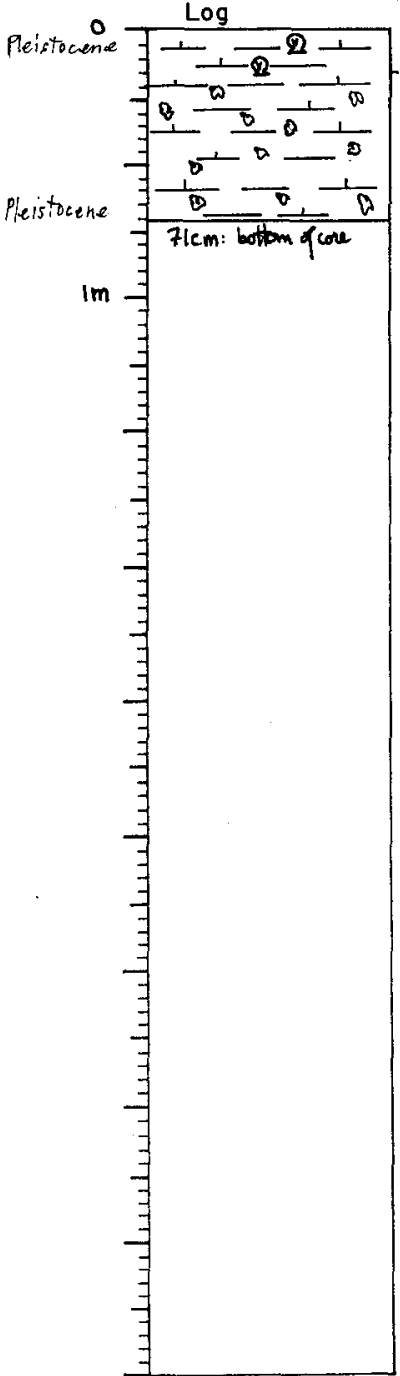

\section{Description}

\section{HLY CALC CLAY}

dry, crumbled lutite, forams common, several mollusc

shell fradents

sediment has shrunk, less than $71 \mathrm{~cm}$ remsining

$15-71$

HLY CALC CLAY

$5 Y 5 / 2$ olive gray

extensive fine scale mottling, it olive gray; mot tling, burrowing

dry, cracked lutite, fewer forams and shell fragments than end of core 


\section{0}

SMEAR SLIDE DESCRIPTIONS - W.H.O.I. SEDIMENT CORES

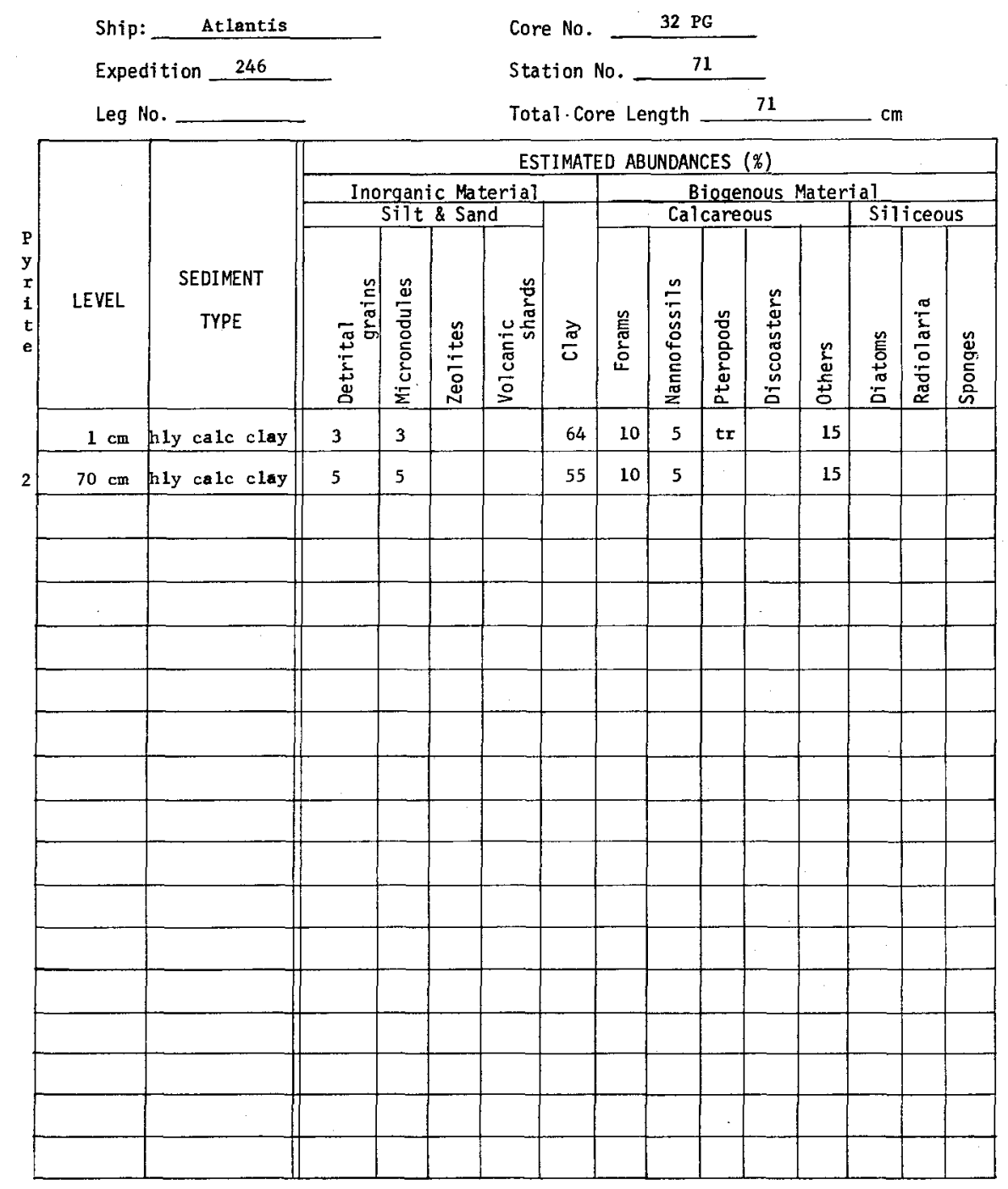

0151

VISUAL CORE DESCRIPTION

Page 1 of 1

Ship A Cruise 246 Leg Sta. 214 Core No. $33 \mathrm{PG}$ Total Length $110 \mathrm{~cm}$. Lat. $10^{\circ} 15^{\prime} N$ - Long. $71^{\circ} 35^{\prime} w$ Depth $\frac{33 \mathrm{~m}}{6 \mathrm{~m}}$

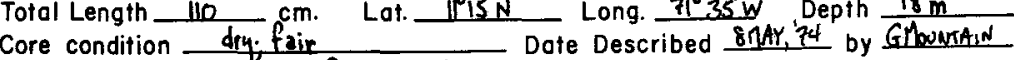
Physiogrophic location Gurf of Vemezual
Lithologic

\section{Log
$0-110$ Detailed Descriptio
UNFOS CLAY/DET
$5 Y$ 6/2 1t ollve gray
extensive fine scale,}

extensive fine scale, irregular mottling, it gray hard, cracked silty lutite, scattered shell fragments considerable sirrinkage; only $70 \mathrm{~cm}$ of sediment remaining

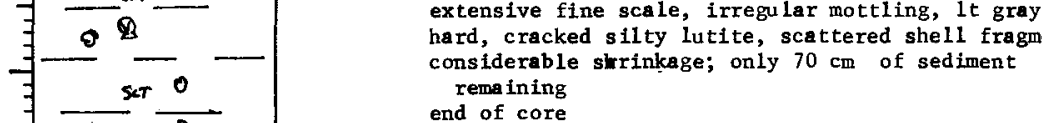

im

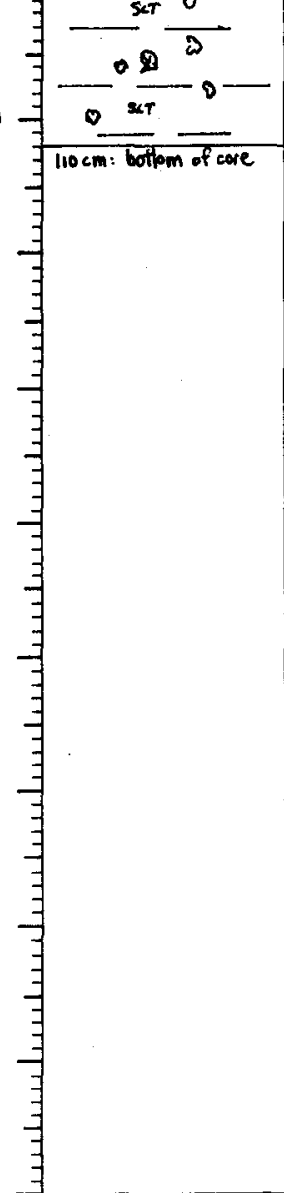

\section{-}

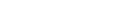




\section{2}

SMEAR SLIDE DESCRIPTIONS - W.H.O.I. SEDIMENT CORES

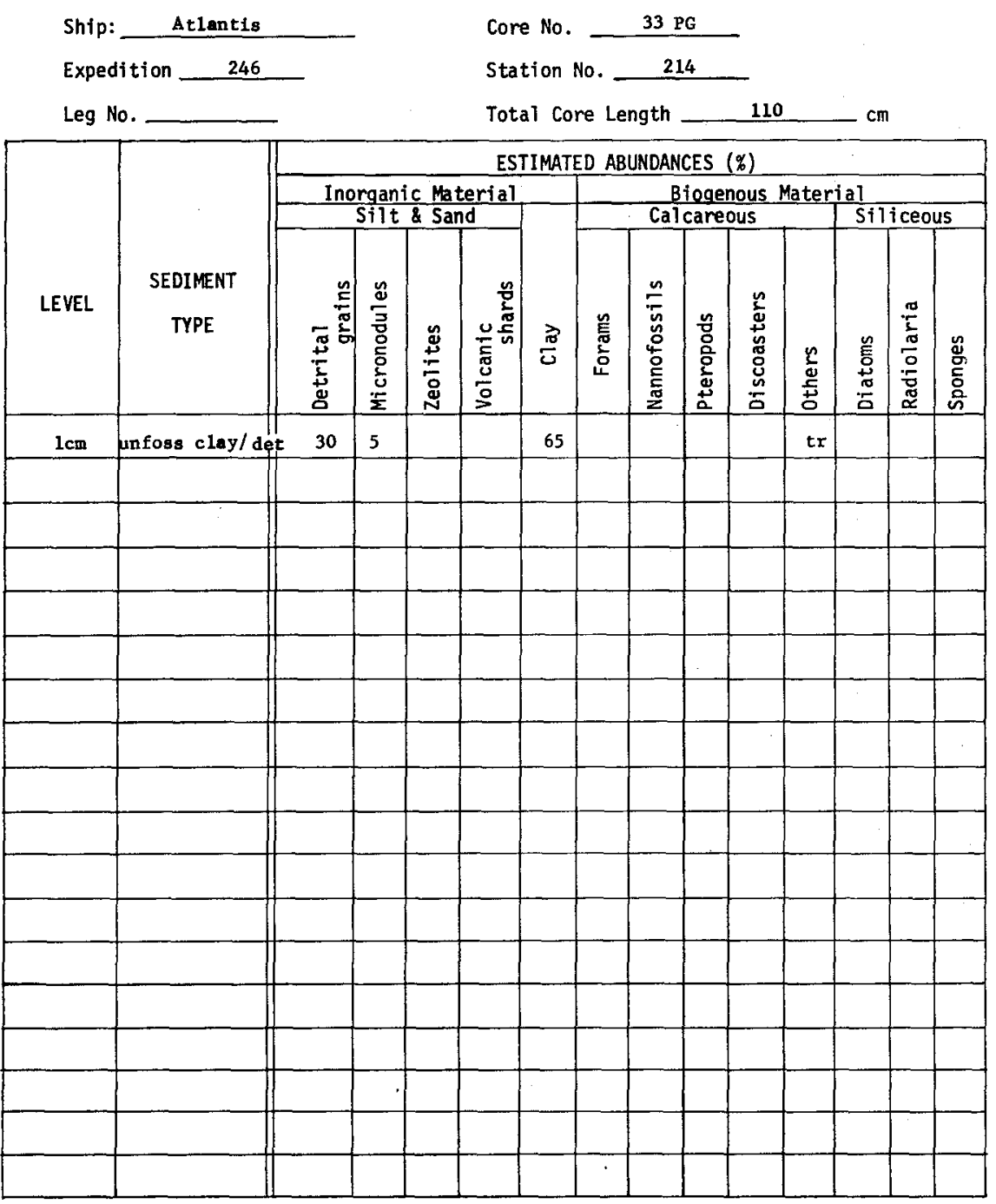

0153

VISUAL CORE .DESCRIPTION

Page 1 of 1

Ship A Cruise 246 Leg Sta. 215 Core No. 34fG Total Length $63 \mathrm{~cm}$. Lat. $111^{\circ} 26 . \mathrm{s}^{\mathrm{N} N \mathrm{~N}}$ Long. $71^{\circ} 36.5^{\prime} \mathrm{w}$ Depth $24 \mathrm{~m}$

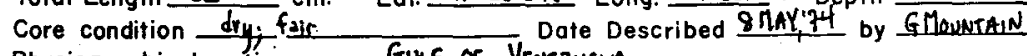
Physiogrophic location Gurf of VENEzUELA

Lithologic

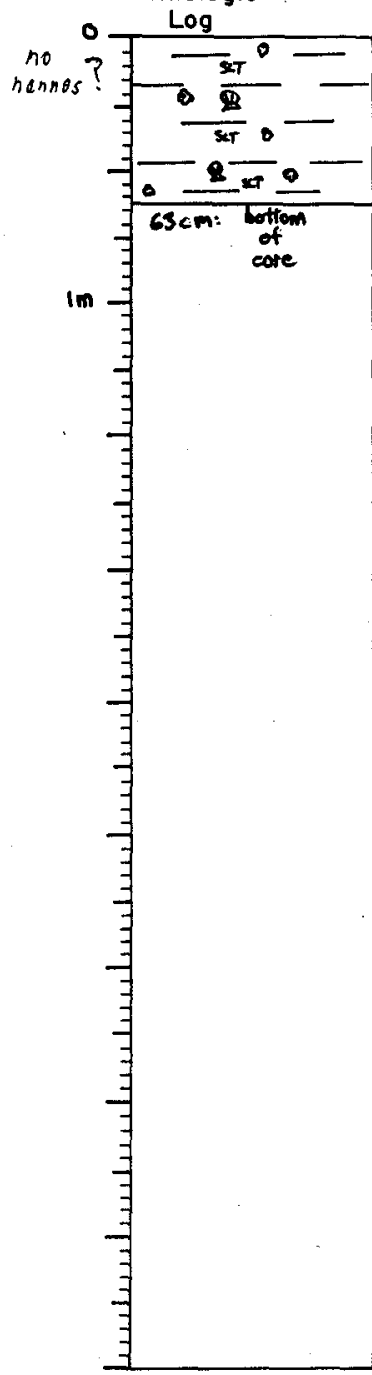

$0-63$

Detailed Description

UNFOSS CLAY/DET

extensive fine scale, Irregular mottling, 1t olive gray lutite, scattered mollusc shell 


\section{4}

SMEAR SLIDE DESCRIPTIONS - W.H.O.I. SEDTMENT CORES

Ship: Atlontis

Core No. $34 \mathrm{PG}$

Expedition 246

Leg No.

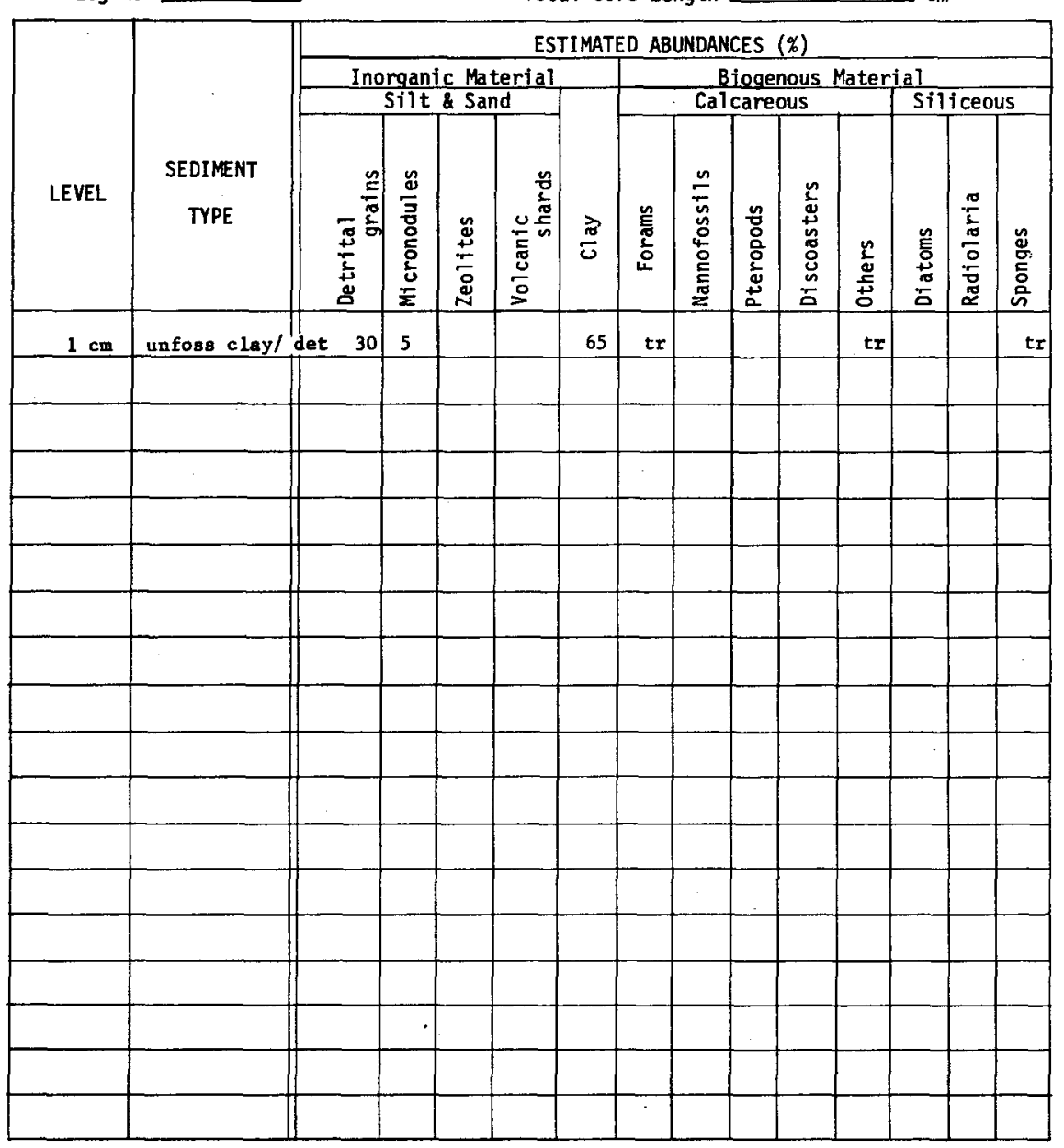

0155

VISUAL CORE . DESCRIPTION

Page 1 of 1 Ship A Cruise 246 Sto. 216 Core No. 35 PG Total Length $28 \mathrm{~cm}$. Lat. $11^{\circ} 34^{\prime} \mathrm{N}$ Long. $71^{\circ} 35.5^{\prime} \mathrm{W}$ Depth $22 \mathrm{~m}$ Core condition dry. fuir Date Described 8 MaY'74 by GibunIAIN Physiogrophic location_ GULF of VENEZUELA

Lithologic

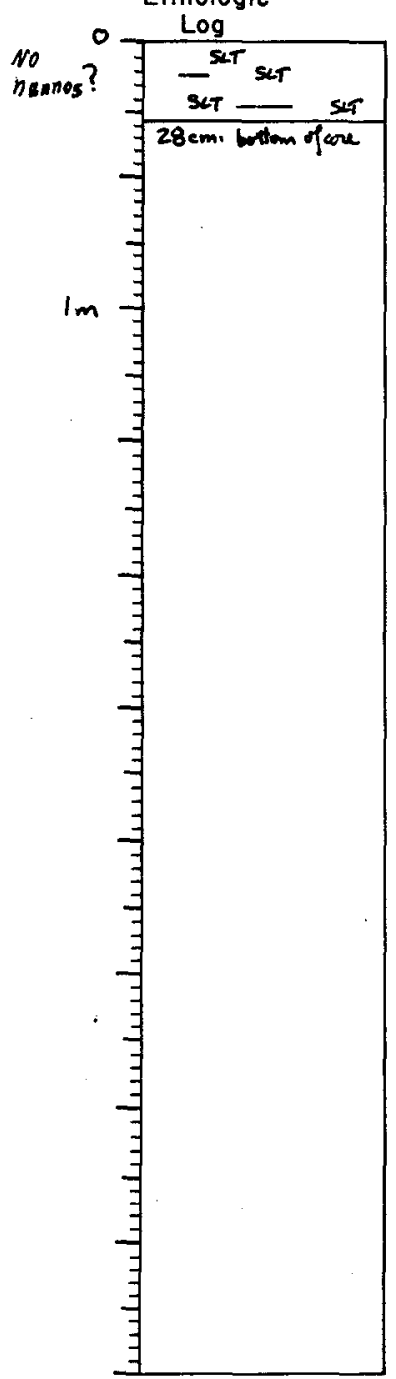

Detailed Description SILT

$5 Y$ 5/2 olive gray

fragment and of core 
0156

SMEAR SLIDE DESCRIPTIONS - W.H.O.I. SEDIMENT CORES

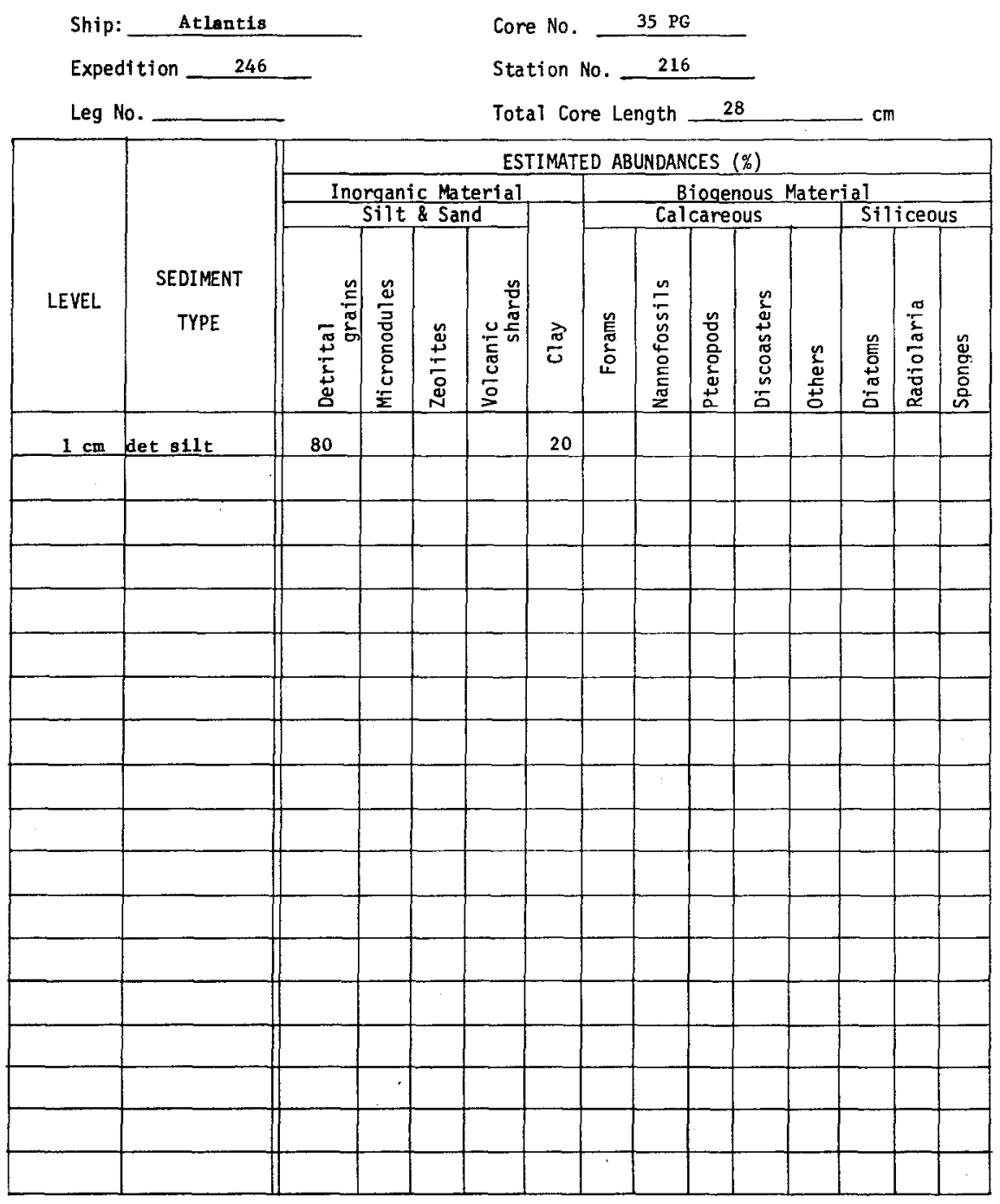

0157

VISUAL CORE .DESCRIPTION.

Page 1 of 1

Ship A Cruise 246 Leg_Sta. 217 Core No. $36 \mathrm{PG}$

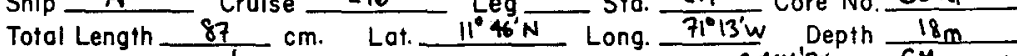
Core condition dry; poop Date Described 9 AnY'74 by GMouraw Physiogrophic locotion Lithologic

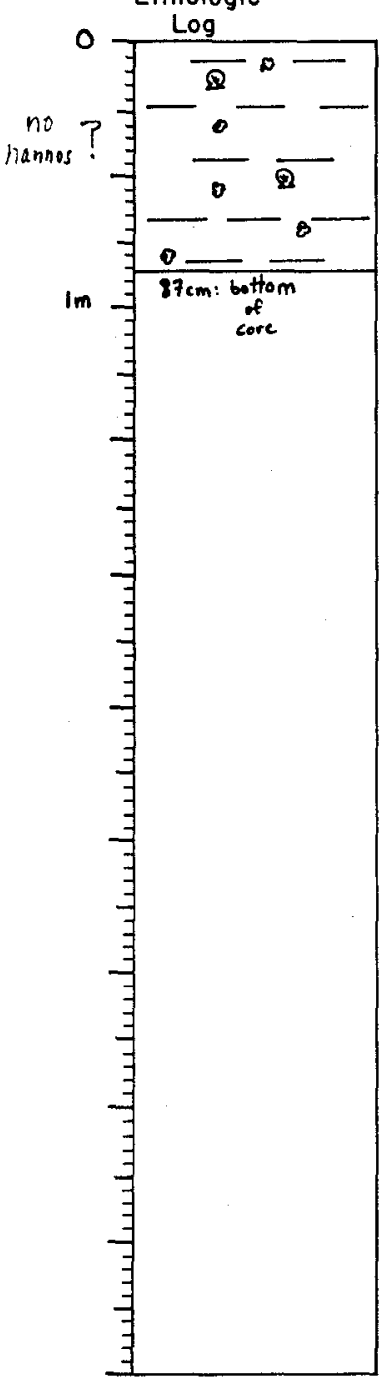

\section{Detailed Description}

$0-87$

UNFOSS CLAY

$5 Y 5 / 2$ olite gray

extensive fine scale, irregular mottling, lt olive gray dy and considerably crumbled silty lutite, scattered shell fragments 
0158

SMEAR SLIDE DESCRIPTIONS - W.H.O.I. SEDIMENT CORES

Ship: Atlantis

Expedition 246

Leg No.

\begin{tabular}{|c|c|c|c|c|c|c|c|c|c|c|c|c|c|c|}
\hline \multirow[b]{4}{*}{ LEVEL } & \multirow[b]{4}{*}{$\begin{array}{c}\text { SEDIMENT } \\
\text { TYPE }\end{array}$} & \multicolumn{13}{|c|}{ ESTIMATED ABUNDANCES (\%) } \\
\hline & & \multirow{2}{*}{\multicolumn{4}{|c|}{$\begin{array}{c}\text { Inorganic Material } \\
\text { Silt \& Sand }\end{array}$}} & & \multirow{2}{*}{\multicolumn{5}{|c|}{$\begin{array}{l}\text { Biogenous Mater } \\
\text { Calcareous }\end{array}$}} & & & \\
\hline & & & & & & & & & & & & \multicolumn{3}{|c|}{ Siliceous } \\
\hline & & 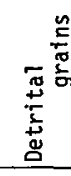 & 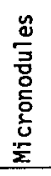 & 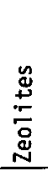 & 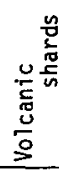 & $\frac{\vec{\pi}}{0}$ & 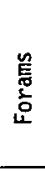 & 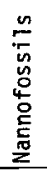 & 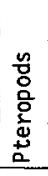 & 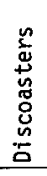 & 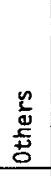 & 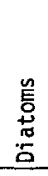 & $\begin{array}{l}\frac{\pi}{2} \\
\frac{\pi}{0} \\
\frac{0}{0} \\
\frac{\pi}{0} \\
\tilde{\pi} \\
\end{array}$ & 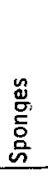 \\
\hline $1 \mathrm{~cm}$ & unfoss clay & 15 & 5 & & & 80 & & & & & $\mathrm{tr}$ & & & tr \\
\hline & & & & & & & & & & & & & & \\
\hline & & & & & & & & & & & & & & \\
\hline & & & & & & & & & & & & & & \\
\hline & & & & & & & & & & & & & & \\
\hline & & & & & & & & & & & & & & \\
\hline & & & & & & & & & & & & & & \\
\hline & & & & & & & & & & & & & & \\
\hline & & & & & & & & & & & & & & \\
\hline & & & & & & & & & & & & & & \\
\hline & & & & & & & & & & & & & & \\
\hline & & & & & & & & & & & & & & \\
\hline & & & & & & & & & & & & & & \\
\hline & & & & & & & & & & & & & & \\
\hline & & & & & & & & & & & & & & \\
\hline & & & & & & & & & & & & & & \\
\hline & & & & & & & & & & & & & & \\
\hline & & & & & & & & & & & & & & \\
\hline & & & & & & & & & & & & & & \\
\hline
\end{tabular}

0159

VISUAL CORE DESCRIPTION

Page 1 of 1

Ship A Cruise 246 Leg Sta. 218 Core No. 37PG Total Length $87 \mathrm{~cm}$. Lat. $11^{\circ} 46^{\prime} \mathrm{N}-$ Long. $71^{\circ} 05^{\circ} \mathrm{W}$ Depth $16 \mathrm{~m}$

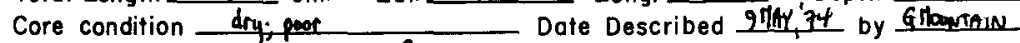

Physiographic location GULF of VENEZUELA

Lithologic

No 2 Lannes?

\section{Detailed Description}

$0-87$ UNFOSS CIAY

extensive fine gray dry, crumbled, silty lutite; pteropod and pelecypod fragments

only $60 \mathrm{~cm}$ of sediment remsining end of core

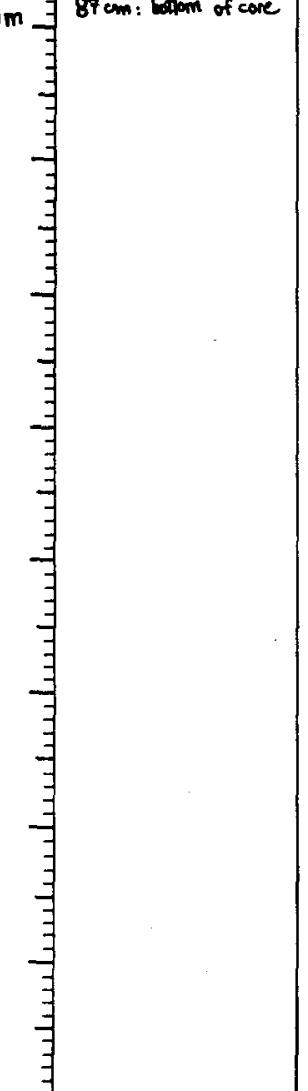


0160

SMEAR SLIDE DESCRIPTIONS - W.H.O.I. SEDIMENT CORES

Ship: Atlantis

Expedition 246

Leg No.

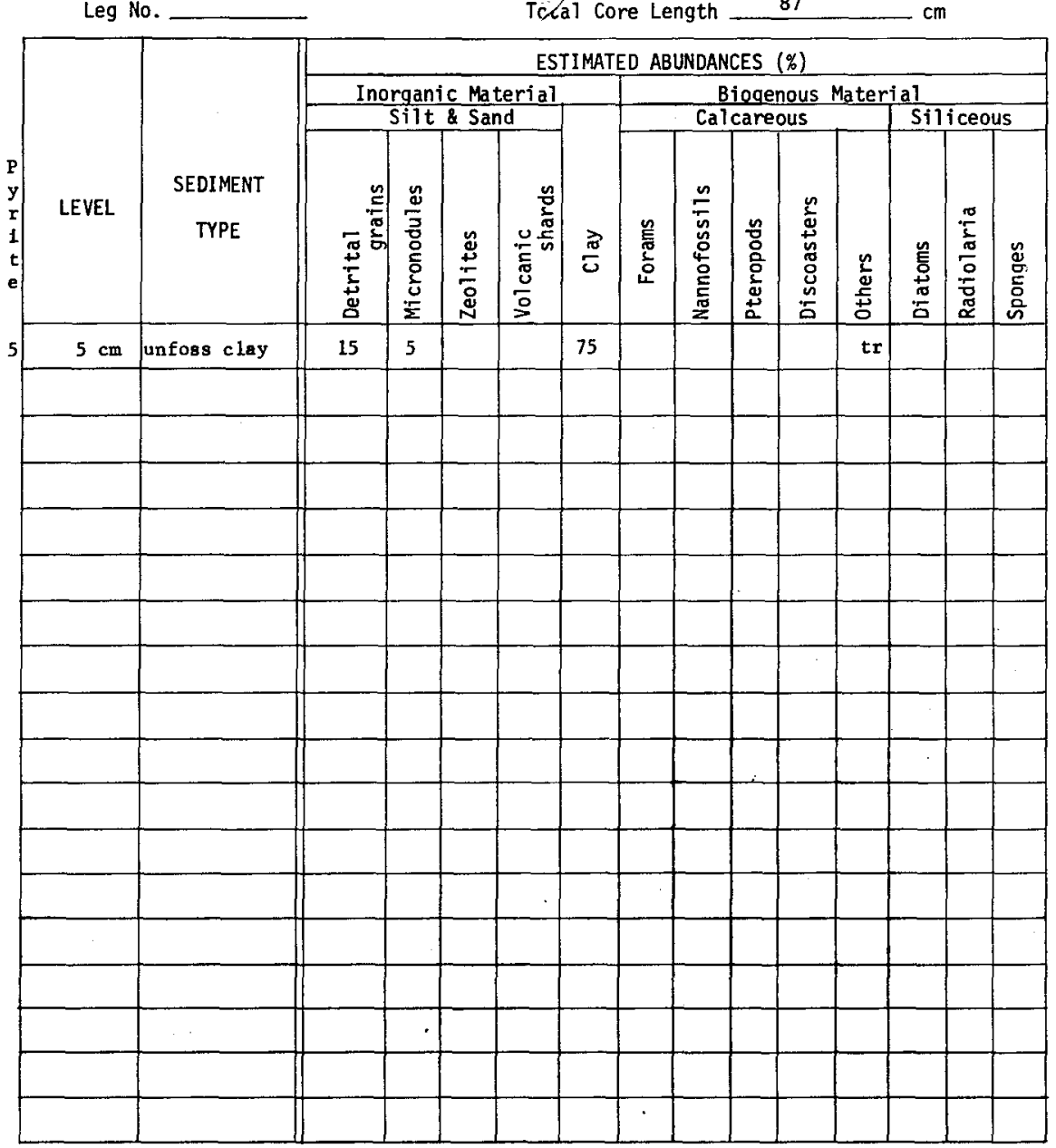

0161

VISUAL CORE DESCRIPTION

Page 1 of 1 Ship $\frac{A}{A}$ Cruise $\frac{246}{10}$ Leg Sta. $\frac{219}{70^{\circ} 40^{\prime} \mathrm{w}}$ Core No. $\frac{38 \mathrm{PG}}{37 \mathrm{~m}}$ Total Length $-55 \mathrm{~cm}$. Lat. $11^{\circ} 45^{\prime} \mathrm{N}$ Long. $70^{\circ} 49^{\prime} \mathrm{W}$, Depth $\frac{37 \mathrm{~m}}{\mathrm{C}}$ Core condition dry foir Physiographic location GULF of VENE2UELA

Lithologic

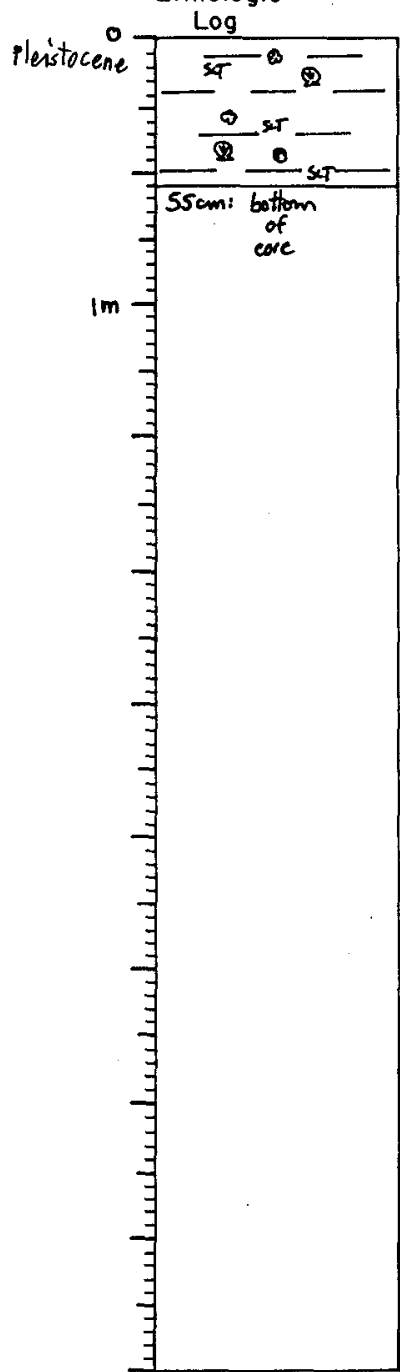

UNFOSS CLAY/DET SILT

SY $6 / 2$ lt olive gray irregular mottling, olive gray hard, crumbled; scattered pelecypod fragments end of core 
SMEAR SLIDE DESCRIPTIONS - W.H.O.I. SEDIMENT CORES

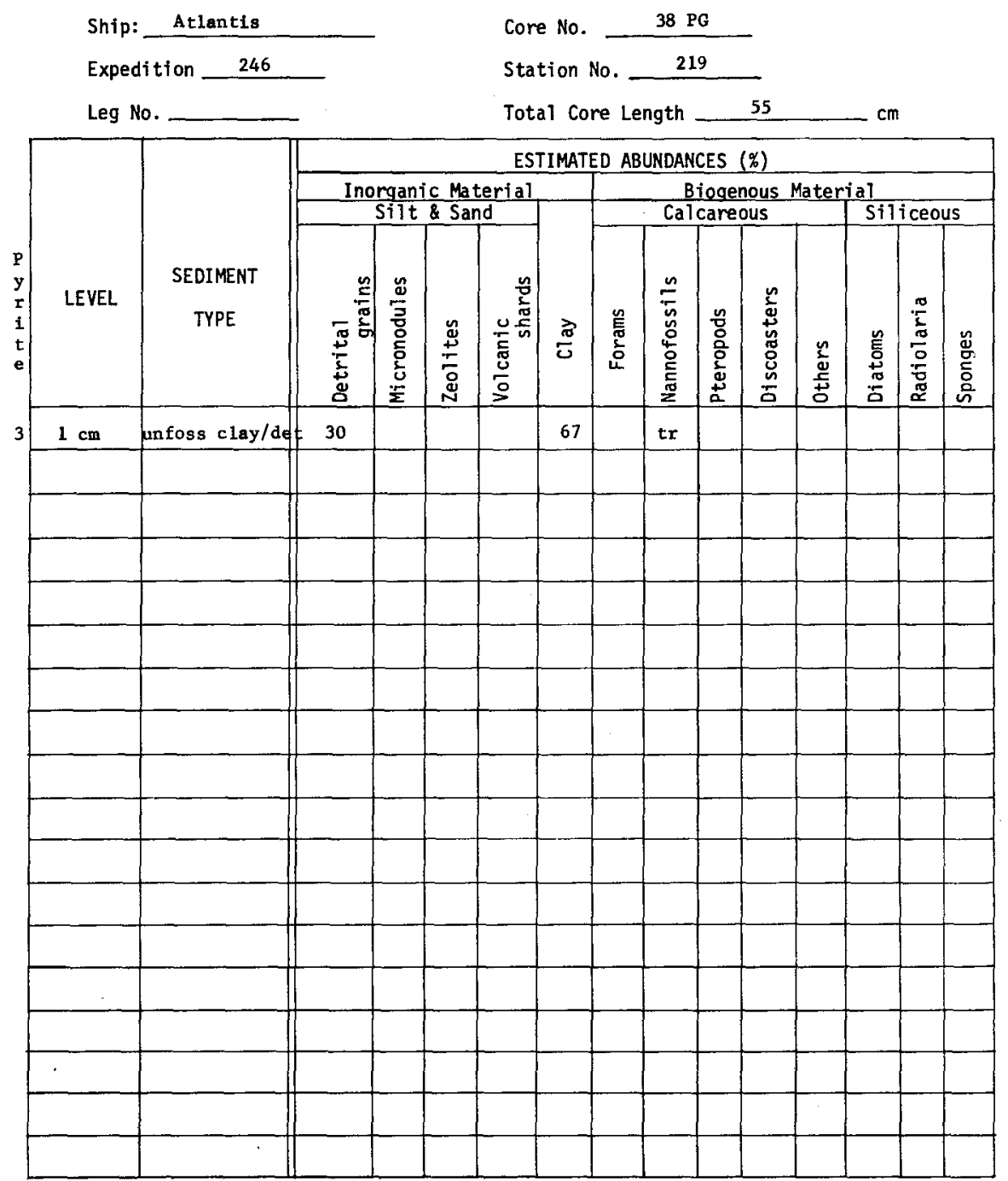




\section{$U \perp 0 u$}

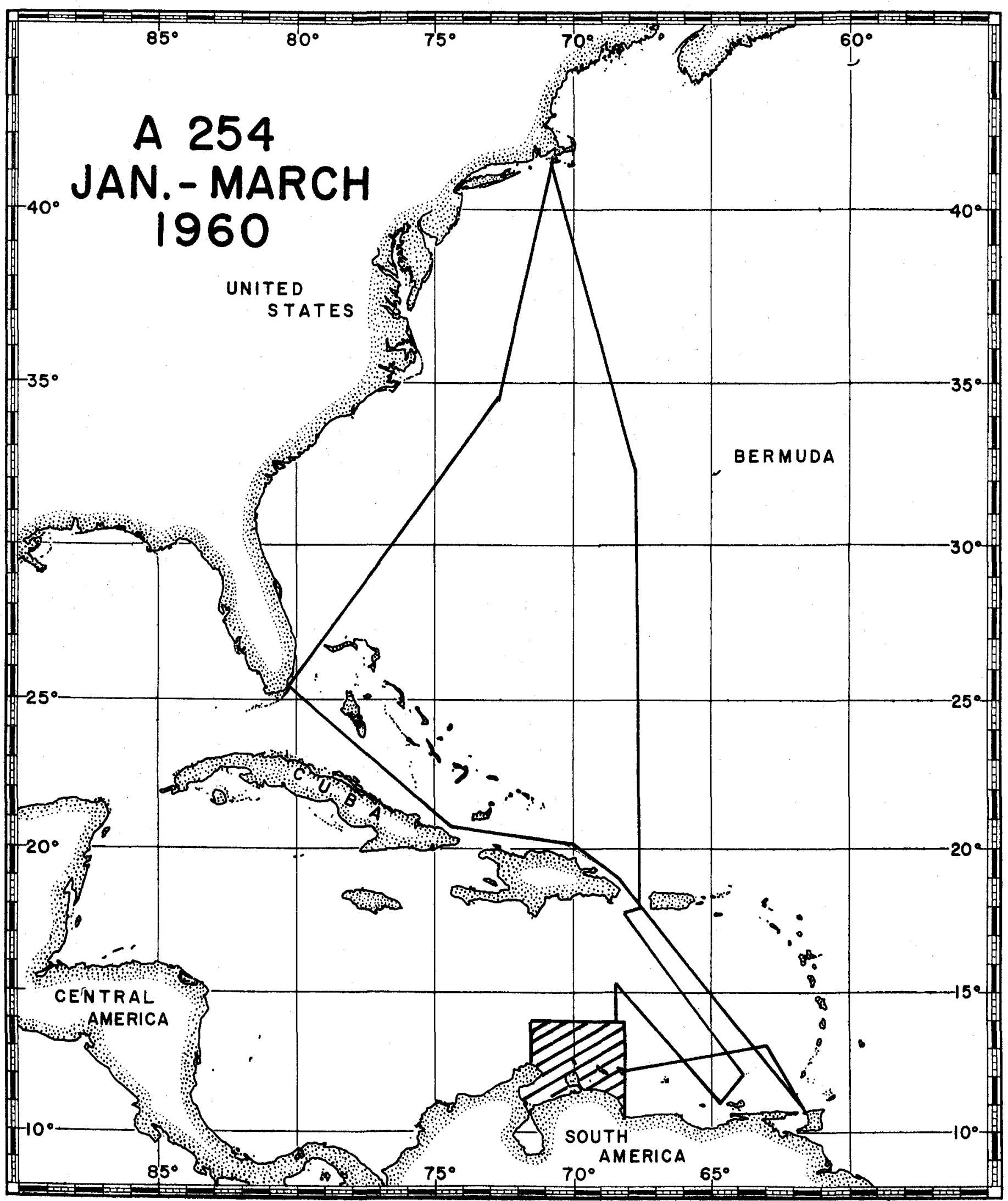



0165

VISUAL CORE DESCRIPTION

Ship A Cruise 254 Leg 3 Sta. 324 Core No. JPC

Total Length $601 \mathrm{~cm}$. Lat. $31^{\circ} 32^{\circ} \mathrm{N}$ Long. $83^{\circ} 0^{\prime} \mathrm{w}$ Depth 3655 correm.

Core condition FAR ...(andudemed died) Dote Described BMAY14 by J.BRODA.

Physiographic location YWLATAN BASIN

Lithologic (mearly all contact concere ufwards... somewhat desturbed).

Lithologic

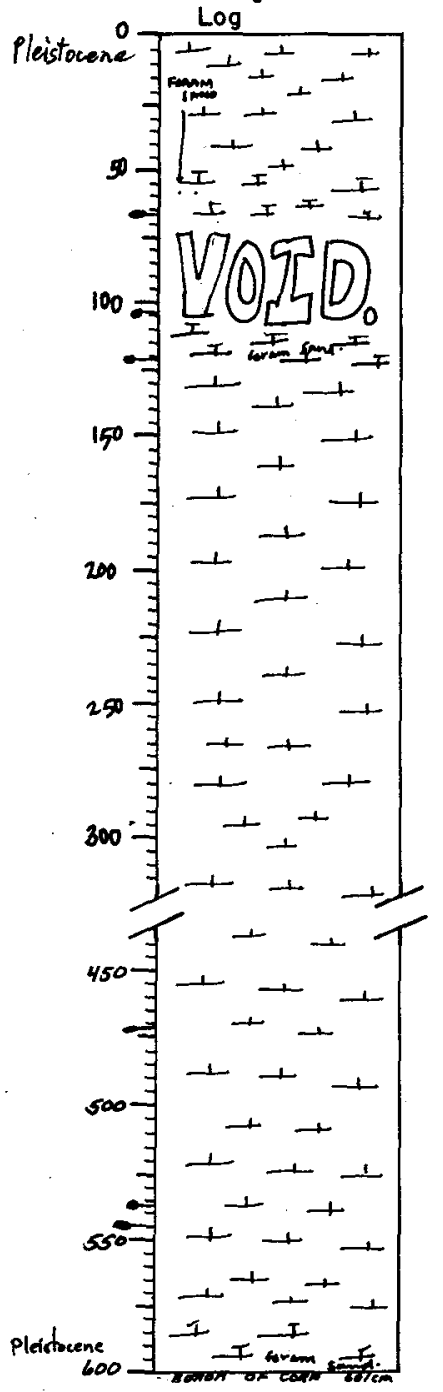

0-67 FORAM SAND CALC OOZE

( scattered pteropod

$67-104$

104-121

CALC OOZE FORAM SAND

LOXR $7 / 4 \mathrm{v}$. pale brow

extremely abunt. forams in silty lutite S, horizonta

121-472

CALC OOZE

alternating faint bands. of calc ooze, concave upward distortions

forams scattered throughout lutite

$s$, dried and cracked

472-537

CAIC OOZE, FRRAM SAND

10YR $7 / 4$ v. pale brown

extrememy abut. forams

some verylarge foram tes
$537-545$

VOTD

$545-601$

CALC OOZE

1OYR $7 / 2$ white

scattered forams in lutite

lithfications, white, ve pale brown, 1/4-1/2 cm diamete

scattered
0166

SMEAR SLIDE DESCRIPTIONS - W.H.O.I. SEDIMENT CORES

Ship: Atlantis

Expedition 254

Leg No. 3

1

Station No. 324

Total Core Length 601

\begin{tabular}{|c|c|c|c|c|c|c|c|c|c|c|c|c|c|c|}
\hline \multirow[b]{3}{*}{ LEVEL } & \multirow[b]{3}{*}{$\begin{array}{l}\text { SEDIMENT } \\
\text { TYPE }\end{array}$} & \multicolumn{13}{|c|}{ ESTIMATED ABUNDANCES $(\%)$} \\
\hline & & \multicolumn{4}{|c|}{$\frac{\text { Inorganic Material }}{\text { Silt \& Sand }}$} & \multirow[b]{2}{*}{$\stackrel{\frac{\pi}{\omega}}{\sigma}$} & \multicolumn{5}{|c|}{$\begin{array}{l}\text { Biogenous Mater } \\
\text { Calcareous }\end{array}$} & & Siliceous & \\
\hline & & 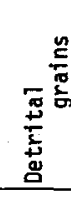 & 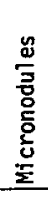 & 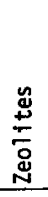 & 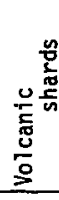 & & $\begin{array}{l}\text { 唇 } \\
\text { ¿े }\end{array}$ & 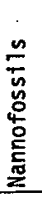 & 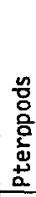 & 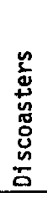 & 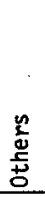 & 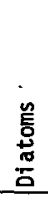 & 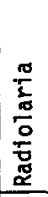 & 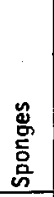 \\
\hline $1 \mathrm{~cm}$ & calc ooze & & & & & 20 & 45 & 27 & & tr & 8 & & & tr \\
\hline $105 \mathrm{~cm}$ & calc ooze & tr & & & & 30 & 35 & 30 & & 3 & 2 & & & $\mathrm{tr}$ \\
\hline $125 \mathrm{~cm}$ & calc ooze & & & & & 12 & 15 & 68 & & $t x$ & 5 & & & $\mathrm{tr}$ \\
\hline $205 \mathrm{~cm}$ & calc ooze & 2 & & & & 40 & 7 & 45 & & & 6 & & & \\
\hline $306 \mathrm{~cm}$ & calc ooze & & & & & 30 & 5 & 55 & & 2 & 8 & & & tr \\
\hline $405 \mathrm{~cm}$ & calc ooze & & & & & 35 & 8 & 50 & & & 7 & & & \\
\hline $506 \mathrm{~cm}$ & ce lc ooze & & & & & 10 & 60 & 28 & & tr & 2 & & & \\
\hline $601 \mathrm{~cm}$ & calc ooze & & & & & 40 & 9 & 45 & & tr & 6 & & & tr \\
\hline & & & & & & & & & & & & & & \\
\hline & & & & & & & & & & & & & & \\
\hline & & & & & & & & & & & & & & \\
\hline & & & & & & & & & & & & & & \\
\hline & & & & & & & & & & & & & & \\
\hline & & & & & & & & & & & & & & $\ldots$ \\
\hline & & & & & & & & & & & & & & \\
\hline & & & & & & & & & & & & & & \\
\hline & & & & & & & & & & & & & & \\
\hline & & & & & & & & & & & & & & \\
\hline
\end{tabular}


Ship A Cruise 254 Leg 3 sta. 325 Core No. 2 PC Total Length $248 \mathrm{~cm}$. Lat. $21^{\circ} 11.0^{\circ} \mathrm{N}$ Long. $82^{\circ} 50.0^{\circ} \mathrm{W}$ Depth 4463 corr.meters Core condition DRY CRAYED. IFARO. Dote Described SMAY 74 by J.BRPA.

Physiographic location YULATAN BASIN

Lithologic

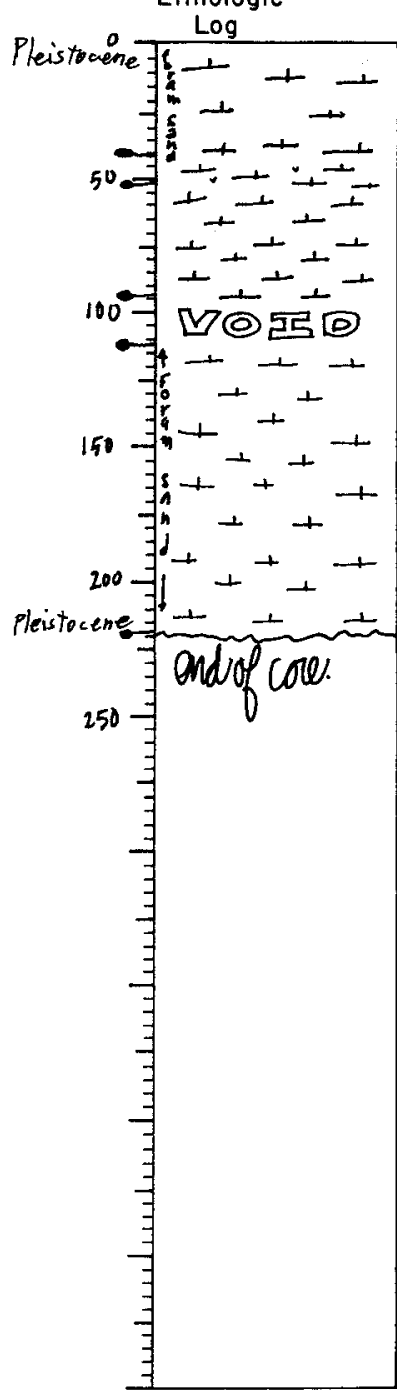

Detailed Description

$0-41$ CALC OOZE (FORAM SAND) IOYR $8 / 3 \mathrm{v}$. pale brown fragments

41-51

CALC OOZE

LOYR $8 / 3$ v. pale brown

common smail motthing, lt gray

1 ty in mottles

$51-94$

CALC OOZE

tered shell fragment

94-111

VOID
$11-218$

CALC OOZE (FORAM SAND)

10YR $8 / 2$ white

loram sand

end of core

SMEAR SLIDE DESCRIPTIONS - W.H.O.I. SEDIMENT CORES

Ship: Atlantis

Expedition 25
Leg No. 3

Core No. 2 PC

Station No. 325

Total Core Length $218 \quad \mathrm{~cm}$

ESTIMATED ABUNDANCES (\%)

\begin{tabular}{|c|c|c|c|c|c|c|c|c|c|c|c|c|c|c|}
\hline \multirow[b]{4}{*}{ LEVEL } & \multirow[b]{4}{*}{$\begin{array}{c}\text { SEDIMENT } \\
\text { TYPE }\end{array}$} & \multicolumn{13}{|c|}{ ESTIMATED ABUNDANCES (\%) } \\
\hline & & \multirow{2}{*}{\multicolumn{4}{|c|}{$\begin{array}{c}\text { Inorganic Material } \\
\text { Silt \& Sand } \\
\end{array}$}} & \multirow[b]{3}{*}{$\frac{\mathrm{E}}{0}$} & \multirow{2}{*}{\multicolumn{5}{|c|}{ Calcareous }} & & & \\
\hline & & & & & & & & & & & & \multicolumn{3}{|c|}{ Siliceous } \\
\hline & & 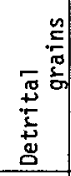 & 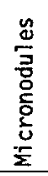 & 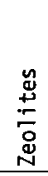 & 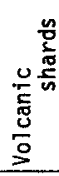 & & 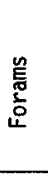 & 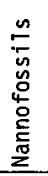 & 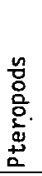 & 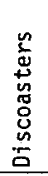 & $\begin{array}{l}\frac{n}{2} \\
\frac{5}{5} \\
0\end{array}$ & 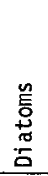 & 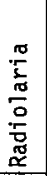 & 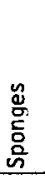 \\
\hline $1 \mathrm{~cm}$ & calc ooze & & & & & 10 & 55 & 30 & & $\operatorname{tr}$ & 5 & & & tr \\
\hline $44 \mathrm{~cm}$ & calc ooze & & & & tr $\mathbf{r}$ & 40 & 12 & 38 & & $\operatorname{tr}$ & 5 & & $\operatorname{tr}$ & 5 \\
\hline $112 \mathrm{~cm}$ & calc ooze & & & & & 10 & 55 & 30 & & tr & 5 & & & \\
\hline $217 \mathrm{~cm}$ & calc ooze & & & & & 6 & 60 & 30 & & $\operatorname{tr}$ & 4 & & & $\operatorname{tr}$ \\
\hline & & & & & & & & & & & & & & \\
\hline & & & & & & & & & & & & & & \\
\hline & & & & & & & & & & & & & & \\
\hline & & & & & & & & & & & & & & \\
\hline & & & & & & & & & & & & & & \\
\hline & & & & & & & & & & & & & & \\
\hline & & & & & & & & & & & & & & \\
\hline & & & & & & & & & & & & & & \\
\hline & & & & & & & & & & & & & & \\
\hline & & & & & & & & & & & & & & \\
\hline & & & & & & & & & & & & & & \\
\hline & & & & & & & & & & & & & & \\
\hline & & & & & & & & & & & & & & \\
\hline & & & & & & & & & & & & & & \\
\hline
\end{tabular}


0169

VISUAL CORE DESCRIPTION

Page__ _ of _ـ 1

Ship A Cruise 254 Leg 3 sta. $\frac{325}{220}$ Core No. 2PG

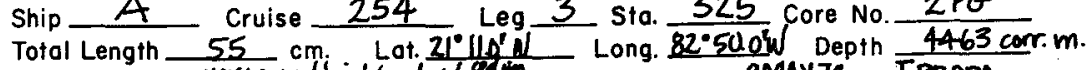

Total Length $\frac{55}{\mathrm{~cm}} \mathrm{~cm}$. Lot.

Physiographic location YULATAN QBASIN.

Lithologic

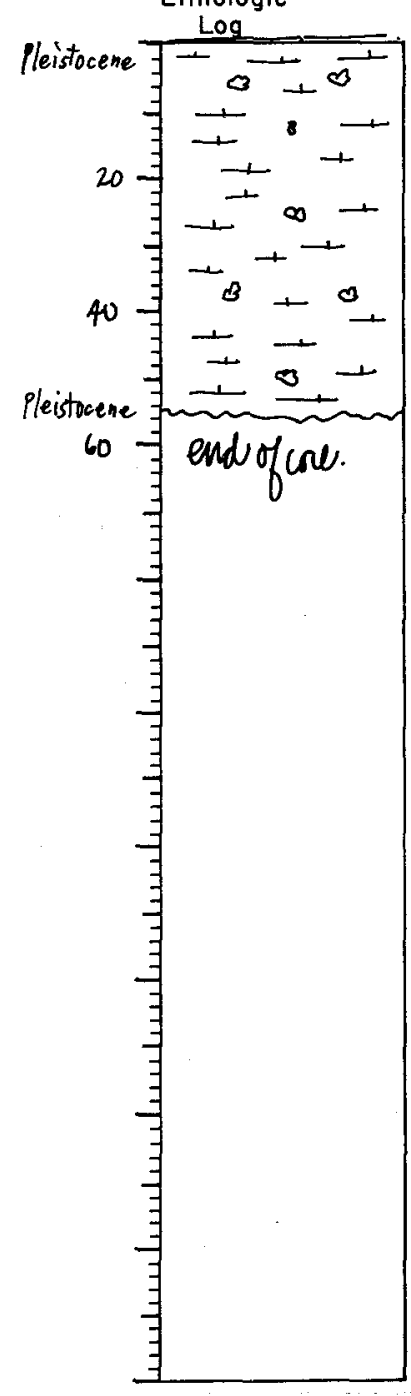

-55 Detoiled Description

CALC OOZE

white

forams in silty lutite

foccur throughout core, $15 \mathrm{~cm}$ altogether but impossible to determine their exact orientaion. end of core $s 1$ mottling, white and $v$, pale brown due to the sample's condition when it was split it io

\section{0}

SMEAR SLIDE DESCRIPTIONS - W.H.O.I. SEDIMENT CORES

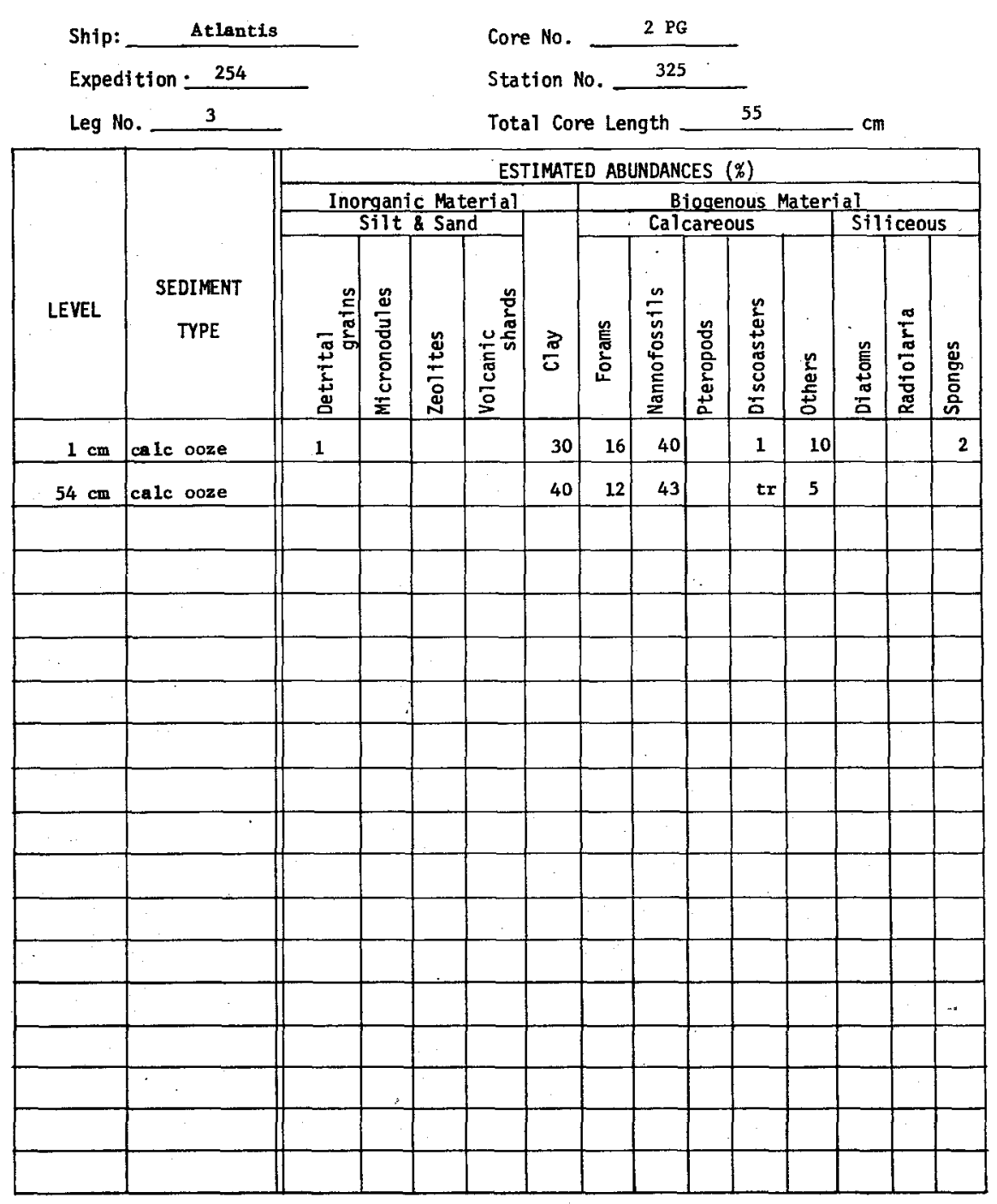


0171

VISUAL CORE DESCRIPTION

Poge 1 af 1

Ship $\bar{A}$ Cruise 254 Leg 3 sto. 326 Core No. 3PC

Total Length $537 \mathrm{~cm}$. Lat. $21^{\circ} 11.0^{\prime} \mathrm{N}$ Long. $82^{\circ} 500^{\circ} \mathrm{W}$ Depth $3596 \mathrm{~m} / \mathrm{cose}$

Core condition FAIR.. INTAC BUT DRIED?m-Date Described 9MAV74 by J.RROCA.

Physiographic location MMALL SEATMOUNT. YUCATIN BASIN

Lithologic

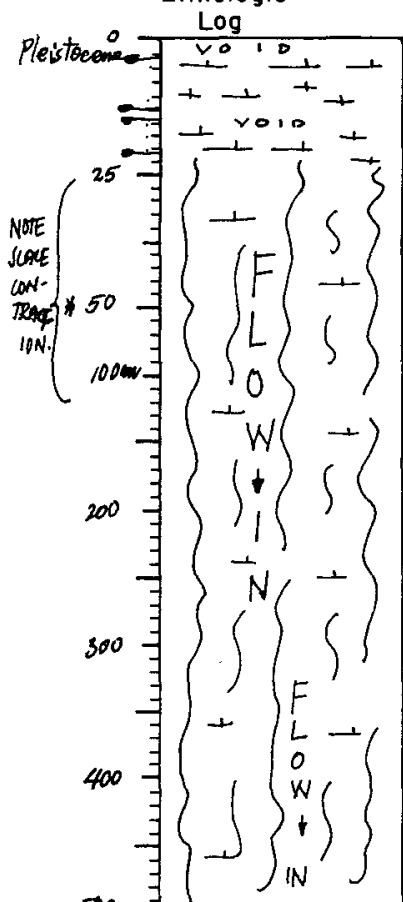

\section{Detailed Description}

$0-2$ VoID

$2-13$ CALC 002

1OYR $8 / 4$ v. pale brown

com and motting, pale browa

scattered forams in silty lutite

5 with void

13-16

$16-21$

CALC OOZE

1OYR $8 / 3 \mathrm{v}$. pale brown

distorted lams concave upward, pale brown

S horizontal

FLOW I

end of core

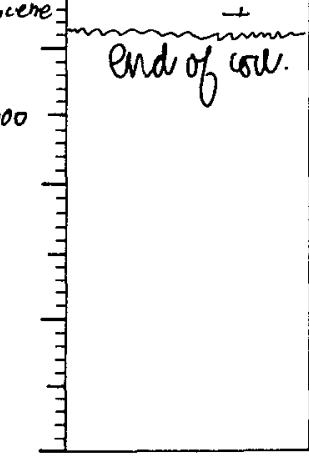

0172

SMEAR SLIDE DESCRIPTIONS - W.H.O.I. SEDIMENT CORES

Ship: Atlantis

Expedition 254

Leg No.

\begin{tabular}{|c|c|c|c|c|c|c|c|c|c|c|c|c|c|c|}
\hline \multirow[b]{4}{*}{ LEVEL } & \multirow[b]{4}{*}{$\begin{array}{l}\text { SEDIMENT } \\
\text { TYPE }\end{array}$} & \multicolumn{13}{|c|}{ ESTIMATED ABUNDANCES $(\%)$} \\
\hline & & \multirow{2}{*}{\multicolumn{4}{|c|}{$\frac{\text { Inorganic Material }}{\text { Sijt \& Sand }}$}} & \multirow[b]{3}{*}{$\frac{\vec{\pi}}{0}$} & \multirow{2}{*}{\multicolumn{5}{|c|}{$\begin{array}{l}\text { Biogenous Materi } \\
\text { Calcareous }\end{array}$}} & \multirow{2}{*}{\multicolumn{3}{|c|}{ Siliceous }} \\
\hline & & & & & & & & & & & & & & \\
\hline & & 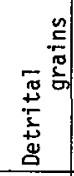 & 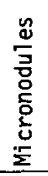 & 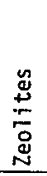 & 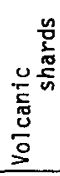 & & 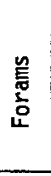 & 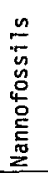 & 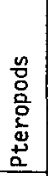 & 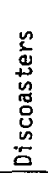 & 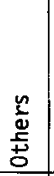 & 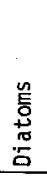 & 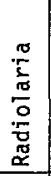 & 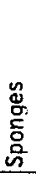 \\
\hline $2 \mathrm{~cm}$ & calc ooze & 2 & & & & 30 & 25 & 30 & & & 10 & & & 3 \\
\hline $20 \mathrm{~cm}$ & calc oos e & & & & & 50 & 15 & 30 & & & 5 & & & \\
\hline $535 \mathrm{~cm}$ & calc ooze & & & & & 45 & 35 & 35 & & & 5 & & & \\
\hline & & & & & & & & & & & & & & \\
\hline & & & & & & & & & & & & & & \\
\hline & & & & & & & & & & & & & & \\
\hline & & & & & & & & & & & & & & \\
\hline & & & & & & & & & & & & & & \\
\hline & & & & & & & & & & & & & & \\
\hline & & & & & & & & & & & & & & \\
\hline & & & & & & & & & & & & & & \\
\hline & & & & & & & & & & & & & & \\
\hline & & & & & & & & & & & & & & . \\
\hline & & & & & & & & & & & & & & \\
\hline & & & & & & & & & & & & & & \\
\hline & & & & & & & & & & & & & & \\
\hline & & & & & & & & & & & & & & \\
\hline
\end{tabular}

Core No. $\quad 3 \mathrm{PC}$

Station No.

Total Core Length $\quad 537 \quad \mathrm{~cm}$

\section{ESTIMATED ABUNDANCES (g)}


0173

VISUAL CORE DESCRIPTION

Page 1 of 1

Ship $A$ Cruise 254 Leg 3 sta. 326 Core No. $3 P G$

Total Length $56 \mathrm{~cm}$. Lat. $21^{\circ} 11.0^{\prime} \mathrm{N}$ Long. $82^{\circ} 50.0^{\circ} \mathrm{W}$ Depth $\frac{3596 \text { corr }}{9}$

Core condition PDOR DRIED ILAUESD Date Described 9MAY 74 by J.RROPA.

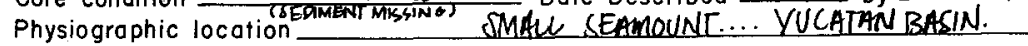

Lithologic

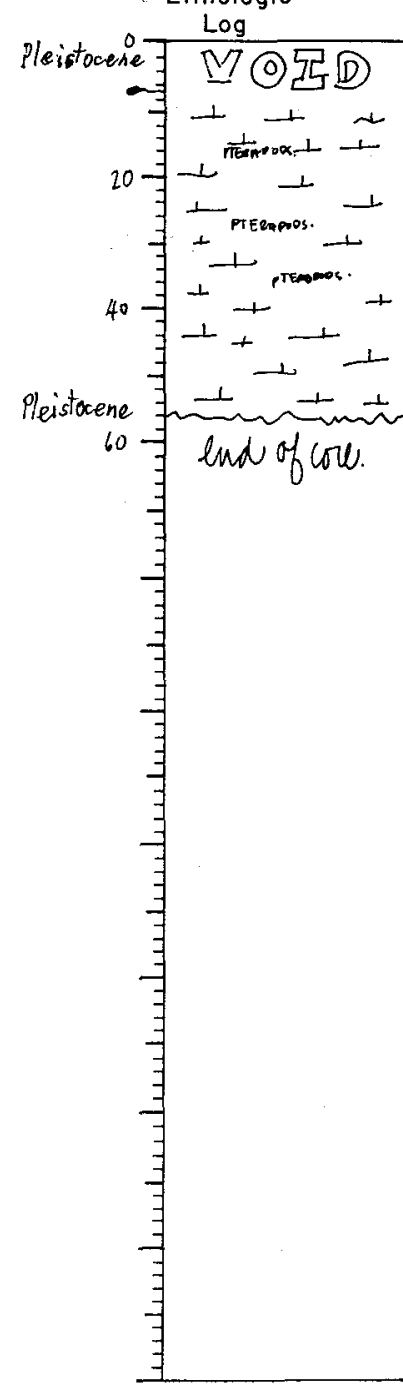

$0-7$

Detailed Description

VoID

due to condition of this core wher split it is the broken and separated segments of this sample

7-56

ALLC DOZE

IOYR $8 / 3$ v. pale brown

. sl motting, scatlered thin lans of white

scat tered lorams, silty litite

of core

0174

SMEAR SLIDE DESCRIPTIONS - W.H.0.I. SEDIMENT CORES

Ship: Atlantis

Expedition 254

Leg No. 3
Core No. 3 PG

Station No. 326

Total Core Length 56

ESTIMATED ABUNDANCES (\%)

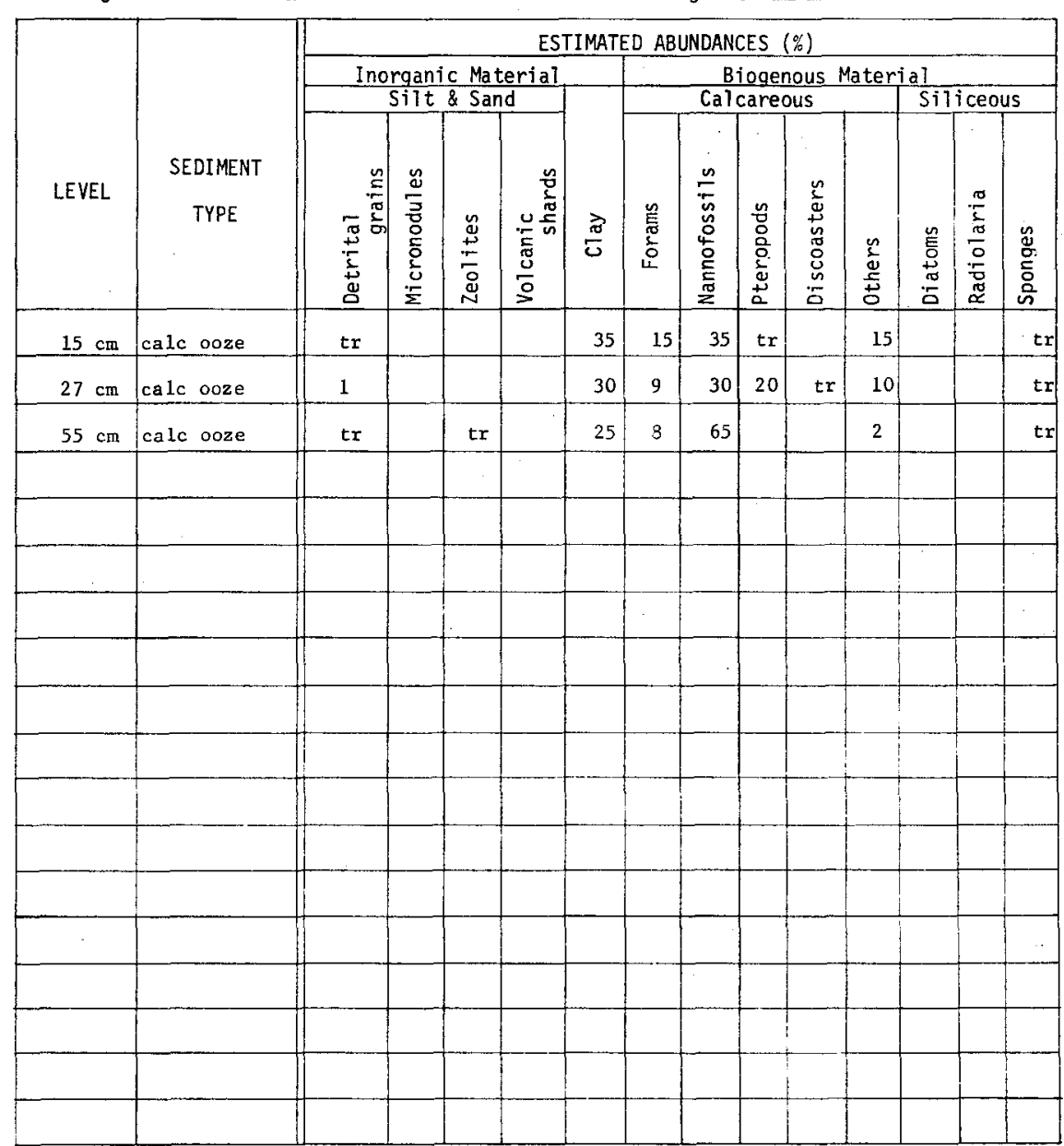


0175

VISUAL CORE DESCRIPTION

Ship A Cruise 254 Leg 3 sta. 327 Core No. 4PC Core condition COOD .. Smwhat duld. Dote Described 9MAY 14 by J.BRODA.

Physiograpic localion Lithologic

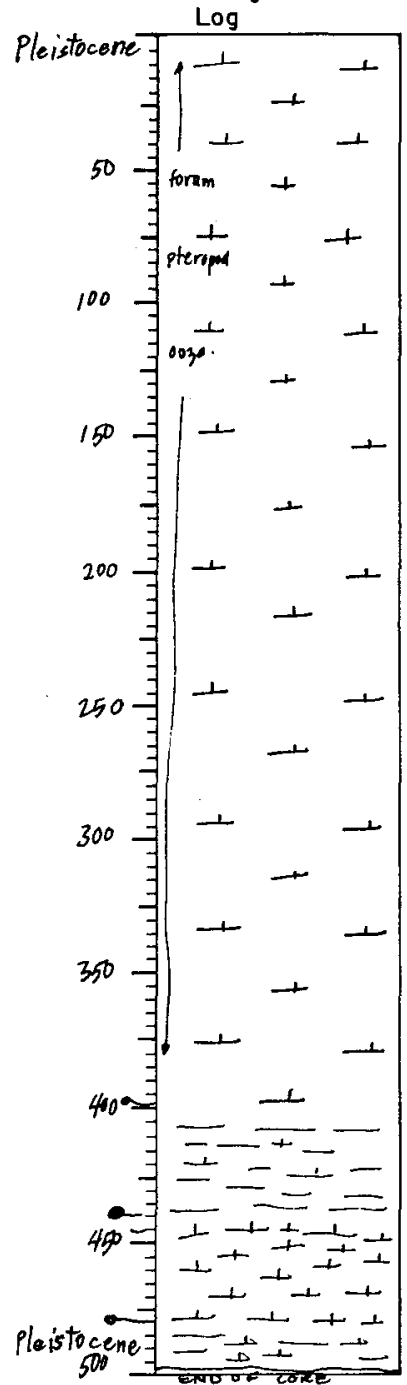

Detailed Description

0-397 CALC OOZE

$2.5 Y 7 / 21 t$ (5)

extremely abunt. forams \& pteropods :more pteropods extensive unit of foram $p$ teropod ooze. the relative amount of the two constituents fluctuates but the integrity of the unit remains constant throughout, wood-like seed-like black inclusions scattered throughout; mos $s$

97-440

SL CALC CLAY

$2.5 \mathrm{Y} 6 / 2$ it brownish gray

sl motting, white

440-480

CAIC DOZE

IOYR $8 / 1$ white

uniformly silty in textur

$\underset{8}{G}$

HLY CALC CLAY

common mottling, white

scattered forams

end of core

ene $=1$
0176

SMEAR SLIDE DESCRIPTIONS - W.H.O.I. SEDIMENT CORES

Ship: Atlantis

Expedition 254

Leg No. 3

327

Total Core Length $\quad 498$

\begin{tabular}{|c|c|c|c|c|c|c|c|c|c|c|c|c|c|c|}
\hline \multirow[b]{3}{*}{ LEVEL } & \multirow[b]{3}{*}{$\begin{array}{c}\text { SEDIMENT } \\
\text { TYPE }\end{array}$} & \multicolumn{13}{|c|}{ ESTIMATED ABUNDANCES (\%) } \\
\hline & & \multicolumn{4}{|c|}{$\begin{array}{c}\text { Inorganic Material } \\
\text { Silt \& Sand } \\
\end{array}$} & & \multicolumn{5}{|c|}{$\begin{array}{l}\text { Biogenous Mater } \\
\text { Calcareous }\end{array}$} & \multicolumn{3}{|c|}{ Siliceous } \\
\hline & & 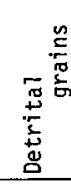 & 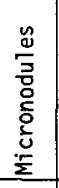 & 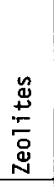 & 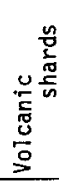 & $\stackrel{\pi}{\sigma}$ & $\begin{array}{l}\text { 号 } \\
\text { 产 } \\
\text { o }\end{array}$ & 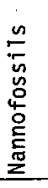 & 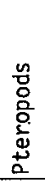 & 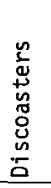 & $\begin{array}{l}0 \\
\frac{2}{2} \\
\frac{a}{2} \\
0 \\
\end{array}$ & 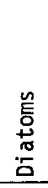 & 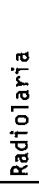 & 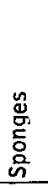 \\
\hline $1 \mathrm{~cm}$ & calc ooze & 8 & & - & & 30 & 25 & 15 & 15 & tr & 10 & & & \\
\hline $100 \mathrm{~cm}$ & calc ocze & 2 & & & & 18 & 45 & 12 & 20 & tr & 3 & & & \\
\hline $115 \mathrm{~cm}$ & ("wood" chip) & canr & lot b & sur & & & & & & & & & & \\
\hline $200 \mathrm{~cm}$ & calc ooze & 3 & & & & 13 & 40 & 10 & 28 & & 6 & & & \\
\hline $305 \mathrm{~cm}$ & calc ooze & 3 & & & & 25 & 33 & 12 & 22 & & 5 & & & \\
\hline $400 \mathrm{~cm}$ & calc clay & 3 & & 1 & & 80 & 2 & 8 & & & 6 & & & \\
\hline $455 \mathrm{~cm}$ & calc ooze & $\mathrm{tr}$ & & & & 30 & 45 & 8 & 2 & & 15 & & & \\
\hline $497 \mathrm{~cm}$ & calc ooze & 2 & & 1 & & 63 & 3 & 22 & tr & $\operatorname{tr}$ & 10 & & & $\mathrm{tr}$ \\
\hline & & & & & & & & & & & & & & \\
\hline & & & & & & & & & & & & & & \\
\hline & & & & & & & & & & & & & & \\
\hline & & & & & & & & & & & & & & \\
\hline & & & & & & & & & & & & & & \\
\hline & & & & & & & & & & & & & & \\
\hline & & & & & & & & & & & & & & \\
\hline & & & & & & & & & & & & & & \\
\hline & & & & & & & & & & & & & & \\
\hline & & & & & & & & & & & & & & \\
\hline
\end{tabular}


VISUAL CORE DESCRIPTION

Ship A Cruise 254 Leg 3 sta. -328 Core No. 5 PG Total Length $72 \mathrm{~cm}$. Lat. $20^{\circ} \mathrm{Oez} . \mathrm{W}$ Long. $84^{\circ} 11^{\circ} \mathrm{W}$ Depth 4568 cope $\mathrm{m}$

Core condition POOR-CRACKEDQRRED. Date Described IOMAYza by - Brodal Physiographic location VULATAN SASIN.

Lithologic

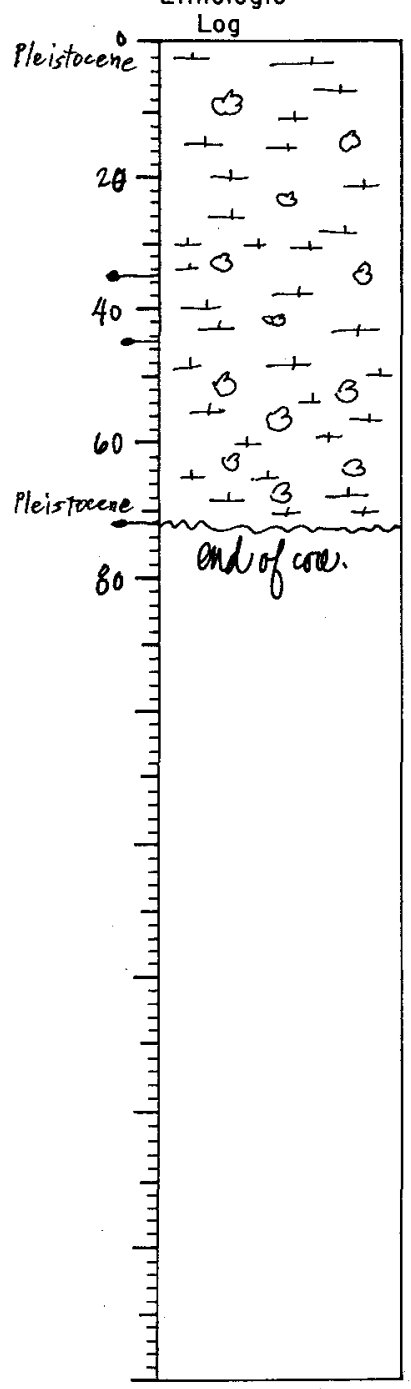

\section{Detailed Description}

v. sl mottling, with forams
0177

\section{8}

SMEAR SLIDE DESCRIPTIONS - W.H.O.I. SEDIMENT CORES

Note; Voids occur throughout this core and due to the core's condition when it was split it is impossible

core was described with the $25 \mathrm{~cm}$ of void spread

throughout with the assumption that the core has shrunk uniformly over 1ts legnth.

$0-35$

CALC OOZE

10YR $8 / 2$ white
sl mottling, v. pale brown

sl mottling,

few forams
$G$ textural

$35-45$

CALC OOZE

10YR $8 / 3$ v. pa le brown

abunt. forams

45-72

cate

IOYR $8 / 2$ whit

common mottling, v. pale brown

few forams

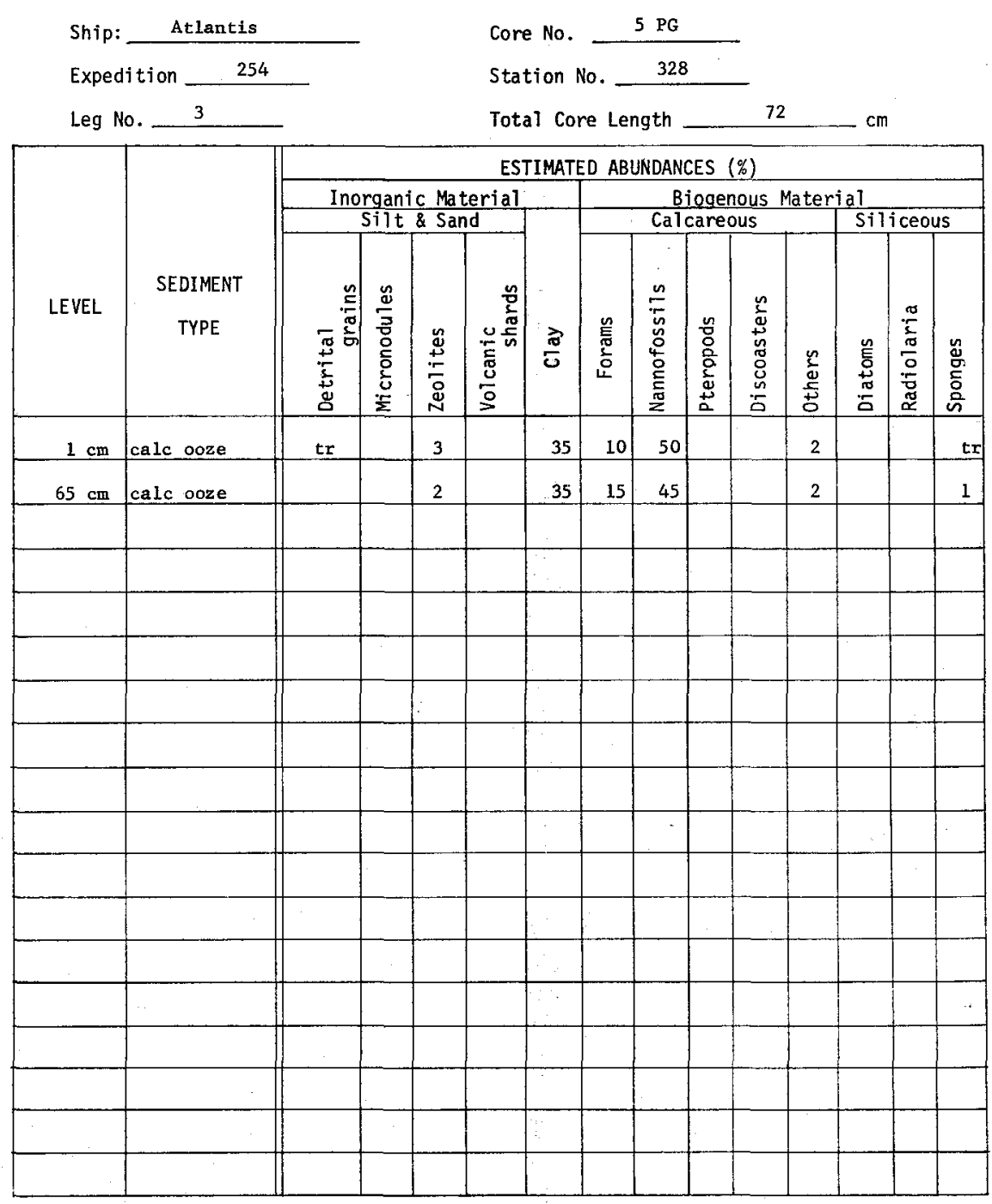


0179

VISUAL CORE DESCRIPTION

Page 1 of 1

Ship $\frac{A}{\text { Cruise } 254}$ Leg 3 sta. 330 Core No. 6 PC

Total Length $605 \mathrm{~cm}$. Lot. $19^{\circ} 35^{\prime N}$ Long. $84^{\circ} 51$ ' W D Depth 4579 conmma

Core condition 6000 Date Described IOMAY 74 by J.BROPA.

Physiographic location N. OF SWAN ISLANDS

Lithologic

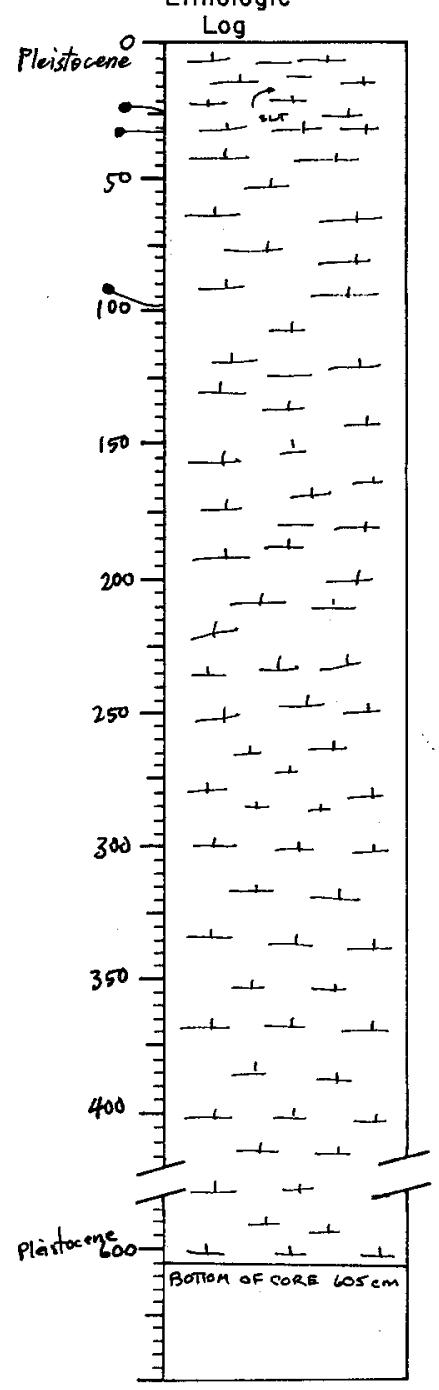

Detailed Description

$0-24$

CALC OOZE

(16-24 cm $\mathrm{S}, \mathrm{I} 5^{\circ}$

4-32

CALC OOZE

10YR $7 / 2$ It gray

somewhat silty

$32-96$

CALC OOZE

1OYR $8 / 2$ whit

smooth lutite

fafnt white laminations, $1 / 2 \mathrm{~cm}$ in thickness

$G$
-605

CAIC OOZE

$2.5 \mathrm{Y} 7 / 21 \mathrm{t}$ gray

sediment between $287-300 \mathrm{~cm}$ missing

Do lithologic features of any sort. suspect there's a

possibility of flow-in here although no sign of vertical striations are visible.

end of core

SMEAR SLIDE DESCRIPTIONS - W.H.O.1 SEDIMENT COPES

Ship: Athntis

Expedition 254

Leg No. 3

\begin{tabular}{|c|c|c|c|c|c|c|c|c|c|c|c|c|c|c|}
\hline \multicolumn{2}{|c|}{ Leg No. 3} & \multicolumn{13}{|c|}{ Total Core Length } \\
\hline \multirow[b]{3}{*}{ LEVEL } & \multirow[b]{3}{*}{$\begin{array}{c}\text { SEDIMENT } \\
\text { TYPE }\end{array}$} & \multicolumn{13}{|c|}{ ESTIMATED ABUNDANCES $(\%)$} \\
\hline & & \multicolumn{4}{|c|}{$\begin{array}{c}\text { Inorganic Material } \\
\text { Silt \& Sand }\end{array}$} & \multirow[b]{2}{*}{$\frac{\pi}{\sigma}$} & \multicolumn{5}{|c|}{$\begin{array}{l}\text { Biogenous Mater } \\
\text { Calcareous }\end{array}$} & \multicolumn{3}{|c|}{ Siliceous } \\
\hline & & 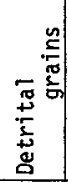 & 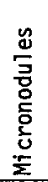 & $\begin{array}{l}\stackrel{y}{ \pm} \\
\frac{0}{8} \\
\text { N }\end{array}$ & 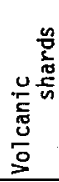 & & 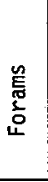 & 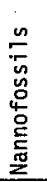 & 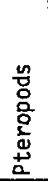 & 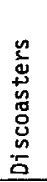 & 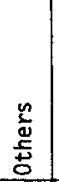 & 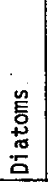 & 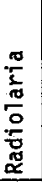 & 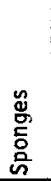 \\
\hline $1 \mathrm{~cm}$ & calc ooze & & & 2 & & 40 & 18 & 35 & & tr & 4 & & & 1 \\
\hline $28 \mathrm{~cm}$ & calc ooze & $\operatorname{tr}$ & & 2 & & 40 & 7 & 40 & & $\operatorname{tr}$ & 11 & & & $\mathrm{tr}$ \\
\hline $90 \mathrm{~cm}$ & calc ooze & & & 1 & & 60 & 5 & 30 & & & 4 & & & $t x$ \\
\hline $250 \mathrm{~cm}$ & calc ooze & tr & & 2 & $\operatorname{tr}$ & 45 & 3 & 46 & & 1 & 3 & & & $\operatorname{tr}$ \\
\hline $500 \mathrm{~cm}$ & calc ooze & & & 1 & & 45 & 5 & 45 & & 1 & 3 & & & $\mathrm{tr}$ \\
\hline $605 \mathrm{~cm}$ & calc ooze & $\operatorname{tr}$ & & 1 & & 35 & 2 & 35 & & tr & 2 & & & $\mathrm{tx}$ \\
\hline & & & & & & & & & & & & & & \\
\hline & & & & & & & & & & & & & & \\
\hline & & & & & & & & & & & & & & \\
\hline & & & & & & & & & & & & & & \\
\hline & & & & & & & & & & & & & & \\
\hline & & & & & & & & & & & & & & \\
\hline & & & & & & & & & & & & & & \\
\hline & & & & & & & & & & & & & & $\ldots$ \\
\hline & & & & & & & & & & & & & & \\
\hline & & & & & & & & & & & & & & \\
\hline & & & & & & & & & & & & & & \\
\hline & & & & & & & & & & & & & & \\
\hline
\end{tabular}

Core No. 6 PC

Core No.

Station No. 330

Total Core Length 605

ESTIMATEO ABUNDANCES $(\%)$ 


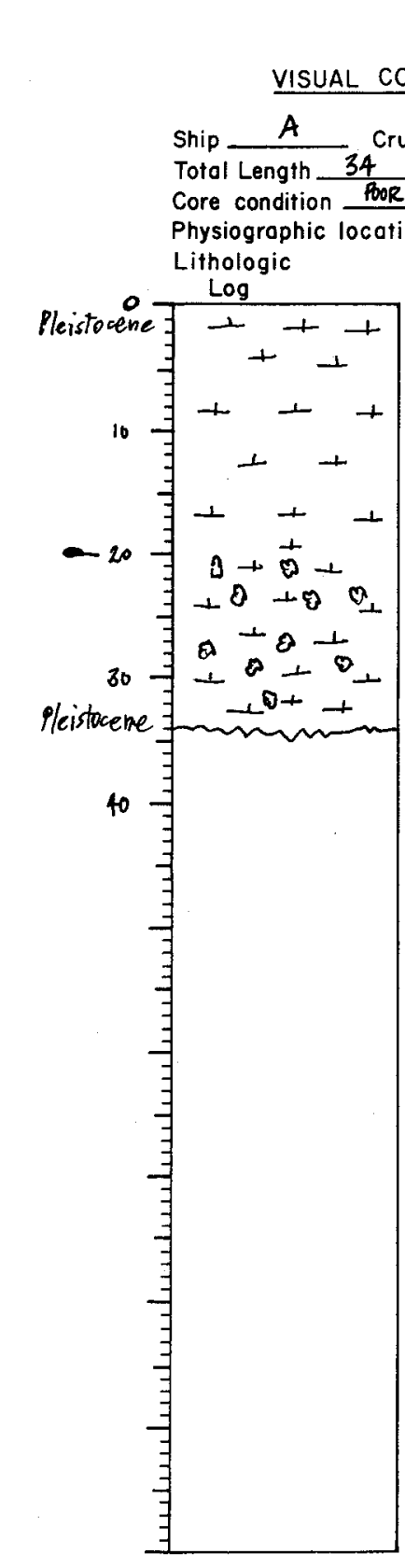

Page _ _ I of _ 1

Total Length $34 \quad \mathrm{~cm}$. Lat. $19^{\circ} 35^{\mathrm{rN}}$ Long. $\frac{35 \mathrm{~S} / \mathrm{W}}{\mathrm{c}}$ Depth 4579 merere Core condition PBOR..ORY. CRAxEQ Date Described 13MY74 by T.BRODA.

Detailed Description

$0-20$

CALC OOZE

in lutite, no foram

$S$ concave upward

20-34 CA LC OOZE

1OYR $7 / 2$ 1t gray

ing, 1t brownish gray

ed foram

end of core
SMEAR SLIDE DESCRIPTIONS - W.H.O.I. SEDIMENT CORES

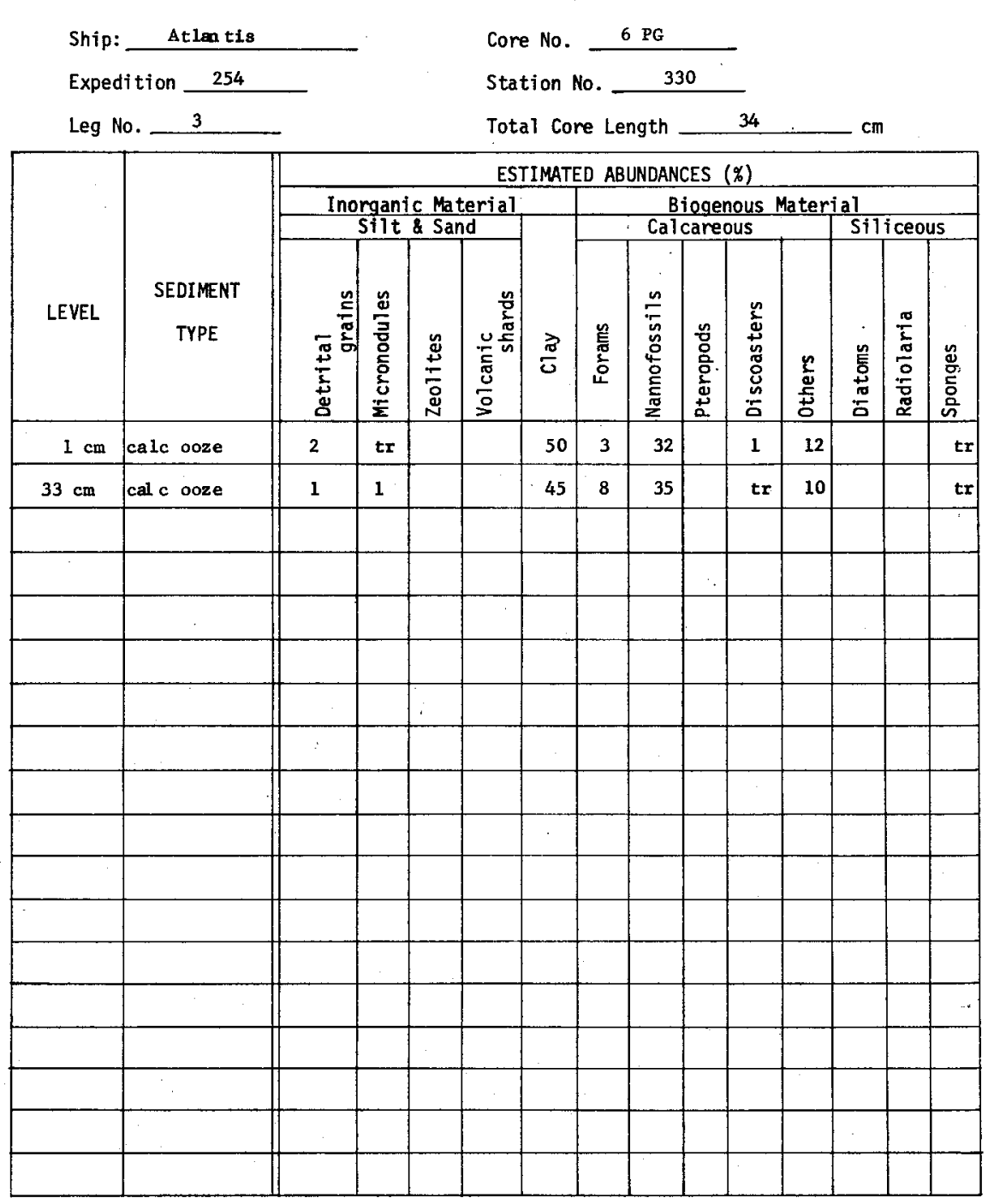


VISUAL CORE DESCRIPTION Ship A Cruise 254 Leg 3 sta. 331 Core No. $7 \mathrm{PC}$

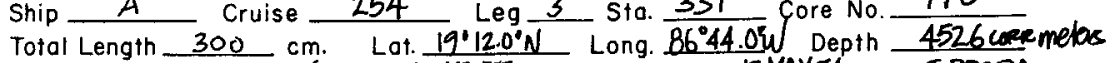

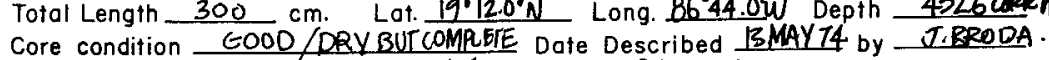
Physiographic location OFF YUCATAN PENINSUCA.

Lithologic

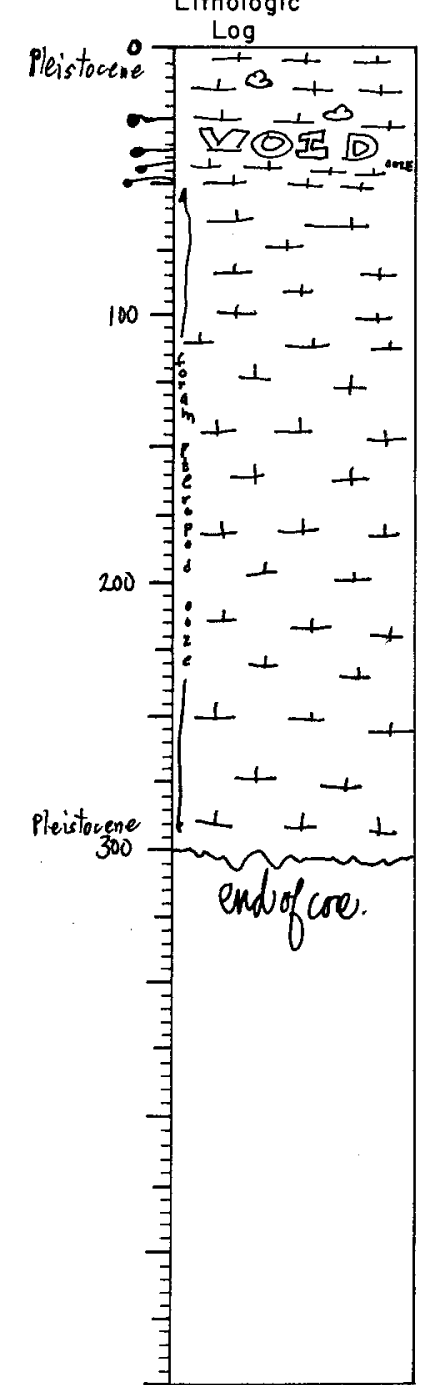

32-42 CATC OOZ

thin lams, 1t brownish gray

1OYR $8 / 2$ thite
0183

Page 1 of 1

Detailed Description

0184

SMEAR SLIDE DESCRIPTIONS - W.H.O.I. SEDIMENT CORES

$0-25$

CAIC OOZE

IOYR $8 / 3 \mathrm{v}$. pale brown

s1 mottling, white

scattered forams in somewhat silty lutite

VOID

CAIC OOZE

10YR $8 / 2$ white

foram ptexopod ooze

42-47

CAIC OZE

1OYR $8 / 2$ white

$S$ inclined $25^{\circ}$

47-300

CALC OOZE

forsm pteropod ooze, a bit coarser $220-300 \mathrm{~cm}$, more

end of core
Ship: At1amtis

Expedition 254

Leg No. 3

\begin{tabular}{|c|c|c|c|c|c|c|c|c|c|c|c|c|c|c|}
\hline Leg & 3 & & & & Tot: & $1 \mathrm{Co}$ & Le & gth & & 00 & & $\mathrm{~cm}$ & & \\
\hline \multirow[b]{3}{*}{ LEVEL } & \multirow[b]{3}{*}{$\begin{array}{c}\text { SEDIMENT } \\
\text { TYPE }\end{array}$} & \multicolumn{13}{|c|}{ ESTIMATEO ABUNDANCES (\%) } \\
\hline & & \multicolumn{4}{|c|}{$\frac{\text { Inorganic Material }}{\text { Silt \& Sand }}$} & \multirow[b]{2}{*}{$\stackrel{\frac{\pi}{\sigma}}{\sigma}$} & \multicolumn{5}{|c|}{$\begin{array}{l}\text { Biogenous Mater } \\
\text { Calcareous }\end{array}$} & \multicolumn{3}{|c|}{$\frac{21}{\text { Siliceous }}$} \\
\hline & & 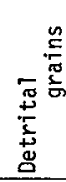 & 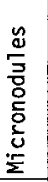 & 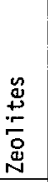 & 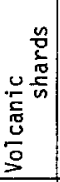 & & 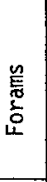 & 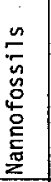 & 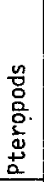 & 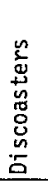 & 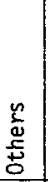 & 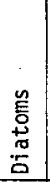 & 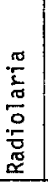 & 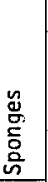 \\
\hline $2 \mathrm{~cm}$ & cale ooze & & & tr & & 25 & 12 & 55 & 5 & & 3 & & & \\
\hline $45 \mathrm{~cm}$ & calc ooze & $\operatorname{tr}$ & 1 & & & 45 & 5 & 42 & $\operatorname{tr}$ & & 7 & & & \\
\hline $100 \mathrm{~cm}$ & calc ooze & 1 & $\operatorname{tr}$ & & & 15 & 35 & 10 & 30 & 1 & 8 & & & \\
\hline $200 \mathrm{~cm}$ & calc ooze & 1 & & & & 10 & 40 & 10 & 35 & & 4 & & & $\mathrm{tr}$ \\
\hline $298 \mathrm{~cm}$ & calc ooze & & & & & 6 & 35 & 4 & 40 & $\operatorname{tr}$ & 15 & & & $\mathrm{tr}$ \\
\hline & & & & & & & & & & & & & & \\
\hline & & & & & & & & & & & & & & \\
\hline & & & & & & & & & & & & & & \\
\hline & & & & & & & & & & & & & & \\
\hline & & & & & & & & & & & & & & \\
\hline & & & & & & & & & & & & & & \\
\hline & & & & & & & & & & & & & & \\
\hline & & & & & & & & & & & & & & \\
\hline & & & & & & & & & & & & & & \\
\hline & & & & & & & & & & & & & & \\
\hline & & & & & & & & & & & & & & \\
\hline & & & & & & & & & & & & & & \\
\hline & & & & & & & & & & & & & & \\
\hline
\end{tabular}

Core No. 7 PC

Station No. 331

Station No.

1 Core Length 300 
0185

VISUAL CORE DESCRIPTION

Page 1 of 1

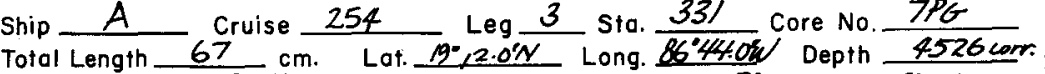

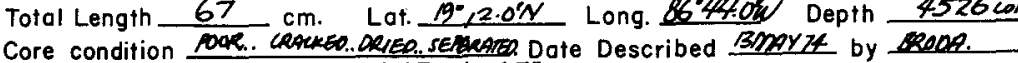

Physiographic location OFF YUCATAN PENINSUCA.

Lithologic

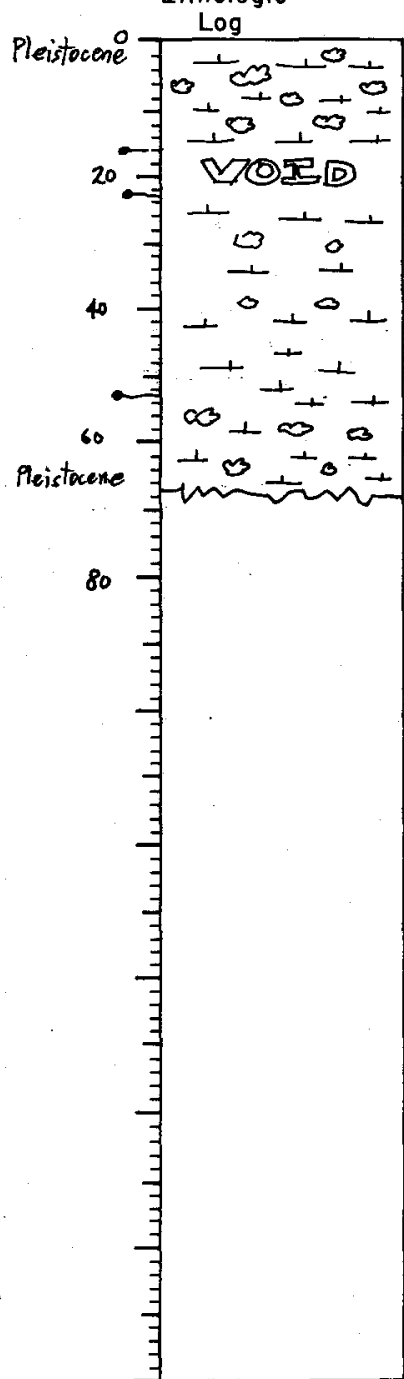

CAIC OOZE

chray

scattered forams in lutite

16-23 VOID

23-53

CAIC OOZE

IOYR $7 / 2$ It gray

abute extremely abunt. $S, I 5$

CALC OOZE

IOYR $7 / 3 \mathrm{v}$. pale brown

extensive mottling, white, v. pale brown..lutite end of core

\section{Detailed Description}

al mottling, white with forams

\section{6}

SMEAR SLIDE DESCRIPTIONS - W.H.O.I. SEDIMENT CORES

\begin{tabular}{|c|c|}
\hline Ship: Atlantis & Core No. \\
\hline Expedition $\quad 254$ & Station No. \\
\hline
\end{tabular}

Leg No. 3 Total Core Length $\quad 67 \quad \mathrm{~cm}$

\begin{tabular}{|c|c|c|c|c|c|c|c|c|c|c|c|c|c|c|}
\hline \multirow[b]{4}{*}{ LEVEL } & \multirow[b]{4}{*}{$\begin{array}{c}\text { SEDIMENT } \\
\text { TYPE }\end{array}$} & \multicolumn{13}{|c|}{ ESTIMATED ABUNDANCES $(\%)$} \\
\hline & & \multirow{2}{*}{\multicolumn{4}{|c|}{$\begin{array}{c}\text { Inorganic Material } \\
\text { Silt \& Sand }\end{array}$}} & \multirow[b]{3}{*}{$\frac{\vec{\epsilon}}{\sigma}$} & \multirow{2}{*}{\multicolumn{5}{|c|}{$\begin{array}{l}\text { Biogenous Mater } \\
\text { Calcareous }\end{array}$}} & & & \\
\hline & & & & & & & & & & & & \multicolumn{3}{|c|}{ Siliceous } \\
\hline & & 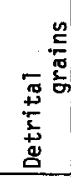 & 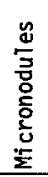 & 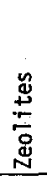 & 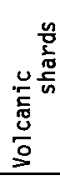 & & 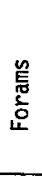 & 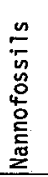 & 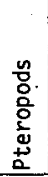 & 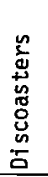 & 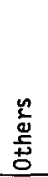 & 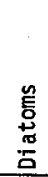 & $\begin{array}{l}\frac{\pi}{2} \\
\frac{\pi}{\pi} \\
\overline{0} \\
\frac{0}{\pi} \\
\tilde{x} \\
\end{array}$ & 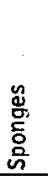 \\
\hline $3 \mathrm{~cm}$ & calc ooze & & & & & 55 & 20 & 15 & tr & & 10 & & & \\
\hline $32 \mathrm{~cm}$ & calc ooze & tr & & & & 25 & 35 & 20 & 15 & & 5 & & & tr \\
\hline $.66 \mathrm{~cm}$ & calc ooze & & & & & 55 & 10 & 30 & $\mathrm{tr}$ & & 3 & & & 2 \\
\hline & & & & & & & & & & & & & & \\
\hline & & & & & & & & & & & & & & \\
\hline & & & & & & & & & & & & & & \\
\hline & & & & & & & & & & & & & & \\
\hline & & & & & & & & & & & & & & \\
\hline & & & & & & & & & & & & & & \\
\hline & & & & & & & & & & & & & & \\
\hline & & & & & & & & & & & & & & \\
\hline & & & & & & & & & & & & & & \\
\hline & & & & & & & & & & & & & & \\
\hline & & & & & & & & & & & & & & \\
\hline & & & & & & & & & & & & & & \\
\hline & & & & & & & & & & & & & & \\
\hline & & & & & & & & . & & & & & & \\
\hline & & & & & & & & & & & & & & \\
\hline
\end{tabular}


0187

VISUAL CORE DESCRIPTION

Page 1 of 2

Ship $A$ Cruise 254 Leg 3 sta. 333 Core No. 8 PC Total Length $292 \mathrm{~cm}$. Lat. $18^{\circ} 29.0^{\prime} \mathrm{N}$ Long. $86^{\circ} 20.0^{\prime} \mathrm{W}$ Depth $4402 \mathrm{~m} \mathrm{Caen}$

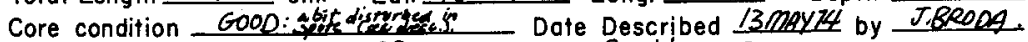
Physiographic location of Hendues Cacibhen Sea

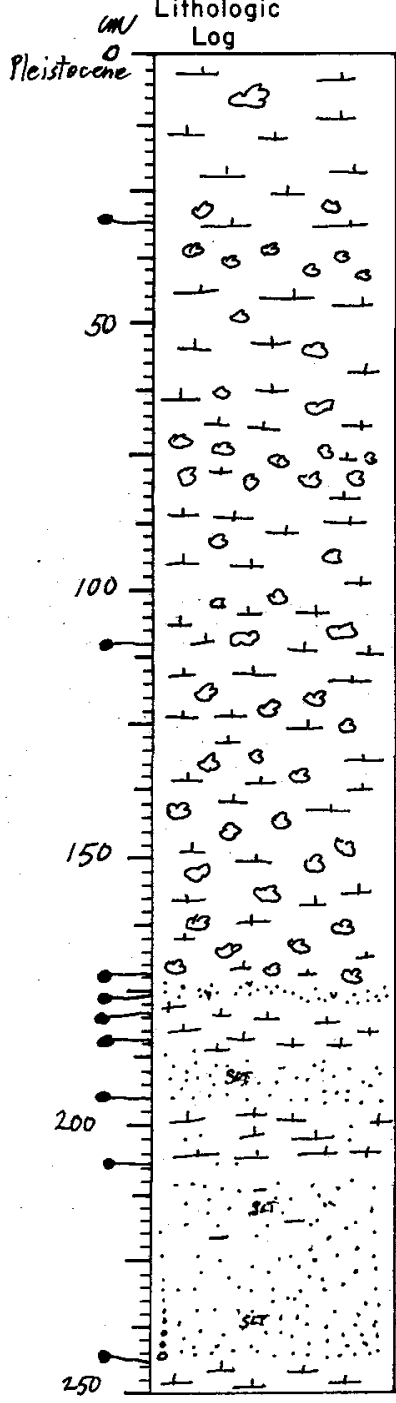

Detailed Description

$0-31$

CAIC OOZE

$10 x$ mot

forams except 21-30 cm where v. abunt.

31-11

CALC

1OYR $7 / 3$ v. pa le browa

extensive mottling, $31-40,71-80 \mathrm{~cm}$, white, common eksawhere

flecks, 104-110 ca

CAIS OOZ

1OYR $7 / 2$ it gray

extensive small mottling throughout, white, pale brown

scattered forams

scattered shell fragments

175

\section{$5 Y 6 / 2$ 1t oifive gray}

fine det sand

$S$ horizontal

175.5-179

IOYR $8 / 1$ whit

silty lutite

S, concave upward

179-184

CALC OOZE

10YR $7 / 2$ it gray

v. sandy lut

184-195

DET

1OYR $7 / 4 \mathrm{v}$. pale brown, $8 / 1$ white

note this portion of core disturbed and broken laxge $G$

95-207

CALC OOZE

silty lutite with white fleck

some lams of silt

207-247

$2.5 \times 6 / 4$ lt yellowish brown

disturbed due to the dry
0188

ISUAL CORE DESCRIPTION

Page 2 of 2

Ship $A$ Cruise 254 Leg 3 Sta. 333 Core No. 8R
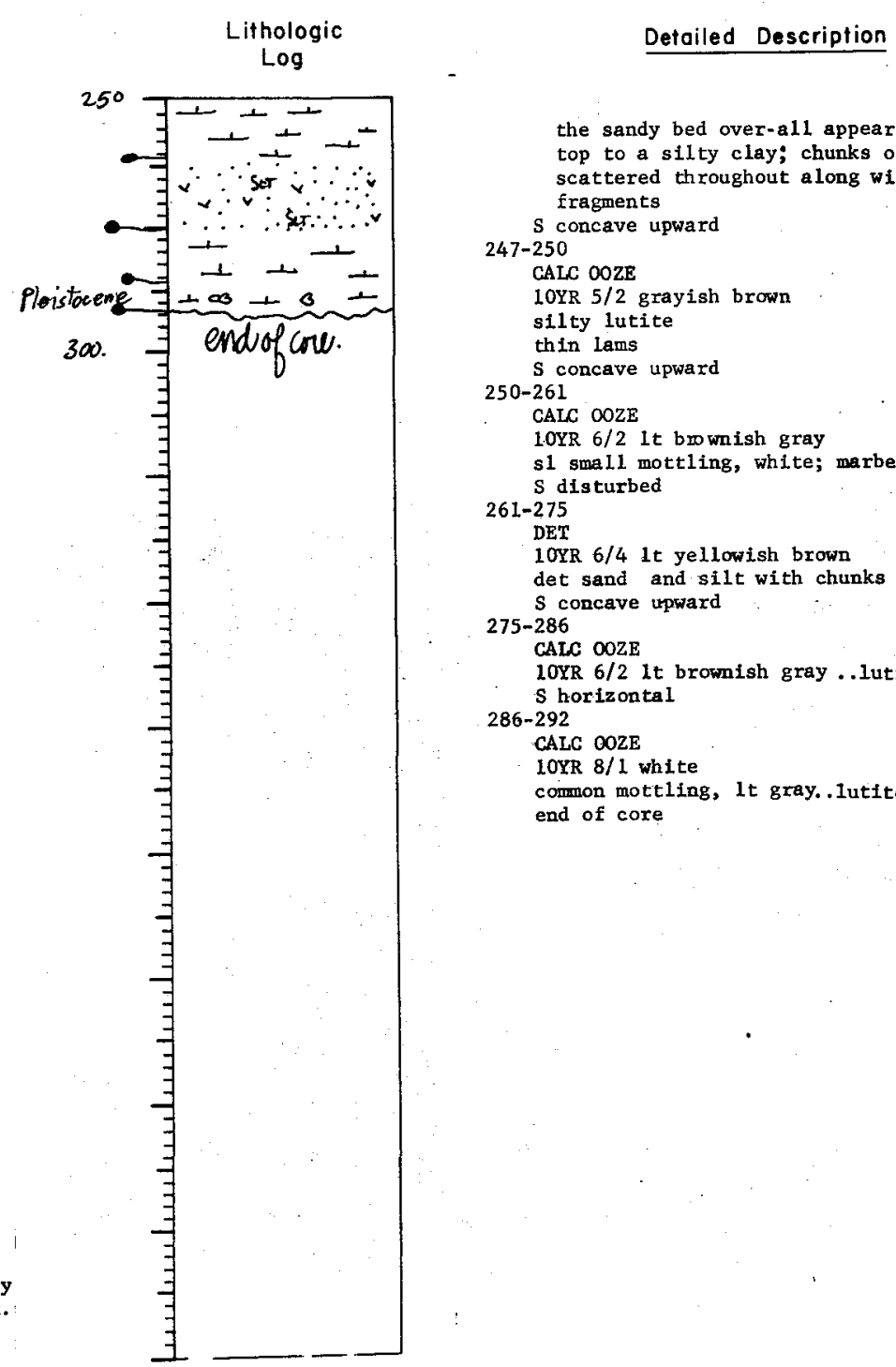

the sandy bed over-all appears to be graded coarse on top to a silty clay; chunk of calc ooze and clay are fragments

247-250 concave upwar

CALC $00 Z$

1OYR $5 / 2$ grayish brown

silty lutit thin lams

S concave upward

CAIC OOZE

1.OYR $6 / 2$ 1t brownish gray

s1 smaIl mottling, white; marbelling...lutite

261-275

1OYR 6/4 1t yellowish brown

det sand and silt with chunks of clay scattered throughou $S \cos$
$75-286$

CAIC OOZE

10YR $6 / 2$ it brownish gray ..lutite

$S$ horizontal

286-292

CALC OOZE

1OYR 8/1 white

common mottling, it gray..lutite 


\section{3}

SMEAR SLIDE DESCRIPTIONS - W.H.O.I. SEDIMENT CORES

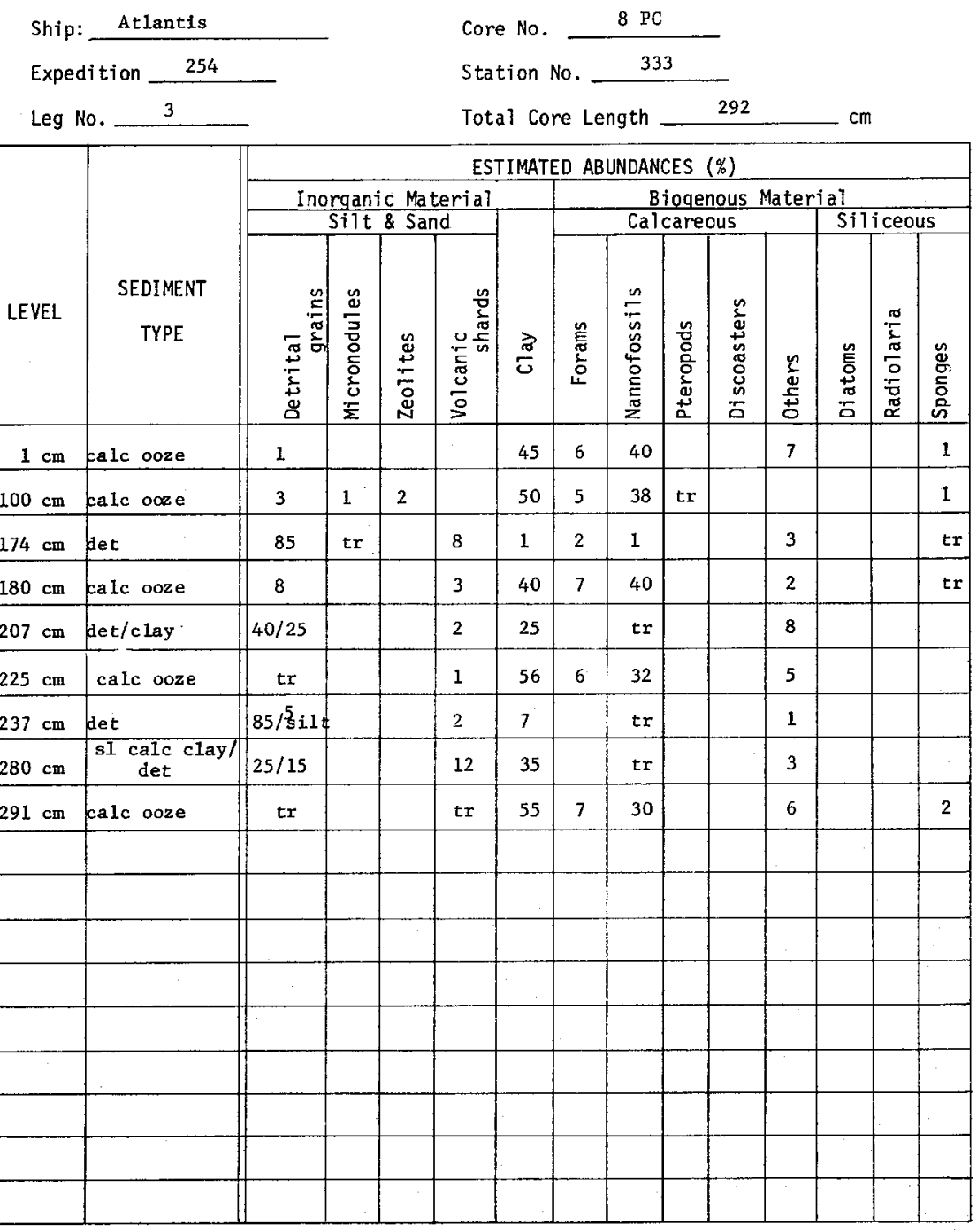

0190

VISUAL CORE DESCRIPTION

Poge 1 of 1

Ship A Cruise 254 Leg 3 sta. 333 Core No. 8PG Total Length $76 \mathrm{~cm}$. Lat. $18^{\circ} 29^{\circ} . \mathrm{N}$ Long. $86^{\circ} 20.0 \mathrm{~N}$ Depth $4402 \mathrm{~m}$. ConR.

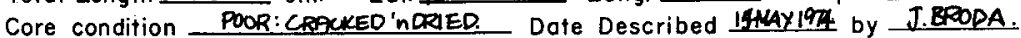

Physiographic location off Handuras, Caribbean Sea Lithologic

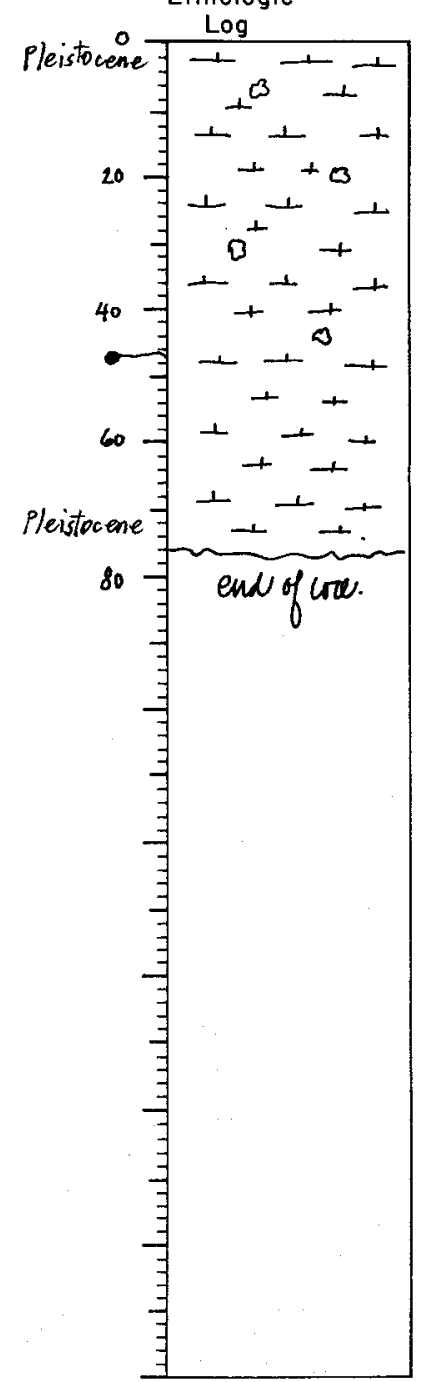

Detailed Description $0-47$ CALC OOZE IOYR $8 / 2$ white abunt. forams $0-18 \mathrm{~cm}$, scattered $19-47 \mathrm{~cm}$

47-76 CAIC OOZE

pale brown scattered forams and large shell fragments nd of core 
0191

SMEAR SLIDE DESCRIPTIONS - W.H.O.I. SEDIMENT CORES

Ship: Athntis

Core No. $\quad 8$ PG

Expedition 254

Leg No. 3

Station No. 333

Total Core Length 76

\begin{tabular}{|c|c|c|c|c|c|c|c|c|c|c|c|c|c|c|}
\hline \multirow[b]{4}{*}{ LEVEL } & \multirow[b]{4}{*}{$\begin{array}{c}\text { SEDIMENT } \\
\text { TYPE }\end{array}$} & \multicolumn{13}{|c|}{ ESTIMATED ABUNDANCES $(\%)$} \\
\hline & & \multirow{2}{*}{\multicolumn{4}{|c|}{$\begin{array}{c}\text { Inorganic Material } \\
\text { Silt \& Sand } \\
\end{array}$}} & \multirow[b]{3}{*}{$\frac{\pi}{0}$} & \multirow{2}{*}{\multicolumn{5}{|c|}{$\begin{array}{l}\text { Biogenous Mate } \\
\text { Calcareous }\end{array}$}} & \multirow{2}{*}{\multicolumn{3}{|c|}{ SiTiceous }} \\
\hline & & & & & & & & & & & & & & \\
\hline & & 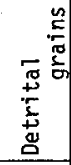 & 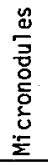 & 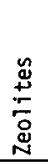 & 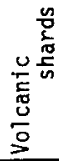 & & 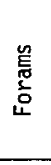 & 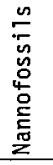 & 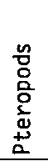 & 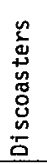 & 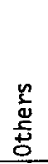 & 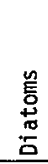 & $\begin{array}{l}\frac{\pi}{2} \\
\frac{\pi}{0} \\
\frac{0}{0} \\
\frac{\pi}{\alpha} \\
\end{array}$ & 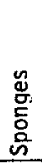 \\
\hline $1 \mathrm{~cm}$ & calc ooze & & & & & 50 & 15 & 30 & & tr & 4 & & & 1 \\
\hline $75 \mathrm{~cm}$ & calc ooze & tr & & tr & & 28 & 12 & 55 & & & 4 & & & 1 \\
\hline & & & & & & & & & & & & & & \\
\hline & & & & & & & & & & & & & & \\
\hline & & & & & & & & & & & & & & \\
\hline & & & & & & & & & & & & & & \\
\hline & & & & & & & & & & & & & & \\
\hline & & & & & & & & & & & & & & \\
\hline & & & & & & & & & & & & & & \\
\hline & & & & & & & & & & & & & & \\
\hline & & & & & & & & & & & & & & \\
\hline & & & & & & & & & & & & & & \\
\hline & & & & & & & & & & & & & & \\
\hline & & & & & & & & & & & & & & \\
\hline & & & & & & & & & & & & & & \\
\hline & & & & & & & & & & & & & & \\
\hline & & & & & & & & & & & & & & \\
\hline & & & & & & & & & & & & & & \\
\hline
\end{tabular}

0192

VISUAL CORE DESCRIPTION

Page__ 1 of 1

Ship A 254 Chise 334 Sore No Total Length $61^{1} \mathrm{~cm}$. Lat. $17^{\circ} 52.0^{2} \mathrm{~N}$ Long. $\frac{36^{\circ} 15.04 \mathrm{~N}}{\text { Depth }} 2440 \mathrm{~cm}$ Core condition POOR. CRACKEO \&DRIEO. Date Described 15MAY74 by J.froda.

Physiographic location off Honduras, Caribbean Sea
Lithologic
Detailed Description

Pleistocene $\frac{\log }{1}$

\section{$1+1,0-61$ CALC OOZE \\ IOYR $8 / 2$ white}

scattered forams throughout, except 18-34 cm and 41-43 cm

where they are abunt with pteropod fragments

$20\}+1$

end of core

$1+-1$

40

$+-1+$

Pleistocene

60 expof core

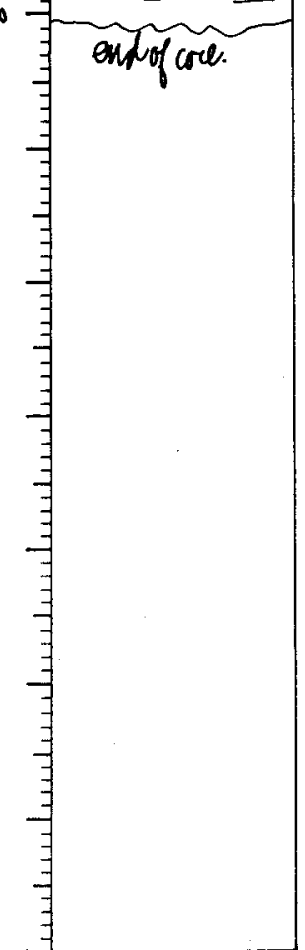


0193

SMEAR SLIDE DESCRIPTIONS - W.H.O.I. SEDIMENT CORES

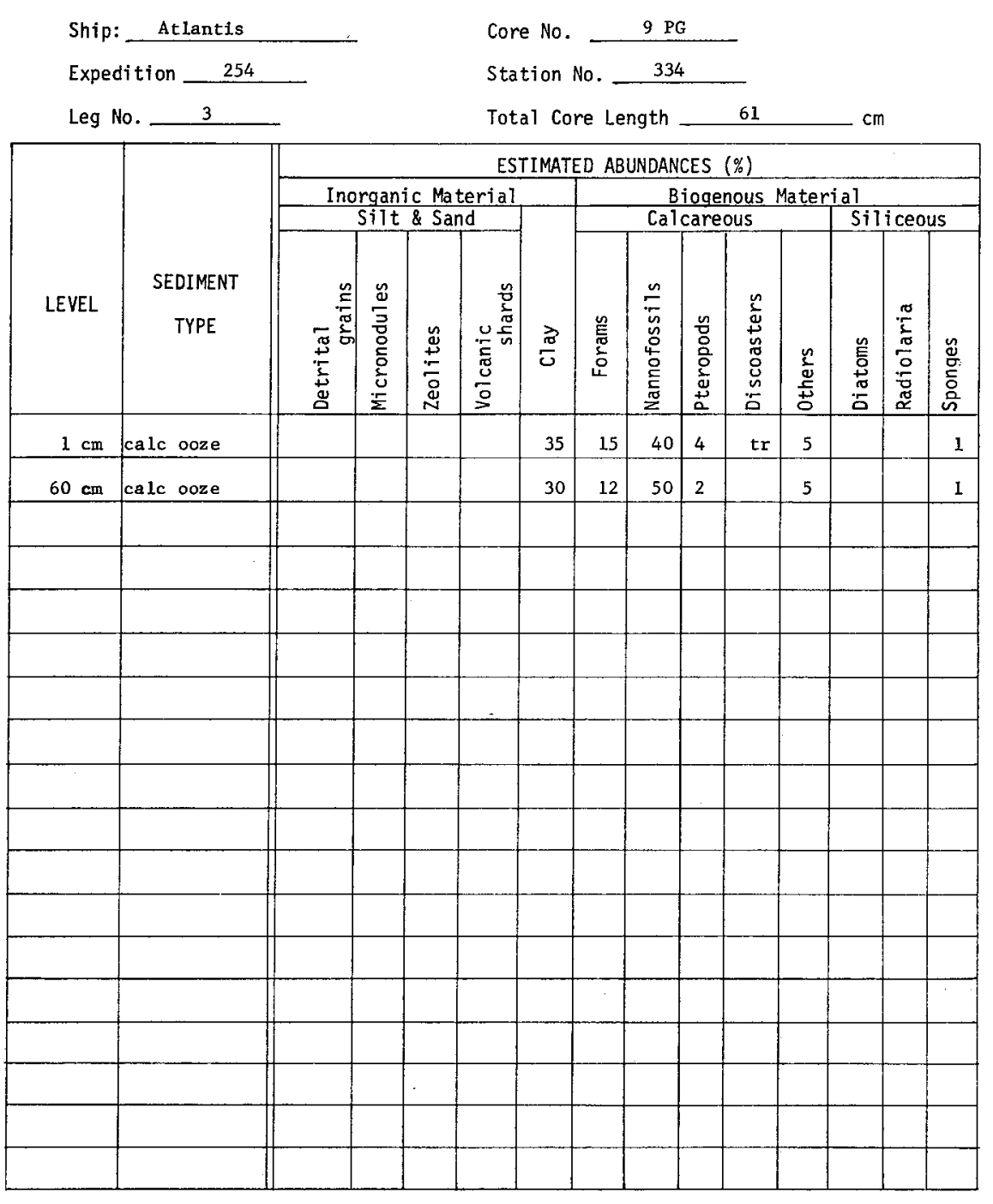

0194

VISUAL CORE DESCRIPTION

Page 1 of 1

Ship A Cruise 254 Leg 3 sta. 335 Core No. 10 PG Total Length $66 \quad \mathrm{~cm}$. Lat. $16^{\circ} 29.0^{\circ} \mathrm{N}$ Long. $86^{\circ} 34.5^{\circ} \mathrm{W}$ Depth 2791 con.m. Core condition POOR.. DRIED.. CRACKED. Dote Described ISMAY74 by J.RROOA. Physiographic location off Honduras, Caribbean Sea

Lithologic

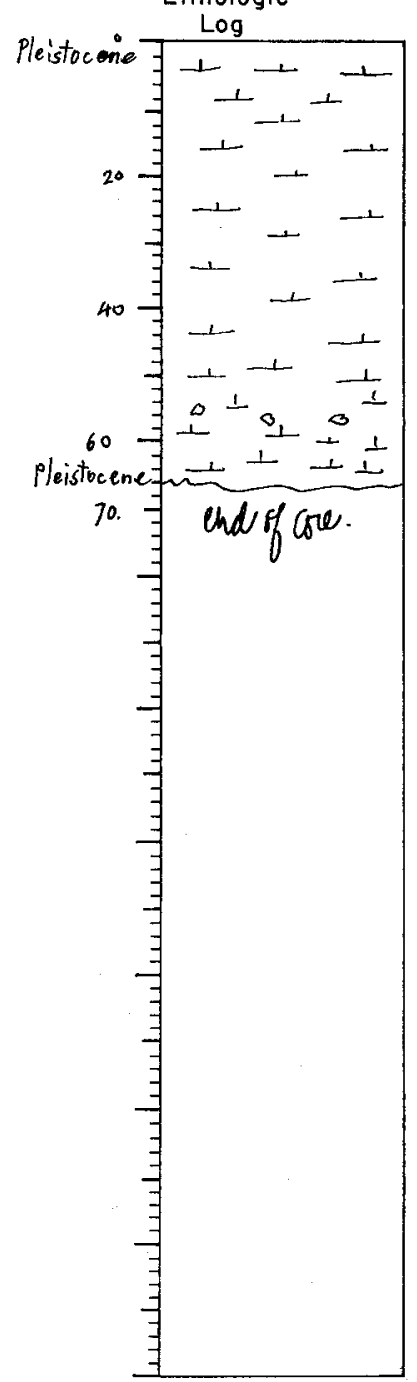

$0-66$

\section{Detailed Description}

CALC OOZE

OYR $7 / 3$ v. pale brown

1 1 sma 11 mottling 55-60 cm, white

scattered $0-29 \mathrm{~cm}$ abund. $29-50 \mathrm{~cm}$, and small shell fragments 
SMEAR SLIDE DESCRIPTIONS - W.H.O.I. SEDIMENT CORES

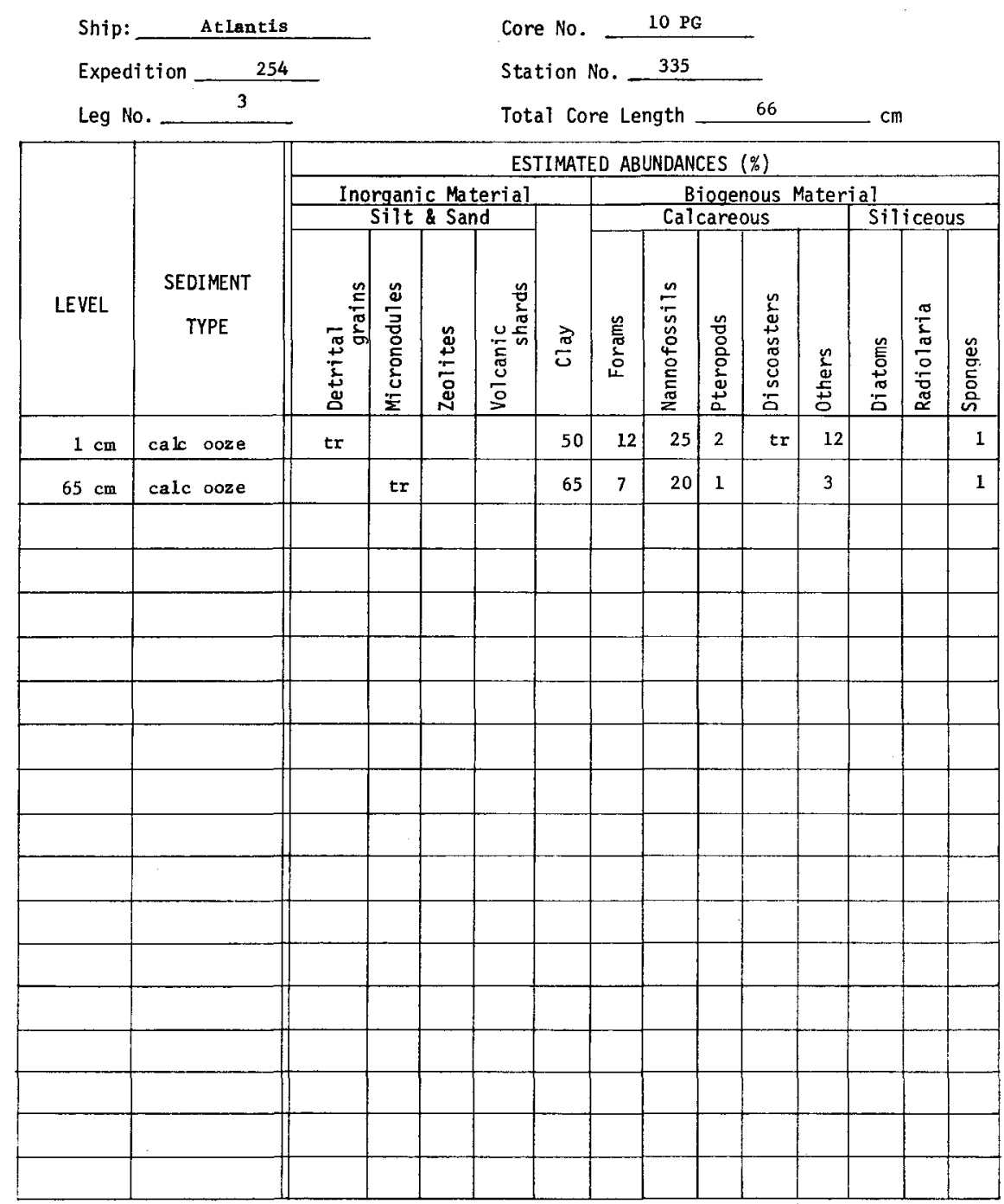




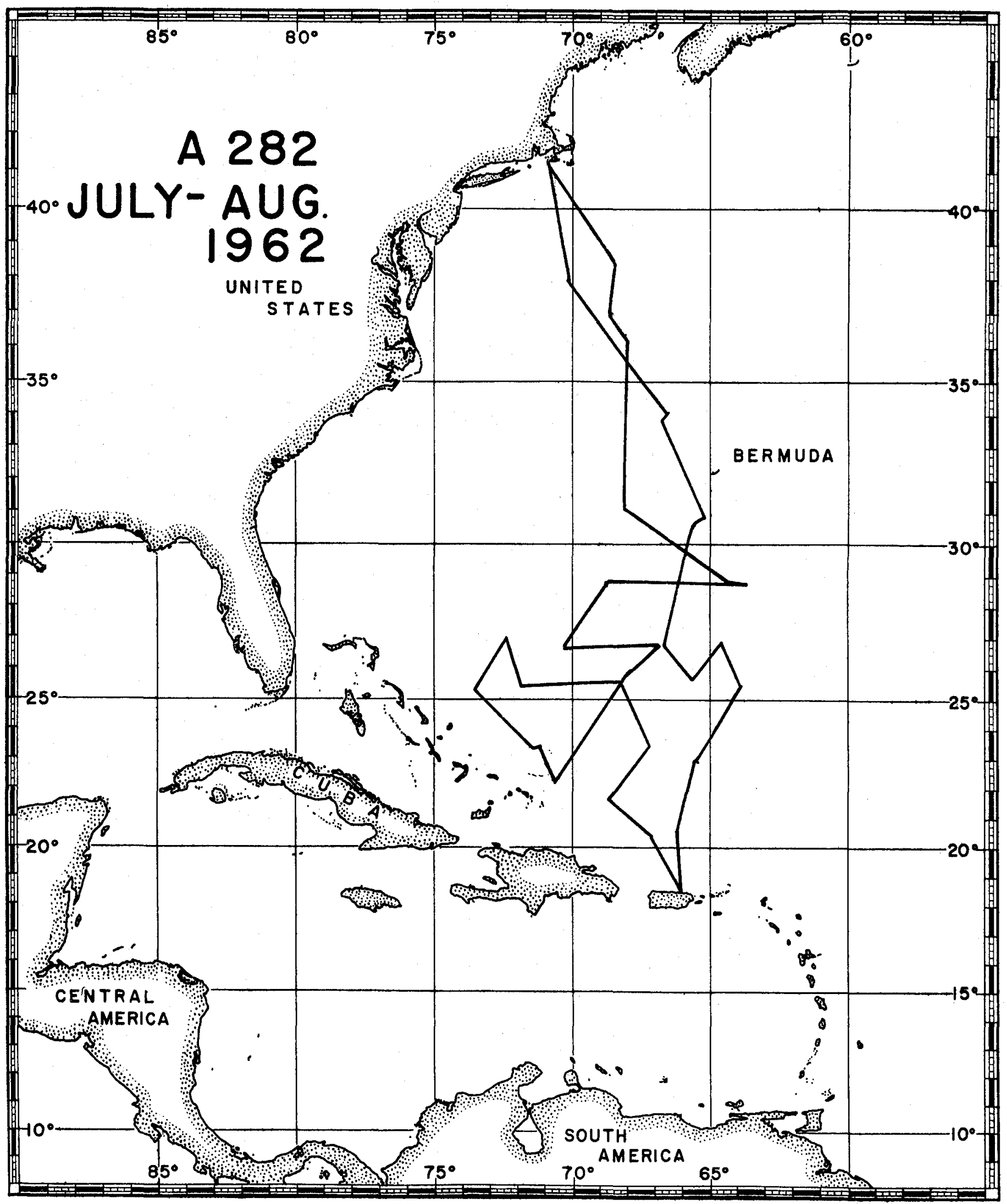


$\forall * * * * * * * * * * * * *$

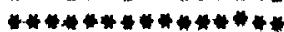

STATION OATA RETRIEYAL

DATE: O6132 MAY 13,175 $\forall * * * * * * * * * * * * * *$

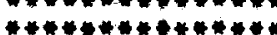

PAGE 1 OF I

* WHO I**

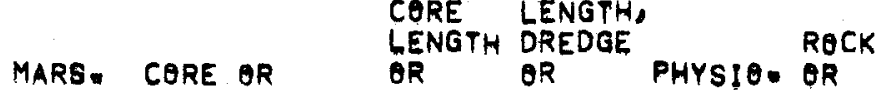

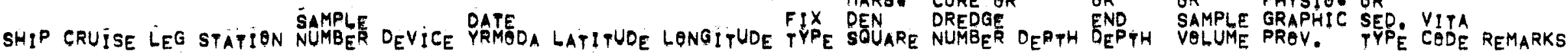

\begin{tabular}{|c|c|c|c|}
\hline $\begin{array}{l}282 \\
282 \\
282 \\
282 \\
282 \\
282 \\
282 \\
282 \\
282 \\
282 \\
282 \\
282 \\
282 \\
282 \\
282 \\
282 \\
282 \\
282 \\
282 \\
282 \\
282 \\
282 \\
282 \\
282 \\
282 \\
282\end{array}$ & $\begin{array}{l}1 \\
1 \\
1 \\
1 \\
1 \\
1 \\
1 \\
1 \\
1 \\
1 \\
1 \\
1 \\
1 \\
1 \\
1 \\
1 \\
1 \\
1 \\
1 \\
1 \\
1 \\
1 \\
1\end{array}$ & $\begin{array}{l}0001 \\
0001 \\
0002 \\
0003 \\
0005 \\
0009 \\
0007 \\
0008 \\
0009 \\
0010 \\
0011 \\
0012 \\
0013 \\
0014 \\
0015 \\
0019 \\
0017 \\
0018 \\
0019 \\
0020 \\
0021 \\
0022 \\
0023\end{array}$ & $\begin{array}{l}0000 \\
0000 \\
0000 \\
0000 \\
0000 \\
0000 \\
0000 \\
0000 \\
0000 \\
0000 \\
0000 \\
0000 \\
0000 \\
0000 \\
0000 \\
0000 \\
000 \\
0000 \\
0000 \\
0000 \\
0000 \\
0000 \\
0000 \\
0000 \\
0000\end{array}$ \\
\hline
\end{tabular}

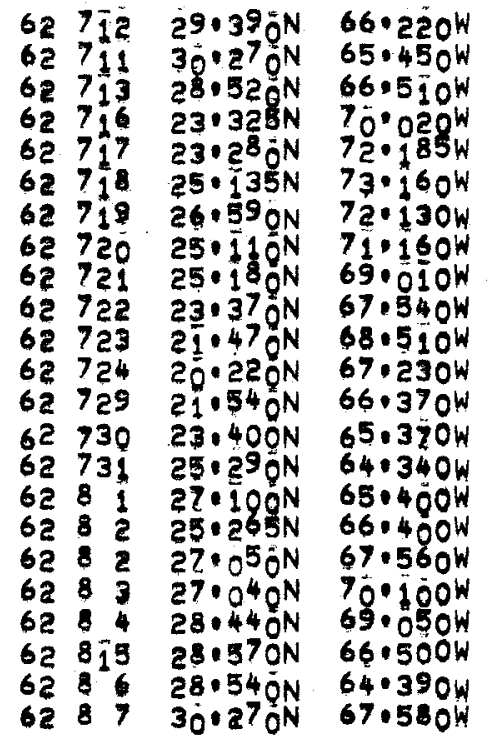

79.960001
115.050014
79.860002
80.300003
80.320005
80.530006
80.620007
80.510008
79.590009
79.370010
79.180011
79.070012
79.160013
79.350014
79.540015
79.750016
79.560017
79.770018
80.700019
79.890020
79.860021
79.840022
115.070023

$\begin{array}{lll}5128: & 209: & 0000 \\ 4945: & 269: & 0000 \\ 5451: & 190: & 0000 \\ 5492: & 120: & 0000 \\ 5287: & 164: & 0000 \\ 5316: & 188: & 0000 \\ 5154: & 228: & 0000 \\ 5520: & 30: & 0000 \\ 5593: & 196: & 0000 \\ 5668: & 171: & 0000 \\ 55130 & 185: & 0000 \\ 5416: & 155: & 0000 \\ 5653: & 131: & 0000 \\ 5771: & 243: & 0000 \\ 5706: & 245: & 0000 \\ 5413: & 197: & 0000 \\ 5602: & 254: & 0000 \\ 5195: & 246: & 0000 \\ 5482: & 113: & 0000 \\ 5325: & 243: & 0000 \\ 5306: & 224: & 0000 \\ 4846: & 301: & 0000 \\ 5188: & 437: & 0000\end{array}$

13
13
13
10
10
10
10
10
10
10
10
10
10
10
10
13
13
13
13
13
13
13
13

$\begin{array}{ll}4129 & 0 \\ 3869 & 0 \\ 3459 & 0 \\ 4841 & 0 \\ 4149 & 0 \\ 4859 & 0 \\ 4129 & 0 \\ 4869 & 0 \\ 4159 & 0 \\ 4159 & 0 \\ 1859 & 0 \\ 4143 & 0 \\ 4159 & 0 \\ 1159 & 0 \\ 1159 & 0 \\ 1453 & 0 \\ 4153 & 0 \\ 1449 & 0 \\ 4249 & 0 \\ 4129 & 0 \\ 1429 & 0 \\ 3469 & 0 \\ 2379 & 0\end{array}$

THERE WERE 23 ITEMS THAT MET YOUR REQUIREMENTS,

THANK YOU FER USING PREGRAM MUDDIE.

"STOP* THAT IS ALL FOR NOW RUN 
VISUAL CORE DESCRIPTION

Ship A Cruise 282 Leg_ Sta. $\frac{1}{1}$ Core No. $\frac{1 G C}{528}$ Total Length $209 \mathrm{~cm}$. Lat. $29^{\circ} 39 \mathrm{~N}$ Long. $\overline{66^{\circ} 22 . \mathrm{W}}$ Depth $5128 \mathrm{mCORR}$ Core condition dry. foir Date Described IZAPRI'74 by GMOUNTAN

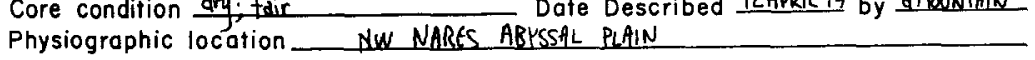

Lithologic

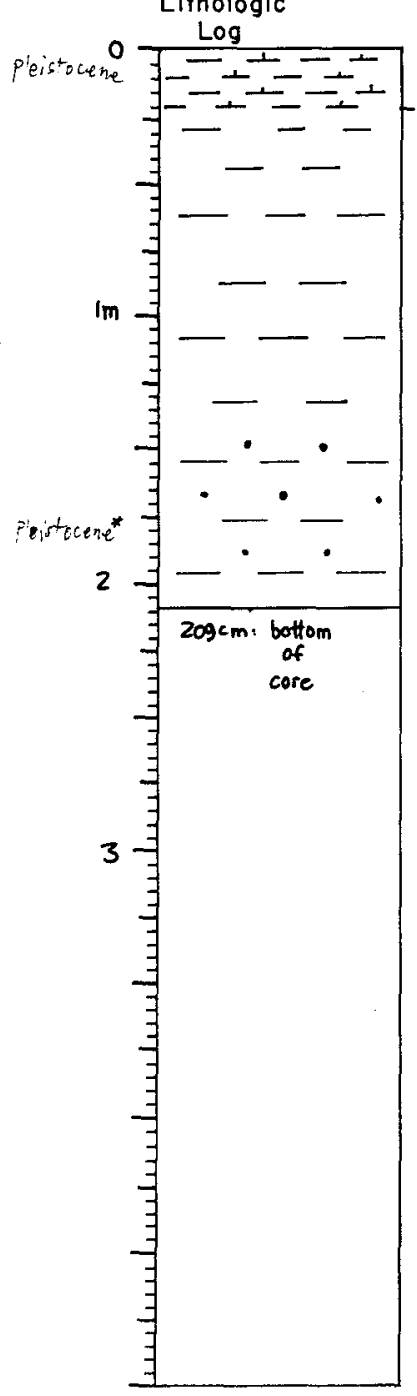

0198

Page _ 1 of__ _.

0193

SMEAR SLIDE DESCRIPTIONS - W.H.O.I. SEDIMENT CORES
Ship: Atlantis

Expedition 282

Leg No.
Core No. 16C

HLY CALC CLAY

1OYR $5 / 3$ brown

total core is homogeneous except for scattered v. dk gray specks (Mn?)

26- 209

UNFOSS CIAY WITH Mn

10YR $3 / 3 \mathrm{dk}$ brown

this unit grad

grades $s 1 \mathrm{dk}$ with depth

end of core

\begin{tabular}{|c|c|c|c|c|c|c|c|c|c|c|c|c|c|c|}
\hline & 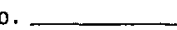 & & & & Tot & & & & & & & $-\mathrm{cm}$ & & \\
\hline \multirow[b]{4}{*}{ LEVEL } & \multirow[b]{4}{*}{$\begin{array}{c}\text { SEDIMENT } \\
\text { TYPE }\end{array}$} & \multicolumn{13}{|c|}{ ESTIMATED ABUNDANCES $(\%)$} \\
\hline & & \multirow{2}{*}{\multicolumn{4}{|c|}{$\begin{array}{c}\text { Inorganic Material } \\
\text { Silt \& Sand } \\
\end{array}$}} & & \multirow{2}{*}{\multicolumn{5}{|c|}{$\begin{array}{l}\text { Biogenous Mater } \\
\text { Calcareous }\end{array}$}} & & & \\
\hline & & & & & & & & & & & & \multicolumn{3}{|c|}{ Siliceous } \\
\hline & & 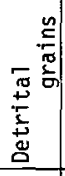 & 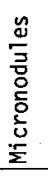 & 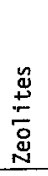 & 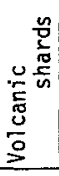 & $\frac{\widehat{\pi}}{\sigma}$ & 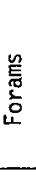 & 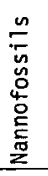 & 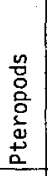 & 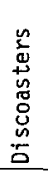 & 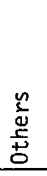 & 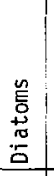 & 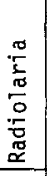 & 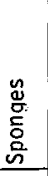 \\
\hline $3 \mathrm{~cm}$ & hly calc clay & & 10 & $\operatorname{tr}$ & & 62 & 5 & 20 & & $\operatorname{tr}$ & 3 & & & \\
\hline $100 \mathrm{~cm}$ & $\begin{array}{l}\text { unfoss clay } \\
\text { with Mn }\end{array}$ & $\operatorname{tr}$ & 20 & 2 & & 78 & & & & & & & & \\
\hline $206 \mathrm{~cm}$ & \begin{tabular}{|l|} 
unfoss clay \\
with Mn \\
\end{tabular} & $\operatorname{tr}$ & 20 & 2 & & 78 & & & & & & & & \\
\hline & & & & & & & & & & & & & & \\
\hline & & & & & & & & & & & & & & \\
\hline & & & & & & & & & & & & & & \\
\hline & & & & & & & & & & & & & & \\
\hline & & & & & & & & & & & & & & \\
\hline & & & & & & & & & & & & & & \\
\hline & & & & & & & & & & & & & & \\
\hline & & & & & & & & & & & & & & \\
\hline & & & & & & & & & & & & & & \\
\hline & & & & & & & & & & & & & & \\
\hline & & & & & & & & & & & & & & \\
\hline & & & & & & & & & & & & & & \\
\hline & & & & & & & & & & & & & & \\
\hline & & & & & & & & & & & & & & \\
\hline & & & & & & & & & & & & & & \\
\hline
\end{tabular}


0200

VISUAL CORE DESCRIPTION

Page 1 of 1

Ship $A$ Cruise 282 Leg_ Sto. $1 A$ Core No. $196 C$ Total Length $369 \mathrm{~cm} \mathrm{~cm}$. Lot. $30^{\circ} 27^{\circ} \mathrm{N}$ Long. $65^{\circ} 45^{\prime} \mathrm{W}$ Depth $4945 \mathrm{CoRRm}$ Core condition dry, hard; fair Dote Described 8 May 74 by $B$ MeGirr

Physiographic location $N W$ Nares Abyssal Plain

Lithologic

Log Forams

Defailed Description

F-1. CALC OOZE

1OYR $4 / 2$ dk grayish brown

common mottling throughout, white

sl silty lutite, few foran

dry throughour, somewhat crumbly at lower end

手

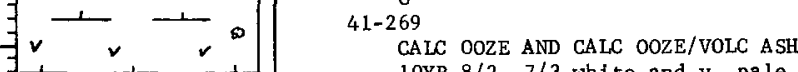

(Oتn

- $\longrightarrow$ -

$\operatorname{lm}$

unit v, dry and somewhat crumbly throughout, it and $\mathrm{dk}$ colors randomly mixed

s colors randomly mixed
Note; The volc ash found in the smear slides may
macroscopically exist as thin, volc ash beds or la

macroscopically exist as thin, volc ash beds or iarger

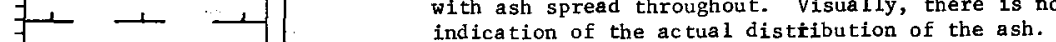
end of core
0201

SMEAR SLIDE DESCRIPTIONS - W.H.O.I. SEDIMENT CORES$$
\text { ㄴ․ }
$$$$
\text { 2月ㄷㄴ }
$$$$
\text { - }-1
$$$$
\exists-\frac{1}{v}
$$$$
\text { 我 } 1 \text { ㄴ. }
$$

Yeistocent $=v-1$ -

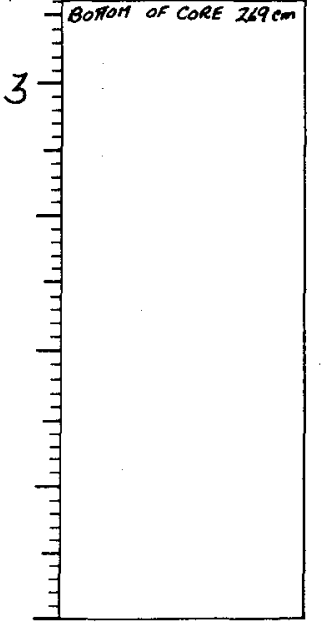


0240

VISUAL CORE DESCRIPTION

Page 1 of 1

Ship A Cruise 282 Leg_L Sta. 23 Core No. $226 \mathrm{C}(\mathrm{HF})$ Totol Length $301 \mathrm{~cm}$. 1 at. $28^{\circ} 54^{\circ} \mathrm{N}$ Long. $64^{\circ} 39^{\prime} \mathrm{W}$ Depth $4846 \mathrm{cosem}$ Core condition dry, hard; fair Date Described $5 / 15 / 74$ by $B$ MCGirm

Physiographic location Bermuda Rise

Lithologic $\underset{\substack{\text { Foranes } \\ \rightarrow \infty}}{ }$

Detailed Description

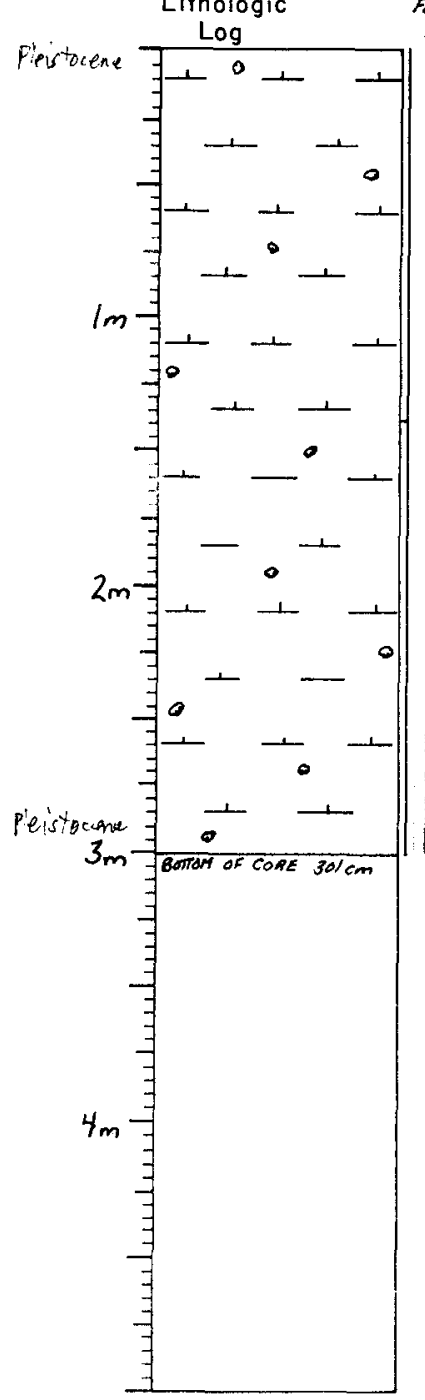

$0-139$

1OYR $7 / 3 \mathrm{v}$. pale brown

scattered mottling throughout

$139-301$

HLY CALC CLAY AND CALC OOZE

TR $3 / 3,4 / 3$ dk brown

cottered nottling throughout, brown

somewhat dry, sl silty lutite
0241

SMEAR SLIDE DESCRIPTIONS - W.H.O.I. SEDIMENT CORES

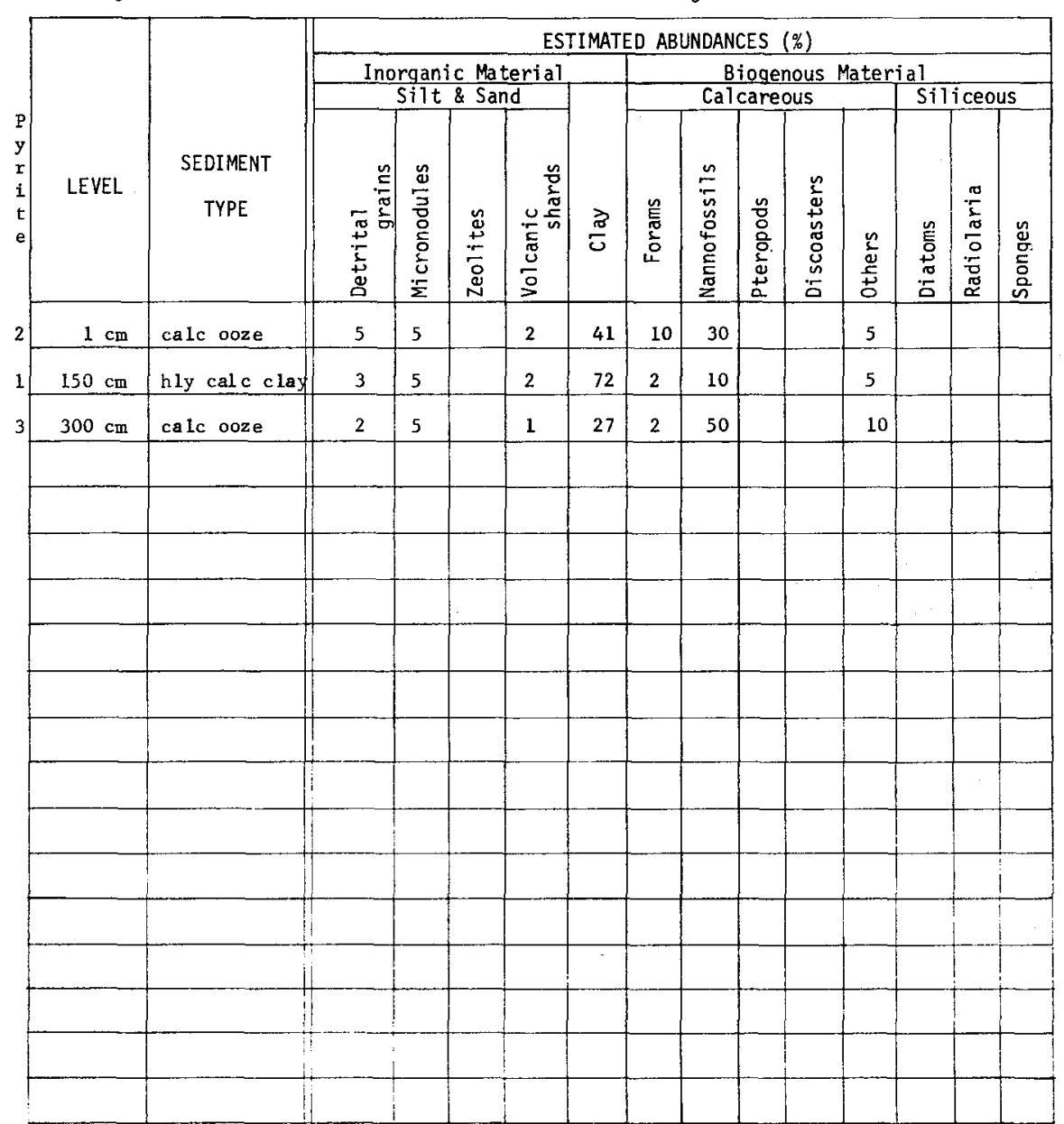

Ship: Atlentis

Expedition 282

Leg No.

Core No. 22 GC (HF)

Station No 22

Total Core Length 30

Total Core Length

rat 
0238

VISUAL CORE DESCRIPTION

Page__ 1 of

Ship $A$ Cruise 282 Leg_L Sta. 21 Core No. $21 G C(H F)$ Total Length $224 \mathrm{~cm}$. Lat. $28^{\circ} 51^{\circ} \mathrm{N}$ Long. $66^{\circ} 50^{\circ} \mathrm{W}$ Depth $5306 \mathrm{Corem}$ Core condition very dy, hard; fair Dote Described 24 Mey 24 by $B$ MCGirr

Physiographic location Bermude_Rise

Lithologic

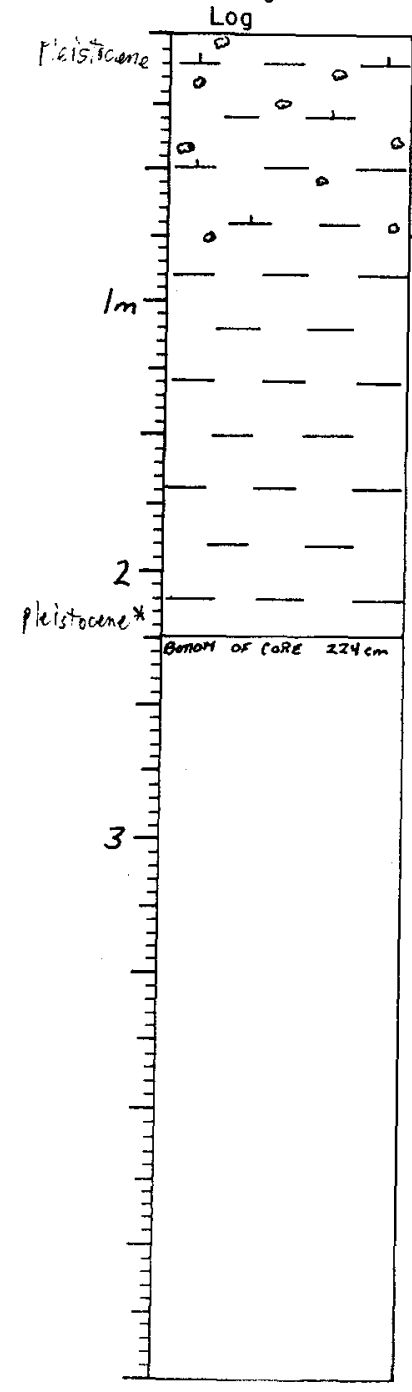

0-75 Detailed Description

CALC CLAY

common mottling $0-40 \mathrm{~cm}$, scattered $40-75 \mathrm{~cm}$

dry hard lutite $75-224$

UNFOSS CIAY

10YR $6 / 3$ pale brown

dry hard lutite entire core is dry, hard and pretty much crumbled
0233

SMEAR SLIDE DESCRIPTIONS - W.H.O.I. SEDIMENT CORES

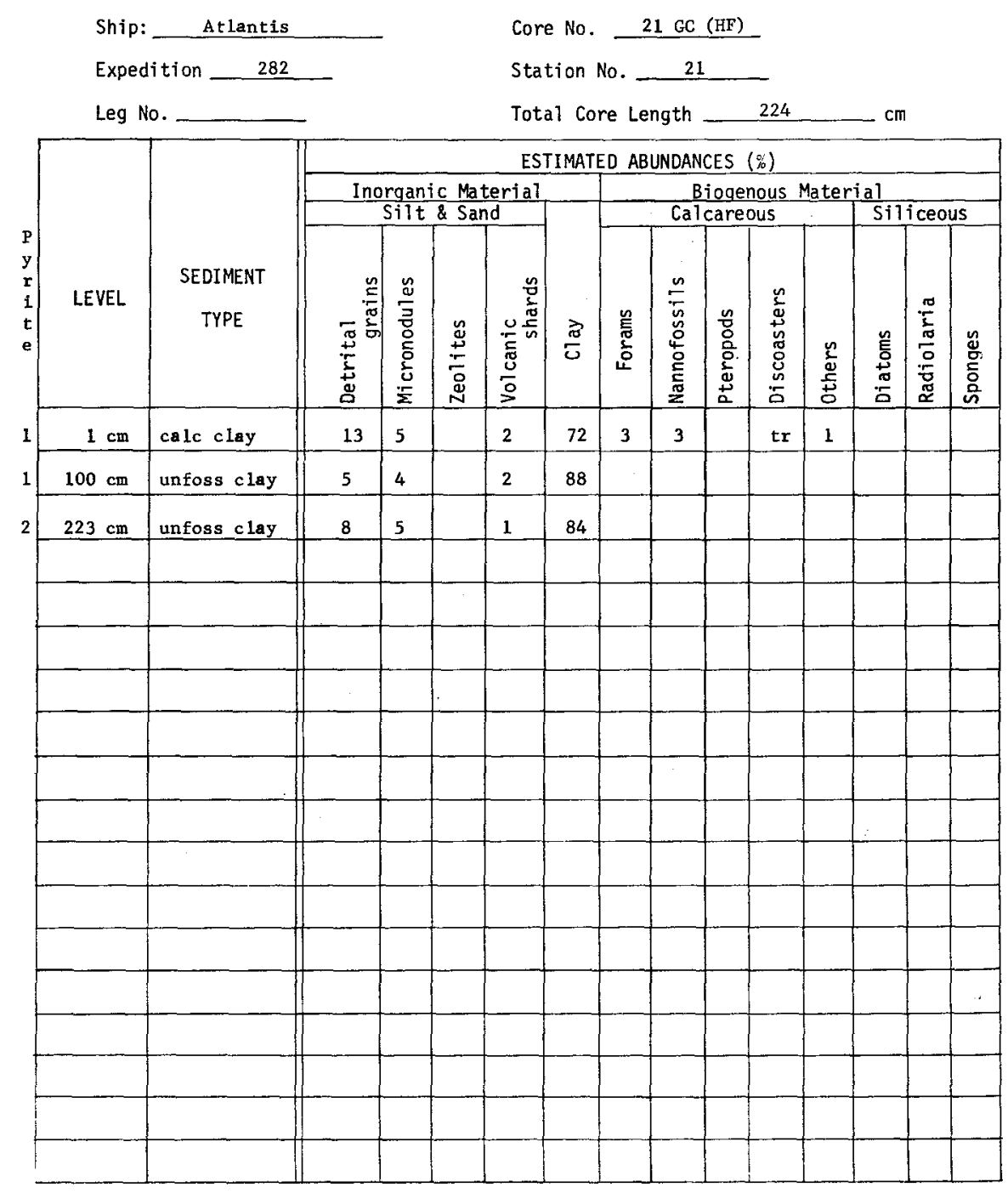


0236

VISUAL CORE DESCRIPTION

Page _ L of _ 1

Ship A Cruise 282 Leg Sta 20 Core No. 20 GC (HF) Total Length $243 \mathrm{~cm}$. Lot. $28^{\circ} 44^{\prime} \mathrm{N}$ Long. $69^{\circ} \mathrm{O} 5^{\prime} \mathrm{W}$ Depth $5325 \mathrm{CoRRm}$ Core condition dry, herd; faic Date Described 14 May 74 by $B$ MCGicr

Physiographic location Bermuda Rise

Lithologic

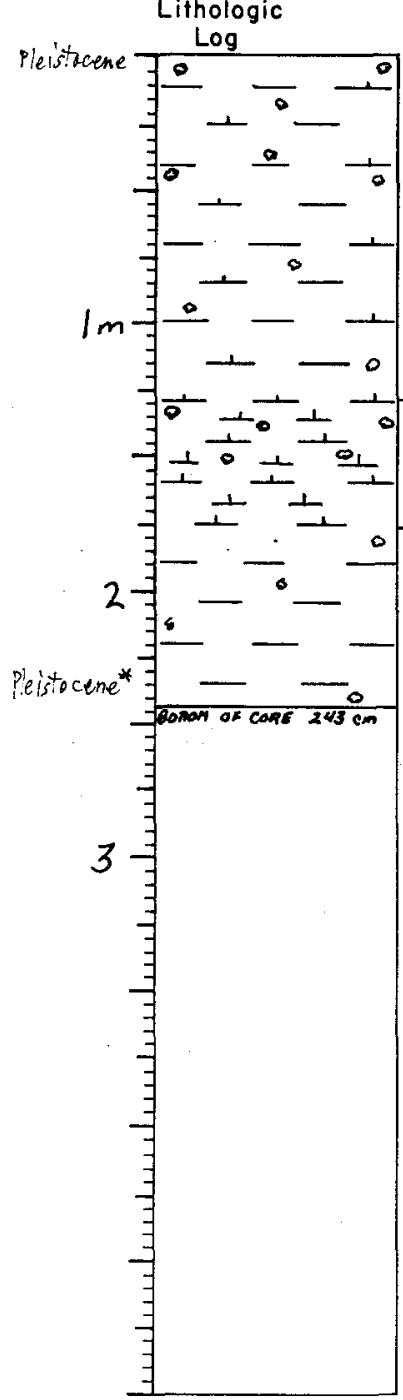

$0-128$

Detailed Description LOYR 6/3 PEI brown mottling throughout, common $0-40 \mathrm{~cm}$ dry hard lutite

128-176

CALC OOZE

scattered mottling throughout, common 130-150 cm

$176-243$

Foss CTAY

IOYR $6 / 3$ pale brown

scattered mottling throughou

dry, hard lutit
0237

SMEAR SLIDE DESCRIPTIONS - W.H.O.I. SEDIMENT CORES

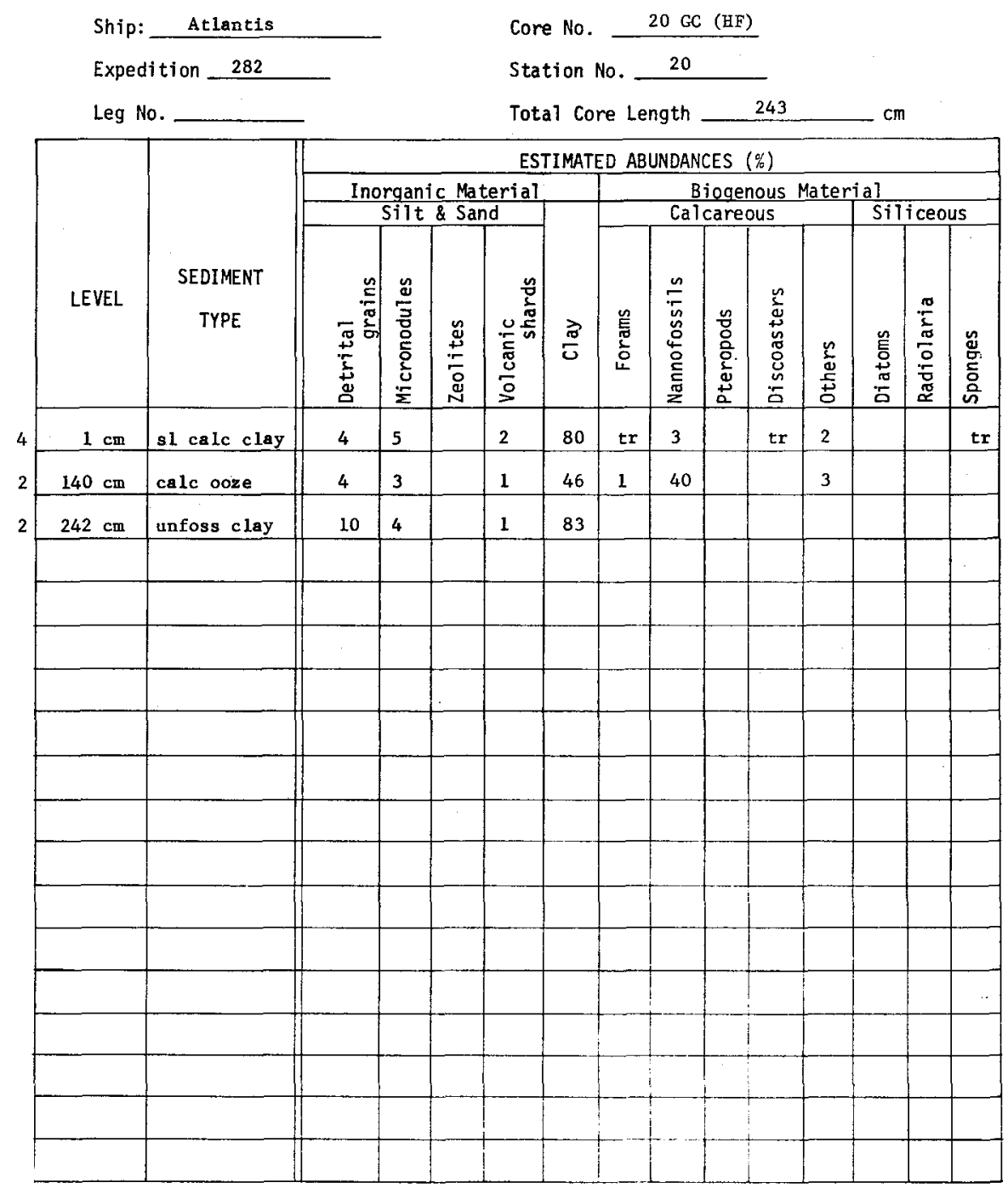


0234

VISUAL CORE DESCRIPTION

Page 1 of 1

Ship_A Cruise 282 Leg__ Sta. 19 Core No. $196 \mathrm{CC}$ (HE) Total Length $1133 \mathrm{~cm}$. Lat. $27^{\circ} 04^{\prime} \mathrm{N}$ Long. $20^{\circ} 10^{\circ} \mathrm{W}$ Depth $5482 \mathrm{CaRe}$ Core condition Verydry, hardifair Date Described 14 Mer 74 by BMCGir

Physiographic location Becmuda Rise

Lithologic

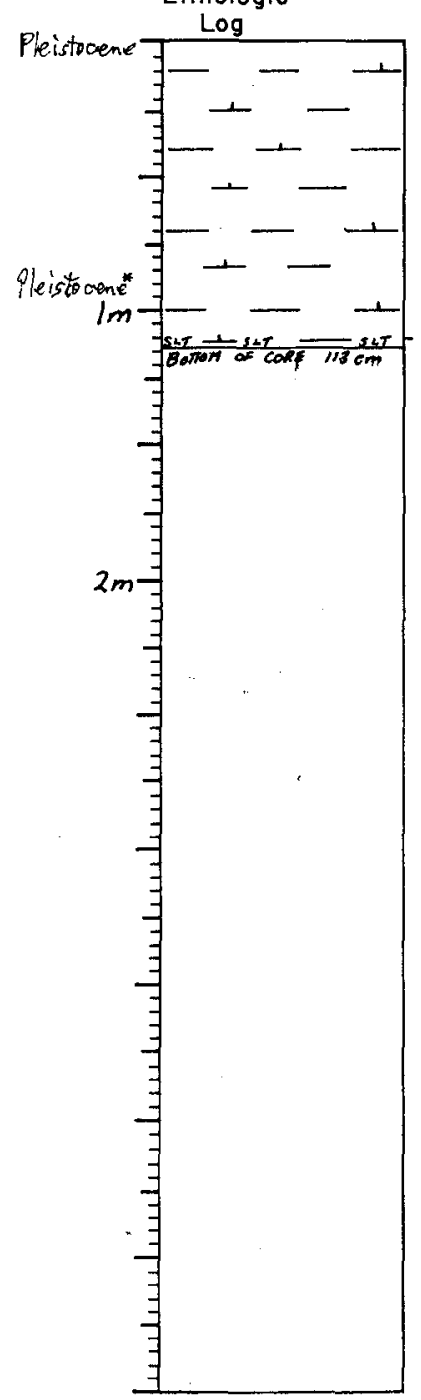

Detailed Description

SL CALC CLAY

$2.5 \mathrm{Y} 6 / 21 \mathrm{t}$ brownish gray

faint, thin horizontal lams $0-40 \mathrm{~cm}$, core v. dry and hard somewhat crumbly throughout

SL CALC CLAY/DET

$2.5 \mathrm{Y} 6 / 2$ it brownish gray

dry, hard silty lutite

0235

SMEAR SLIDE DESCRIPTIONS - W.H.O.I. SEDIMENT CORES

Ship: Atlantis

Expedition 282

Leg No.

\begin{tabular}{|c|c|c|c|c|c|c|c|c|c|c|c|c|c|c|}
\hline \multirow[b]{4}{*}{ LEVEL } & \multirow[b]{4}{*}{$\begin{array}{c}\text { SEDIMENT } \\
\text { TYPE }\end{array}$} & \multicolumn{13}{|c|}{ ESTIMATED ABUNDANCES $(\%)$} \\
\hline & & \multirow{2}{*}{\multicolumn{4}{|c|}{$\begin{array}{c}\text { Inorganic Material } \\
\text { Silt \& Sand } \\
\end{array}$}} & & \multirow{2}{*}{\multicolumn{5}{|c|}{$\begin{array}{l}\text { Biogenous Mater } \\
\text { Calcareous } \\
\end{array}$}} & \multirow{2}{*}{\multicolumn{3}{|c|}{ Siliceous }} \\
\hline & & & & & & & & & & & & & & \\
\hline & & 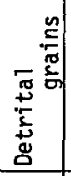 & 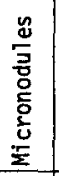 & $\begin{array}{l}\tilde{y} \\
\stackrel{+}{*} \\
\bar{g} \\
\stackrel{N}{N}\end{array}$ & 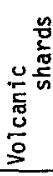 & $\frac{\pi}{6}$ & $\begin{array}{l}\text { ñ } \\
\text { 产 } \\
\text { ơ }\end{array}$ & 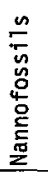 & 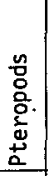 & 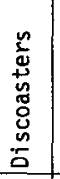 & 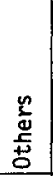 & 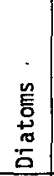 & 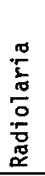 & \begin{tabular}{|l|}
0 \\
0 \\
$\mathbf{u}$ \\
0 \\
0 \\
0 \\
0 \\
0
\end{tabular} \\
\hline $1 \mathrm{~cm}$ & sl calc clay & 6 & 4 & & 2 & 79 & & 4 & & $\mathrm{tr}$ & 1 & & & $\mathrm{tr}$ \\
\hline $100 \mathrm{~cm}$ & sl calc clay & 8 & 2 & & 1 & 82 & & 2 & & & & & & \\
\hline $112 \mathrm{~cm}$ & $\begin{array}{l}\text { si calc clay } \\
\text { det }\end{array}$ & 70 & 1 & & 2 & 17 & 1 & 2 & & & 2 & & & \\
\hline & & & & & & & & & & & & & & \\
\hline & & & & & & & & & & & & & & \\
\hline & & & & & & & & & & & & & & \\
\hline & & & & : & & & & & & & & & & \\
\hline & & & & & & & & & & & & & & \\
\hline & & & & & & & & & & & & & & \\
\hline & & & & & & & & & & & & & & \\
\hline & & & & & & & & & & & & & & \\
\hline & & & & & & & & & & & & & & \\
\hline & & & & & & & & & & & & & & \\
\hline & & & & & & & & & & & & & & 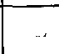 \\
\hline & & & & & & & & & & & & & & \\
\hline & & & & & & & & & & & & & & \\
\hline & & & & & & & & & & & & & & \\
\hline & & & & & & & & & & & & & & \\
\hline
\end{tabular}

Core No. $\quad 19 \mathrm{GC}(\mathrm{HF})$

Station No. 19

Total Core Length 113

113 ESTIMATED ABUNDANCES (\%) 
0232

VISUAL CORE DESCRIPTION

Page 1 of 1

Ship $A$ Cruise 282 Leg sto. 18 Core No. 18 GC (HF)

Total Length $246 \mathrm{~cm}$. Lot. $27^{\circ}$ O5'N Long. $67^{\circ} 56 \mathrm{~W}$ Depth $5195 \mathrm{CoRsm}$

Core condition very dry, hard; fair-poor. Dote Described $\angle 4$ May 74 by $B M^{C} G$ Gir

Physiographic location Bermuda Rise

Lithologic

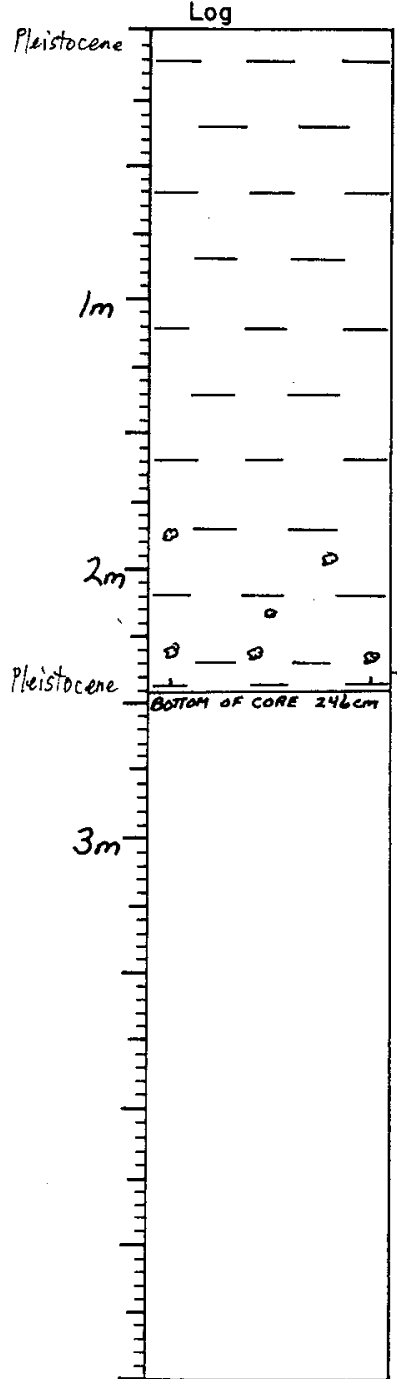

\section{Detailed Description}

UNFOSS CIAY

LOYR $4 / 3,3 / 2$ brown and $v, d k$ grayish brown

scattered mottling increasing to abunt., pale brown

dry, hard lutite

132-200 cm, entin core someshat crumbly and dis turbed

$S$ irregular

HIY CAIC CIAY

10YR 6/3 pale brow dry, hard lutite
0233

SMEAR SLIDE DESCRIPTIONS - W.H.O.I. SEDIMENT CORES

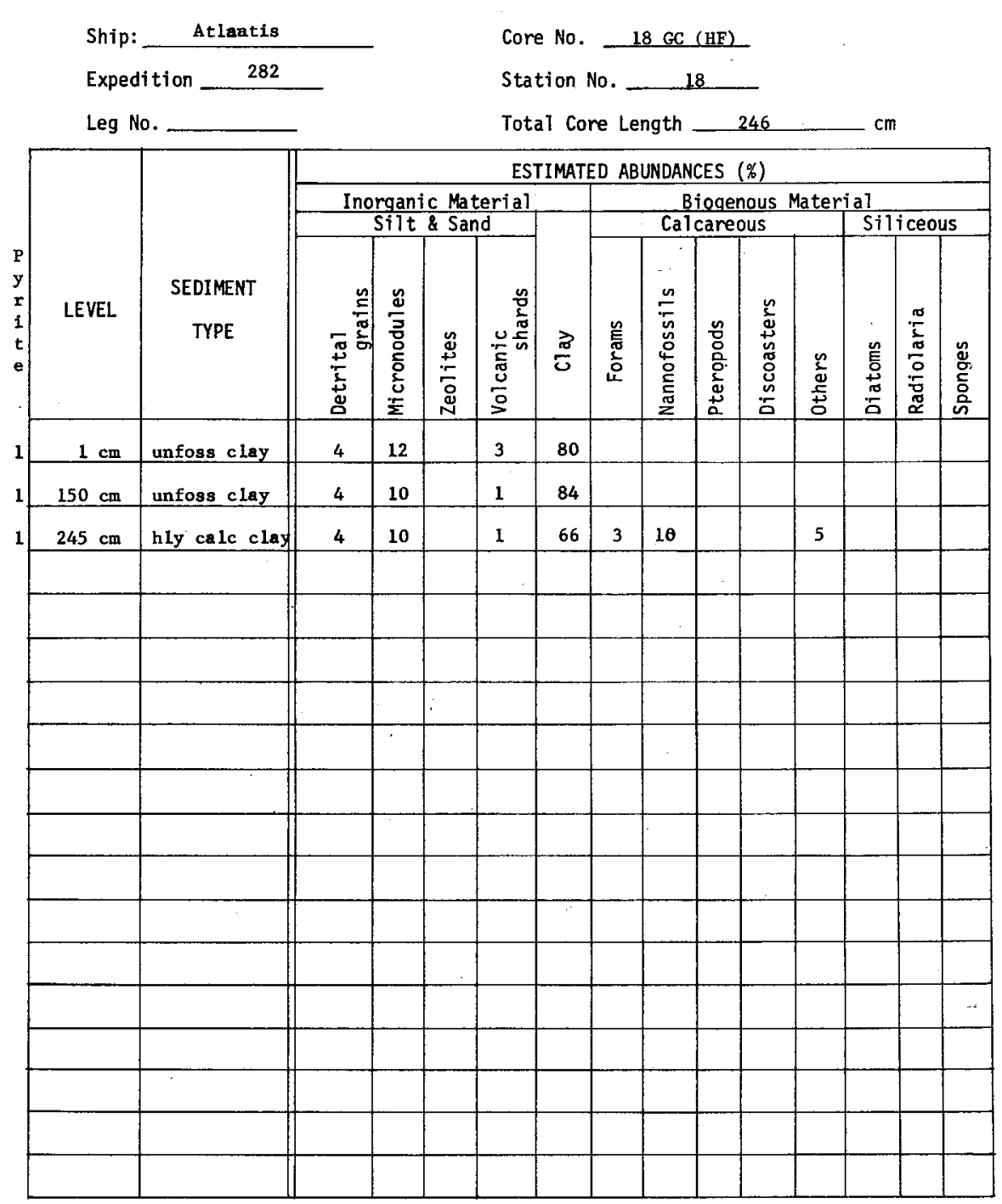


0230

VISUAL CORE DESCRIPTION

Poge_L of _ L

Ship $A$ Cruise 282 Leg 17 Core No.17GC(HF) Total Length $254 \mathrm{~cm}$. Lat. $25^{\circ} \mathrm{zb} .5^{\prime} \mathrm{N}$ Long. $66^{\circ} 40 \mathrm{~W}$ Depth 5602 corem

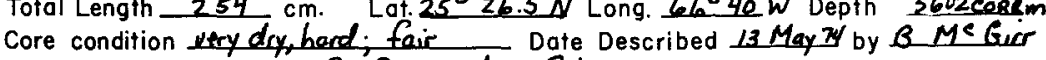
Physiographic location $S$ Bermuda Rise

Lithologic

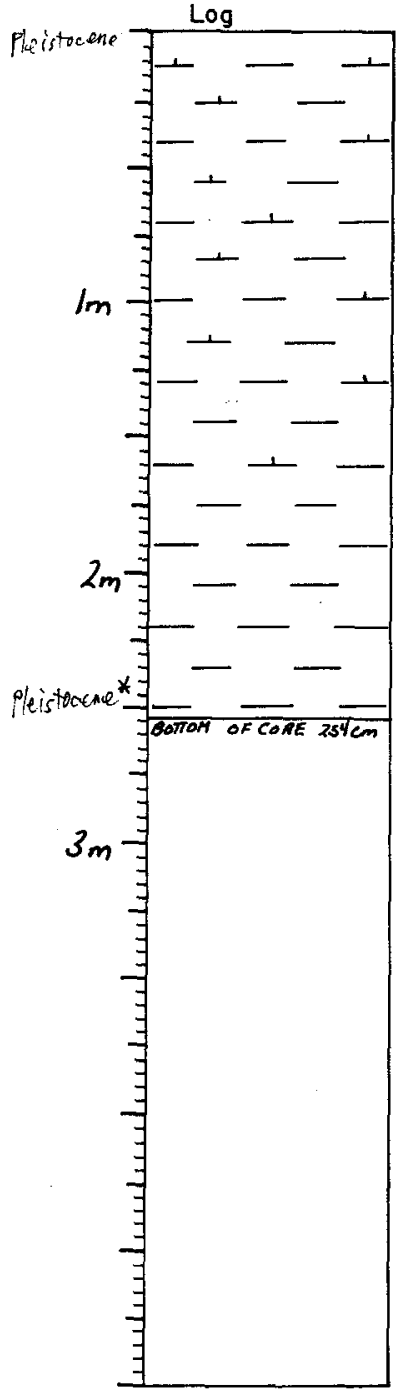

$0-254$

dxy, hard lutite

core disturbed and mask core features
0231

SMEAR SLIDE DESCRIPTIONS - W.H.O.I. SEDIMENT CORES

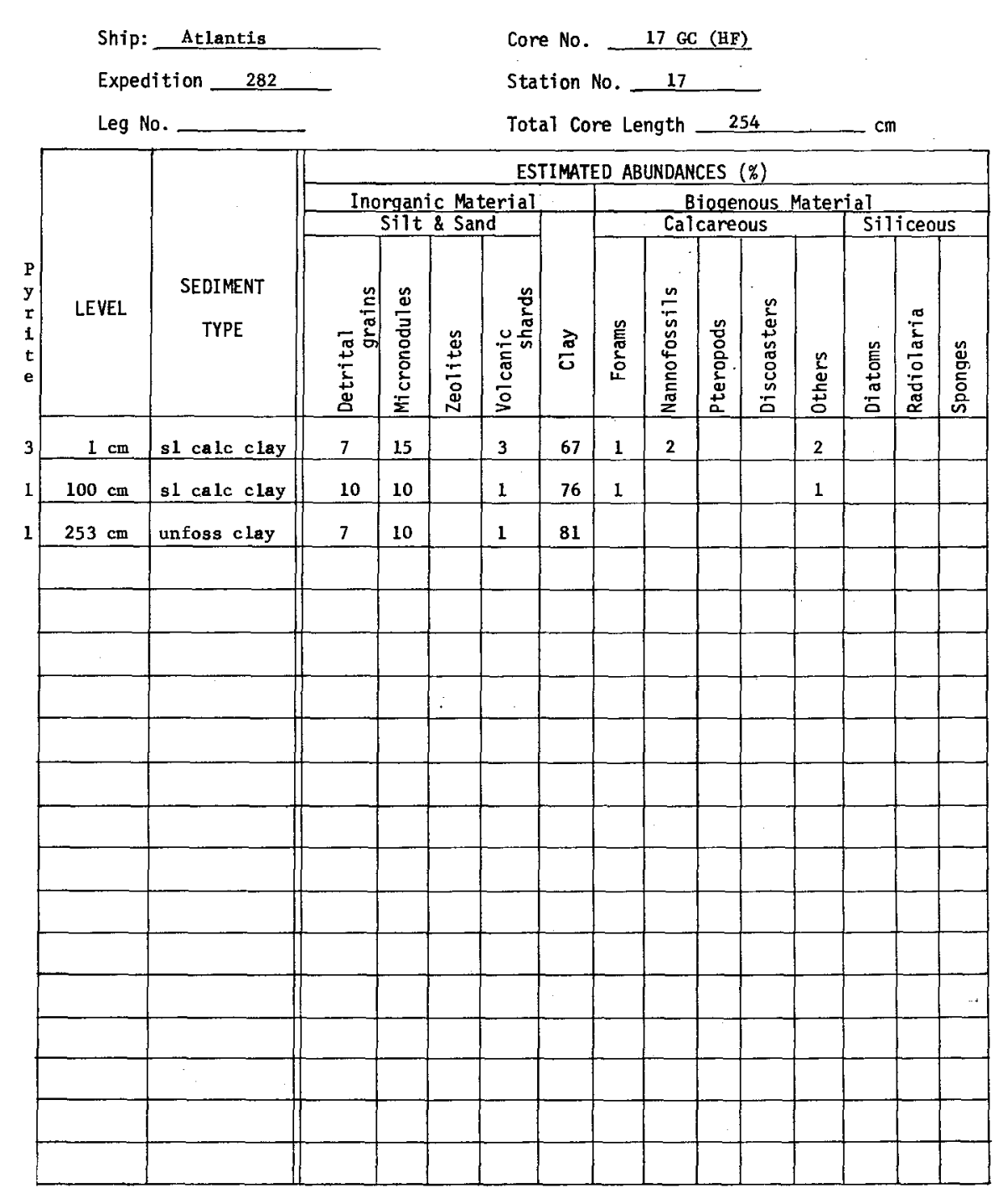


0228

VISUAL CORE DESCRIPTION

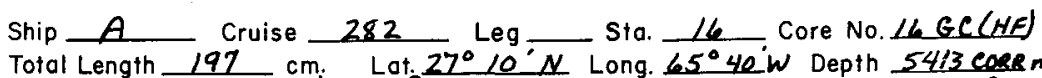
Core condition very dry, hard; fair-paer. Date Described 13 May 24 by $B$ MeGirr

Physiographic location $S$ Bermuda Rise

Lithologic

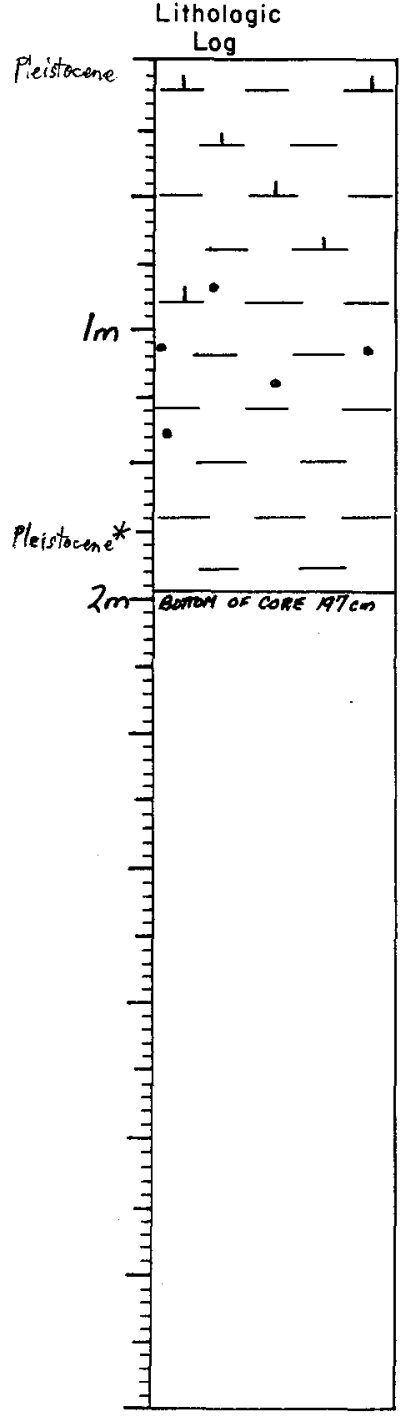

Page 1 of 1

0229

SMEAR SLIDE DESCRIPTIONS - W.H.O.I. SEDIMENT CORES

Detailed Description

\section{CALC CIAY TO CLAY}

IOYR $5 / 3$ brown

dry hard si silty lutite

Wn nodules scattered $80-135 \mathrm{~cm}$, upto $1 \mathrm{~cm}$ in diameter, micros to bottom of cor

Leg No.

Ship: $\frac{\text { Atlantis }}{282} \quad \begin{aligned} & \text { Core No. } \frac{16 \text { GC (BF) }}{26} \\ & \text { Expedition }\end{aligned}$

Total Core Length $197 \quad \mathrm{~cm}$

\begin{tabular}{|c|c|c|c|c|c|c|c|c|c|c|c|c|c|c|}
\hline \multirow[b]{4}{*}{ LEVEL } & \multirow[b]{4}{*}{$\begin{array}{c}\text { SEDIMENT } \\
\text { TYPE }\end{array}$} & \multicolumn{13}{|c|}{ ESTIMATED ABUNDANCES (\%) } \\
\hline & & \multirow{2}{*}{\multicolumn{4}{|c|}{$\frac{\text { Inorganic Material }}{\text { Silt \& Sand }}$}} & & \multirow{2}{*}{\multicolumn{5}{|c|}{$\begin{array}{l}\text { Biogenous Mater } \\
\text { Calcareous }\end{array}$}} & \multirow{2}{*}{\multicolumn{3}{|c|}{ Siliceous }} \\
\hline & & & & & & & & & & & & & & \\
\hline & & 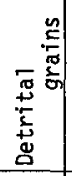 & 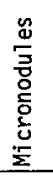 & $\begin{array}{l}\stackrel{0}{ \pm} \\
\stackrel{ \pm}{0} \\
\stackrel{N}{N}\end{array}$ & 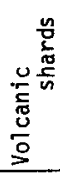 & $\frac{\vec{\pi}}{\tilde{\sigma}}$ & 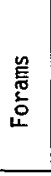 & 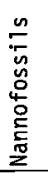 & 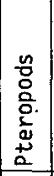 & 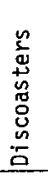 & 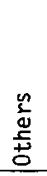 & 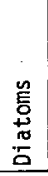 & $\begin{array}{l}\frac{\pi}{2} \\
\pi \\
\frac{\pi}{0} \\
\frac{\pi}{\pi} \\
\tilde{x} \\
\end{array}$ & 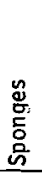 \\
\hline $1 \mathrm{~cm}$ & calc clay & 5 & 2 & & $\mathrm{tr}$ & 80 & 2 & 5 & & & 3 & & & \\
\hline $100 \mathrm{~cm}$ & unfoss clay & 5 & 4 & & $\mathrm{tr}$ & 89 & & & & & & & & \\
\hline $196 \mathrm{~cm}$ & unfoss clay & 2 & 8 & & tx & 89 & $\mathrm{tr}$ & & & & & & & \\
\hline & & & & & & & & & & & & & & \\
\hline & & & & & & & & & & & & & & \\
\hline & & & & & & & & & & & & & & \\
\hline & & & & & & & & & & & & & & \\
\hline & & & & & & & & & & & & & & \\
\hline & & & & & & & & & & & & & & \\
\hline & & & & & & & & & & & & & & \\
\hline & & & & & & & & & & & & & & \\
\hline & & & & & & & & & & & & & & \\
\hline & & & & & & & & & & & & & & \\
\hline & & & & & & & & & & & & & & . \\
\hline & & & & & & & & & & & & & & \\
\hline & & & & & & & & & & & & & & \\
\hline & & & & & & & & & & & & & & \\
\hline & & & & & & & & & & & & & & \\
\hline
\end{tabular}


VISUAL CORE DESCRIPTION

Ship $A$ Cruise 282 Leg_ Sta. 15 Core No. 15 GC(MF) Total Length $245 \mathrm{~cm}$. Lat. $25^{\circ} 29^{\circ} \mathrm{N}$ Long. $64^{\circ} 34^{\prime \prime} \mathrm{W}$ Depth $5706 \mathrm{CoRRm}$ Core condition very dry, hard; fair-peorDate Described 13 May 74 by $B$ MCGier Physiographic location - Naces Abyssal Plain

Lithologic

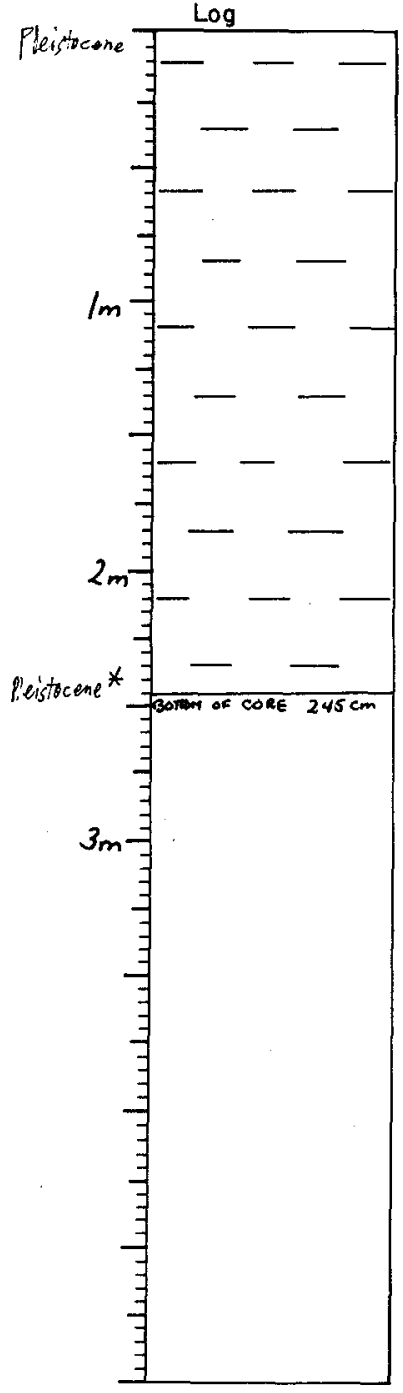

0226

Poge $\quad l$ of

0227

SMEAR SLIDE DESCRIPTIONS - W.H.O.I. SEDIMENT CORES

$0-245$

UNFOSS CLAY

10YR $5 / 3,4 / 2$ brown and dk grayish brown $80-120 \mathrm{~cm}$

firm $s 1$ silty lutite

core v. dry and ha rd, somewhat crumbly, core condition may mask special features
Detailed Description

Ship: Atlantis

Expedition 28

Leg No.

Core No. $15 \mathrm{GC}(\mathrm{HF})$

Station No. 15

Total Core Length 245

$\mathrm{cm}$

\begin{tabular}{|c|c|c|c|c|c|c|c|c|c|c|c|c|c|c|}
\hline \multirow[b]{4}{*}{ LEVEL } & \multirow[b]{4}{*}{$\begin{array}{c}\text { SEDIMENT } \\
\text { TYPE }\end{array}$} & \multicolumn{13}{|c|}{ ESTIMATED ABUNDANCES (\%) } \\
\hline & & \multirow{2}{*}{\multicolumn{4}{|c|}{$\frac{\text { Inorganic Material }}{\text { Silt \& Sand }}$}} & & \multirow{2}{*}{\multicolumn{5}{|c|}{$\begin{array}{l}\text { Biogenous Mater } \\
\text { Ca1careous }\end{array}$}} & \multirow{2}{*}{\multicolumn{3}{|c|}{ Siliceous }} \\
\hline & & & & & & & & & & & & & & \\
\hline & & 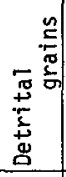 & 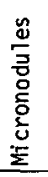 & 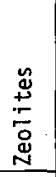 & 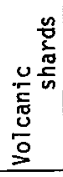 & $\stackrel{\frac{\pi}{\sigma}}{0}$ & 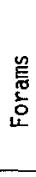 & 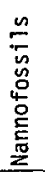 & 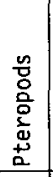 & 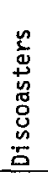 & 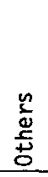 & 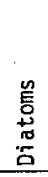 & 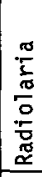 & 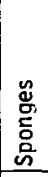 \\
\hline $1 \mathrm{~cm}$ & unfoss clay & 8 & 4 & & 1 & 84 & & & & & & & & \\
\hline $100 \mathrm{~cm}$ & unfoss clay & 7 & 8 & & tr & 83 & & & & & & & & \\
\hline $244 \mathrm{~cm}$ & unfoss clay & 4 & 5 & & tr & 87 & & & & & & & & \\
\hline & & & & & & & & & & & & & & \\
\hline & & & & & & & & & & & & & & \\
\hline & & & & & & & & & & & & & & \\
\hline & & & & & & & & & & & & & & \\
\hline & & & & & & & & & & & & & & \\
\hline & & & & & & & & & & & & & & \\
\hline & & & & & & & & & & & & & & \\
\hline & & & & & & & & & & & & & & \\
\hline & & & & & & & & & & & & & & \\
\hline & & & & & & & & & & & & & & \\
\hline & & & & & & & & & & & & & &. \\
\hline & & & & & & & & & & & & & & \\
\hline & & & & & & & & & & & & & & \\
\hline & & & & & & & & & & & & & & \\
\hline & & & & & & & & & & & & & & \\
\hline
\end{tabular}




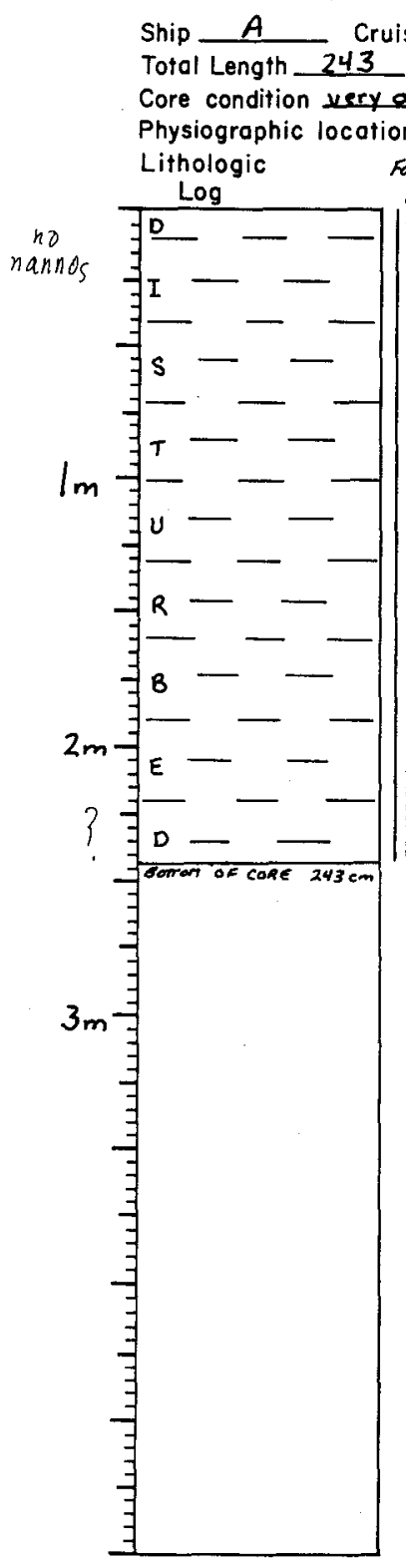

SMEAR SLIDE DESCRIPTIONS - W.H.O.I. SEDIMENT CORES

Ship A Cruise 282 Leg_Sta. 14 Core No. 14 GC (HF)

$23^{\circ} 40^{\prime} \mathrm{N}$ Long. $45^{\circ} 37 \mathrm{~W}$ Depth 5721 CoRR m very dey; poor Date Described $4 / 12 / 74$ by $B$ MrGic

locotion Nares Abyssal Plain

\section{Torams \\ UNFOSS CLAY}

10YR $5 / 3$ brown

some indication of small black mottles throughout, core really too dry to tell except for a few $\mathrm{cm}^{\prime} \mathrm{s}$ at top of each section

entire core is extremely dry and hard, color is lt brown where core is drier, disturbed throughout, core liner is split in half, but core has only small slice taken out, essentially entire core has not been split

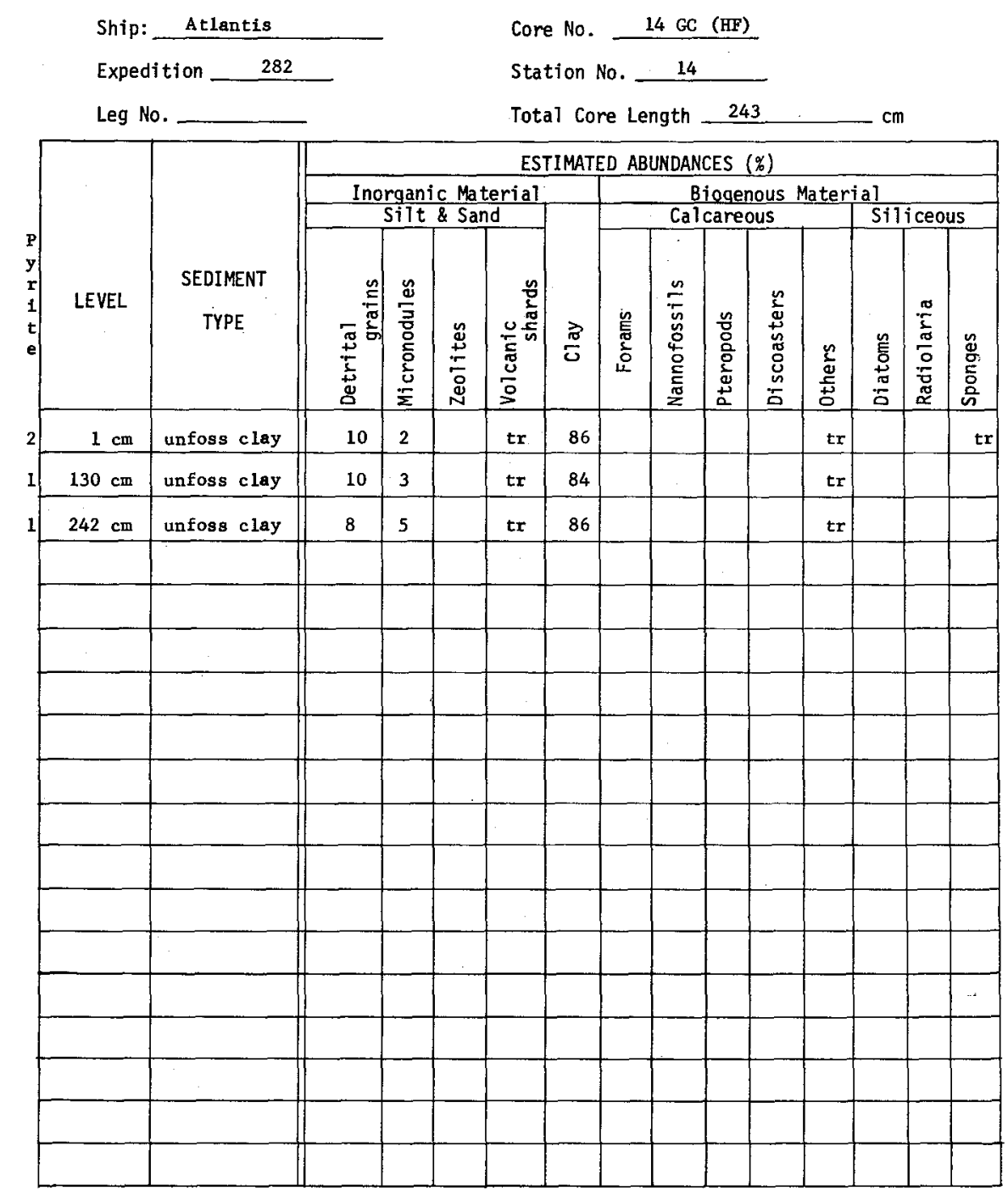


VISUAL CORE DESCRIPTION

Ship $A$ Cruise 282 Leg_Sta. $\frac{13}{42}$ Core No. $13 G C(H F)$ Total Length $13 / \mathrm{cm}$. Lat. $21^{\circ} 54^{\prime} \mathrm{N}$ Long. $44^{\circ} 37^{\circ} \mathrm{W}$ Depth $5653 \mathrm{coxem}$ Core condition wery dry: fair-poer qate Described loMay 74 by $B$ McGire
Physiogrophic location. Nares Abyssal Plain Lithologic

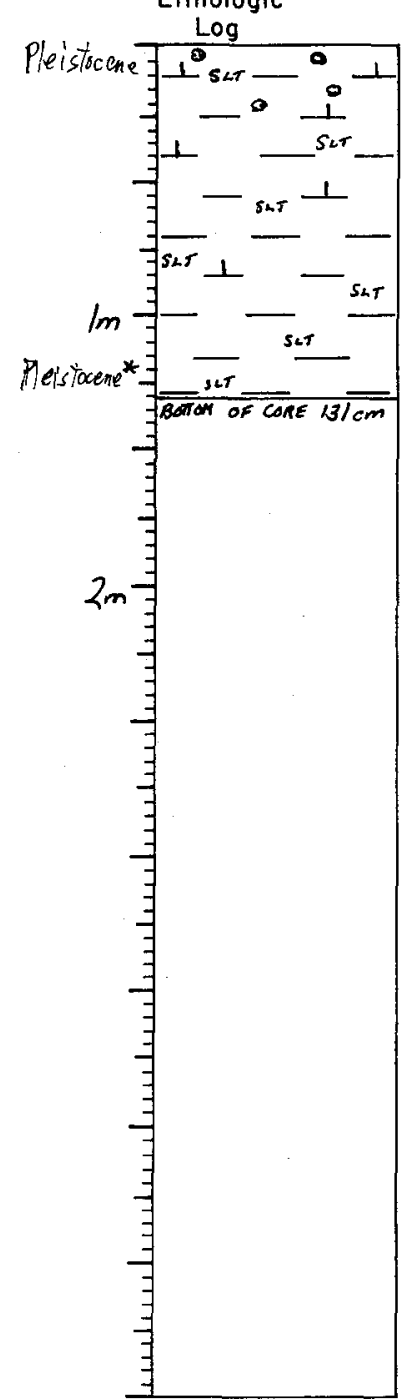

0222

0223

SMEAR SLIDE DESCRIPTIONS - W.H.0.I. SEDIMENT CORES

\section{$0-131 \quad$ Detoiled Description}

CALC CIAY WITH DET TO CLAY WITH DET 10YR $4 / 2,5 / 3$ dk grayish brown and brown common mottling, $0-20 \mathrm{~cm}$

dk brown occurs in central strip, with 1 t color along outside edges

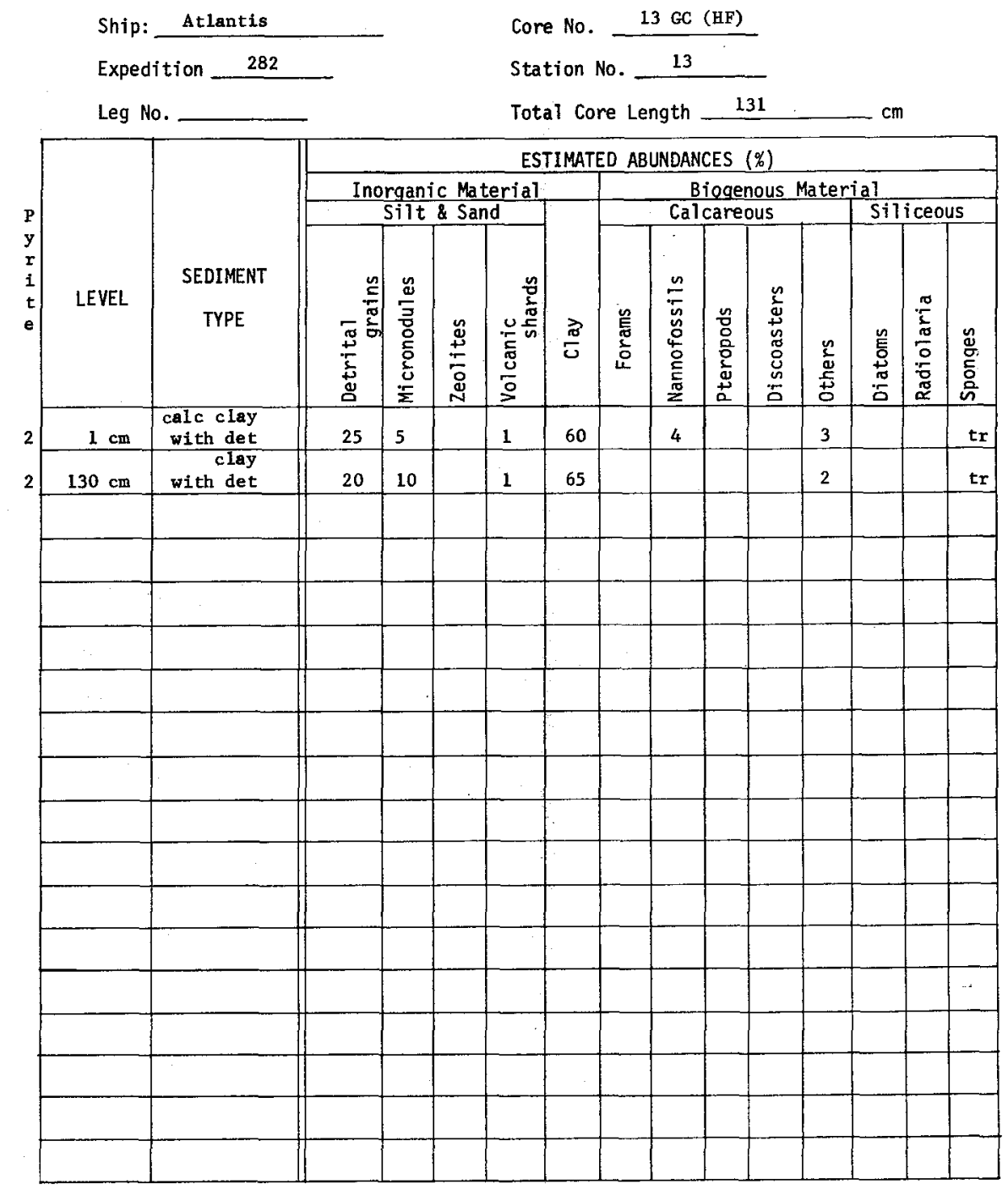


0220

VISUAL CORE DESCRIPTION

Ship $A$ Cruise 282 sta. 12 Core No. $\angle 26 C$ (HF) Totol Length $155 \mathrm{~cm}$. Lat. $20^{\circ} 22^{\prime} \mathrm{N}$ Long. $62^{\circ} 23^{\prime} \mathrm{W}$ Depth 5416 CORR Core condition very dry; fair-peor Date Described 10 May 74 by $B$ MeGier Physiographic location Nares Abyssal Plain

Lithologic

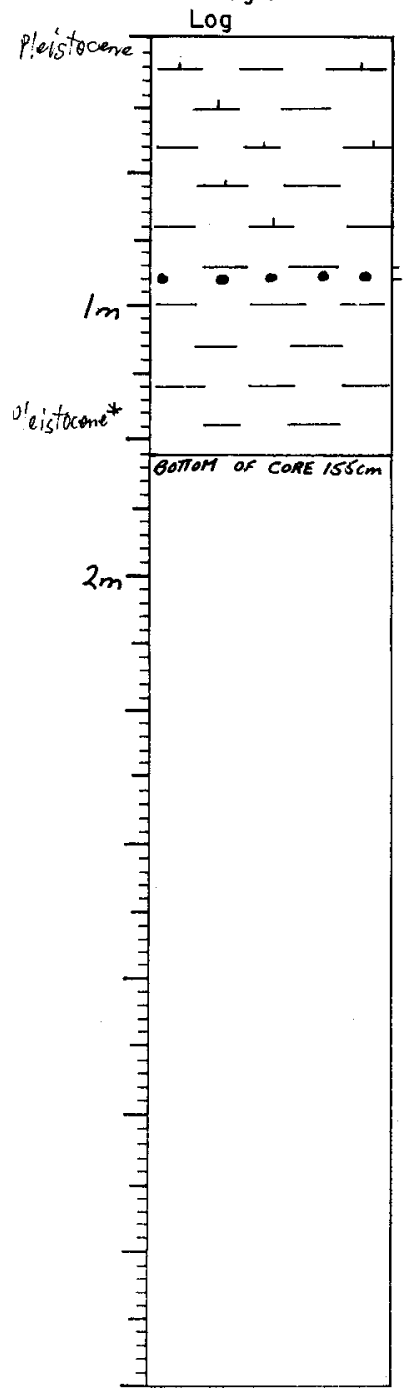

\section{Detoiled Description}

CALC CLAY

10YR $6 / 3$ pale brow

sl silty lutite

hat crumbly, ii fficult to see any feature

irregulax

Mn NODULES

small pebbles, up to $1 \mathrm{~cm}$ in diameter

S irregular

$89-155$

NFOSS CLAY

10YR $6 / 3$ pale brown

sl silty lutite

man poor condition may mask some

features small, b lack specks at 90-100 cm, (broken Mn)
0221

SMEAR SLIDE DESCRIPTIONS - W.H.O.I. SEDIMENT CORES

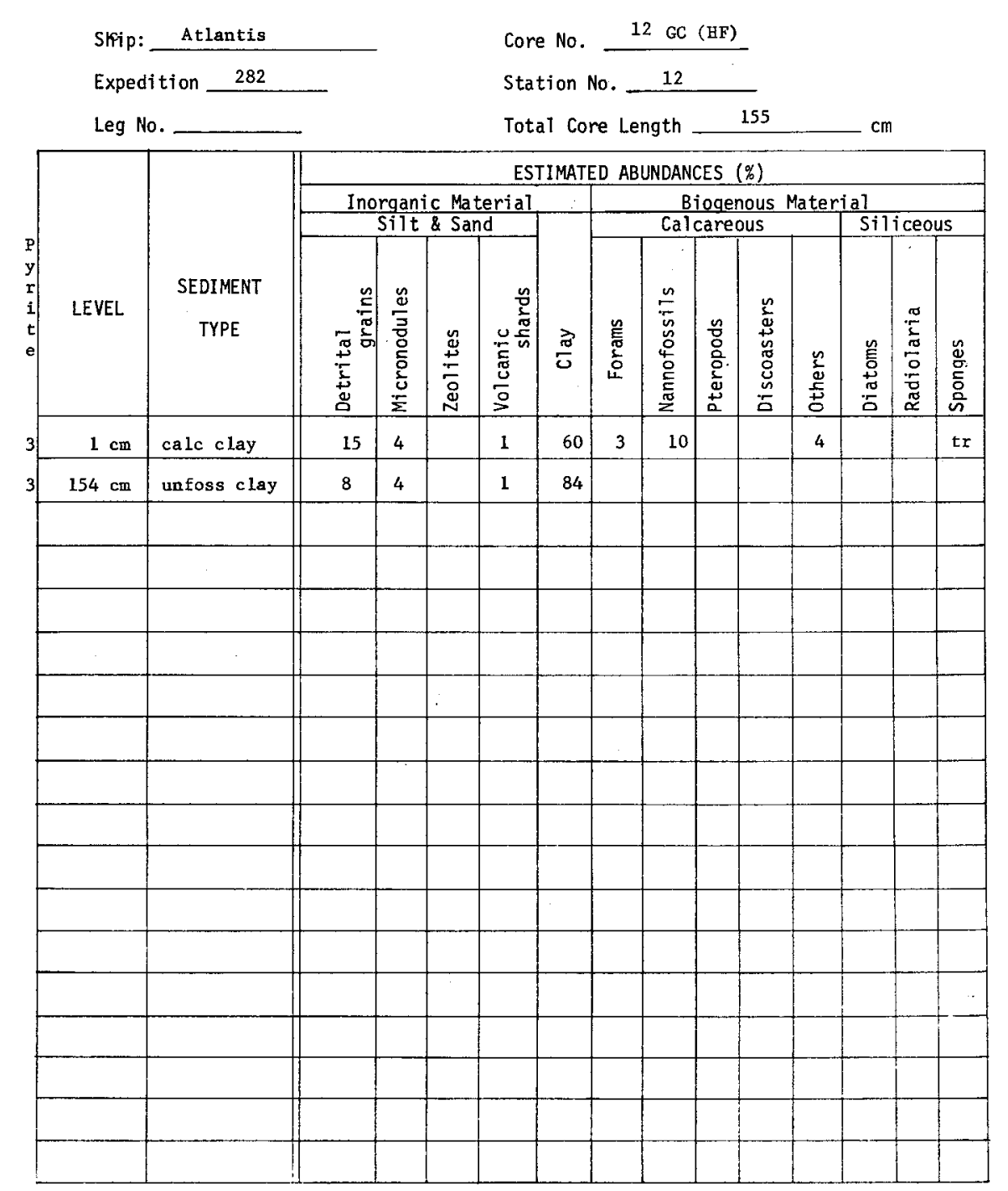


0218

VISUAL CORE DESCRIPTION

Ship A

Cruise 282

28 Leg Sta. $\frac{11}{68^{\circ} 51^{\prime}}$ Core No. $\frac{11 \mathrm{GC}(\mathrm{HF})}{5513 \mathrm{mCOQR}}$

Total Length $185 . \quad \mathrm{cm}$. Lof. $21^{\circ} 47.0^{\prime} \mathrm{N}$ Long. $68^{\circ} 51^{\prime} \mathrm{W}$ Depth $5513 \mathrm{mCopR}$

Core condition dry fregunented; poor Date Described I2APR'74 by GMoumain

Physiographic location NARES ABYSSAL PLAIN

Lithologic

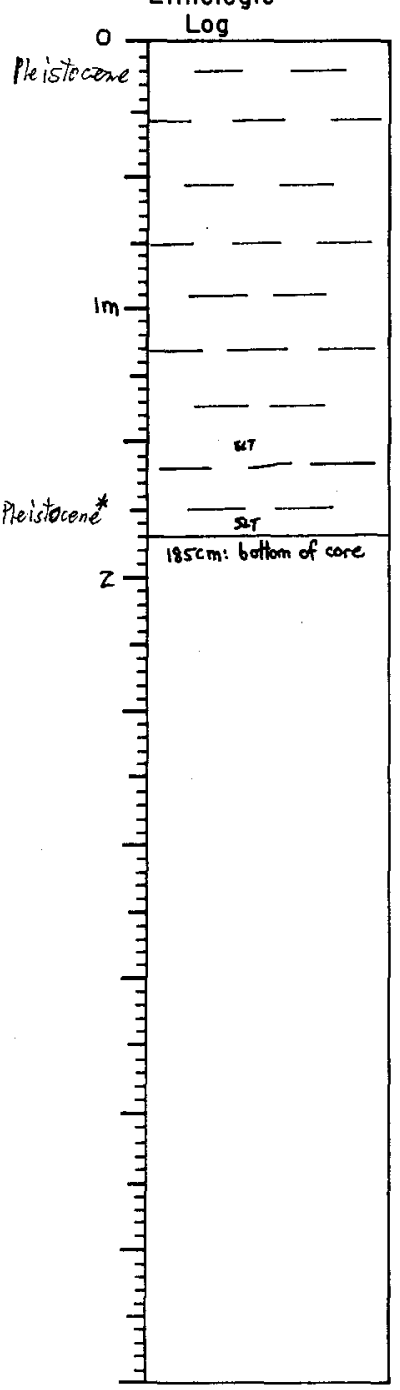

$-185$

UNFOSS CLAY WITH DET SILT INCREASING DOWNWARDS 1OYR $5 / 3$ brown

to see it value
0219

SMEAR SLIDE DESCRIPTIONS - W.H.0.I. SEDIMENT CORES

scattered mottling, pale brown, mottling, where possible

cre $v$. dry and fragmented, visual description of minimal

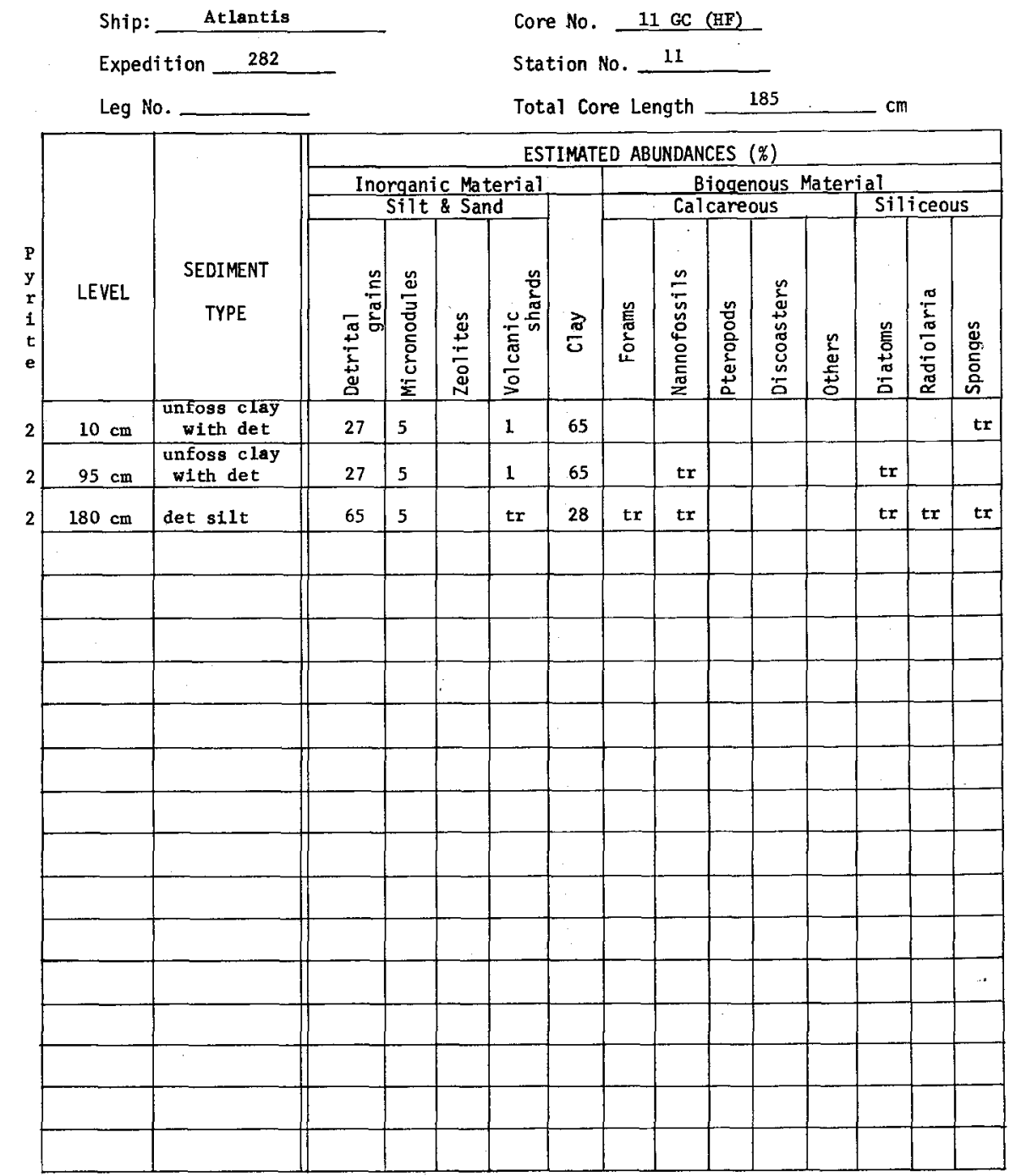


0216

VISUAL CORE DESCRIPTION

Ship A Cruise 282 Leg Sta. 10 Core No. 20 GC(HE) Total Length $I I I \mathrm{~cm}$. Lat. $23^{\circ} 37^{\mathrm{N} N}$ Long. $67^{\circ} 54^{\prime} \mathrm{W}$ Depth 5668 Corr $\mathrm{m}$ Core condition verydy; Poor Date Described 9 May 74 by $B$ MCGice Physiographic location Nares Abyssal Plain

Lithologic

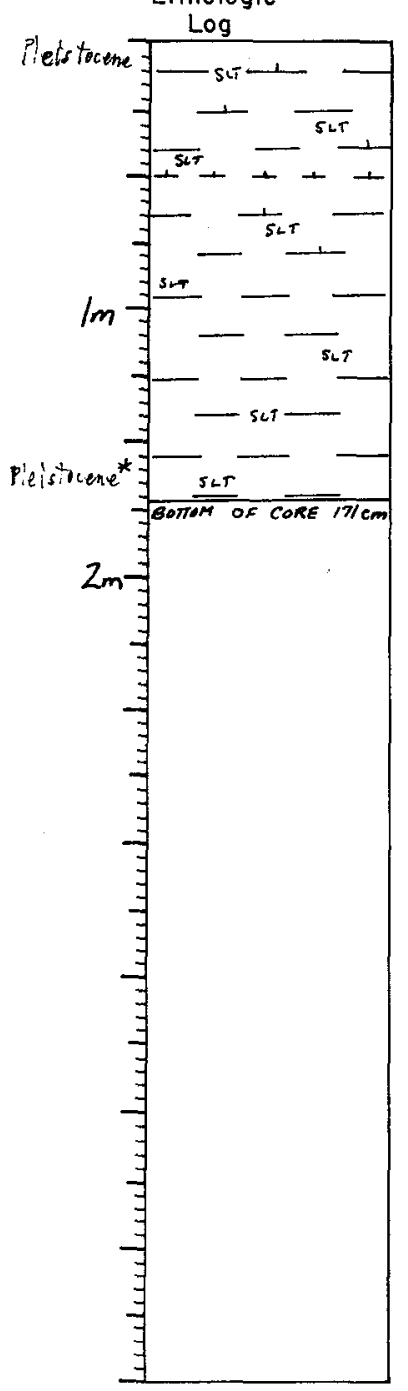

\section{Detailed Description}

$0-171$ SL CALC CLAY WITH DET TO UNFOSS CIAY WITH DET 10YR $6 / 3$ pale brown

dry, hard sl silty lutite original features due to poor condition, white lutite laye $49-50 \mathrm{~cm}$ sherp contacts

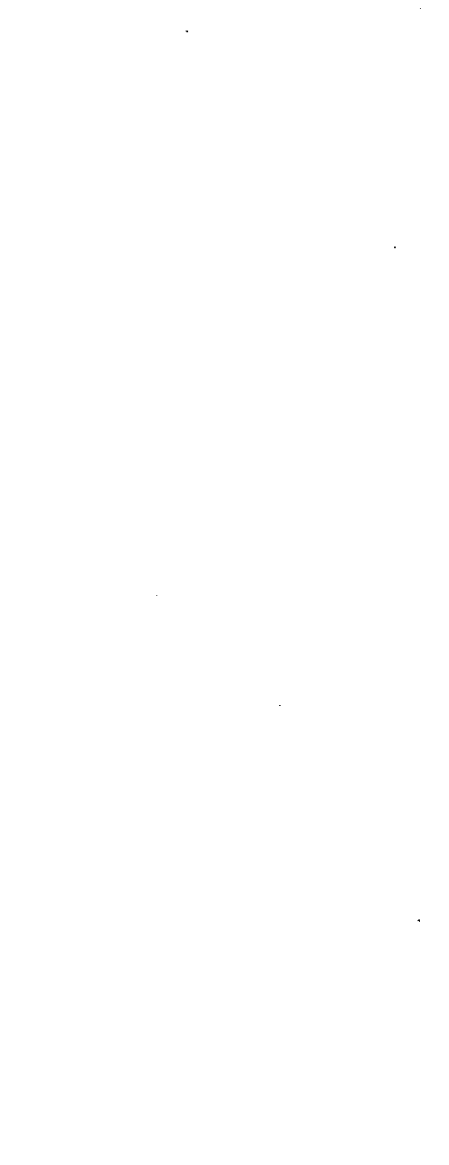

0217

SMEAR SLIDE DESCRIPTIONS - W.H.O.I. SEDIMENT CORES

Ship: Atlantis

Expedition 282

Leg No.

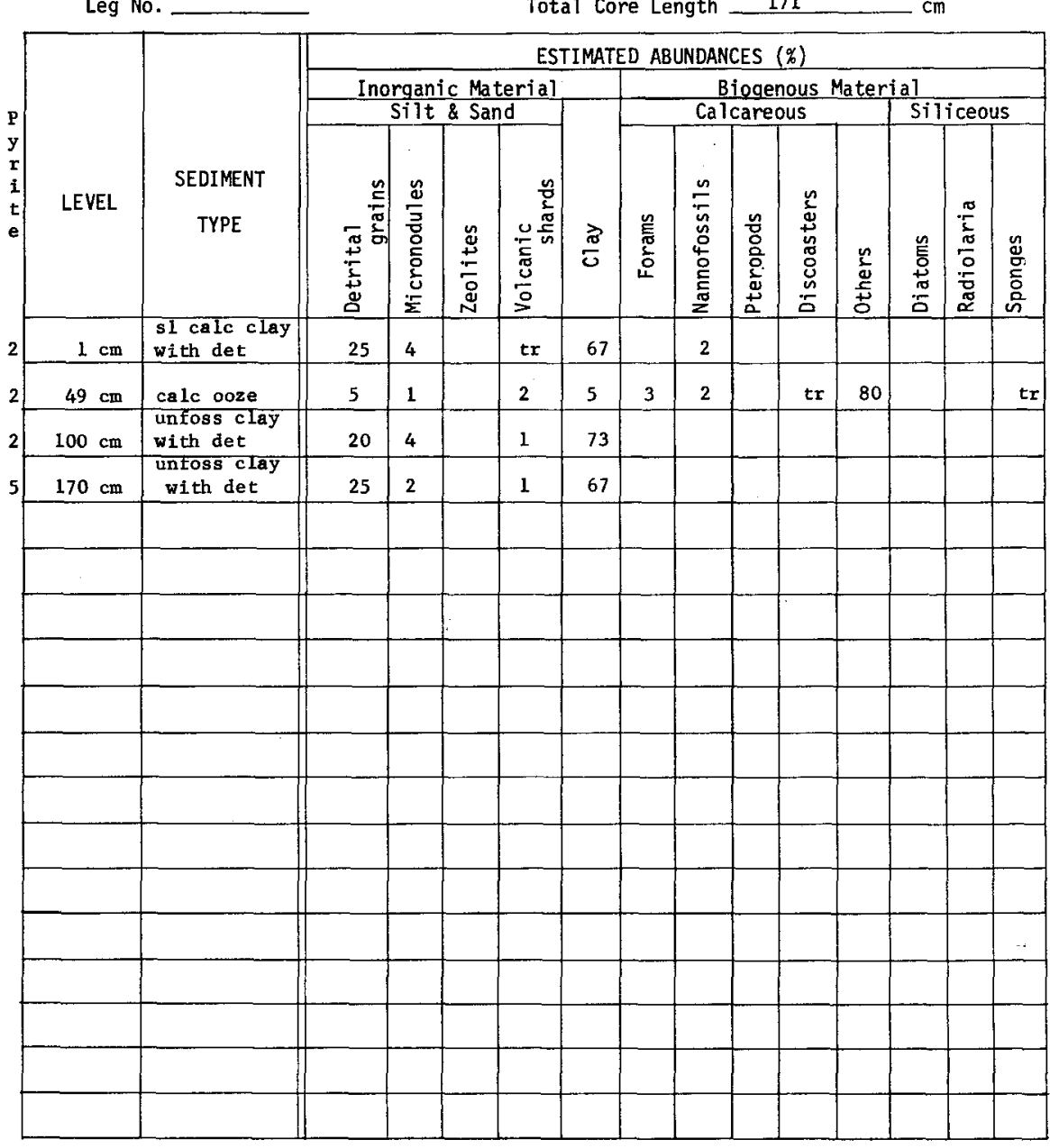

Core No. $10 \mathrm{GC} \mathrm{(HF)}$

Station No.

Station No.

Total Core Length _ 17

Total Core Length 
0214

VISUAL CORE DESCRIPTION

Page __ of _ _ 1

Ship $A$ Cruise 282 Leg__ Sta. $\frac{9}{9}$ Core No. $26 C$ (HE) Total Length $196 \mathrm{~cm}$. Lat. $25^{\circ} / 8^{\circ} \mathrm{N}$ Long. $69^{\circ}$ o $\mathrm{W}$ Depth $5593 \mathrm{CoRRm}$ Core condition verydry, herd; faic-poorDate Described 9 May 74 by $B$ MCGucr

Physiographic location. Nares Abyssal Plain
Lithologic

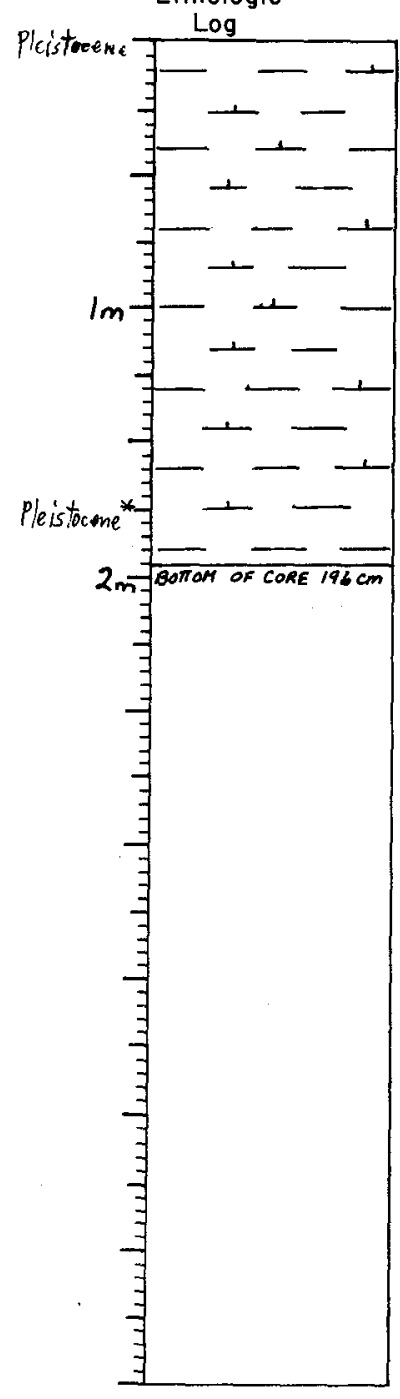

0-196

Detailed Description

SL CALC CIAY TO UNFOSS CLAY 10YR $7 / 11 \mathrm{t}$ gray

dry, silty lutite core split previously but two halves since welded back
together, core v. dry and hard, and real stratigraphic markers may be hidden by the core oondition
0215

SMEAR SLIDE DESCRIPTIONS - W.H.O.I. SEDIMENT CORES
Ship: Atlantis

Expedition 282

Leg No.

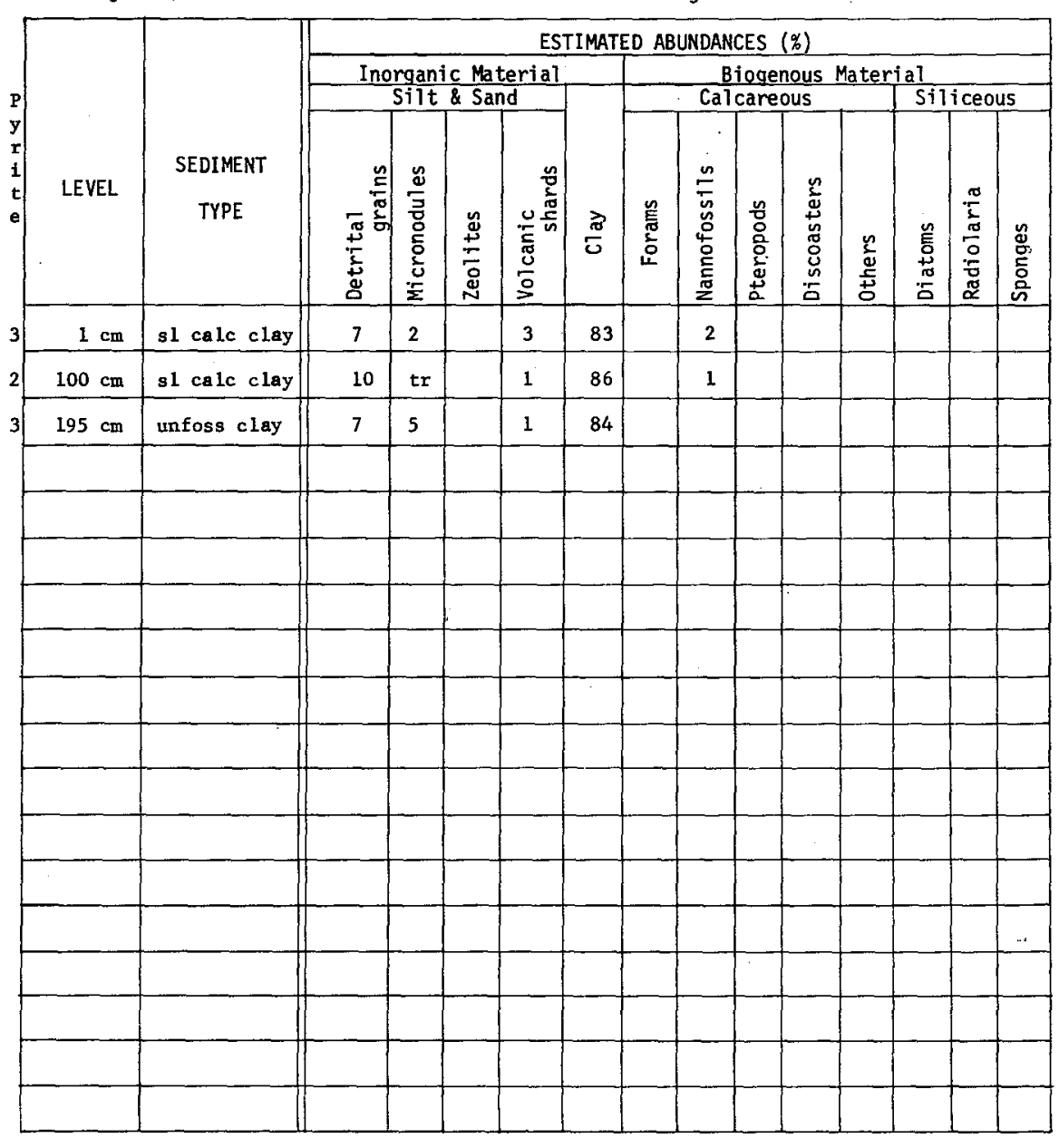

Station No. 9

Total Core Length 196
Core No. $9 \mathrm{GC}(\mathrm{HF})$

Station No. 
0212

VISUAL CORE DESCRIPTION

Page 1 of 1

Ship A Cruise 282 Leg Sta. 8 Core No. 8 GC(HF) Total Length $30 \mathrm{~cm}$. Lat. $25^{\circ}, 1 / \mathrm{N}$ Long. $71^{\circ} 16^{\circ} \mathrm{W}$ Depth 5520 Cogr m

Core condition fairlymoist; gaod Date Described $4 / 23 / 24$ by B MeGire

Physiographic location Neac boundary, Hatteras - Nares Abyssal Plains.

Lithologic

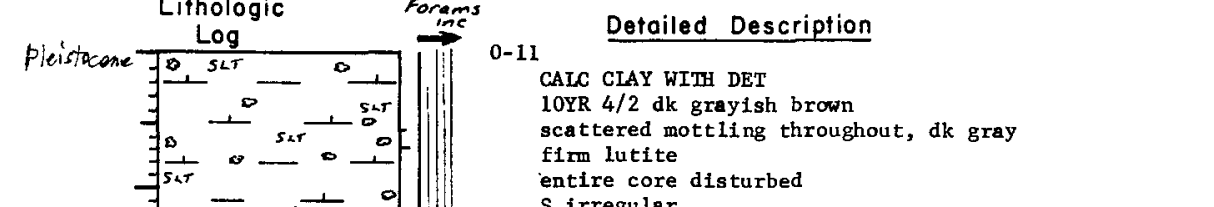
CALC CLAY WITH DET

attered mottling throughout, dk gray

$11-30$

CALC CLAY WITH DET

10xk $4 / 2$ dk grayish browa

scattered motting throughout
0213

SMEAR SLIDE DESCRIPTIONS - W.H.O.I. SEDIMENT CORES

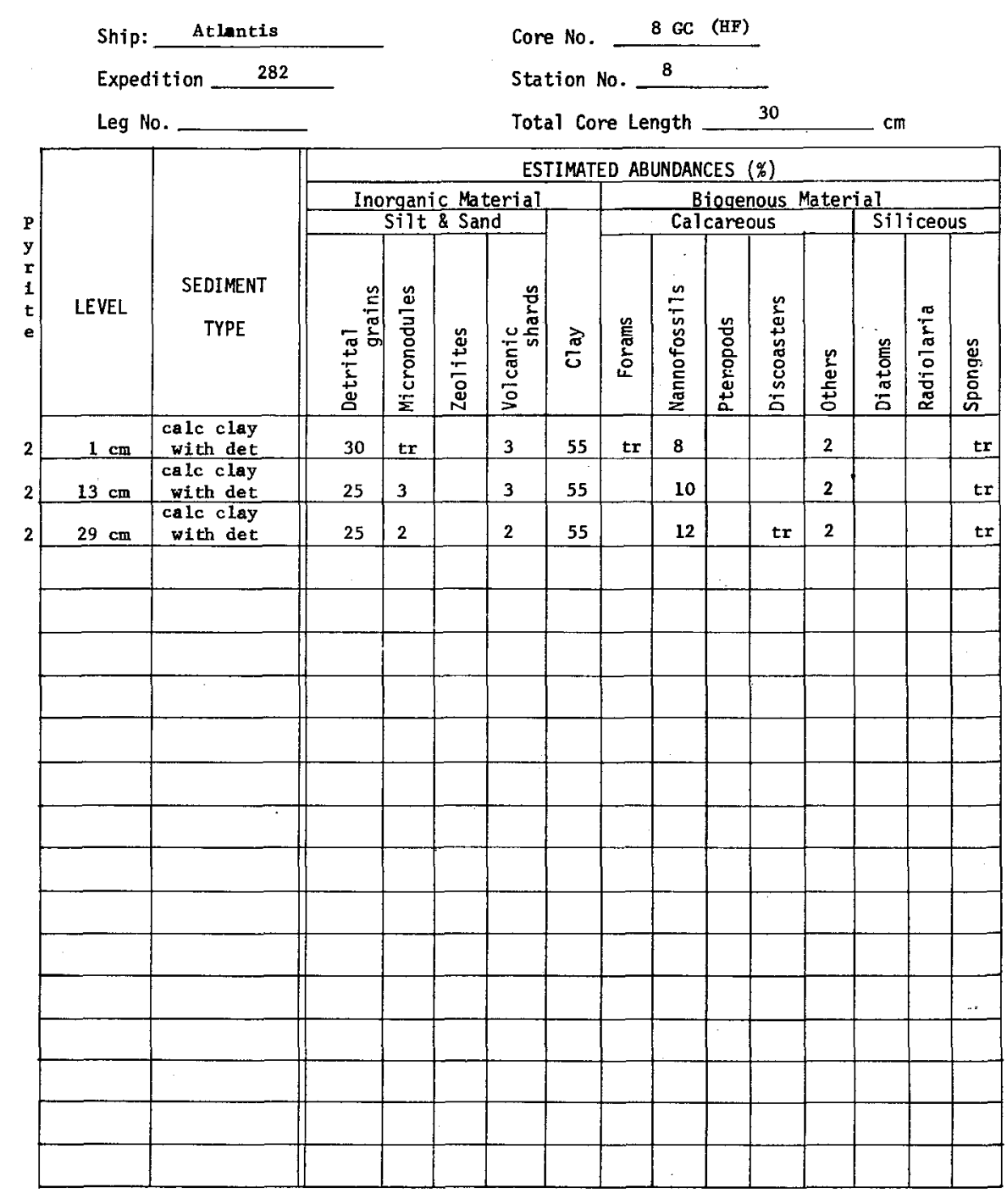


0210

VISUAL CORE DESCRIPTION

Page__ of

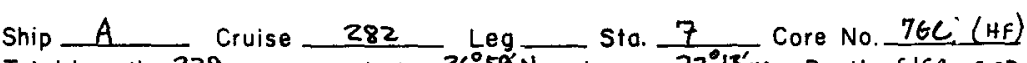
Total Length $228 \mathrm{~cm}$. Lat. $26^{\circ} 59 \mathrm{~N}$ Long. $72^{\circ} 13^{\prime} w$ Depth $5154 \mathrm{mcorR}$ Core condition dry, frimmented, poor —Date Described 12APRk 74 by GMoventAw

Physiogrophic location HATERAS ABYSSRL PLAIN

Lithologic

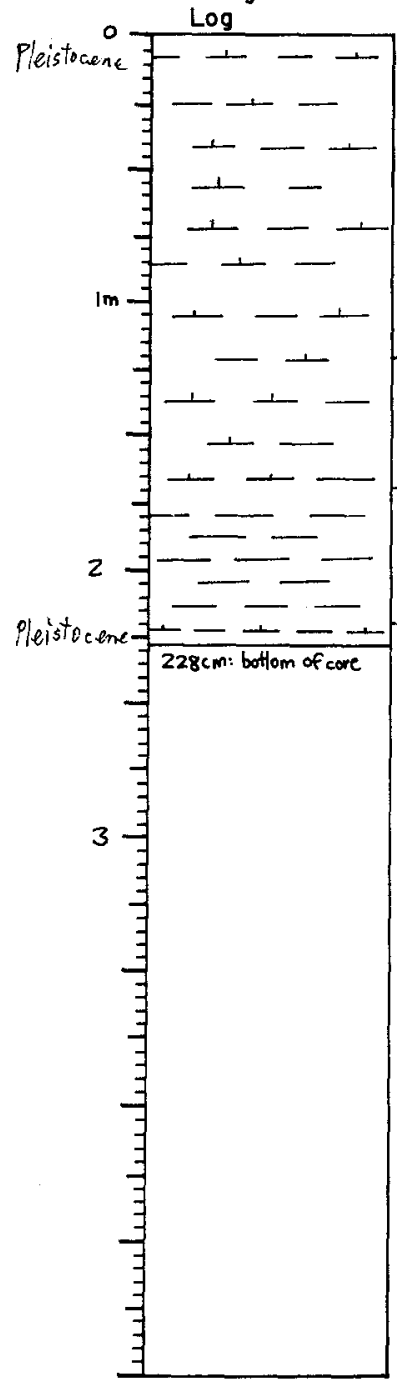

\section{Detailed Description}

$0-121$ HLY CALC CLAY WITH DET

v. difficult to see mottling; v. fine scale $1 \mathrm{t} / \mathrm{dk}$ mottles $70-80$ at least
dry, hard drumbly

colors are secondary, visual description of minimal value numerous fine drying cracks indicate fine lams at $30-40 \mathrm{~cm}$ HI-170 HLY CALC CLAY

fain grayish brown

firm s1 moist, silty lutite

G

UNFOSS CLAY

10YR $4 / 1$ dk gray

silty lutite

omogeneous

220-228

1OYR $7 / 1$ lt gray
dry, hard crumbling silty lutite
HLY CALC CLAY WITH DET

0211

SMEAR SLIDE DESCRIPTIONS - W.H.O.I. SEDIMENT CORES

Ship: Atlantis

Expedition $\quad 282$

Leg No.

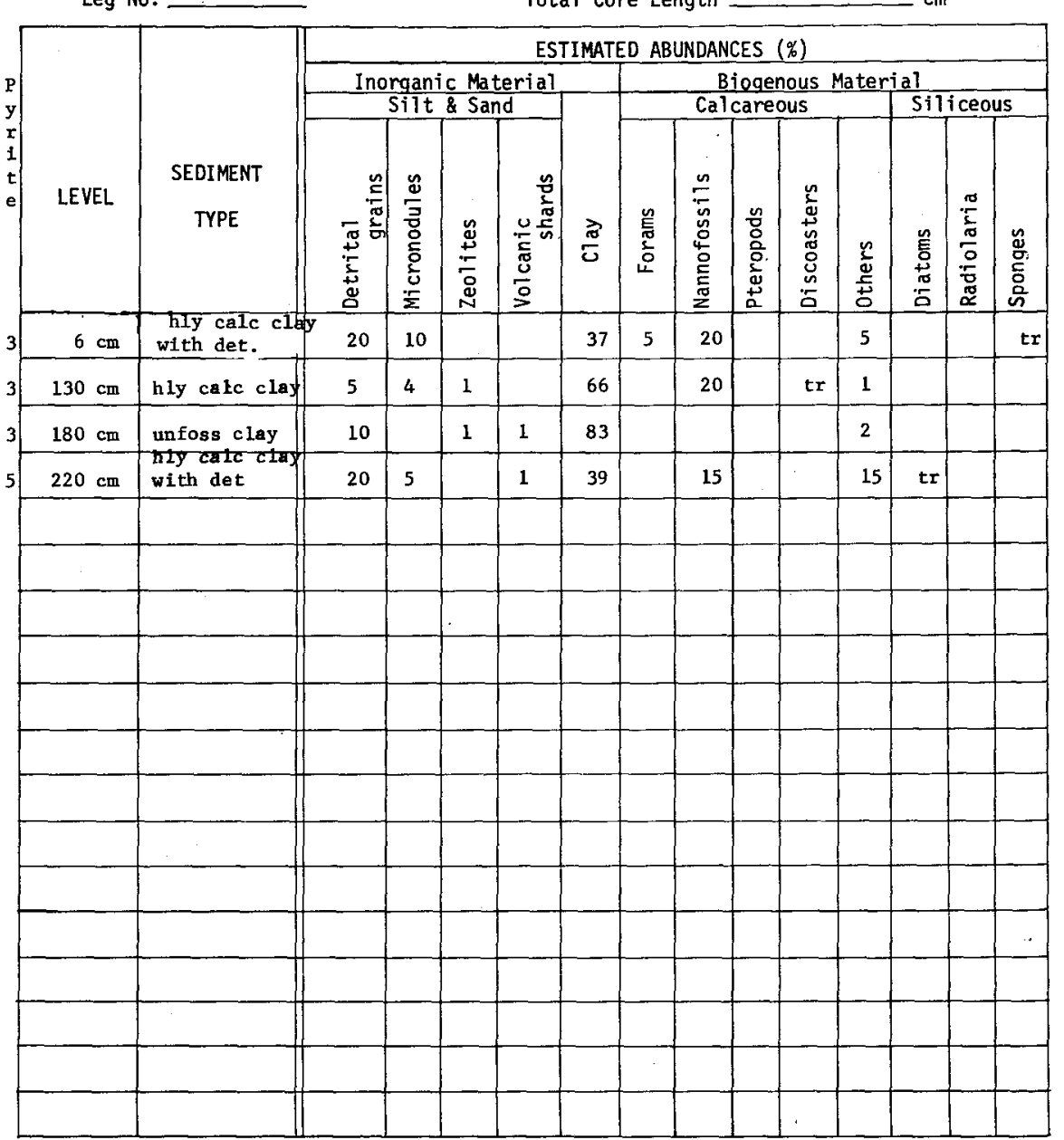

Core No. $7 \mathrm{GC}$ (HF)

Station No.

Station No. 7 (a)

Total Core Length 228

ESTIMATED ABUNDANCES (\%) 
Core condition verydry, hard: paor Date Described $4 / 2 / 24$ by $B M^{\circ}$ Girc

Physiogrophic location Hattaces Abyssel Plain, NE of Bahamas.

Lithologic

Pleistocene

Log

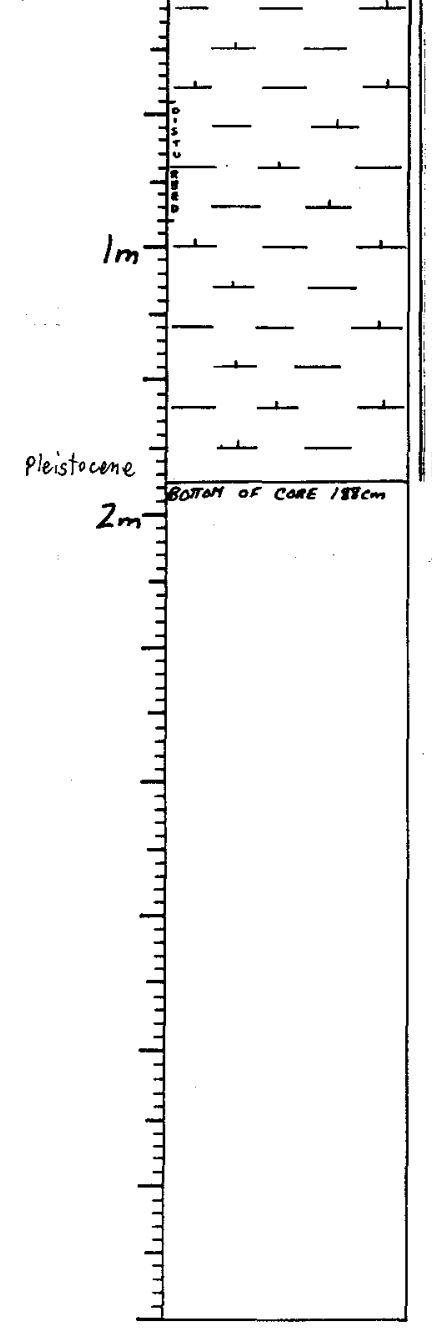

\section{Detailed Description}

\section{$0-188$ Detailed Description}

CALC CIAY WITH DET TO SL CALC CLAY WITH DET 10YR $6 / 2$ it brownish gray

$590 \mathrm{~cm}$, dk thick layer at $128 \mathrm{~cm}, \mathbf{s} 1$ colo variations throughout core
SMEAR SLIDE DESCRIPTIONS - W.H.O.I. SEDIMENT CORES




0206

VISUAL CORE DESCRIPTION

Page 1 of 1

Ship A Cruise 282 Leg__ Sto. 5 Core No. 5 GC (HE) Total Length $164 \mathrm{~cm}$. Lat. $23^{\circ} 28^{\circ} \mathrm{N}$ Long. $22^{\circ} 18.5^{\circ} \mathrm{W}$ Depth 5287 Corem Core condition doy, hard; fair Date Described 9 May 74 by $\mathcal{B} M \cdot$ Gior

Physiographic location North of Eastern Bahomes

Lithologic

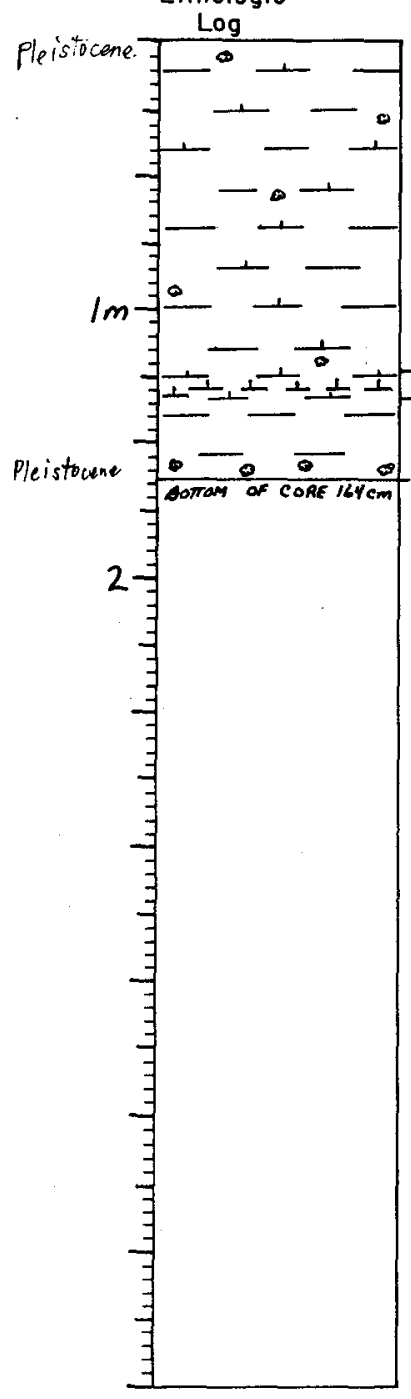

Detailed Description

$0-123$

CAIC CLAY

$10 \mathrm{YR} 7 / 26 / 3,1 \mathrm{t}$ gray and pale brown scattered mottling in places

Dresent in places, unit homogeneous in other places

S3-134

CALC OOZE

CALC OOZE

dry silt

$S$ irregular

134-164

INFOSS CIAY

10YR $6 / 2$ 1t brownish gray

extensive mottling, $155-164 \mathrm{~cm}$ $\mathrm{d}$, s1 silty lutite

0207

SMEAR SLIDE DESCRIPTIONS - W.H.O.I. SEDIMENT CORES

\begin{tabular}{|c|c|c|c|c|c|c|c|c|c|c|c|c|c|c|}
\hline \multirow[b]{4}{*}{ LEVEL } & \multirow[b]{4}{*}{$\begin{array}{c}\text { SEDIMENT } \\
\text { TYPE }\end{array}$} & \multicolumn{13}{|c|}{ ESTIMATED ABUNDANCES $(\%)$} \\
\hline & & \multirow{2}{*}{\multicolumn{4}{|c|}{$\frac{\text { Inorganic Material }}{\text { Silt \& Sand }}$}} & & \multirow{2}{*}{\multicolumn{5}{|c|}{$\begin{array}{l}\text { Biogenous Mater } \\
\text { Calcareous }\end{array}$}} & & & \\
\hline & & & & & & & & & & & & \multicolumn{3}{|c|}{ Siliceous } \\
\hline & & 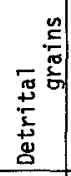 & 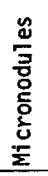 & 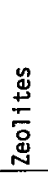 & 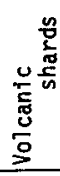 & $\frac{\mathrm{d}}{\mathrm{U}}$ & 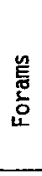 & 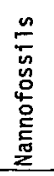 & 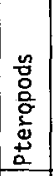 & 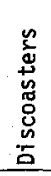 & 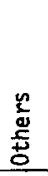 & 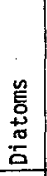 & 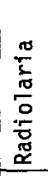 & 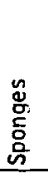 \\
\hline $1 \mathrm{~cm}$ & calc clay & 12 & 3 & & tr & 70 & 1 & 10 & & & 2 & & & \\
\hline $115 \mathrm{~cm}$ & calc clay & 15 & 1 & & 1 & 69 & tr & 10 & & & 1 & & & \\
\hline $130 \mathrm{~cm}$ & calc ooze & 10 & & & $\mathrm{tr}$ & 5 & 30 & & $\mathrm{tr}$ & & 55 & & & tx \\
\hline $164 \mathrm{~cm}$ & unfoss cley & 15 & 1 & & $\mathrm{tr}$ & 79 & & & & & & $\mathrm{tr}$ & & $\operatorname{tr}$ \\
\hline & & & & & & & & & & & & & & \\
\hline & & & & & & & & & & & & & & \\
\hline & & & & & & & & & & & & & & \\
\hline & & & & & & & & & & & & & & \\
\hline & & & & & & & & & & & & & & \\
\hline & & & & & & & & & & & & & & \\
\hline & & & & & & & & & & & & & & \\
\hline & & & & & & & & & & & & & & \\
\hline & & & & & & & & & & & & & & \\
\hline & & & & & & & & & & & & & & \\
\hline & & & & & & & & & & & & & & \\
\hline & & & & & & & & & & & & & & \\
\hline & & & & & & & & & & & & & & \\
\hline & & & & & & & & & & & & & & \\
\hline
\end{tabular}

Ship: Atlantis

Expedition 282

Leg No.

Core No. 5 GC (HF)

Station No.

Total Core Length 164 
0204

VISUAL CORE DESCRIPTION

Page _ _ of _ـ 1

Ship $A$ Cruise 282 Leg 3 Core No $3 \mathrm{GC}$ (HF) Total Length $120 \mathrm{~cm}$. Lat. $23^{\circ} 32.5^{\circ} \mathrm{N}$ Long. $70^{\circ} 02^{\circ} \mathrm{W}$ Depth 5492 EorR m Core condition fairly moist; good Date Described 9 May 74 by $B$ Me Gier Physiographic location Hatteres Abyssal Plain

Lithologic

Preistiocene

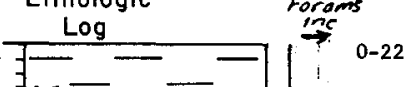

0-22 Detoiled Description

UNFOSS CIAY (DET $15-16 \mathrm{~cm}$ )

10YR $4 / 2$ dk grayish brown

Eirm lutice

$\left.\bar{Z}-{ }_{s_{L T}}\right]^{\bar{s} T}$

$\exists-\quad$ Str

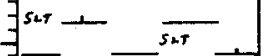

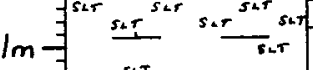

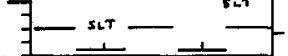

Pleistucene

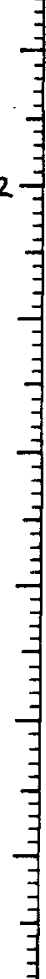

$$
\text { v. thin horizontal lams }
$$

2-81

81

10YR 4/1 dk gray

firm lutite

81-92

$2.5 \mathrm{Y} 6 / 2$ 1t brownish gray

vitury

faint, $v$. thin wavy lams throughout, possible graded beddin S irregular

92-112

SL CALC CLAY WITH DET

10XR $5 / 2$ grayish brown

firm lutite

112-120

CAIC OOZE

10YR $7 / 2$ it gray

Ilty lutite

end of core
0205

SMEAR SLIDE DESCRIPTIONS - W.H.O.I. SEDIMENT CORES

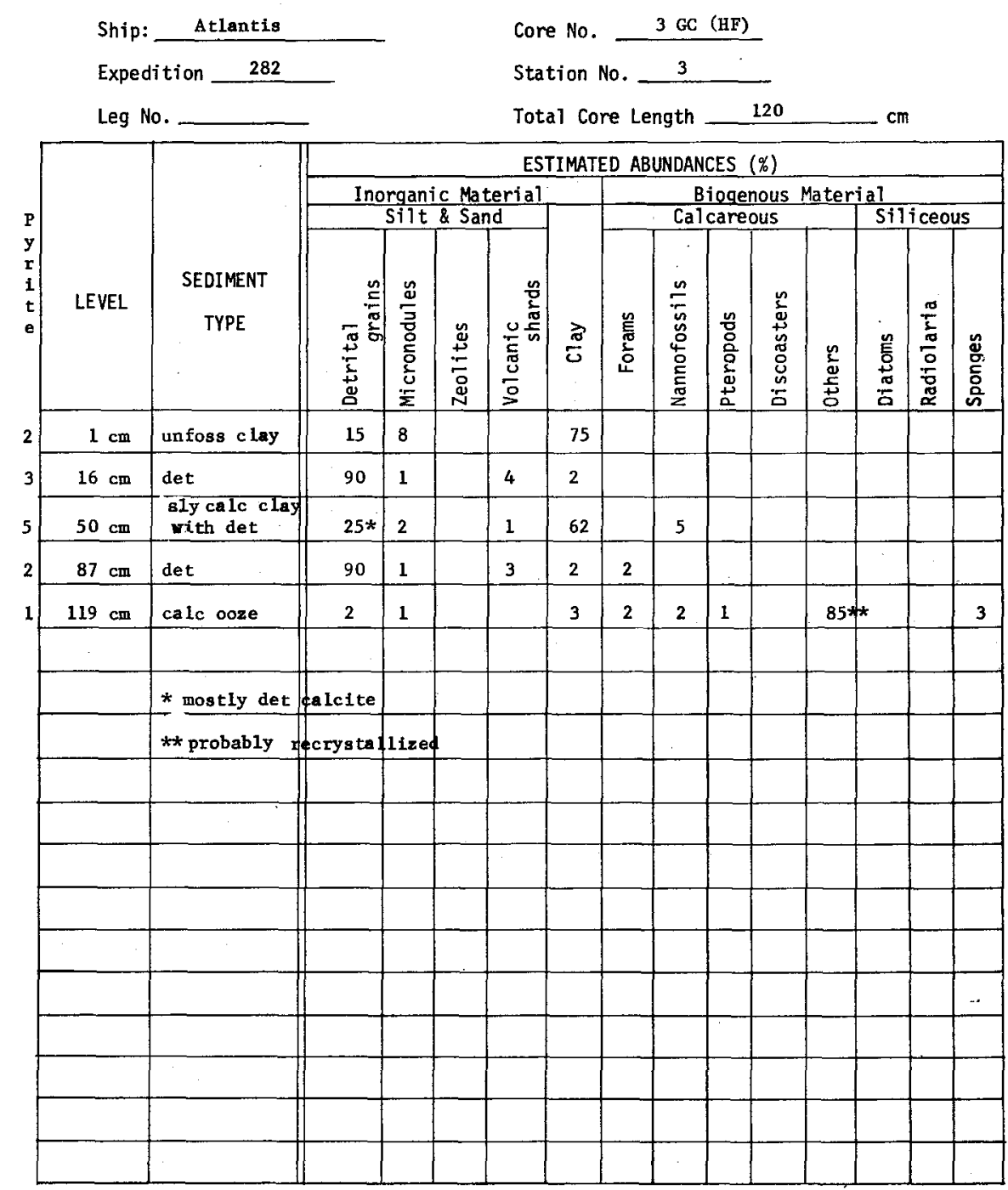


VISUAL CORE DESCRIPTION

Ship $A$ Cruise 282 Leg Sto. 2 Core No. 2GC (HF) Total Length $190 \mathrm{~cm}$. Lot. $28^{\circ} 52^{\circ} \mathrm{N}$ Long. $66^{\circ} 51^{\circ} \mathrm{W}$ Depth 5451 CORR Core condition dry; hand; fair Date Descriped 8 May 74 by BM $B$ C Gir

Physiographic location.Nares Abyssal Plain (W Margin)

Lithologic

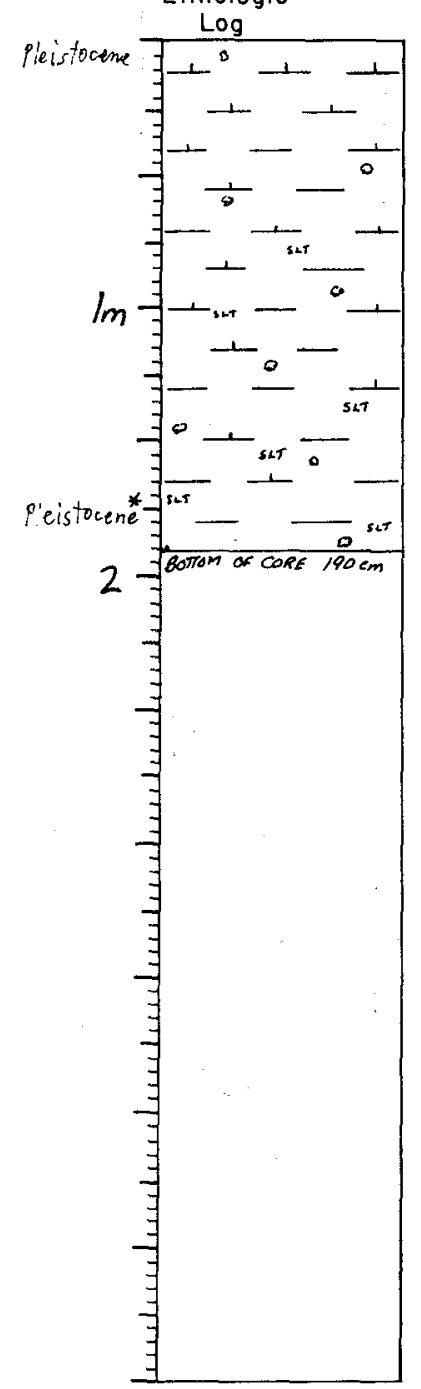

0202

Page _ 1 of $\_1$

0203

SMEAR SLIDE DESCRIPTIONS - W.H.O.I. SEDIMENT CORES

Detailed Description

CALC OOZE TO SL CALC CLAY TO SL CALC GIAY WITH DETRITUS 10YR $5 / 3$ brown

scattered mottling throughout

dry, silty lutite

dry, hard, crumbly core, somewhat disturbed

Note; Visually no change in lithology was noted. However the smear slide analysis suggests at least three different units. The basic change, from calc ooze to calc clay, may take place at about $25 \mathrm{~cm}$, where the sediment becomes $\mathrm{v}$. sl dk than above.

\begin{tabular}{|c|c|c|c|c|c|c|c|c|c|c|c|c|c|c|}
\hline Ship & Atlantis & & & & Core & No. & 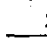 & GC $(1$ & & & & & & \\
\hline Expe & ition 282 & & & & Stat & ion 1 & o. - & 2 & & & & & & \\
\hline Leg & . & & & & Tota & 1 Cor & e Le & gth & 1 & & 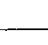 & $-\mathrm{cm}$ & & \\
\hline & & & & & & IMATE & $D A B$ & NDANC & ES & & & & & \\
\hline & & & rgani & $c \mathrm{Ma}$ & erial & & & & Qger & $M$ & teri & & & \\
\hline $\mathrm{P}$ & & & Si]t & $\& \mathrm{Sa}$ & & & & Calc & ares & & & Sil & iceo & \\
\hline LEVEL & $\begin{array}{l}\text { SEDIMENT } \\
\text { TYPE }\end{array}$ & 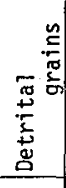 & 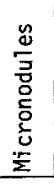 & 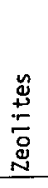 & 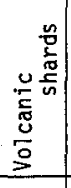 & $\frac{\vec{m}}{\omega}$ & 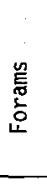 & 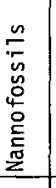 & 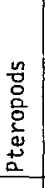 & 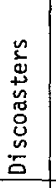 & 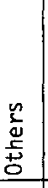 & 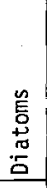 & 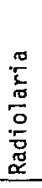 & 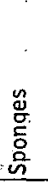 \\
\hline $1 \mathrm{~cm}$ & calc ooze & 10 & 5 & & 1 & 32 & 5 & 30 & & $\mathrm{tr}$ & 15 & & & \\
\hline $100 \mathrm{~cm}$ & s1 calc clay & 10 & 8 & & 1 & 75 & 1 & 1 & & & 1 & & & \\
\hline \begin{tabular}{l|l}
2 & $187 \mathrm{~cm}$ \\
\end{tabular} & $\begin{array}{l}\text { si calc clay } \\
\text { with det }\end{array}$ & 20 & 3 & & 1 & 72 & & 1 & & & 1 & & $\because$ & \\
\hline & & & & & & & & & & & & & & \\
\hline & & & & & & & & & & & & & & \\
\hline & & & & & & & & & & & & & & \\
\hline & & & & & & & & & & & & & & \\
\hline & & & & & & & & & & & & & & \\
\hline & & & & & & & & & & & & & & \\
\hline & & & & & & & & & & & & & & \\
\hline & & & & & & & & & & & & & & \\
\hline & & & & & & & & & & & & & & \\
\hline & & & & & & & & & & & & & & \\
\hline & & & & & & & & & & & & & & . \\
\hline & & & & & & & & & & & & & & \\
\hline & & & & & & & & & & & & & & \\
\hline & & & & & & & & & & & & & & \\
\hline & & & & & & & & & & & & & & \\
\hline
\end{tabular}


0242

VISUAL CORE DESCRIPTION

Ship $A$ Cruise 282 Leg_ Sta. $\frac{23}{2}$ Core No. 23 PC Total Length $437 \mathrm{~cm}$. Lat. $30^{\circ} 27^{\prime} \mathrm{N}$ Long. $67^{\circ} 58^{\prime} \mathrm{W}$ Depth $5188 \mathrm{cosem}$ Core condition dry fair Date Described 15 May 74 by $B$ MCGuir

Physiographic location Bermuda Rise

Lithologic

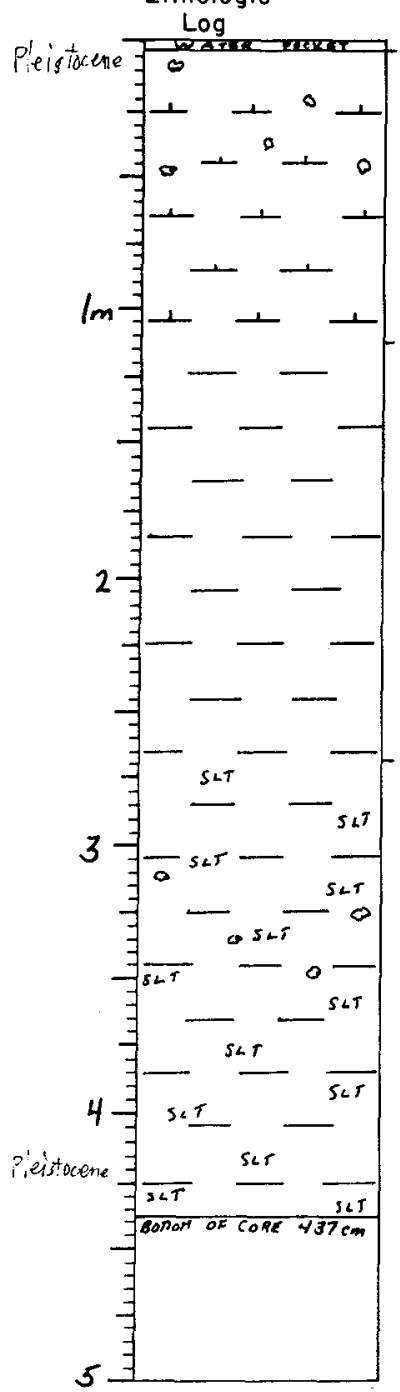

10YR 4/2 dk grayish brown
Poge _L L

0243

SMEAR SLIDE DESCRIPTIONS - W.H.O.I. SEDIMENT CORES

\section{Detailed Description}

WATER POCKET

CALC OOZE

IOYR $7 / 2$ 1t gray

(1) pale brown

dry, hard lutite, also crumbly

113-268

UNFOSS CLAY

farly dry, sil siley lutite

somewhat crumbly and disturbed

268-437

UNFOSS CLAY/DET

10YR $6 / 3$ pale brown

scattered mottling $300-350 \mathrm{~cm}$

dxy, hard silty lutite

somewhat crumbly and disturbed

$\mathrm{S}$

Expedition 282

Leg No.
Ship: Atlantis

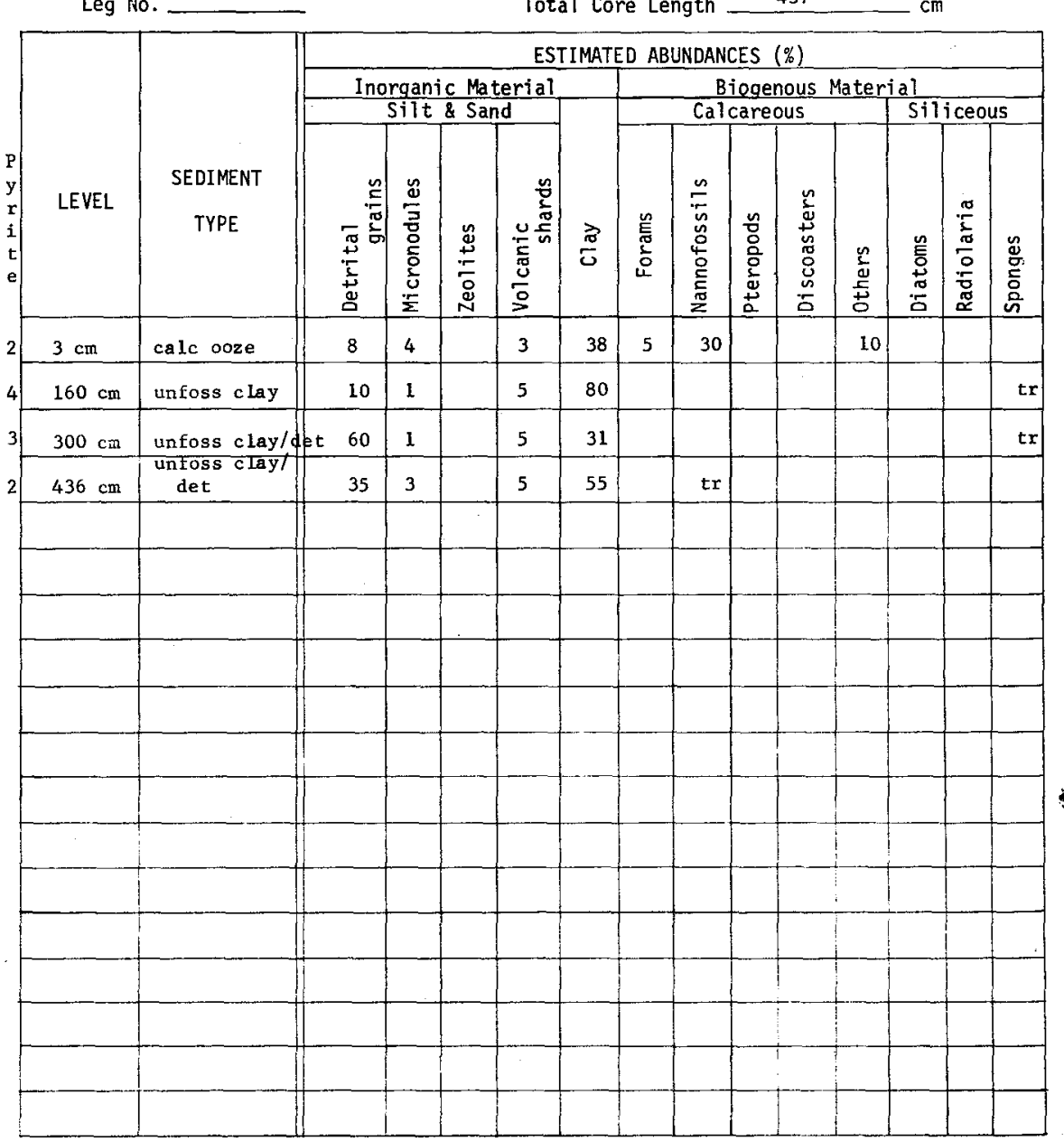

Core No. 23 PC

Station No. 23 


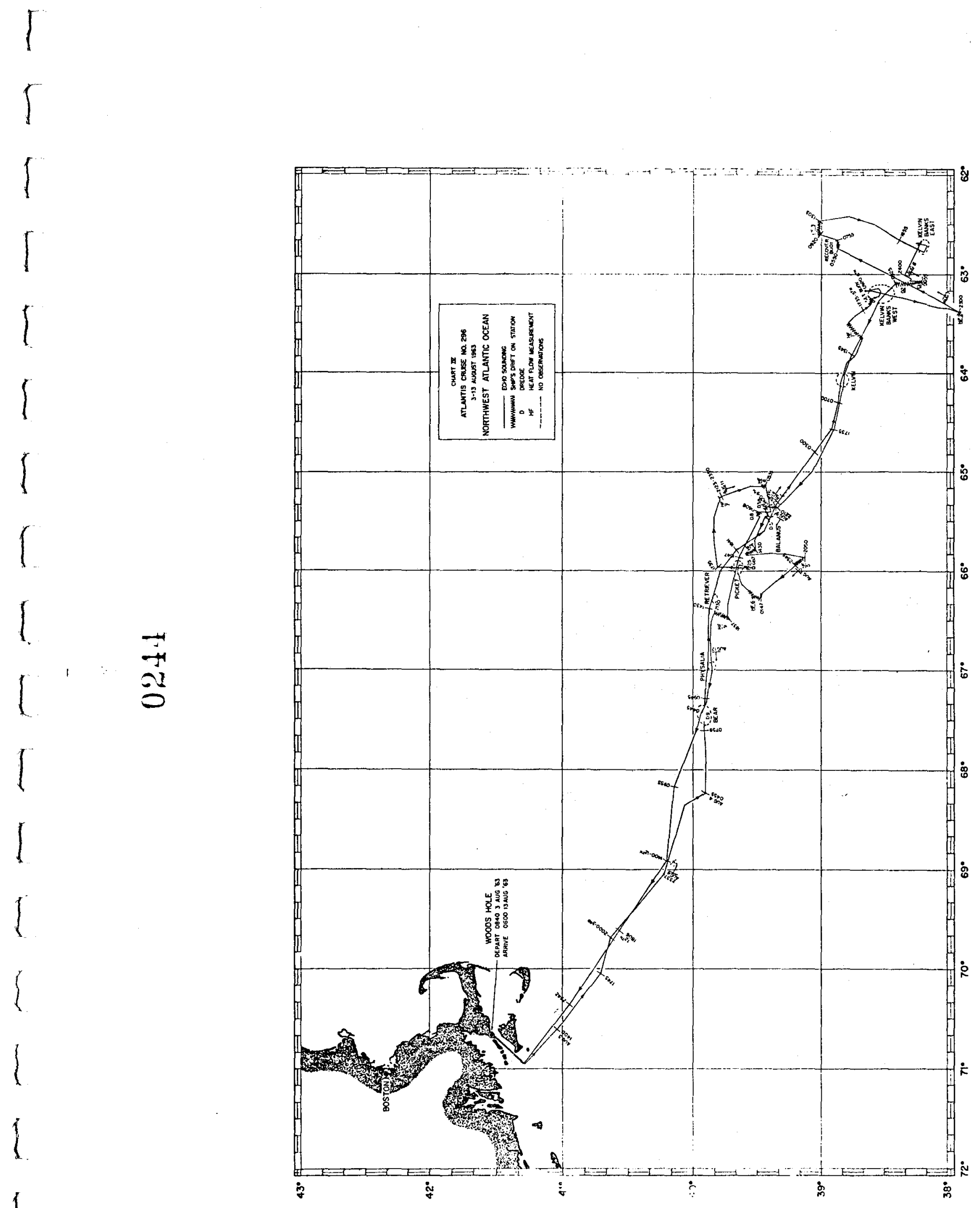



0246

VISUAL CORE DESCRIPTION

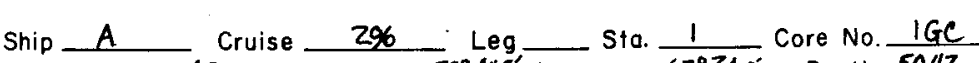

Total Length $63 \mathrm{~cm}$. Lot. $38^{\circ} 44.5^{\circ} \mathrm{N}$ Long. $63^{\circ} 34.0^{\circ} \mathrm{N}$ Depth $5043 \mathrm{mCor}$

Core condition dry, crumblect. fair Date Described 10 MAY74 by GMlountain

Physiogrophic location BETWEEN KELVIN SEAMOUNT + KELVIN BANKS

Lithologic

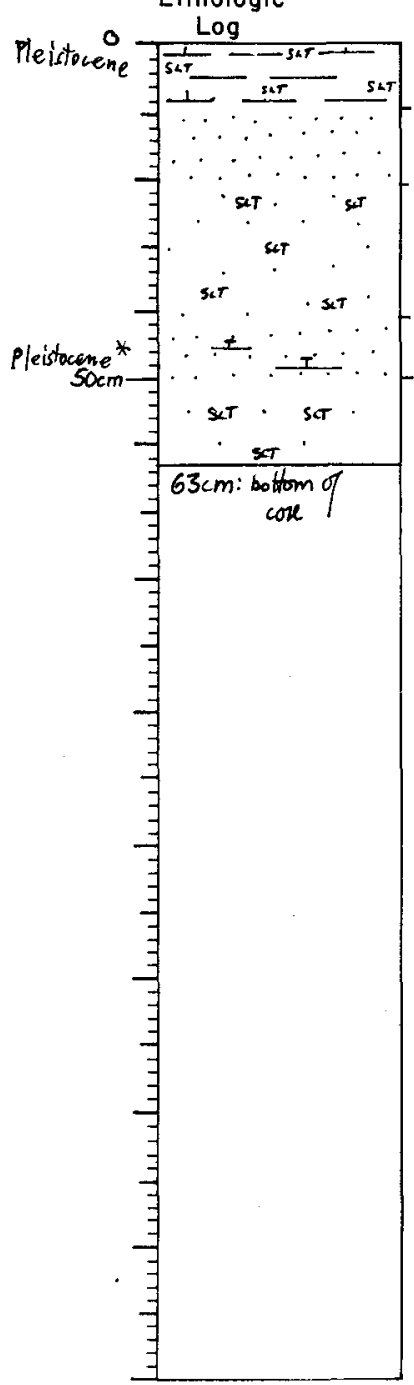

\section{Detailed Description}

C-9 CALC CIAY/DET

7.5YR $6 / 2$ pinkish gray

hard lutite

$S$ convex upward

$9-21$

DET SILT AND SAND

7.5YR $6 / 2$ pinkish gray with variously color sand grains

$1-41$

DET SILT AND SAND

10YR $7 / 3 \mathrm{v}$. pale brown $S$

$41-50$

DET SAND

LOYR $7 / 2$ 1t gray with various sand colors medium to coarse sand, with scattered forams G

DET SILT AND SAND

DET SILT AND SAND
10YR $7 / 3$ v. pale brown

coarse silt to v. fine sand

end of core medium sand in fine matrix

\section{7}

SMEAR SLIDE DESCRIPTIONS - W.H.O.I. SEDIMENT CORES

Ship: Atlantis

Expedition 296

Leg No.

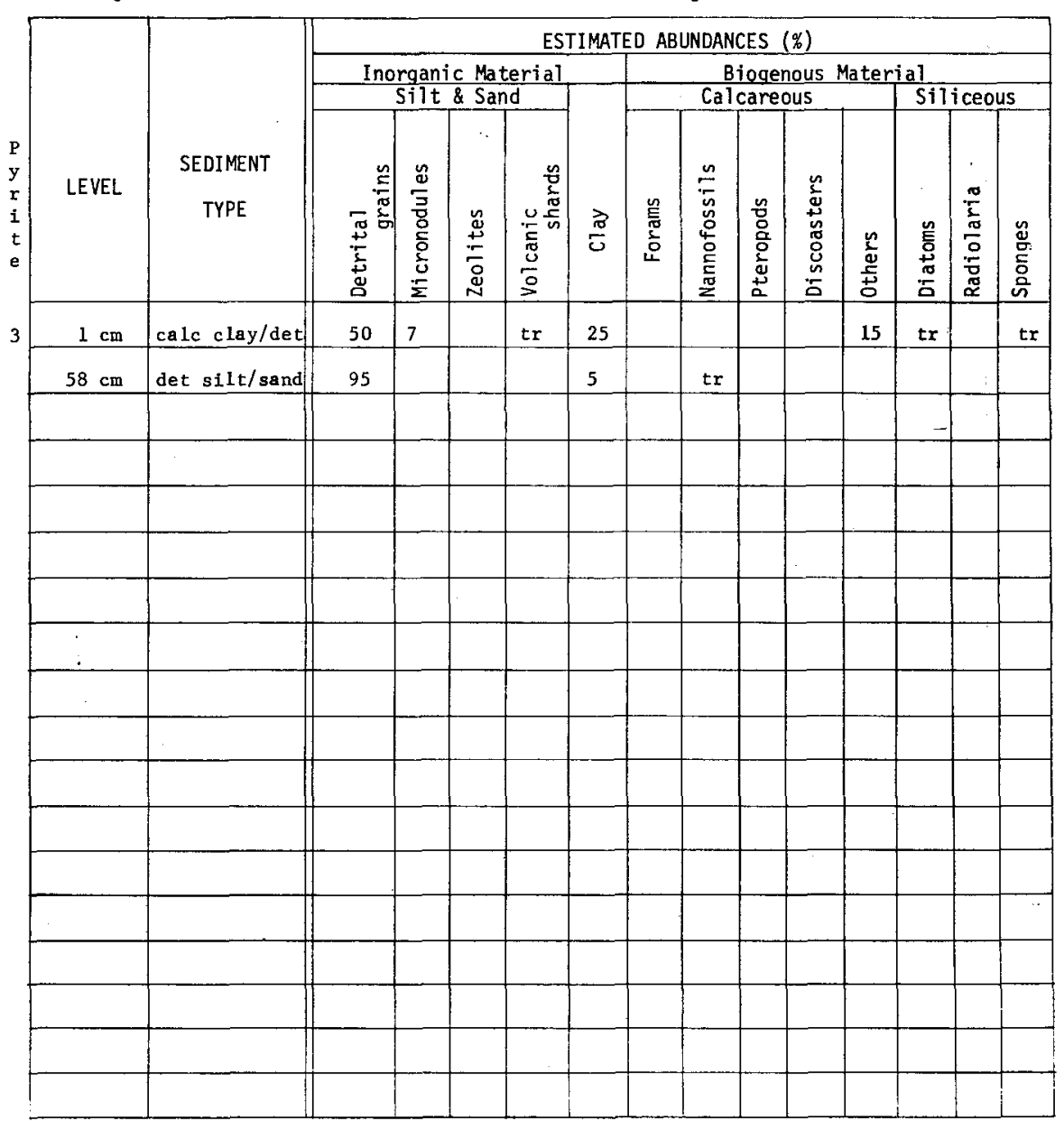




\section{VISUAL CORE DESCRIPTION}

Page 1 of 1

Ship A Cruise 296 Leg__ Sto. 2 _ Core No. 2GC Total Length 46 cm. Lat. $37^{\circ} 53.0^{\circ} \mathrm{N}$ Long. $63^{\circ} 22.0^{\circ} \mathrm{W}$ Depth $5044 \mathrm{mcar}$ Core condition dry. foir Date Described 131th 74 by Gilountan

Physiographic location Sount of KELVIN BAoks

Lithologic

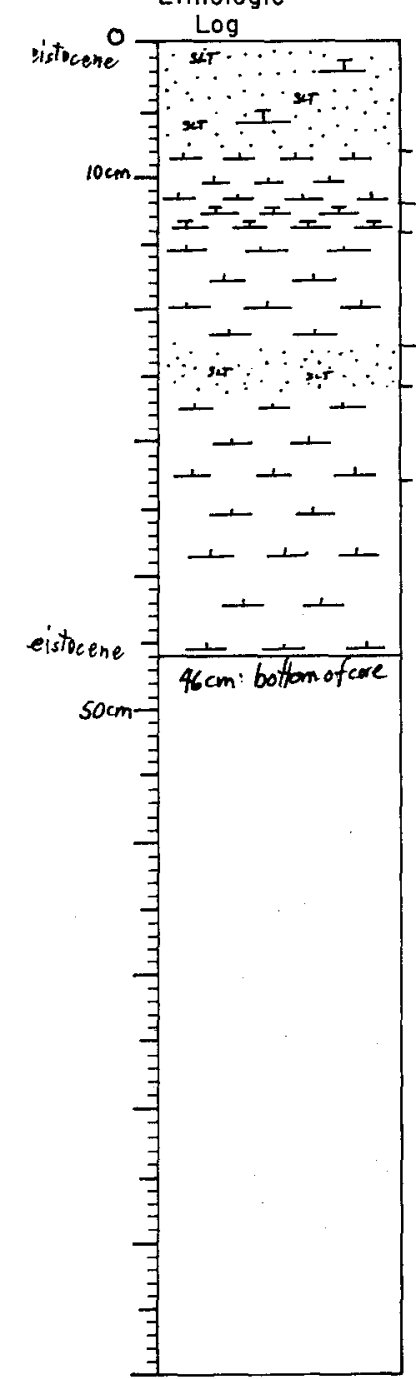

\section{Detailed Description}

$0-8$

DET SILT/SAND; FORAMS

OYR $7 / 2$ lt gray silty fine sand
$S$ irregular

8-12

CALC OOZE

10YR $7 / 3$ v. pale brown

indurated silty, $v$, fine sand

s,

FORAM SAND

LOYR $7 / 2$ 1t gray

$S$ irregular

14-23

CALC OOZE

.5YR $6 / 2$ pinkish gray

dry, cracked silty, $v$. fine sand

3-26 irregula

DET SAND

1OYR $7 / 2$ it gray

fine sand, forams commo

26-33,

10YR $6 / 3$ pale brown

ary, crumbled silty, v. fine sand

CALC OOZE

10YR $7 / 2$ it gray

dry, crumbled silty, fine sand, forams common end of core

NOTE: present condition is marginal for visual description. the above description is taken from a visual the above description is taken from a vis
CALC OOZE

SMEAR SLIDE DESCRIPTIONS - W.H.O.I. SEDIMENT CORES

Ship: Atlantis

Expedition 296

Leg No.
Core No. $2 \mathrm{GC}$

Station No. 2

Total Core Length 46 ESTIMATED ABUNDANCES (\%)

\begin{tabular}{|c|c|c|c|c|c|c|c|c|c|c|c|c|c|c|}
\hline \multirow[b]{4}{*}{ LEVEL } & \multirow[b]{4}{*}{$\begin{array}{c}\text { SEDIMENT } \\
\text { TYPE }\end{array}$} & \multicolumn{13}{|c|}{ ESTIMATED ABUNDANCES $(\%)$} \\
\hline & & \multirow{2}{*}{\multicolumn{4}{|c|}{$\begin{array}{l}\text { Inorganic Material } \\
\text { Silt \& Sand }\end{array}$}} & & \multirow{2}{*}{\multicolumn{5}{|c|}{$\begin{array}{l}\text { Biogenous Mater } \\
\text { Calcareous }\end{array}$}} & \multirow{2}{*}{\multicolumn{3}{|c|}{ Siliceous }} \\
\hline & & & & & & & & & & & & & & \\
\hline & & 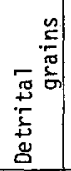 & 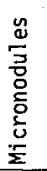 & 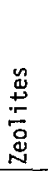 & 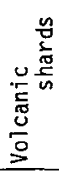 & $\frac{\pi}{\omega}$ & 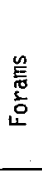 & 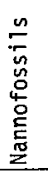 & 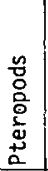 & 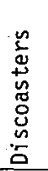 & $\frac{n}{2}$ & 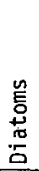 & 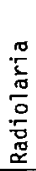 & 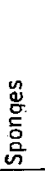 \\
\hline $1 \mathrm{~cm}$ & $\begin{array}{l}\text { det silt/ } \\
\text { sand; forams }\end{array}$ & 75 & & & & $\operatorname{tr}$ & 25 & $\operatorname{tr}$ & & & & & & \\
\hline $11 \mathrm{~cm}$ & calc ooze & 10 & 3 & & & 37 & 10 & 20 & & & 20 & & & tr \\
\hline & & & & & & & & & & & & & & \\
\hline & & & & & & & & & & & & & & \\
\hline & & & & & & & & & & & & & & \\
\hline & & & & & & & & & & & & & & \\
\hline & & & & & & & & & & & & & & \\
\hline & & & & & & & & & & & & & & \\
\hline & & & & & & & & & & & & & & \\
\hline & & & & & & & & & & & & & & \\
\hline & & & & & & & & & & & & & & \\
\hline & & & & & & & & & & & & & & \\
\hline & & & & & & & & & & & & & & \\
\hline & & & & & & & & & & & & & & .. \\
\hline & & & & & & & & & & & & & & \\
\hline & & & & & & & & & & & & & & \\
\hline & & & & & & & & & & & & & & \\
\hline & & & & & & & & & & & & & & \\
\hline
\end{tabular}


0250

VISUAL CORE DESCRIPTION

Page 1 of 1

Ship Atlantis Cruise 296 Leg_Sto. 3 Core No. 3 GC(He) Total Length 1 TAR $\mathrm{cm}$. Lot. $39^{\circ}$. Ol.5' N Long. $62^{\circ} 32.5^{\prime} \mathrm{W}^{\prime}$ Depth 5044 CoRR Core condition I JAR - hard, not oriented Date Described $1 / 1 / 1 / 73$ by JW Shaw

Physiographic location North of New England Seamount Chan Lithologic

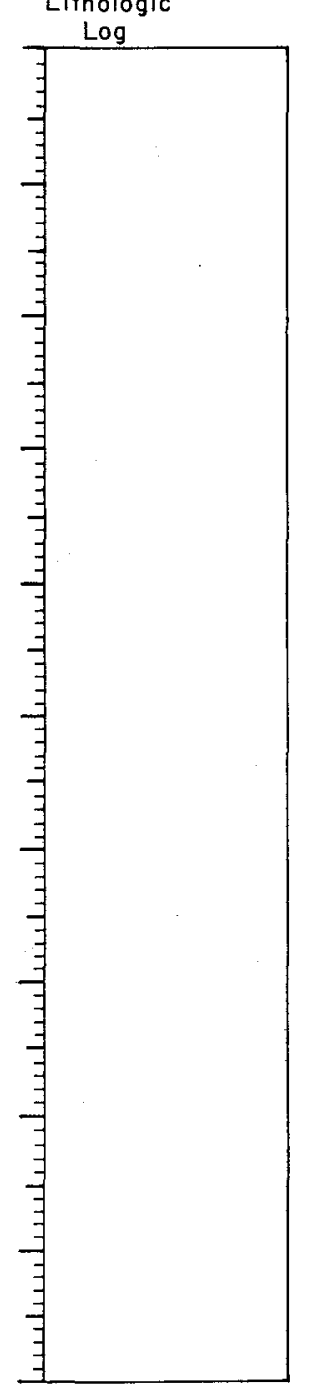

Detailed Description

Few grams of lutite and silt from catcher stored in 1 container.

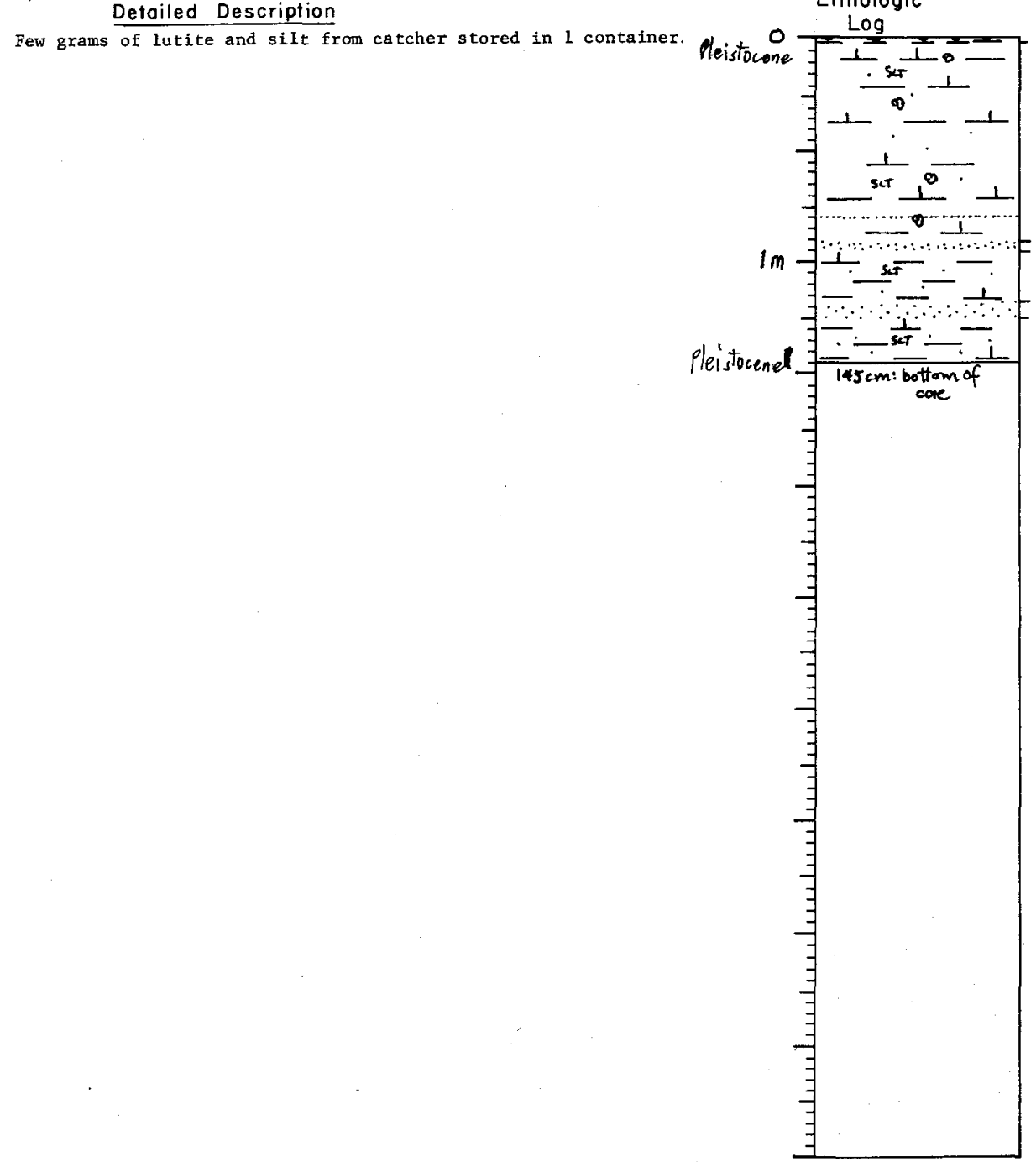

0251

VISUAL CORE DESCRIPTION

Page 1 of 1

Ship A Cruise 296 Leg__ Sta. 4 Core No. $4 \mathrm{GC}$ Total Length $145 \mathrm{~cm}$. Lat. $39^{\circ} 32.0^{\circ} \mathrm{N}$ Long. $65^{\circ} 49.5^{\circ} \mathrm{W}$ Depth $-4348 \mathrm{mcon}$. Core condition dy; fair Dote Described 13 MAY'74 by GHounTAin

Physiographic location Between Bactulus + Picket Seanounts

Lithologic
Detailed Description

Note; Description taken from one done sometime earlier. FORAM SAND

10YR $7 / 3$ v. pale brown

2-91 HLY CALC CLAY WITH DET $2.5 \mathrm{Y} 6 / 2$ 1t brownish when core was moist, scattered irregular mottles were probably present; now $v$. fain dry, crumbled silty lutite 91-95 DET SAND

$2.5 \mathrm{Y} 6 / 2$ it brownish gray when

SL CALC CLAY WITH DET SILT/SAND $2.5 \mathrm{Y} 5 / 2$ grayish brown interbedded thin lenses $(.1 \mathrm{~cm})$ of $v$. fine sand

DET SAND

(t) brownish gray

$125-145$

SL CALC CLAY WITH DET SILT/SAND

$2.5 Y 5 / 2$ grayish brown

列 $v$. fine sand end of core 
SMEAR SLIDE DESCRIPTIONS - W.H.O.I. SEDIMENT CORES

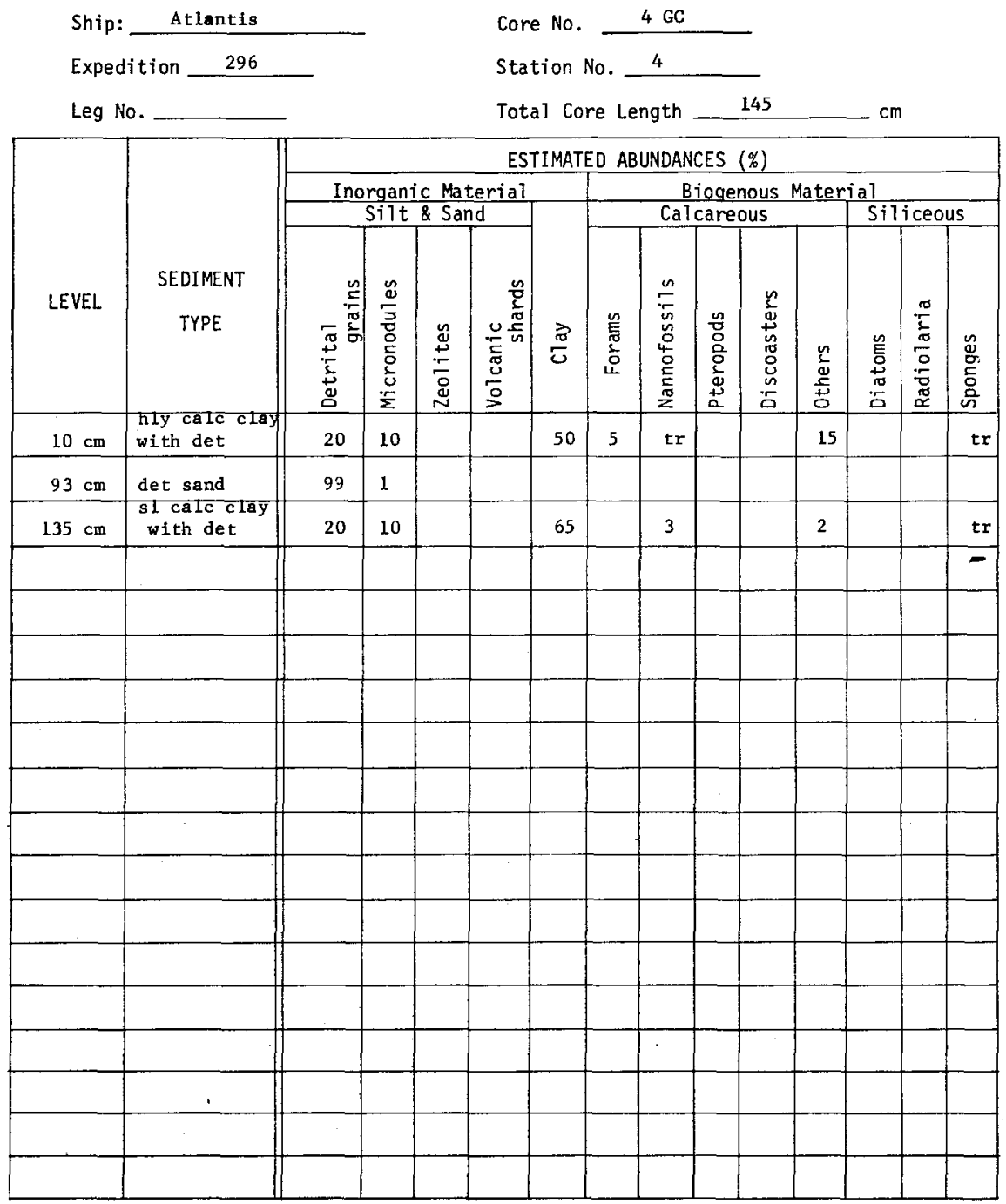

VISUAL CORE DESCRIPTION

Page 1 of 1

Ship A Cruise 2xo sta 5 Core No 5GC

Total Length $197 \mathrm{~cm}$. Lat. $39^{\circ} 09^{\prime} \mathrm{ON}$ Long. $65^{\circ} 54.0^{\circ}$ WDepth. 4616 meon. Core condition dry; fir Date Described $13 \pi(1) 74$ by Groontain Physiographic location 30 MILES SOUTH of PICKET SEANOONT

Lithologic

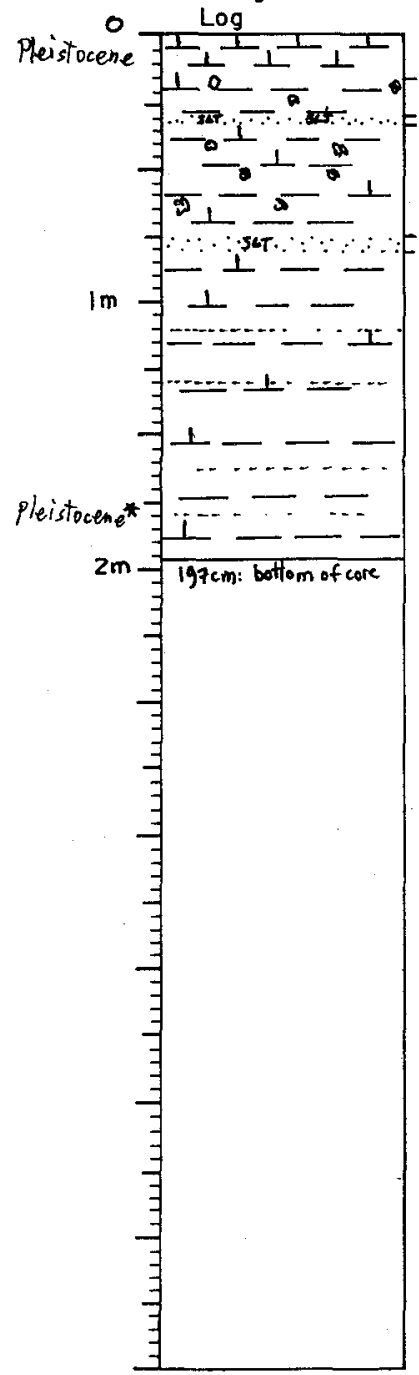

\section{Detailed Description}

$0-15$

CALC OOZE WITH DET

10YR $6 / 3$ pale brown

$S$ mottled

$15-2$

SL CALC CLAY WITH DET

IOYR $3 / 3 \mathrm{dk}$ brown

mottling, yellowish brown, scattered

in upper mottled areas

29-3 -33 inclined

DET SILT/SAND

LOYR $6 / 2$ it brownish gray

silty v. fine sand

Sincline

SL CALC CLAY WITH DET

1OYR $4 / 2$ dk grayish brown

extensive fine scale mottling, pale brown

dry, cracked silty lutite

74-77

DET SAND

$2.5 \mathrm{Y} 6 / 2$ it brownish gray

dry, v. fine sand

$\mathrm{S}$,
$77-197$

\section{SL CALC CLAY WITH DET}

grays brown grades to brown in indurated unit, $170-197 \mathrm{~cm}$

te gray coarse silt/v. fine sand interbedded lenses 100 $197 \mathrm{~cm}$ intensely lamed 
0254

SMEAR SLIDE DESCRIPTIONS - W.H.O.I. SEDIMENT CORES

Ship: Atlantis

Expedition 296

Leg No.

Core No. $5 \mathrm{GC}$

\begin{tabular}{|c|c|c|c|c|c|c|c|c|c|c|c|c|c|c|}
\hline \multirow[b]{4}{*}{ LEVEL } & \multirow[b]{4}{*}{$\begin{array}{c}\text { SEDIMENT } \\
\text { TYPE }\end{array}$} & \multicolumn{13}{|c|}{ ESTIMATED ABUNDANCES (\%) } \\
\hline & & \multirow{2}{*}{\multicolumn{4}{|c|}{$\begin{array}{l}\text { Inorganic Material } \\
\text { Silt \& Sand }\end{array}$}} & \multirow[b]{3}{*}{$\frac{\pi}{\omega}$} & \multirow{2}{*}{\multicolumn{5}{|c|}{$\frac{\text { Biogenous Mater }}{\text { Calcareous }}$}} & & & \\
\hline & & & & & & & & & & & & \multicolumn{3}{|c|}{ Siliceous } \\
\hline & & " & 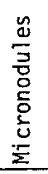 & 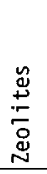 & 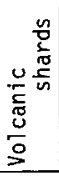 & & 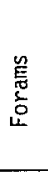 & 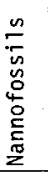 & 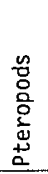 & 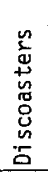 & 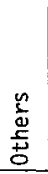 & 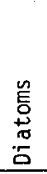 & 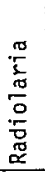 & 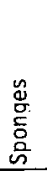 \\
\hline $1 \mathrm{~cm}$ & $\begin{array}{l}\text { calc ooze } \\
\text { with det }\end{array}$ & 25 & 3 & & & 17 & 40 & 15 & & & & tr & & $\operatorname{tr}$ \\
\hline $120 \mathrm{~cm}$ & $\begin{array}{l}\text { si calc clay } \\
\text { with det }\end{array}$ & 20 & 3 & & & 65 & & $\operatorname{tr}$ & & & 5 & tr & & tr \\
\hline $130 \mathrm{~cm}$ & det silt/sand & 79 & 1 & & 5 & 10 & & $\mathrm{tr}$ & & & tr & & & $\mathrm{tr}$ \\
\hline $190 \mathrm{~cm}$ & \begin{tabular}{|c|} 
s1 calc clay \\
with det
\end{tabular} & 15 & 3 & & $\operatorname{tr}$ & 70 & & 2 & & & 3 & & & tr \\
\hline & & & & & & & & & & & & & & \\
\hline & & & & & & & & & & & & & & \\
\hline & & & & & & & & & & & & & & \\
\hline & & & & & & & & & & & & & & \\
\hline & & & & & & & & & & & & & & \\
\hline & & & & & & & & & & & & & & \\
\hline & & & & & & & & & & & & & & \\
\hline & & & & & & & & & & & & & & \\
\hline & & & & & & & & & & & & & & \\
\hline & & & & & & & & & & & & & & \\
\hline & & & & & & & & & & & & & & \\
\hline & & & & & & & & & & & & & & \\
\hline & & & & & & & & & & & & & & \\
\hline & & & & & & & & & & & & & & \\
\hline
\end{tabular}

0255

VISUAL CORE DESCRIPTION

Page 1 of 1

Ship A Cruise 296 Leg_o Sto. 6 Core No. GGC Total Length $187 \mathrm{~cm}$. Lat. $39^{\circ} 33,0^{\prime} \mathrm{N}$ Long. $66^{\circ} 17.0^{\prime} \mathrm{W}$ Depth 4340 mcer Core condition dey foir Date Described 14MAr 74 by GMountaw Physiogrophic location SW OF PICKET SEAMOUNT

Lithologic

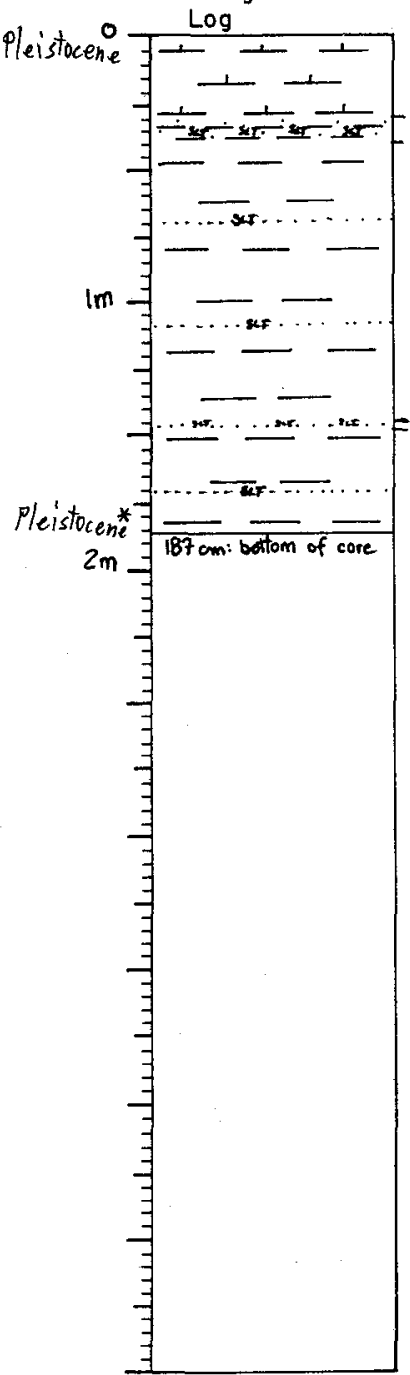

\section{Detailed Description} CALC OOZE silty lutite, gray forams $S$ irregular

29-39 UNFOSS CLAY/DET IOYR $6 / 4$ it yellowish brown silty lutite
S irregular

$39-145$ UNFOSS CLAY 10YR $5 / 21$ t brownish gray hard, cracked silty lutite v. finely lam, with lenses of it gray, reddish brown Lams 80-100 cD

S convex upward

145-147 DET SAND DET SAND
1OYR $5 / 2$ it brownish gray $\checkmark$ fine sand

UNFOS

$2.5 \mathrm{Y} 5 / 2$ grayish brown

hard silty lutite

extensively lam with $1 \mathrm{t}$ brownish gray, $v$ fine sand/sil end of cor
$S$ convex upwa
147-187 


\section{0}

SMEAR SLIDE DESCRIPTIONS - W.H.O.I. SEDIMENT CORES

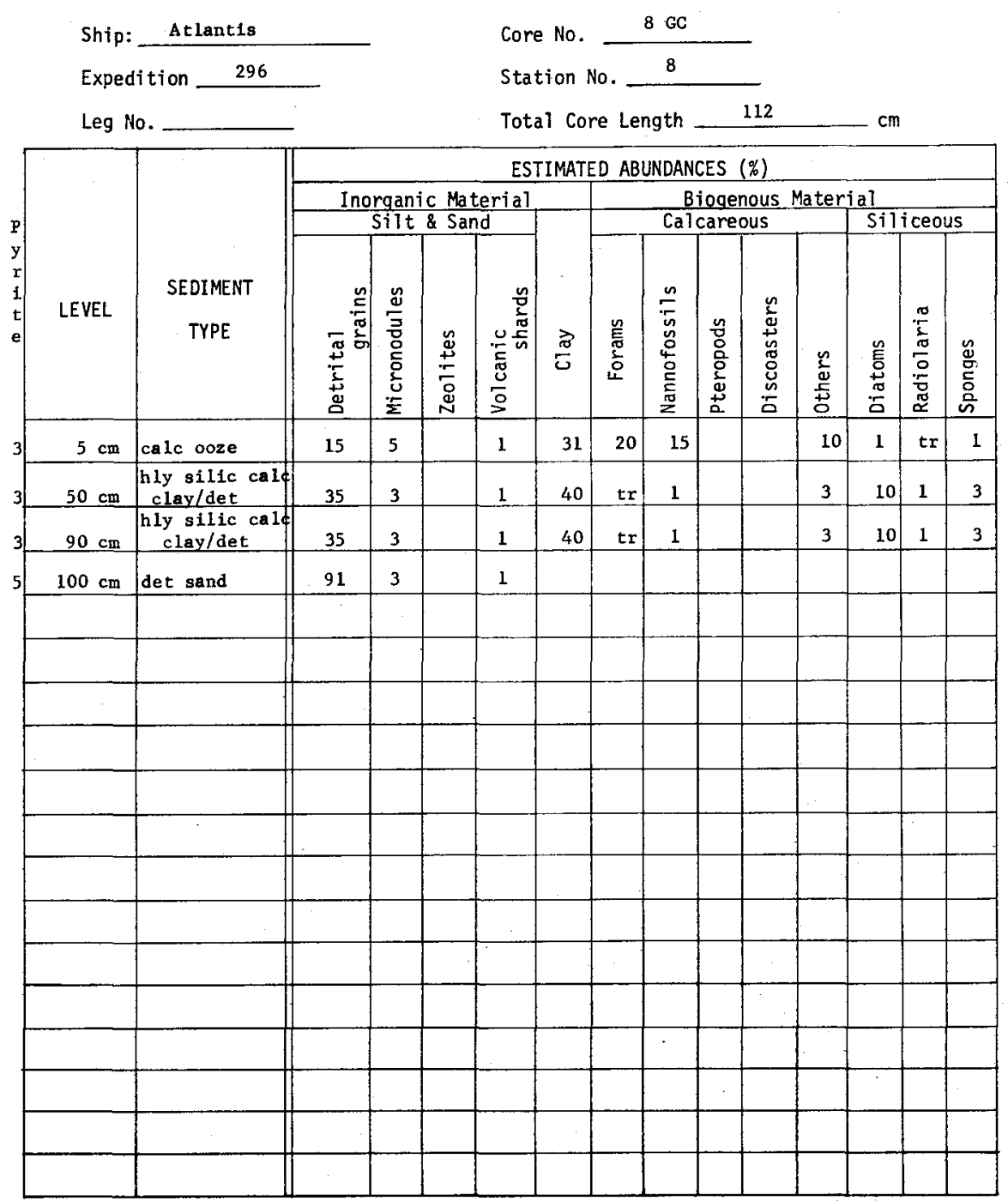

0261

VISUAL CORE DESCRIPTION

Page 1 of 1

Ship A Cruise 296 Leg_Sta. 9 Core No. gGe Tatal Length $180 \mathrm{~cm}$. Lat. $39^{\circ} 46.0^{\circ} \mathrm{N}$ Long. $66^{\circ} 28.0^{\circ} \mathrm{W}$ Depth $3940 \mathrm{mcar}$ Cory fair $15 \mathrm{MAr} 74$ by Gllountain Core condition $\frac{d r y}{\text { Physiographic location 5-10 MLES SW OF RETREIUER SEAMUUNT }}$

Lithologic

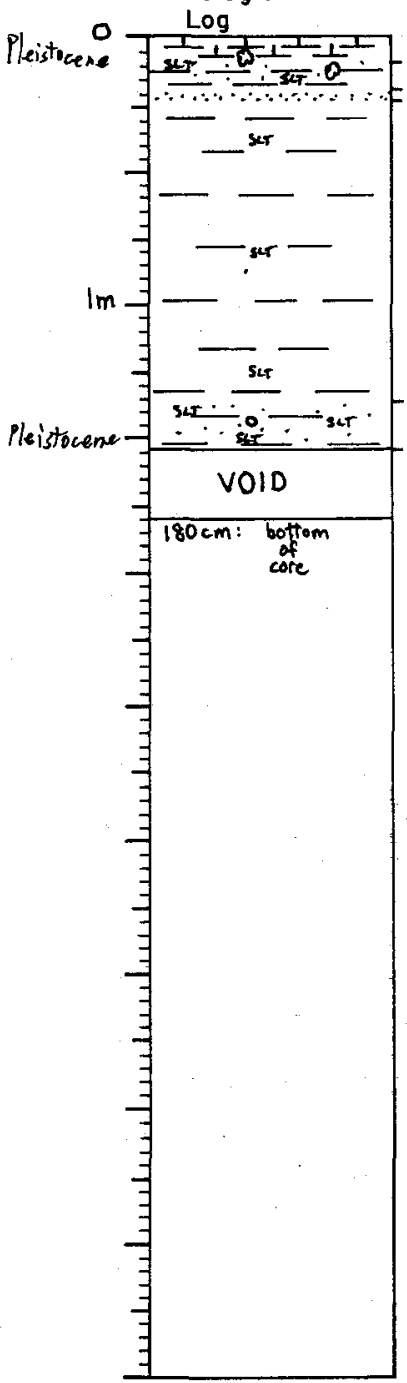

\section{CALC OOZE}

1OYR $6 / 21 \mathrm{t}$ brownish gray dry, silty lutite, v, abunt. forams $S$ irregular

9-19 dry silt

19-23

DET SAND

10YR $6 / 2$ It brownish gray

$s$ irregular

23-136

UNFOSS CLAY WITH DE

LOYR 6/2 It brownish gray

layers of reddish brown lutite, esp. $50-80 \mathrm{~cm}$ S irregular

136-154

UNFOSS CLAY/DET

dry

(thinor amounts of coarse sand and pebbles (ice rafted?)

154-180

end of core

\section{Detailed Description}

UNFOSS CIAY/DET

scattered mottling, grayish brown and brown 


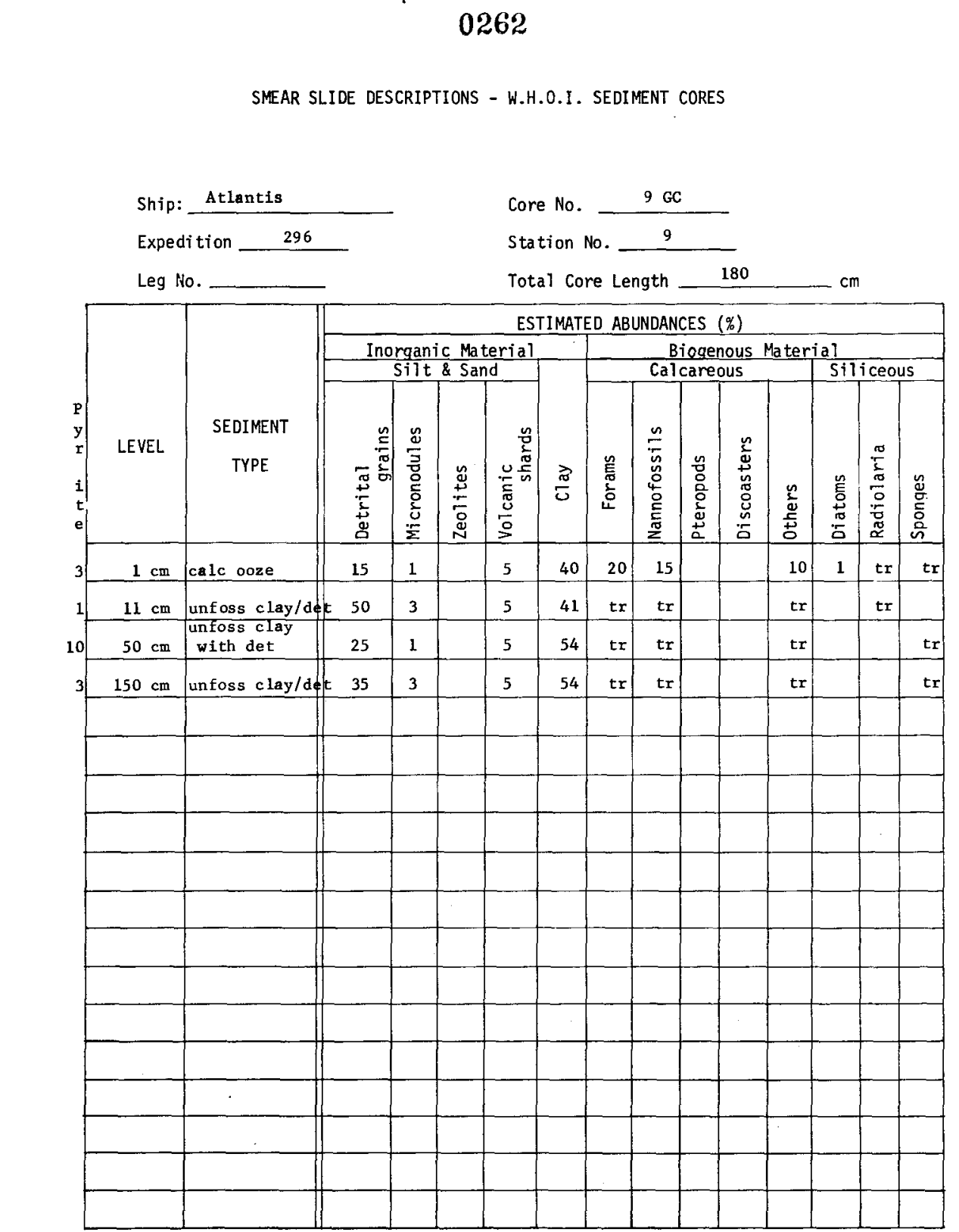




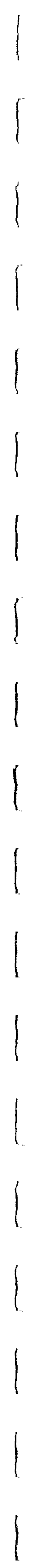




\section{4}

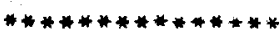

$* * * * * * * * * * * * * *$
STATIEN DATA RETRIEVAL DATE: $17: 43$ JAN 19,175

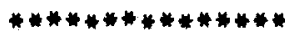

$* * * * * * * * * * * * * *$
PAGE 1 OF

* *WHOI**

MARS. CORE GR LENGTH DREDGE PHYSIO. ROCK

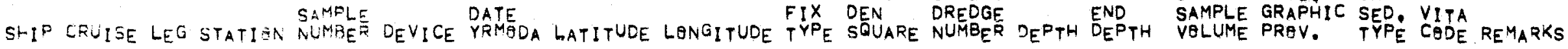

\begin{tabular}{|c|c|c|c|c|c|c|c|c|c|c|c|c|c|c|c|c|c|}
\hline $\begin{array}{l}\text { ATL } \\
\text { ATL } \\
\text { ATL } \\
\text { ATL } \\
\text { ATL } \\
\text { ATL } \\
\text { ATL }\end{array}$ & $\begin{array}{l}297 \\
297 \\
297 \\
297 \\
297 \\
297 \\
297\end{array}$ & $\begin{array}{l}1 \\
1 \\
1 \\
1 \\
1 \\
1 \\
1\end{array}$ & $\begin{array}{l}6369 \\
6371 \\
6372 \\
6375 \\
6377 \\
6380 \\
6381\end{array}$ & $\begin{array}{l}0000 \\
0200 \\
0000 \\
0000 \\
0000 \\
0000 \\
0000\end{array}$ & $\begin{array}{l}13 \\
13 \\
13 \\
13 \\
13 \\
13 \\
13\end{array}$ & $\begin{array}{l}63 \\
63 \\
63 \\
63 \\
63 \\
63 \\
63\end{array}$ & $\begin{array}{l}818 \\
818 \\
818 \\
819 \\
819 \\
823 \\
824\end{array}$ & $\begin{array}{l}36 \cdot 400^{N} \\
37 \cdot 100^{N} \\
37 \cdot 155 \mathrm{~N} \\
36 \cdot 505 \mathrm{~N} \\
36 \cdot 470^{N} \\
37 \cdot 250^{N} \\
37 \cdot 410^{N}\end{array}$ & $\begin{array}{l}70 \cdot 110 \mathrm{~W} \\
70 \cdot 250 \mathrm{w} \\
70 \cdot 070 \mathrm{w} \\
69 \cdot 360 \mathrm{w} \\
69 \cdot 530 \mathrm{~W} \\
69 \cdot 28 \mathrm{~W} \\
69 \cdot 335 \mathrm{~W}\end{array}$ & $\begin{array}{l}0 \\
0 \\
0 \\
0 \\
0 \\
0 \\
0\end{array}$ & $\begin{array}{l}116.60 \\
116.70 \\
116.70 \\
115.69 \\
115.69 \\
115.79 \\
115.79\end{array}$ & $\begin{array}{l}0001 \\
0002 \\
0003 \\
0005 \\
0006 \\
000^{7} \\
000^{8}\end{array}$ & $\begin{array}{l}4418^{\circ} \\
42610^{\circ} \\
4243^{\circ} \\
4396^{\circ} \\
4401^{\circ} \\
4270^{\circ} \\
4110^{\circ}\end{array}$ & $\begin{array}{l}1110^{\circ} \\
40 \\
210^{\circ} \\
58 \\
60 \\
39 \\
117\end{array}$ & $\begin{array}{l}0000 \\
0000 \\
0000 \\
0000 \\
0000 \\
0000 \\
0000\end{array}$ & $\begin{array}{l}2 \\
2 \\
2 \\
2 \\
2 \\
2 \\
2\end{array}$ & $\begin{array}{l}3839 \\
4459 \\
3359 \\
4459 \\
4459 \\
3359 \\
3359\end{array}$ \\
\hline
\end{tabular}

THERE WERE 7 ITEMS THAT MET YQUR REQUIREMENTS.

THANK YGU FER USING PRGGRAM MUDDIE.

-STEP* THAT IS ALL FGR NGW

RUN 
0265

VISUAL CORE DESCRIPTION

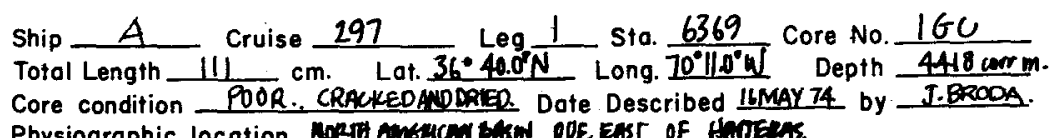

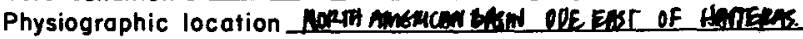

Lithologic

Meitacene $\frac{\log }{-1-}$

Log

更 1

$25 \exists+$ sex.

$1+1+$

$\exists+\longrightarrow$

so

$+\frac{1}{1}+$

I $1+1$

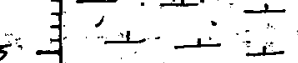

-

$\exists \frac{1}{1} \frac{1}{2} \frac{1}{2}$

$100 \mathrm{~F} \div 30, \cdots$

Heistocene $1+\perp$

1257 end of cone.

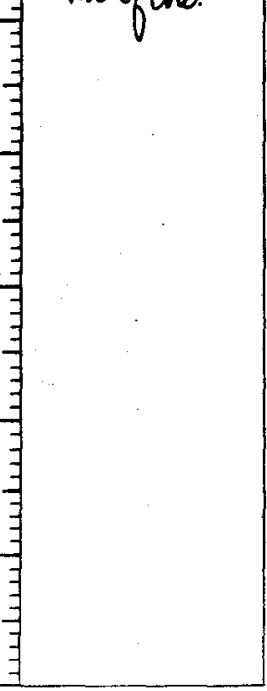

$0-111$

\section{Detailed Description}

\section{CALC OOZE/DET}

10YR $6 / 2$ it olive gray

tayers, $20-22 \mathrm{~cm}, 93-95 \mathrm{~cm}, 82-84 \mathrm{~cm}$ end of core 2
0266

SMEAR SLIDE DESCRIPTIONS - Y.H.O.I. SEDIMENT CORES

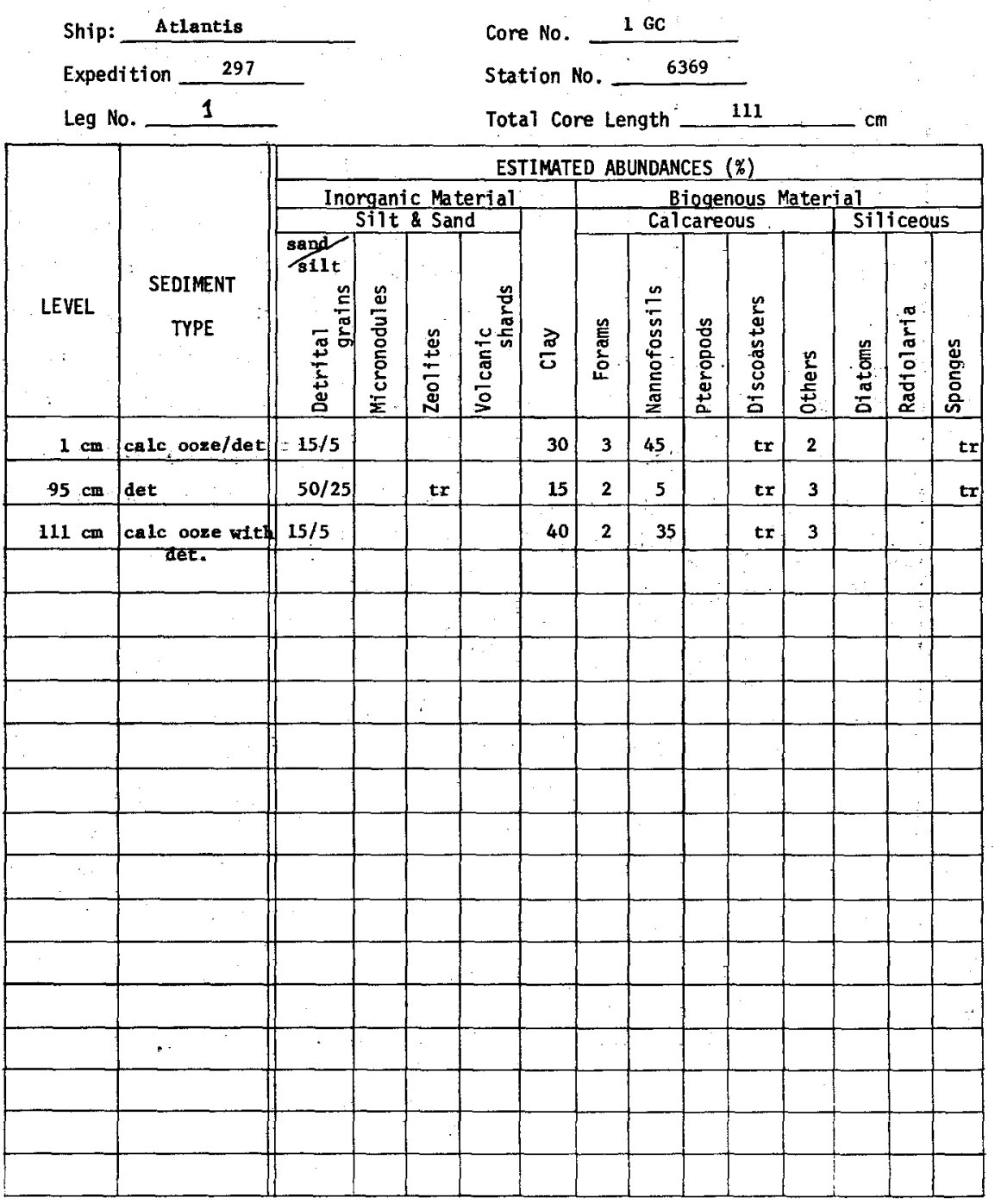


VISUAL CORE DESCRIPTION

Ship A Cruise 297 Leg 1 sta. 6371 Core No. 2GC Total Length to $\mathrm{cm}$. Lat. $37^{\circ} 10.0^{\circ} \mathrm{N}$ Long. $70^{\circ} 25.0^{\prime} \mathrm{W}$ Depth 4261 cokr Core condition POOR... CRACKEO. DRIED. Date Described 14Max Z4 by I Srede Physiographic location N.AMERICAN BASIN.

Lithologic

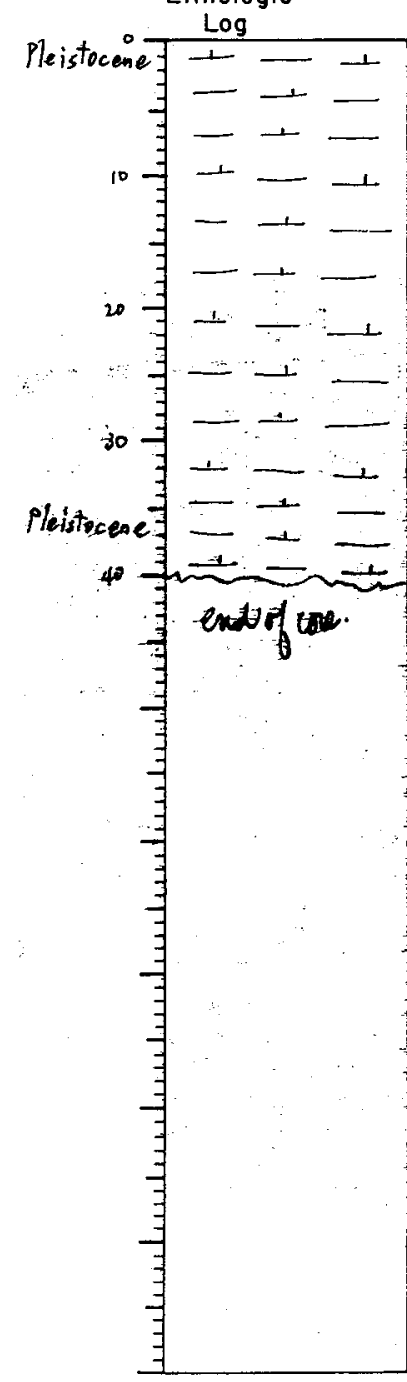

0267

Detailed Description

\section{8}

SMEAR SLIDE DESCRIPTIONS - W.H.O.I. SEDIMENT CORES

$0-40$

HLY CALC CLAY

abunt forams in dry compact lutite

end of core

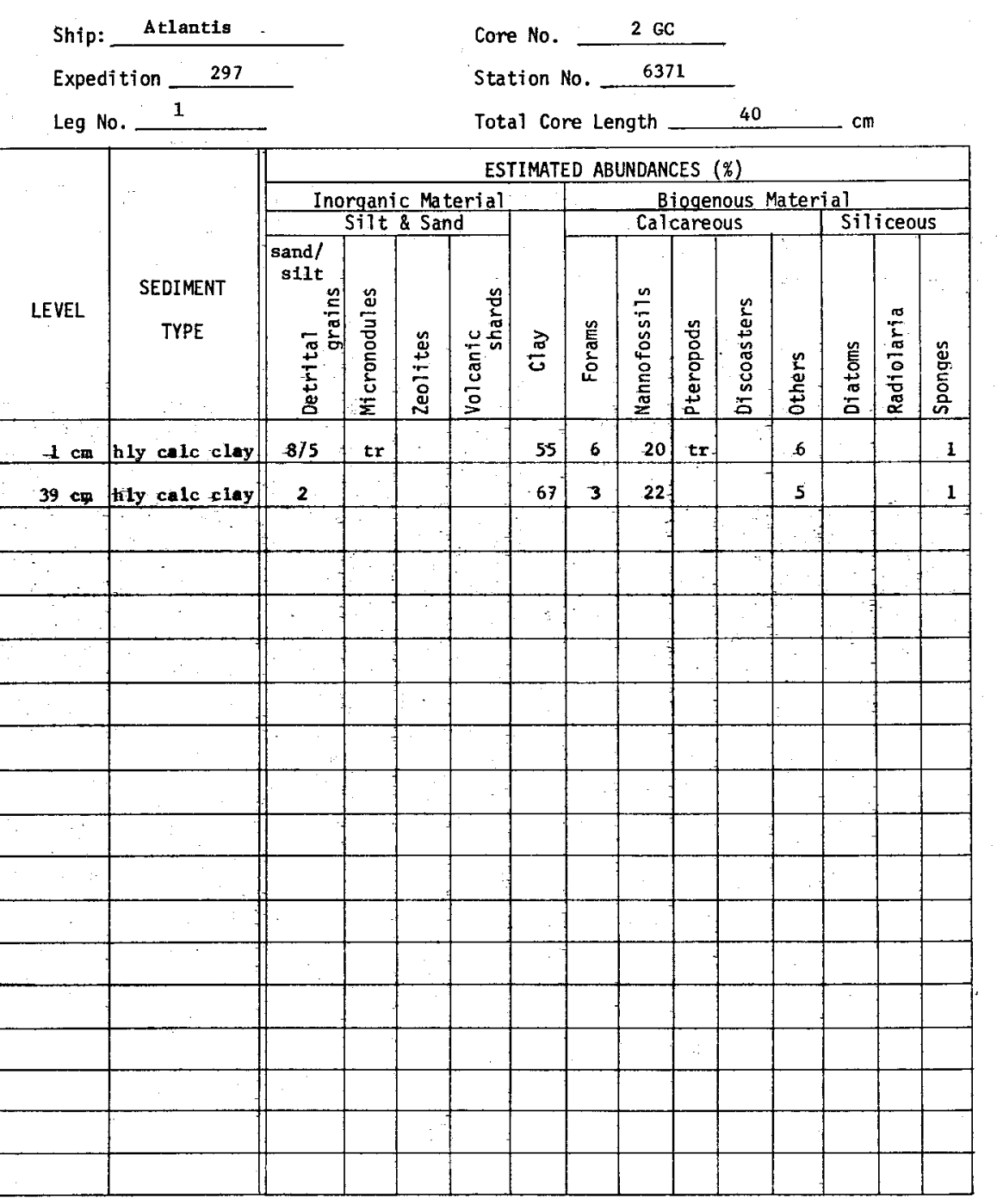




\section{4}

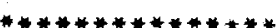

$* * * * * * * * * * * * * *$
STATION DATA RETRIEVAL OATE: $17: 43$ JAN 19,175

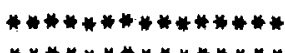

$* * * * * * * * * * * * * * *$
PAGE 1 OF 1

**WHOI**

\section{MARS. CORE $A R$ LENGTH DREDGE PHYSIO, ROCK}

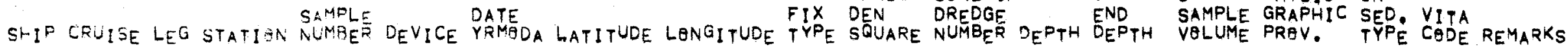

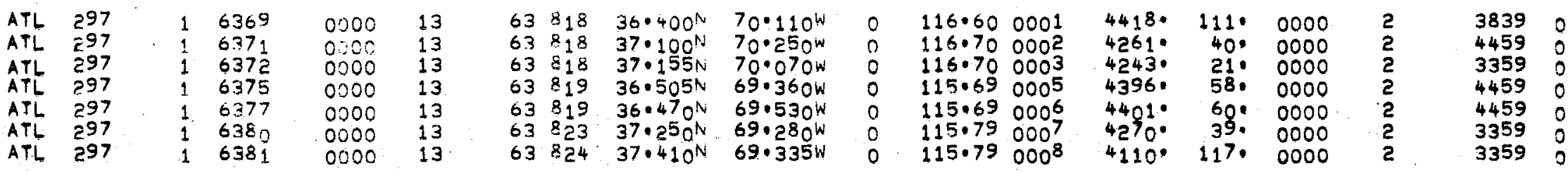

THERE WERE 7 ITEMS THAT MET YQUR REQUIREMENTS.

THANK YOU FER USING PRGGRAM MUDDIE.

*STEP* THAT IS ALL FGR NGH

RUN 
0265

VISUAL CORE DESCRIPTION

Page 1 of 1

Ship $A$ Cruise 297 Leg $\frac{1}{1}$ Sta. 6369 Core No. 160

Total Length $11 \mathrm{~L} \mathrm{~cm}$. Lat. $36^{\circ} 40.0^{\circ} \mathrm{N}$ Long. $70^{\circ} 110^{\circ} \mathrm{W}$ Depth $4418 \mathrm{corm}$ Core condition POOR. CRACKEDANDDRIED. Date Described ILMAY 74 by I. PROOA.

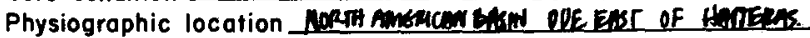

Lithologic

Detailed Description

$0-111$

CALC OOZE/DET

(OTh $6 / 2$ 1t ollve gray

silty layers, $20-22 \mathrm{~cm}, 93-95 \mathrm{~cm}, 82-84 \mathrm{~cm}$

end of cor
0266

SMEAR SLIDE DESCRIPTIONS - W.H.O.I. SEDIMENT CORES




0267

VISUAL CORE DESCRIPTION

Page 1 of 1

Ship A Cruise 297 Leg 1 sta. 6371 Core No. 2GC Total Length to $\mathrm{cm}$. Lat. 37\%10.0 N Long. $70^{\circ} 25.0^{\prime} \mathrm{W}$ Depth 4261 conR Core condition POOR... CRACEEO. DRIED. Date Described $16 M_{4 y} 74$ by I Prede Physiographic location N.AMERICANal BACIN.

Lithologic

Pleistecenet Log

$\stackrel{\log }{-1}-1$

百- - -

$10 \exists+\ldots$

手 - -

$\exists-\longrightarrow-$

$207+\div$

A $-\cdots-1$

so $-\div$

$\exists+\cdots$

Pleistocene $\square+1$

$40 \div=$

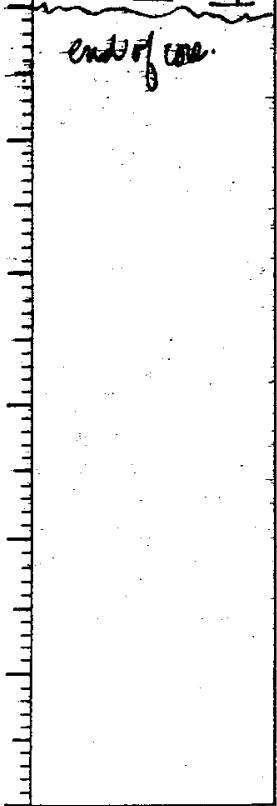

Detailed Description

$0-40$

HLY CALC CLAY

abunt. forams, in dry compact lutite

end of core
0268

SMEAR SLIDE DESCRIPTIONS - W.H.O.I. SEDIMENT CORES

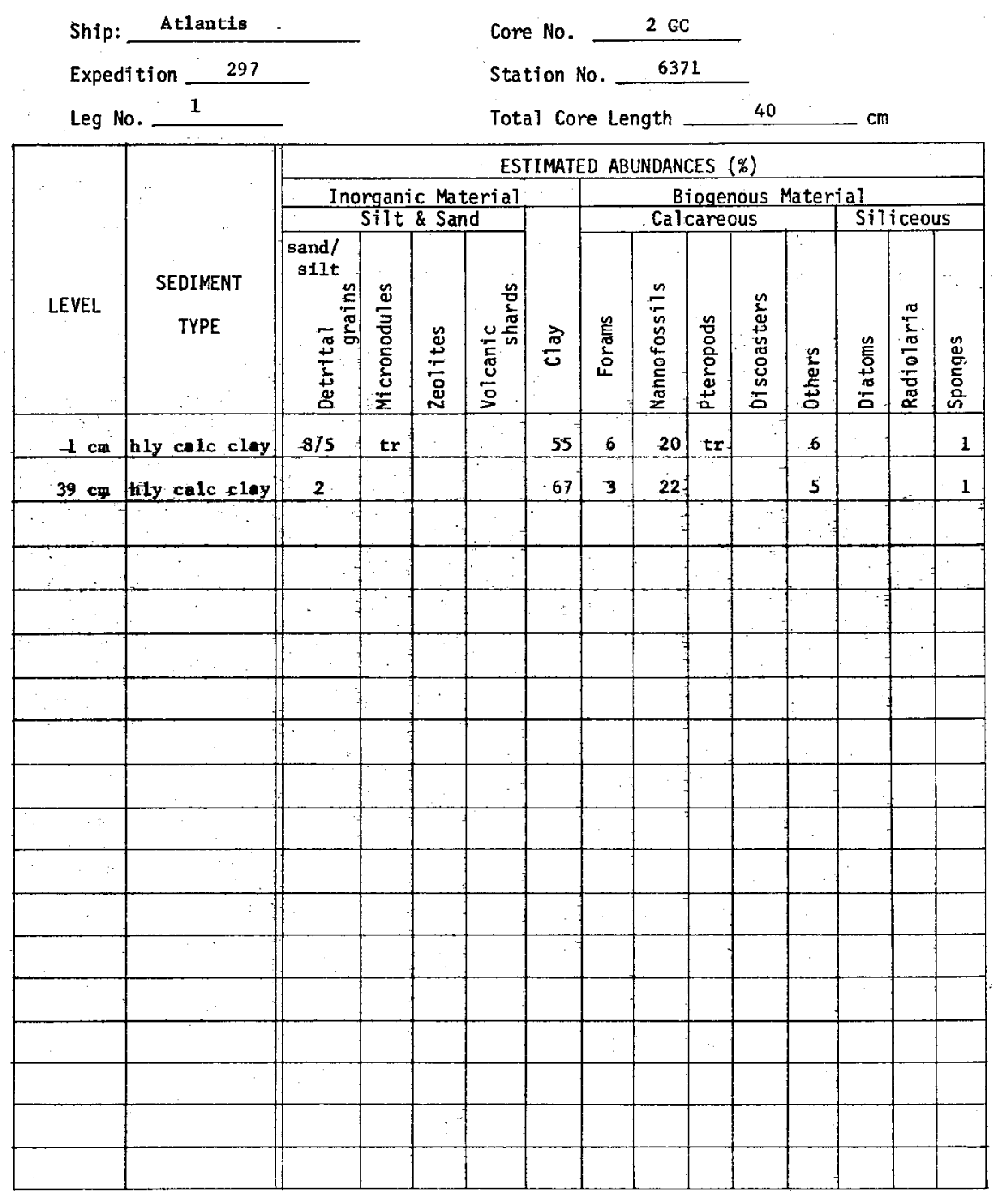


0269

VISUAL CORE DESCRIPTION

Poge 1 of 1

Ship A Cruise 297 sto. 6372 Core No. $36 \mathrm{CC}$ Total Length 21 Lat. 37.15.5' N Long. $70^{\circ} 07.0^{\circ} \mathrm{W}$ Depth 4243 corr $\mathrm{m}$

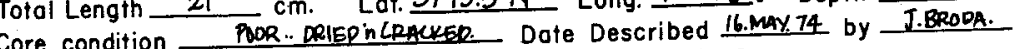
Physiogrophic location N.AMERICAN BASIN .. OFF HATTERAS.

Lithologic

- $\frac{\log }{1+}$

L $1-1$

$\exists+1$

$10 \exists+1,+$

- $1+1$

$+1$

pleistrenenc $1-1 \div$

25

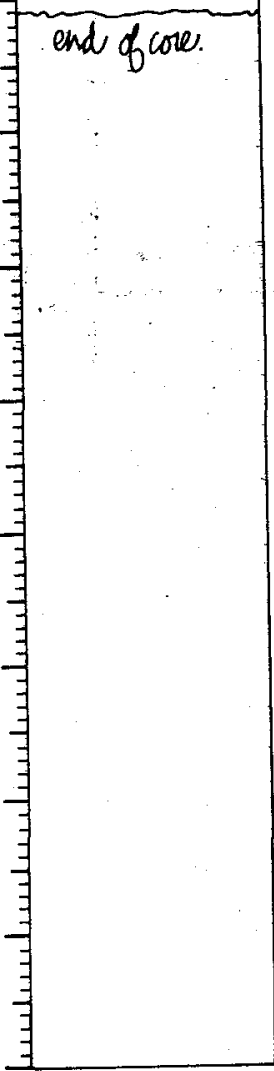

\section{Detailed Description}

0-21

CALC OOZE

IOYR $7 / 1$ 1t gray

end of core
0270

SMEAR SLIDE DESCRIPTIONS - W.H.O.I. SEDIMENT CORES

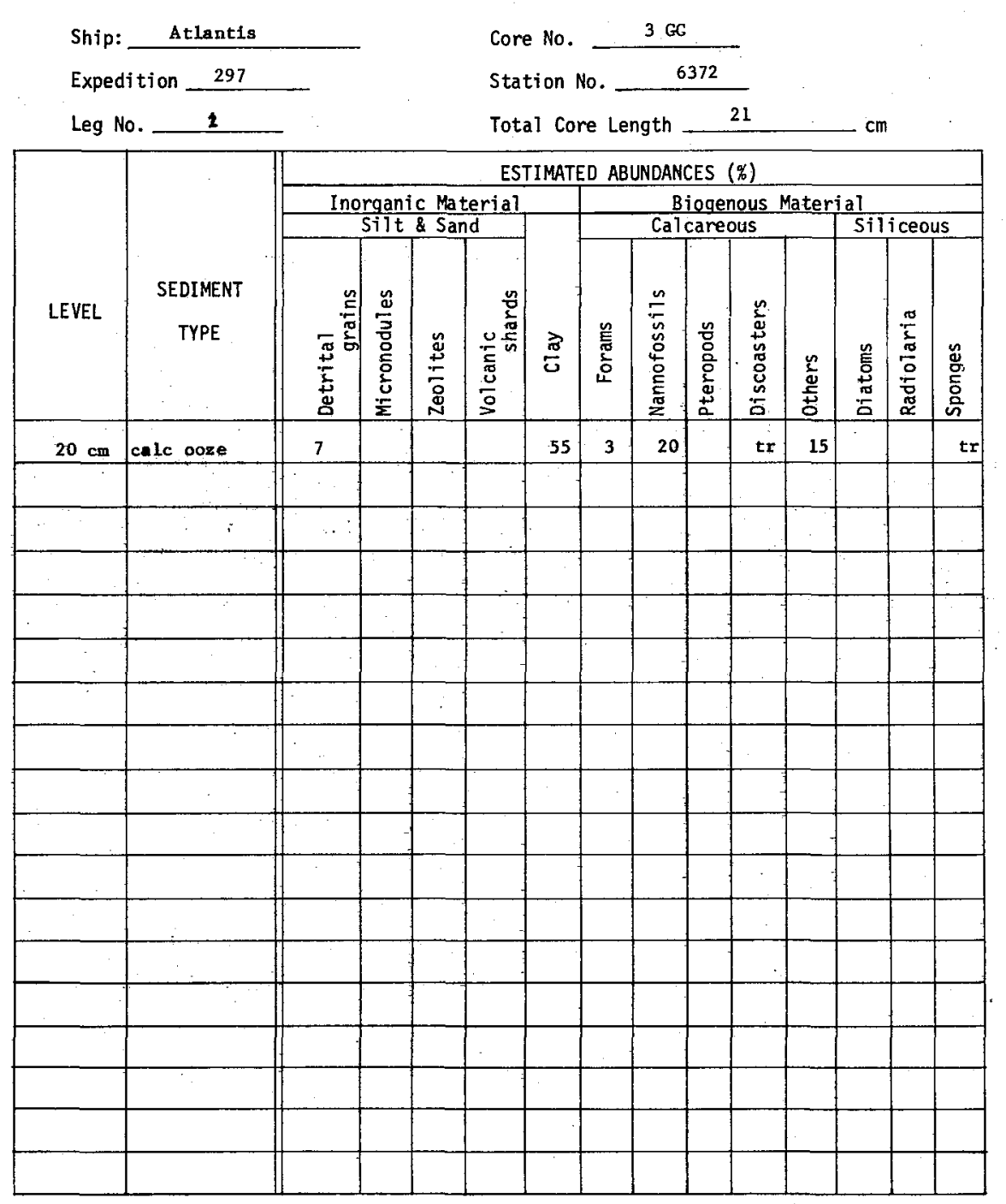




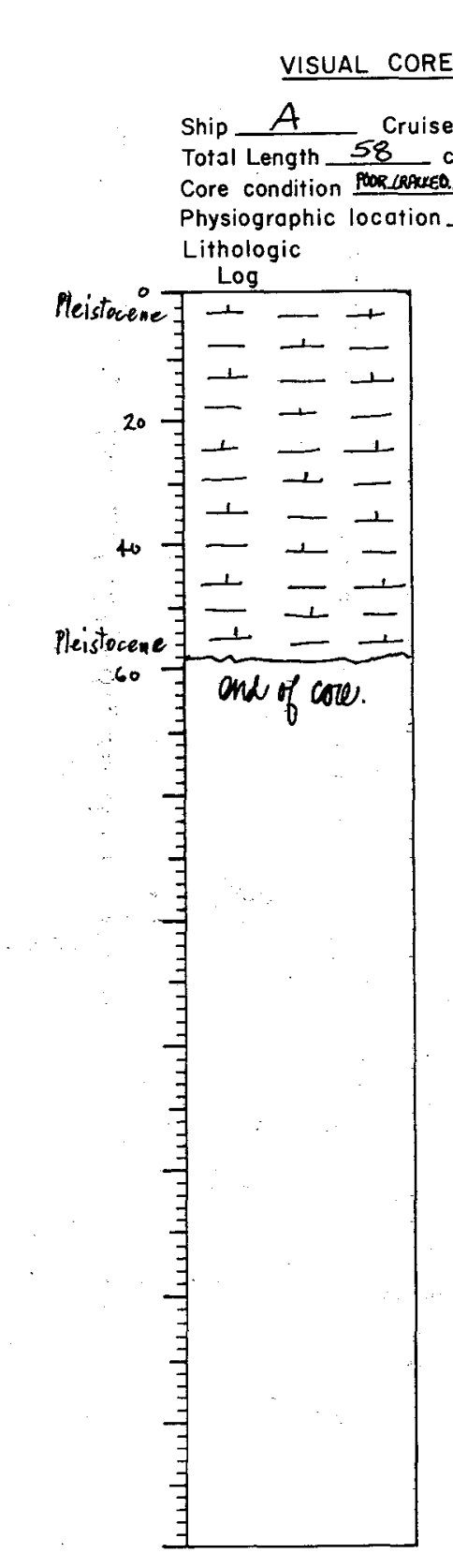

Page _ _ 1 of 1 6375 Core No. $56 C$ 4396 corm Date Described I7MAY 74 by J. BRopa. North Ammican Basin Detailed Description

$0-58$ HLY CALC CIAY $2.5 \mathrm{y} 7 / 21 \mathrm{t}$ gray dry cracked 1 end of core

\section{2}

SMEAR SLIDE DESCRIPTIONS - W.H.O.I. SEDIMENT CORES

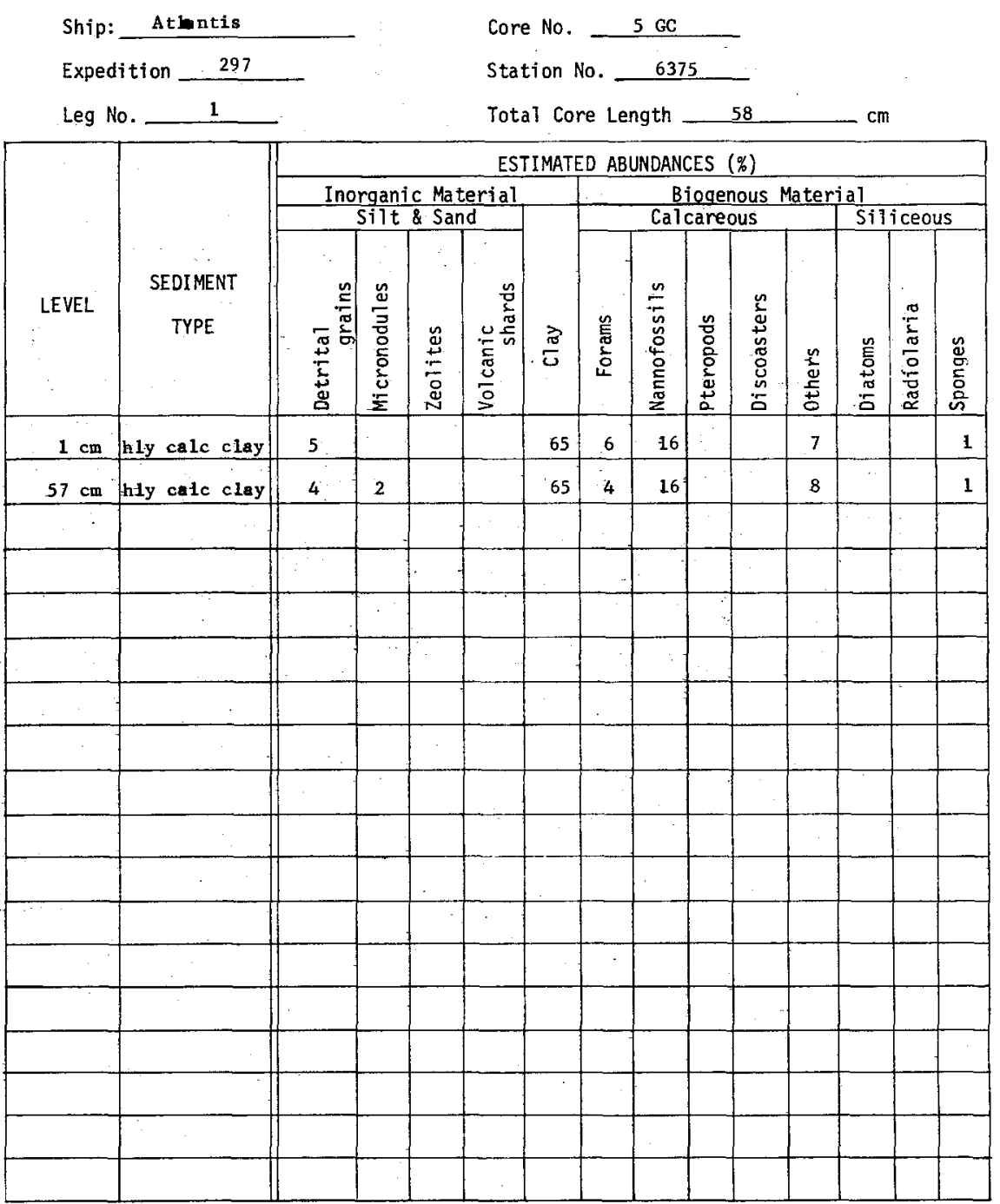


